Nevada.

Environmental

Restoration

Project

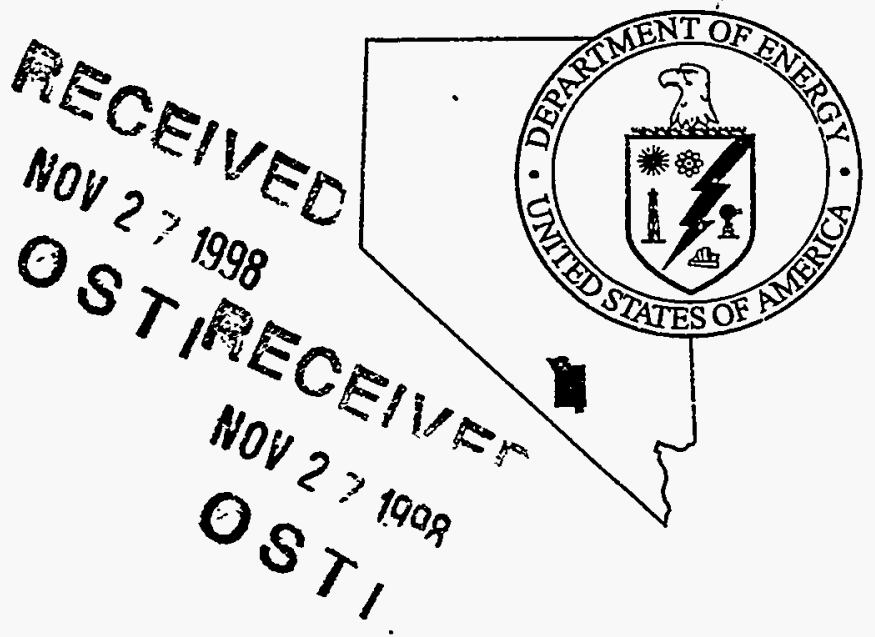

\title{
Closure Report for CAU 93: Area 6 Steam Cleaning Effluent Ponds Nevada Test Site
}

\section{Volume II}

Controlled Copy No UNCONTROLR FP COPY

Revision: 0

November 1997

\section{Environmental Restoration}

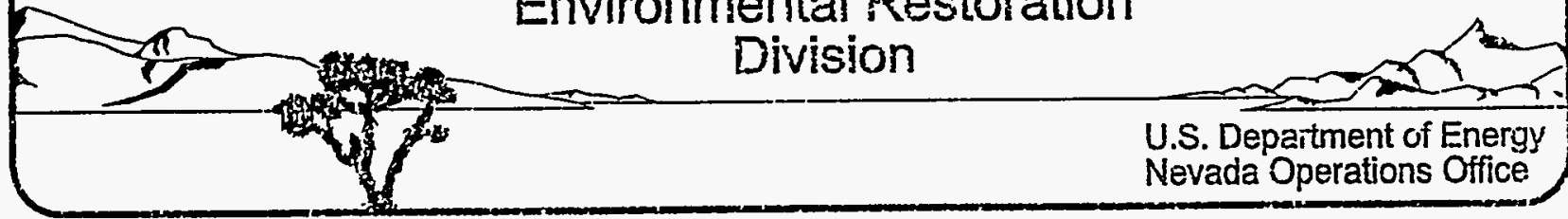


This report has been reproduced from the best available copy.

DOE and DOE contractors can obtain copies of this report from the Office of Scientific and Technical Information, P.O. Box 62, Oak Ridge, TN 37831, (423) 576-8401.

This report is publicly available from the National Technical Information Service, U. S. Department of Commerce, 5285 Port Royal Road, Springfield, VA, 22161, (703) 487-4650. 


\section{APPENDIX B}

\section{DATA SUMMARY SHEETS}

\section{DISCLAIMER}

This report was prepared as an account of work sponsored by an agency of the United States Government. Neither the United States Government nor any agency thereof, nor any of their empleyees, makes any warranty, express or implied, or assumes any legal liability or responsibility for the accuracy, completeness, or usefulness of any information, apparatus, product, or process disclosed, or represents that its use would not infringe privately owned rights. Reference herein to any specific commercial product, process, or service by trade name, trademark. manufacturer, or otherwise does not necessarily constitute or imply its endorsement, recommendation, or favoring by the United States Government or any agency thereof. The views and opinions of authors expressed herein do not necessarily state or reflect those of the United States Government or any agency thereof. 


\section{DISCLAIMER}

Portions of this document may be illegible in electronic image products. Images are produced from the best available original document. 
Appendix B provides all of the laboratory summary data sheets, this information is not provided within Volume I of the report. The laboratory data summary sheets have not been included in Volume I. This information is provided in Volume II of the Closure Report for CAU 93: Area 6 Steam Cleaning Effluent Ponds, Nevada Test Site and is available upon request.

Appendix B is composed of all of the laboratory data summary sheets for the Area 6 SECPs. closure activities. Appendix $B$ has been organized so that all of the analytical data for the closure of each component of CAU 93 are grouped together. At the start of each section a summary table has been provided. If specific $Q A / Q C$ information is required, the complete analytical data packages are available upon request through the DOE/NV Environmental Restoration Division. 


\section{VOLUME 2 APPENDIX B}

TABLE 1 ANALYTICAL DATA 
TABLE 1: SUMMARY OF NORTH SCEP PIPELINE VERIFICATION RESULTS

\begin{tabular}{|c|c|c|c|c|c|c|c|}
\hline (5) & 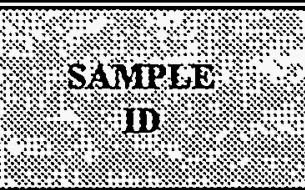 & $\begin{array}{l}\text { SAMPLE } \\
\text { oEPTH } \\
\text { (am fuches) }\end{array}$ & 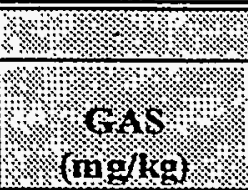 & 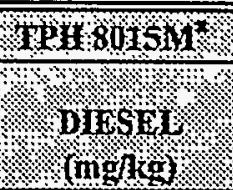 & (6.) & 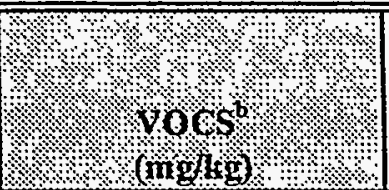 & 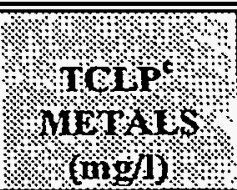 \\
\hline \multicolumn{8}{|c|}{ BUILDING 6-623 STEAM CLEANING PAD SAMPLES COLLECTED JULY 17, 1997} \\
\hline $\begin{array}{l}\text { Collected Below } \\
\text { Pipe Joint }\end{array}$ & NSCEP-SP-001 & $\begin{array}{c}0-15.2 \\
{[0-6]}\end{array}$ & ND & ND & ND & NA & NA \\
\hline $\begin{array}{l}\text { Collected Below } \\
\text { Pipe Joint }\end{array}$ & NSCEP-SP-002 & $\begin{array}{c}0-15.2 \\
{[0-6]}\end{array}$ & ND & ND. & ND & NA & NA \\
\hline $\begin{array}{l}\text { Collected Below } \\
\text { Pipe Joint }\end{array}$ & $\begin{array}{l}\text { NSCEP-SP-003 } \\
\text { (Duplicate of } 002 \text { ) }\end{array}$ & $\begin{array}{c}0-15.2 \\
{[0-6]}\end{array}$ & ND & ND & 36 & NA & NA \\
\hline \multicolumn{8}{|c|}{ LAYOUT PAD GREASE TRAP SAMPLES COLLECTED JULY 21-22, 1997} \\
\hline $\begin{array}{c}\text { Below West } \\
\text { Pipe Joint }\end{array}$ & NSCEP-LP-001 & $\begin{array}{c}0-15.2 \\
{[0-6]}\end{array}$ & ND & ND & ND & NA & NA \\
\hline $\begin{array}{c}\text { Below East } \\
\text { Pipe Joint }\end{array}$ & NSCEP-LP-002 & $\begin{array}{c}0-15.2 \\
{[0-6]}\end{array}$ & ND & ND & ND & NA & NA \\
\hline $\begin{array}{c}\text { Below West End } \\
\text { of Grease Trap }\end{array}$ & $\begin{array}{l}\text { NSCEP-LP-003 } \\
.\end{array}$ & $\begin{array}{c}0-15.2 \\
{[0-6]}\end{array}$ & ND & $\mathrm{ND}$ & ND & NA & NA \\
\hline $\begin{array}{l}\text { Below East End } \\
\text { of Grease Trap }\end{array}$ & NSCEP-LP-004 & $\begin{array}{c}0-15.2 \\
{[0-6]}\end{array}$ & ND & ND & ND & NA & NA \\
\hline \multicolumn{8}{|c|}{ TEST PAD SAMPLES COLLECTED JULY 22, 1997} \\
\hline $\begin{array}{c}\text { Below North } \\
\text { Pipe Joint }\end{array}$ & NSCEP-TP-001 & $\begin{array}{c}0-15.2 \\
{[0-6]}\end{array}$ & ND & $\mathrm{ND}$ & ND & $\mathrm{NA}$ & NA \\
\hline $\begin{array}{l}\text { Below North } \\
\text { Pipe Joint }\end{array}$ & NSCEP-TP-002 & $\begin{array}{c}0-15.2 \\
{[0-6]}\end{array}$ & ND & ND & ND & NA & NA \\
\hline \multicolumn{8}{|c|}{ BUILDING 6-623 GREASE TRAP SAMPLES COLLECTED MAY 19, 1997} \\
\hline $\begin{array}{c}\text { Below West Bottom } \\
\text { of Grease Trap }\end{array}$ & 6-623-GT-01 & $\begin{array}{c}304 \\
{[120]}\end{array}$ & ND & ND & ND & ND & $\begin{array}{ll}\mathrm{Ba} & 0.552 \\
\mathrm{Ag} & 0.026\end{array}$ \\
\hline $\begin{array}{l}\text { Below West Bottom } \\
\text { of Grease Trap }\end{array}$ & $\begin{array}{c}\text { 6-623-GT-02 } \\
\text { (Dulicate of -01) }\end{array}$ & $\begin{array}{c}304 \\
{[120]}\end{array}$ & ND & ND & ND & ND & $\begin{array}{l}\mathrm{Ba} 0.556 \\
\mathrm{Ag} \quad 0.020\end{array}$ \\
\hline
\end{tabular}


TABLE 1: SUMMARY OF NORTH SCEP PIPELINE VERIFICATION RESULTS

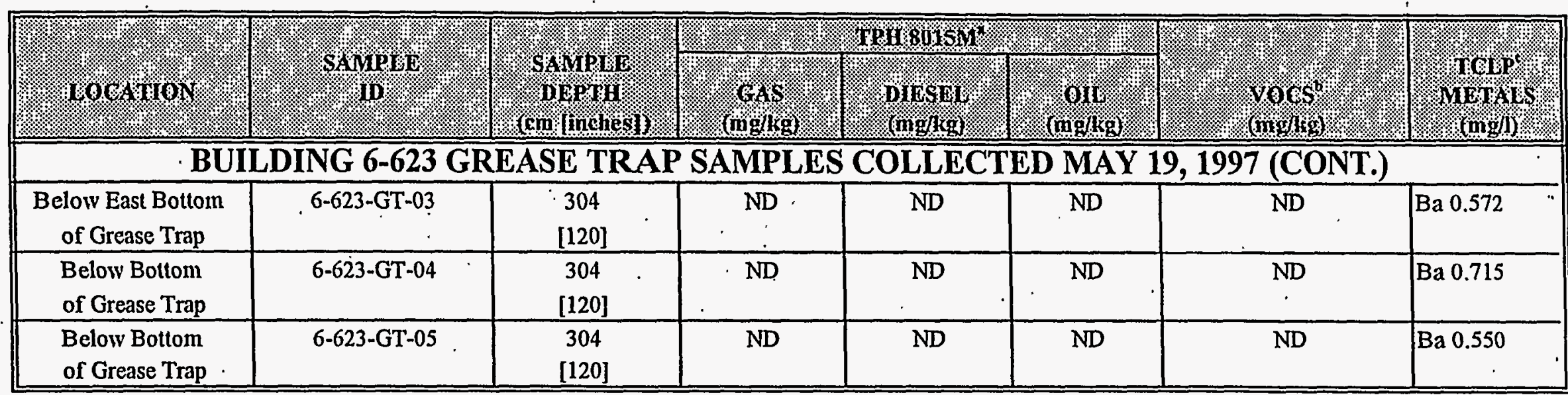

Notes:

"TPH 8015M: Total Petroleum Hydrocarbons Method 8015 Modified.

${ }^{b}$ VOCs: Volatile Organic Compounds.

c TCLP: Toxicity Characteristic Leaching Procedure.

Ba Barium

Ag Silver

SCEP: Steam Cleaning Effluent Pond

ND: Not Detected

NA: Not Analyzed

cm: centimeter

mg/kg: milligrams per kilogram

mg/l: milligrams per liter 
1D

LBH ANALYYSIS DATA SHEET
EPA SAMPLE NO.

NSCEP-SP-1 ao Name: Quanterra, MO

$\therefore$ Code: ITMO Case No.:

ucrix : (soil/water) SOIL .

: ple wt/vol: $1.0 \quad(\mathrm{~g} / \mathrm{ml}) \_G$

trel: (low/med) LOW

loisture: not dec.

AS NO.

Compound

LBH (1)
Contract: 625.09

SAS NO.:

SDG No.: V253

Lab Sample ID: 15396-001

Lab File ID:

Date Sampled: 07-17-97

Date Analyzed: 07-31-97

Dilution Factor: 1.0

CONCENTRATION UNITS:

$(\mathrm{ug} / \mathrm{L}$ or $\mathrm{ug} / \mathrm{Kg})$ UG/KG

Q 500 $u$

U: Concentration of analyte is less than the value given.

$\because \quad$ Pattern reasonably matches gasoline

: Pattern appears to be multi-peaks, but does not match gasoline

-) Concentration is primarily from a single peak

(1): Low.Boiling Hydrocarbon (LBH) is quantitated as if it is Gasoline. 
1D

HBE ANALYSIS DATA SHEET
EPA SAMPLE NO.

NSCEP-SP-1
Iab Name: OUANTERRA KO

Iب̣b Code: ITMO Case No.:

SAS NO.:

contract:

625.01

SDG No.: V253

Iab Sample ID:

15396-001

Iab File ID:

sample wt/vol: $20.2(\mathrm{~g} / \mathrm{ml}) \cdot \mathrm{G}$

Level: (low/med) LOW

Date Sampled:

$07-17-97$

8. Moisture: not dec. dec.

Date Extracted:

$07-31-97$

Extraction: (SepF/Cont/Sonc/Shak) SHAR

GPC Cleanup: $(Y / N)$ N

Date Analyzed:

$08-08-97$

Dilution Factor:

1

CONCENTRATION UNITS:

CAS NO. Compound

DIESEL

HASTB OII

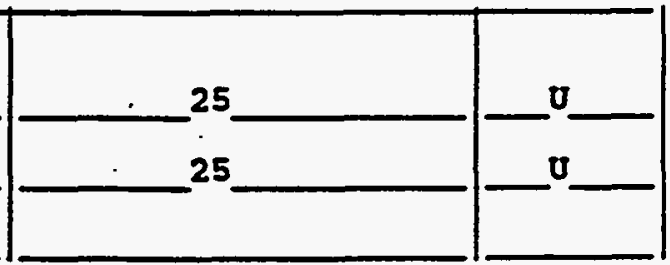

U: Concentration of analyte is less than the value given. 
EPA SAMPLE NO.

NSCEP-SP-2
Lao Name: Quanterra, MO

L'? Code: ITMO Case No.:

Mucrix : (soil/water) SOIL

\& nple wt/vol: $1.0(\mathrm{~g} / \mathrm{ml}) \mathrm{G}$

ut rel: (low/med) LOW

. Moisture: not dec.

:AS NO.

Compound

LBH (1)
Contract: $\quad 625.01$

SAS No.: SDG No.: V253

Lab Sample ID: 15396-002

Lab File ID:

Date Sampled: . 07-17-97

Date Analyzed: 07-31-97

Dilution Factor: 1.0

CONCENTRATION UNITS:

(ug/L or ug/Kg) UG/KG

$Q$

\begin{tabular}{|l|l|l|}
\hline${ }^{\text {LBH }}(1) \ldots$ & $\cdot \ldots 500$ \\
\hline
\end{tabular}

U: Concentration of analyte is less than the value given.

\%. Pattern reasonably matches gasoline

Pattern appears to be multi-peaks, but does not match gasoline

" Concentration is primarily from a single peak

(1): Low.Boiling Hydrocarbon (LBH) is quantitated as if it is Gasoline. 
ID

HBH ANALYSIS DATA SHEET
EPA SAMPLE NO. NSCEP-SP-2
Iab Name: OUANTERRA MO

Iab Code: ITMO Case No.:

soII

Matrix : (Boil/water) $20.1+(9 / m 1)$ G

Sample wt/vol:

Level: (Iow/med) LOH

* Moisture: not dec. dec.

Extraction: (Sepr/Cont/Sonc/Shak) SHAR GPC Cleanup: $(Y / N)+N$ Contract: SAS No.: Iab Sample ID: 625.01 Lab File ID: Date Sampled: Date Extracted: $07-31-97$

Date Analyzed:

Dilution Factor: 1 CONCENTRATION UNITS: CAS No. Compound (mg/I or $\mathrm{mg} / \mathrm{kg}$ ) $\operatorname{ma} / \mathrm{kg}$

$-{ }^{25}-{ }^{25}-\frac{0}{0}$

U: Concentration of analyte is less than the value given. 
1D

LBH ANALYSIS DATA SHEET
EPA SAMPLE NO.

NSCEP-SP-3 sab Name: Quanterra, MO

i Code: ITMO Case No.:

fatrix : (soil/water) SOIL

j nple wt/vol:

9.0 $(g / m l)$

evel: (low/med) LOW

10isture: not dec.

CAS NO. .
Contract: $\cdot 625.01$

SAS No.:

SDG No.: V253

Lab Sample-ID: 15396-003

Lab File ID:

Date Sampled: 07-17-97

Date Analyzed: 07-31-97

Dilution Factor: $1: 0$

CONCENTRATION UNITS:

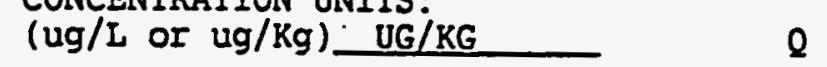

LBH (1)

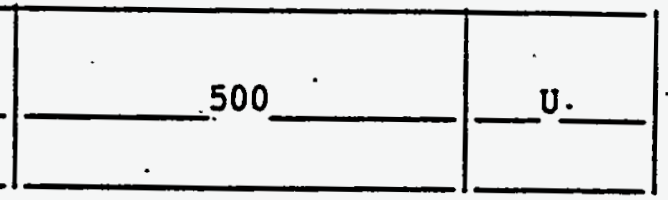

v: Concentration of analyte is less than the value given.

Pattern reasonably matches gasoline

Pattern appears to be multi-peaks, but does not match gasoline

$z:$ Concentration is primarily from a single peak

1): Low Boiling Hydrocarbon (LBH) is quantitated as if it is Gasoline. 


\section{-1D \\ HBH ANALYSIS DATA SHEET}

EPA SAKPIE NO. NSCEP-SP-3

\begin{abstract}
Lab Name: OUANTERRA HO
Lab code: ITMO Case No.:

Hatrix : (goil/water)

SOIL

Sample wt/vol: $20.0 \quad(9 / \mathrm{ml})$ G -
\end{abstract} Level: (low/med) ION

* Moisture: not dec. dec. Extraction: (SepF/Cont/Sonc/Shak) ... SHAK GPC Cleanup: $(\mathrm{Y} / \mathrm{N})$ N $\mathrm{NH}$ CAS NO. Compound

625.01

SAS NO.: SDG No.: $\quad$ V253

Iab Sample ID: $+15396-003$ Lab File ID: Date sampled: $07-17-97$ pate Extracted: $07-31-97$ Date Analyzed: $08-08-97$ Dilution Factor: 1. CONCENTRATION UNITS: (mg/t or $\mathrm{mg} / \mathrm{Kg}$ ) _. mg/kq 2

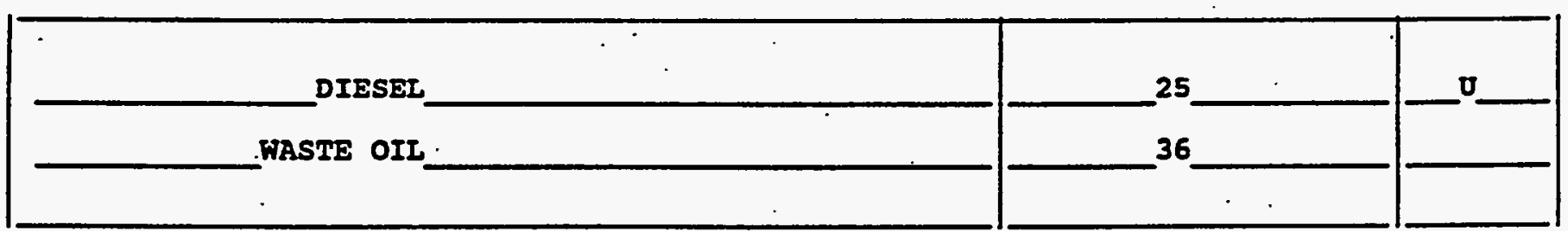

U: Concentration of analyte is less than the value given. 
Lab Name: Quanterra, MO

I b Code: ITMO Case No.:

Matrix : (soil/water) SOIL

i mple wt/vol:

$1.0 \quad(\mathrm{~g} / \mathrm{ml})$

G

Level: (low/med)

LOW

Moisture: not dec.

CAS NO.

Compound
Contract: 625.01

SAS NO.:

SDG No.: V256

Lab Sample ID: 15408-001

Lab File ID:

Date Sampled: $\quad 07-22-97$

Date Analyzed: 08-01-97

Dilution Factor: 1.0
CONCENTRATION UNITS:

$(u g / \mathrm{L}$ or $\mathrm{ug} / \mathrm{Kg})$ UG/KG

Concentration of analyte is less than the value given.

Pattern reasonably matches gasoline

1: Pattern appears to be multi-peaks, but does not match gasoline
2: Concentration is primarily from a single peak

(1): Low Boiling Hydrocarbon (LBH) is quantitated as if it is Gasoline. 
10

HBH ANATLYSIS DATA SHEET
EPA SAMPLE NO. NSCEP-TP-1

Lab Name: OUANTERRA MO

Contract:

625.07

Lab Code: Irmo Case No.:

SAS NO.:

SDG NO.: V256

Matrix : (soil/water)

SOIL

Iab Sample ID:

15408-001

Sample wt/vol:

$20.1(g / \mathrm{ml})$

G

Lab File ID:

Ievel: (low/med)

LOW

Date Sampled:

$07-22-97$

Moisture: not dec.

dec.

Date Extracted:

$08-05-97$

Extraction: (SepF/Cont/Sonc/Shak) ___ SHAR

Date Analyzed:

08-18-97

GPC Cleanup: $(\mathrm{Y} / \mathrm{N})+\mathrm{N}$ PH:

Dilution Factor:

1.

CAS NO.

Compound

CONCENTRATION UNITS:

(mg/L or $\mathrm{mg} / \mathrm{Rg}$ ) $\mathrm{mg} / \mathrm{kg}$

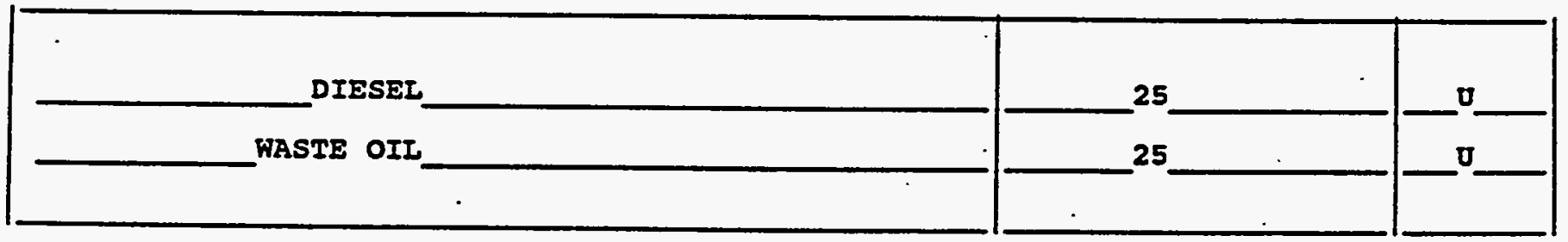

U: Concentration of analyte is 1 esg than the value given. 
1D

LBH ANALYSIS DATA SHEET
EPA SAMPLE NO.

NSCEP-TP-2 ia Name: Quanterra,Mo

is Code: ITMO Case No.:

lacrix : (soil/water) solL

i nple wt/vol:

$1.0(\mathrm{~g} / \mathrm{ml})$

wel: (low/med) LOW

Moisture: not dec.

ZAS NO.

Compound

CONCENTRATION UNITS:

(ug/L or $\mathrm{ug} / \mathrm{Kg}$ ) UG/KG

Q

\begin{tabular}{|l|l|}
\hline & $500 \_-3$ \\
\hline
\end{tabular}

U: Concentration of analyte is less than the value given.

$\checkmark$ Pattern reasonably matches gasoline

Pattern appears to be multi-peaks, but does not match gasoline

-: Concentration is primarily from a single peak

11): Low. Boiling Hydrocarbon (LBH) is quantitated as if it is Gasoline. 
1D

HBH ANALYSIS DATA SHEET
EPA SAMPLE No. NSCEP-IP-2
Lab Name: - OUANTERRA NO

Lạb Code: ' Iтxo Case No.:

Matrix : (soil/water)

soIL

Sample wt/vol: $20.2(\mathrm{~g} / \mathrm{m} 1)$

G

Level: (low/med)

LOF

Soisture: not dec. dec.

Extraction: (SepF/Cont/Sone/Shak) SHAR GPC Cleanup: $(Y / N)$ N

Contract: 625.01

SAS NO.:

SDG NO.:

Lab Sample ID:

15408-002

Iab File ID:

Date Sampled:

$07-22-97$

Date Extracted: 08-05-97

Date. Analyzed:

$08-18-97$

Dilution Factor:

1

CONCENTRATION UNITS:

CAS No.

compound

(mg/I or $\mathrm{mg} / \mathrm{Kg}$ ) $\mathrm{mg} / \mathrm{kg}$

$\mathbf{Q}$

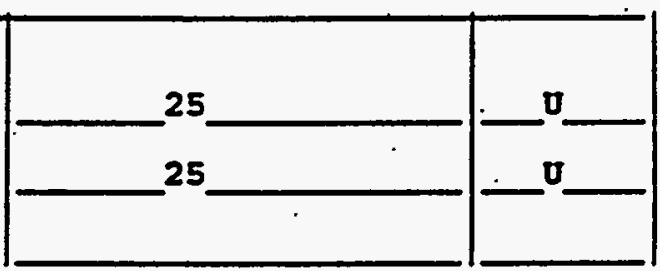

U: Concentration of analyte is legs than the value given. 
sab Name: Quanterra, MO

\& o Code: ITMO Case No.: vatrix : (soil/water) SOIL

j nple wt/vol: $(g / m 1)$

Level: (low/med) LOW

Ioisture: not dec.

LAS NO.
Contract: $\quad 625.09$

SAS. NO. :
Lab Sample ID: 15397-001

Lab File ID:

Date Sampled: 07-21-97

Date Analyzed: 08-01-97

Dilution Factor: 1.0

CONCENTRATION UNITS:

(ug/L or $u g / K g)$ UG/KG

$Q$

LBH (1)

500

$\mathbf{U}$

U: Concentration of analyte is less than the value given.

Pattern reasonably matches gasoline

Pattern appears to be multi-peaks, but does not match gasoline

i: Concentration is primarily from a single peak

-1): Low Boiling Hydrocarbon (LBH) is quantitated as if it is Gasoline. 
10

HBH ANATYSIS DATA SHEET
EPA SAMPLE NO. NSCEP-LP-1

Lab Name:

OUANTERRA MO

Contract:

625.01

Lab Code: ITMO Case No.:

SAS NO.:

SDG NO.: V254

Hatrix : ' (soil/water)

SOIL

Lab Sample ID:

15397-001

Sample. wt/vol:

$20.3(\mathrm{~g} / \mathrm{ml})$

Lab File ID:

Level: (low/med)

IOW

Date Sampled:

07-21-97

\& Moisture: not dec. dec.

Date Extracted:

$08-04-97$

Extraction: (SepF/Cont/Sonc/Shak)__ SHAR

Date Analyzed:

08-05-97

GPC Cleanup: $(\mathrm{Y} / \mathrm{N})$ N $\mathrm{NH:}$

Dilution Factor:

1

CONCENTRATION UNITS:

CAS NO.

Compound

(mg/L or $\mathrm{mg} / \mathrm{kg}$ )

$\mathrm{ma} / \mathrm{ko}$

$\mathbf{Q}$
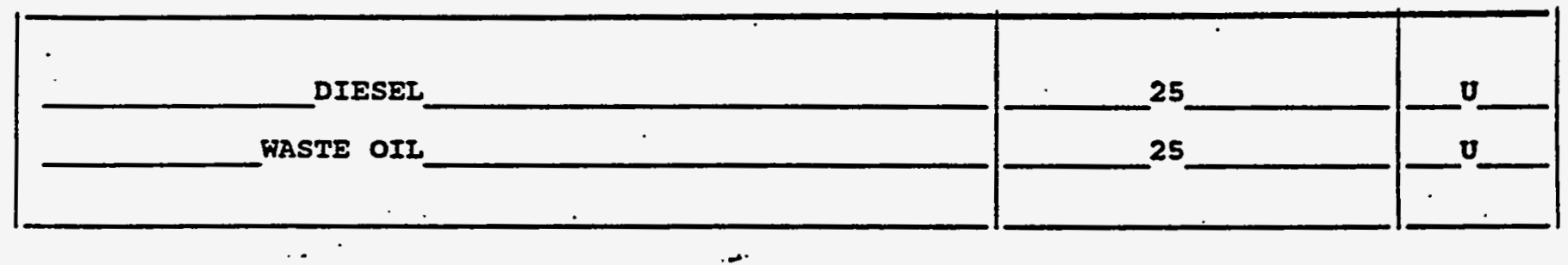

$U$ : Concentration of analyte is less than the value given. 
Lab Name:

L ) Code: ITHO Case No.:

MatIix : (soil/water) soIr

S. iple wt/vol:

$1.0 \quad(\mathrm{~g} / \mathrm{ml})$

G

Level: (low/med) LOW

q loisture: not dec.

CAS NO.

Compound
Contract: 625.01

SAS No.:

SDG No.: V254.

Lab Sample ID: 15397-002

Lab File ID:

Date Sampled: $\quad 07-21-97$

Date Analyzed: 08-01-97

Dilution Factor: 1.0

CONCENTRATION UNITS:

(ug/L or $u g / \mathrm{kg}$ ) UG/KG

2

LBH (1)

500

U

U: Concentration of analyte is less than the value given.

Pattern reasonably matches gasoline

pattern appears to be multi-peaks, but does not match gasoline

$\mathrm{z}$ : Concentration is primarily from a single peak

1): Low Boiling Hydrocarbon (LBH) is quantitated as if it is Gasoline. 
10

HBH ANATYSIS DATA SHEET
EPA SAMPLE NO.

NSCEP-IP-2

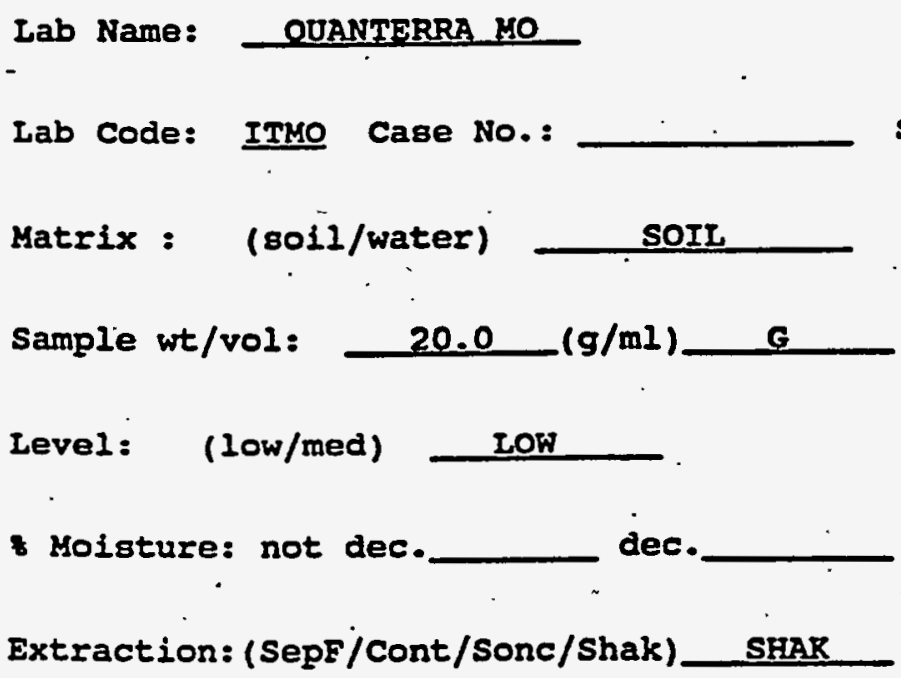
GPC Cleanup: $(Y / N)+N \quad \mathrm{~N}$ contract: SAS NO.:
625.01

SDG NO.: $\quad$ V254

Iab Sample ID: $15397-002$

Iab File ID:

Date sampled: $\quad$ 07-21-97

Date Bxtracted:

$08-04-97$

Date Analyzed:

$08-05-97$

Dilution Pactor:

1

CAS No.

Compound

CONCENTRATION UNITS:

(mg/L or $\mathrm{mg} / \mathrm{Kg}) \ldots \mathrm{mg} / \mathrm{kg}$

$\mathbf{Q}$

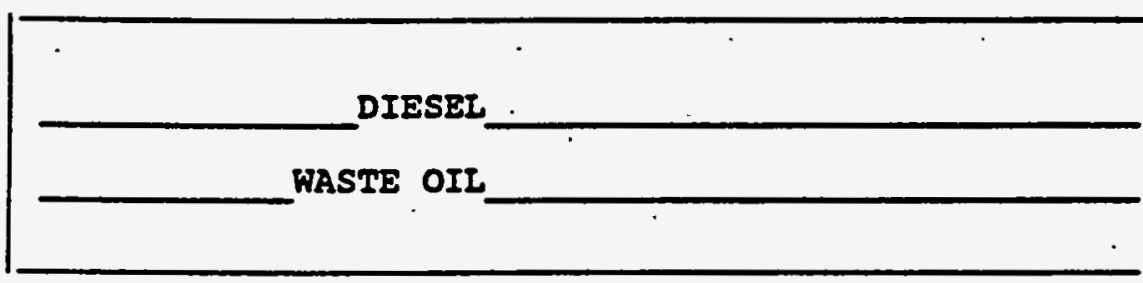

\begin{tabular}{|r|l|}
\hline 25 & 25 \\
\hline$\quad 0$ & 0 \\
\hline & 0 \\
\hline
\end{tabular}

U: Concentration of analyte is lesg than the value given. 


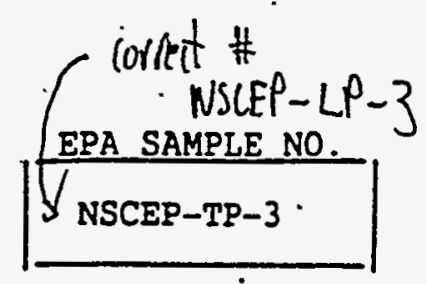

sab Name: $\frac{\text { Quanterra,MO }}{i \text { J Code: }{ }_{\text {ITMO }} \text { Case No.: }}$

yarrix : (soil/water) SOIL

j nple wt/vol: $1.0 \quad(\mathrm{~g} / \mathrm{ml}) \mathrm{G}$

sevel: (low/med) LOW

z loisture: not dec.

Contract: 625.01

SAS No.:

SDG No.: V256

Lab Sample ID: 15408-003

Lab File ID:

Date Sampled: 07-22-97

Date Analyzed: 08-04-97

Dilution Factor: 1.0

CONCENTRATION UNITS:

$(\mathrm{ug} / \mathrm{L}$ or $\mathrm{ug} / \mathrm{Kg})$ UG/KG,$Q$

Compound 500 u.

LBH (1)

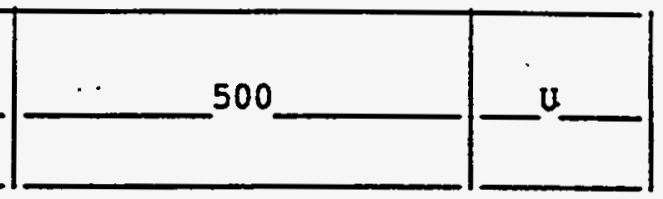

$\mathrm{U}$ : Concentration of analyte is less than the value given.

Pattern reasonably matches gasoline

Pattern appears to be multi-peaks, but does not match gasoline

Concentration is primarily from a single peak

1): Low Boiling Hydrocarbon (LBH) is quantitated as if it is Gasoline. 


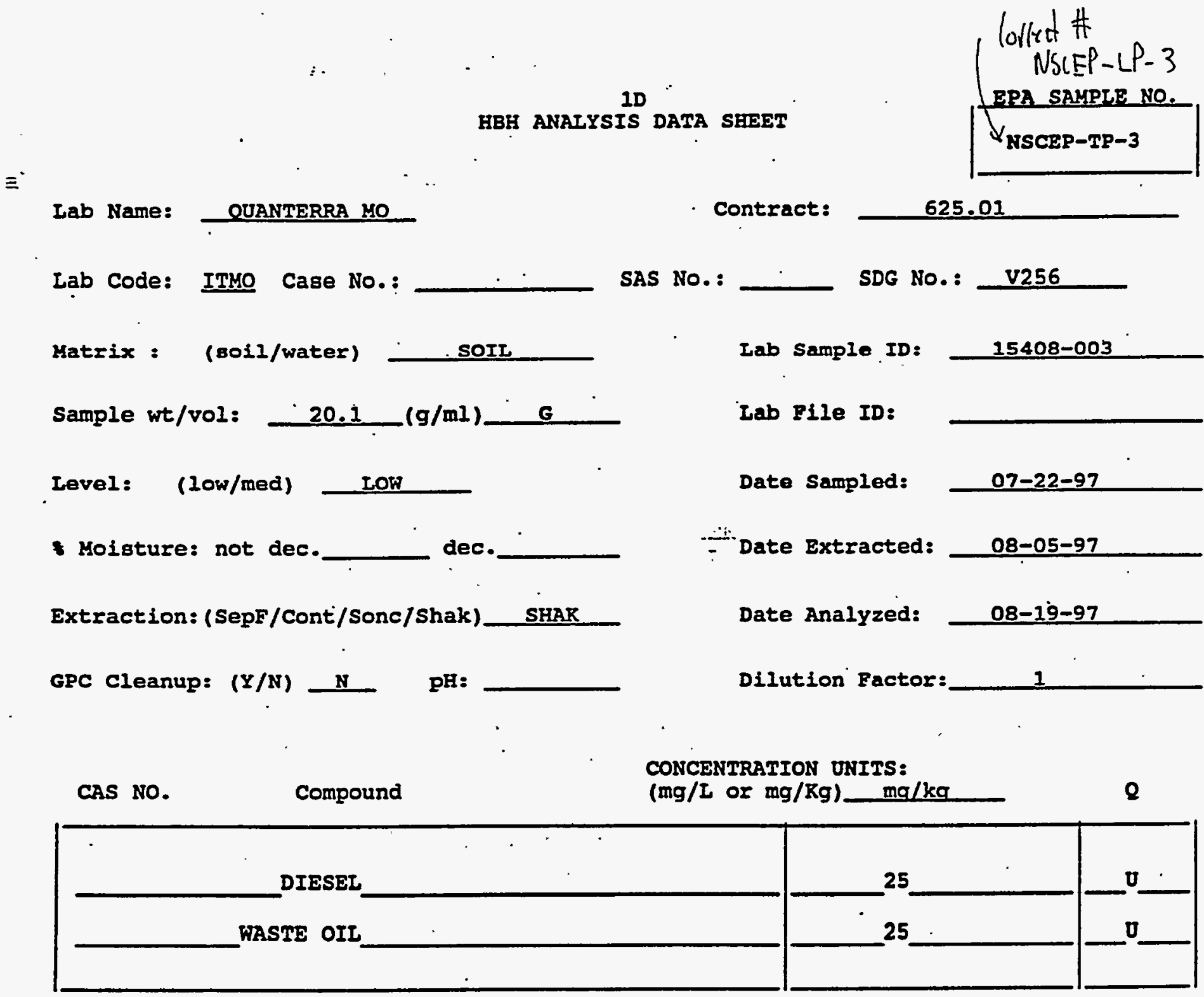

U: Concentration of analyte is less than the value given. 
Lab Name: Quanterra, MO

- ab Code: ITMO Case No.:

.atrix : (soil/water) SOIL

cample wt/vol: 1.0

Level: (low/med) LOW

- Moisture: not dec.

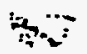

NSIEP-LP -4

EPA SAMPLE NO

NSCEP-TP-4

Contract: 625.01

SAS NO.:

SDG No.: V256

Lab Sample ID: 15408-004

Lab File ID:

Date Sampled:

Date Analyzed: 08-01-97

Dilution Factor: 1.0

CAS NO.

Compound

CONCENTRATION UNITS:

(ug/L or ug/ $\mathrm{kg})$ UG/KG

Q

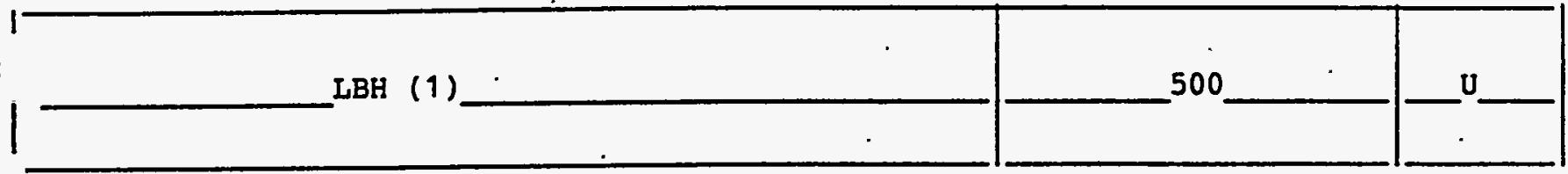

U: Concentration of analyte is less than the value given.

X: Pattern reasonably matches gasoline

$\mathrm{y}$ : Pattern appears to be multi-peaks, but does not.match gasoline

Z: Concentration is primarily from a single peak

(1): Low Boiling Hydrocarbon ( $\mathrm{LBH}$ ) is quantitated as if it is Gasoline.

FORM I LBH 
1D

HBH ANALYSIS DATA SHEET

$$
\text { Lolfert \# }
$$

NSEP - LP -Y

EPA SAMPLE NO.

NSCEP-TP-4

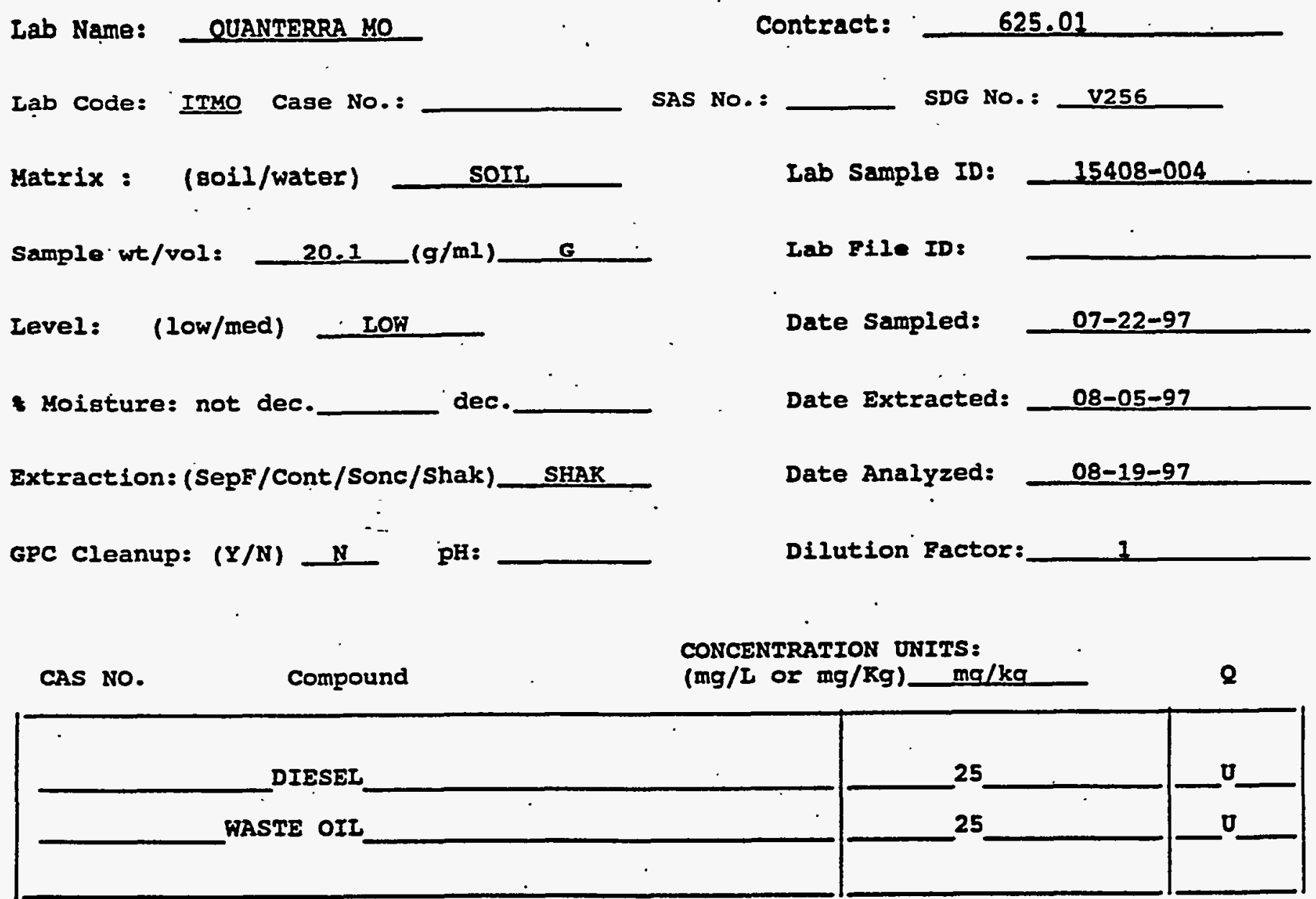

D: Concentration of analyte is 1888 than the value given.

FORY I EBB 


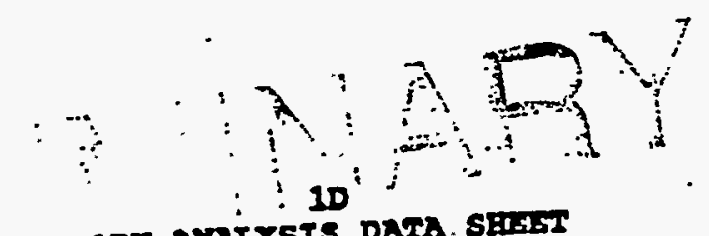

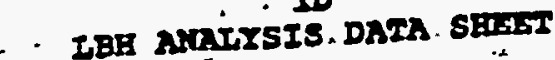

FPA SAYPL 10.

.. ${ }^{2}$.

contract: 625,01

I b Nama: Ouantertako

i b coder ITPOO Case NO.:

patrix : (Boil/water) soII

i mple wt/vol: $1,0^{\circ}(g / \mathrm{ml}) \quad 0$

Level: (low/mad) LOH

Moisture: not dec.

-.

cas ro.

compound

LBB

(I)
SAs RO.:

SDG X0.: V216

Iab Sample ID: 14697-001

Iab File ID:

Date Sampled: 05-19-97

Date analyzed: $05-27-97$

Dilution Factor: 1.0
CONCBLIRATIOR URIIS:

(ug/L or ug/Rg) Ue/Re $\cdot \mathbf{2}$

$U$ : Concentration of analyte is 1 ees than the value given.

X:Pattern reasonably matcher gasolina

but does not wateh gasoline

Y i Pattorn appears to be marliy from a minglo peak

(1) sow Boșing Eydrocarbon (LBE) is quantitated as if it is Gasoline.

FORY I IBBE 


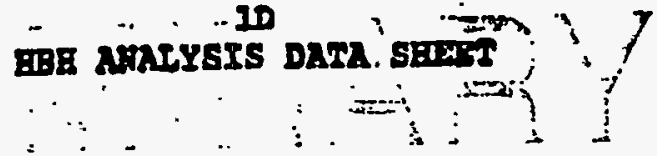

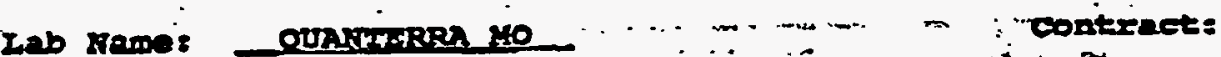

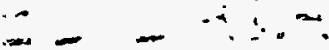

Iab codes Imro Cage Ho.:

SAS NO.:

Inb Snmple ID:

14697-001

Iab File ID:

Sample wt/vols -

$20.2(g / m)$

$G$

Level: (10w/med)

T.OA.

Date surpleds

Date sxtracted:

$05-27-97$

Date Analyzed:

$05-28-97$
T Molgture: not dec. dec.
Extraction: (Sepp/Cont/Sone/Shalk)_ SHax

GPC Cleanup: (Y/X) L PE:

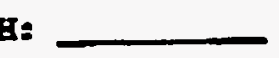

625.02
SDG $10 .:-7216$
TPA SAYRTR KO. $6-623-02-01$
Dilution Factor:

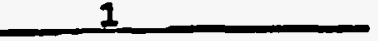

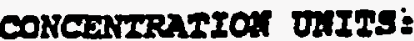

cas ro.

compound

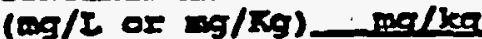

Q

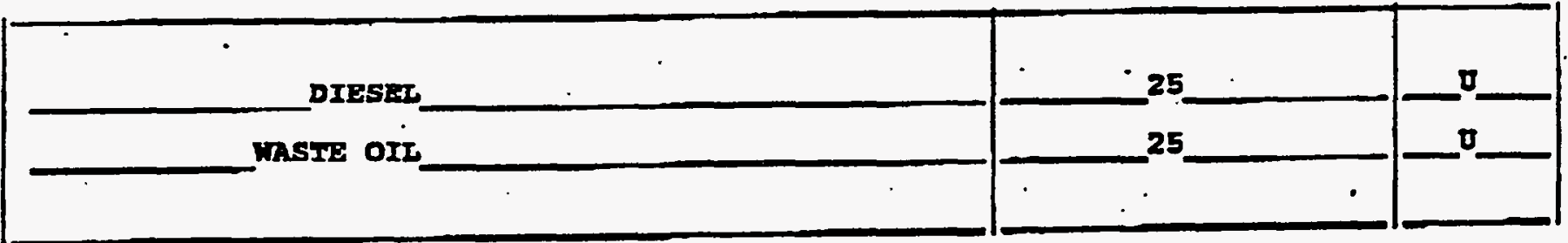

U: Concentration of analyte is less than the value given. 
F'MTE: QUANTERRA MO

Code: ITMO Case No.: V6970I SAS No.: $\therefore \therefore$ (so1l/water) soIL

Ile wt/vol: . $5.00(\mathrm{~g} / \mathrm{mI}) \mathrm{G}$

$\therefore \quad$ (low/med) LOW

ifsture: not dec.

X_Lmn: RTX-502.2 ID: 0.530 (mm)

(uI)
SDG NO.: V216
Contract: $625-01$

Iab Sample ID: 14697-001

Iab File ID: G6545

Date Received: $05 / 21 / 97$

Date Analyzed: 05/31/97

Dilution Factor: $\quad 1.0$

Soil Aliquot volume: ' (ur) ixtract volume:

- Ixtiract Volume: 
$I A$

VOLATILE ORGANICS ANATYSIS DATA SHEET
Lab Name: QUANTERRA MO

Iab Code: ITMO

Case No.: V6970I

Matr1x: (so1I/water) SOII

Sample wt/vol:

$5.00(\mathrm{~g} / \mathrm{mI}) \mathrm{G}$

Level: (low/med) IOW

f Moisture: not dec.

GC Column: RTX-502.2 ID: 0.530 (mm)

Soil Extract Volume:

(ur)
Contract: $625-01$

SAS NO.:

SDG NO.: V216

$$
6-623-G T-01
$$

Lab Sample ID: 14697-001

Lab File ID: $\quad \mathbf{G 6 5 4 5}$

Date Received: 05/21/97

Date Analyzed: 05/31/97

Dilution Factor: . 1.0

Soil Aliquot volume:

CONCENTRATION UNITS:

CAS NO.

COMPOUND

$Q$

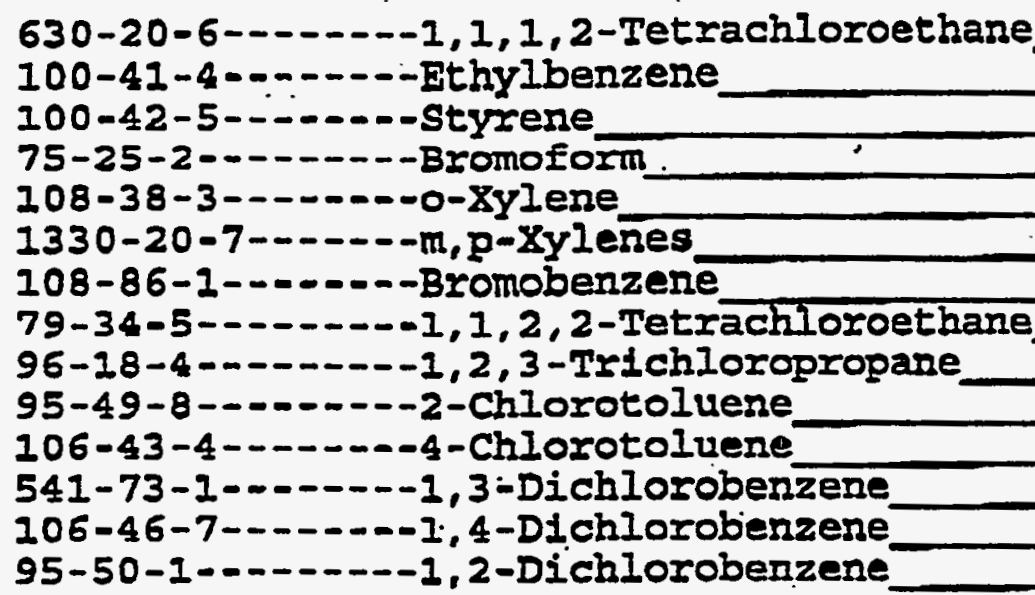




\section{ס.S. EPA - CLP}

1 INORGANIC ANAIYSES DATA SHEET
EPA SAMPIE NO.

$$
6-623-G T-01
$$

it ne: QUANTERRA_MO

snile: ITMO

Case No.:

¿. (80il/water) : WATER

. (Iow/med) :

LOW

Contract: 625.01

SAS NO.:

SDG NO.: V216

ida :

$-0.0$

Concentration Unfts (ug/L or $\mathrm{mg} / \mathrm{kg}$ dry weight) : $0 G / \mathrm{L}_{-}$

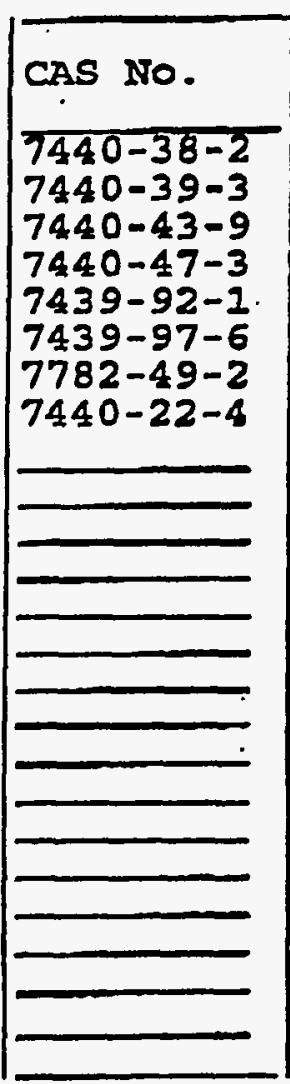

: 3efore:

= After:

:: $: \mathbf{s :}$

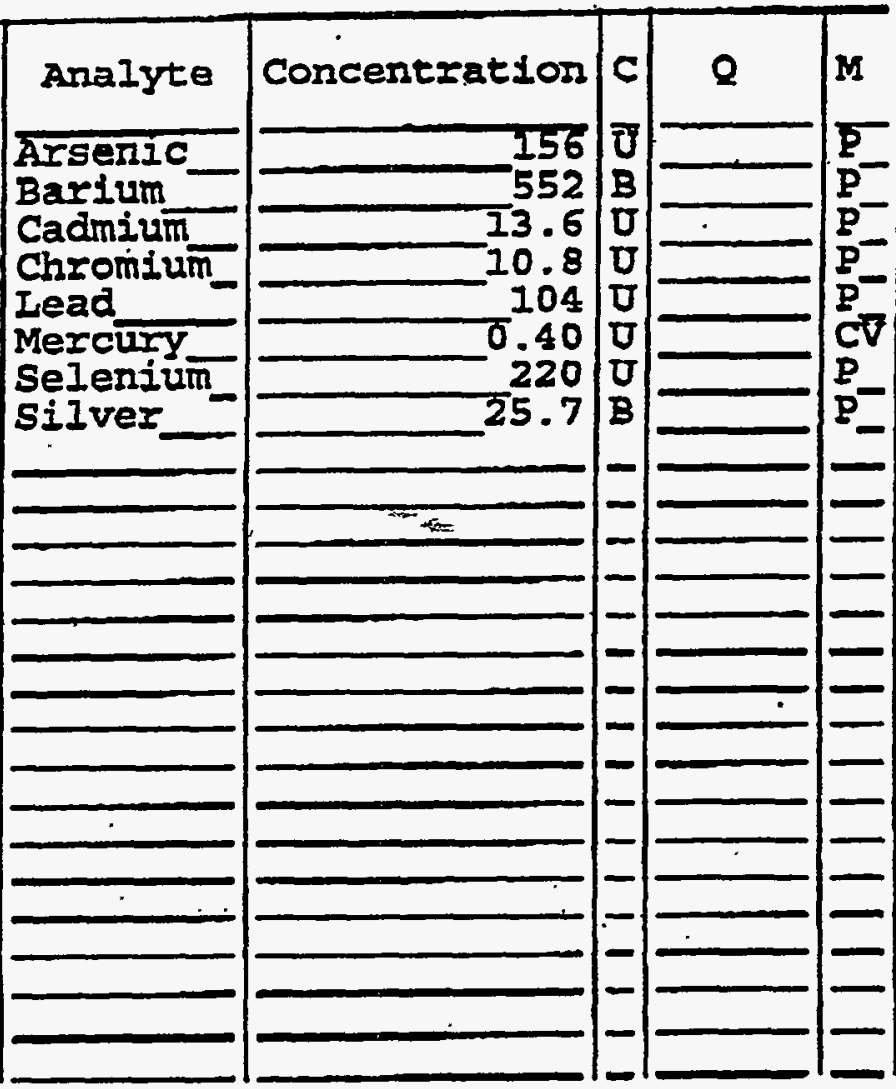

Clarity Before:

Texture:

clarity After:
Iab Sample ID: P14697-001

- Date'Received: 05/21/97 
Lab Name:

Iub coder

Hateix

Sample wt/vols

(sol1/water) soirt

Leval: (20w/mad)

1.0 $(9 / m)$

c

/ Moisture: not dec.

rogis

ans. ro.

Compound
Contract: 625.01

sas No.:

SDG KO.2 บ216

Inb sample ID: 14697-002

Iab File to:

Date Gampleds

$05-19-97$

Date Annyzeds :05-27-97

D1lutson Eactor: 1.0

\section{CONCENmRATIOH UAITS:}

(ug/L or ug/Kg) De/KG

$\boldsymbol{Q}$ 500 t

LBI (1)

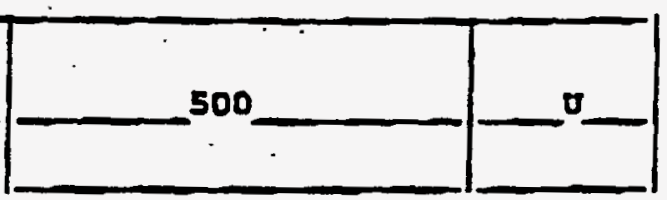

UsConcentration of analyte is less chan the valun glven. X:Pattern zeasonably matches gasoline

Yipattern appears to be multi-peaks, but.does not match gagolina

$z$ zconcentretion is primnzily from a oingle pers:

(1) Iow Boiling Bydxocarbon (IBB) 18 guantitated al if it is Gaboline. 
10

EBE ARRAYSIS DATA SELET

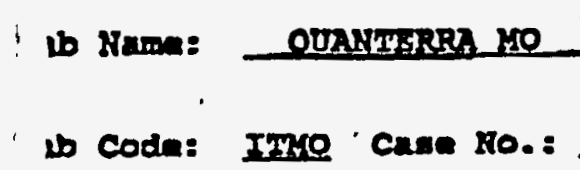

Anterise: (0011/untens)

Lample wt/001:

$20.0 \quad\left(9 / \mathrm{m}^{2}\right)$

sort

vels (low/mad)

Ion

- Moletures not dec. dec.

•.

ExtractLon: (SepF/Cont/sone/Shak) _. SEAK

PC cleanup: $(\Psi / N)-\mathrm{R}$

Contrict:

SAS No. 8

SDE RO.:

Lab Sampla ID: 14697-002

Iab File IDe

pate Srupled:

$05-19-97$

Date seractod:

$05-27-97$

Date analyzed:

$05-28-97$

DLIution Factors

1

CAS No.

compound

coxceniturerton urtTs:

(mg/t or $\mathrm{mg} / \mathrm{hg})$ ma/ke

$\mathbf{q}$

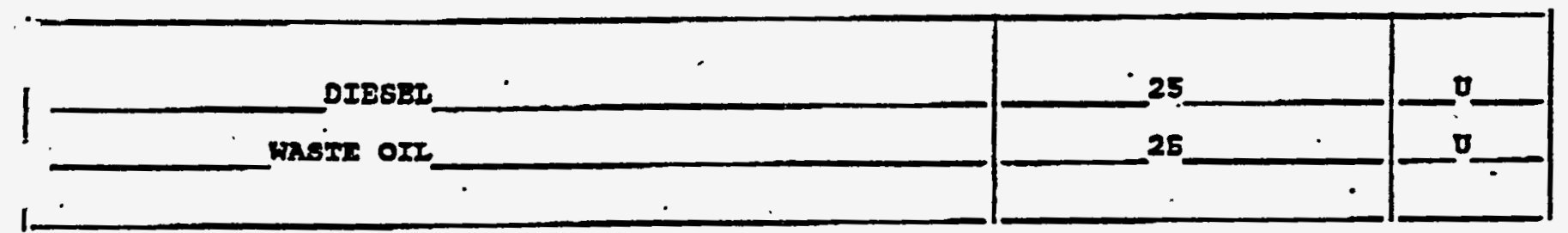

D: Concentration of analyte 18 10sa than the value glven.

PORY I HBE 
$1 A$

VOLATIIE ORGANICS ANALYSIS DATA SHEET
EPA SAMPLE NO.

$6-623-G T-02$
Lab Name: QUANTERRA MO

Lab Code: ITMO

Case No.: V69701

Matrix: (soil/water) soII

Sample wt/vol: $\quad 5.00(\mathrm{~g} / \mathrm{mI}) \mathrm{G}$

LeveI: (Iow/med) LOW

\% Moisture: not dec.

GC Column: RTX-502.2 ID: 0.530 (mm)

Soil Extract Volume:

(นL)
Contract: $625-01$

SAS NO.:

SDG NO.: V216

Iab Sample ID: 14697-002

Lab File ID: $\quad \mathbf{6} 6546$

Date Received: 05/21/97

Date Analyzed: 05/31/97

Dilution Factor: $\quad 1.0$

Soil Aliguot Volume:

(ut)

CONCENITRATION UNITS:

CAS NO.

COMPOUND.

75-71-8--..--Dichlorodifluoromethane

$74-83-9$ Bromomethane

75-0I-4-.-.-.--Vinyl Chloride

75-00-3-...-.-.-Chloroethane

75-09-2-...-.-Methylene ChIoride

75-69-4-.....-. TrI ch I or of Iuoromethane

75-34-3--..-1, 1-Dichloroethane

156-60-5-.--.-.-trans-1, 2-Dichloroethene

256-59-2-...--cis-1, 2-Dichloroethene

107-06-2--..--1, 2-Dichloroethane

594-20-7--.---2, 2-Dichloropropane

74-97-5-...---Bromochloromethane

$71-55-6 \ldots-\ldots-1,1,1-$ Trichloroethane

78-93-3-.-.----2-Butanone

56-23-5-....-.-Carbon Tetrachloride

563-58-6------1, 1-Dichloropropene

75-27-4-....--Bromodichloromethane

78-87-5--..--1, 2-Dichloropropane

10061-01-5-----cis-1, 3-Dichloropropene

79-01-6-........Trichloroethene

79-00-5--.-.-1, 1, 2-Trichloroethane

71-43-2-.--.---Benzene

74-95-3--..---Dibromomethane

10061-02-6--.--trans-1,3-Dich loropropene

106-93-4---n---1,2-Dibxomoethane

127-18-4--.---Tetrachloroethene

142-28-9-....--1,3-DiChIoropropane

124-48-1--.----Dibromochloromethane

108-88-3-...--Toluene

108-90-7-...--Chlorobenzene

\begin{tabular}{|l|l|}
\hline 10 & $U$ \\
10 & $U$ \\
10 & $U$ \\
10 & $U$ \\
2 & $B J$ \\
5 & $U$ \\
5 & $U$ \\
5 & $U$ \\
5 & $U$ \\
5 & $U$ \\
5 & $U$ \\
5 & $U$ \\
5 & $U$ \\
20 & $U$ \\
5 & $U$ \\
5 & $U$ \\
5 & $U$ \\
5 & $U$ \\
5 & $U$ \\
5 & $U$ \\
5 & $U$ \\
5 & $U$ \\
5 & $U$ \\
5 & $U$ \\
5 & $U$ \\
5 & $U$ \\
5 & $U$ \\
5 & $U$ \\
5 & $U$ \\
5 & $U$ \\
& \\
\hline
\end{tabular}


IA

VOLATILE ORGANICS ANALYSIS DATA SHEET.
EPA SAMPIE NO.

6-623-GT-02
NAME: QUANTERRA MO

Lode: ITMO

Case No.: V69701

; ix: (soil/water) soIL

ple wt/vol:

$5.00(\mathrm{~g} / \mathrm{mL}) \mathrm{G}$

i.: (low/med) Low

oisture: not dec.

(IIUmn: RTX-502.2. ID: 0.530 (mm)

? Extract Volume:

(นI)
Contract: $625-01$

SDG NO.: V216

Lab Sample ID: 14697-002

Lab File ID: $\quad 66546$

Date Received: 05/21/97

Dare Analyzed: 05/31/97

Dilution Factor:

2.0

Soil Aliquat Volume:

(uL)

CAS NO COMPOUND

\begin{tabular}{|c|c|c|}
\hline 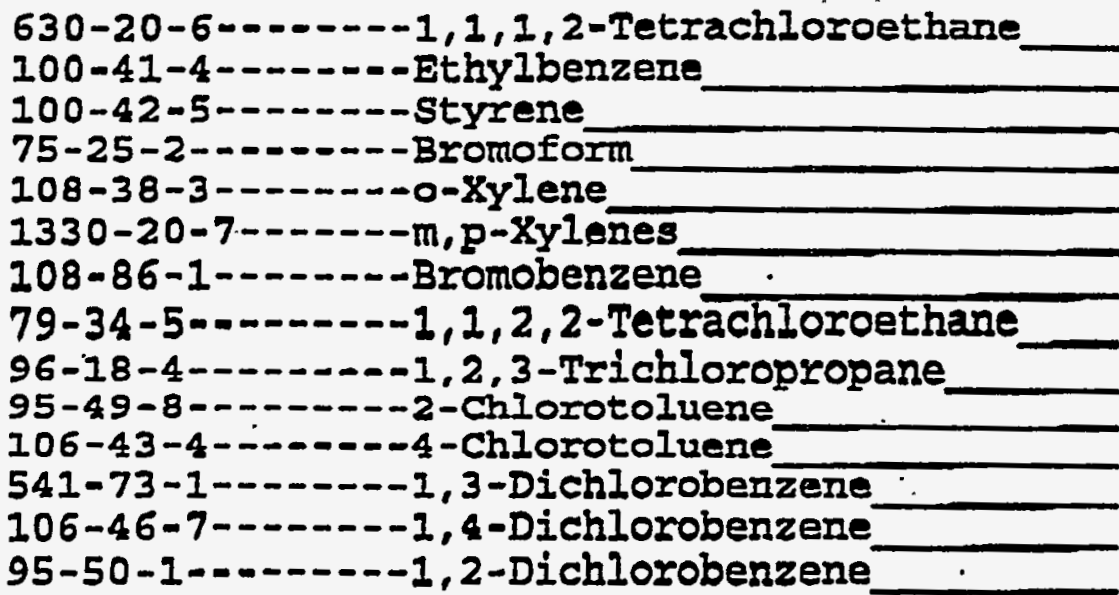 & $\begin{array}{l}5 \\
5 \\
5 \\
5 \\
5 \\
5 \\
5 \\
5 \\
5 \\
5 \\
5 \\
5 \\
5 \\
5\end{array}$ & $\begin{array}{l}0 \\
0 \\
0 \\
0 \\
0 \\
0 \\
0 \\
0 \\
0 \\
0 \\
0 \\
0 \\
0 \\
0 \\
0 \\
0\end{array}$ \\
\hline
\end{tabular}




\section{J.s. EPA - CLP}

\section{INORGANIC ANAIY'YSES DATA SHEET}

ab Name: QUAANTERRA_MO

ab Code: ITMO_ Case No.: atrix (soil/water): WATER svel (low/med): LOW_
Contract: 625.01 SAS NO.:
EPA SAMPLE NO.

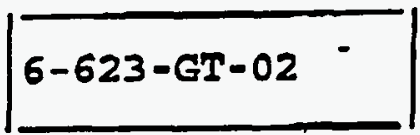

SDG NO.: V216

Lab Sample ID: PI46997-002

Date Received: 05/21/97

Solids :

0.0

Concentration Units (ug/L or $\mathrm{mg} / \mathrm{kg}$ dry weight): UG/L_

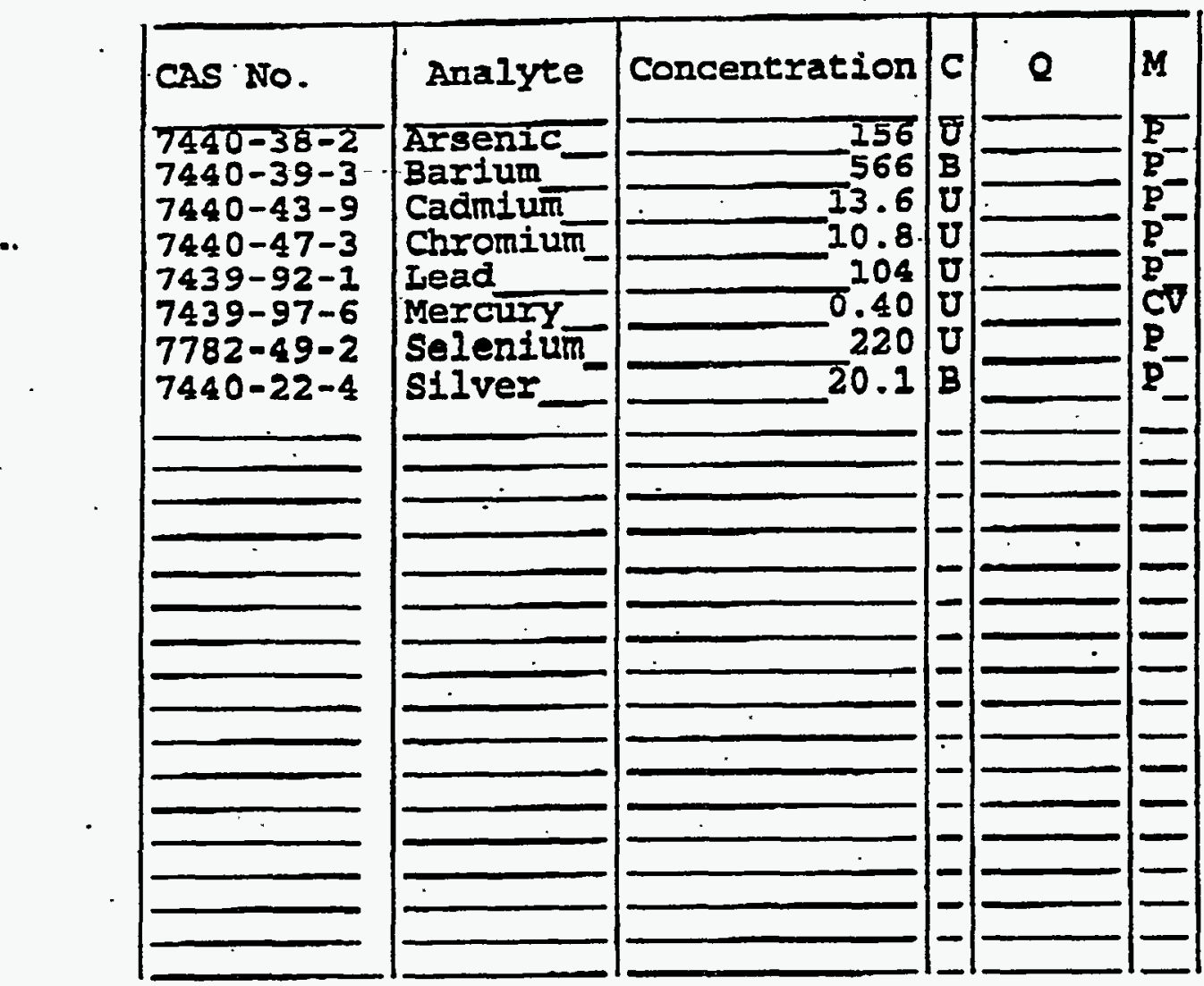

olor Before:

olor After: omments:
Clarity Before:

Clarity After:
Texture:

Artifacts: 
an Hames ouanterra,ko

i. Code: Inyo cane Ho.:

-tix :. (8012/water) sort

' íle wt/vo1: ' $19 / \mathrm{ml}) \quad r$

orra 1: (Low/med) ron

olstures not dec.

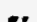

CAS NO. contract: 625.01

SAS No.:

SDe Ho.: V216

Iab sample ID: 14697-003

In FLIE ID:

Date sampled: 05-19-97

Date annlyzed: - 05-27-97

DLIution Factor: 1.0

\section{CORCBLHRATIOR UAITS:}

(ug/I, or ug/Rg) 0e/ke

Q

riconcentration of analyte in lese than the value givin.

ipattexn raamonably watches gacoline

- - Pattorn appears to be multi-park, but dose not mateb gavoline.

8:Concentration io primarily from a eingle pouk

1):Low Boiling Aydrócarbon (LBA) is quantitated ne is it is Gasoline. 
HPA SAMPLE NO. $6-623-6 T-03$

Inb Name: OURWIERBR MO

coneracts

625.09

Inb Code: Inyo case Mo.:

sns 10.8

SDE HO.: . Y216

Matrix : (sol2/water)

sorf

Iab sample ID:

14697-003

Sample wt/vol:

$20.2(9 / 212)$

G

Iab File ID:

Iovel: (10w/mad)

- ros

Date sampled:

$0 \dot{5-19-97}$

Date Extrectad:

05-27-97

\% Hoigtures not dec.

dec.

Extraction: (SepF/Cont/Sonc/Shak)

SPRx

GPC Cleanup: $(X / N) \perp X$

Daze Analyzed:

$05-28-97$

Dilution Fastors

1

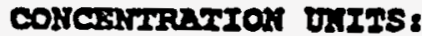

CAS No.

compound

$(\mathrm{mg} / \mathrm{L}$ or $\mathrm{mg} / \mathrm{Lg})$

molko

$\mathbf{9}$

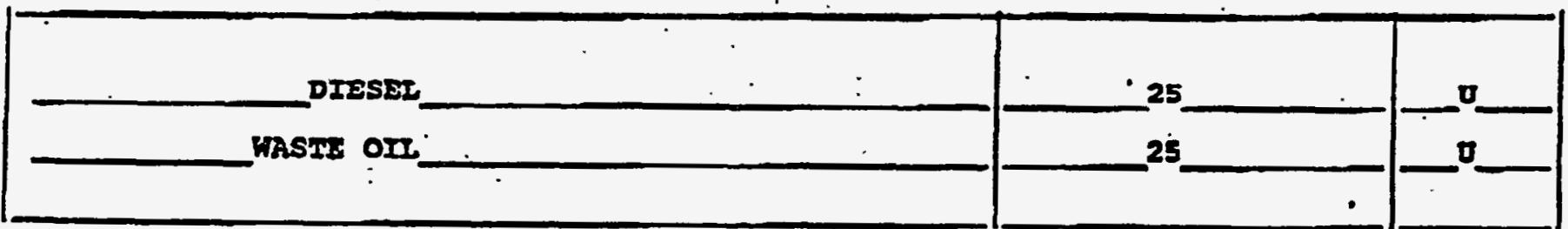

U: Concentration of andyte is leas than the value glvan.

EORY I IAE 
$1 A$

VOLATILE ORGANICS ANALYSIS DATA SHEET
EPA SAMPIE NO.

$$
\text { 6-623-GT-03 }
$$

NAme: QUANTERRA MO

cude: ITMO

Case No.: V69701

:: (so11/water) soIJ

le wt/vol:

$5.00(\mathrm{~g} / \mathrm{mL}) \mathrm{G}$

(1ow/med) IOW

isture: not dec.

:i. 'umn: RTX-502.2 ID: 0.530 (mm)

3xtract Volume:

(UI)
Contract: $625-01$

SAS NO.:

SDG NO.: V216

Lab Sample ID: 14697-003

Lab File ID: $\quad \mathbf{6 5 5 4 7}$

Date Received: 05/21/97

Date Analyzed: 05/31/97

Dilution Factor: $\quad 1.0$

Soil Aliquot Volume: (UI)

CONCBNTRATION ONITS:

CAS NO.

COMPOUND

(ug/L or ug/Rg) UG/KG

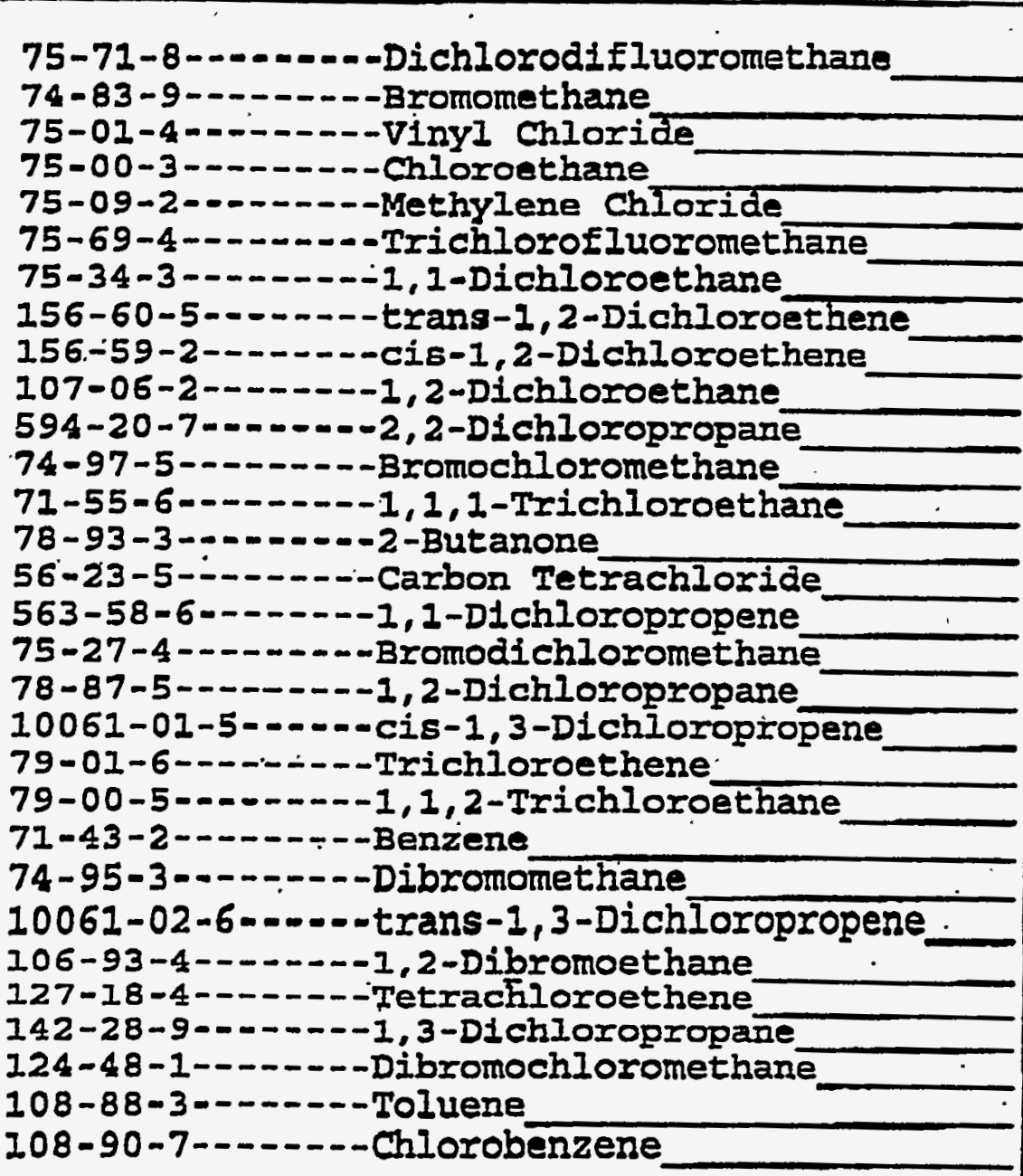

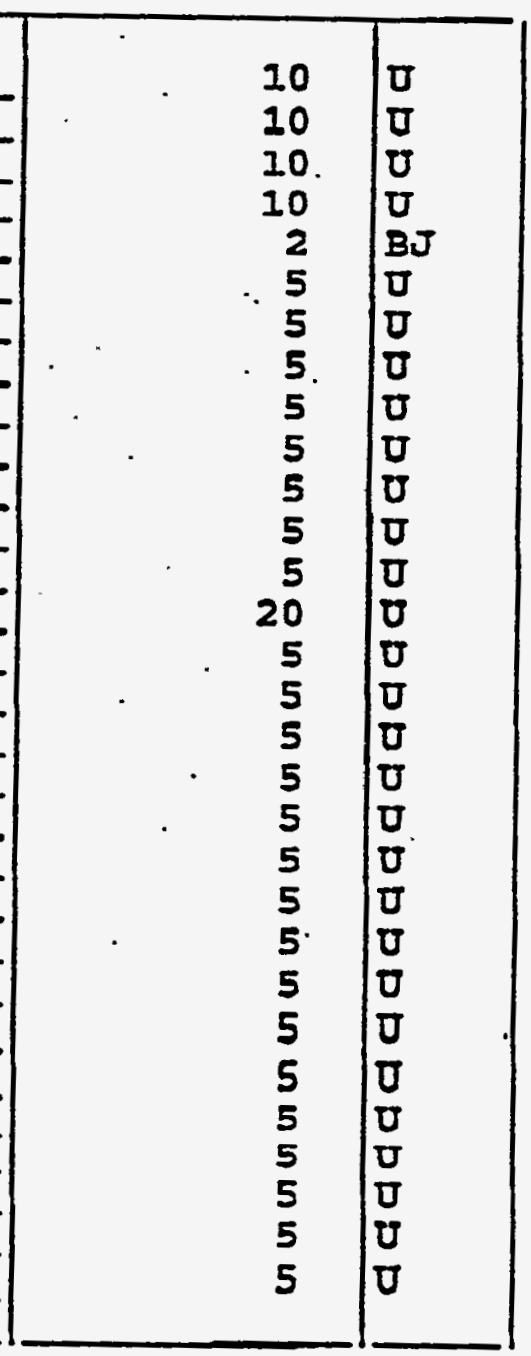


$1 A$

VOLATIIE ORGANICS ANALYSIS DATA SHEET
EPA SAMPLE NO.

$6-623-\mathrm{CT}-03$
Lab Name: QUANTERRA MO

Lab code: ITMO

Matrix: (soil/water) SOII

Sample wt/vol:

$5.00(\mathrm{~g} / \mathrm{mL}) \mathrm{G}$

Level: (Iow/med) LOW

Folsture: not dec.

GC Column: RTX-502.2 ID: $0.530(\mathrm{~mm})$

soil Extract Volume:

(UI)
Contract : 625-01

SAS HO. :

SDG NO.: V216

Lab Sample ID: 14697-003

Iab File ID: 66547

Date Received: 05/21/97

Date Analyzed: 05/31/97

Dilution Factor: $\quad 1.0$

Soil Aliquot volume:

(uIL) CONCENTRATION UNITS:

CAS NO.

COMPOUND

(ug/I or ug/Rg) UG/RG

Q

$630-20-6-\cdots---1,1,1,2-$ Tetrachloroethane

$100-41-4-------$ - thylbenzene

100-42-5--....--styrene

75-25-2--.-.-.-Bromoform

108-38-3-0.----o-Xylene

1330-20-7-...--m,p-xylenes

108-86-1-.-...-Bromobenzene

79-34-5--.-.-1, 1, 2, 2-Tetrachloroethane

96-18-4---n---1,2, 3-Trichloropropane

95-49-8-0.-.-.-2-Chlorotoluene

106-43-4--..--4-Chlorotoluene

541-73-1-..---1,3-Dichlorobenzene

106-46-7-....--1,4-D1chlorobenzene

95-50-1--.---1,2-Dichlorobenzene

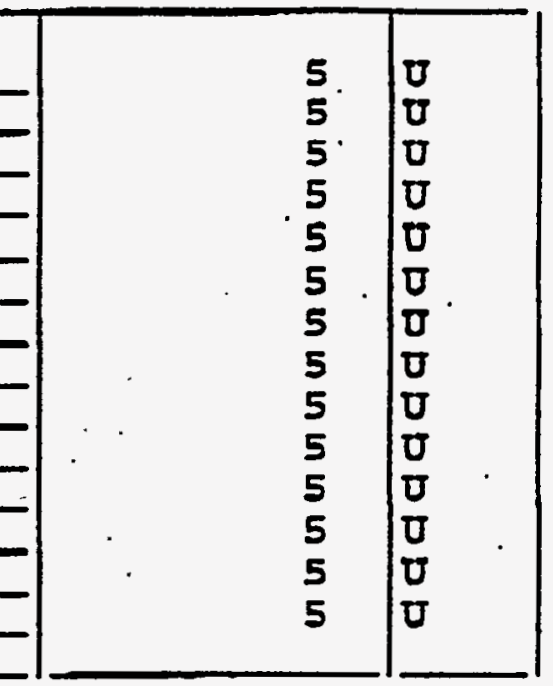


EPA SAMPLE NO.

N_me: QUANTERRA_MO C-de: ITMO_ Case No.: I. (soil/water): WATER

I (low/med): LOW

I'Ids: $-0.0$
$6-623-G T-03$ Contract: 625.01 SAS NO.:

SDG NO.: V216

Lab Sample ID: P14697-003

Date Received: 05/21/97

Concentration Units (ug/L or $\mathrm{mg} / \mathrm{kg}$ dry weight) : UG/L_

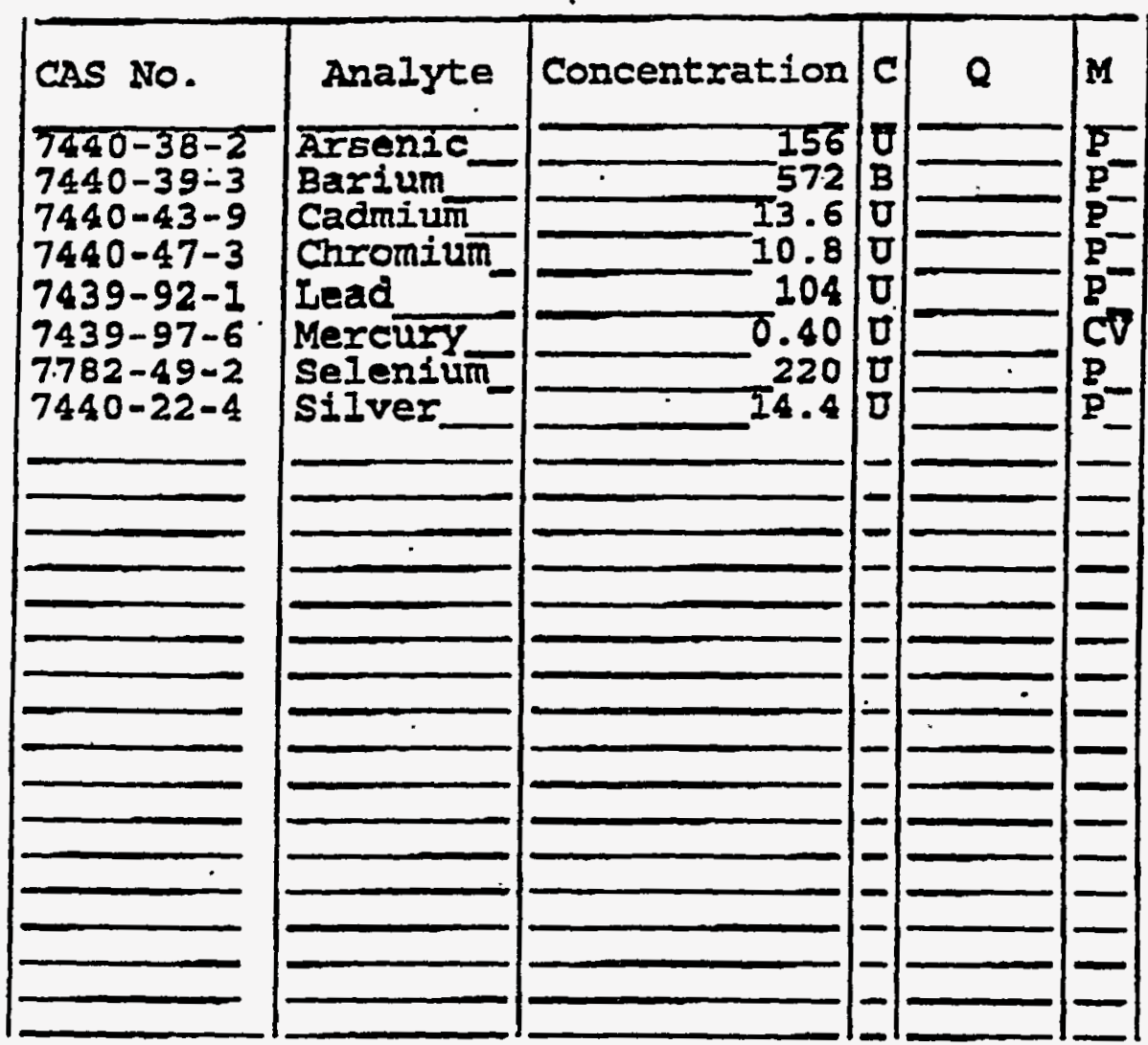

J. Before:

or After:

ntas:
Clarity Before:

Clarity After:
Texture:

Artifacto: 
10

Lag aratysts DAgA stherT
GER SAYPLE KO

$6-623-6 x-04$
Lab Name:

Inb code:

Materix

sample wt/rol:

Leve1: (Low/med)

4 Molstura: not dec.

contract: 625.01

SAS MO.: - SDG MO.: V216

Lab Sample ID: 14697-004

Iab File ID:

Date sampled;

05-19-97

Dnte Analyzed: 05-27-97

Dllution Bactor: 1.0

COKCSNIRATIOA untrs:

(ug/L of ug/Lg) De/Re 500

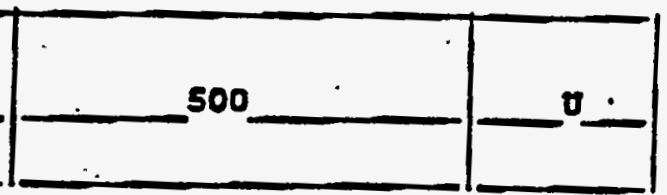

U:Concentzation of andite lo less than the value given. X: Pattern reasonably watehes gasoline

Y:Pateern appearg to be mults-poaks; but does not atach gasoline

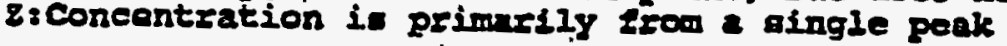

(2):Low Boiling fyerocerbon (IBE) is quantitated as if it in Gasoline. 


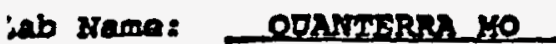

contracts

625.01

in coder Iryle cane Ko.s

Sh5 40.:

me No.: v216

Lntrix : (noil/water)

Inb sampla I0:

14697-004

sample ut/rols

$20.1 \cdot(9 / \mathrm{ml})$

Lab File mo

hrel: (10w/med)

IOST

Date sampled:

$05-19-97$

7 Malatures not dec. dac.

Dare Bxtructedz

$05-27-97$

•.

Extraction: (SepR/Cont/Sone/Shak)

$\operatorname{sen}$

Date Analyzed:

$05-28-97$

IFC Cleanup: (Y/N) N PE:

D1luelon Fnetor:

1

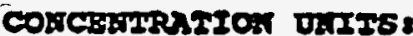

(wg/L or $\mathrm{mg} / \mathrm{Rg})$ _.

$\mathbf{2}$

cas no.

compound

$\begin{array}{r}\cdot 25 \\ \hline 25 \\ \hline\end{array}$

o

DIRST

HASTE OIT
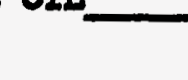

2.

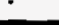

$U$ : Concentration of analyte is lave than the value given. 
IA.

VOLATILE ORGANICS ANALYSIS DATA SHEET
EPA SAMPLS NO.

$6-623-G T-04$
Iäb Name: QUANTERRA MO

L尚 Code: ITMO

Case No.:. V69701

Matrix: (soil/water) SoII

Sample wt/vol: $\quad 1.00(\mathrm{~g} / \mathrm{mL}) 0$

Level: (Low/med) IOW

\% Moisture: not dec.

GC Column: RTX-502.2 ID: $0.530 .(\mathrm{mm})$

Soil Extract Volume:

(uL)
Contract : $625-01$

SAS NO.:

SDG NO.: VZ16
Iab Sample ID: 14697-004

Iab File ID: G6585

Date Received: 05/21/97

Date Arialyzed: 06/02/97

Dilution Factor: $\quad 1.0$

Soll Aliquot Volume:
CONCENTRATION- ONITS:

(ug/I or $\mathrm{ug} / \mathrm{Kg}$ ) UG/KG

ciss No.

COMPOUND

Dichlorodifluoromethane

75-71-8--.-.--Dichlorodiflu

75-01-4-------Vinyl Chloride

75-00-3-...--.-Chloroethane.

75-09-2-....-.-Methylene Chloride

75-69-4-..---Trichlorofluoromethane

75-34-3-....--1,1-Dichloroethane

156-60-5---n.-trans-1,2-Dichloroethene

156-59-2--.---cis-1,2-Dichloroethene

107-06-2-...--1,2-Dichloroethane

594-20-7--n-- 2,2-Dich?oropropane

74-97-5-....... Bromochloromethane

71-55-6--.-.-1, 1, 1-Trichloroethane

78-93-3-...-.--2-Butanone.

56-23-5-....-.-Carbon Tetrachloride

563-58-6------ 1, 1-D1chloropropene

75-27-4-...-. - - Bromodichloromethane

78-87-5-..---1, 2-Dichloropropane

10061-01-5--.-cis-1,3-Dichloropropene

79-01-6-....-Trichloroethene

79-00-5-....--1,1,2-Trichloroethane

$71-43-2--\ldots----B e n z e n e$

74-95-3 - - - - Dibromomethane

10061-02-6--.--trans-1, 3-Dichloropropene

106-93-4-...--1,2-Dibromoethane

127-18-4----.--TetrachIoroethene

142-28-9-...--1,3-Dichloropropane

124-48-1-2.-.--Dibromochloromethane

108-88-3-...---Toluene.

108-90-7-.....-.-Chlorobenzene

50

50

50

50

6

25

25

25

25

25

25

25

25

17

25

25

25

25

25

25

25

25

25

25

25

25

25

25

25

25 
Name: QUANTERRA MO

Cude: ITMO , Case NNO.: V69701. SAS NO.: i : (soil/water) soIL

le, wt/vol:

$1.00(\mathrm{~g} / \mathrm{mL}) \theta$

i) (Iow/med) LOW

isture: not dec.

if .umn: RTX-502.2 ID: 0.530 (mm)

Extract Volume:

(uIs)
Contract: 625-01

SDG NO.: V216

Lab Sample ID: 14697-004

Lab FIIe ID: . G6585

Date Received: .05/21/97

Date Analyzed: 06/02/97

Dilution Factor: $\quad 1.0$

Soil Aliquot volume:

(uL)

CONCENTRATION UNITS:

CAS NO. C COMPOUND $(\mathrm{ug} / \mathrm{L}$ or ug/ $\mathrm{Kg}$ ) UG/RO

Q

630-20-6-n-.-1,1,1,2-Tetrachloroethane

100-42-4...-..--Ethylbenzene

100-42-5-...-.--styrene

75-25-2-..- - Bxomoform

$108-38-3-\ldots---0-x y l e n e$

1330-20-7---.--m,p-XYlenes

108-86-1-n-.----Bromobenzene

79-34-5-n...-1,1,2,2-Tetrachloroethane

96-18-4-...-.-1,2,3-Trichloropropane

95-49-8-......-2-Chlorotoluene

.106-43-4---n-4-chlorotoluene

541-73-1-2.-- - 1, 3-Dichlorobenzene

106-46-7-n..--1,4-Dichlorobenzene

95-50-1-....-1,2-Dichlorobenzene

\begin{tabular}{l|l}
25 & $\square$ \\
25 & $U$ \\
25 & $U$ \\
25 & $U$ \\
25 & $U$ \\
25 & $U$ \\
25 & $U$ \\
25 & $U$ \\
25 & $U$ \\
25 & $U$ \\
25 & $U$ \\
25 & $U$ \\
25 & $U$ \\
25 & $U$
\end{tabular}




\section{U.S. EPA - CLP}

1

INORGANIC ANALYSES DATA SHEET

Contract: 625.01

SAS NO.:

EEA SAMPLE NOO.

Jab Name: QUANTERRA_MO.

ab Códe: ITMO - Case No.:

latrix (soil/water) : WATER

sevel (low/med) : IOW_

; Solids:

0.0

Concentration Dnits (ug/I or $\mathrm{mg} / \mathrm{kg} d r y$ weight) : $\sigma G / L_{-}$

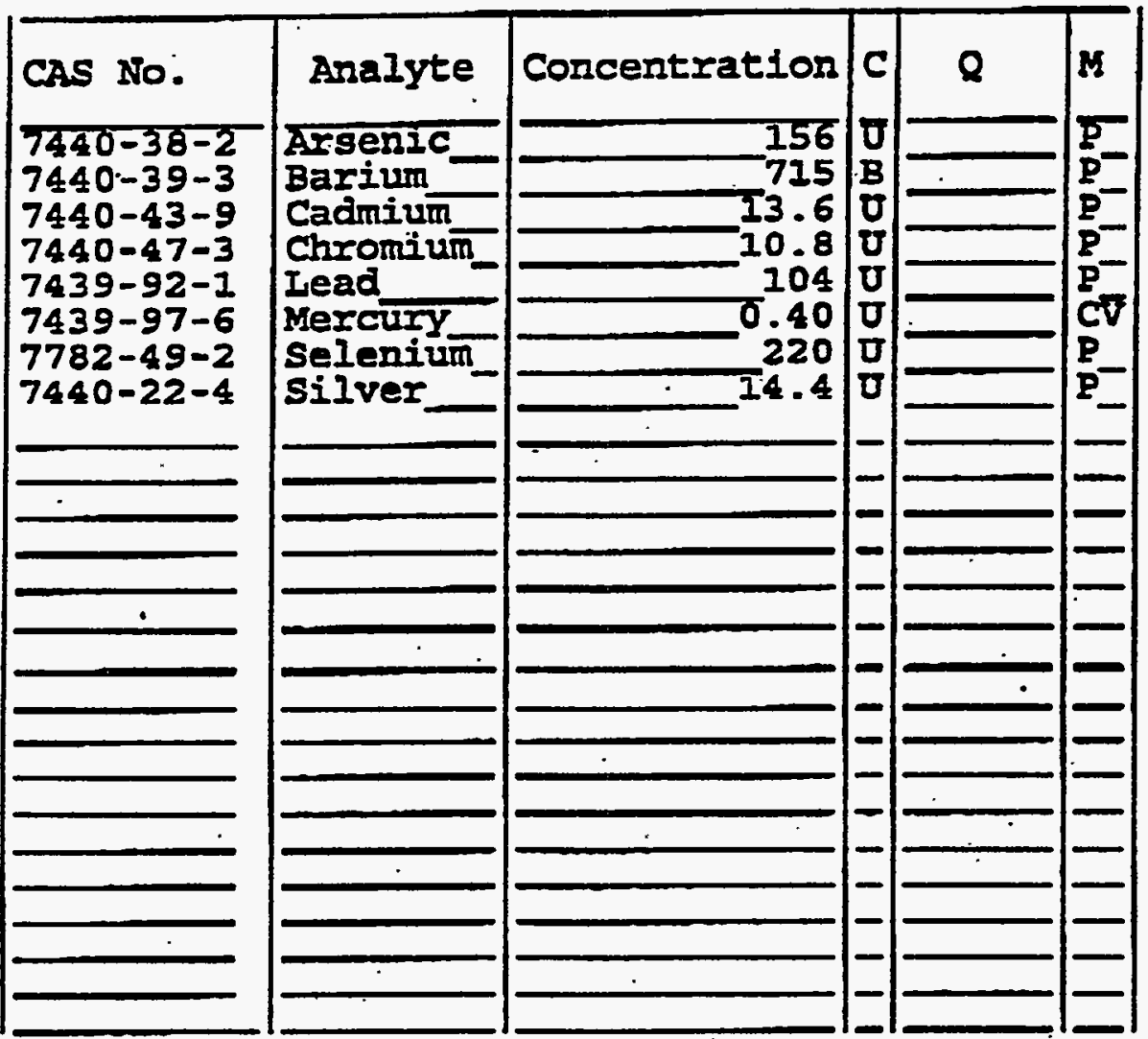

olor Before:

Clarity Before:

Clarity After:
Lab Sample ID: P1469̣7-004

Date Received: 05/21/97 olor After:

omments : 
PPA SAYPRT No. $6-623-6 x-05$

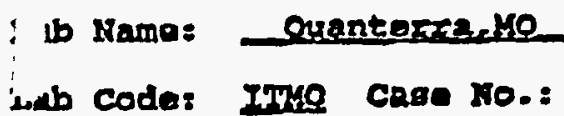

"itrix : (8011/water) sorf

-imple wt/vol: $2,0 \quad\left(g / \mathrm{ml}^{\circ} \mathrm{G}\right.$

- ovedi (low/mad) I0si

Yolstura: not doc.

$\bullet$

Chs no. contract: 625.01

SDG $80 .=$ Y216

SAS No.:

tab sample ID: 14697-005

Iab rile ID:

Date sumpled: 05-19-97

Date Analyzed: 05-28-97

Dilution Eactor: 1 le

IBE ' (1)

500

iv

CORCRATRATIOA ONITS:

$(u g / L$ or ug/Rg) De/RT

8

$500+5$

U: Concentration of analyte is lese than the value given.

$X$ : Pattorn reasonably matcher ganoline

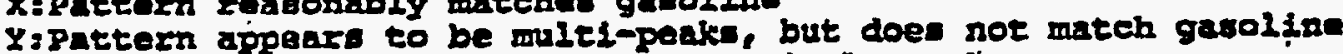

$z$ : Concencrattion is primarily fxom a single pear

(1) LLow Boiling Bydrocarbon (2BE) is quantitated an if it is Gasoline. 
ID

HeB MNRISIS DAM SHEET
GPA SAYPRE Ro. 6-623-62-05

Inb Name: ODARPERRA KO

Contract: 625.01

Iab coder Inyo care Ho.:

ans Ho.:

SDE 110.2 v216

Iab Sample ID:

24697-005

Matrix \& (noil/wator)

sotr.

Iab FL1e ID:

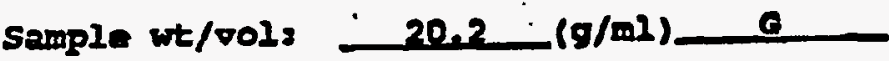

Ieval: (10w/mad)

Ion:

Deee sarpleds

05-19-97

Date Exeracted:

$05-27-97$

- Mosoture: not dec. dac.

Date Analyzed:

$05-28-97$

Extractionj (SepF/Cont/Sone/shar) senR

Dilution Factorz

1

COACHNTRATIOA DAITS:

CAS NO.

Compound

(mg/L or mg/Tg)

molkeg

$\boldsymbol{Q}$

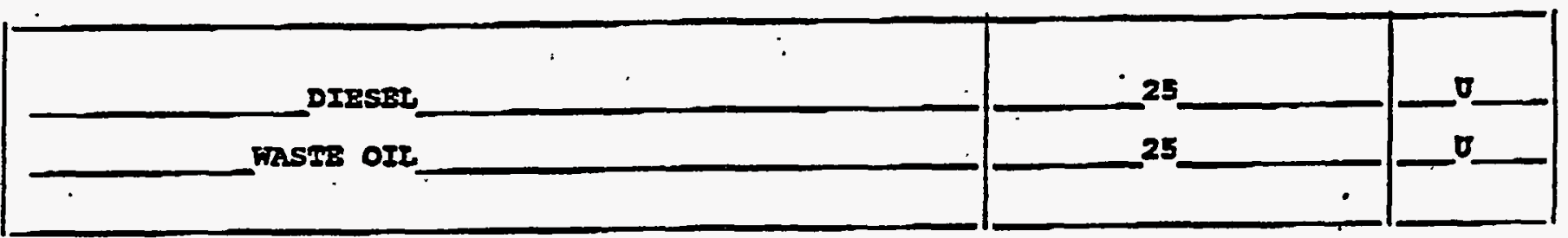

0: Concantration of analyte if $18 s$ than the value given. 
Name: QUANTERRA MO

Lrde: ITMO

Case NO.: V69701

rf s: (goil/water) soII

sle wt/vol:

$1.00(\mathrm{~g} / \mathrm{mL}) \mathrm{G}$

$\therefore:$ (Iow/med) LOW

jisture: not dec.

I Lumn: RTX-502.2 ID: 0.530 (m)

L Extract Volume:

(uI)
Contract: $625-01$

SAS NO.:

SDG NO.: V2:16

Iab Sample ID: 14697-005

Iab File ID: $\quad \mathbf{6 6 5 8 6}$

Date Received: $05 / 21 / 97$

Date Analyzed: 06/02/97

Dilution Factor: $\quad 1.0$

Soll Aliguot Volume:

(uL)

CONCENTRATION ONITS:

CAS N NO.

COMPOUND

(ug/I or ug/Rg) UG/RG

\begin{tabular}{|c|c|c|}
\hline 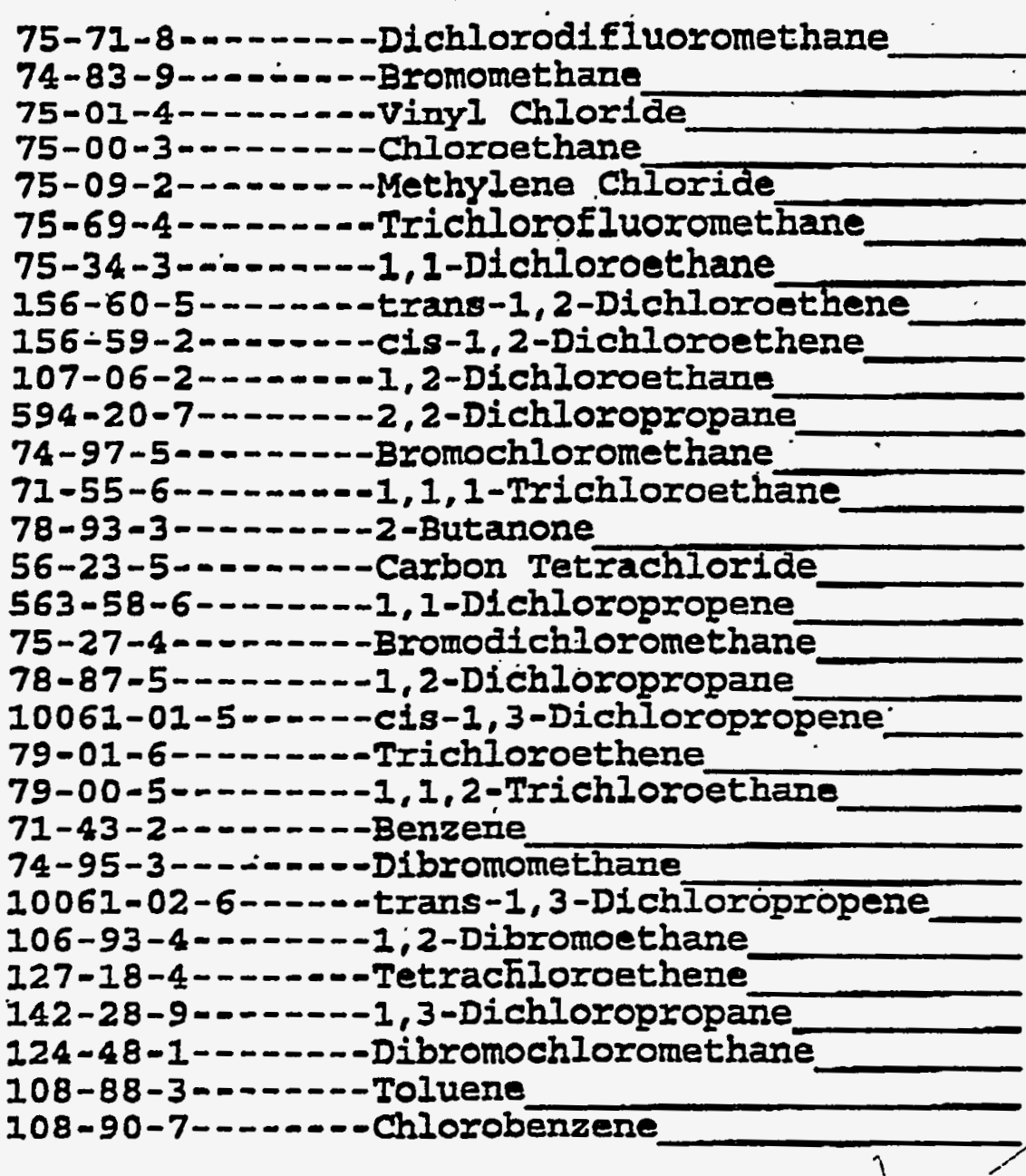 & $\begin{array}{cc} & 50 \\
& 50 \\
f_{1} & 50 \\
& 50 \\
& 25 \\
& 25 \\
& 25 \\
25 \\
25 \\
25 \\
25 \\
25 \\
25 \\
25 \\
100 \\
25 \\
25 \\
25 \\
25 \\
25 \\
28 \\
25 \\
25 \\
25 \\
25 \\
25 \\
25 \\
25 \\
25 \\
25 \\
25\end{array}$ & $\begin{array}{l}0 \\
0 \\
U \\
0 \\
0 \\
0 \\
0 \\
0 \\
0 \\
0 \\
0 \\
0 \\
0 \\
0 \\
U \\
0 \\
0 \\
0 \\
0 \\
x \\
0 \\
0 \\
0 \\
0 \\
0 \\
0 \\
U \\
0 \\
u \\
0\end{array}$ \\
\hline
\end{tabular}


$1 A$.

VOLATILE. ORGANICS ANALYSIS DATA SHEET
EPA SAMPIE NO.

$6-623-G T-05$
Lab Name: QUANTERRA MO

Lab Code: ITMO

Case No.: V69701

Matrix: (soil/water)-soII

Sample wt/vol:

$1.00(\mathrm{~g} / \mathrm{mL}) \mathrm{G}$

Ievel:

(low/med) LOW

f Moisture: not dec.

GC Column: RTX-502.2 ID: $0.530^{\circ}$ (mm)

Soil Extract Volume:

CAS NO.

630-20-6-....--1,1,1,2-Tetrachloroethane

100-41-4-...--.-Ethylbenzene

100-42-5-...-.--Styrene

75-25-2--.-.---Bromoform

108-38-3----- - - - Xylene

$1330-20-7-\cdots--m, p-x y l e n e s$

108-86-1-.-----Bromobenzene

79-34-5-..---1, 1, 2,2-Tetrachloroethane

96-18-4-...---1,2,3-Trichloropropane

95-49-8-..----2-Chlorotoluene

106-43-4--.---4-Chlorotoluene

541-73-1-------1,3-Dichlorabenzene

106-46-7------1,4-Dichlorobenzene

95-50-1-.---- 1, 2-Dichlorobenzerie
Contract: $625-01$

SDE NO.: V216

Iab Sample ID: 14697-005

Lab File ID: $\quad$ G6586

Date-Received: $\quad 05 / 21 / 97$

Date Analyzed: $06 / 02 / 97$

Dilution Factor: $\quad 1.0$

Soil Aliquot Volume:

(UI)
CONCENTRATION UNITS:

(ug/I or ug/Kg) UG/KG

$\mathbf{Q}$

\begin{tabular}{l|l}
25 & $U$ \\
25 & $U$ \\
25 & $U$ \\
25 & $U$ \\
25 & $U$ \\
25 & $U$ \\
25 & $U$ \\
25 & $U$ \\
25 & $U$ \\
25 & $U$ \\
25 & $U$ \\
25 & $U$ \\
25 & $U$ \\
25 & $U$ \\
\end{tabular}


オ.S. EPA - CLP

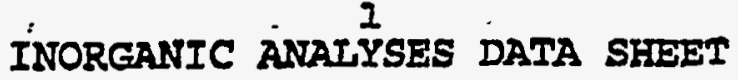

Laule: QUANTERRA_MO

ic le: INMO Case No.:

;. (soil/water) : .WATER

- (Iow/med) : IOW_

ids :

$-0.0$

Concentration Units. (ug/I or $\mathrm{mg} / \mathrm{kg} \mathrm{dry}$ weight) : UG/L_

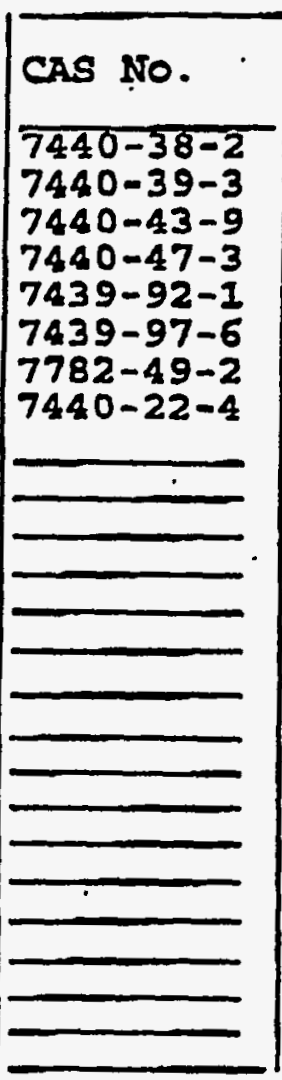

I Before:

$r$ After:

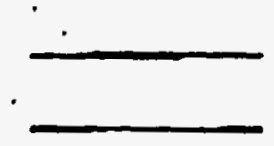

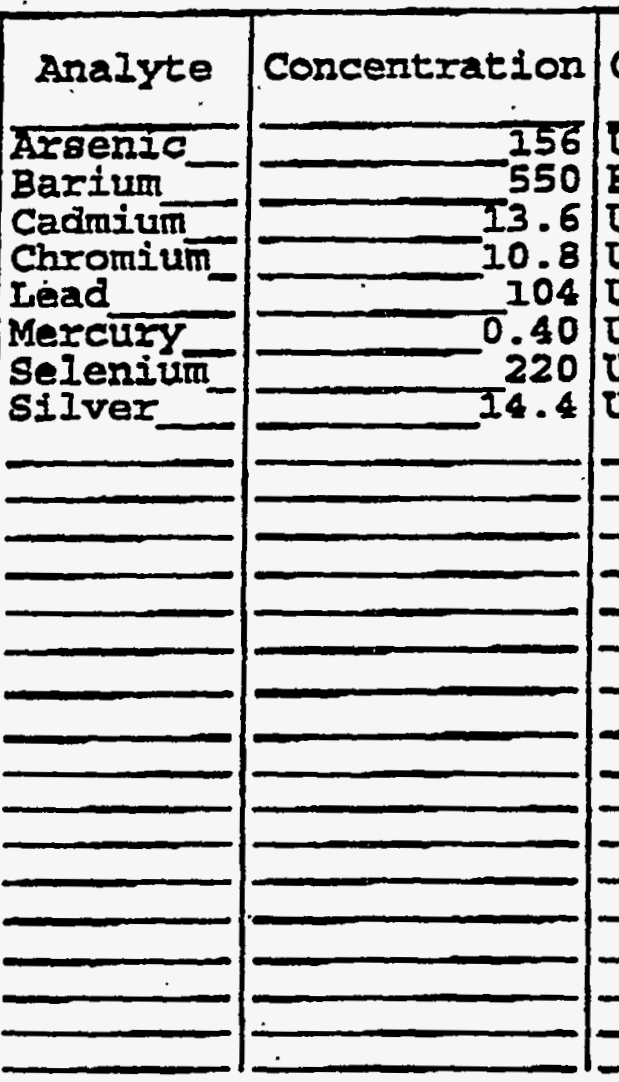

clarity Before:

Clarity After:
EPA SAMPIE NO.

$6-623-G T-05$ sas No. -

SDG NO.: V216

Iab Sample ID: P14697-005

Date Received: 05/21/97.

\section{c. JtE:}




\section{VOLUME 2 APPENDIX B}

TABLE 2 ANALYTICAL DATA 
TABLE 2: SUMMARY OF NORTH SCEP VERIFICATION RESULTS

\begin{tabular}{|c|c|c|c|c|c|}
\hline 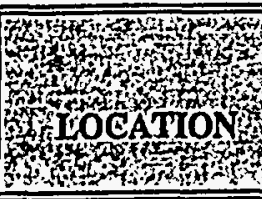 & 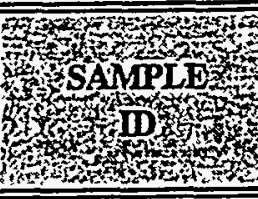 & 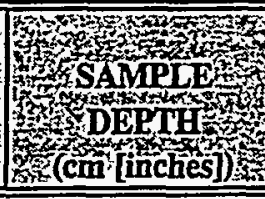 & 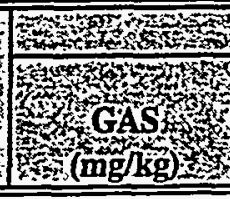 & 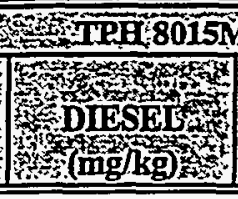 & 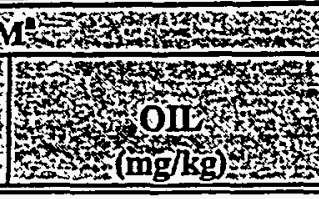 \\
\hline & SAMLLE & COLLECTE & D AUGUSI & 27,1997 & \\
\hline Cell No. 3 & NSCEP-V1-3 & $\begin{array}{c}0-15.2 \\
{[0-6]} \\
\end{array}$ & ND & ND & ND \\
\hline Cell No. 90 & NSCEP-V1-90 & $\begin{array}{c}0-15,2 \\
{[0-6]}\end{array}$ & ND & 25.5 & 148 \\
\hline , & NSĊEP-V2-90 & $\begin{array}{c}0-15.2 \\
{[0-6]} \\
\end{array}$ & ND* & ND* & 36 \\
\hline Cell No. 102 & NSCEP-V1-102 & $\begin{array}{c}0-15.2 \\
{[0-6]} \\
\end{array}$ & ND & ND & $\mathrm{ND}$ \\
\hline Cell No. 106 & $\begin{array}{l}\text { NSCEP-V1-106 } \\
\end{array}$ & $\begin{array}{c}0-15.2 \\
{[0-6]} \\
\end{array}$ & ND & ND & ND \\
\hline Cell No. 145 & NSCEP-V1-145 & $\begin{array}{l}0-15.2 \\
{[0-6]} \\
\end{array}$ & ND & ND & ND \\
\hline Cell No. 176 & NSCEP-V1-176 & $\begin{array}{c}0-15.2 \\
{[0-6]} \\
\end{array}$ & ND & 25.1 & 120 \\
\hline & NSCEP-V2-176 & $\begin{array}{c}0-15.2 \\
{[0-6]} \\
\end{array}$ & ND & ND & ND \\
\hline $\begin{array}{l}\text { Cell No. } 178 \\
\end{array}$ & NSCEP-V1-178 & $\begin{array}{c}0-15.2 \\
{[0-6]} \\
\end{array}$ & ND & ND & ND \\
\hline Cell No. 187 & NSCEP-V1-187 & $\begin{array}{c}0-15.2 \\
{[0-6]}\end{array}$ & $\mathrm{ND}$ & ND & ND \\
\hline Cell No. 208 & NSCEP-V1-208 & $\begin{array}{l}0-15.2 \\
{[0-6]} \\
\end{array}$ & ND & ND & ND \\
\hline Cell No. 226 & NSCEP-V1-226 & $\begin{array}{c}0-15.2 \\
{[0-6]} \\
\end{array}$ & ND & ND & ND \\
\hline Cell No. 231 & NSCEP-V1-231 & $\begin{array}{l}0-15.2 \\
{[0-6]} \\
\end{array}$ & ND & $\mathrm{ND}$ & ND \\
\hline Cell No. 235 & NSCEP-V1-235 & $\begin{array}{c}0-15.2 \\
{[0-6]} \\
\end{array}$ & ND & ND & ND \\
\hline Cell No. 261 & NSCEP-V1-261 & $\begin{array}{c}0-15.2 \\
{[0-6]} \\
\end{array}$ & ND & ND & ND \\
\hline Cell No. 283 & NSCEP-V1-283 & $\begin{array}{c}0-15.2 \\
{[0-6]} \\
\end{array}$ & ND & ND & ND \\
\hline Cell No. 286 & NSCEP-V1-286 & $\begin{array}{c}0-15.2 \\
{[0-6]} \\
\end{array}$ & ND & ND & ND \\
\hline Cell No. 287 & NSCEP-V1-287 & $\begin{array}{c}0-15.2 \\
{[0-6]}\end{array}$ & ND & ND & ND \\
\hline Cell No. 300 & NSCEP-V1-300 & $\begin{array}{c}0-15.2 \\
{[0-6]}\end{array}$ & ND & ND & ND \\
\hline
\end{tabular}


TABLE 2: SUMMARY OF NORTH SCEP VERIFICATION RESULTS

\begin{tabular}{|c|c|c|c|c|c|}
\hline 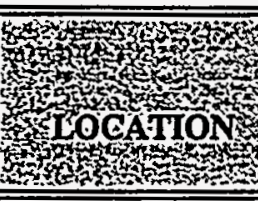 & 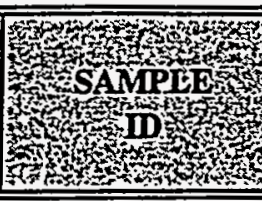 & 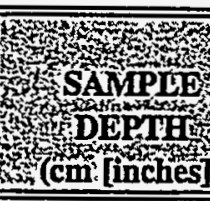 & 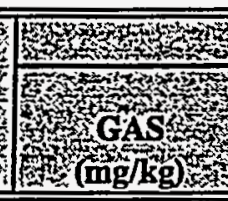 & 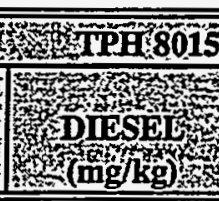 & 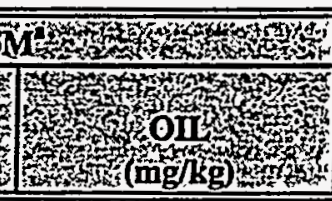 \\
\hline & SAMPLE & $\overline{\text { COLLEC }}$ & D AUGUS & {$[.27,1997$} & \\
\hline Cell No. 322 & NSCEP-V1-322 & $\begin{array}{c}0-15.2 \\
{[0-6]}\end{array}$ & $\mathrm{ND}$ & $\mathrm{ND}$ & ND \\
\hline Cell No. 323 & NSCEP-V1-323 & $\begin{array}{c}0-15.2 \\
. \quad[0-6] \\
\end{array}$ & ND & ND & ND \\
\hline Cell No. 332 & NSCEP-V1-332 & $\begin{array}{l}0-15.2 \\
{[0-6]}\end{array}$ & ND & ND & $\mathrm{ND}$ \\
\hline & NSCEP-V2-332 & $\begin{array}{c}0-15.2 \\
{[0-6]}\end{array}$ & ND & ND & ND \\
\hline Cell No. 368 & NSCEP-V1-368 & $\begin{array}{l}0-15.2 \\
{[0-6]}\end{array}$ & ND & ND & $\overline{N D}$ \\
\hline Cell No. 384 & NSCEP-V1-384 & $\begin{array}{c}0-15.2 \\
{[0-6]}\end{array}$ & ND & ND & ND \\
\hline
\end{tabular}

Notes:

'TPH 8015M: Total Petroleum Hydrocarbons Method 8015 Modified.

SCEP: Steam Cleaning Effluent Pond

ND: Not Detected

cm: centimeter

$\mathrm{mg} / \mathrm{kg}$ : milligrams per kilogram

$\mathrm{ND}^{*}$ : Analyzed 2 days after holding time due to sample extraction bottle breaking. 


\section{" LABORATORIES}

\section{PETROLEUM HYDROCARBONS (TPH)}

$M$ - TPH

\begin{tabular}{ll}
\hline lient Sample ID: & NSCEP-VI-003 \\
Date Collected: & $27-A U G-97$ \\
Date Analyzed: & $19-$ SEP-97 02:54 \\
ate Extracted: & $06-$ SEP-97 \\
atrix: & Soil \\
Percent Moisture: & 7.68
\end{tabular}
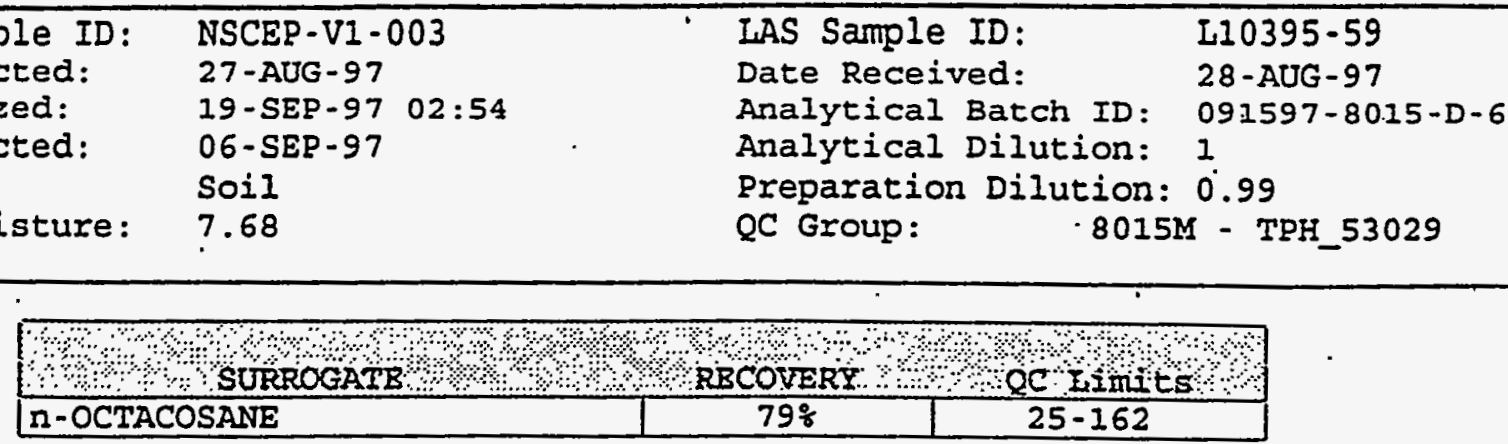

\begin{tabular}{|c|c|c|c|}
\hline $\begin{array}{l}\text { iel Range Organics } \\
\text {,1 ine Range Organics } \\
\text { y OIL }\end{array}$ & TPH & $\begin{array}{l}<32 \\
<32 \\
<32\end{array}$ & 32 \\
\hline
\end{tabular}




\section{. LABORATORIES}

\section{6}

D. PETROLEUM HYDROCARBONS (TPH)

is. - TPH

\begin{tabular}{llll}
\hline Iient Sample ID: & NSCEP-V2-90 & LAS Sample ID: & L10479-11 \\
late Collected: & 09-SEP-97 & Date Received: & 10-SEP-97 \\
Date Analyzed: & $08-0 C T-97$ 09:42 & Analytical Batch ID: 100597-8015-D-4 \\
Date Extracted: & $25-$ SEP-97 & Analytical Dilution: 1 \\
latrix: & Soil & Preparation Dilution: 1.0 \\
'ercent Moisture: & 6.88 & QC Group: & 8015M-TPH_53917 \\
\hline
\end{tabular}
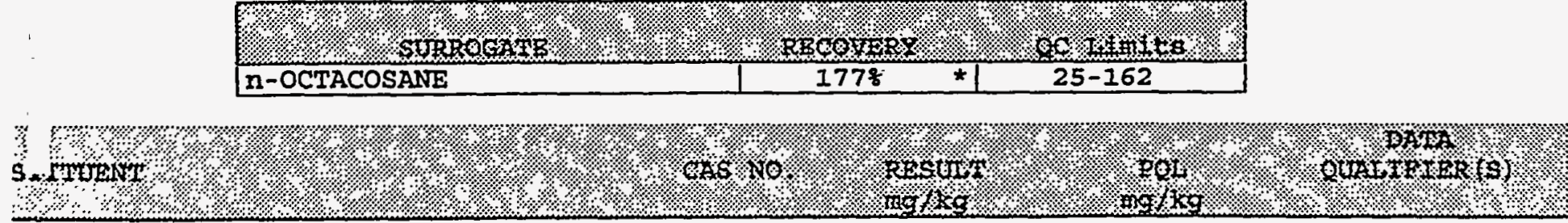

s I Range Organics oline Range Organics OR OIL
TPH

$<32$ $<32$ 260<smiles>CCCCC</smiles>

32

32.

32.

H

H

H

\section{see duth following pases}

Sample whs done using

penk to park integantion

charged to $36.3 \mathrm{mg} / \mathrm{kg}$ 


\section{Bechtel Nevada}

Facsimile No. $702-295-7996$ or -4773

Confirmation No. $702-295-7220$

\section{FACSIMILE TRANSMISSION}

Date: November 03, 1997

To: Kraig Knapp \& Jeff Smith

Company: $\mathrm{BN}-\mathrm{RP}$

Fax No. $\quad 5-7761$

From: Ted Redding Analytical Services Labọatory (702) 295-7220

No. of Pages Including Cover: 4

Message:

Kraig,

Following is the chromatogram for sample NSCEP-V2:90 reprocessed under a different integration mode; "peak to peak" instead of "common baseline". This looks to be the way several other samples were integrated, so it is a valid process:

I spoke with the GC analyst and he indicated that they could not manually integrate the baseline rise seen in all samples, so the effect of that rise on sample values cannot be determined.

Let me know how you want to proceed. If we want the revised values reported, the lab will need to submit a new Form I for the sample.

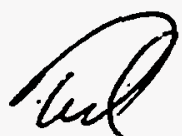




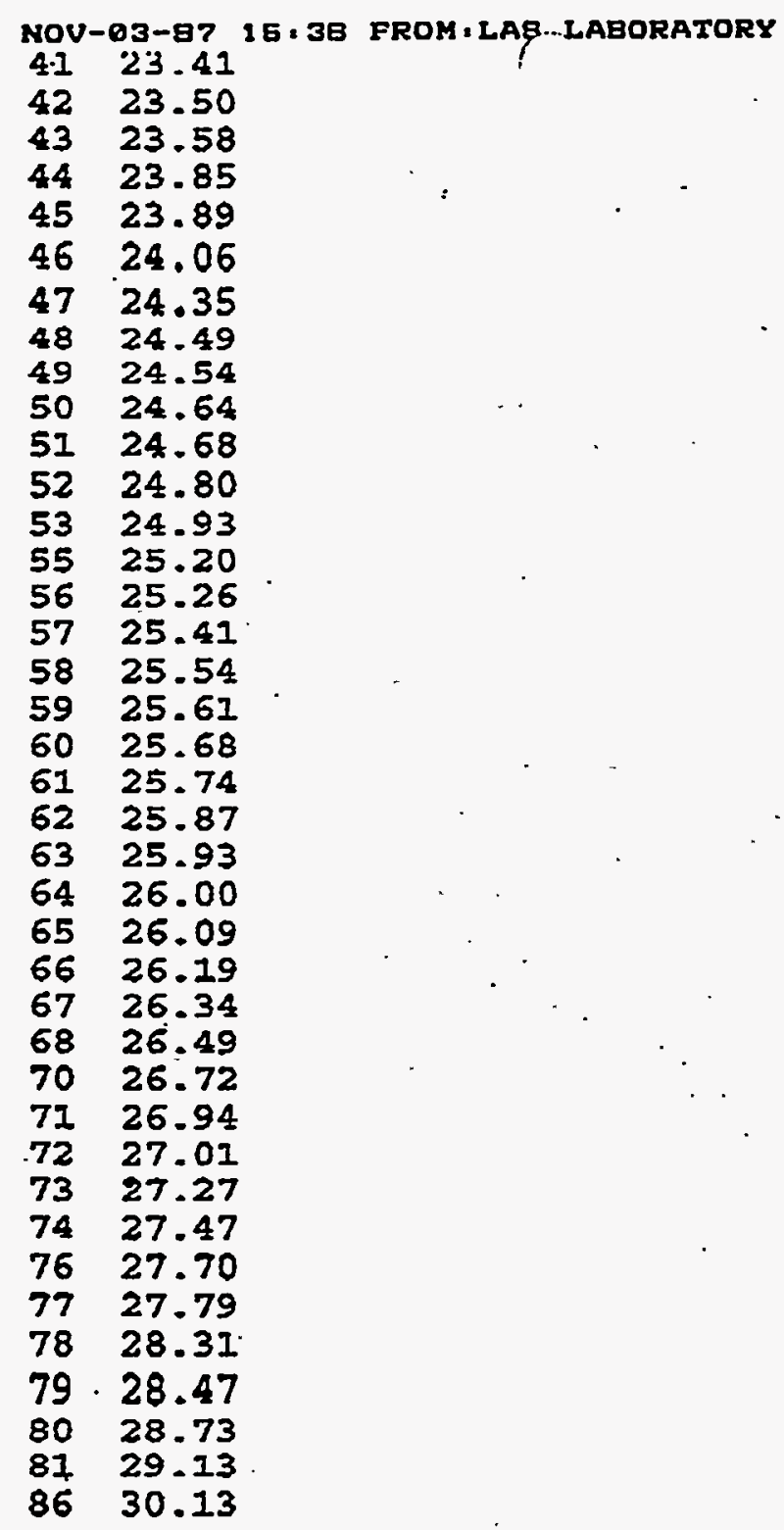

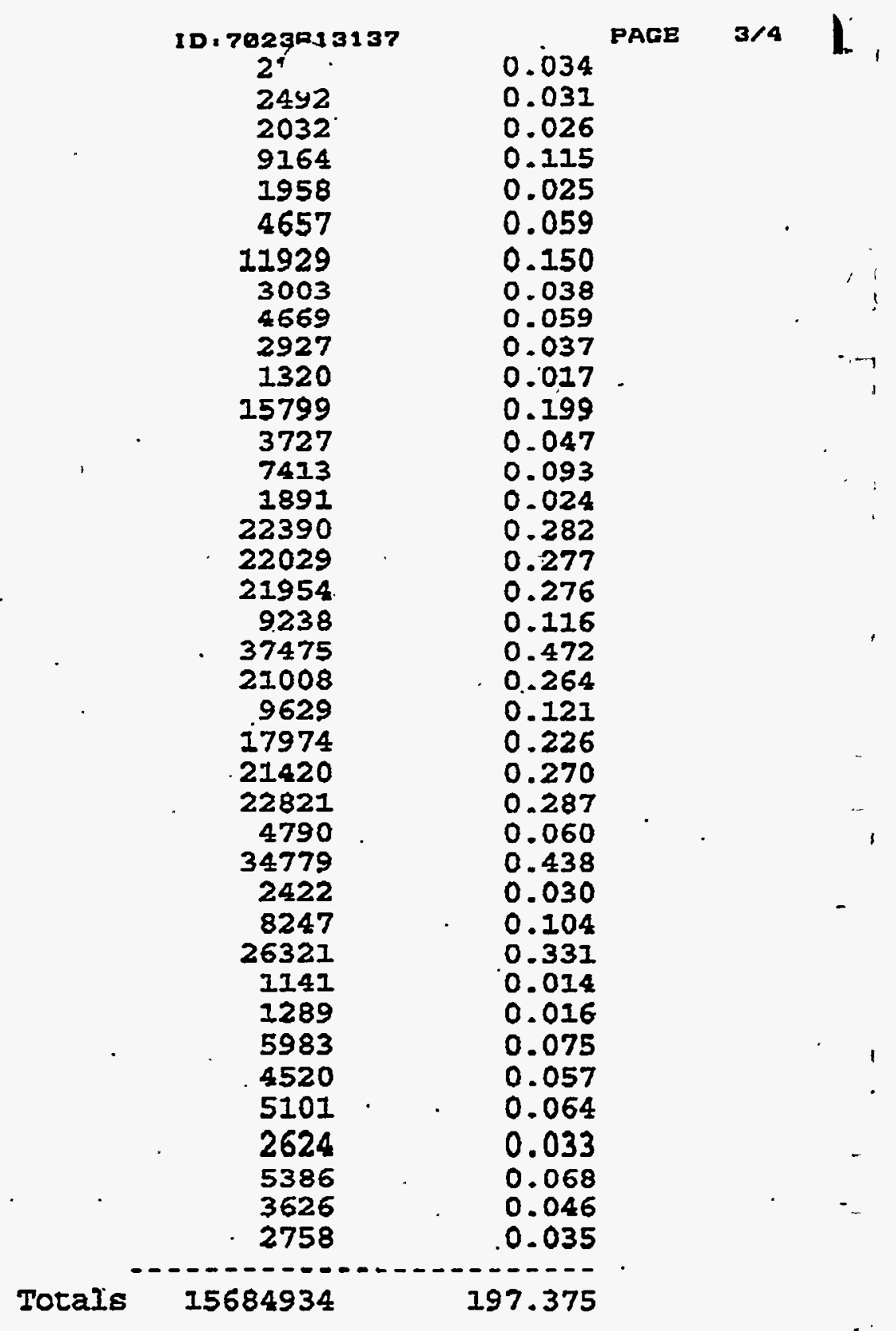

\begin{tabular}{llrr} 
\# Group Name & Amount & Area & Areaf \\
\hline 1 GASOLINE-RANGE-ORG & 0.2745 & 22032 & $0.13 \%$ \\
2 JP5-RANGE-ORG & 0.0999 & 8403 & $0.05 \%$ \\
3 KEROSENB-RANGB-ORG & 0.0370 & 3160 & $0.02 \%$ \\
4 MOTOR OIL & 5.5971 & 452031 & 2.648 \\
& 36.3 & &
\end{tabular}

$\rho A 11 / 3 / 91$

perdient roquest peark to penk intgration was used inssted of common baseline.

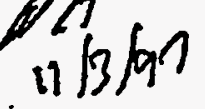


- U-03-87 15.3B FROM.LAR- TABORATORY

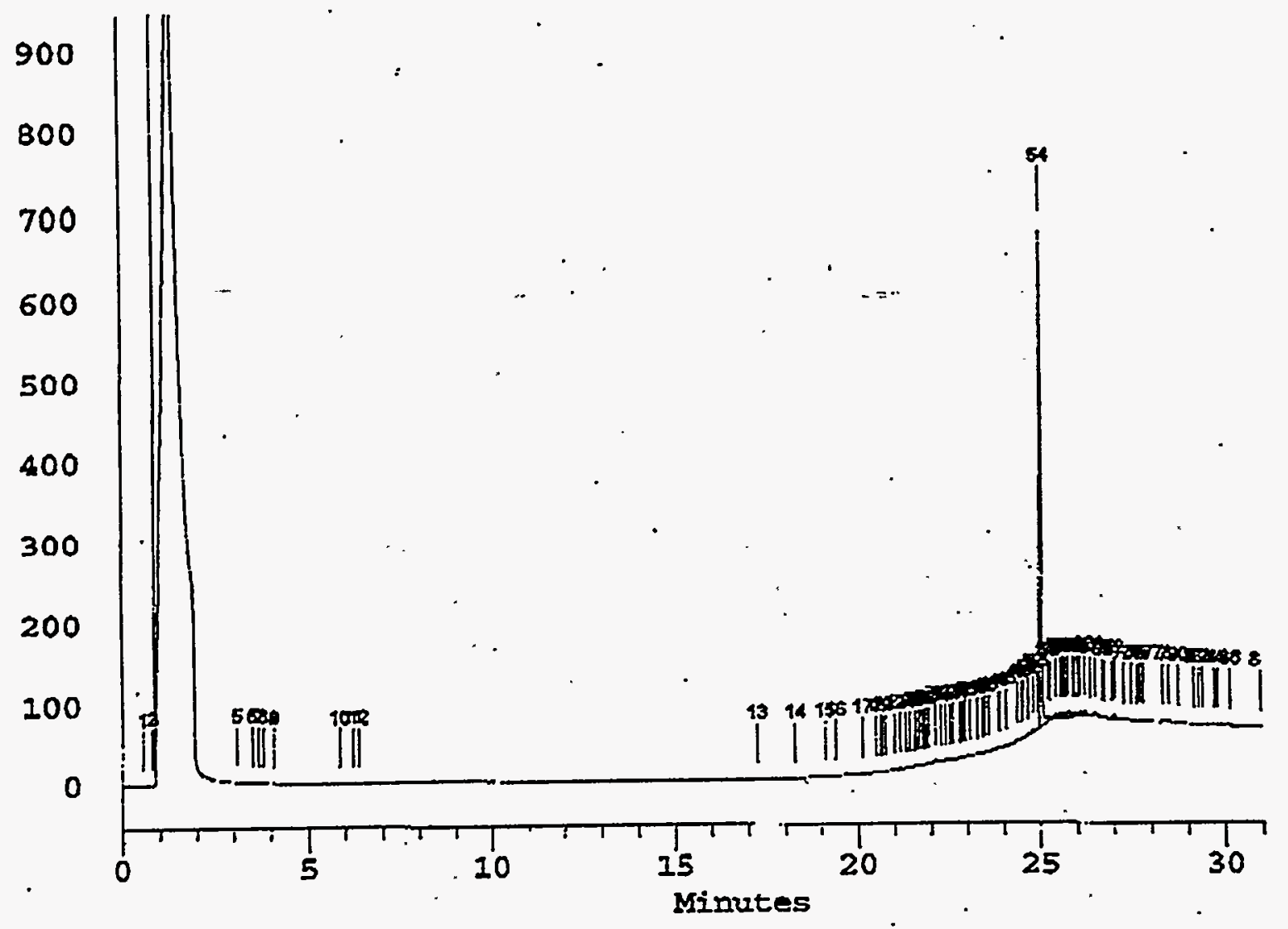


TOTAL PETROLEUM HYDROCARBONS (TPH) $8015 \mathrm{M}-\mathrm{TPH}$

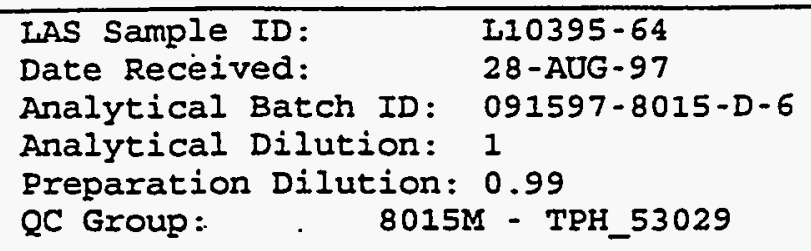

QC Group: . $\quad 8015 M$ - TPH_53029

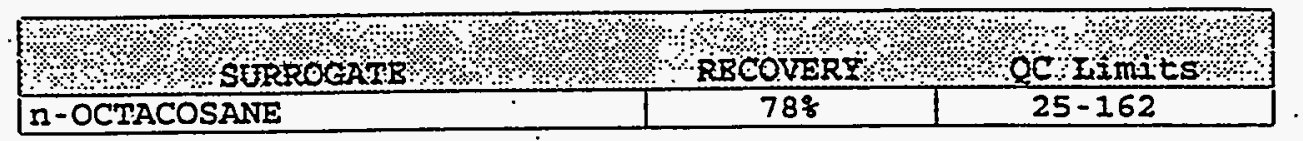

\%

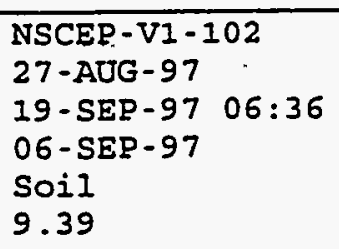

9.39

Diesel Range Organics Gasoline Range Organics MOTOR OII
TPH

$<33$.

$<33$.

$<33$.
33.

33.

33. 
I PETROLEUM HYDROCARBONS (TPH)

jW - TPH

$\begin{array}{ll}\text { Ilient Sample ID: } & \text { NSCEP-V1-106 } \\ \text { Date Collected: } & 27-\text { AUG-97 } \\ \text { Date Analyzed: } & 17-\text { SEP-97 11:05 } \\ \text { Date-Extracted: } & 07-\text { SEP-97 } \\ \text { Matrix: } & \text { Soil } \\ \text { Percent Moisture: } & 2.16\end{array}$

IAS Sample ID:

Date Received:

Analytical Batch ID: 091597-8015-D-4

Analytical Dilution: 1

Preparation Dilution: 0.99

QC Group:
L10395-82

28 -AUG - 97

$8015 M$ - TPH_53132

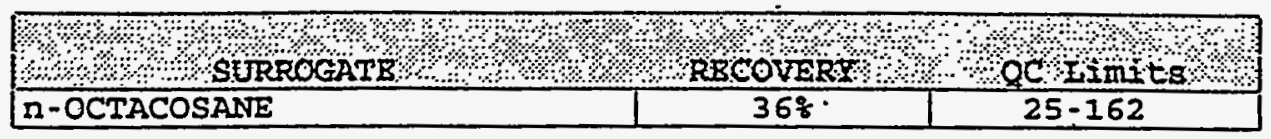

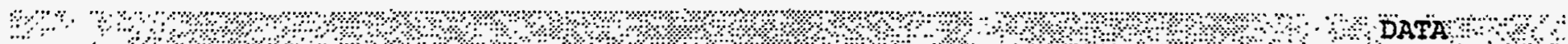
SITUENT:

CAS $10 .+$ pesuts. PQLT: QUTATIPIER $(S)$

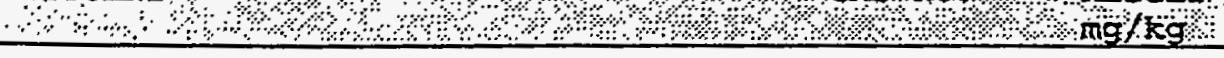
$\mathrm{mig} / \mathrm{kg}$

- I Range Organics oline Range Organics 'QR OIL
TPH

$<30$. $<30$. $<30$.
30 .

30 .

30 . 


\section{LAS LABORATORIES}
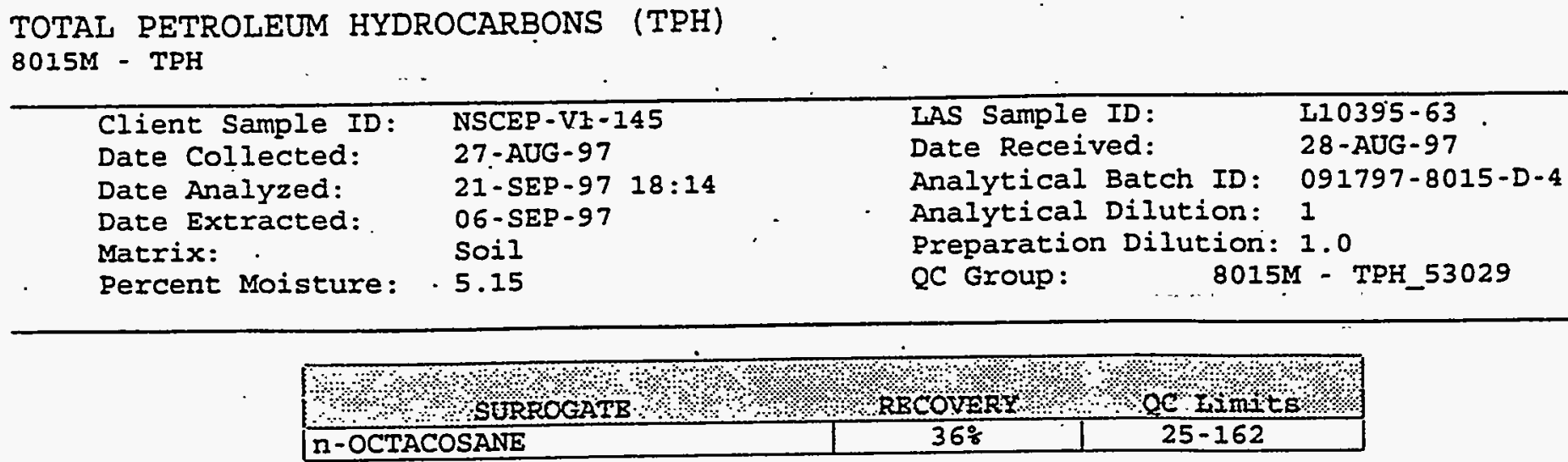

Diesel Range Organics Gasoline Range Organics MOTOR OIL
TPH

$<32$.

$<32$.

$<32$.

32.

32 .

32 . 


\section{LABORATORIES}
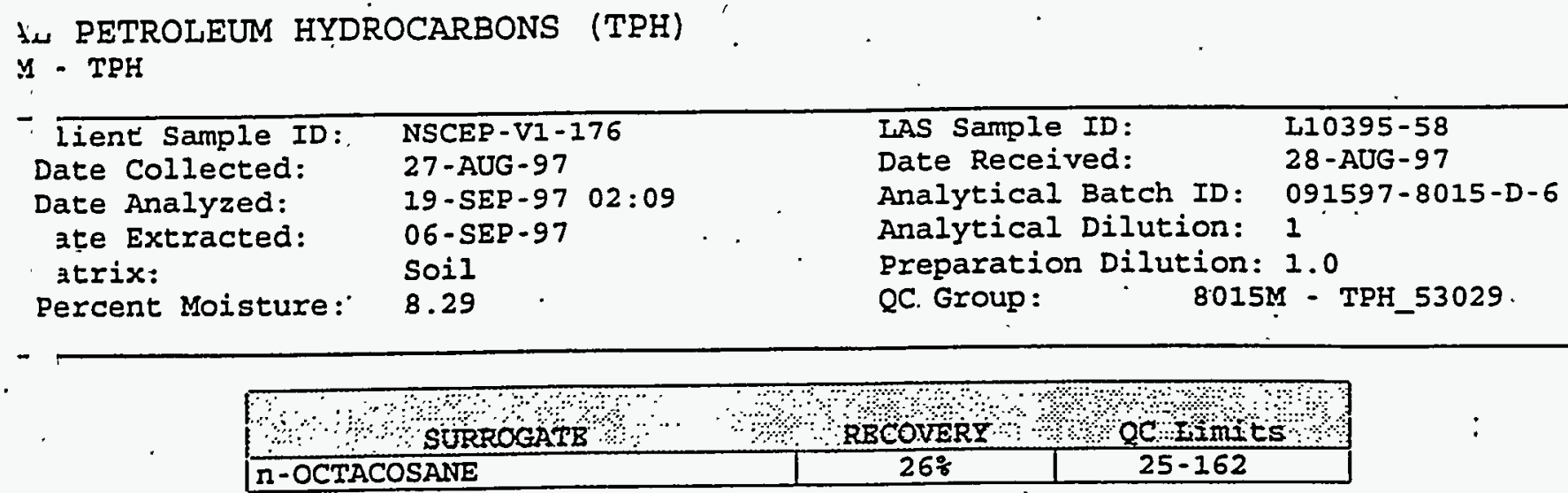

el Range Organics

I ine Range Organics

F OII
TPH

66.
$<33$.
130

33.

33.

33. 
TOTAL PETROLEUM HYDROCARBONS (TPH) BO15M - TPH $=$

$\begin{array}{ll}\text { Client Sample ID: } & \text { NSCEP-V2-176 } \\ \text { Date Collected: } & 09-\text { SEP-97 } \\ \text { Date Analyzed: } & 25-\text { SEP-97 } \\ \text { Date Extracted: } & 12-\text { SEP-97 } \\ \text { Matrix: } & \text { Soil } \\ \text { Percent Moisture: } & 12.24\end{array}$

IAS Sample ID:

Date Received:

Analytical Batch ID: 091797-8015-D-6

Analytical Dilution: 1

Preparation Dilution: 0.99

QC Group:
$8015 \mathrm{M}$ - TPH_53397
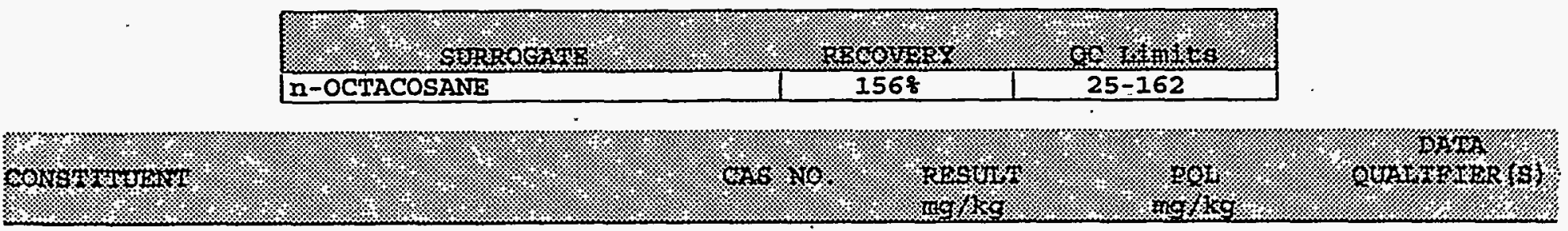

Diesel Range Organics Gasoline Range Organics MOTOR OIL
TPH

$$
\begin{aligned}
& <34 . \\
& <34 . \\
& <34 .
\end{aligned}
$$

34.

34.

34. 


\section{Ij LABORATORIES}

TAL PETROLEUM HYDROCARBONS (TPH)

5M - TPH

- Client Sample ID: Date Collected: Date Analyzed:

Date Extracted:

Matrix:

Percent Moisture:

NSCEP-V1-178
27 -AUG- 97
$19-$ SEP- $97 \quad 03: 38$
06 -SEP-97
SOI1
8.89

NSCEP - V1-178

27-AUG-97

19-SEP-97 $03: 38$

Soil

8.89

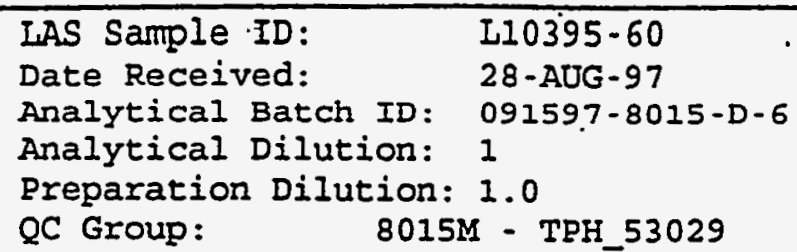

IAS Sample ID:

Date Received:

Analytical Batch ID: 091597-8015-D-6

Analytical Dilution: 1

Preparation Dilution: 1.0

QC Group:

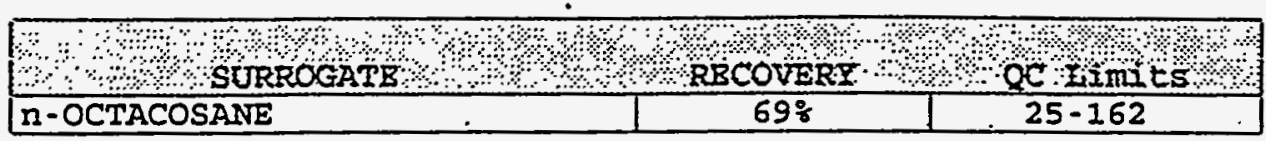

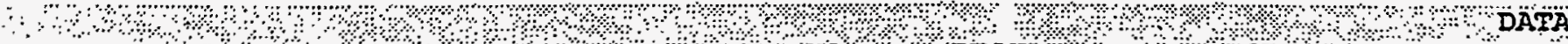
ISTITUENT

RESULT

PQ4 3 . QUAUTEIER (S)

$-$

ssel Range Organics s' ine Range Organics

$\mathrm{mg} / \mathrm{kg}$

$Y$ ! OII

$\begin{array}{cll}\text { TPH } & <33 . & 33 . \\ \cdot & <33 . & 33 . \\ & <33 . & 33 .\end{array}$


TOTAL PETROLEUM. HYDROCARBONS (TPH) 8015M - TPH

$\begin{array}{ll}\text { Client Sample ID: } & \text { NSCEP-V1-187 } \\ \text { Date Collected: } & 27-\text { AUG }-97 \\ \text { Date Analyzed: } & 18-\text { SEP-97 11:12 } \\ \text { Date Extracted: } & 07-\text { SEP-97 } \\ \text { Matrix: } & \text { Soil } \\ \text { Percent Moisture: } & 7.26\end{array}$

LAS Sample ID: Date Received: Analytical Dilution: 1 QC Group:

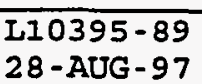

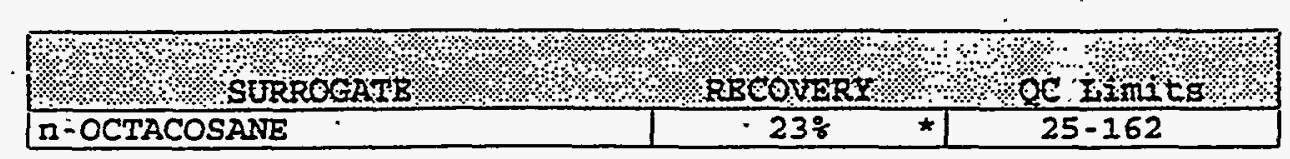

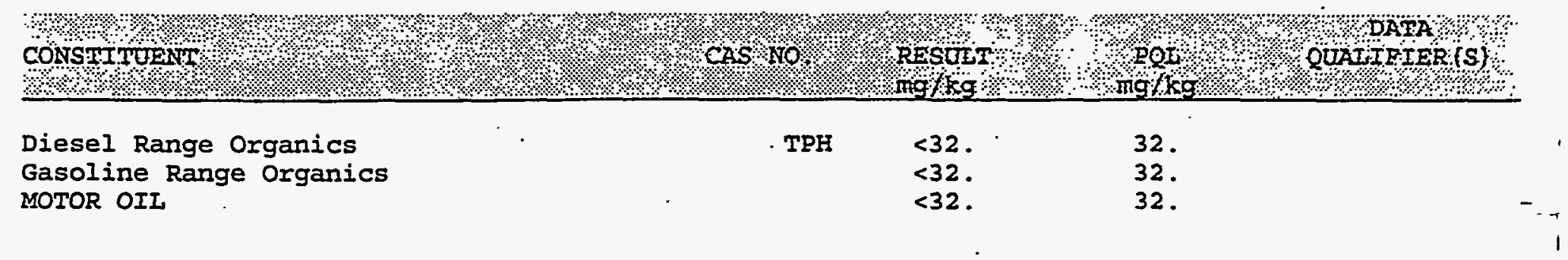

\begin{tabular}{lllll}
\hline IJ7682STANDARD & N & R20472 & Page 1 & .
\end{tabular}




\section{¿ LABORATORIES}

L PETROLEUM HYDROCARBONS (TPH)

1. TPH

- Iient Sample ID: Date Collected:

nzte Analyzed: ite Extracted: atitix:

percent Moisture:

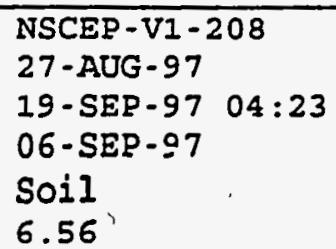

6.56

LAS Sample ID: II0395-6I

Date Received: - 28-AUG-97

Analytical Batch ID: 091597-8015-D-6

Analytical Dilution: 1

Preparation Dilution: 1.0

QC Group: $\quad 8015 M$ - TPH_53029

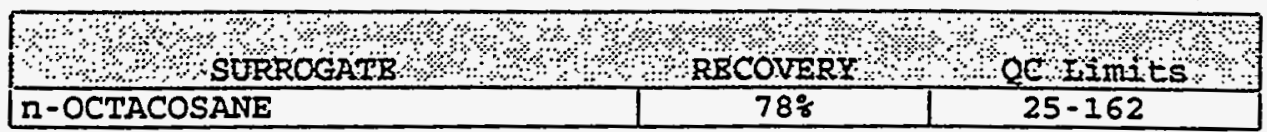

$\because \because 3$

TTiving

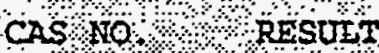

DATAัด

-

el Range Organics

1. 'ne Range Organics

1 OIL
TPH

$<32$.

$<32$.

$<32$. $25-162$

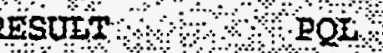

32.

32 .

32 . 


\section{LAS LABORATORIES}

TOTAL PETROLEUM HYDROCARBONS (TPH)

8015M - TPH

$\begin{array}{ll}\text { Client Sample ID: } & \text { NSCEP-VI-226 } \\ \text { Date Collected: } & 27-\text { AUG-97 } \\ \text { Date Analyzed: } & 19-\text { SEP-97 05:07 } \\ \text { Date Extracted: } & 06-\text { SEP-97 } \\ \text { Matrix: } & \text { SOil } \\ \text { Percent Moisture: } & 6.14\end{array}$
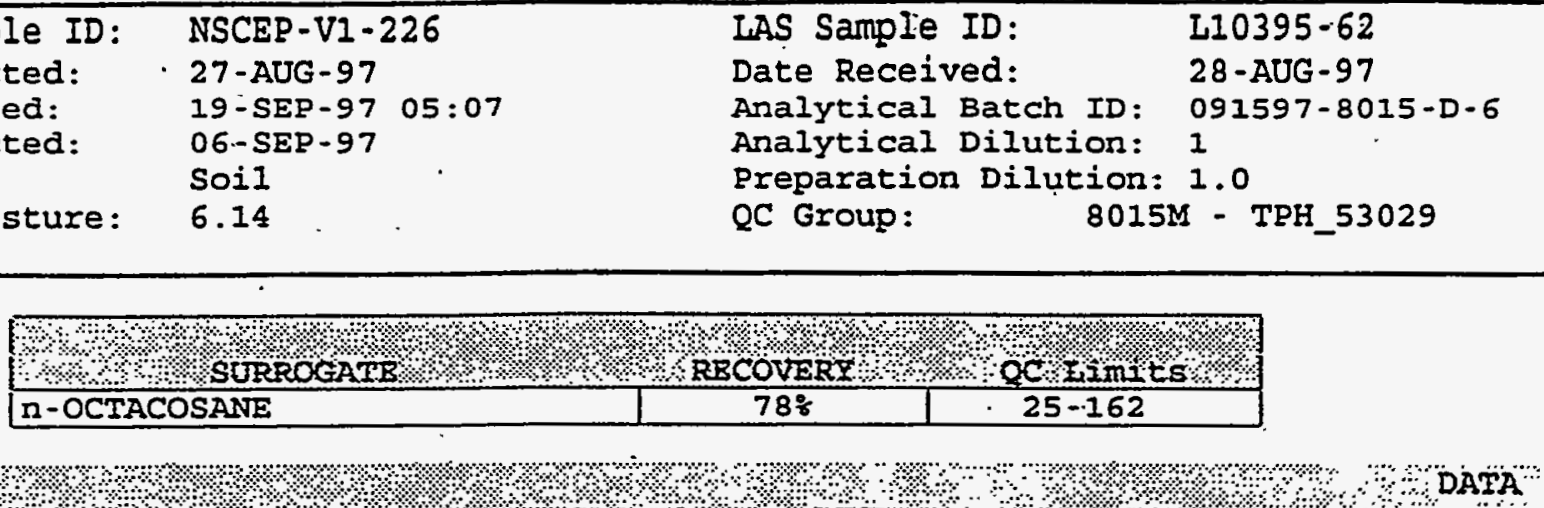

$<32$.

$<32$.

$<32$.

32 .

32 .

32 . 


\section{^`LABORATORIES}

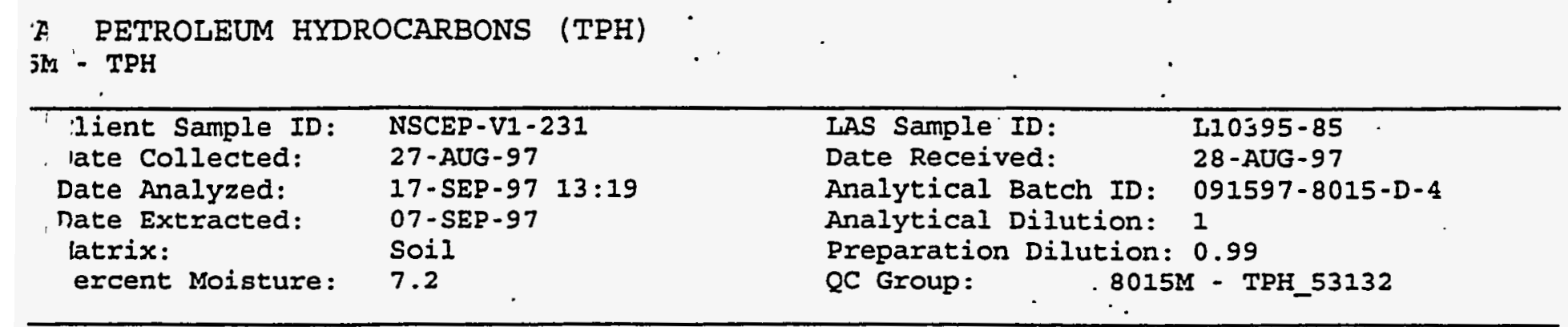

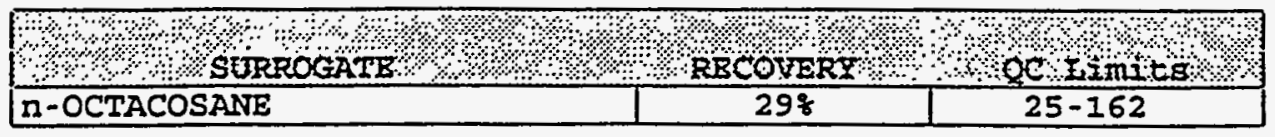

S

s . Range Organics

oline Range Organics

OD OIL
TPH

$<32$.

$<32$.

$<32$.

32.

32 .

32 . 


\section{LAS LABORATORIES}

TOTAL PETROLEUM HYDROCARBONS (TPH) 9015M - TPH

Client Sample ID: Date Collected: . Date Analyzed: Date Extracted: Matrix:

Percent Moisture:

NSCEP-VI-235

$27-A U G-97$

17-SEP-97 $15: 32$

$07-S E P-97$

Soil

3.24
IAS Sample ID:

Date Received:

Analytical Batch ID:

Analytical Dilution:

Preparation Dilution: 0.99

QC Group:

\section{L10395-88}

28 -AUG - 9.7

$091597-8015-D-4$

1

8015M - TPH_53132

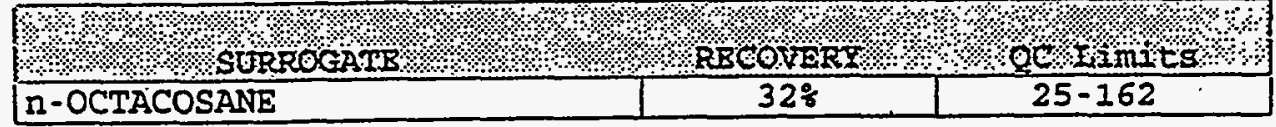

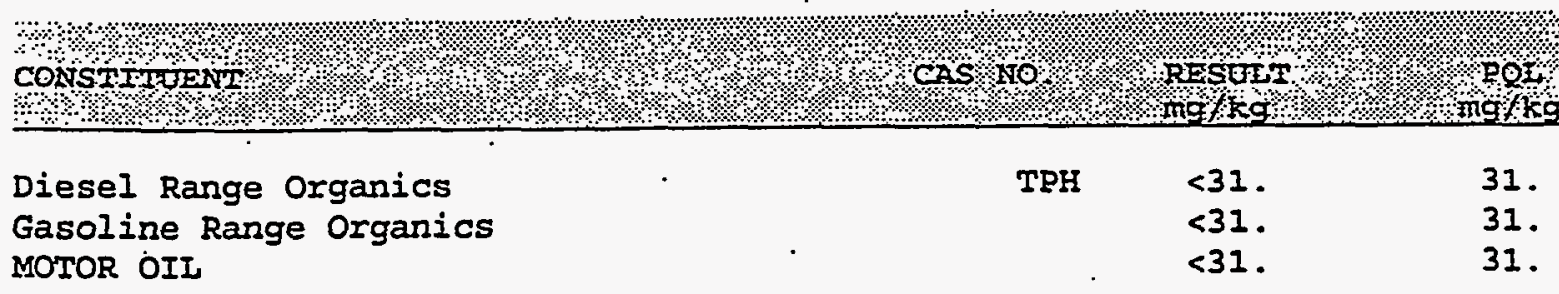

31.

31. 


\section{1 ; LABORATORIES}

$j$ PETROLEUM HYDROCARBONS . (TPH)
iM - TPH

\begin{tabular}{ll}
\hline :lient Sample ID: & NSCEP-V1-261 \\
Jace Collected: & $27-$ AUG-97 \\
Date Analyzed: & $20-$ SEP-97 19:36 \\
Jate Extracted: & $07-$ SEP-97. \\
latrix: & SOil \\
Lercent Moisture: & 5.87
\end{tabular}

I.AS Sample ID:

Date Received:

Analytical Batch ID:

Analytical Dilution:

Preparation Dilution: 0.99

QC Group: $\quad 8015 \mathrm{M}$ - TPH_53158.

L10395-94

28 -AUG-97

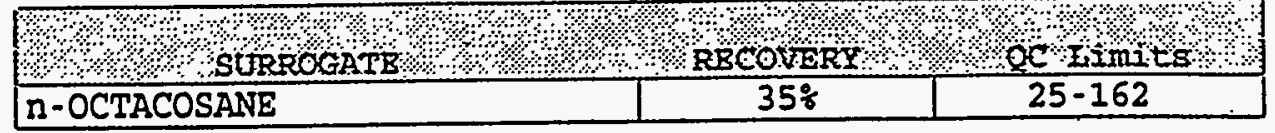

$091797-8015-D-2$

S- 1 Range Organics Jline Range Organics

I OIL

\section{TPH}

$<32$

$<32$.

$<32$. 


\section{LAS LABORATORIES}

TOTAL PETROLEUM HYDROCARBONS (TPH)

8015M - TPH

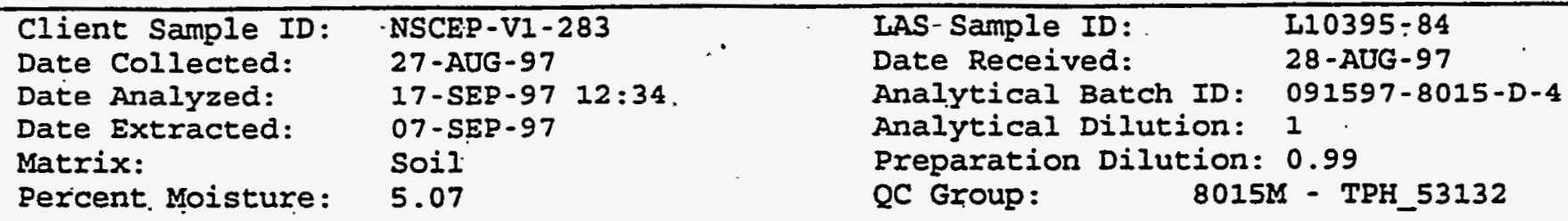

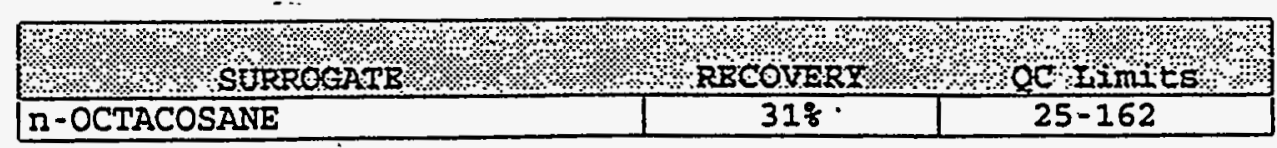

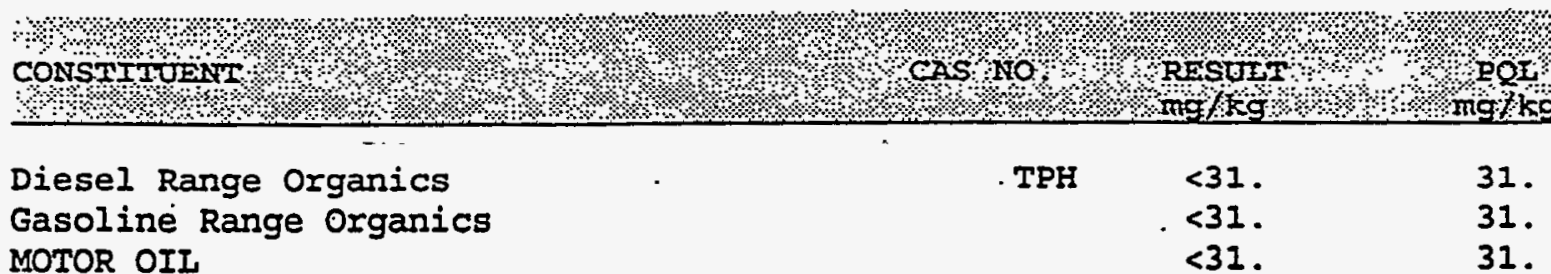

31.

31. 


\section{I ) LABORATORIES}

$\therefore$ PETROLEUM HYDROCARBONS (TPH)

5.. - TPH

\begin{tabular}{ll}
\hline Elient Sample ID: & NSCEP-V1-286 \\
Date Collected: & $27-A U G-97$ \\
Date Analyzed: & $17-$ SEP-97 14:48 \\
Date Extracted: & $07-$ SEP-97 \\
Matrix: & Soil \\
Percent Moisture: & 2.6
\end{tabular}

IAS Sample ID:

Date Received:

Analytical Batch ID: 091597-8015-D-4

Analytical Dilution: 1

Preparation Dilution: 1.0

QC Group: $\quad 8015 \mathrm{M}$ - TPH_53132

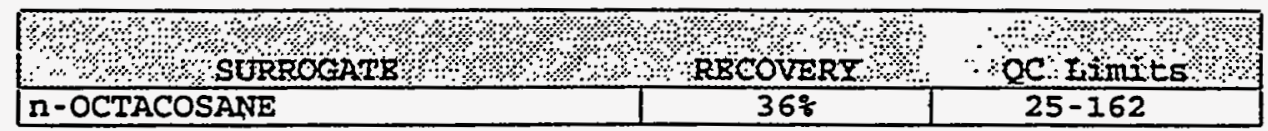

i. ITENT

Rasity

H०००ै००००० औ\%

: I Range Organics ing $/ \mathrm{kg}$

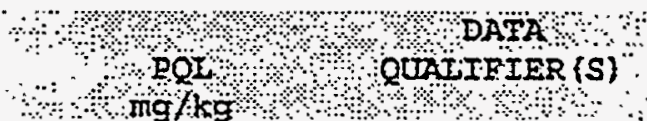

oline Range Organics

TPH

$<31$.

$<31$.

$<31$.

31.

31.

OR OII

31.

\begin{tabular}{llll}
\hline 682 STANDARD & N & R20472 & Page I
\end{tabular}




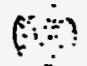

TOTAL PETROLEUM HYDROCARBONS (TPH)

8015M - TPH

$\begin{array}{ll}\text { Client Sample ID: } & \text { NSCEP-V1-287 } \\ \text { Date Collected: } & 27-A U G-97 \\ \text { Date Analyzed: } & 17-\text { SEP-97 14:04 } \\ \text { Date Extracted: } & 07-\text { SEP-97 } \\ \text { Matrix: } & \text { Soil } \\ \text { Percent Moisture: } & 4.07\end{array}$

IAS Sample ID:

Date Received:

Analytical Batch ID:

Analytical Dilution:

Preparation Dilution: 0.99

QC Group:
I10395-86

28-AUG-97

$091597-8015-D-4$

1

8015M - TPH_53132
Diesel Range Organics Gasoline Range Organics MOTOR OIL
$\mathrm{CAS} 110$

PESTIII

DATA

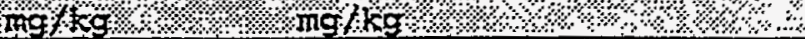

TPH

$<31$.

$<31$.

31.

$<31$.

31 .

31. 


\section{¿ LABORATORIES}

1. PETROLEUM HYDROCARBONS (TPH)

- TPH

- lient Sample ID: NSCEP-VI-300 uate Collected: 27-AUG-97

Date Analyzed: 20-SEP-97 18:51

2te Extracted: 07-SEP-97

itrix:

Soil

Percent Moisture:' 5.6

IAS Sample ID:

Date Received:

Analytical Batch ID: 091797-8015-D-2

Analytical Dilution: 1

Preparation Dilution: 0.99

QC Group:

$8015 \mathrm{M}$ - TPH_53158
L10395-93

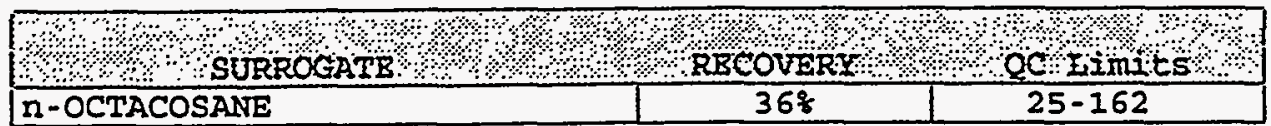

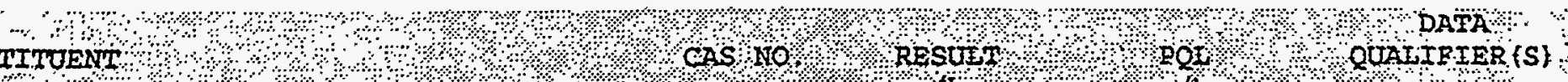

\section{*it: $\because \cdots$}

é. Range Organics line Range Organics

E DIL
TPH

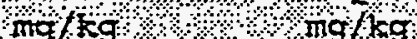

$-$ 


\section{LAS LABORATORIES}

TOTAL PETROLEUM HYDROCARBONS (TPH)

$8015 M$ - TPH

$\begin{array}{ll}\text { Client Sample ID: } & \text { NSCEP-V1-322 } \\ \text { Date Collected: } & 27-A U G-97 \\ \text { Date Analyzed: } & 18-\text { SEP-97 11:57 } \\ \text { Date Extracted: } & 07-\text { SEP-97 } \\ \text { Matrix: } & \text { Soil } \\ \text { Percent Moisture: } & 8.65\end{array}$

IAS Sample ID:

L10395-90

Date Received: 28-AUG-97

Analytical Batch ID: 091597-8015-D-5

Analytical Dilution: 1

Preparation Dilution: 0.99

Percent Moisture: 8.65

QC Group:

$8015 \mathrm{M}$ - TPH_53132

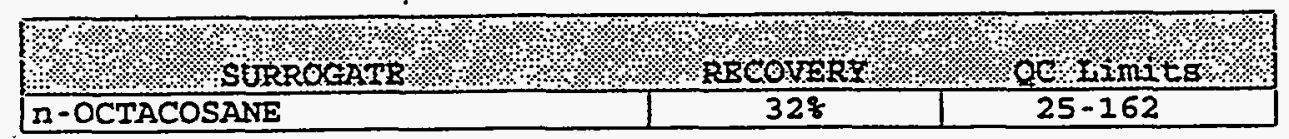

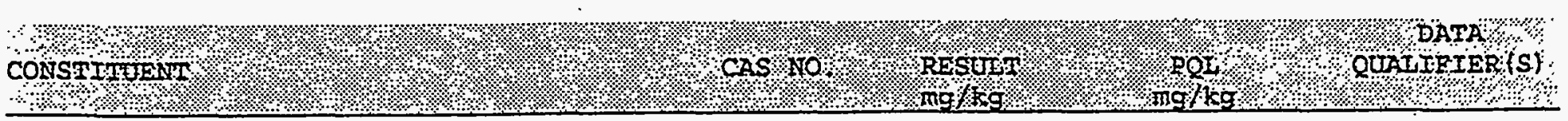

Diesel Range Organics Gasoline Range Organics MOTOR OII
TPH

$<32$.

$<32$.

$<32$.

32.

32 .

32. 

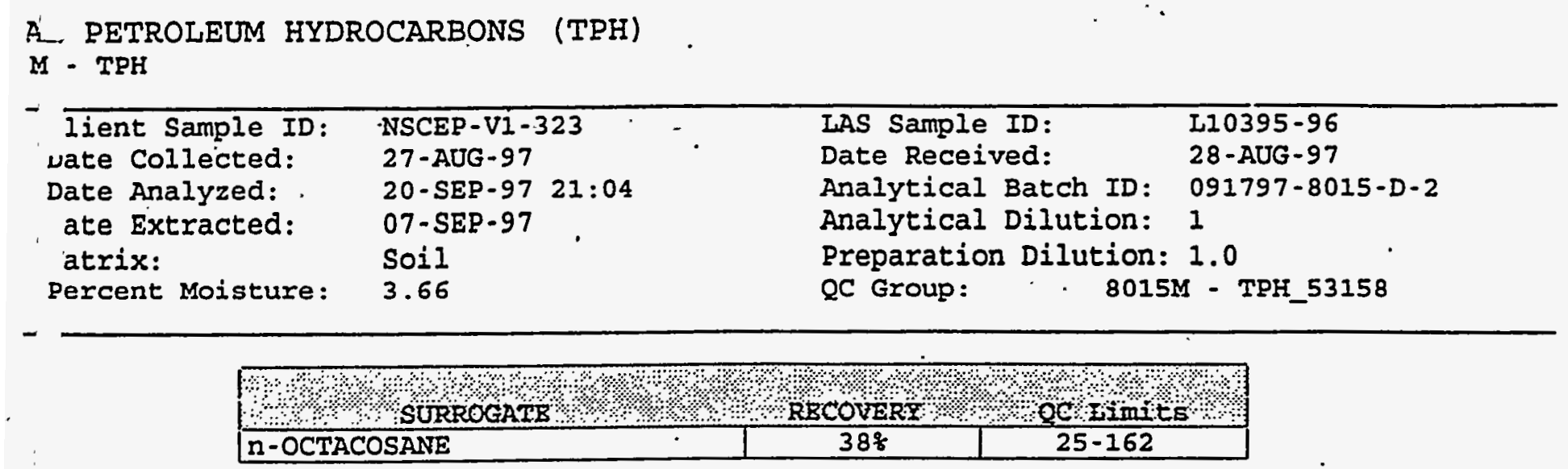

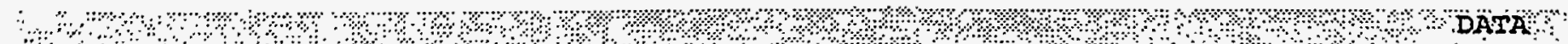

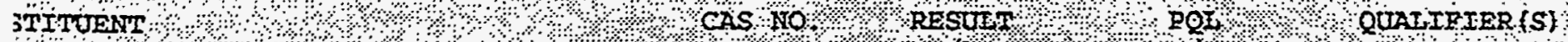
-

sel Range Organics Jline Range Organics 3 OIL
TPH

$\begin{array}{ll}<32 . & 32 . \\ <32 . & 32 . \\ <32 . & 32 .\end{array}$




\section{LAS LABORATORIES}

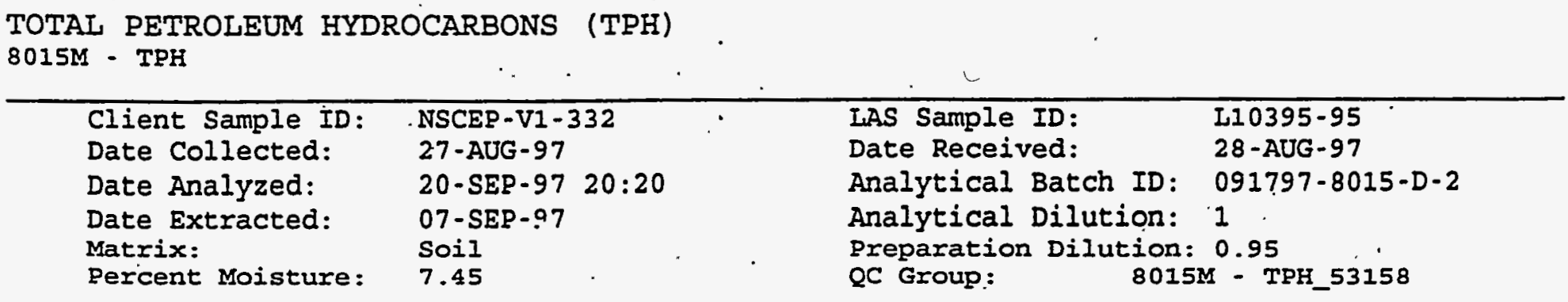

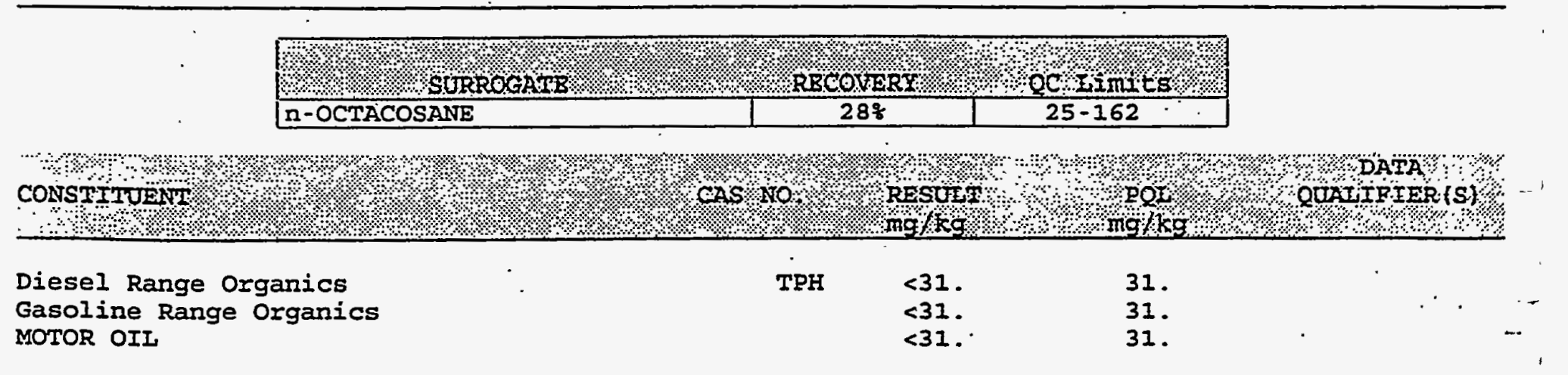


1. PETROLEUM HYDROCARBONS (TPH)

$\checkmark$ - TPH

\begin{tabular}{llll}
\hline Client Sample ID: & NSCEP-V2-332 & IAS Sample ID: & L10479-3 \\
ate Collected: & $09-S E P-97$ & Date Received: & $10-S E P-97$ \\
ate Analyzed: & $.25-$ SEP-97 & Analytical Batch ID: $091797-8015-D-6$ \\
Date Extracted: & $12-S E P-97$ & Analytical Dilution: 1 \\
"atrix: & Soil & Preparation Dilution: 1.0 \\
ercent Moiature: & 11.61 & QC Group: & 8015M - TPH_53397 \\
\hline
\end{tabular}
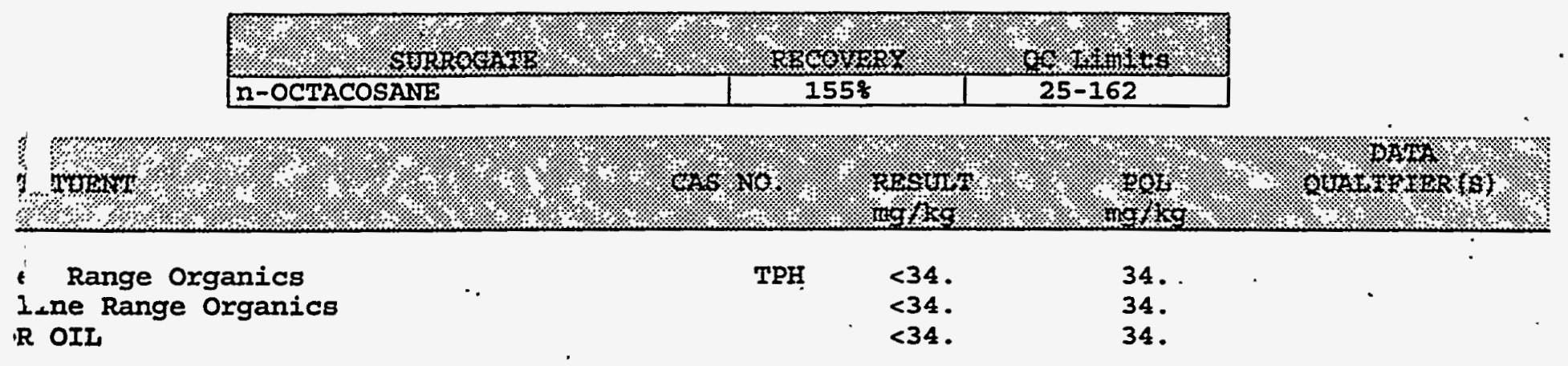
TOTAL PETROLEUM HYDROCARBONS (TPH) $8015 \mathrm{M}$ - .TPH

Client Sample ID: NSCEP-V1-337

Date Collected: 27-AUG-97

Date Analyzed: 17-SEP-97 11:50

Date Extracted:

Matrix:

Percent Moisture:
I.AS Sample ID: L10395-83

Date Received: 28-AUG-97

Analytical Batch ID: 091597-8015-D-4

Analytical Dilution: 1

Preparation Dilution: 1.0

QC Group: $\quad 8015 M$ - TPH_53132

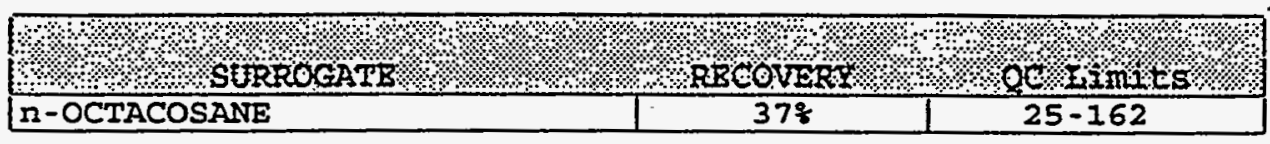

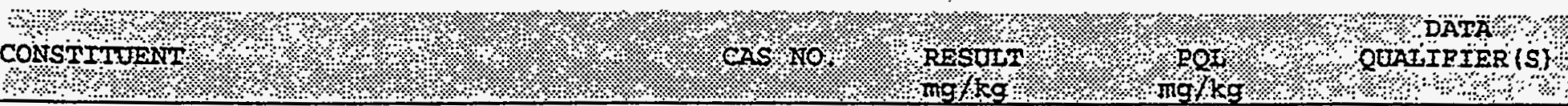

Diesel Range Organics Gasoline Range Organics MOTOR OIL

\section{TPF:}

$<32$.

$<32$.

$<32$.

32 .

32 .

32 . 
(.):

\section{¿ LABORATORIES}

\section{PETROLEUM HYDROCARBONS (TPH)}

M - TPH

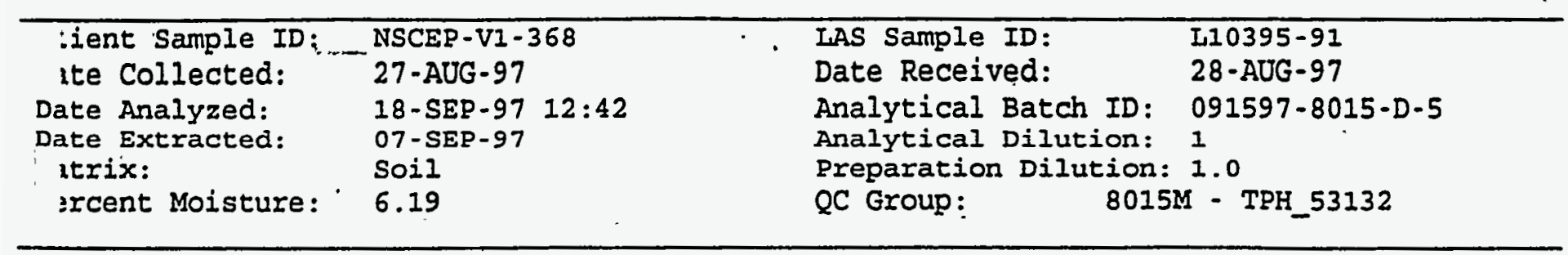

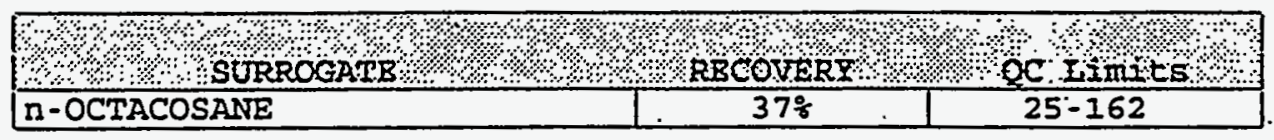

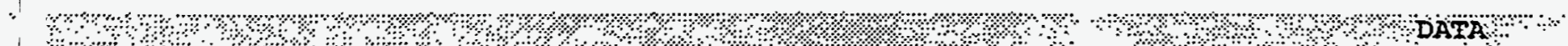
TIVENTE

Asto $\%$ RFSUTS

pori:

QUATIRIER:(S).

- U

e Range Organics

line Range Organics

IR OIL
TPH

$<32$.

$<32$.

$<32$.
32.

32 .

32 . 


\section{LAS LABORATORIES}

\begin{tabular}{llll} 
TOTAL PETROLEUM HYDROCARBONS (TPH) & & \\
8015M - TPH. & & & \\
\hline Client Sample ID: & NSCEP-VI-384 & LAS Sample ID: & LI0395-92 \\
Date Collected: & $27-$ AUG-97 & Date Received: & 28-AUG-97 \\
Date Analyzed: & $20-$ SEP-97 18:06 & Analytical Batch ID: 091797-8015-D-2 \\
Date Extracted: & $07-$ SEP-97 & Analytical Dilution: I & \\
Matrix: & Soil & Preparation Dilution: 0.99 \\
Percent Moisture: & 5.9 & QC Group: &
\end{tabular}

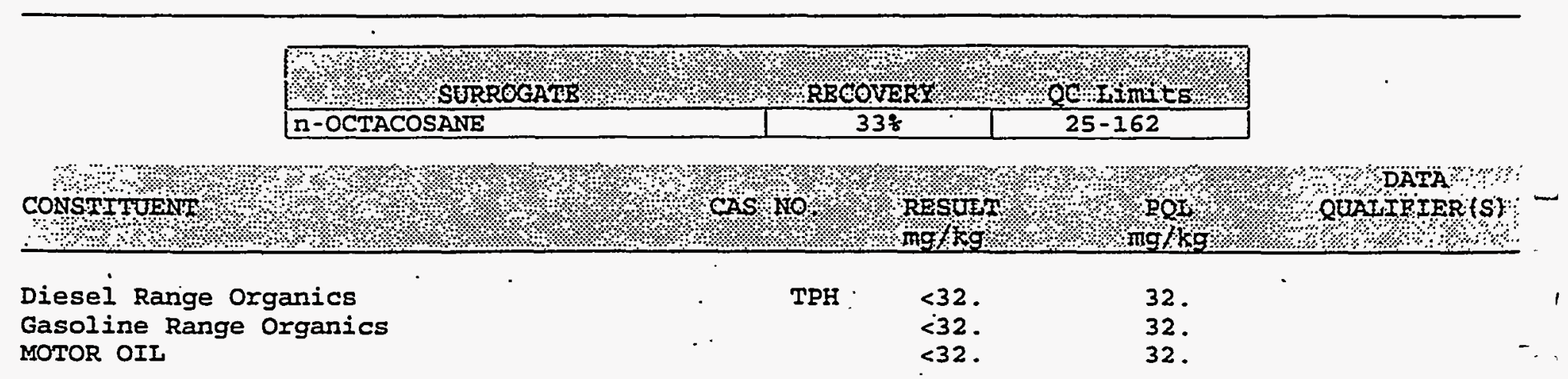




\section{VOLUME 2 APPENDIX B}

TABLE 3 ANALYTICAL DATA 


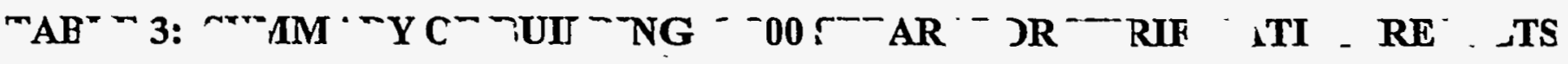

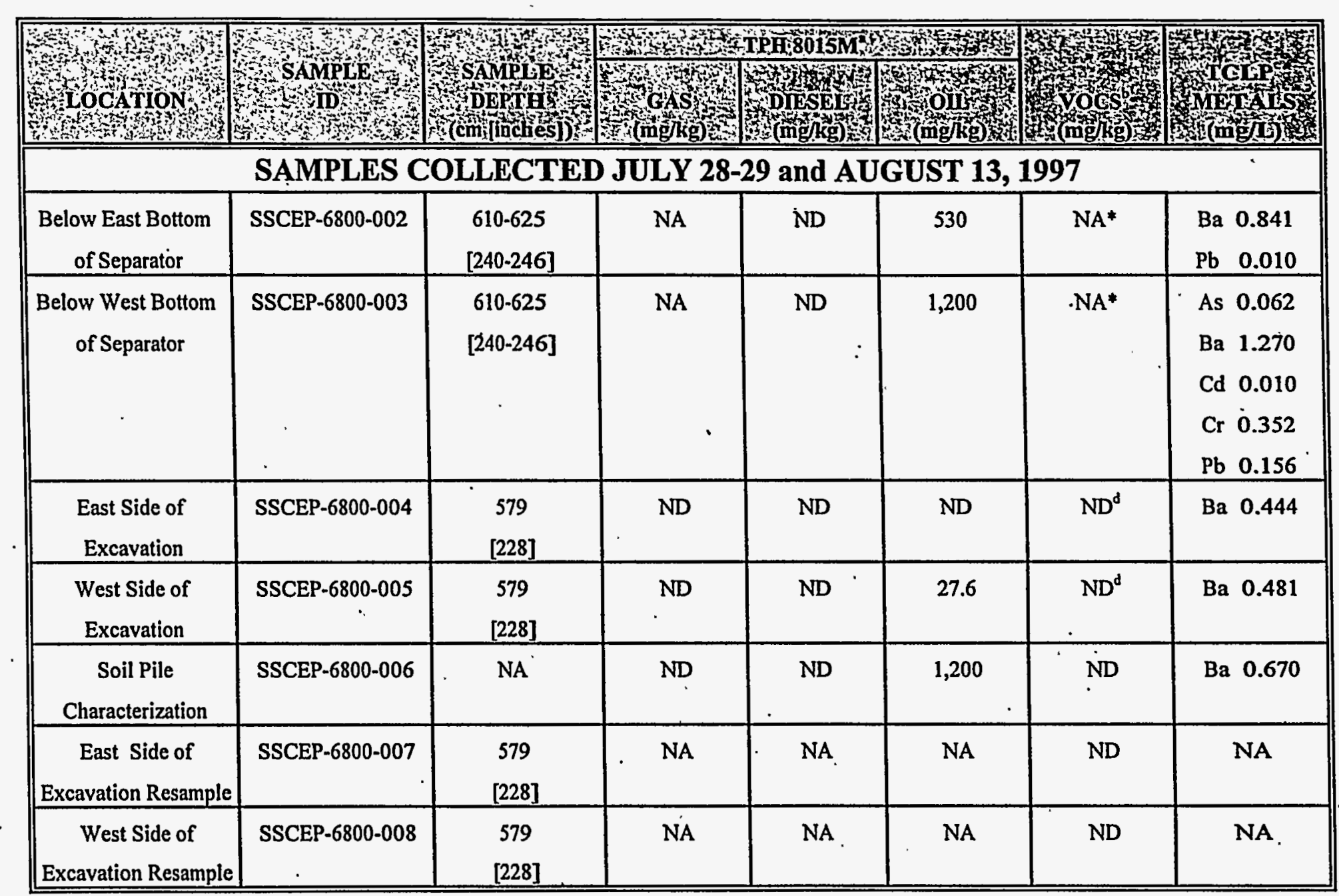

\section{Notes:}

- TPH 8015M: Total Petroleum Hydrocarbons Method 8015 Modified.

' VOCs: Volatile Organic Compounds.

'TCLP: Toxicity Characteristic Leaching Procedure

ND': While no detectable VOCs were found within the sample, the sample had been analyzed 1-day after the CLP manadated holding time.

SCEP: Steam Cleaning Effluent Pond

ND: Not Detected

NA: Not Analyzed

$\mathrm{NA}^{\star}$ : VOC analysis cancelled due to samples were received at 27 degrees Celsius 
10

HBH ANALYSIS DATA SHEET
BPA SAYPLE NO. SSCEP-6800-2

Lab Name: QUANTERRA MO

Contract:

625.01.

Lạb Code: ITMO Case No.:

SAS NO.: :

SDG No.: $\quad$ v264

Matrix.: (soll/water)

SOIL

Iab Sample ID:

15474-001

Sample wt/vol:

20.1

$G^{\circ}$

Lab File ID:

Level: (low/med)

IOW

Date Sampled:

07-29-97

Date Extracted:

$08-05-97$

Moisture: not dec. dec.

Date Analyzed:

$08-18-97$

GPC Cleanup: $(\mathrm{Y} / \mathrm{N})+\mathrm{N} \cdot \mathrm{PH}$

Dilution Factor:

1

CEITIRATION ONITS:

CAS NO.

Compound

(mg/L or $\mathrm{mg} / \mathrm{kg})$

$\mathrm{mg} / \mathrm{kg}$

$\mathbf{Q}$

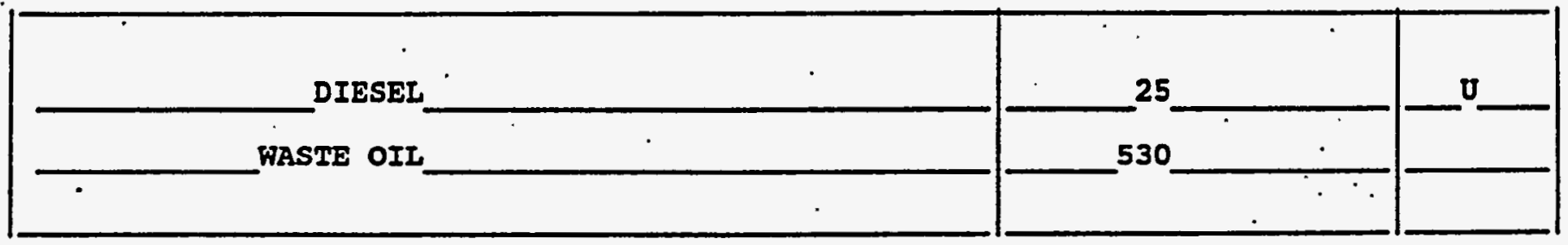

U: Concentration of analyte is less than the value given.

FORM I HBE 
1

\section{INORGANIC ANALYSES DATA SHEET}

Lab Name: QUANTERRA_MO

Lab Code: ITMO_ Case No.:

Matrix (soil/water) : WATER

Level (low/med):

LOW

Contract: 625.01

SAS NO.:

SDG No.: V264

Lab Sample ID: P15474-001

Date Received: 08/04/97
EPA SAMPLE NO.

SSCEP68002

: Solids:

0.0

Concentration Units (ug/L or $\mathrm{mg} / \mathrm{kg}$ dry weight) : UG/L_

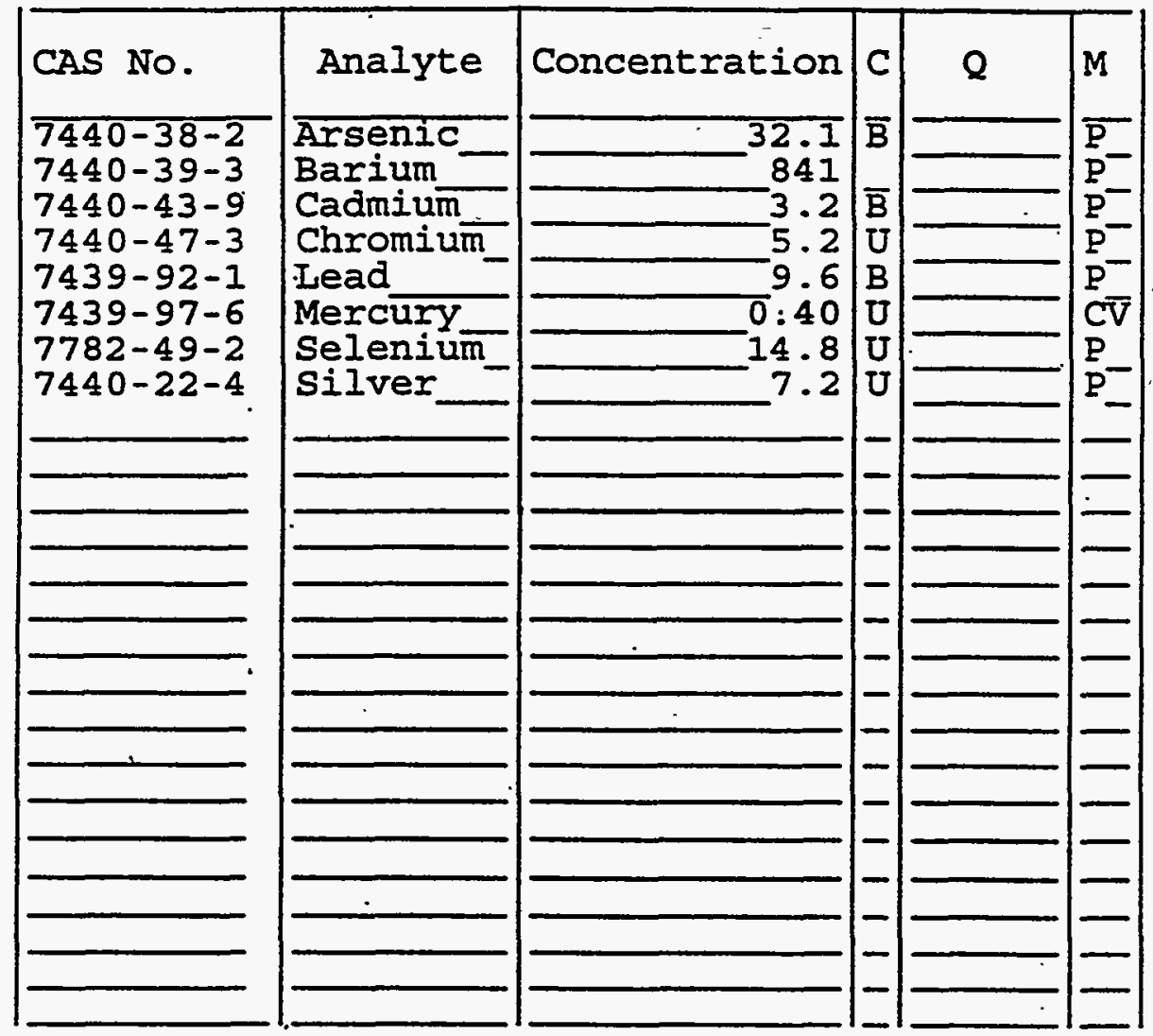

Color Before:

Color After:
Clarity Before:

Clarity After:

Comments :

FORM I - IN 


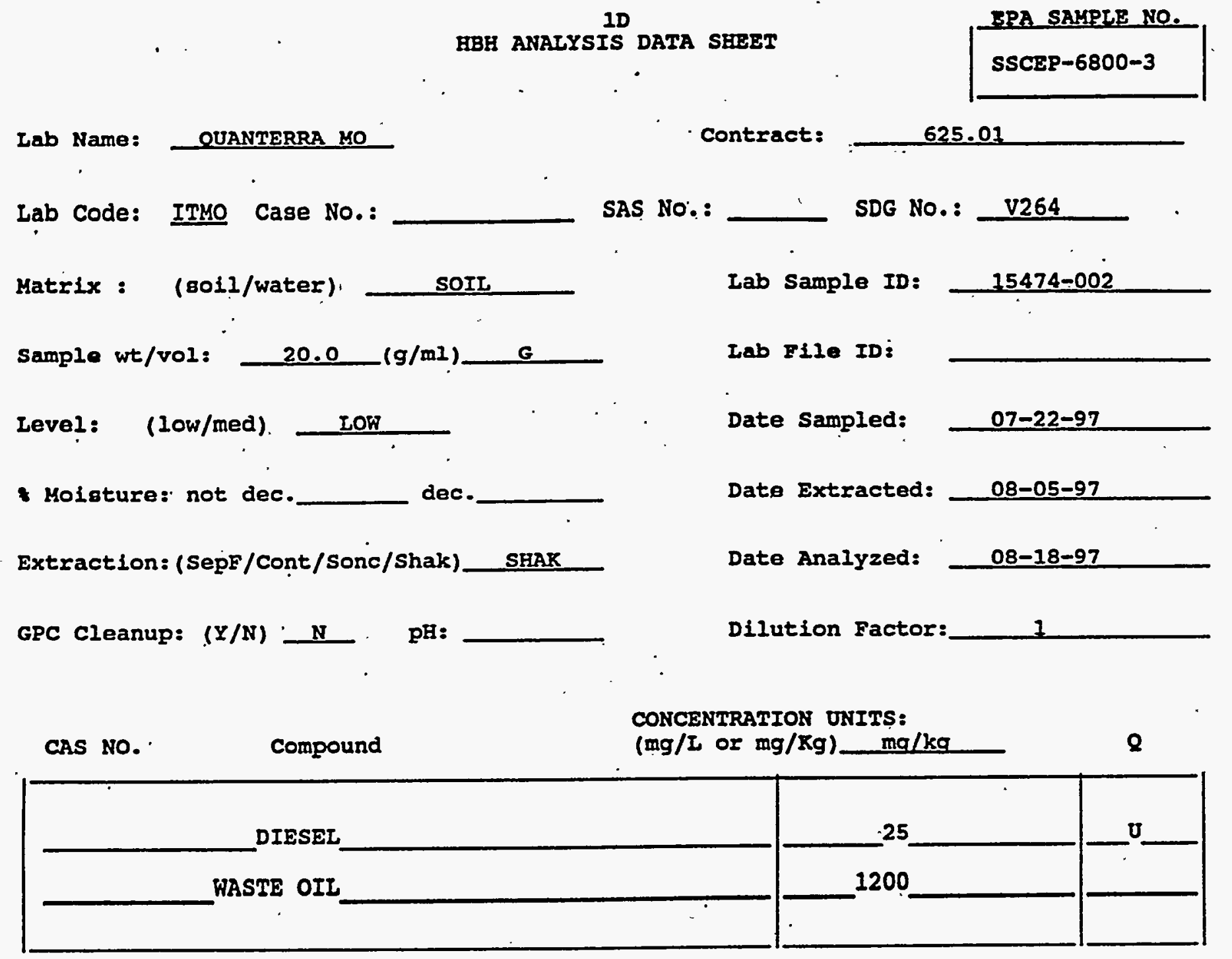

U: Concentration of analyte is less than the value given.

FORA I ABE 


\section{U.S. EPA - CLP}

\section{INORGANIC ANAIYSES DATA SHEET}

Lab Name: QUANTERRA_MO

Lab Code: ITMO_ Case No.:

Matrix (soil/water): WATER

Level (low/med): LOW

\% Solids :
Contract: 625.01

SAS NO.:
EPA SAMPLE NO.

\section{SSCEP 68003}

SDG No.: V264

Lab Sample ID: P15474-002

Date Received: 08/08/97

Concentration Units (ug/L or img/kg dry weight): UG/L_

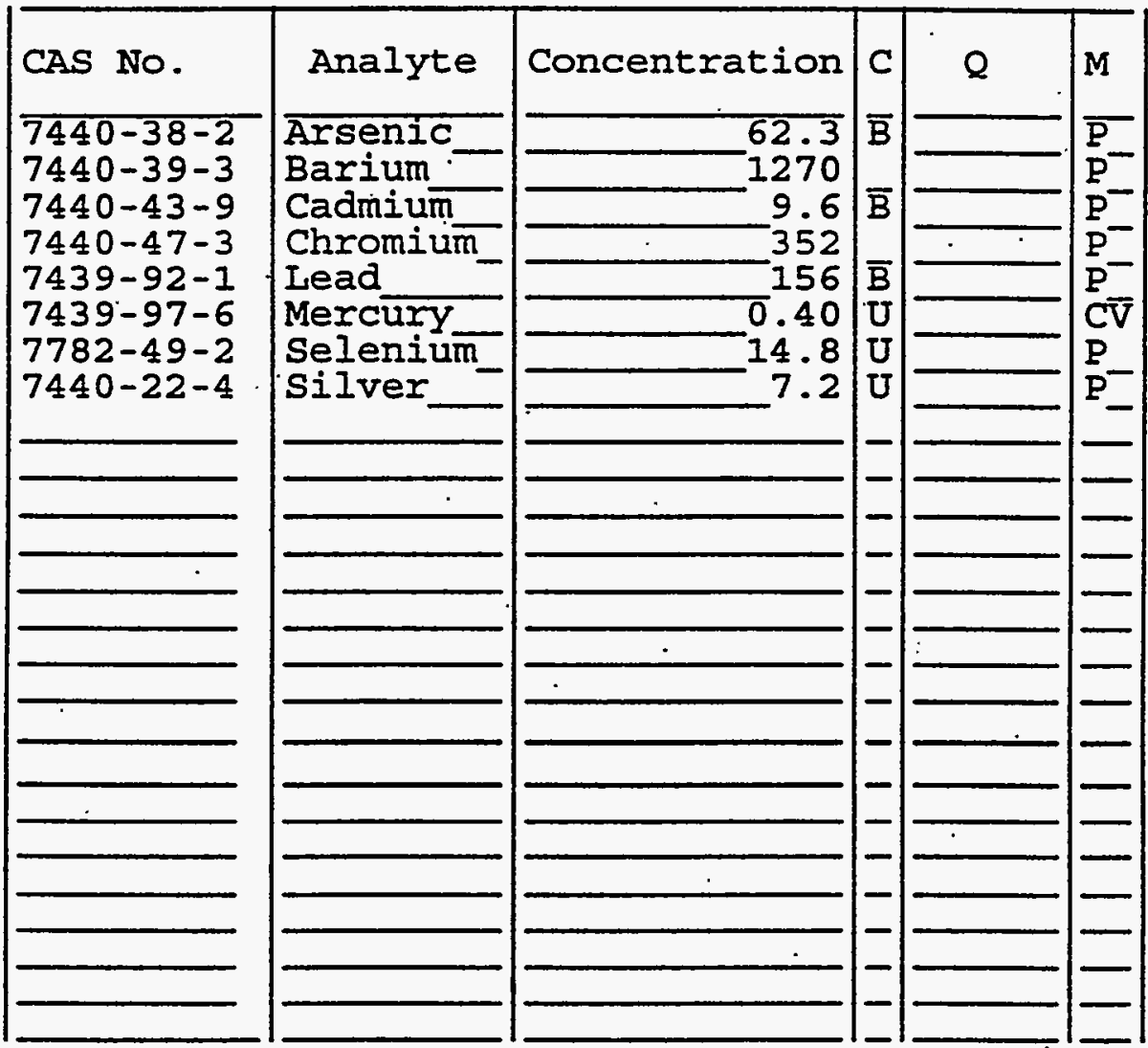

Color Before:

Color After:

Comments :
Clarity Before:

Clarity After:
Texture :

Artifacts : 


\section{A S LABORATORIES}

: II PETROLEUM HYDROCARBONS (TPH)

1 - TPH

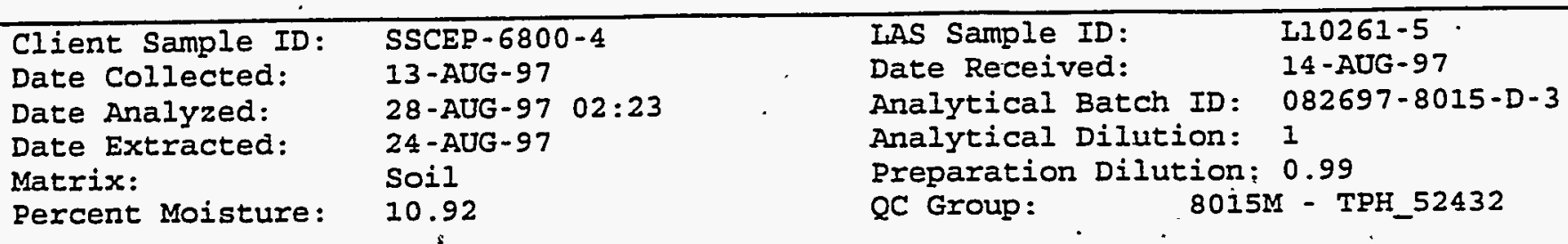

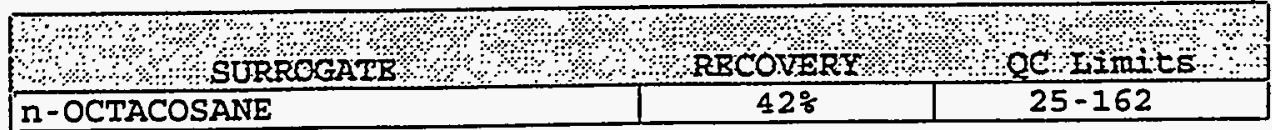

$<33$.

$<33$.

33.

33 . 


\section{LAS LABORATORIES}

VOLATILE ORGANICS BY GC/MS 8260 VOLATILES

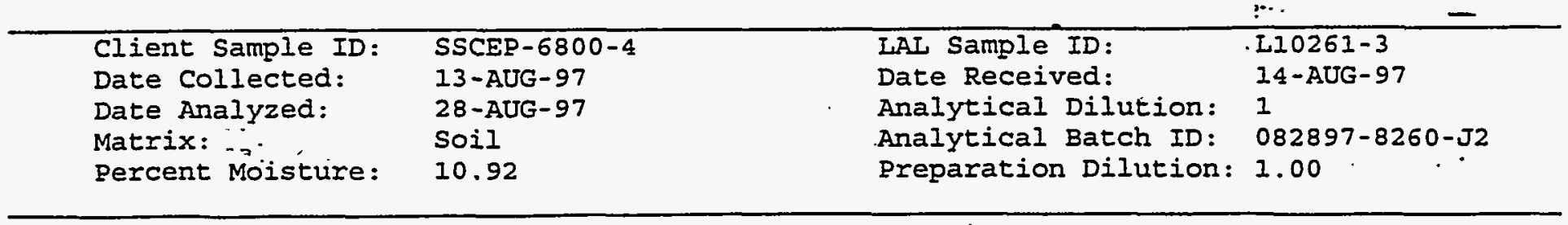

\begin{tabular}{|c|c|c|}
\hline मै। & \%ैRBCove & क्लिम \\
\hline 1,2-Dichloroethane-d4 & $84 \%$ & $77-127$ \\
\hline Toluene-d8 & $86 \%$ & $84-120$ \\
\hline Bromofluorobenzene & $88 \frac{8}{8}$ & $78-125$ \\
\hline
\end{tabular}

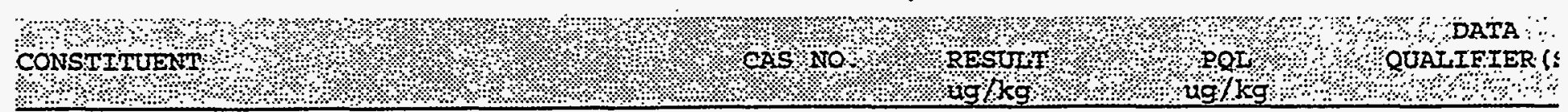

\section{Chloromethane}

vinyl Chloride

Bromomethane

Chloroethane

Trichlorofluoromethane

Acetone

1,1-Dichloroethene

Carbon Disulfide

Methylene ChIoride

trans-1,2-Dichloroethene

Vinyl Acetate

1,1-Dichloroethane

2-Butanone

Cis-1,2-Dichloroethene

Chloroform

2-Hexanone

1,1,1-Trichloroethane

Carbon tetrachloride

1,2-Dichloroethane

Benzene

Trichloroethene

1,2-Dichloropropane

Bromodichloromethane

2-Chloroethyl vinylether

4-Methyl-2-Pentanone

cis-1,3-Dichloropropene

Toluene

trans-1,3-Dichloropropene

1,1,2-Trichloroethane

Tetrachloroethene

Dibromochloromethane

Chlorobenzene

Ethylbenzene

m, p-xylene

o-XYlene

Styrene

Bromoform

1, 1, 2,2-Tetrachloroethane

1,3-Dichlorobenzene

1,4-Dichlorobenzene

1,2-Dichlorobenzene
$<5.6$

$<5.6$

$<5.6$

$<5.6$

$<5.6$

7.4

$<5.6$

$<5.6$

$<5.6$

$<5.6$

$<11$.

$<5.6$.

$<11$.

$<5.6$

$<5.6$

<II.

$<5.6$

$<5.6$

$<5.6$

$<5.6$

1. 5

$<5.6$

$<5.6$

$<22$.

$<1 I$.

$<5.6$

$<5.6$

$<5.6$

$<5.6$

$<5.6$

$<5.6$

$<5.6$

$<5.6$

$<5.6$

$<5.6$

$<5.6$

$<5.6$

$<5.6$

$<5.6$

$<5.6$

$<5.6$
5.6

5.6

5.6

5.6

5.6

11 .

5.6

5.6

5.6

5.6

11 .

5.6

11.

5.6

5.6

11 .

5.6

5.6

5.6

5.6

5.6

5.6

5.6

22.

11.

5.6

5.6

5.6

5.6

5.6

5.6

5.6

5.6

5.6

5.6

5.6

5.6

5.6

5.6

5.6

5.6
H

H

F

H

H

HJB

H

H

H

H

H

H

H

H

HI

H

H.

H

H

$\mathrm{H}$

HJ

H

H

H

H

H

H

H

H

HI

H

$\mathrm{H}$

H

H

H

F

F

H

H

E

H 
1

INORGANIC ANALYSES DATA SHEET
Name : I.A.S b Code: LOCK Case NO.: 811BNE2 SAS NO.:
CLIENT ID NO.

CEP-6800-4

Contract: BECHTEL-NV

SDG No.: V278

Lab Sample ID: I10261-6

Date Received: 08/14/97

vel (low/med): LOW

olids :

Concentration Units (ug/I or $\mathrm{mg} / \mathrm{kg}$ dry weight): UG/L_

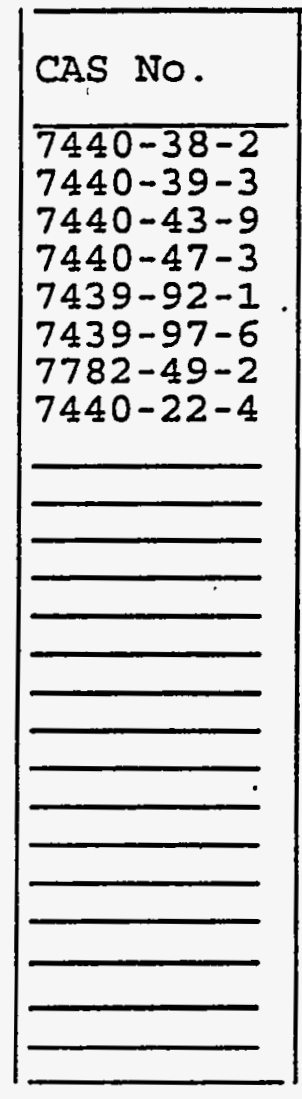

J or Before: COLORLESS

IIor After: COLORLESS

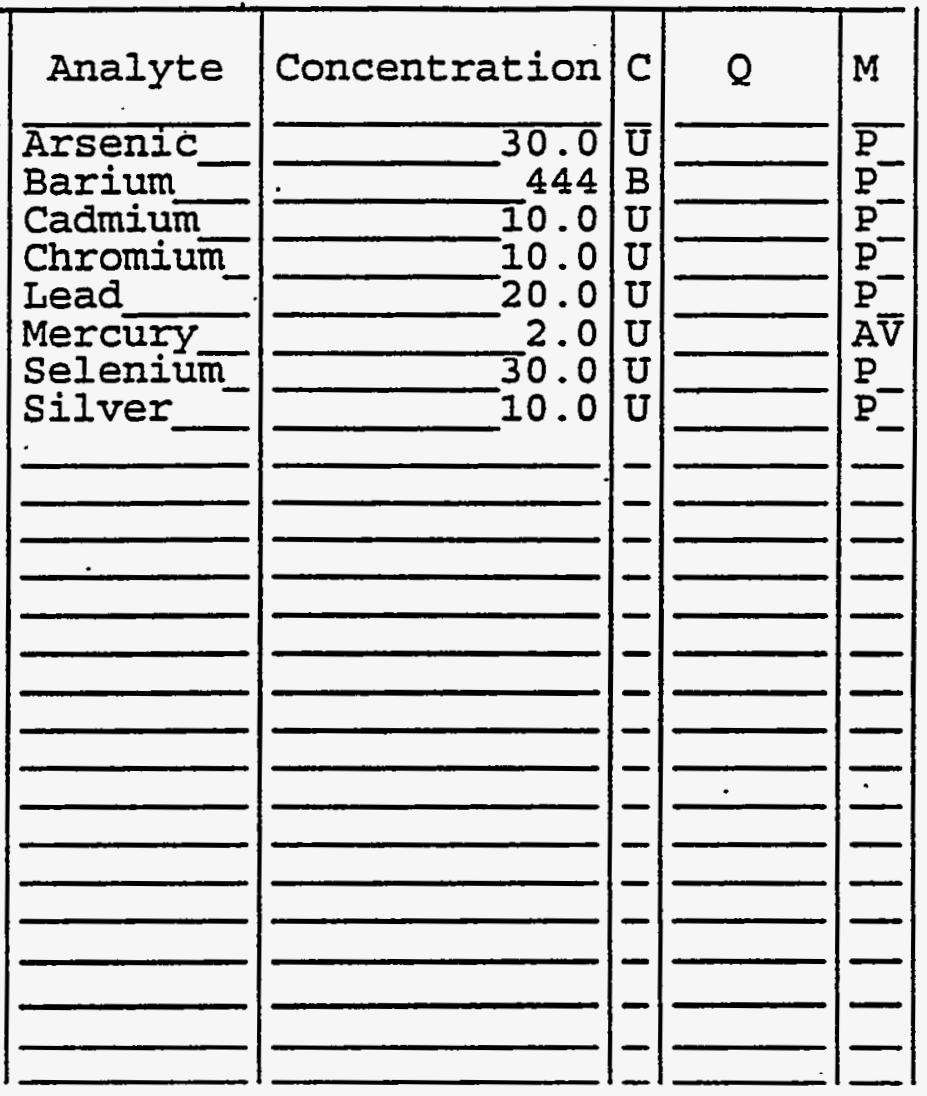

Clarity Before: CLEAR_

Texture :

Clarity After: CLEAR

Artifacts : 


\section{LAS LABORATORIES}

TOTAL PETROLEUM HYDROCARBONS (TPH) $8015 \mathrm{M}$ - TPH

\begin{tabular}{llll}
\hline Client Sample ID: & SSCEP-6800-5 & LAS Sample ID: & I10261-9 \\
Date Collected: & 13-AUG-97 & Date Received: & $14-A U G-97$ \\
Date Analyzed: & 28 -AUG-97 03:07 & Analytical Batch ID: 082697-8015-D-3 \\
Date Extracted: & $24-$ AUG-97 & Analytical Dilution: 1 \\
Matrix: & Soil & Preparation Dilution: 0.99 \\
Percent Moisture: & 10.13 & QC Group: & 8015M-TPH_52432 \\
\hline
\end{tabular}

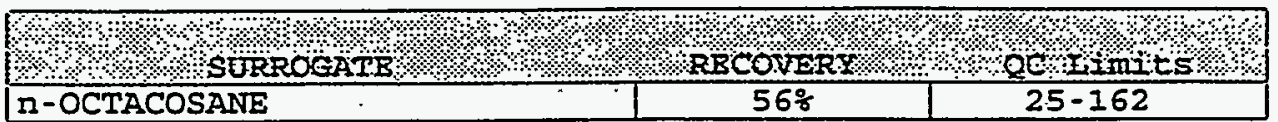

$<33$. 33 .




\section{، IS LABORATORIES}

\section{JLATILE ORGANICS BY GC/MS}

\section{! ) VOLATILES}

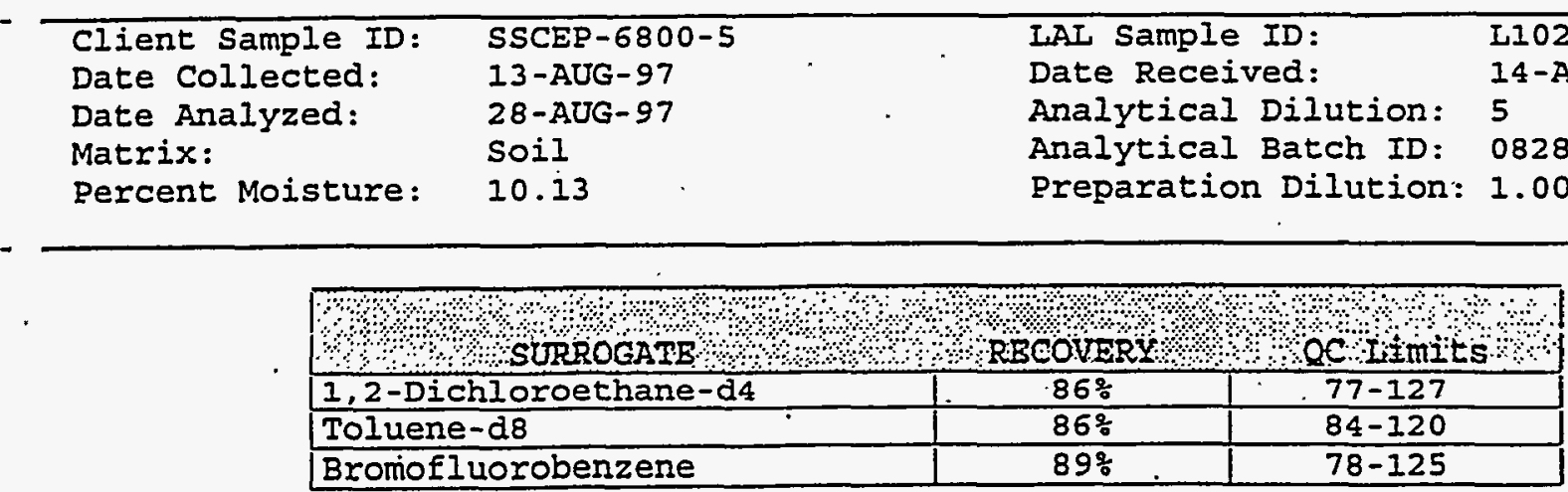

\section{TT

2 Iromethane $\ldots l$ Chloride comomethane $3^{-}$oroethane

- :hlorofluoromethane zecone

. 1-Dichloroethene

z son Disulfide

$\geq$ lylene Chloride

ans-1, 2-Dichloroethene

inyl Acetate

- Dichloroethane

- dtanone

is-I, 2-Dichloroethene

$2^{7}$ oroform

- ixanone

,-,1-Trichloroethane

arbon tetrachloride

,- Dichloroethane

$\geq$ :ene

ichloroethene

, 2-Dichloropropane

= nodichloromethane

- lloroethylvinylether

-Methyl-2-Pentanone

is-1,3-Dichloropropene

2 zene

$=$ is-1,3-Dichloropropene

, 1,2-Trichloroethane

e-rachloroethene

i comochloromethane

isurobenzene

thylbenzene

- -xylene

- rlene

zyrene

comoform

, ,2,2-Tetrachloroethane

- Dichlorobenzene

,4-Dichlorobenzene

, ᄀ-Dichlorobenzene

$\begin{aligned} & 74-87-3<28 . \\ & 75-01-4<28 . \\ & 74-83-9<28 . \\ & 75-00-3<28 . \\ & 75-69-4<28 . \\ & 67-64-1<56 . \\ & 75-35-4<28 . \\ & 75-15-0 .<28 . \\ & 75-09-2<28 . \\ & 156-60-5<28 . \\ & 108-05-4<56 . \\ & 75-34-3<28 . \\ & 78-93-3<56 . \\ & 156-59-2<28 . \\ & 67-66-3<28 . \\ & 591-78-6<56 . \\ & 71-55-6<28 . \\ & 56-23-5<28 . \\ & 107-06-2<28 . \\ & 71-43-2<28 . \\ & 79-01-6<28 . \\ & 78-87-5<28 . \\ & 75-27-4<28 . \\ & 110-75-8<110 \\ & 108-10-1<56 . \\ & 10061-01-5<28 . \\ & 108-88-3<28 . \\ & 10061-02-6<28 . \\ & 79-00-5<28 . \\ & 127-18-4<28 . \\ & 124-48-1<28 . \\ & 108-90-7<28 . \\ & 100-41-4<28 . \\ & 75-35-25-2<28 . \\ & 106-46-7<28 . \\ & 95-50-1<28 . \\ & 100-42-5<28 . \\ & 757-61-2\end{aligned}$

pQL QUAYIIFIER (S) $\mathrm{ug} / \mathrm{kg}$
110261-7 4-AUG- 97

$82897-8260-\mathrm{J} 2$ 
1

INORGANIC ANALYSSS DATA SHEET

Lab Name: L.A.S

Contract: BECHTEL-NV

Lab Code: LOCK

Case No.: 811BNE2 SAS No.:
CLIENT ID NO.

CEP-6800-5
Matrix (soil/water): WATER

Level (low/med):

LOW

\% Solids :

Concentration Units (ug/L or $\mathrm{mg} / \mathrm{kg}$ dry weight) : UG/I_

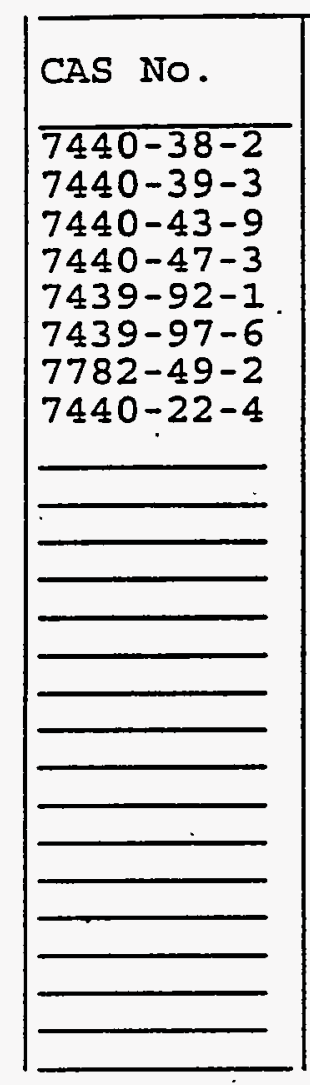

Color Before: COLORLESS Color After: COLORLESS

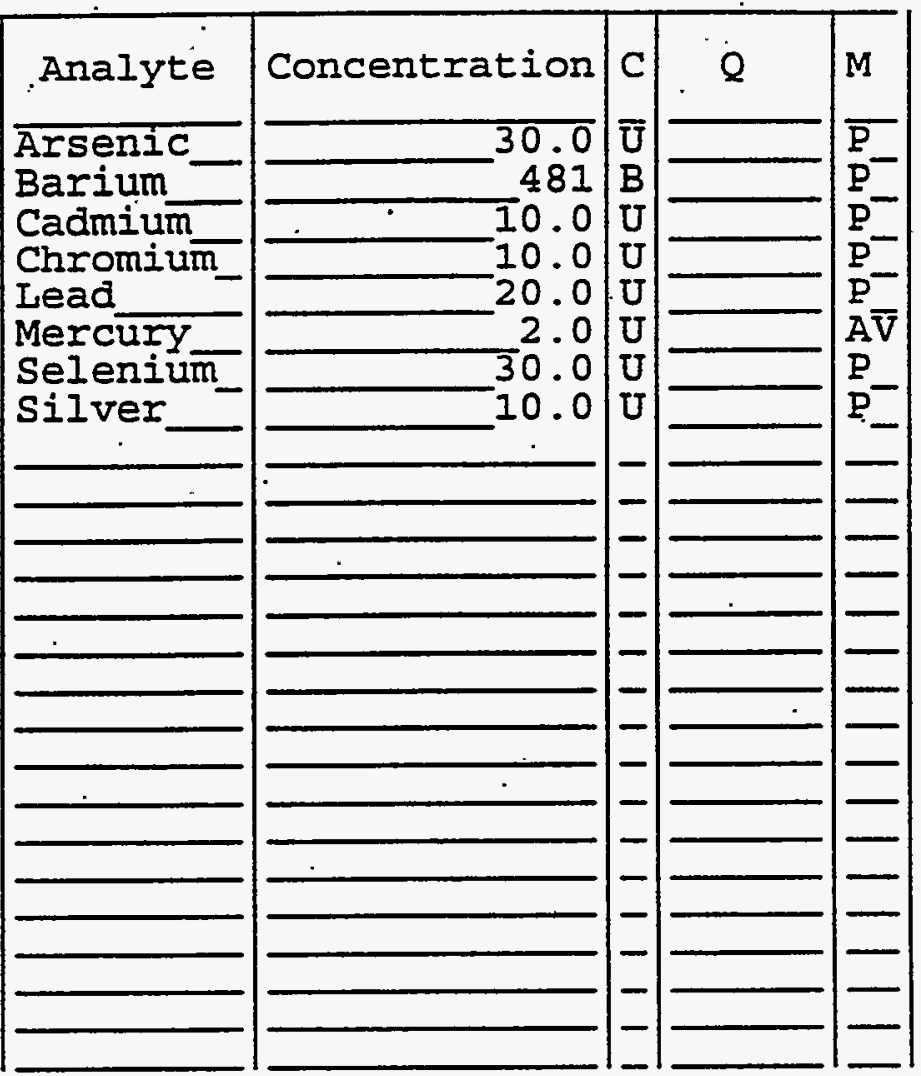

Clarity Before: CLEAR

Texture:

Clarity After: CLEAR

Artifacts : 
P\&T GAS/BTEX

T'T GAS/BTEX

- Client Sample ID: Date Collected: Date Analyzed: Date Extracted: Matrix:

Percent Moisture:

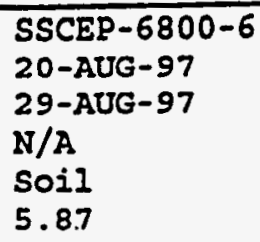

$N / A$

Soil

5.8 .7

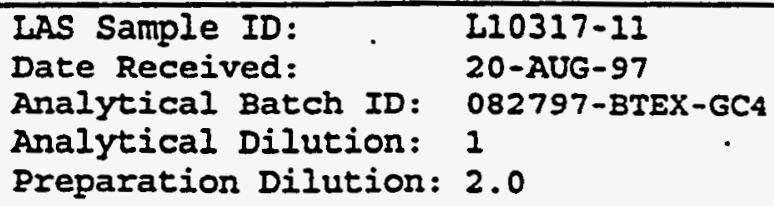

.
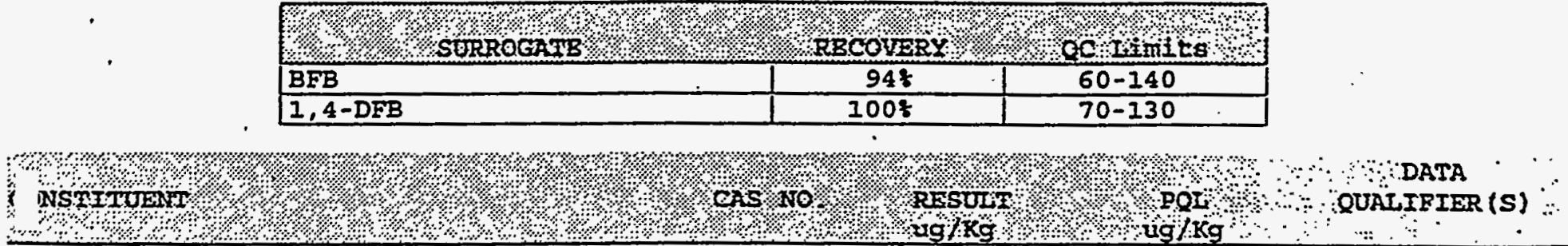

$r$,soline Range Organics

$8006-61-9$

$<210$

210 


\section{LAS LABORATORIES}

TOTAI PETROLEUM HYDROCARBONS (TPH)

8015M - TPH

Client Sample ID: SSCEP-6800-6

Date Collected:

Date Analyzed:

Date Extracted:

Matrix:

Percent Moisture:
20-AUG-97

$18-$ SEP-97

28-AUG-97

Soil

5.87
IAS Sample ID:

Date Received:

Analytical Batch ID: 091597-8015-D-5

Analytical Dilution:

Prepáration Dilution: 0.98

QC Group:
8015M - TPH_52800
L10317:11

20-AUG-97

10

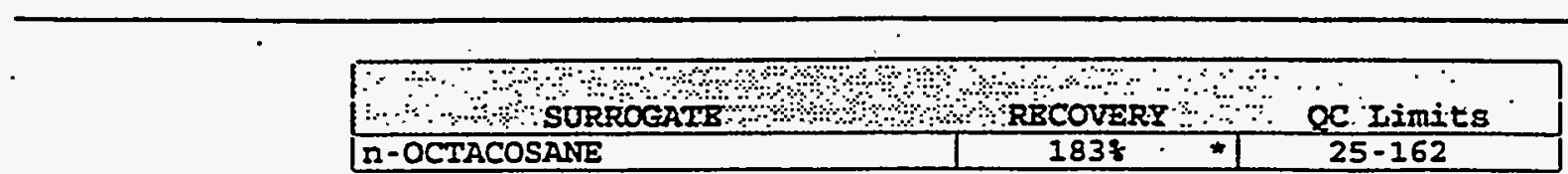

CONSTITUENT

- . m \% $\because \because$

3

$\therefore \because$

Diesel Range Organics

MOTOR OIL

\section{5-162}




\section{¿AS LABORATOR IS}

\section{MS FOR VOLATILE ORGANICS}

20 VOLATILES

$\begin{array}{llll}\text { Client Sample ID: } & \text { SSCEP-6800-6 } & \text { IAI Sample ID: } & \text { L10317-5 } \\ \text { Date Collected: } & \text { 20-AUG-97 } & \text { Date Received: } & 20-A U G-97 \\ \text { Date Analyzed: } & 03-\text { SEP-97 } & \text { Analytical Dilution: } & \text { I } \\ \text { Matrix: } & \text { Soil } & \text { Analytical Batch ID: 090397-8260A-DI } \\ \text { Percent Moisture: } & 5.87 & \text { Preparation Dilution: } 1.00\end{array}$

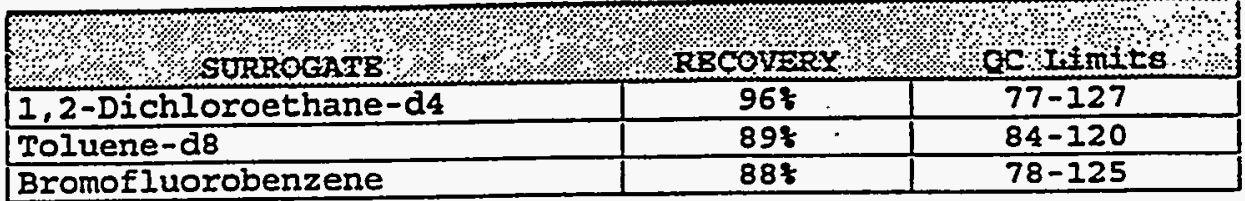

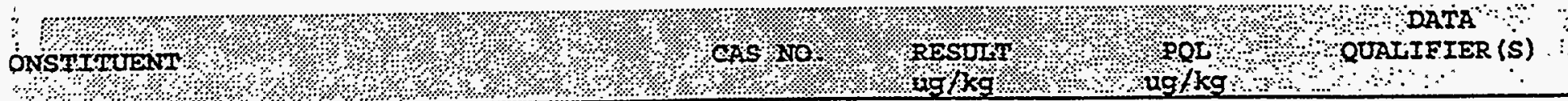

1 oromethane

inyl chloride

romomethane

1 oroethane

2 chlorofluoromethane

cetone

, -Dichloroethene

a bon Disulfide

e-hylene Chloride

rans-1, 2-Dichloroethene

j-yl Acetate

-Dichloroethane

- outanone

is-1, 2-Dichloroethene

l. oroform

- exanone

,1,1-Trichloroethane

arbon tetrachloride

- -Dichloroethane

$\epsilon$ zene

richloroethene

, -Dichloropropane

I modichloromethane

- hloxoethylvinylether

-Methyl-2-Pentanone

j=-1,3-Dichloropropene

(; uene

I,ns-1,3-Dichloropropene

, 1,2-Trichloroethane

t rachloroethene

j romochloromethane

hiorobenzene

thylbenzene

- -xylene

+ ylene

tyrene

romoform

, 2,2-Tetrachloroethane

- -Dichlorobenzene

, 4-Dichlorobenzene

, ?-Dichlorobenzene
$<5.3 \quad 5.3$

$<5.3 \quad 5.3$

$<5.3 \quad 5.3$

$<5.3 \quad 5.3$

$<5.3 \quad 5.3$

<11. 11.

$<5.3 \quad 5.3$

$<5.3 \quad 5.3$

$<5.3 \quad 5.3$

$<5.3 \quad 5.3$

$<11$. 11.

$<5.3 \quad 5.3$

$<11$. 11.

$<5.3 \quad 5.3$

$<5.3 \quad 5.3$

<11. 11 .

$<5.3 \quad 5.3$

$<5.3 \quad 5.3$

$<5.3 \quad 5.3$

$<5.3 .5 .3$

$<5.3 \quad 5.3$

$<5.3$. 5.3

$<5.3 \quad 5.3$

$<21$. 21 .

$<11$. $\quad 11$.

$<5.3 \quad 5.3$

$<5.3 \quad 5.3$

$<5.3 \quad 5.3$

$<5.3 . \quad 5.3$

$<5.3 \quad 5.3$

$<5.3$. 5.3

$<5.3 \quad 5.3$

$<5.3 \quad 5.3$

$<5.3 \quad 5.3$

$<5.3 \quad 5.3$

$<5.3 \quad 5.3$

$<5.3 \quad 5.3$

$<5.3 .5 .3$

$<5.3 \quad 5.3$

$<5.3 \quad 5.3$

$<5.3 \quad 5.3$ 


\section{CLP}

\section{INORGANIC ANALYSES DATA SHEET}

CLIENT ID. NO.

Lab Name: I.A.S

SSCEP-6800-6

Lab Code: LOCK

Case NO.: 820BNE SAS No.:

SDG NO.: L10317

Matrix (soil/water) : WATER

Level (low/med): LOW.

Lab Sample ID: L10317-11_.

Solids :

Date Received: 08/20/97

0

Concentration Units. (ug/L or $\mathrm{mg} / \mathrm{kg}$ dry weight) : UG/L_

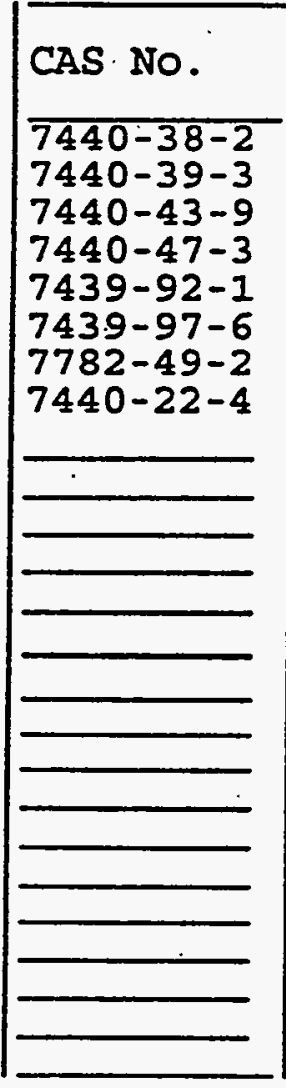

COLORLESS

COLORLESS

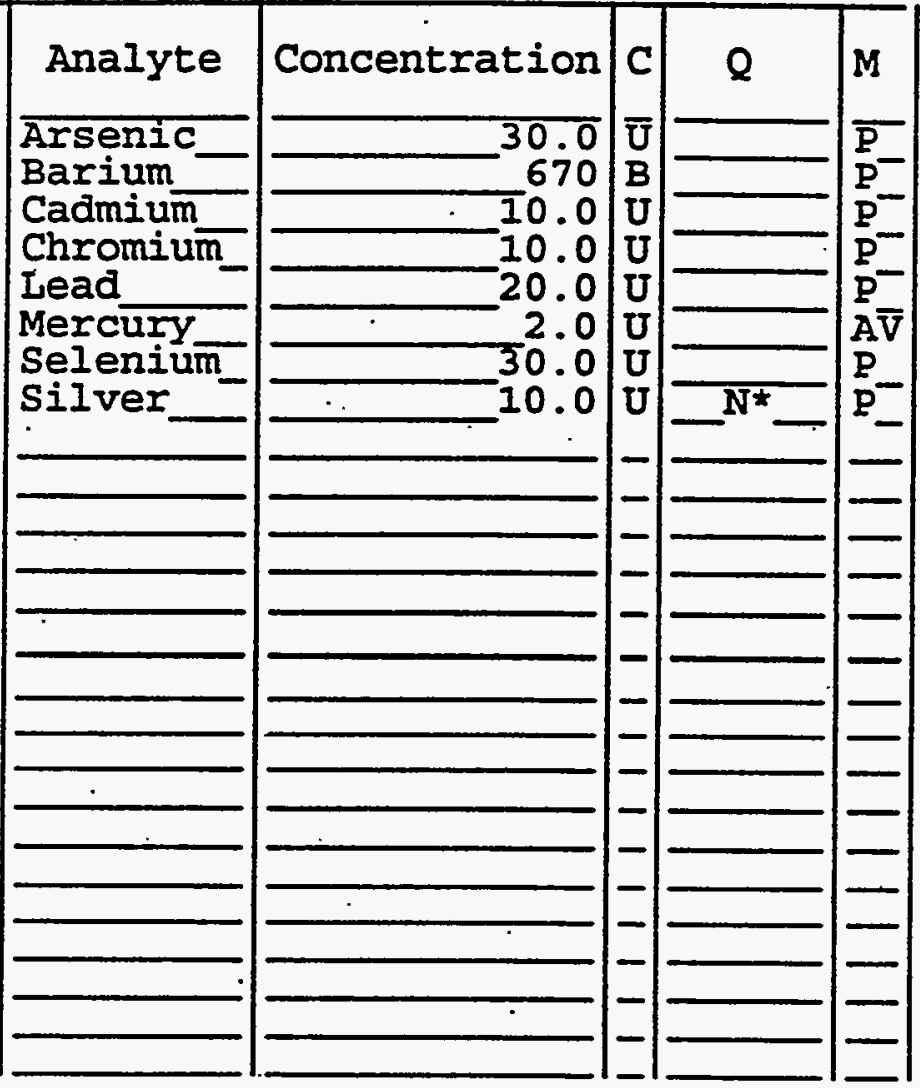

Clarity Before: CLEAR

Texture :

Clarity After: CLEAR_

Artifacts; Eomments :

FORM I - IN 


\section{AS LABORATORIES}

I TILE ORGANICS BY GC/MS 60 VOLATILES

\begin{tabular}{llll}
\hline Client Sample ID: & SSCEP-6800-7 & LAL Sample ID: & L10799-3 \\
Date Collected: & $21-0 C T-97$ & Date Received: & $22-0 C T-97$ \\
Date Analyzed: & $23-0 C T-9710: 45$. & Analytical Dilution: & 1 \\
Matrix: & Soil & Analytical BatCh ID: I02397-8260-EI \\
Percent Moisture: $/ A$ & Preparation Dilution: $1: 00$
\end{tabular}

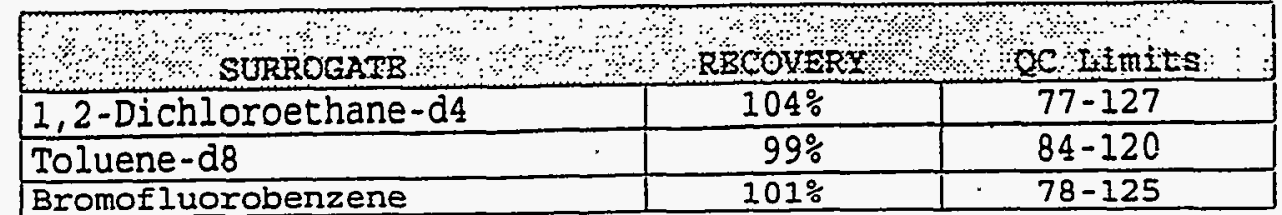

Iloromethane

imil Chloride

$\because$ nomethane

3 soroethane

ichlorofluoromethane

zone

, -Dichloroethene

zrbon Disulfide

əthylene Chloride

c ns-1,2-Dichloroethene

i. Il Acetate

i-Dichloroethane

-ritanone.

i $4-1,2-D i c h l o r o e t h e n e$

7-aroform

-Hexanone

, 1-Trichloroethane

¿- oon tetrachloride

, 4 -Dichloroethane

enzene

i chloroethene

- -Dichloropropane romodichloromethane - Chloroethylvinylether

- ethyl-2-Pentanone

j $-1,3$-Dichloropropene

oluene

! ns-1,3-Dichloropropene

, 2-Trichloroethane

ecrachloroethene

ibromochloromethane

1 - orobenzene

i ylbenzene

, p-Xylene

-xylene

i rene

$\therefore$ imoform

, 1,2,2-Tetrachloroethane

3-Dichlorobenzene.

-Dichlorobenzene

, - -Dichlorobenzene

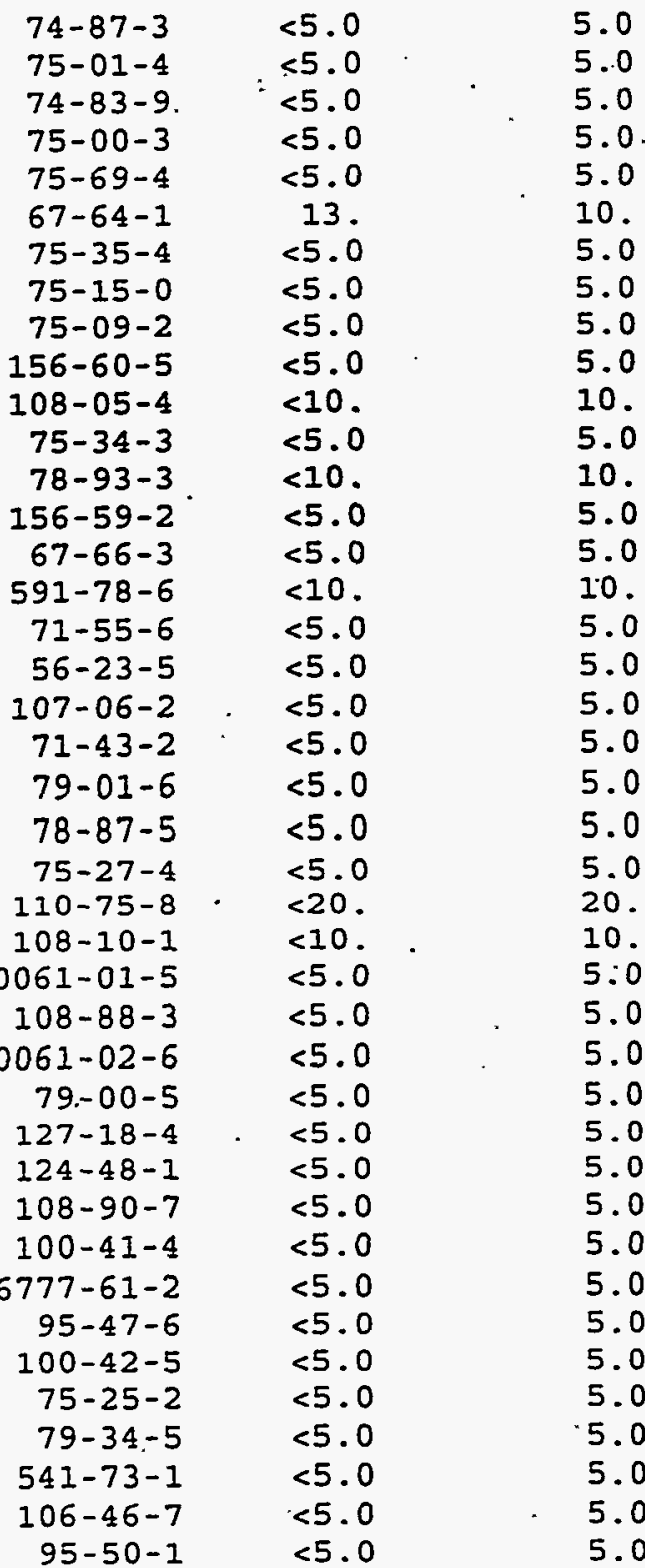

5.0

5.0

5.0

5.0

5.0

5.0

5.0

10.

5.0

5.0

5.0

10.

5.0

5.0

5.0

5.0

20 .

10 .

5.0

5.0

5.0

5.0

5.0

5.0

5.0

5.0

5.0

5.0

5.0

5.0 


\section{LAS LABORATORIES}

VOLATILE ORGANICS BY GC/AS

8260 VOIATILES

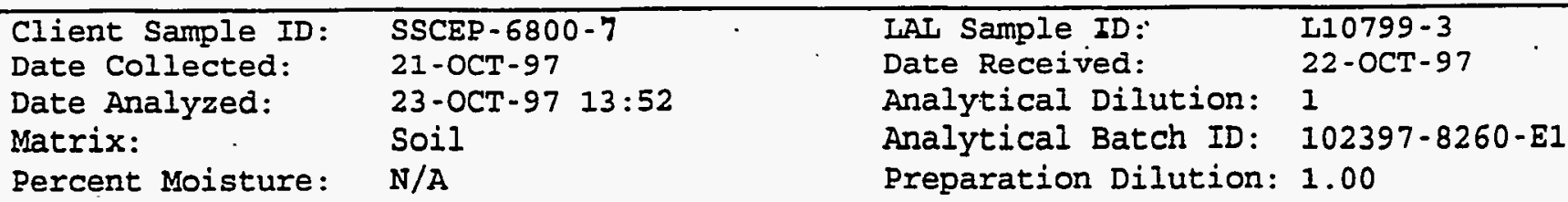

\begin{tabular}{|c|c|c|}
\hline H४২, & 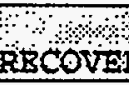 & \\
\hline 1,2-Dichloroethane-d4 & $102 \%$ & $77-127$ \\
\hline Toluene-d8 & $100 \%$ & $84-120$ \\
\hline Bromofluorobenzene & $100 \%$ & $78-125$ \\
\hline
\end{tabular}

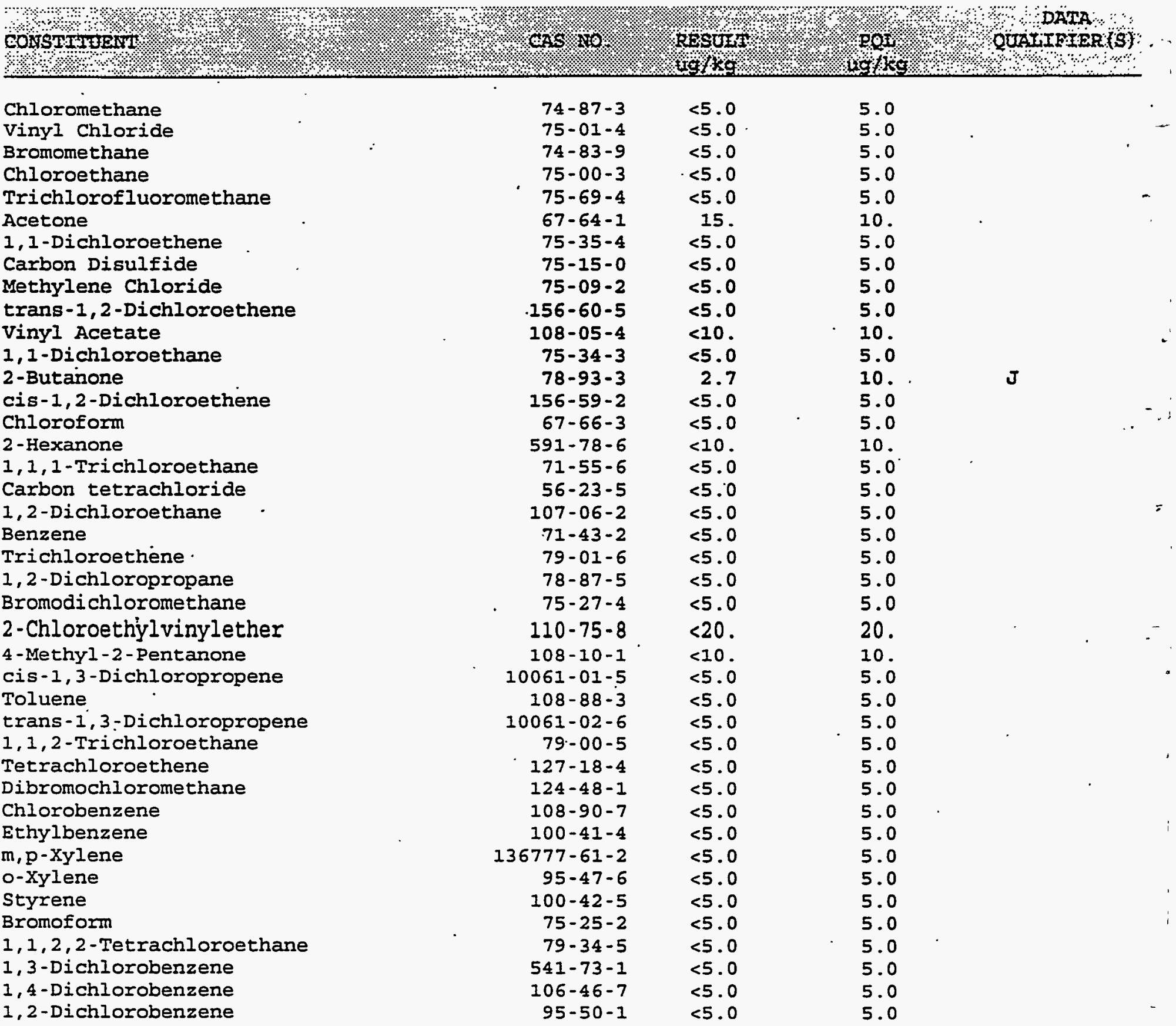




\section{¿AS LABORATORIES}

$0:$ ITILE ORGANICS BY GC/MS : 260 VOLATILES

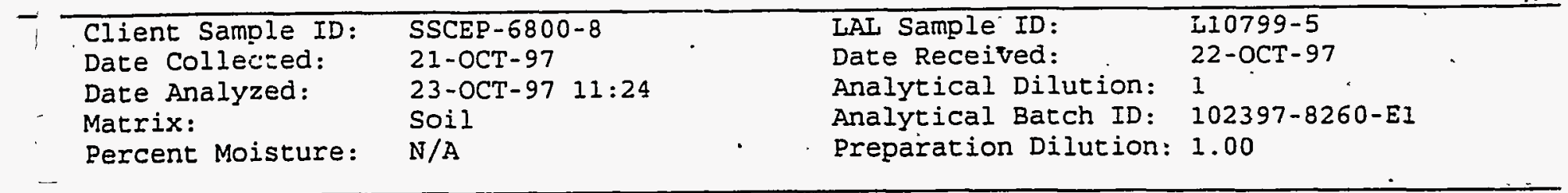

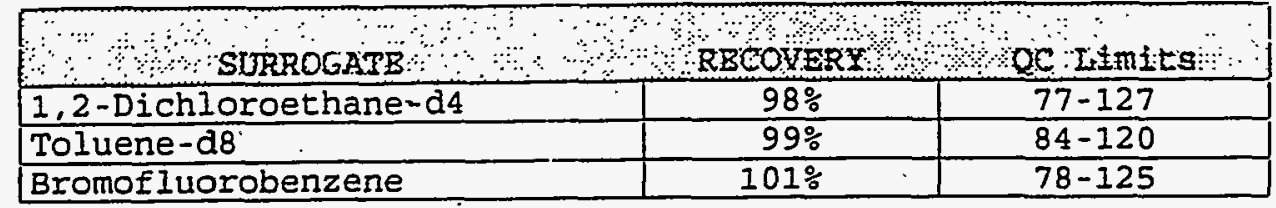

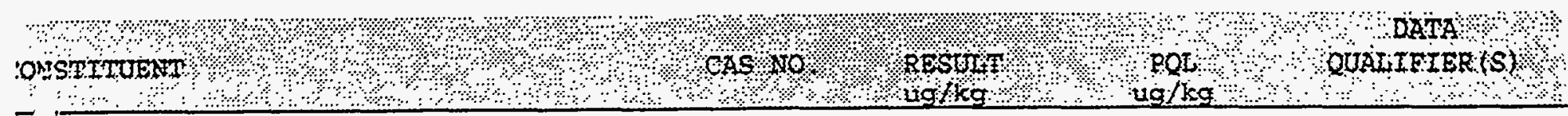

hloromethane

$r_{j}-y l$ Chloride

ir momethane

h.oroethane

'richlorofluoromethane

ic tone

., -Dichloroethene

arbon Disulfide

lerhylene Chloride

:I ns-1,2-Dichloroethene

Ij $y$ I Acetate

1-Dichloroethane

?- Dutanone

$\therefore \quad-1,2-D i c h l o r o e t h e n e$

31. oroform

?-Hexanone

L, ,1-Trichloroethane

$\therefore$ bon tetrachloride

1, L-Dichloroethane

3enzene

I. chloroethene

1. -Dichloropropane

3romodichloromethane

2-Shloroethylvinylether

4. lethyl-2-Pentanone

I. - 1, 3-Dichloropropene

Toluene

- ins-1,3-Dichloropropene

1 , 2-Trichloroethane

Teirachloroethene

Jibromochloromethane

$\approx$.orobenzene

a lylbenzene

$n, p$-xylene

o-Xylene

$\mathrm{S}$ rrene

$\mathrm{B}$ imoform

1, 1,2,2-Tetrachloroethane

13 -Dichlorobenzene

I' 1 -Dichlorobenzene

1,2-Dichlorobenzene
$<5.0$

$<5.0$

$<5.0$

$<5.0$

$<5.0$

$<10$.

$<5.0$

$<5.0$

$<5.0$

$<5.0$

$<10$.

$<5.0$

$<10$.

$<5.0$

$<5.0$

$<10$.

$<5.0$

$<5.0$

$<5.0$

$<5.0$

$<5.0$

$<5.0$

$<5.0$

$<20$.

$<10$.

$<5.0$

$<5.0$

$<5.0$

$<5.0$

$<5.0$

$<5.0$

$<5.0$

$<5.0$

$<5.0$

$<5: 0$

$<5.0$

$<5.0$

$<5.0$

$<5.0$

$<5.0$

$<5.0$
5.0

5.0

5.0

5.0

5.0

10.

5.0

5.0

5.0

5.0

10.

5.0

10.

5.0

5.0

10.

5.0

5.0

5.0

5.0

5.0

5.0

5.0

20.

10 .

5.0

5.0

5.0

5.0

5.0

5.0

5.0

5.0

5.0

5.0

5.0

5.0

5.0

5.0

5.0

5.0 


\section{VOLUME 2 \\ APPENDIX B}

\section{TABLE 4 ANALYTICAL DATA}




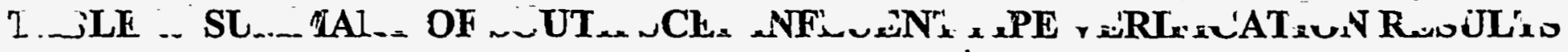

\begin{tabular}{|c|c|c|c|c|c|c|c|}
\hline 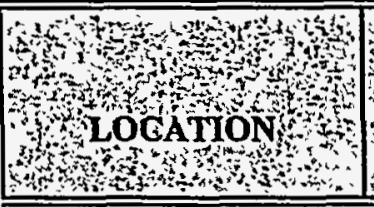 & 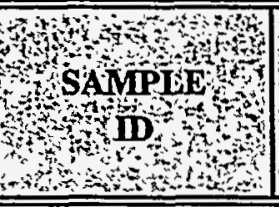 & 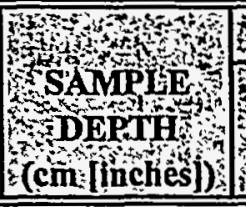 & 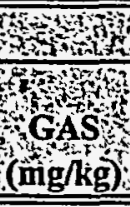 & 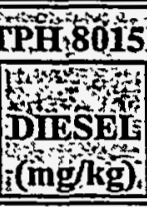 & 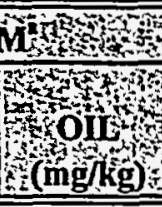 & 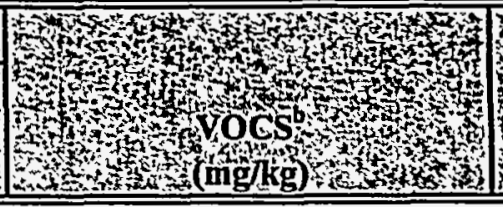 & 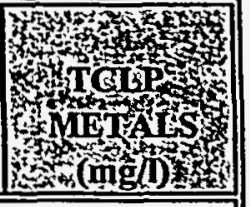 \\
\hline \multicolumn{8}{|c|}{ SAMPLES COLLECTED JUNE 23, JULY 15, 16, \& 29, AND OCTOBER 9, 1997} \\
\hline West of South Pond & SSCEP-PIPE-1 & $91-106[36-42]$ & ND & ND & ND & $\cdot N D$ & Ba 0.994 \\
\hline West of South Pond & SSCEP-PIPE-2 & $|91-106[36-42]|$ & ND & 70 & ND & ND & $\mathrm{Ba} 1.130$ \\
\hline $\begin{array}{l}\text { End of Iron Pipe } \\
\text { East of 6-05 Road }\end{array}$ & SSCEP-PIPE-3 & $91-106[36-42]$ & ND. & ND & ND & ND & $\mathrm{Ba} 0.620$ \\
\hline $\begin{array}{c}\text { End of Pipe Joint } \\
\text { East of Boxcar }\end{array}$ & SSCEP-PIPE-4 & $|91-106[36-42]|$ & ND & ND & 96 & Methylene Chloride $\quad 0.008$ & $\mathrm{Ba} 0.633$ \\
\hline $\begin{array}{c}\text { End of Pipe Joint } \\
\text { East of Boxcar }\end{array}$ & SSCEP-PIPE-4B & $91-106[36-42]$ & NA & NA & NA & ND & NA \\
\hline $\begin{array}{l}\text { 7th Iron Ring Joint } \\
\text { SE.6-800 Separator }\end{array}$ & SSCEP-PIPE-5 & $37-152[54-60$ & ND & ND & ND & ND & $\begin{array}{l}\mathrm{Ba} 0.816 \\
\mathrm{Ag} \quad 0.017\end{array}$ \\
\hline $\begin{array}{l}\text { 5th Iron Ring Joint } \\
\text { SE 6-800 Separator }\end{array}$ & SSCEP-PIPE-6 & $37-152[54-60$ & ND & ND & 47 & ND & Ba 0.545 \\
\hline $\begin{array}{c}\text { Intersection Trench } \\
6-800 \text { and } 6-621 \\
\end{array}$ & SSCEP-PIPE-7 & $37-152[54-60]$ & ND & ND & 110 & ND & Ba 0.727 \\
\hline $\begin{array}{l}\text { Sample Point } 7 \text { After } \\
\text { Additional Excavation }\end{array}$ & SSCEP-PIPE-7B & $98-213[78-84$ & ND & ND & ND & NA & NA \\
\hline $\begin{array}{l}\text { 2nd pipe elbow } \\
\text { SE 6-800 Separator }\end{array}$ & SSCEP-PIPE-8 & $37-152[54-60]$ & ND & ND & ND & ND & $\mathrm{Ba} 0.813$ \\
\hline
\end{tabular}


TABLE 4: SUMMARY OF SOUTH SCEP INFLUENT PIPE VERIFICATION RESULTS

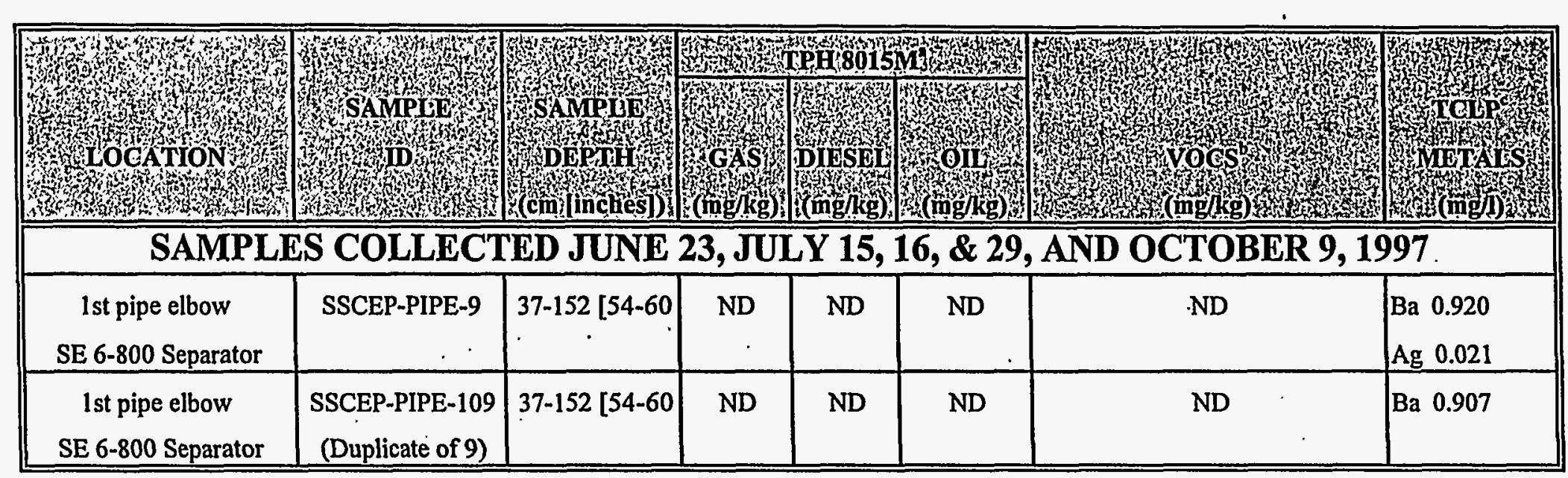

Notes:

'TPH 8015M: Total Petroleum Hydrocarbons.Method 8015 Modified.

bOCs: Volatile Organic Compounds.

' TCLP: Toxicity Characteristic Leaching Procedure.

Ba Barium

Ag Silver

SCEP: Steam Cleaning Effluent Pond

ND: Not Detected

NA: Not Analyzed

cm: centimeter

mg/kg: milligrams per kilogram

mg/l: milligrams per liter 
EPA SAMPIE NO. SSCEP-PIPEI

\begin{abstract}
ab Name: Quanterra, MO
Lab code: ITMO Case No.: Htrix : (soil/water) soII

Sample wt/vol: $\quad 1.0^{\circ}(\mathrm{g} / \mathrm{ml}) \quad \mathrm{G}$

इvel: (low/med) LOW
\end{abstract}

soisture: not dec.

CAS NO.

Compound .

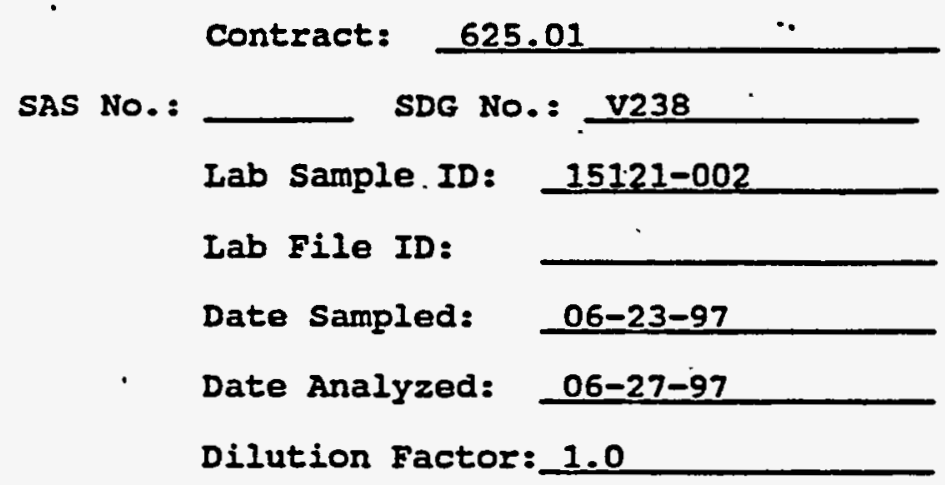

CONCENTRATION UNITS:

(ug/L or ug/Rg) UG/RG

IBH (1)

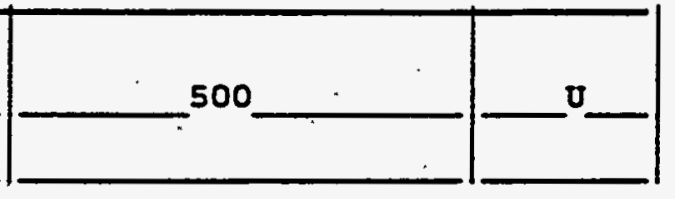

J:Concentration of analyte is less than the value given. $X:$ Pattern reasonably matches gasoline 6: Pattern appears to be multi-peaks, but does not match gasoline ::Concentration is primarily from a single peak

(1): Low Boiling Hydrocarbon (LBE) is quantitated as if it is Gasoline. 
ID

EPA SAMPLE NO.

SSCEP-PIPE-I
Iab Name: OUANTERRA MO

Iab Code: ITMO Case No.:

SOIL

Matrix : . (soil/water)

Sample wt/vol:

$20.0 \quad(\mathrm{~g} / \mathrm{ml})$

G

SAS NO.:

Contract :

625.01

Level: (low/med)

IOW

Moisture: not dec. dec.

Extraction: (SepF/Cont/Sonc/Shak)

SHAR

GPC Cleanup: $(\mathrm{Y} / \mathrm{N}) \mathrm{N} \quad \mathrm{pH}$ :

$\longrightarrow$
Lab Sample ID:

$15121-002$

Lab File ID:

Date Sampled:

$06-23-97$

Date Extracted:

$06-27-97$

Date Analyzed:

$06-28-97$

Dilution Factor:

1

CAS NO.

Compound

CONCENTRATION UNITS

$\begin{array}{r}\hline \text { DIESEI__ } \\ \hline \text { WASTE OIL_ }\end{array}$

*: Sample has an unknown hydrocarbon at approximately $26 \mathrm{mg} / \mathrm{kg}$.

U: Concentration of analyte is less than the value given. 
i. Name: QUANTERRA MO

Case No.: V12101

it Code: ITMO
Contract: $625-01$

SAS NO.:

SSCEPPIPE2

2) :ix: (soil/water) soIL

imple wt/vol:

$5.00(\mathrm{~g} / \mathrm{mL}) \mathrm{G}$

*I: (low/med) LOW

Mnisture: not dec.

: column: RTX-502.2 ID: 0.530 (mm)

: . Extract Volume:

(UL)

Lab Sample ID: 15121-003

Lab File ID: G7259

Date Received: 06/25/97

Date Analyzed: 07/.04/97

Dilution Factor: $\quad 1.0$

Soil Aliquot Volume:

(UI)

CONCENTRATION UNITS:

CAS NO.

COMPOUND

(ug/I or $u g / \mathrm{kg}$ ) UG/KG 


\section{U.S. EPA - CLP}

1 INORGANIC ANAIYSES DATA SHEET

Lab Name: QUANTERRA_MO Lab Code: ITMO_ Case No.:

Contract: 625.01 SAS NO . :

EPA SAMPLE NO.

\section{SSCEPPIPE2}

Matrix (soil/water) : WATER

Level (low/med): LOW
SDG NO.: V238

Lab Sample ID: P15121-003

Date Received: $06 / 25 / 97$

क्षे Solids :

0.0

Concentration Units (ug/I or $\mathrm{mg} / \mathrm{kg}$ dry weight) : UG/I_

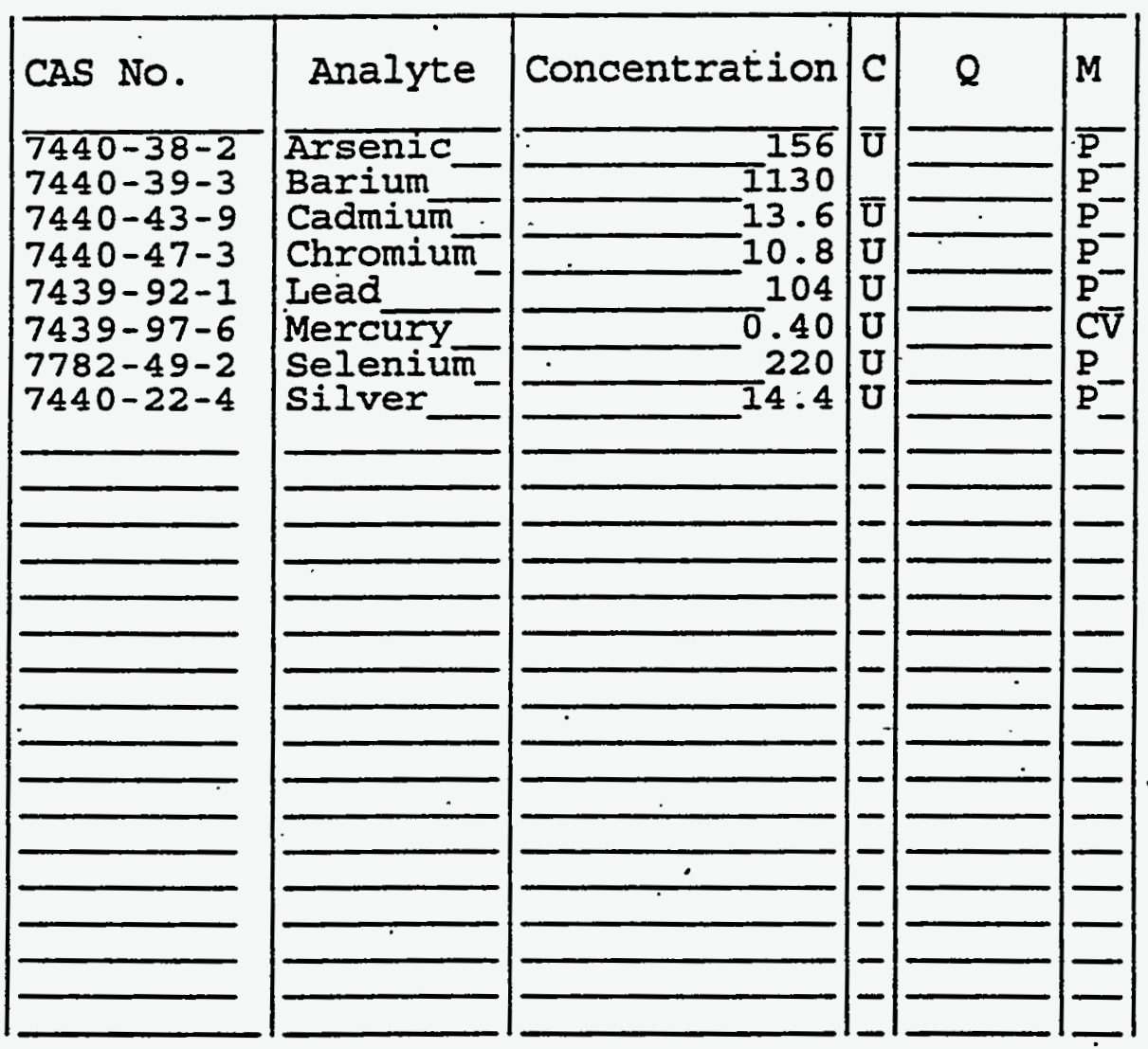

Color Before:

Color After:

Comments :
Clarity Before:

Clarity After:
Texture:

Artifacts : 


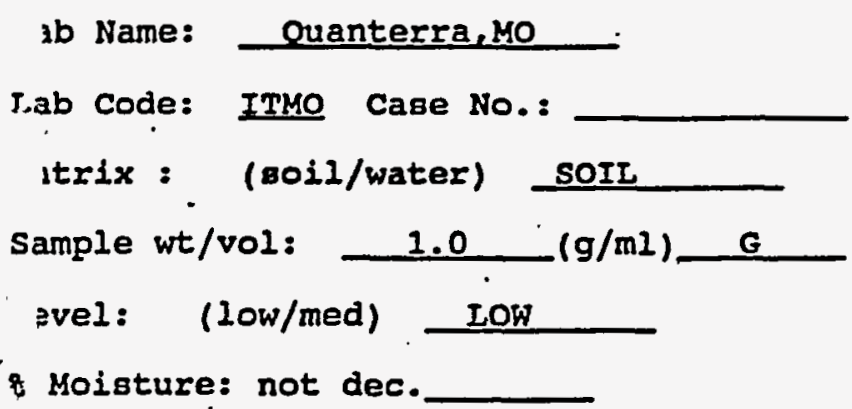

Contract: 625.01 SAS NO.: SDG NO.: V238

Iab Sample ID: 15121-003

Lab File ID:

Date Sampled: $\quad 06-23-97$

Date Analyzed: $06-27-97$

Dilution Factor: 1.0
CONCENTRATION UNITS:

(ug/I or ug/Rg) UG/KG
- $\mathbf{Q}$

\begin{tabular}{ll|l|l|}
\hline LBH (1)___ & \\
\hline
\end{tabular}

$U$ : Concentration of analyte is less than the value given.

$\mathrm{X}$ : Pattern reasonably matches gasoline

$\psi$ : Pattern appears to be multi-peaks, but does not match gasoline

$z$ :Concentration is primarily from a single peak

(1): Low Boiling Hydrocarbon (LBH) is quantitated as if it is Gasoline. 
1D

HBH ANALYSIS DATA. SHEET
EPA SAMPLE NO. SSCEP-PIPE-2
Lab Name: OUANTERRA MO

Lab Code: ITMO Case No.:

Matrix : (soil/watex)

SOIL

Sample wt/vol:

$20.0 \quad(\mathrm{~g} / \mathrm{ml})$

G

Level: (low/med)

LOW

? Moisture: not dec. dec.

Extraction: (SepF/Cont/Sonc/Ṣhak)

SHAK

GPC Cleanup: $(\mathrm{Y} / \mathrm{N})$ N $\mathrm{N}$

CAS NO.

Compound

CONCENTRATION UNITS :

$(\mathrm{mg} / \mathrm{L}$ or $\mathrm{mg} / \mathrm{Kg}$ ) $\mathrm{mg} / \mathrm{kg}$

$\mathbf{Q}$

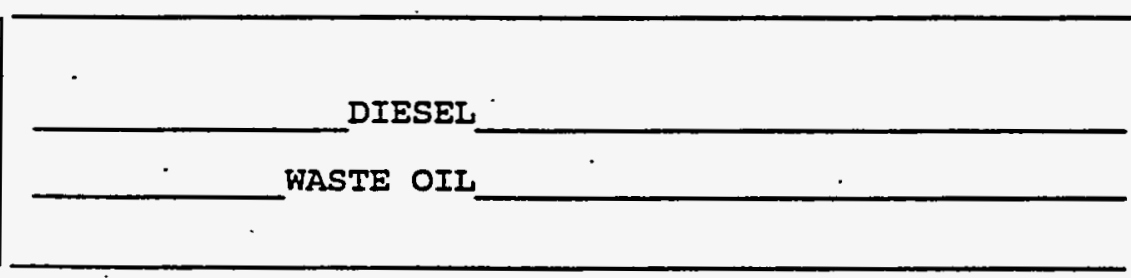

$U$ : Concentration of analyte is less than the value given.
625.01

SDG NO.: $\quad$ V238

Iab File ID:

Date Sampled:

$06-23-97$

Date Extracted:

$06-27-97$

Date Analyzed:

$06-28-97$

Dilution Factor: 1
15121-003

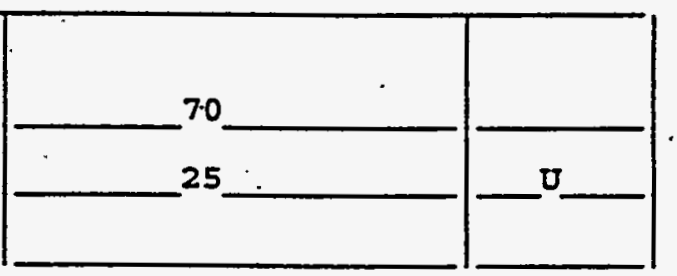

FORM I HBE 
b Name: QUANTERRA MO

j Code: ITMO

trix: (soil/water) soIL

tuple wt/vol:

$5.00(\mathrm{~g} / \mathrm{mL}) \mathrm{G}$

$\because \geqq 1: \quad$ (low/med) LOW

Moisture: not dec.

: Zolumn: RTX-502.2 ID: 0.530 (mm)

jil Extract Volume:

CAS NO.
'Contract: 625-01

SAS NO.:
SSCEPPIPE!

SDG No.: V238

Lab Sample ID: 15121-002

Lab File ID: $\quad$ G727 $\ddot{3}$

Date Received: 06/25/97

Date Analyzed: 07/04/97

Dilution Factor: $\quad 1.0$

Soil Aliquot Volume:

(uL)

CONCENTRATION UNITS:

(ug/I or $\mathrm{ug} / \mathrm{Kg}$ ) UG/KG

74-87-3--.---Chloromethane

74-83-9-..---Bromomethane

75-01-4-...--.-Vinyl Chloride

75-00-3-..---Chloroethane

75-09-2-...-.-Methylene Chloride

67-64-1-....-.-Acetone

75-15-0--.---Carbon Disulfide

75-35-4-.---1,1-Dichloroethene

75-34-3-...-1,1-Dichloroethane

540-59-0-..---1,2-Di=hloroethene (total)

67-66-3-..----Chloroform

107-06-2-...-1,2-Dichloroethane

78-93-3-...--2-Butanone

$71-55-6 \ldots-1.1,1-$ Trichloroethane

56-23-5-..-----Carbon Tetrachloride

75-27-4-...-.-Bromodichloromethane

78-87-5-...--1, 2-Dichloropropane

10061-01-5-----cis-1,3-Dichloropropene

79-01-6-...--Trichloroethene

124-48-1--.--Dibromochloromethane

79-00-5--.---1, 1,2-Trichloroethane

71-43-2----.--Benzene

10061-02-6-..--trans-1,3-Dichloropropene

75-25-2-..----Bromoform

108-10-1-....--4-Methyl-2-Pentanone

591-78-6--.----2-Hexanone

127-18-4-...--Tetrachloroethene

$79-34-5-\ldots \ldots-1,1,2,2$-Tetrachloroethane

108-88-3-..---Toluene

108-90-7--.---Chlorobenzene

100-41-4------Ethylbenzene

100-42-5---.---Styrene.

$1330-20-7-\ldots .-$ Xylene (total) 


\section{U.S. EPA - CLP}

\section{1}

INORGANIC ANAIYSES DATA SHEET
Lab Name: QUANTERRA_MO

Lab Code: ITMO_ Case No.:

Matrix (soil/water): WATER

Level (low/med): LOW

$\%$ Solids:

$-0.0$
Contract: 625.01 SAS NO.:

SDG NO.: V238

Lab Sample ID: P15121-002

Date Received: 06/25/97

EPA SAMPLE NO.

SSCEPPIPE1

Concentration Units (ug/L or $\mathrm{mg} / \mathrm{kg}$ dry weight): UG/L_

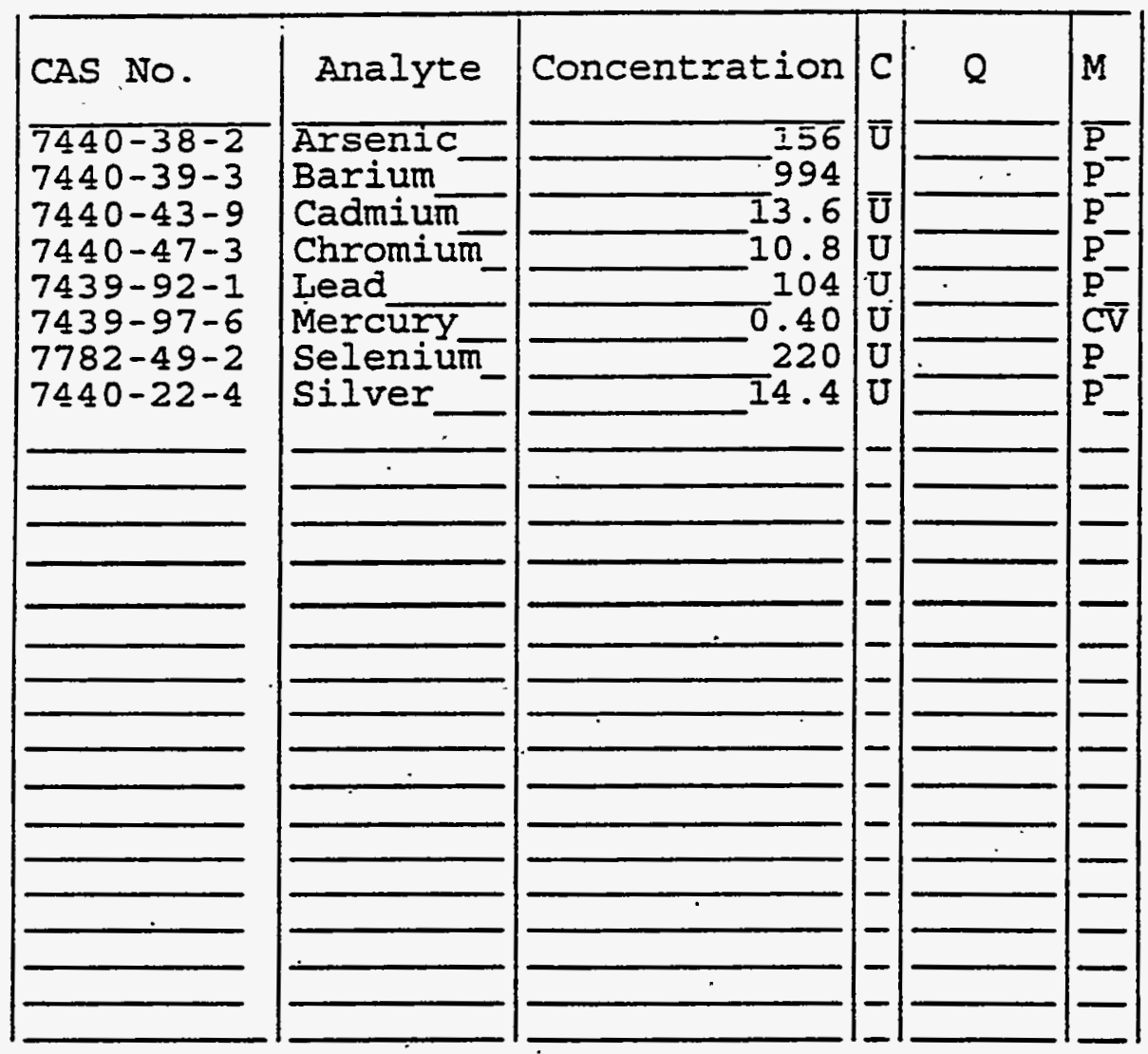

Color Before:

Clarity Before:

Texture:

Clarity After:

Artifacts :

Color After:

Comments : 
ab Name: Quanterra,MO

, J Code: ITMO Case No.:

1atrix : (soil/water) SOIL

; uple wt/vol:

$1.0 \quad(\mathrm{~g} / \mathrm{ml})$ LOW

sevel: (low/med)

; loisture: not dec.

CAS NO.

Compound

CONCENTRATION UNITS:

(ug/L or ug/kg) UG/KG - . Q .

SAS NO.:

SDG No.: V250

Lab Sample ID: 15349-002

Lab File ID:

Date Sampled: $\quad 07-15-97$

Date Analyzed: $07-29-97$

Dilution Factor: 1.0

LBH (1)

Contract: 625.01

LEH (1)__

U: Concentration of analyte is less than the value given.

Pattern reasonably matches gasoline

Pattern appears to be multi-peaks, but does not match gasoline

Concentration is primarily from a single peak

1): Low Boiling Hydrocarbon ( $L B H$ ) is quantitated as if it is Gasoline. 


\section{D \\ HBH ANALYSIS DATA SHEET}

EPA SAMPLE NO. SSCEP-PIPE-3
Lab Name: OUANTERRA MO

Lab Code: ITMO Case No.:

Matrix : (soil/water)

SOIL

Sample wt/vol:

20.

$(g / m I)$

G

Level: (low/med)

LOW

\& Moisture: not dec. dec.

Contract:

SAS NO.:

Lab Sample ID:

SDG NO.: V250

Lab File ID:

Date Sampled:

Date Extracted:

Date Analyzed:

Dilution Factor:

CONCENTRATION UNITS:

CAS NO.

Compound

. $(\mathrm{mg} / \mathrm{L}$ or $\mathrm{mg} / \mathrm{kg})$

DIESEL

WASTE OIL

*: Sample is a mixture of an early unknown hydrocarbon (approx. $1500 \mathrm{mg} / \mathrm{kg}$ ) and waste oil.

$\mathrm{U}$ : Concentration of analyte is less than the value given.

FORM I HBH
$07-15-97$

07-22-97

$07-24-97$

1

$\mathrm{mg} / \mathrm{kg}$

$\mathbf{Q}$

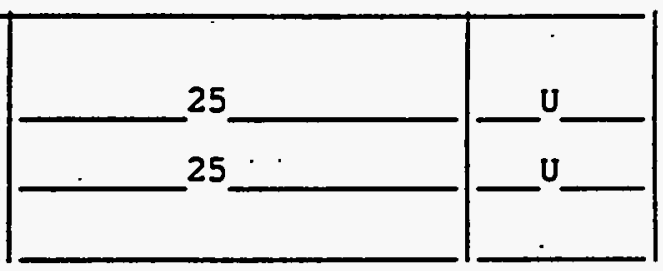


VOLATILE ORGANICS ANALYSIS DATA SHEET

Name: QUANTERRA MO

Code: ITMO

Case No.: V34901

ix: (soil/waterr) SoIL

iple wt/vol:

$5.00(\mathrm{~g} / \mathrm{mL}) \quad \mathrm{G}$

r L: (low/med) LOW

Inisture: not dec.

wolumn: RTX-502.2 ID: 0.530 (mm)

Extract Volume:

(uL)
Contract: $625-01$

SAS NO.:
SSCEPPIPE3

SDG No.: V250
Lab Sample ID: 15349-002

Lab File ID: F0951

Date Received: 07/17/97

Date Analyzed: $07 / 28 / 97$

Dilution Factor: $\quad 1.0$

Soil Aliquot Volume:

(uL)

CÁS NO.

COMPOUND

CONCENTRATION UNITS:

(ug/L or ug/Kg) UG/KG

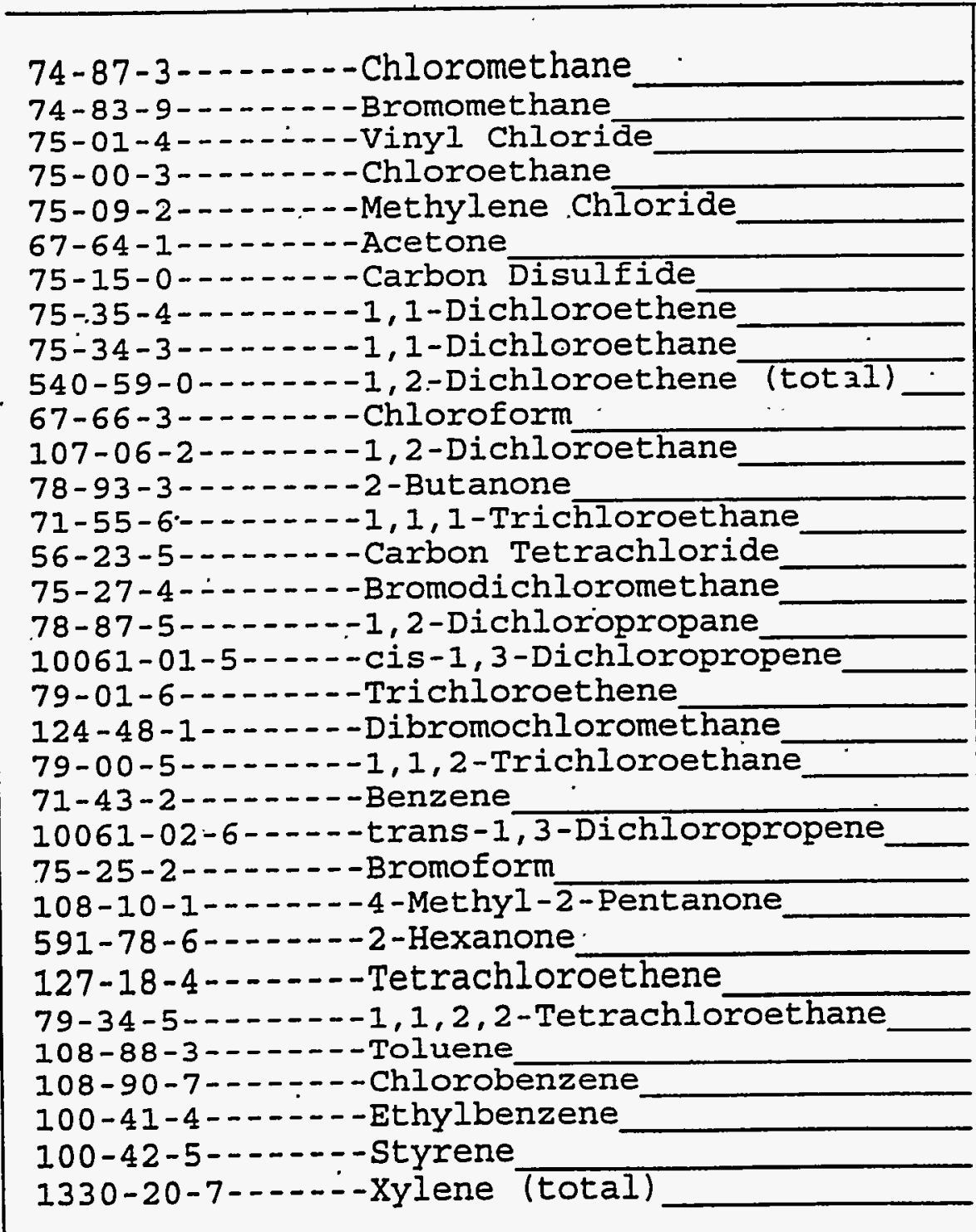


Lab Name: QUANTERRA_MO

Lab Code: ITMO

Case No.:

Matrix (soil/water) : WATER

Level (low/med): LOW

Solids :
Contract: 625.01 SAS NO.:

SDG NO.: 'V250

Lab Sample ID: P15349-002

Date Received: 07/17/97
EPA SAMPLE NO.

SSCEP-PIPE-3 
Lab Name:

Quanterra, Mo

t b Code: ITMO Case No.:

Matrix : (soil/water) SOIL

I mple wt/vol:

$1.0(\mathrm{~g} / \mathrm{ml})$

G

Level: (low/med)

LOH

q Moisture: not dec.
Contract: $\quad 625.01$

SAS NO.:

$$
\text { SDG NO.: V251 }
$$

Lab Sample ID: 15351-002

Lab File ID:

Date Sampled: 07-16-97

Date Analyzed: 07-29-97

Dilution Factor: 1.0

CONCENTRATION UNITS:

(ug/I or ug/Kg) UG/KG $Q$

LBH (1)

500

$\mathrm{u}$

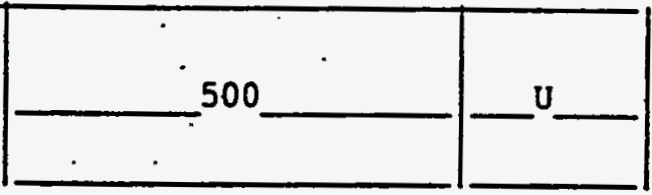

U: Concentration of analyte is less than the value given.

Pattern reasonably matches gasoline

Pattern appears to be multi-peaks, but does not match gasoline

Concentration is primarily from a single peak

1): Low Boiling Hydrocarbon (LBH) is quantitated as if it is Gasoline. 
Lab Name: QUANTERRA MO

Contract:

625.01

Lab Code: ITMO Casé No.: SAS NO.: SDG NO.: V251

Matrix : (soil/water)

SOIL

Sample wt/vol: 20.0 $(g / m l)$ G

Level: (low/med) LOW

\& Moisture: not dec. dec. Extraction: (SepF/Cont/Sonc/.Shak) ____HAK GPC Cleanup: $(\mathrm{Y} / \mathrm{N})+\mathrm{N} \quad \mathrm{pH}$ : CAS No. Compound UNITS (mg/L or $\mathrm{mg} / \mathrm{Kg}$ ) $\mathrm{mg} / \mathrm{kq}$

]$^{25}+96$

$\mathbf{U}$ DIESEL WASTE OIL

W DASTE OIL_

Lab Sample ID: $15351-002$ Lab File ID: Date Sampled: $07-16-97$

Date Extracted:

Date Analyzed:

Dilution Factor: $\quad 1$ LeT $q 1 / 5 / 9 z^{\cdots}$

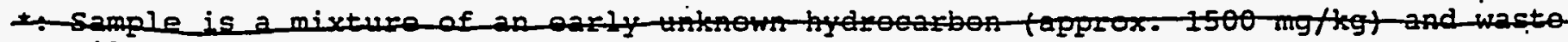
Q $9 / 1,5 / 97$

u: Concentration of analyte is less than the value given.
} 


\section{LAS LABORATOKES}

\section{$\checkmark$ LATILE ORGANICS BY GC/MS}

8260 VOLLATILES

\begin{tabular}{llll}
\hline Client Sample ID: & SSCEP-PIPE-4B & IAS Sample ID: & LI0691-3 \\
Date Collected: & $07-0 C T-97$ & Date Received: & $09-0 C T-97$ \\
Date Analyzed: & $13-0 C T-9718: 16$ & Analytical Dilution: 1 \\
Matrix: & Soil & Analytical Batch ID: 101397-8260-C2 \\
Percent Moisture: & 7.91 & Preparation Dilution:-1.00 \\
\hline
\end{tabular}

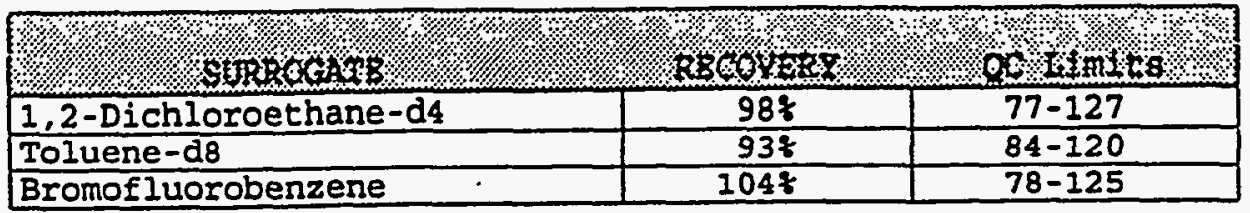

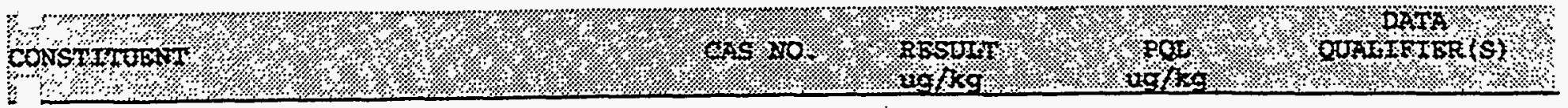

cnloromethane

Vinyl Chloride

f omomethane

I loroethane

Trichlorofluoromethane

Asetone

1-Dichloroethene

'. irbon Disulfide

Methylene Chloride

r.ans-1,2-Dichloroethene

- nyl Acetate

-1-Dichloroethane

2-Butanone

-1s-1,2-Dichloroethene

lloroform

- -Hexanone

1,1,1-Trichloroethane

- irbon tetrachloride

2-Dichloroethane

senzene

Trichloroethene

,2-Dichloropropane comodichloromethane

2-Chloroethylvinylether

4-Methyl-2-Pentanone

is-1, 3-Dichloropropene

oluene

trans-1, 3-Dichloropropene

1,1,2-Trichloroethane

etrachloroethene.

ibromochloromethane

Chlorobenzene

thylbenzene

ip-Xylene

-xylene

Styrene

romoform

,1,2,2-Tetrachloroethane

1,3-Dichlorobenzene

1,4-Dichlorobenzene

.,2-Dichlorobenzere
$<5.4$

$<5.4$

$<5.4$

$<5.4$

$<5.4$

7.0

$<5.4$

$<5.4$

$<5.4$

$<5.4$

$<11$.

$<5.4$

$<11$.

$<5.4$

$<5.4$

$<11$.

$<5.4$

$<5.4$

$<5.4$

$<5.4$

$<5.4$

$<5.4$

$<5.4$

$<22$.

$<11$.

$<5.4$

$<5.4$

$<5.4$

$<5.4$

$<5.4$

$<5.4$

$<5.4$

$<5.4$

$<5.4$

$<5.4$

$<5.4$

$<5.4$

$<5.4$

$<5.4$

$<5.4$

$<5.4$
5.4

5.4

5.4

5.4

5.4

11.

5.4

5.4

5.4

5.4

11.

5.4

11.

5.4

5.4

11.

5.4

5.4

5.4

5.4

5.4

5.4

5.4

22.

11.

5.4

5.4

5.4

5.4

5.4

5.4 .

5.4

5.4

5.4

5.4

5.4

5.4

5.4

5.4

5.4

5.4
JB 
Lab Name:

Lab code: ITMO Case No.:

Matrix : (soilfwater) SOIL

Sample wt/vol: 1.0 $(g / m l)$

Ievel: (low/med) LOW

\& Moisture: not dec.

CAS No. Compound .
Contract: $\quad 625.01$

SDG NO.: V238

SAS NO.:

Lab Sample ID: 15121-004

Lab File ID:

Date sampled:

Date Analyzed: 06-27-97

Dilution Factor: 1.0

CONCENTRATION UNITS:

(ug/I or ug/Rg) UG/RG

Q 500 $\mathbf{0}$

$\mathrm{IBH}^{\circ}$ (1)

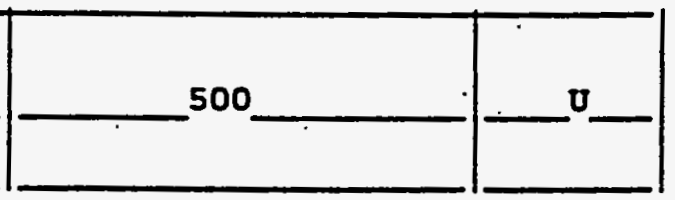

$U$ :Concentration of analyte is 2 ess than the value given. $X:$ Pattern reasonably matches gasoline

Y:Pattern appears to be multi-peaks, but does not match gasoline

$\mathrm{z}$ : Concentration is primarily from a single peak

(1):Low Boiling Hydrocarbon (LBH) is quantitated as if it is Gasoline. 
ID

HBH ANALYSIS DATA SHEET
EPA SAMPLE NO.

SSCEP-PIPE- 5

Iab Name: OUANTERRA MO

Contract:

625.01

iab Code: ITMO Case No.:

SAS NO.:

SDG NO.: V238

itix

(soil/water)

SOIL

Lab Sample ID:

$15121-004$

$\Gamma$ mple wt/vol:

$20.1-(\mathrm{g} / \mathrm{ml})$

Iab File ID:

Level: (Low/med)

LOW

Date Sampled:

$06-23-97$

Date Extracted:

06-27-97

Date Analyzed:

$06-28-97$

Dilution Factor:

1

rרc cleanup: $(\mathrm{Y} / \mathrm{N}) \stackrel{\mathrm{N}}{\mathrm{pH}}$ :

1

CONCENTRATION UNITS :

CAS NO.

Compound

(mg/I or $\mathrm{mg} / \mathrm{kg}$ )

$\mathrm{mg} / \mathrm{kg}$

Q

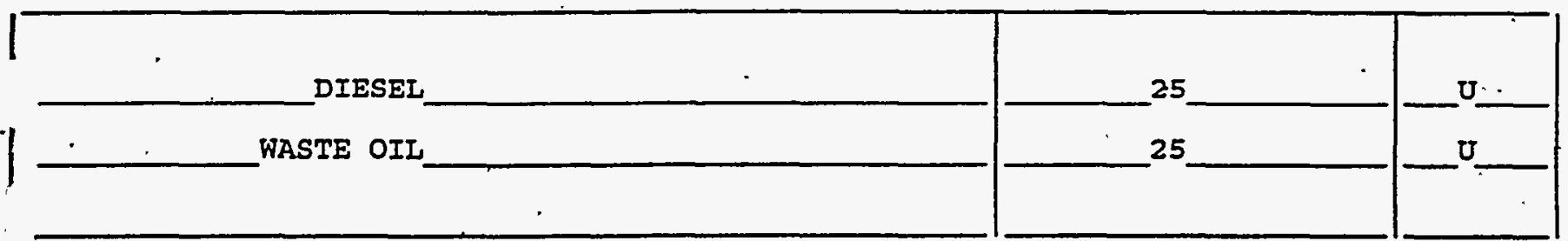

I: Concentration of analyte is less than the value given.

FORM I HBH 
SSCEPPIPE5

Lab Name: QUANTERRA MO

Lab Code: ITMO

Case No.: V12101

Matrix: (soil/water) soIL

Sample wt/vol: $\quad 5.00(\mathrm{~g} / \mathrm{mI}) \mathrm{G}$

Level: (low/med) LOW

\% Moisture: not dec.

Contract: $625-01$

SAS NO.:

SDG No.: V238

Lab Sample ID: 15121-004

Lab File ID: . G730 $\ddot{5}$

Date Received: 06/25/97

Date Analyzed: 07/06/97

Dilution Factor: $\quad 1.0$

Soil Aliquot Volume:

(UI)

CAS NO. COMPOUND (ug/L or $\mathrm{ug} / \mathrm{Kg}) \mathrm{UG} / \mathrm{KG}$

CONCENTRATION UNITS:

\begin{tabular}{|c|}
\hline 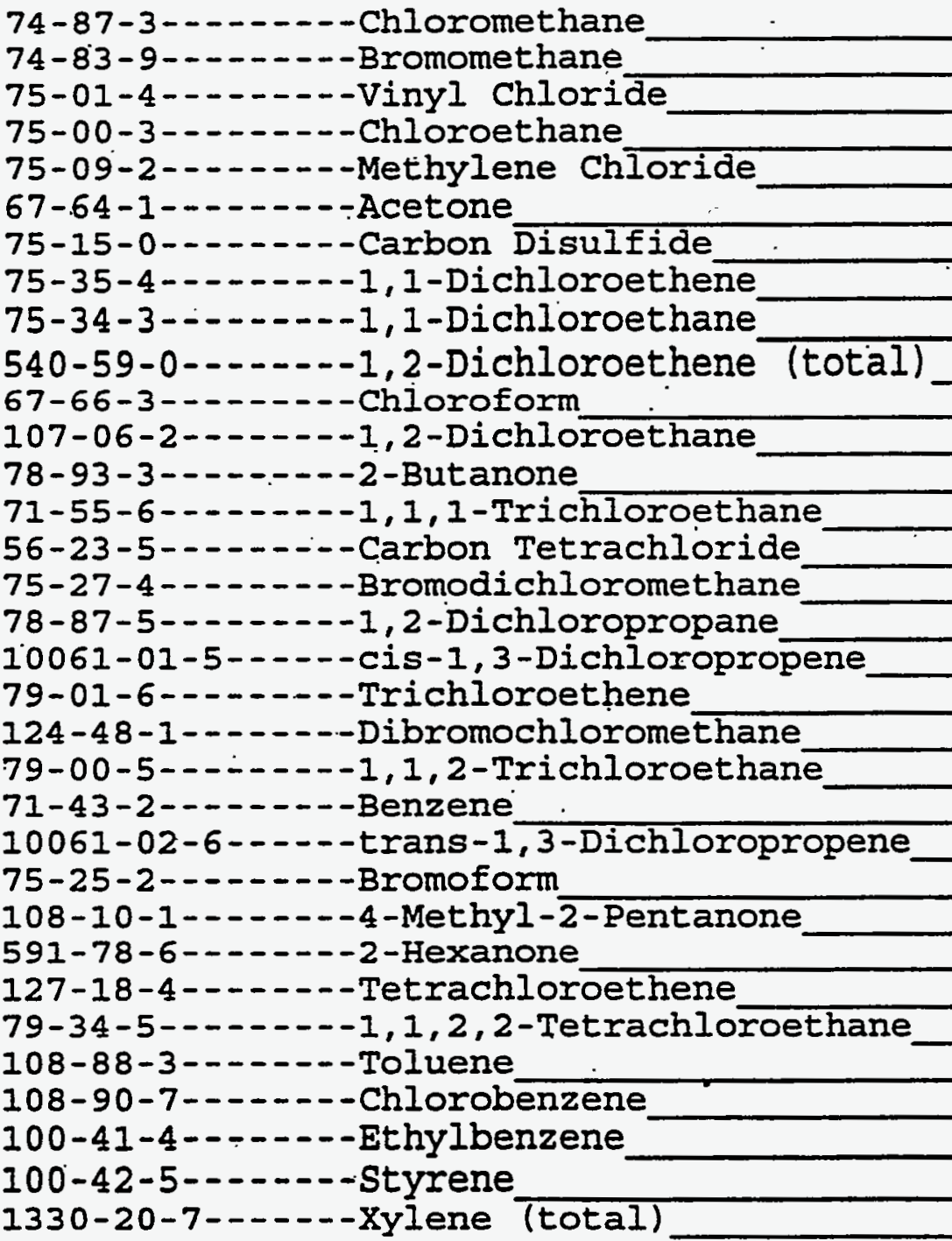 \\
\hline
\end{tabular}


$1 A$

EPA SAMPLE NO.

VOLATILE ORGANICS ANALYSIS DATA SHEET

, Name: 'QUANTERRA MO

, Code: ITMO, Case No.: V35101.

ix: (soil/water) WATER

iple wt/vol:

$5.00(\mathrm{~g} / \mathrm{mL}) \mathrm{ML}$

I: (low $/ \mathrm{med})$ LOW

loisture: not dec.

olumn: RTX-502.2 ID: 0.530 (mm)

.7 Extract Volume:

(uL)
Contract: $625-01$

SAS NO.:
SSCEPPIPE4

SDG NO.: V251

Lab. Sample. ID: 15351-002

Lab File ID: F0953

Date Received: $07 / 17 / 97$

Date Analyzed: 07/28/97

Dilution Factor: $\quad 1.0$.

Soil Aliquot Volume: (UL)

CONCENTRATION UNITS:

CAS NO. COMPOUND (ug/L or ug/Kg) UG/L

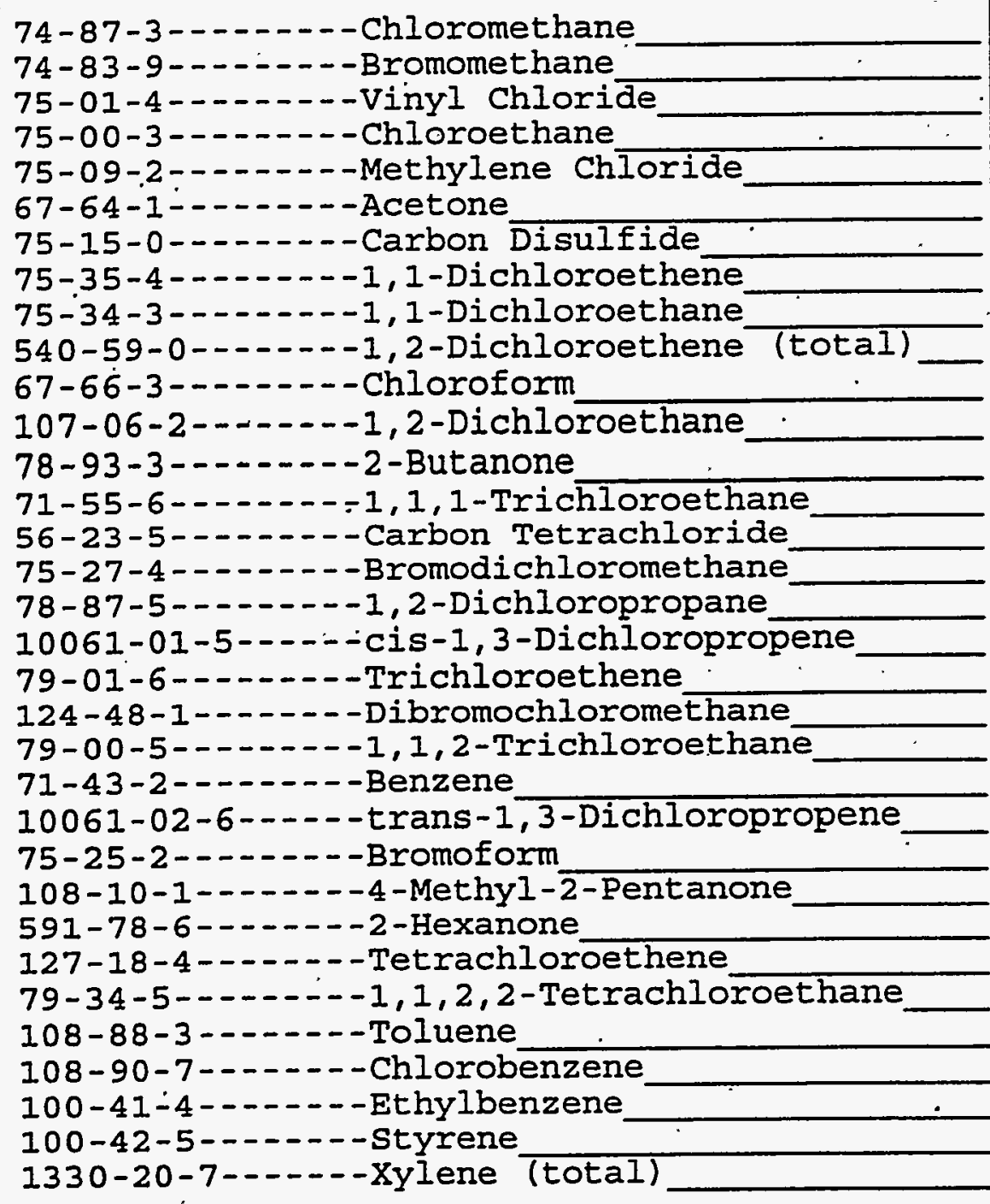

74-87-3--.-.--Chloromethane

74-83-9------Bromomethane

75-01-4-..----Vinyl Chloride

75-00-3--.----Chloroethane

75-09-2.-...--Methylene Chloride

67-64-1--..--Acetone

75-15-0-.-.--Carbon Disulfide

75-35-4--.--1, 1-Dichloroethene

75-34-3-...-1, 1-Dichloroethane

540-59-0--...-1,2-Dichloroethene (total)

67-66-3-...-.-Chloroform

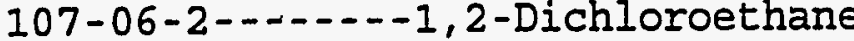

78-93-3--..--2-Butanone

71-55-6-..--1,1,1-Trichloroethane

56-23-5-...---Carbon Tetrachloride

75-27-4---.----Bromodichloromethane

78-87-5------1,2-Dichloropropane

10061-01-5-----cis-1,3-Dichloropropene

79-01-6--..---Trichloroethene

124-48-1--.--Dibromochloromethane

71-43-2--------Benzene

10061-02-6-----trans-1,3-Dichloropropene

75-25-2-....--Bromoform

108-10-1-...--4-Methyl-2-Pentanone

591-78-6-------2-Hexanone

127-18-4--..--Tetrachloroethene

79-34-5--.---1, 1,2,2-Tetrachloroethane

108-88-3-..-- Toluene

108-90-7--.---Chlorobenzene

100-42-5-------styrene

1330-20-7-...--Xylene (total

\begin{tabular}{|c|c|c|}
\hline$\cdot$ & $\begin{array}{r}10 \\
10 \\
10 \\
10 \\
8 \\
20 \\
5 \\
5 \\
5 \\
5 \\
5 \\
5 \\
20 \\
5 \\
5 \\
5 \\
5 \\
5 \\
5 \\
5 \\
5 \\
5 \\
5 \\
5 \\
20 \\
20 \\
5 \\
5 \\
4 \\
4 \\
5 \\
5 \\
5 \\
5\end{array}$ & $\begin{array}{l}U \\
U \\
U \\
U \\
U \\
U \\
U \\
U \\
U \\
U \\
U \\
U \\
U \\
U \\
U \\
U \\
U \\
U \\
U \\
U \\
U \\
U \\
U \\
U \\
U \\
U \\
U \\
U \\
J \\
U \\
U \\
U \\
U\end{array}$ \\
\hline
\end{tabular}


1 INORGANIC ANAIYSES DATA SHEET
EPA SAMPLE NO.

SSCEP-PIIPE-4
Lab Name: QUANTERRA_MO

Lab Code: ITMO_. Case No.:

Matrix (soil/water) : WATER

Level (low/med):

LOW

$-0.0$

Solids:

Contract: 625.01

SAS NO. :

SDG No.: V251

Lab Sample ID: P15351-002

Date Received: 07/17/97

$$
\text { - } 0.0
$$

Concentration Units (ug/L or $\mathrm{mg} / \mathrm{kg}$ dry weight) : UG/I_

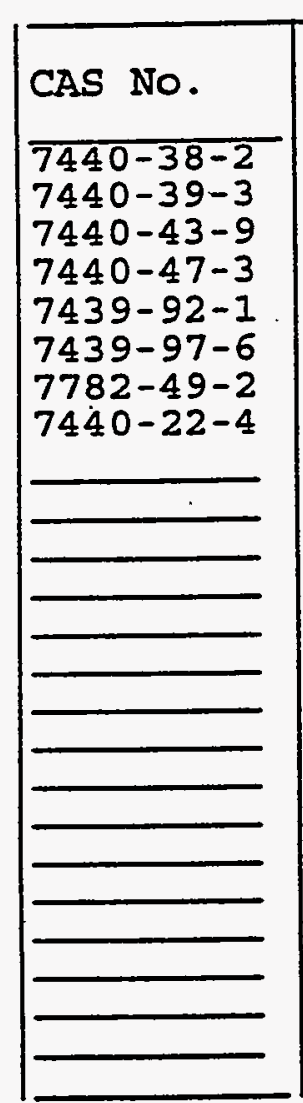

Color Before:

Color After:

Comments :

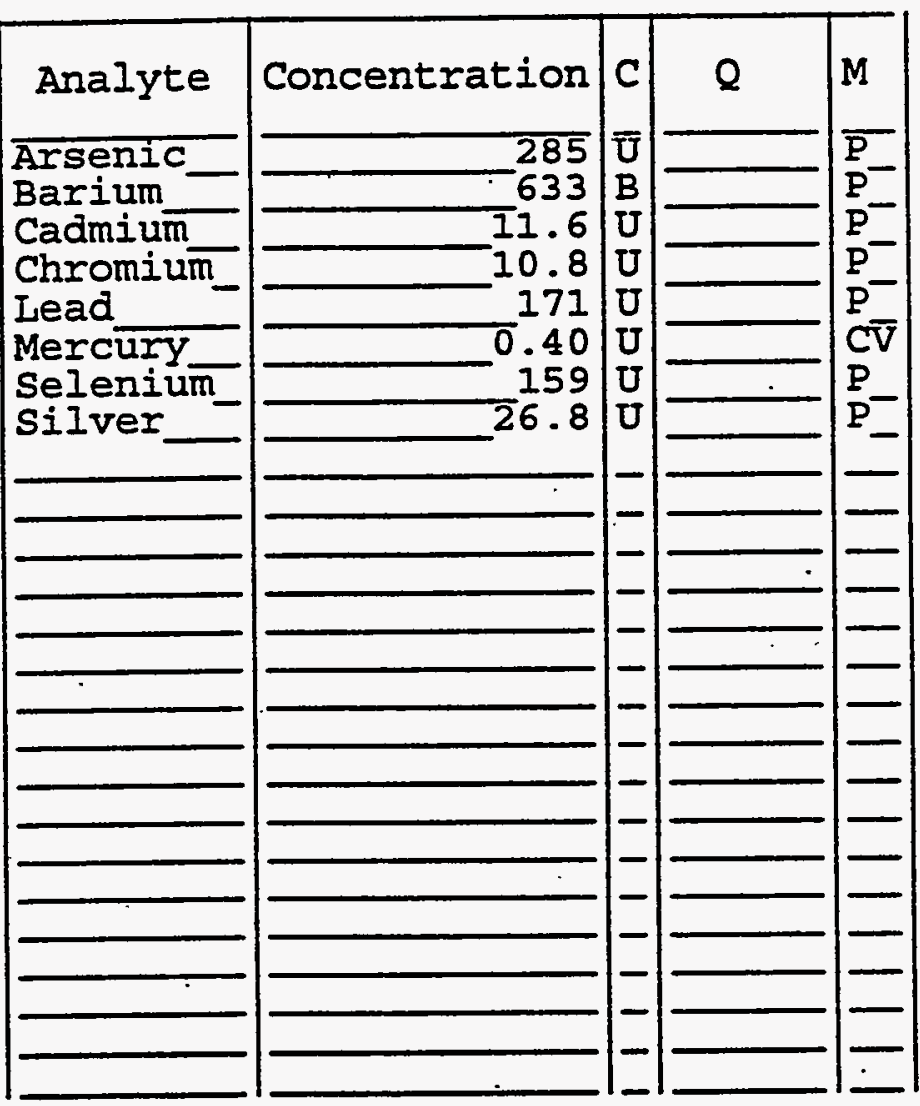

Clarity Before:

Texture:

Clarity After:

Artifacts : 
, Iame: QUANTERRA_MO

, Code: ITMO__. Case No.:

$:$ : $x$ (soil/water) : WATER

rel (low/med) : LOW
Contract: 625.01

SAS NO.:
EPA SAMPLE NO.

\section{SSCEPPIPE5}

ic .ids: 0.0

Concentration Units (ug/I or mg/kg dry weight): UG/L_

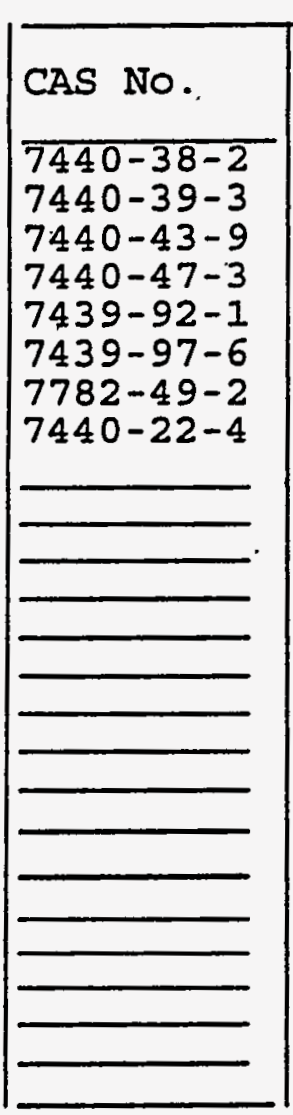

L'r Before: Lor After: $n$ ents:

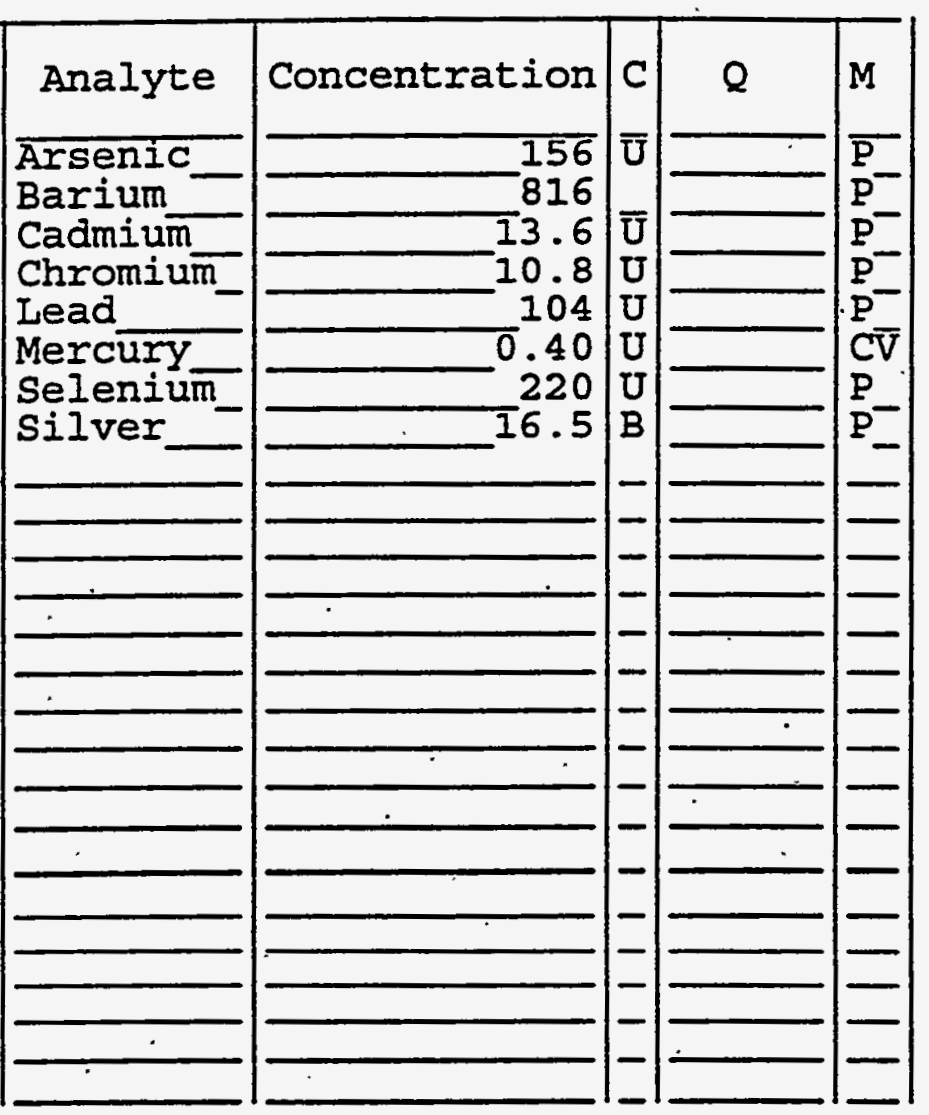

Clarity Before:

Texture :

clarity After:
Lab Sample ID: . P151291-004

Date Received: $06 / 25 / 97$

SDG No.: V238 
Lab Name:

Iab Code:

Matrix

Sample wt/vol:

IMO Case No.:

SOIL

Level: (low/med) LOW .

\& Moisture: not dec.

(Yoisture: not dec.

CAS NO.
Contract: $\quad 625.02$ SDG NO.: V238 SAS NO.: Iab sample ID: 15121-005

Iab File ID:

Date sampled: 06-23-97

Date Analyzed: $06-27-97$

Dilution Factor: 1.0

CONCENTRATION UNITS:

(ug/I or ug/Rg) UG/RG

IBH (1)

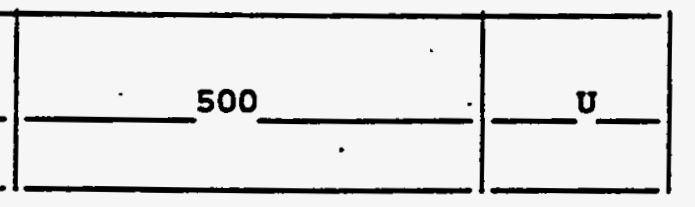

$U$ :Concentration of analyte is less than the value given. $X:$ Pattern reasonably matches gasoline $Y$ :Pattern appears to be multi-peaks, but does not match gasoline $\mathrm{z}$ :Concentration is primarily from a single peak

(1) Low Boiling Hydrocarbon (LBE) is quantitated as if it is Gasoline. 
EPA SAMPLE NO SSCEP-PIPE- 6
Lab Name: OUANTERRA MO

ab Code: ITMO Case No.:

ztrix : (soil/water)

SOIL

cample wt/vol:

$20.2(\dot{g} / \mathrm{ml})$

G

Level: (low/med)

LOW

Moisture: not dec.

dec.

ktraction: (SepF/Cont/Sonc/Shak)

SHAR

sPC Cleanup: $(\mathrm{Y} / \mathrm{N}) \stackrel{\mathrm{N}}{\mathrm{NH}}$

Contract :

625.01

SAS NO.:

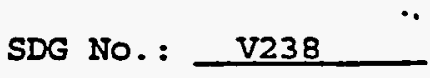

Lab Sample ID:

$15121-005$

Lab File ID:

Date Sampled:

$06-23-97$

Date Extracted:

Date Analyzed: $06-28-97$

Dilution Factor

1

CONCENTRATION UNITS :

CAS NO.

Compound

(mg/L or $\mathrm{mg} / \mathrm{kg}$ ) $\mathrm{ma} / \mathrm{kq}$

$\square^{25}+{ }^{47}$
U DIESEL WASTE OIL

J: Concentration of analyte is less than the value given. 
Lab Name: QUANTERRA MO

Lab Code: ITMO

Case No.: V12101

Matrix: (soil/water) SOIL

Sample wt/vol: $\quad 5.00(\mathrm{~g} / \mathrm{mL}) \mathrm{G}$

Level: (low/med) LOW

$\because$ Moisture: not dec.

GC Column: RTX-502.2 ID: $0.530 .(\mathrm{mm})$

Soil Extract Volume:

(UI)
Contract: $625-01$

SAS NO.:
SSCEPPIPE6

SDG No.: V238

Lab Sample ID: 15121-005

Lab File ID: $\quad$ G727

Date Received: $06 / 25 / 97$

Date Analyzed: 07/04/97

Dilution Factor: $\quad 1.0$

Soil Alịquot Volume:

(UI)

CONCENTRATION UNITS:

CAS NO. COMPOUND (ug/I or ug/Kg) UG/KG

Q

74-87-3-..---Chloromethane

74-83-9-1.-.---Bromomethane

75-01-4-.-----Vinyl Chloride

75-00-3-...---Chloroethane

75-09-2-...-.-Methylene Chloride

67-64-1-..----Acetone

75-15-0-...---Carbon Disulfide

75-35-4-----1, 1-Dichloroethene

75-34-3--.---1,1-Dichloroethane

540-59-0-..--1,2-Dichloroethene (total)

67-66-3--.----Chloroform

107-06-2------1,2-Dichloroethane

78-93-3--.----2-Butanone

$71-55-6 \ldots-\ldots-1,1 ;$ i-Trichloroethane

56-23-5-..----Carbon Tetrachloride

75-27-4-..-- - - Bromodichloromethane

78-87-5--.--1, 2-Dichloropropane

10061-01-5-..--cis-1,3-Dichloropropene

79-01-6-..---.Trichloroethene

124-48-1--.---Dibromochlorome thane

79-00-5---.--1, 1, 2-Trichloroethane

71-43-2--.-----Benzene

10061-02-6--.--trans-1,3-Dichloropropene

75-25-2--.-.--Bromoform

108-10-1-.-.--4-Methyl-2-Pentanone

591-78-6--.----2-Hexanone

127-18-4-...-.-Tetrachloroethene

79-34-5-...-. 1, 1,2,2-Tetrachloroethane

$108-88-3-\cdots---$ Toluene

108-90-7-------Chlorobenzene

100-41-4-.----Ethylbenzene

$100-42-5-\cdots-.-$-Styrene

$1330-20-7-\ldots-.-x y l e n e$ (total)

\begin{tabular}{r|l}
10 & $U$ \\
10 & $U$ \\
10 & $U$ \\
10 & $U$ \\
5 & $U$ \\
20 & $U$ \\
5 & $U$ \\
5 & $U$ \\
5 & $U$ \\
5 & $U$ \\
5 & $U$ \\
5 & $U$ \\
20 & $U$ \\
5 & $U$ \\
5 & $U$ \\
5 & $U$ \\
5 & $U$ \\
5 & $U$ \\
5 & $U$ \\
5 & $U$ \\
5 & $U$ \\
5 & $U$ \\
5 & $U$ \\
5 & $U$ \\
20 & $U$ \\
20 & $U$ \\
5 & $U$ \\
5 & $U$ \\
5 & $U$ \\
5 & $U$ \\
5 & $U$ \\
5 & $U$ \\
5 & $U$ \\
& \\
& \\
\end{tabular}


lame: QUANTERRA_MO

Code: ITMO

Case No.:

; $\mathrm{x}$ (soil/water) : WATER

el (low $/ \mathrm{med})$ : LOW

i ids :
Contract: 625.01

SAS NO.:

SDG No.: V238

Lab Sample ID: P15121-005

Date Received: 06/25/97
EPA SAMPLE NO.

\section{SSCEPPIPE6} $-0.0$

Concentration Units (ug/L or $\mathrm{mg} / \mathrm{kg}$ dry weight) : UG/L_

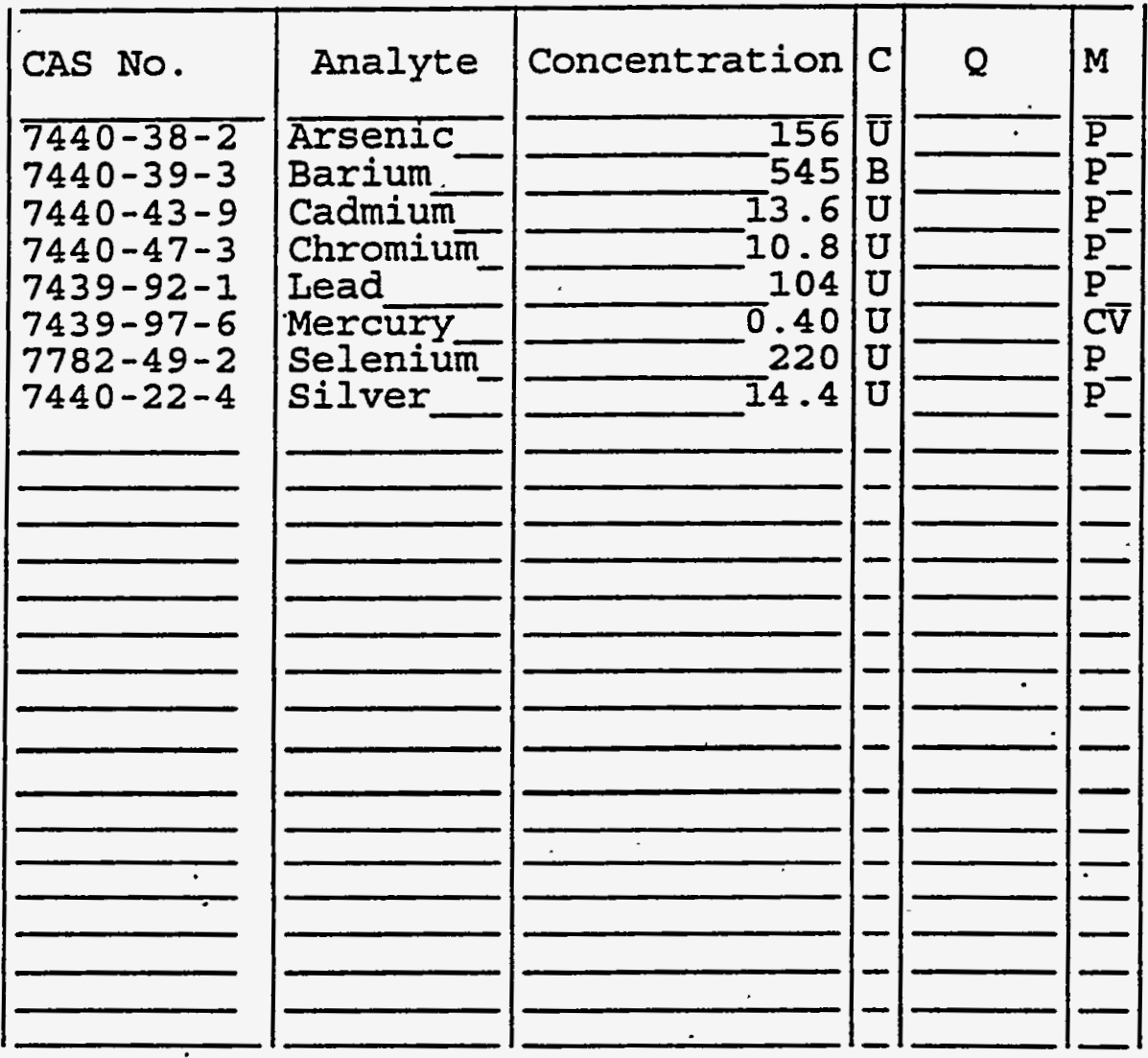

5 Before:

or After:
Clarity Before:

Clarity After:
Texture:

Arțifacts : 1 ents: 
Lab Name:

Lab code: ITMO Case No.:

Matrix : (soil/water) soIL

Sample wt/vol: $1.0 \quad(\mathrm{~g} / \mathrm{ml}) \_$G

Level: (low/med) LOW

$\&$ Moisture: not dec.

Compound .
Contract: -625.01

SDG NO.: V238

SAS NO.:

Lab Sample ID: 15121-006

Lab File ID:

Date sampled: 06-23-97

Date Analyzed: $\quad 06-27-97$

Dilution Factor: 1.0
CONCENTRATION UNITS:

(ug/t or $u g / \mathrm{kg}$ ) UG/KG
$\mathbf{Q}$

LBH $\cdot(1)$ 500

$U$ :Concentration of analyte is less than the value given.

$X:$ Pattern reasonably matches gasoline

$Y:$ Pattern appears to be multi-peaks, but does not match gasoline

$\mathrm{z}$ : Concentration is primarily from a single peak

(1) :Low Boiling Hydrocarbon (LBH) is quantitated as if it is Gasoline. 


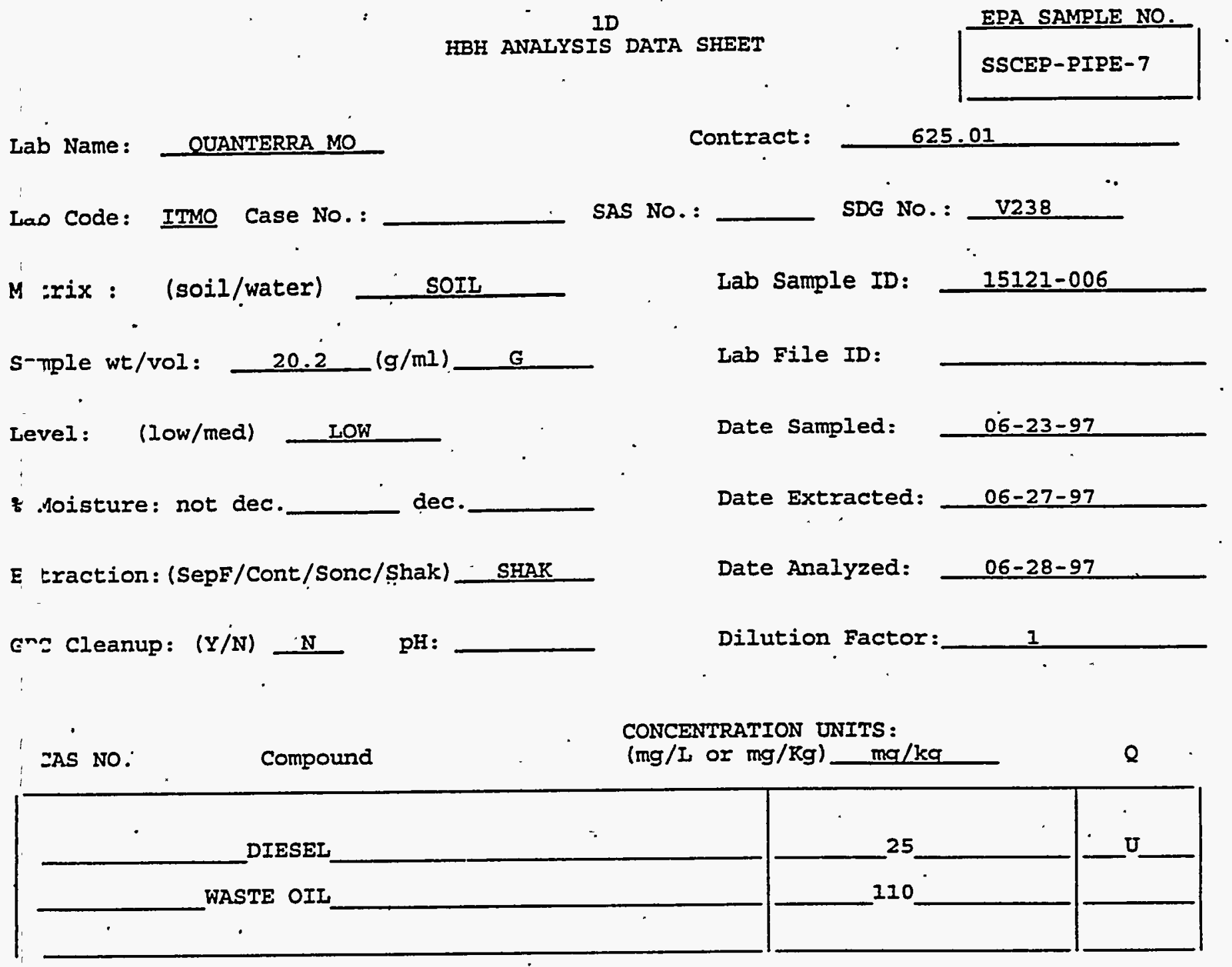

Concentration of analyte is less than the value given.

FORM I HBE 
Lab Name: QUANTERRA MO

Lab Code: ITMO

Case No.: V12101

Matrix: (soil/water) SOIL

Sample wt/vol:

$5.00(\mathrm{~g} / \mathrm{mL}) \mathrm{G}$

Ievel: (low/med) LOW

$\%$ Moisture: not dec.

GC Column: RTX-502.2 ID: $0.530(\mathrm{~mm})$

Soil Extract Volume:

(uI)
Contract : $625-01$

SAS NO.:

SDG NO.: V238

Lab Sample ID: 15121-006

Lab-File ID: G7286

Date Received: 06/25/97

Date Analyzed: 07/05/97

Dilution Factor: $\quad 1.0$

Soil Aliquot Volume:

(UL)

CONCENTRATION UNITS:

CAS NO.

COMPOUND

(ug/L or $\mathrm{ug} / \mathrm{Kg}$ ) UG/KG

74-87-3-..---Chloromethane

74-83-9-....--Bromomethane

75-01-4-....--Vinyl Chloride

75-00-3-..---.-Chloroethane

75-09-2-..---Methylene Chloride

67-64-1------.-Acetone

75-15-0-.-.---Carbon Disulfide

75-35-4-..--1,1-Dichloroethene

75-34-3--.-.--1,1-Dichloroethane

540-59-0-...--1,2-Dichloroethene (total)

67-66-3--.-----Chloroform

107-06-2-...-1, 2-Dichloroethane

78-93-3-.-.---2-Butanone

71-55-6--.--1, 1, 1-Trichloroethane

56-23-5-...-.-Carbon Tetrachloride

75-27-4-..-----Bromodichloromethane

78-87-5------1, 2-Dichloropropane

10061-01-5-----cis-1,3-Dichloropropene

79-01-6--.---.-Trichloroethene

124-48-1--.--Dibromochloromethane

79-00-5---.--1,1,2-Trichloroethane

71-43-2--------Benzene

10061-02-6-----trans-1,3-Dichloropropene

75-25-2-...-.-Bromoform

108-10-1-------4-Methyl-2-Pentanone

591-78-6--.---2-Hexanone

127-18-4-...--Tetrachloroethene

79-34-5 -...-1, 1, 2, 2-Tetrachloroethane

108-88-3--.----Toluene

108-90-7-..--Chlorobenzene

100-41-4-.-.--Ethylbenzene

100-42-5-------Styrene

1330-20-7-...-.xylene (total)

\begin{tabular}{r|r}
10 & $U$ \\
10 & $U$ \\
10 & $U$ \\
10 & $U$ \\
5 & $U$ \\
20 & $U$ \\
5 & $U$ \\
5 & $U$ \\
5 & $U$ \\
5 & $U$ \\
5 & $U$ \\
5 & $U$ \\
20 & $U$ \\
5 & $U$ \\
5 & $U$ \\
5 & $U$ \\
5 & $U$ \\
5 & $U$ \\
5 & $U$ \\
5 & $U$ \\
5 & $U$ \\
5 & $U$ \\
5 & $U$ \\
5 & $U$ \\
20 & $U$ \\
20 & $U$ \\
5 & $U$ \\
5 & $U$ \\
5 & $U$ \\
5 & $U$ \\
5 & $U$ \\
5 & $U$ \\
5 & $U$ \\
&
\end{tabular}


1

INORGANIC ANALYSES DATA SHEET

: Name :- QUANTERRA_MO

, Code: ITMO_ Case No.:

: ix (soil/water): WATER

Contract: 625.01

SAS NO. :

SDG NO.: V238

rel (low/med) : LOW

Lab Sample ID: P15121-006

Date Received: 06/25/97

; lids:

0.0

- Concentration Units (ug/I or $\mathrm{mg} / \mathrm{kg}$ dry weight) : UG/I_

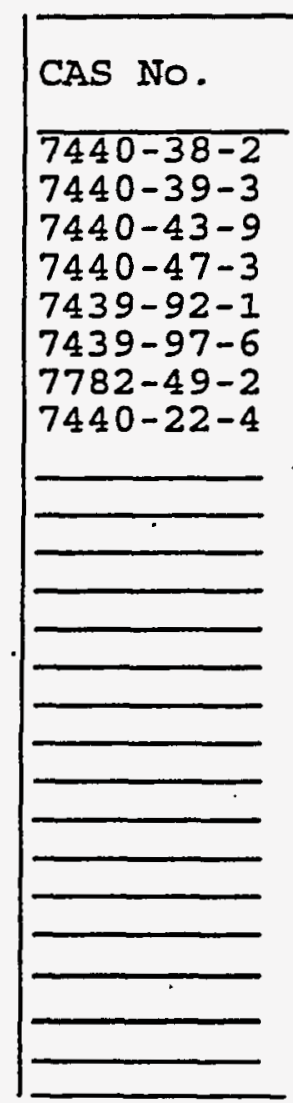

I. is Before:

Lor After:

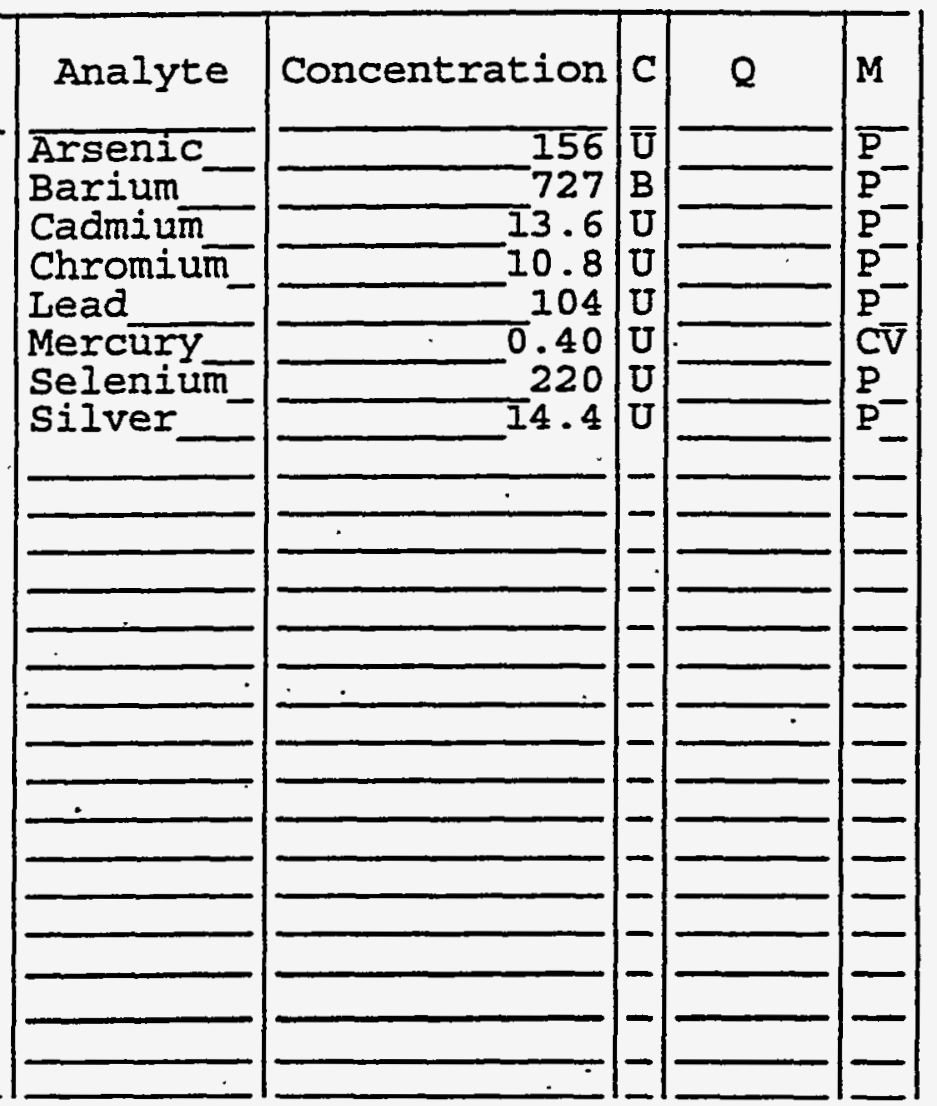

Clarity Before:

Texture :

Clarity After:

Artifacts : 
1D

HBH ANALYSIS DATA SHEERT
BPA SAYPLE NO.

SSCEP-PIPE-7B
Lab Name: OUANTERRA NO

Lab code: IIMO Case No.:

Hatrix : (soil/water)

SOIL

Sample wt/vol: $20.2(9 / \mathrm{ml})$

G

Level: (low/med)

LOW

Molsture: not dec. dec.

Extraction: (SepF/Cont/Sonc/Shak) SHAR GPC Cleanup: $(\mathrm{X} / \mathrm{N}) \perp \mathrm{N}$ pH:
- Contract: 625.01

SAS NO.: SDG NO.: V264

Iab Sample ID: 15474-003

Iab File ID:

Date Sampled: $07-22-97$

Date Extracted:

$08-05-97$

Date Analyzed: 08-19-97

Dilution Factor:

1
CAS No.

Compound

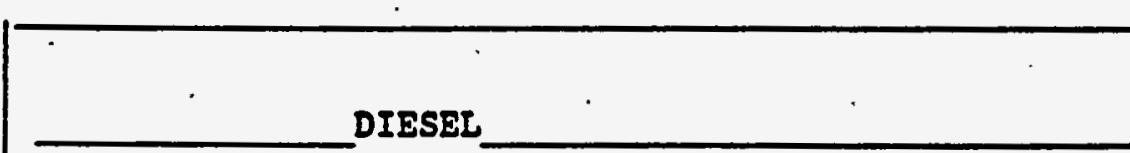

WASTE OIL
CONCENTRATION UNITS: (mg/L or $\mathrm{mg} / \mathrm{kg}$ ) $\mathrm{mg} / \mathrm{kg}$

\begin{tabular}{|c|c|}
\hline 25 & $25 \cdots$ \\
\hline & \\
\hline & \\
\hline
\end{tabular}

U: Concentration of analyte is less than the value given." 
1D

IBH ANAIYSIS DATA SHEET
EPA SAMPLE NO.

SSCEP-PIPE8 -ib Name: Ouanterra, Mo

Tab code: ITMO Case No.:

...trix : (Boil./water) soIL

cample wt/vol: $1.0 \quad(\mathrm{~g} / \mathrm{ml}) \mathrm{G}$

-2ve1: (low/med) LOW

a Moisture: not dec.

CAS NO.
Contract: $\quad 625.01$

SDG NO.: V238

SAS NO.:

Lab Sample ID: 15121-007

Iab File ID:

Date Sampled:

06-23-97

Date Analyzed: $06-27-97$

Dilution Factor: 1.0

CONCENTRATION UNITS:

(ug/L or ug/Rg) UG/RG

$\mathbf{Q}$

IBH (1)

500

0

$\cdot$

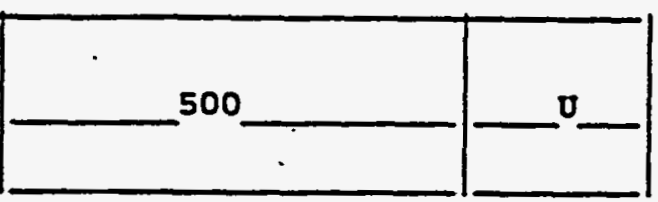

$\mathrm{U}$ : Concentration of analyte is less than the value given.

$X:$ Pattern reasonably matches gasoline

$Y$ : Pattern appears to be multi-peaks, but does not match gasoline

z:concentration is primarily from a single peak

(1):Low Boiling Hydrocarbon (IBH) is quantitated as if it is Gasoline. 


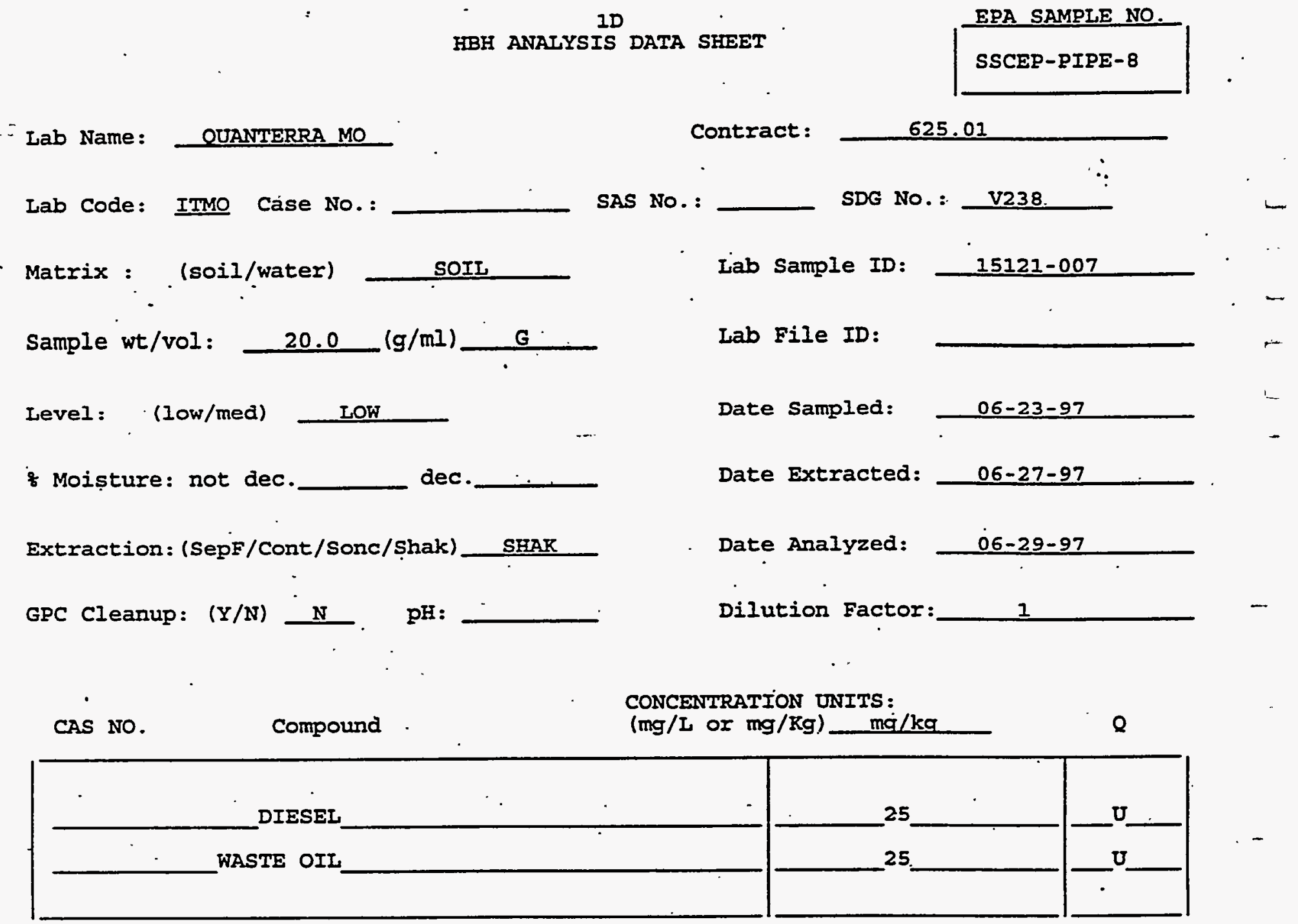

$\mathrm{U}$ : Concentration of analyte is less than the value given.

FORM. I HBH 
ab Name: QUANTERRA MO

इ, Code: ITMO Case No.: V12101

ztrix: (soil/water) soIL

zulple wt/vol:

$5.00(\mathrm{~g} / \mathrm{mL}) \mathrm{G}$

$\equiv$ el: $\quad($ low/med) LOW

Moisture: not dec.

: Column: RTX-502.2 ID: 0.530 (mm)

oil Extract Volume:

(UI)
Contract: $625-01$

SSCEPPIPE8
SAS NO.:

SDG No.: V238
Lab Sample ID: 15121-007

Lab File ID: G728 $\ddot{9}$

Date Received: 06/25/97

Date Analyzed: 07/05/97.

Dilution Factor: $\quad 1.0$

Soil Aliquot Volume:

(UL)

CÁS NO.

COMPOUND

CONCENTRATION UNITS :

(ug/I or ug/Kg) UG/KG

Q

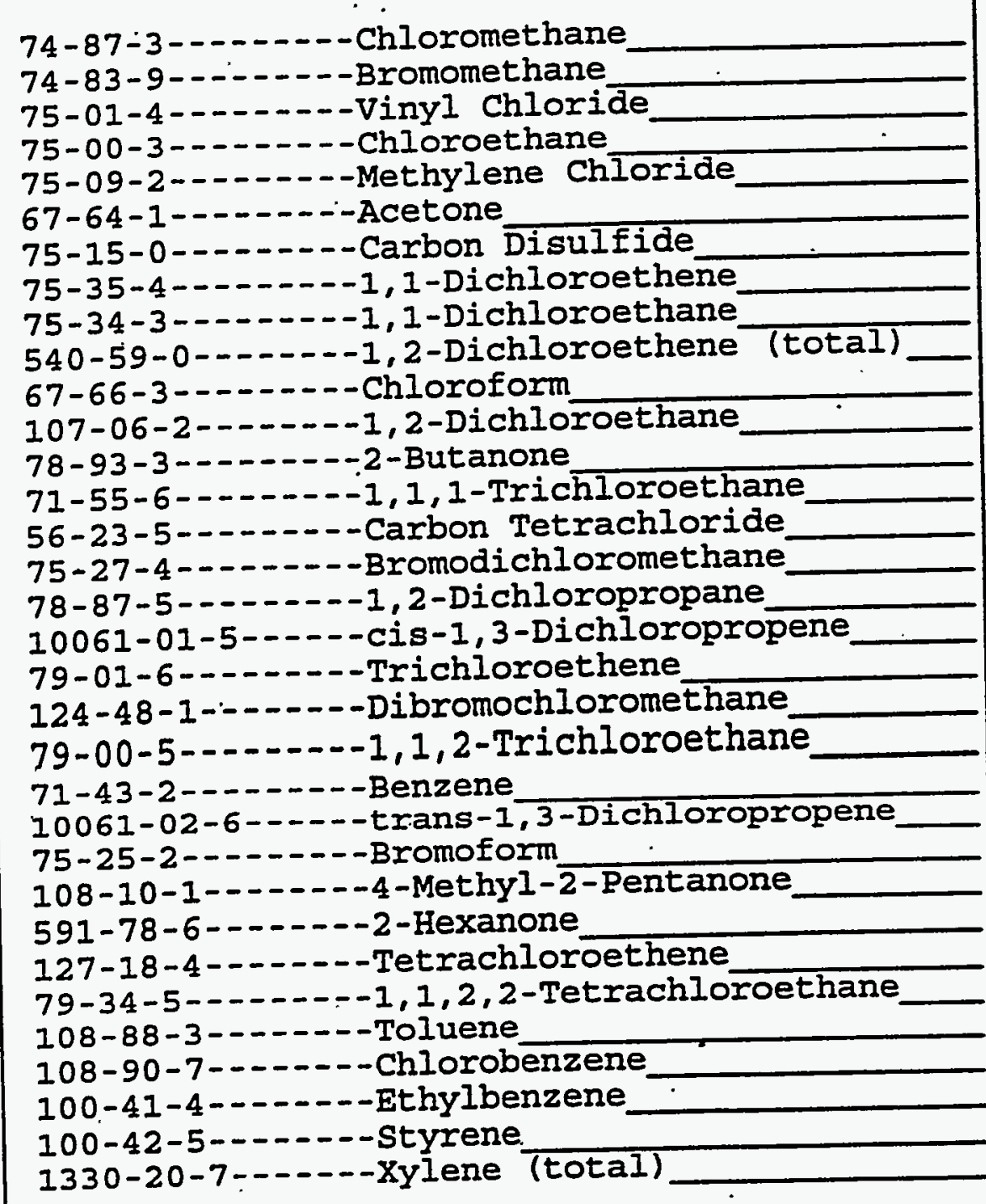


U.S. EPA - CLP

1

INORGANIC ANALY'SES DATA SHEET
EPA SAMPLE NO.

\section{SSCEPPIPE8}

Contract: 625.01

SAS NO.:

SDG NO.: V238

Lab Sample ID: P15121-007

Date Received: 06/25/97

Level (low/med) : LOW.

Solids:

$-0.0$

Concentration Units (ug/L or $\mathrm{mg} / \mathrm{kg}$ dry weight): UG/L_

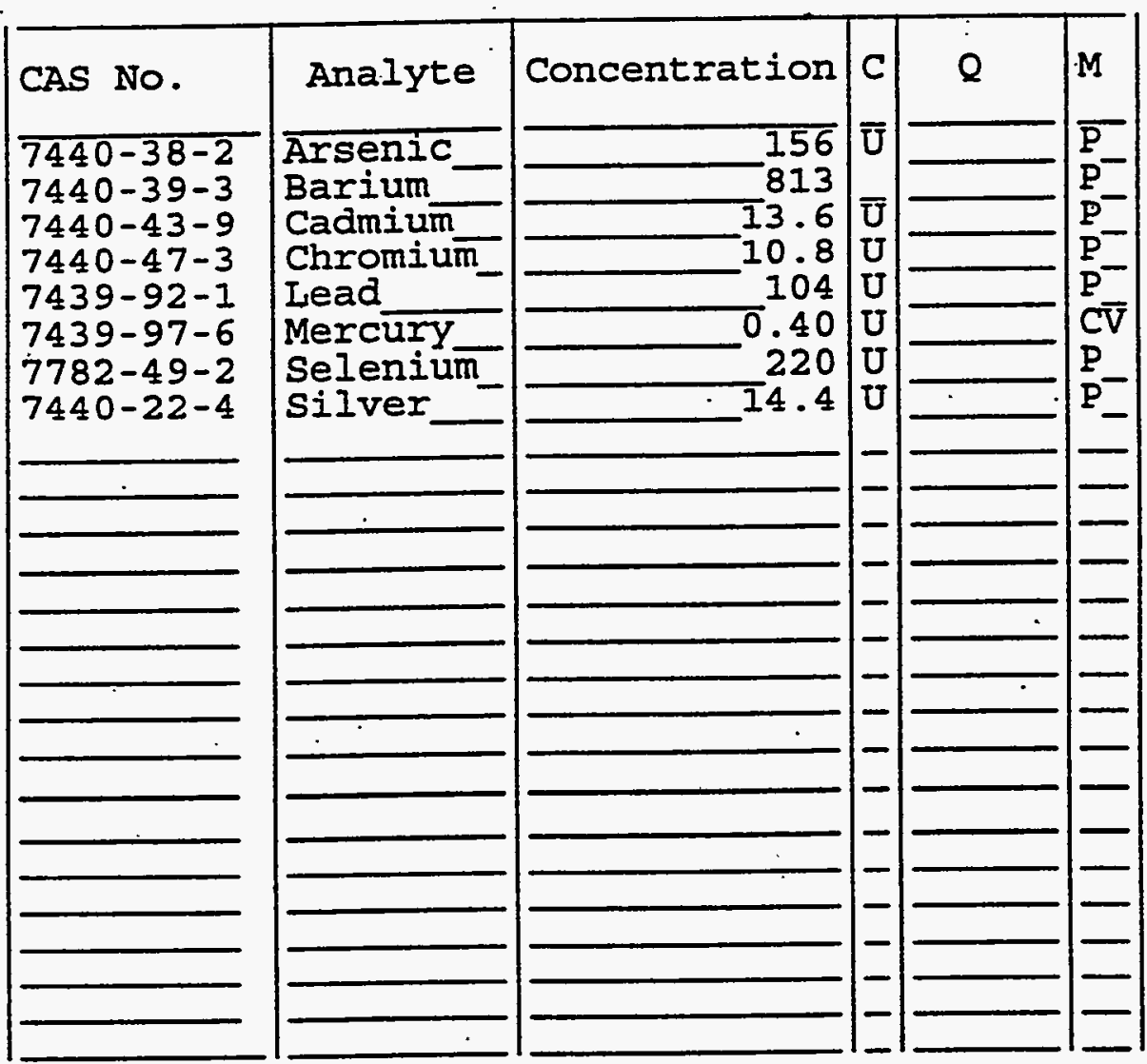

Color Before:

Clarity Before:

Texture:

Clarity After:

Artifacts :

Comments : 
!

Ib Name: Quanterra, yo

T.ab code: ITMO Case No.:

Itrix : (Boilfwater) soII

Sample wt/vol:

$1.0 \quad(\mathrm{~g} / \mathrm{ml})$

evel: (low/med)

LOW

- Moisture: not dec.

Contract: 625.01

SDG No.: $\mathrm{V} 238$

SAS NO.:

Iab Sample ID: 15121-008

Iab File ID:

Date Sampled: 06-23-97

Date Analyzed: 06-27-97

Dilution Factor: 1.0

CAS NO.

Compound .
CONCENTRATION UNITS:

(ug/I or ug/kg) UG/RG
8

0

LBH

(1)

500

U:Concentration of analyte is less than the value given.

$X:$ Pattern reasonably matches gasoline

$Y$ : Pattern appears to be multi-peaks, but does not match gasoline

Z:Concentration is primarily from a single peak

(1):Low Boiling Hydrocarbon (LBH) is quantitated as if it is Gasoline. 
1D

HBE ANALYSIS DATA SHEET
EPA SAMPLE NO.

SSCEP-PIPE-9
Lab Name:

OUANTERRA MO

Lab Code: ITMO Case No.: SOIL

Matrix : (soil/water) 20.1 $(\mathrm{g} / \mathrm{ml})$ $\mathbf{G}$ Sample wt/vol:

Level: (low/med) LOW Contract : SAS NO.:

Iab Sample ID:

625.01

\% Moisture: not dec. dec.

Extraction: (SepF/Cont/Sonc/Shak) SHAK GPC Cleanup: $(\mathrm{Y} / \mathrm{N})+\mathrm{N} \quad \mathrm{pH}:$ : $\longrightarrow$

Lab File ID:

Date Extracted:

Date Analyzed:

Dilution Factor:
SDG NO: : V238

Date Sampled:

$06-23-97$

$06-27-97$

$06-29-97$ 1 CONCENTRATION UNITS : (mg/L or $\mathrm{mg} / \mathrm{Kg}$ ) $\mathrm{mg} / \mathrm{kg}$ Q

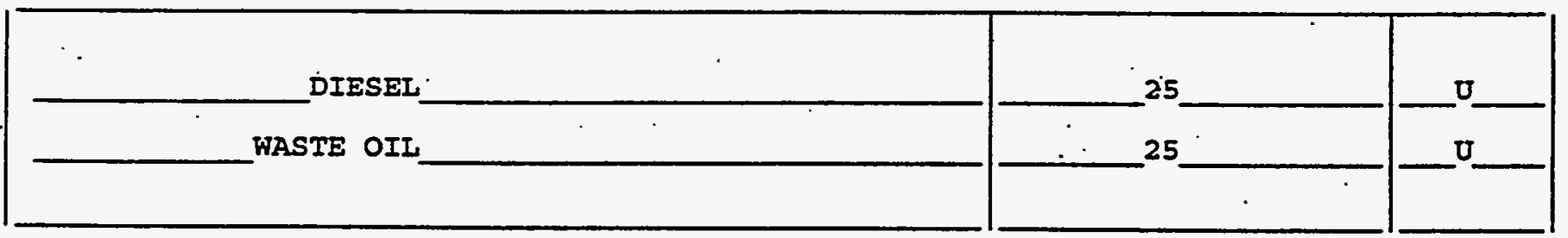

$U$ : Concentration of analyte is less than the value given. 
lb Name: QUANTERRA MO

i code: ITimo

Case No.: V12101

irix: (soil/water) soIL

anple wt/vol:

$5.00(\mathrm{~g} / \mathrm{mL}) \mathrm{G}$

el: (Iow/med) LOW

Moisture: not dec.

: Column: RTX-502.2 ID: 0.530 (mm)

pi,I Extract Volume:

(UI)
Contract: $625-01$

SAS NO.:
SSCEPPIPE9

SDG NO.: V238

Lab Sample ID: 15121-008

Lab File ID: $\quad$ G7290

Date Received: 06/25/97

Date Analyzed: 07/05/97

Dilution Factor: 1.0

Soil Aliquot Volume:

(UI)

CAS NO. COMPOUND

CONCENTRATION UNITS:

(ug/L or $\mathrm{ug} / \mathrm{Kg}$ ) UG/KG

\begin{tabular}{|c|}
\hline 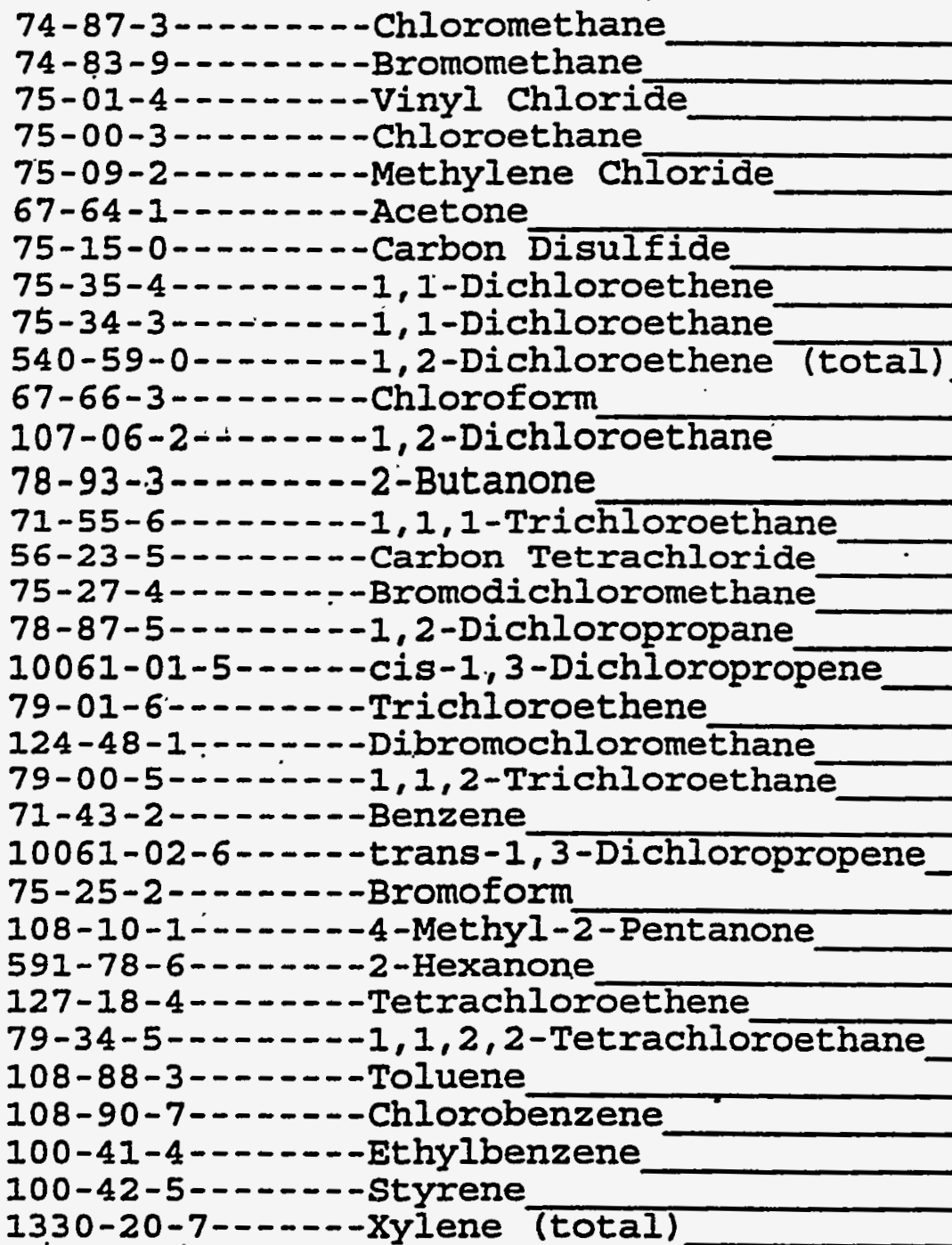 \\
\hline
\end{tabular}


1

INORGANIC ANALYSES DATA SHEET
Lab Name: QUANTERRA_MO

Lab Code: ITMO

Case No.:

Matrix (soil/water): WATER

Level (low/med): IOW

'o'solids :
Contract: 625.01

SAS NO.:
EPA SAMPLE NO.

\section{SSCEPPIPE9}

SDG No.: V238

Lab Sample ID: P15121-008

Date Received: 06/25/97.

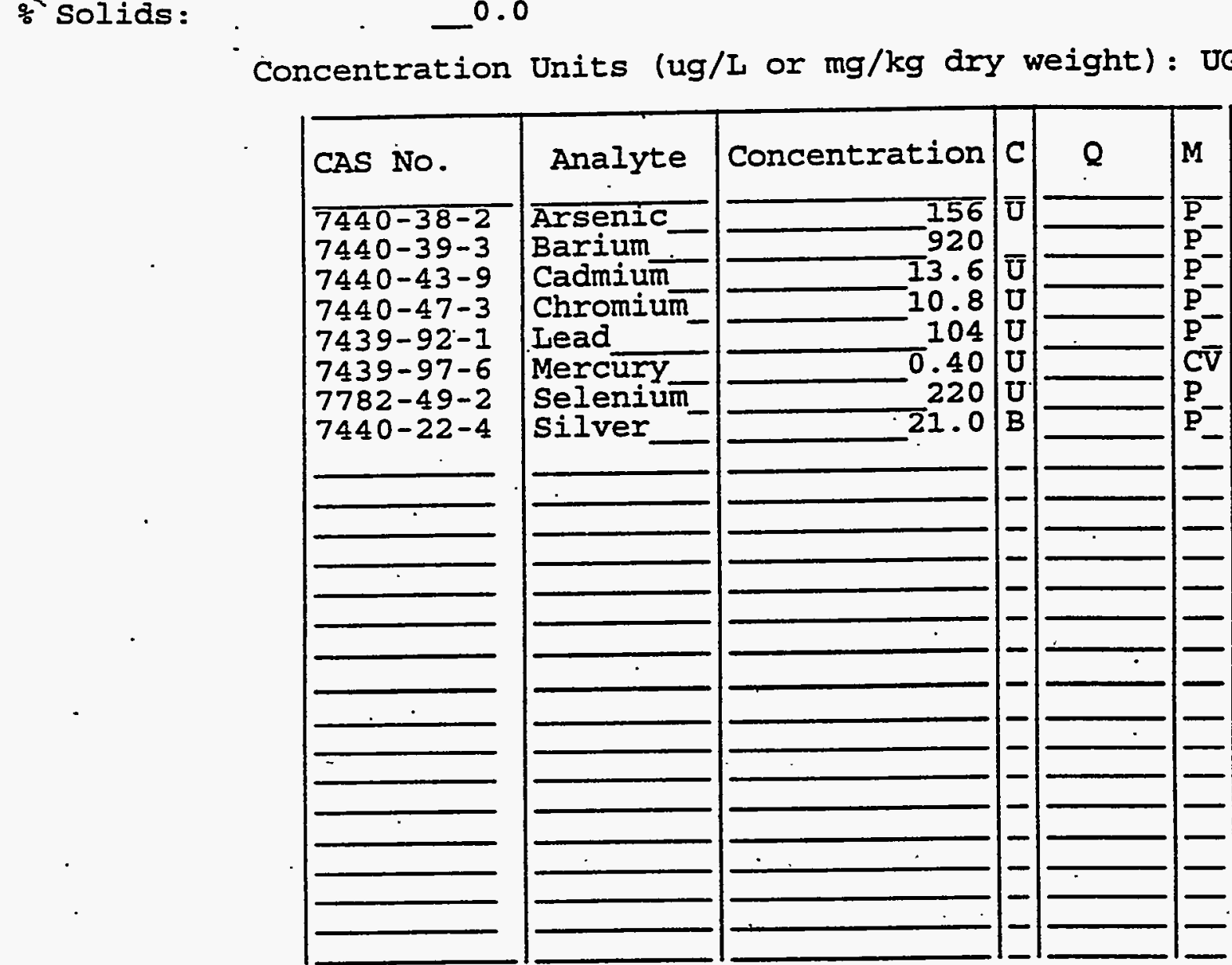

Color Before:

Color After:

Comments :
Clarity Before:

Clarity After:
Texture:

Artifacts : 
: lb Name:

T.ab code: ITMO Case No.:

I ttrix : (so11/water) soIL

sample wt/vol: 1.0 $(g / m l)$

Ivel: (low/med) LOW

Moisture: not dec.

CAS NO.

Compound .

CONCENTRATION UNITS:

(ug/I or $\mathrm{ug} / \mathrm{Kg}$ ) UG/KG

LBE (1)
Contract: $625.01 \quad \therefore$

SDG NO.: v238

Iab Sample ID: 15121-009

Iab Pile ID:

Date Sampled:

Date Analyzed: $06-27-97$

Dilution Factor: 1.0

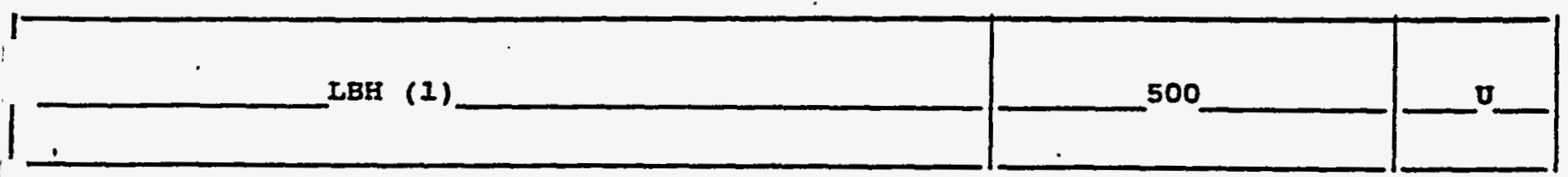

0: Concentration of analyte is less than the value given.

$\mathrm{X}$ : Pattern reasonably matches gasoline

z: Pattern appears to be multi-peaks, but does not match gasoline

$\eta$ :Concentration is primarily from a single peak

(1):Low Boiling Hydrocarbon (LBE) is quantitated as if it is Gasoline. 
Lab Name:

Contract:

625.01

Lab Code: ITMO Case No.:

Matrix : (soil/water)

SOIL

Sample wt/vol:

$20.1 \quad(\mathrm{~g} / \mathrm{ml})$

$\mathbf{G}$

Level: (low/med)

LOW

: Moisture: not dec. dec.

Extraction: (SepF/Cont/Sonc/Shak) SFAK GPC Cleanup: $(\mathrm{Y} / \mathrm{N}) \underline{\mathrm{N}} \mathrm{pH}:$ SAS NO.: Lab Sample ID: $15121-009$ Lab File ID: Date Sampled: $06-23-97$ Date Extracted: $06-27-97$ Date Analyzed: $06-29-97$ Dilution Factor: 1

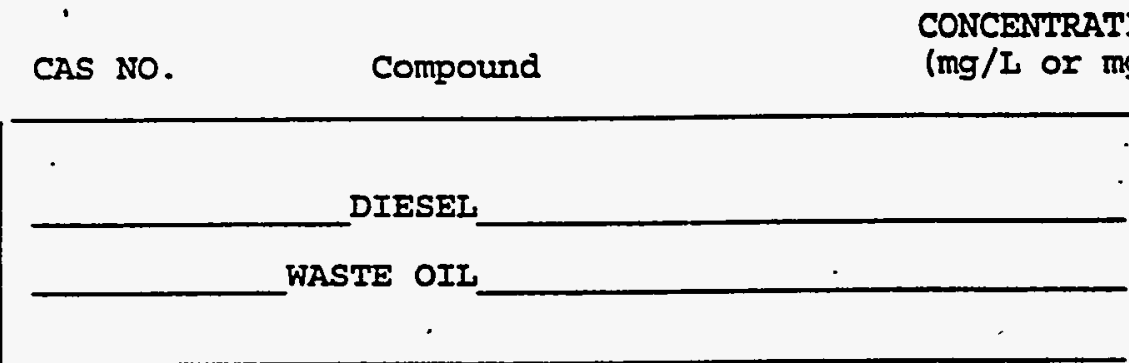

CONCENTRATION UNITS: Or $\mathrm{mg} / \mathrm{Kg}) \mathrm{mg} / \mathrm{kg}$

$U$ : Concentration of analyte is less than the value given. 
ab Name: QUANTERRA MO

¿ , Code: ITMO

Case No.: V12101

$2^{+}$, rix: (soil/water) SOIL

áuple wt/vol:

$5.00(\mathrm{~g} / \mathrm{mL}) \mathrm{G}$

$\epsilon$ 'el: (low/med) Low

Moisture: not dec.

( Column: RTX-502.2 ID: 0.530 (mm)

oil Extract Volume:

(UL)
Contract: $625-01$

SSCEPPIPEIO9 i. Extract Volume:

CAS NO. . COMPOUND
SAS NO.:

SDG NO.: V238
Lab Sample ID: 15121-009

Lab File ID: G7320

Date Received: 06/25/97

Date Analyzed: 07/06/97

Dilution Factor: $\quad 1.0$

Soil Aliquot Volume:

(uI)
CONCENTRATION UNITS :

(ug/L or $\mathrm{ug} / \mathrm{Kg}$ ) UG/KG

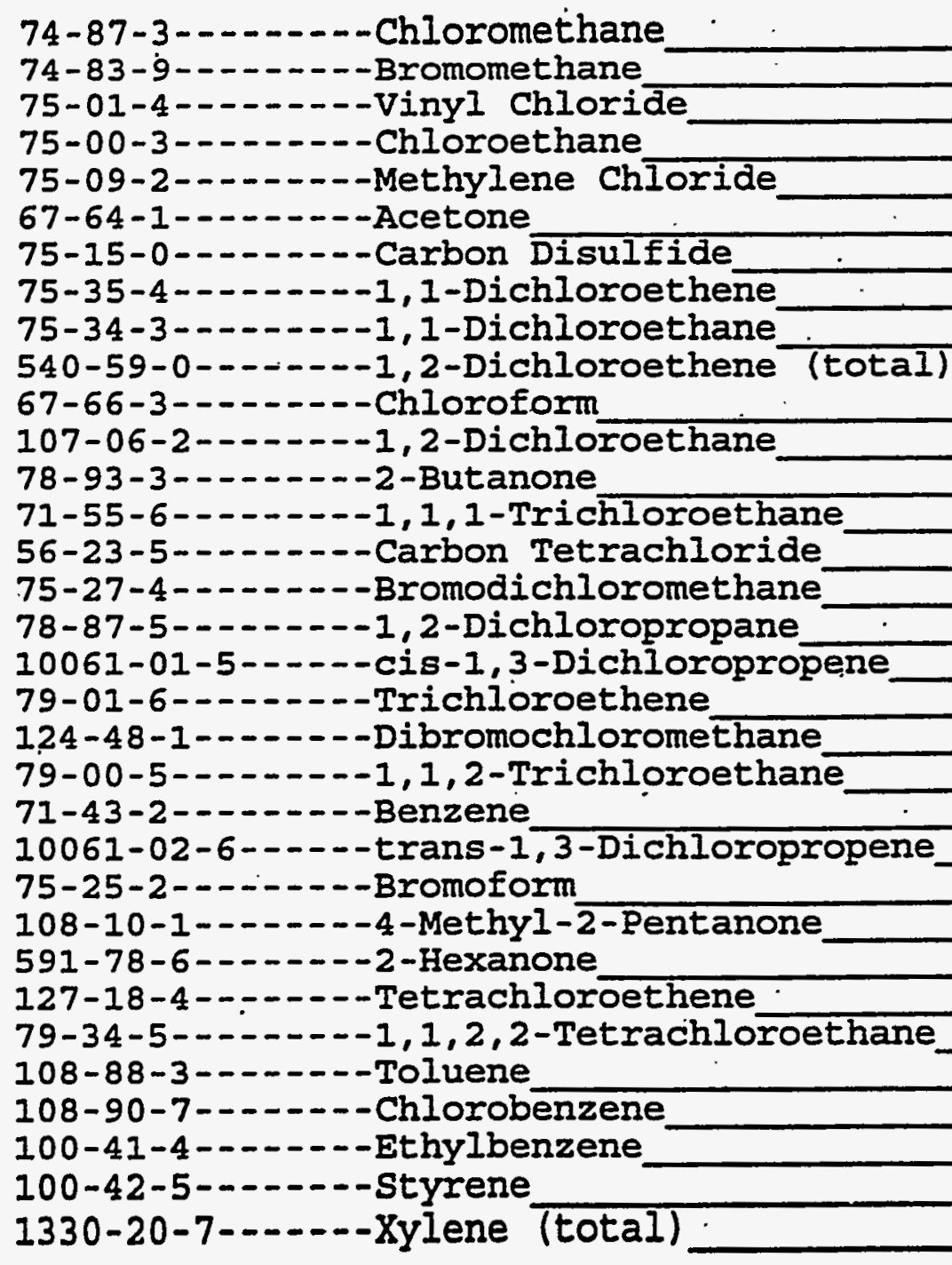


1 INORGANIC ANALYSES DATA SHEET
EPA SAMPLE NO.

\section{SSCEPPIPE109}

Lab Name: QUANTERRA_MO

Lab Code: ITMO Case No.: Matrix (soil/water): WATER

Level (low/med): LOW

$\because$ Solids: 0.0
Contract: 625.01 SAS NO.:

SDG No.: V238

Lab Sample ID: P15121-009

Date Received: 06/25/97 Concentration Units (ug/L or $\mathrm{mg} / \mathrm{kg}$ dry weight): UG/L_

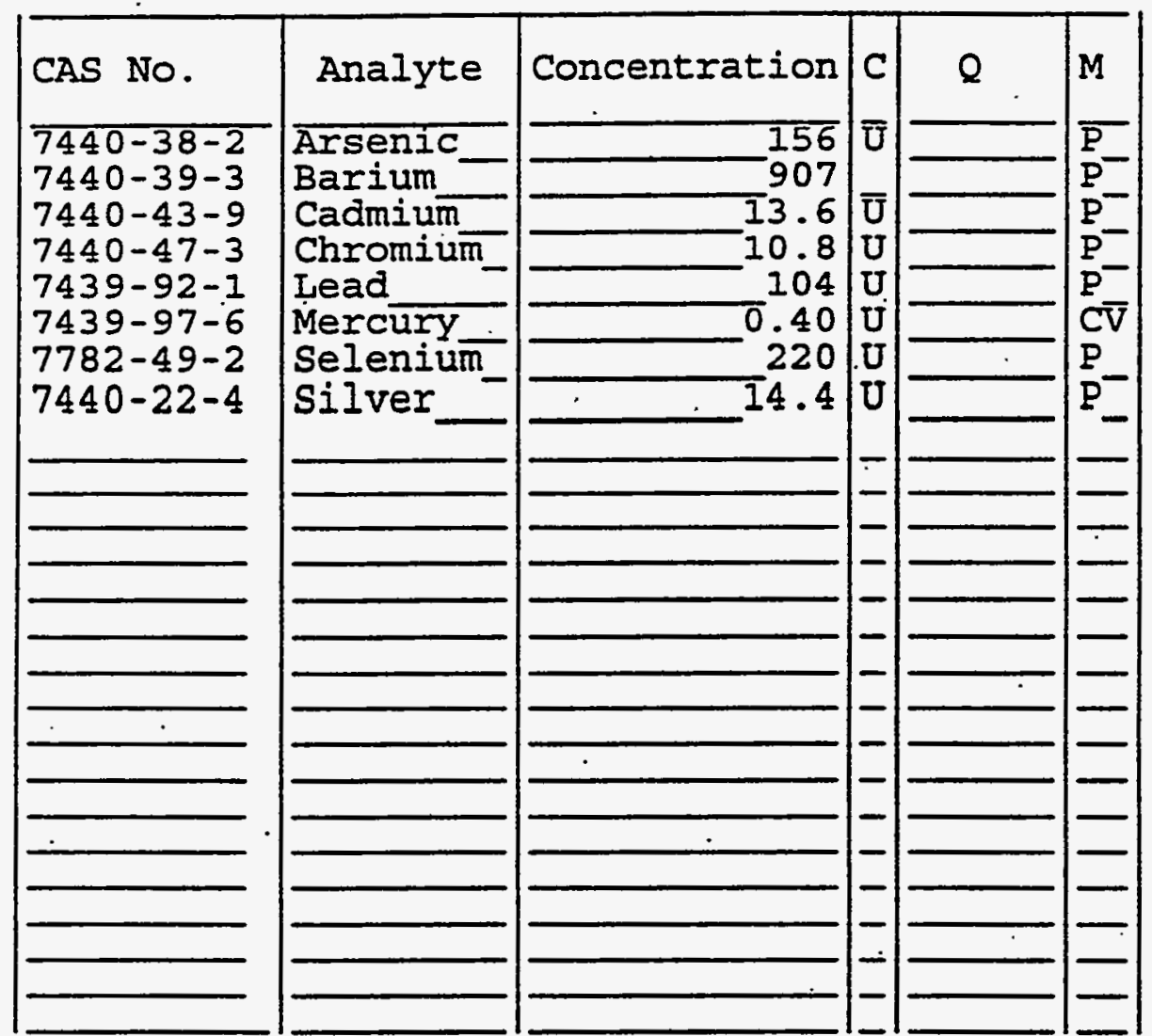

Clarity Before:

Texture:

Clarity After:

Artifacts : Comments : 


\section{VOLUME 2 APPENDIX B}

\section{TABLE 5 ANALYTICAL DATA}




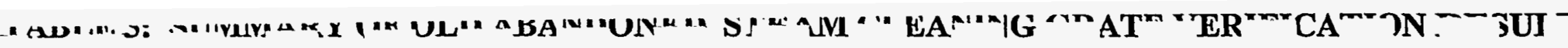

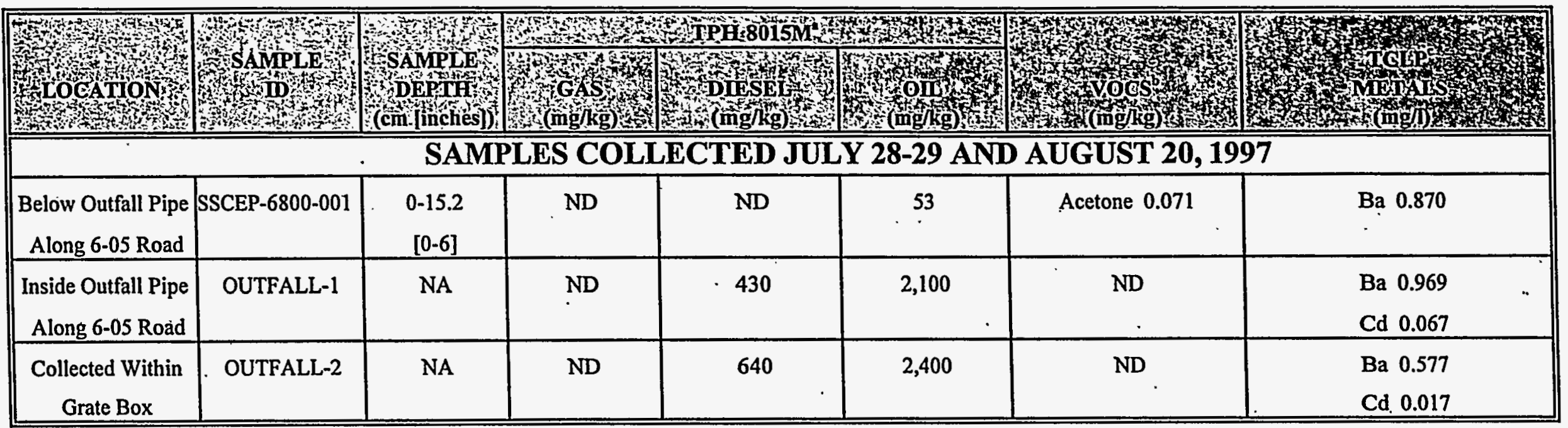

Notes:

'TPH 8015M: Total Petroleum Hydrocarbons Method 8015 Modified.

bOCs: Volatile Organic Compounds.

' TCLP: Toxicity Characteristic Leaching Procedure.

Ba Barium

SCEP: Steam Cleaning Effluent Pond

ND: Not Detected

NA: Not Analyzed/Not Applicable

cm: centimeter

$\mathrm{mg} / \mathrm{kg}$ : milligrams per kilogram

mg/l: milligrams per liter

* : Misidentified as a "6800" sample instead of.a sample from the former steam cleaning grate. 
¿ Name: Quanterra,Mo

ab Code: ITMO Case No.:

$\equiv$ rix: (soil/water) SoIL

mple wt/vol:

21: (low/med)

..oisture: not dec.

rAS NO. Compound
Contract: $\quad 625.01$

SAS No.:

SDE NO.: V262

Lab Sample ID: 15441-002

Lab File J.D:

Date Sampled: $\quad 07-28-97$

Dáte Aralyzed: 08-04-97

Dilution Factor: 1.0
CONCENTRATION UNITS:

(ug/I or ug/ $\mathrm{kg}$ ) UG/KG

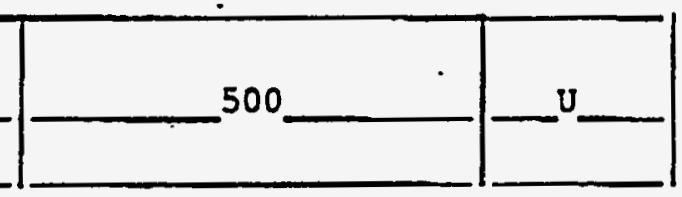

Concentracion of analyte is less than the value given.

Possibly weathered gasoline.

pattern reasonably matches gasoline

Pattern appears to be multi-peaks, but does not match gasoline

Concentration is primarily from a single peak

i,: Low Boiling Hydrocarbon (LBH) is quantitated as if it is Gasoline. 
20

HBE AXALYSIS DATA SEEEM
ERA SAMPLE NO. SSCEP-6800-1
Iab rame:

Iab codas Imo case Ho.:

Matzix : (Bois/water) Sample rt/vol: 20.0 $(g / m l)$ c Level: (Iow/med) IOH

- Moigtures not dec. des.

Extraction: (SepF/Cont/Sone/shax) SFAK SAS No.: contract: 625.01 SDG Ho.: $\quad \sqrt{262}$

Lab sample no: $15441-002$

Iab File ID:

Date Sampled:

$07-28-97$

Date Ixtracted:

$08-01-97$

Date Analyzed: . 08-05-97

Dilution Petor:

1

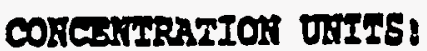

(mg/L as $\mathrm{mg} / \mathrm{Rg}$ ) _ ng/

Q

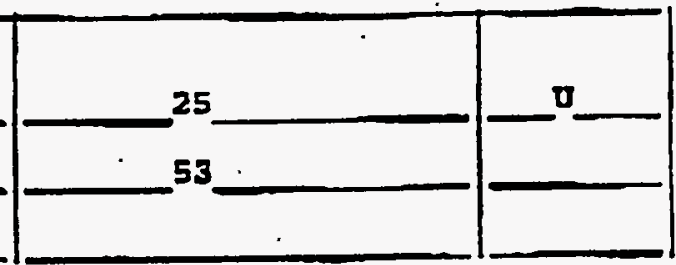

Ds concentration of analyte is less than eno value gloop. 
I Name: QUANTERRA MO

, Code: ITMO ' Case No.: V44101

$\therefore$ ix: (soil/water) SOIL

inle wt/vol: $\quad 5.00(\mathrm{~g} / \mathrm{mL}) \mathrm{G}$

rel: (low/med) LOW

1 isture: not dec.

Column: RTX-502.2 ID: 0.530 (mm)

Extract Volume:

(uL)
Contract: $625-01$

SAS NO.:
SSCEP $6800^{\circ}-1$

SDG No.: V262

Lab Sample ID: 15441-002

Lab File ID: F1199

Date Received: 07/30/97

Date Analyzed: 08/11/97

Dilution Factor: 1.0

Soil Aliquot Volume:

(uL)

CONCENTRATION UNITS:

CAS NO.

COMPOUND

(ug/L or ug/Kg) UG/KG

74-87-3--.---Chloromethane

74-83-9--.----Bromomethane

75-01-4-...-.--vinyl Chloride

75-00-3-...-- Chloroethane

75-09-2--.----Methylene Chloride

67-64-1-..----Acetone

75-15-0-....-.-Carbon Disulfide

75-35-4--.- 1, 1-Dichloroethene

75-34-3--.---1,1-Dichloroethane

540-59-0--.---1,2-Dichloroethene (total)

67-66-3-..---.-Chloroform

107-06-2--.--1, 2-Dichloroethane

78-93-3.-...--2-Butanone

71-55-6------1, 1, 1-Trichloroethane

56-23-5--------Carbon Tetrachloride

75-27-4--.---Bromodichloromethane

78-87-5-..-- 1, 2-Dichloropropane

10061-01-5-----cis-1,3-Dichloropropene

79-01-6--.---.-Trichloroethene

124-48-1------Dibromochloromethane

79-00-5--.---1, 1,2-Trichloroethane

71-43-2--.-.---Benzene

10061-02-6---.-trans-1,3-Dichloropropene

75-25-2--..---Bromoform

108-10-1--.---4-Methyl-2-Pentanone

591-78-6-..----2-Hexanone

127-18-4--.---Tetrachloroethene

79-34-5-...--1, 1,2,2-Tetrachloroethane

108-88-3-..--.-Toluene

108-90-7--..--Chlorobenzene

100-41-4-------Ethylbenzene

100-42-5-.-.---styrene

1330-20-7-...-XYlene (total) 
U.S. EPA - CIP

INORGANIC ANRLIÝSES DATA SHEET

EPA SAMPLE NO.

SSCEP-6800-1

Iab Name: QUANTERRA_MO

Lab Code: ITMO - Case No.: Matrix (soil/water): WATER

Level (low/med) : IOW

* Solids:

0.0
Contract: 625.01

SAS NO: :

SDG NO.: V262

Lab Sample ID: P15441-002

Date Received: 07/30/97

Concentration Units (ug/I or $\mathrm{mg} / \mathrm{kg}$ dry weight) : UG/L_

Color Before:

Color After:

Commests:
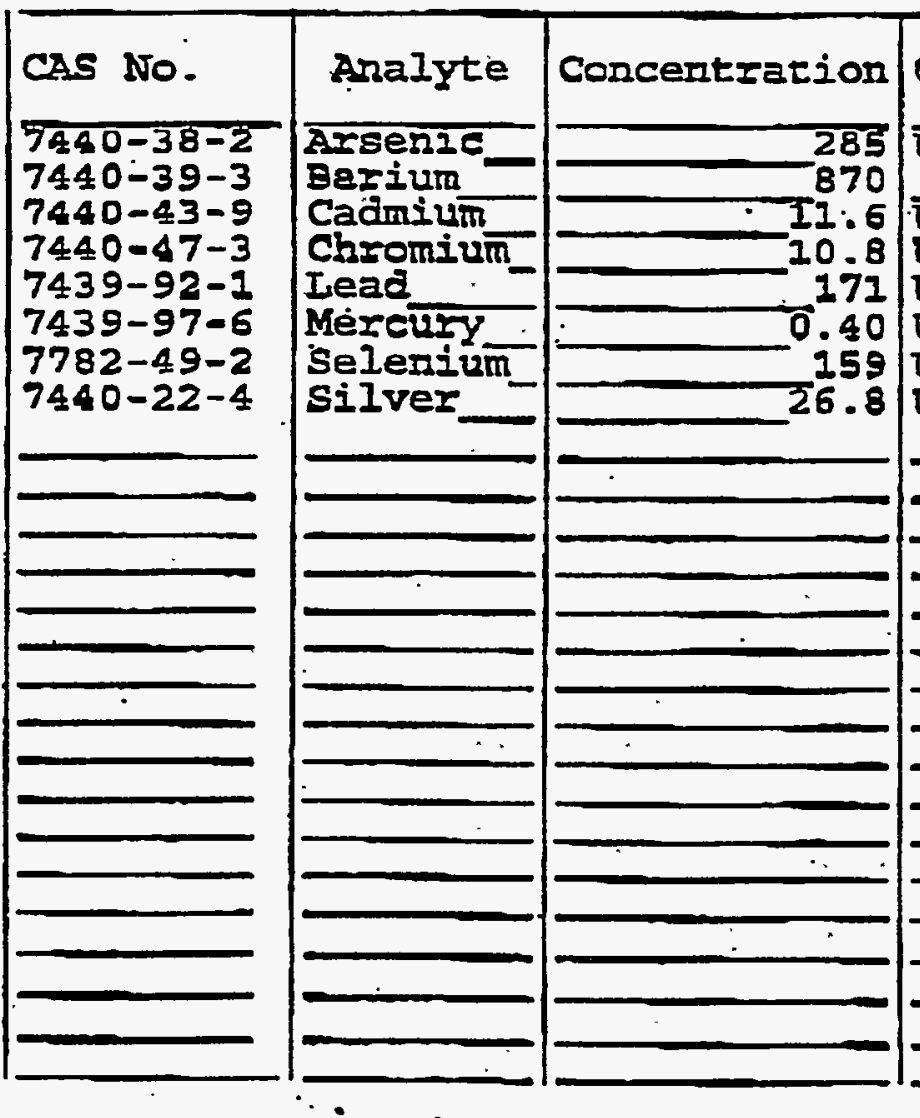

Clarity Before:

Clarity After:

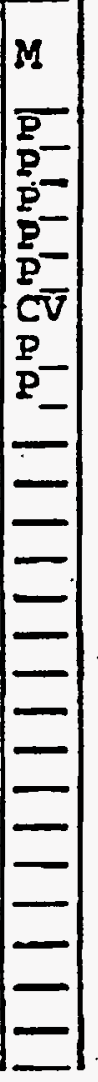

Texture:

Artifacts: 


\section{AS LABORATORES}

\section{\&T GAS/BTEX \\ ; GAS/BTEX}

\begin{tabular}{llll}
\hline Client Sample ID: & OUTFALL-1 & IAS Sample ID: & L10317-9 \\
Date Collected: & $20-$ AUG-97 & Date Received: & $20-A U G-97$ \\
Date Analyzed: & $29-A U G-97$ & Analytical Batch ID: $082797-B T E X-G C 4$ \\
Date Extracted: & N/A & Analytical Dilution: 1 \\
Matrix: & Soil & Preparation Dilution: 2.0 \\
Percent Moisture: & 11.48 &
\end{tabular}

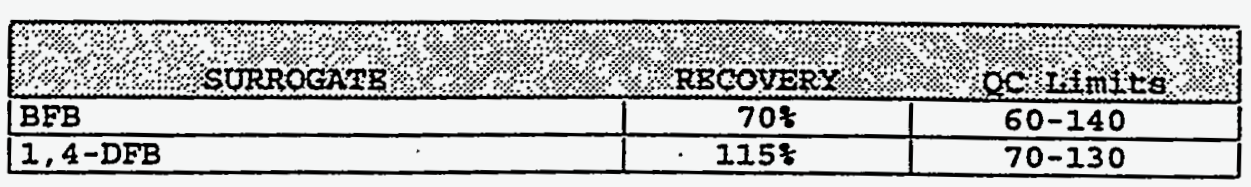

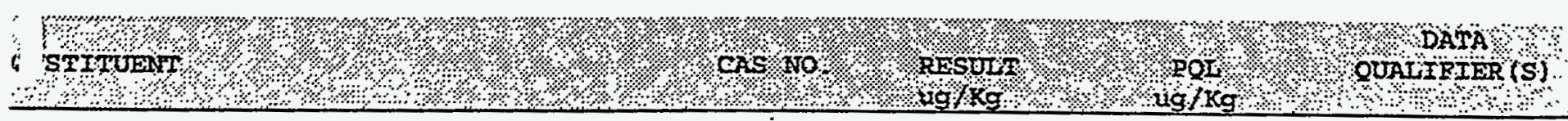

: oline Range Oxganics

$8006-61-9<230$

230

I: 


\section{LAS. LABORATORIES}

TOTAL PETROLEUM HYDROCARBONS (TPH)

8015M - TPH

$\begin{array}{ll}\text { Client Sample ID: } & \text { OUTFAII-1 } \\ \text { Date Collected: } & 20-A U G-97 \\ \text { Date Analyzed: } & 16-\text { SEP-97 } \\ \text { Date Extracted: . } & 28-A U G-97 \\ \text { Matrix: } & \text { Soil } \\ \text { Percent Moisture : } & 11.48\end{array}$

IAS Sample ID:

Date Received:

Analytical Dilution: 10

Preparation Dilution: 0.98

QC Group:

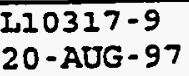

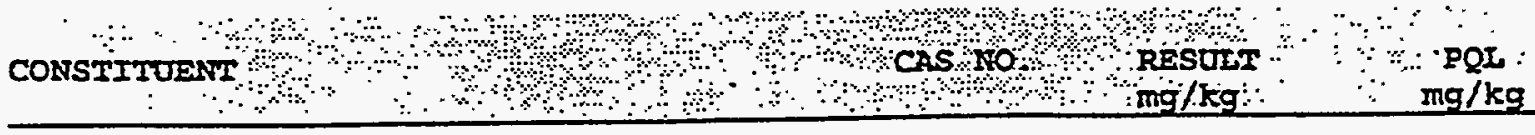

DATA

QUAIIFIER (S)

Diesel Range Organics

TPH .

430

330

MOTOR OIL 
; IMS FOR VOLATILE ORGANICS

3 lo VOLATILES

\begin{tabular}{|c|c|c|c|}
\hline $\begin{array}{l}\text { Client Sample ID: } \\
\text { Date Collected: } \\
\text { Date Analyzed: } \\
\text { Matrix: } \\
\text { Percent Moisture: }\end{array}$ & $\begin{array}{l}\text { OUTFALL-1 } \\
\text { 20-AUG-97. } \\
\text { 03-SEP-97 } \\
\text { SOil } \\
11.48\end{array}$ & $\begin{array}{l}\text { LAI Sample ID: } \\
\text { Date Received: } \\
\text { Analytical Dilution: } \\
\text { Analytical Batch ID: } \\
\text { Preparation Dilution: }\end{array}$ & $\begin{array}{l}\text { L10317-3 } \\
20-A U G-97 \\
1 \\
090397-8260 \text { A } \\
1.00\end{array}$ \\
\hline
\end{tabular}

\begin{tabular}{|c|c|c|}
\hline ২. & R.80OVE & octomits \\
\hline 1,2-Dichloroethane-d4 & $95 t$ & $77-127$ \\
\hline Toluene-d8 & 907 & $84-120$ \\
\hline Bromofluorobenzene & 898 & $78-125$ \\
\hline
\end{tabular}

Loromethane Jinyl Chloride 3romomethane I Loroethane r l.chlorofluoromethane tcetone

L. 2-Dichloroethene

I Ebon Disulfide 1.-chylene Chloride =rans-1,2-Dichloroethene $I^{\prime}$ nyl Acetate

L L-Dichloroethane 2-Butanone

zis-1,2-Dichloroethene

= Loroform

$?$ iexanone

L, 1, 2-Trichloroethane

sarbon tetrachloride

L 2-Dichloroethane

3 :lzene

[richloroethene

L.,2-Dichloropropane

3 modichloromethane

? Ihloroethylvinylether

1-Methyl-2-Pentanone

Iis-1,3-Dichloropropene

c Luene

=-ans-1,3-Dichloropropene

L, 1,2-Trichloroethane

[. :rachloroethene

ᄀ iromochloromethane

Inlorobenzene

:thylbenzene

n ?-Xylene

, Sylene

ityrene

3romoform

1 $1,2,2$-Tetrachloroethane

1 3-Dichlorobenzene

L,4-Dichlorobenzene

1,2-Dichlorobenzene $\begin{array}{lll}74-87-3<5.6 & 5.6\end{array}$

75-01-4 $<5.6 \quad 5.6$

$\begin{array}{lll}74-83-9 & <5.6 & 5.6\end{array}$

$\begin{array}{lll}75-00-3 & <5.6 & 5.6\end{array}$

$75-69-4 \cdot<5.6 \cdot 5.6$

67-64-1 <11. . 11.

$\begin{array}{lll}75-35-4 & <5.6 & 5.6\end{array}$

$\begin{array}{lll}75-15-0 & <5.6 & 5.6\end{array}$

$75-09-2<5.6 \quad 5.6$

$156-60-5<5.6 \quad . \quad 5.6$

$108-05-4<11$.

$\begin{array}{lll}75-34-3 & <5.6 & 5.6\end{array}$

78-93-3 <11. 11.

$\begin{array}{lll}156-59-2 & <5.6 & 5.6\end{array}$

$67-66-3<5.6 \quad 5.6$

591-78-6 <11. 11 .

$\begin{array}{lll}71-55-6 & 2.0 & 5.6\end{array}$

$56-23-5 .<5.6 \cdot 5.6$

207-06-2 <5.6 5.6

$71-43-2<5.6 \quad 5.6$

79-01-6 <5.6. 5.6

$\begin{array}{lll}78-87-5 & <5.6 & 5.6\end{array}$

$\begin{array}{lll}75-27-4 & <5.6 & 5.6\end{array}$

$110-75-8 \quad<23$. 23 .

108-10-1 <11. 11 .

$10061-01-5<5.6 \quad 5.6$

$108-88-3 \quad<5.6 \quad 5.6$

$\begin{array}{lll}10061-02-6 & <5.6 & 5.6\end{array}$

$79-00-5,<5.6 \quad 5.6$

$127-18-4 \quad<5.6 \quad 5.6$

$124-48-1 \quad<5.6 \quad 5.6$

$108-90-7<5.6 \quad 5.6$

$100-41-4 \quad<5.6 \quad 5.6$

$\begin{array}{lll}136777-61-2 & <5.6 & 5.6\end{array}$

$\begin{array}{lll}95-47-6 & <5.6 \quad 5.6\end{array}$

$\begin{array}{lll}100-42-5 & <5.6 & 5.6\end{array}$

$75-25-2<5.6 \quad 5.6$

$79-34-5 \quad<5.6 \quad 5.6$

$\begin{array}{lll}541-73-1 \quad<5.6 & 5.6\end{array}$

$106-46-7 \quad<5.6 \quad 5.6$

$\begin{array}{lll}95-50-1 & <5.6 . & 5.6\end{array}$ 
CLP

1

INORGANIC ANALYSES DATA SHEET

Lab Name: L.A.S

Lab Code: LOCK _

Case NO.: 820BNE SAS NO.:

contract: BECHTEL_--

CLIENT ID NO.

OUTEALI-1
Matrix (soil/water) : WATER

Level (low/med): LOW.

zolids :

Concentration Units (ug/L or $\mathrm{mg} / \mathrm{kg}$ dry. weight): UG/L_

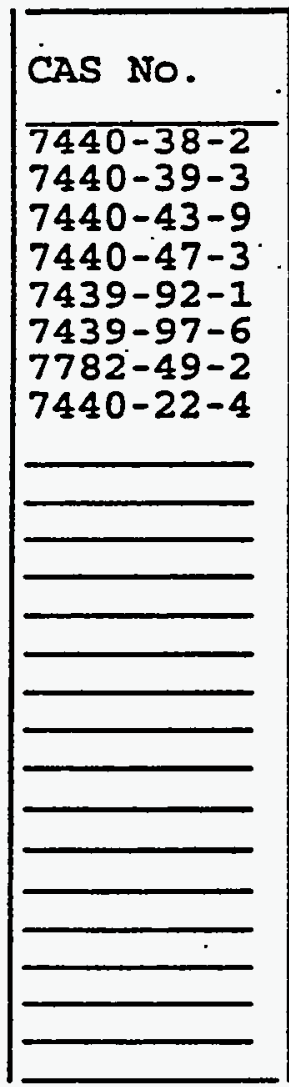

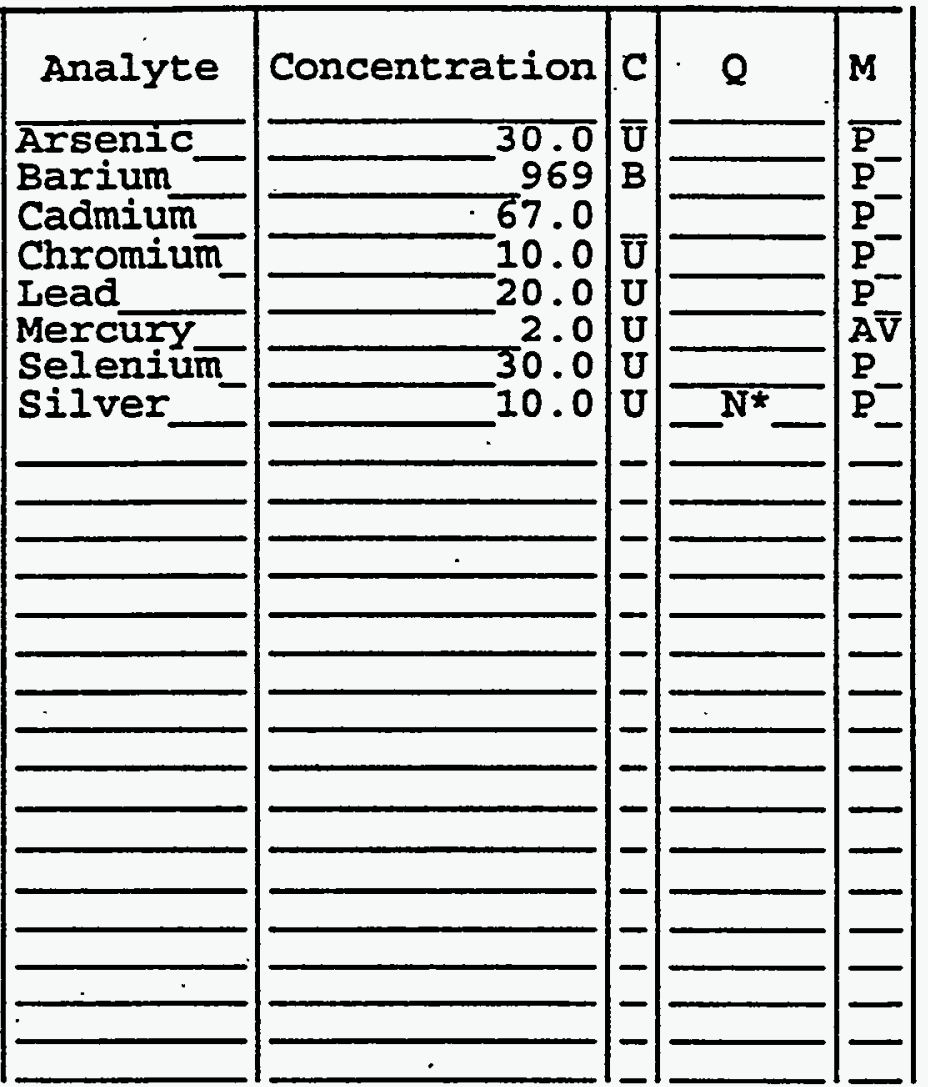

Clarity Before: CLEAR_ Clarity After: CLEAR_
Lab Sample ID: L10317-9

Date Received: 08/20/97
Eolor Before: COLORLESS

zolor After: COLORLESS Zomments :

Texture:

Artifacts : 


\section{¡AS LABORATORIES}

: ITAL PETROLEUM HYDROCARBONS (TPH)

$t=15 M \cdot T P H$

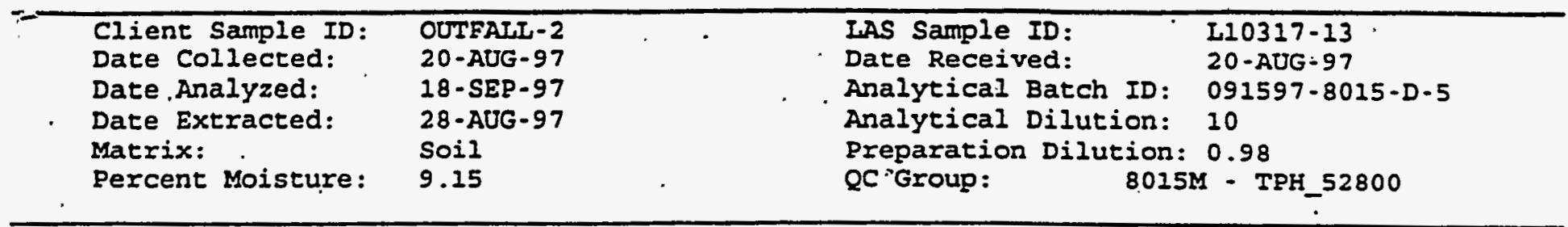

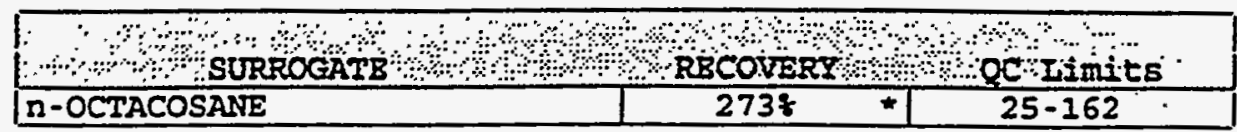

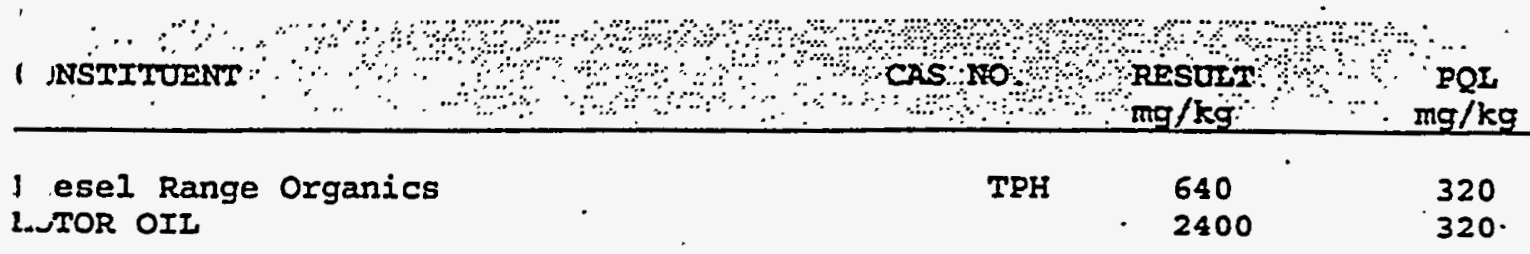




\section{LAS LABORATOKES}

GC/MS FOR VOLATILE ORGANICS

8240 VOIATILES

\begin{tabular}{llll}
\hline Client Sample ID: & OUTFALL-2 & LAL Sample ID: & LI0317-7 \\
Date Collected: & $20-A U G-97$ & Date Received: & $20-A U G-97$ \\
Date Analyzed: & $03-$ SED-97 & Analytical Dilution: 1 & \\
Matrix: & Soil & Analytical Batch ID: 090397-8260A-DI & ni \\
Percent Moisture: & 9.15 & Preparation Dilution: 1.00 &.
\end{tabular}

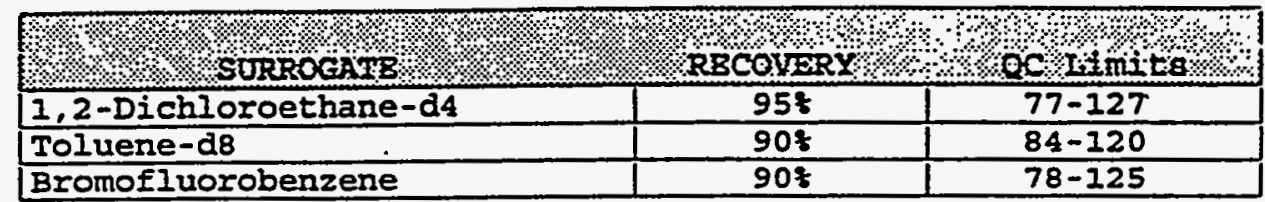

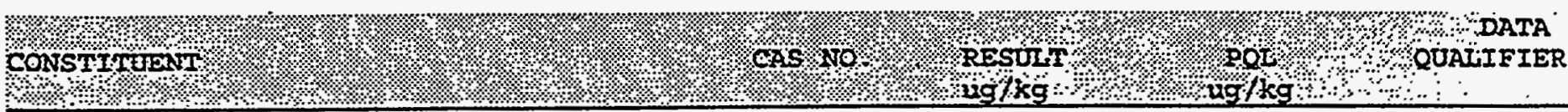

Chloromethane

vinyl Chloride

Bromomethane

Chloroethane

Trichlorofluoromethane

Acetone

1.1-Dichloroethene

Carbon Disulfide

Methylene Chloride

trans-1,2-Dichloroethene

Vinyl Acetate

1,1-Dichloroethane

2-Butanone

cis-1,2-Dichloroethene

Chloroform

2-Hexanone

1,1,1-Trichloroethane

Carbon tetrachloride

1,2-Dichloroethane

Benzene

Trichloroethene

1,2-Dichloropropane

Bromodichloromethane

2-Chloroethylvinylether

4-Methyl-2-Pentanone

cis-1,3-Dichloropropene

Toluene

trans-1,3-Dichloropropene

1,1,2-Trichloroethane

Tetrachloroethene

Dibromochloromethane

Chlorobenzene

Ethylbenzene

$\mathrm{m}, \mathrm{p}$-Xylene

o-xylene

Styrene

Bromoform

1, 1,2,2-Tetrachloroethane

1,3-Dichlorobenzene

1,4-Dichlorobenzene

1,2-Dichlorobenzene

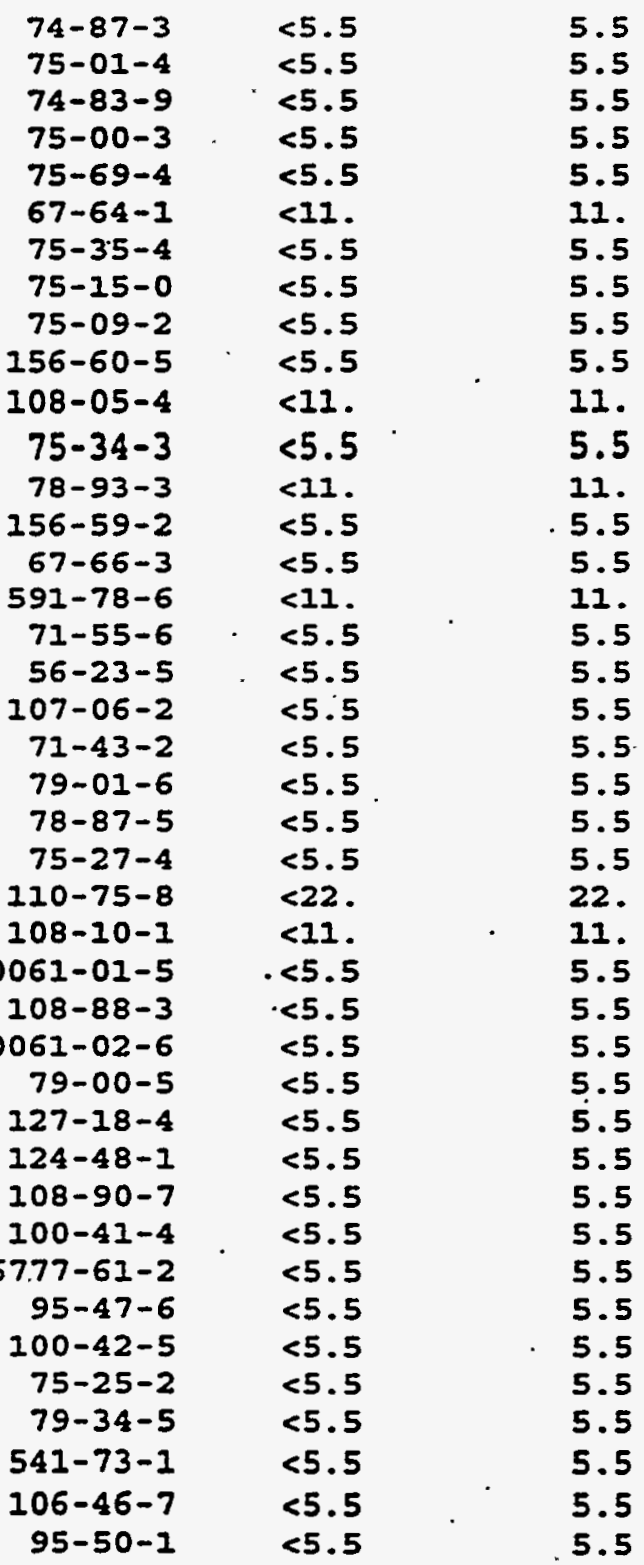




\section{LAS LABORATOR ES}

?\&T GAS/BTEX

?־T GAS/BTEX

$\begin{array}{ll}\text { Client Sample ID: } & \text { OUTFALL-2 } \\ \text { Date Collected: } & 20-A U G-97 \\ \text { Date Analyzed: } & 28-A U G-97 \\ \text { Date Extracted: } & \text { N/A } \\ \text { Matrix: } & \text { SOil } \\ \text { Percent Moisture: } & 9.15\end{array}$

LAS Sample ID:

Date Received:

Analytical Batch ID:

Analytical Dilution:

Preparation Dilution: 2.0
110317-13

20-AUG-97

$082797-B T E X-G C 4$

1

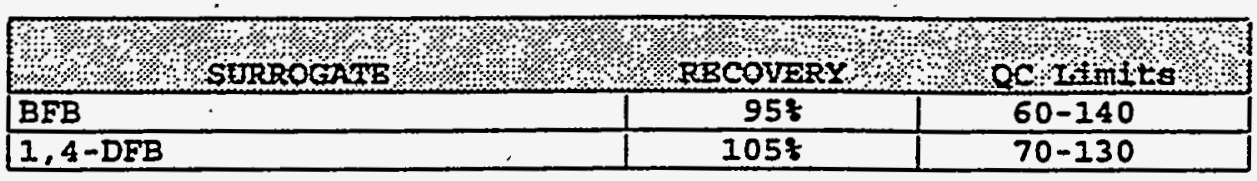

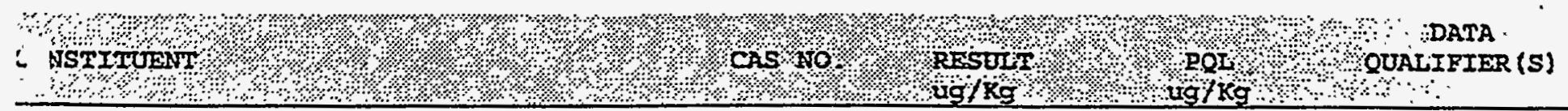

$\begin{array}{llll}\text { s-Boline Range Organics } & 8006-61-9 & <220 & 220\end{array}$

\begin{tabular}{llll}
\hline Y7713STANDARD & N & RI9866 & Page 1
\end{tabular}


1 INORGANIC ANALYSES DATA SHEET
CLIENT ID NO.

\section{OUTEALL-2}

sab Name: 'L.A.S

Contract: BECHTEL.--

دab Code: LOCK

Case No.: 820BNE SAS No.:
SDG No.: L10317 latrix (soil/water): WATER

sevel (low/med): . LOW_

solids :

0
Lab Sample ID: L10317-13_

Date Received: 08/20/97

Concentration Units (ug/L or $\mathrm{mg} / \mathrm{kg}$ dry weight) : UG/L_

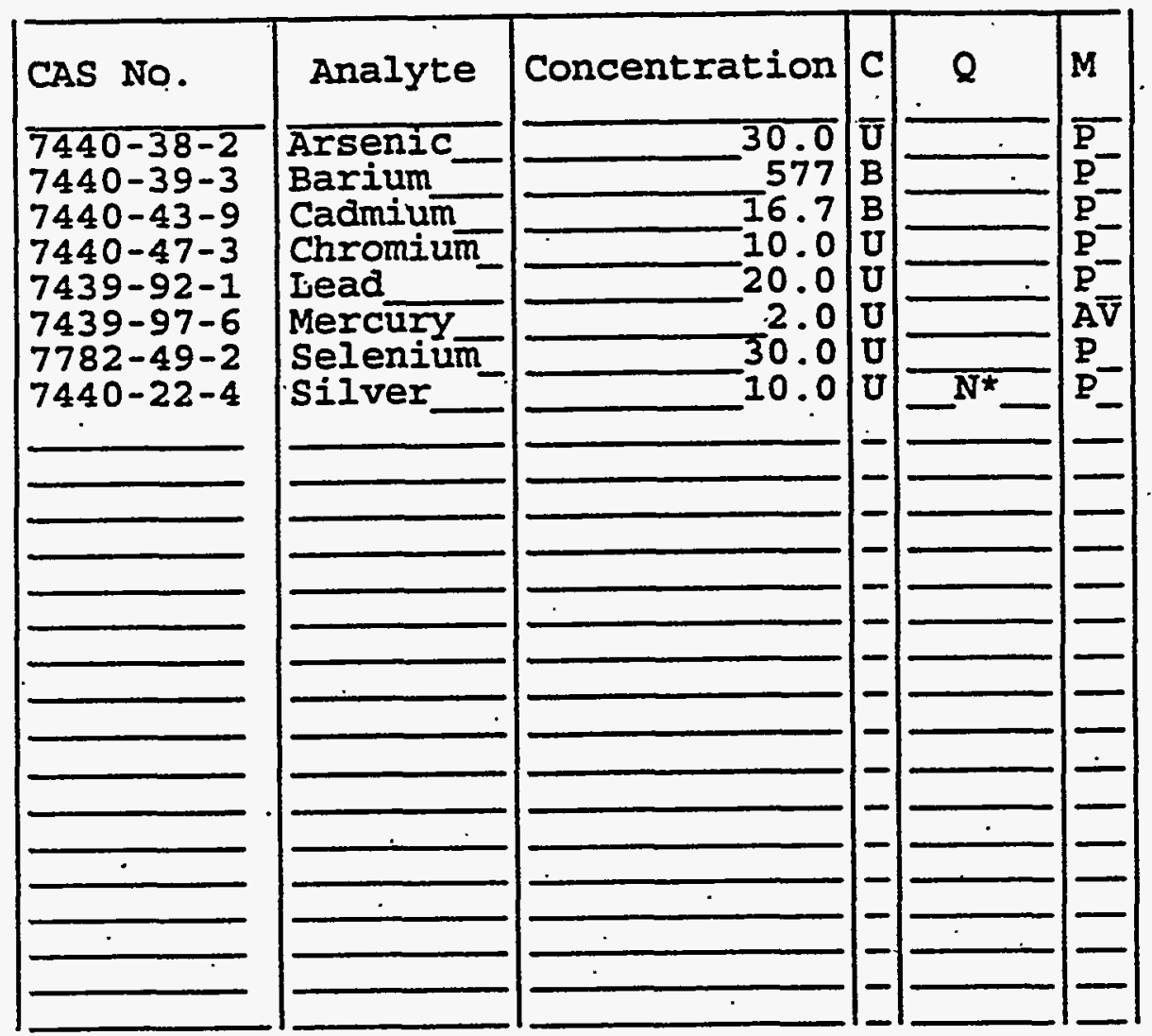

zolor Before: COLORLESS zolor After: COLORLESS

Clarity Before: CLEAR Clarity After: CLEAR_
Texture:

Artifacts: zomments : 


\section{VOLUME 2 APPENDIX B}

TABLE 6 ANALYTICAL DATA 
TABLE 6: SUMMARY OF SOUTH SCEP ZONE 2 VERIFICATION RESULTS

\begin{tabular}{|c|c|c|c|c|c|c|c|c|}
\hline 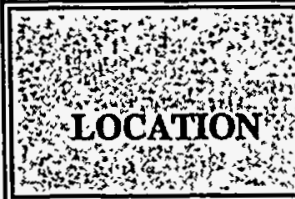 & 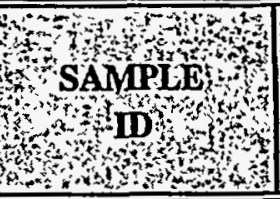 & 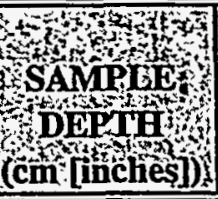 & 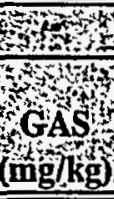 & 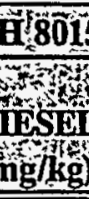 & 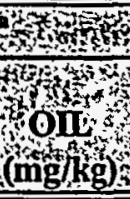 & 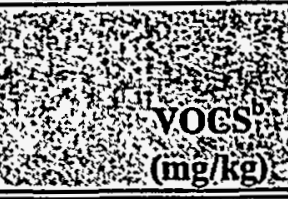 & 48 & 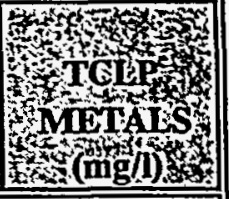 \\
\hline & $\overline{\overline{\mathbf{S A}}}$ & IPLES CC & $\overline{\text { LLEC }}$ & $\overline{\mathbf{E D}}$ & $\overline{Y Y 1}$ & 997 & & \\
\hline Cell No. 1 & SSCEP-V1-1 & $0-15.2[0-6]$ & ND & ND & 3,200 & ND & & $\begin{array}{l}\mathrm{Ba} 0.882 \\
\mathrm{Ag} 0.029\end{array}$ \\
\hline Cell No. 34 & SSCEP-V1-34 & $0-15.2[0-6]$ & ND & ND & 1,200 & ND & & $\mathrm{Ba} 0.628$ \\
\hline Cell No. 36 & $\begin{array}{l}\text { SSCEP-V1-36 } \\
\text { SSCEP-V2-36 }\end{array}$ & $\begin{array}{l}0-15.2[0-6] \\
0-15.2[0-6]\end{array}$ & $\begin{array}{c}7,300 \\
\text { NA }\end{array}$ & $\begin{array}{l}\text { ND } \\
\text { NA }\end{array}$ & $\begin{array}{l}1,600 \\
\text { NA }\end{array}$ & $\begin{array}{l}\text { Acetone } \\
\text { ND }\end{array}$ & 0.038 & $\begin{array}{r}\text { Ba } 0.833 \\
\text { NA }\end{array}$ \\
\hline Cell No. 48 & SSCEP-V1-48 & $0-15.2[0-6]$ & ND & ND & 1,200 & ND & & 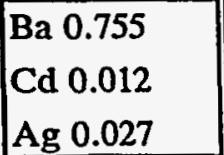 \\
\hline Cell No. 60 & SSCEP-V1-60 & $0-15.2[0-6]$ & ND & ND & 7,100 & ND & & 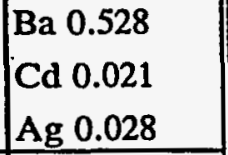 \\
\hline Cell No. 67 & SSCEP-V1-67 & $0-15.2[0-6]$ & ND & ND & 72 & ND & & $\mathrm{Ba} 1.120$ \\
\hline Cell No. 70 & SSCEP-V1-70 & $0-15.2[0-6]$ & ND & ND & 1,300 & ND & & Ba 1.050 \\
\hline Cell No. 76 & $\begin{array}{l}\text { SSCEP-V1-76. } \\
\text { SSCEP-V2-76 }\end{array}$ & $\begin{array}{l}0-15.2[0-6] \\
0-15.2[0-6]\end{array}$ & $\begin{array}{l}\text { ND } \\
\text { NA }\end{array}$ & $\begin{array}{l}\text { ND } \\
\text { NA }\end{array}$ & $\begin{array}{c}2,000 \\
\mathrm{NA}\end{array}$ & $\begin{array}{l}\text { Carbon Disulfide } \\
\text { Acetone }\end{array}$ & $\begin{array}{l}0.008 \\
0.046\end{array}$ & $\begin{array}{r}\mathrm{Ba} 0.621 \\
\mathrm{NA}\end{array}$ \\
\hline Cell No. 79 & $\begin{array}{c}\text { SSCEP-V1-79 } \\
\text { SSCEP-V1-790 } \\
\text { (Duplicate of 79) } \\
\end{array}$ & $\begin{array}{l}0-15.2[0-6] \\
0=15.2[0-6]\end{array}$ & $\begin{array}{l}\text { ND } \\
\text { ND }\end{array}$ & $\begin{array}{l}\text { ND } \\
\text { ND }\end{array}$ & $\begin{array}{l}410 \\
420\end{array}$ & Acetone $^{\text {ND }}$ & 0.074 & 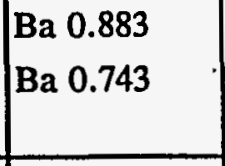 \\
\hline Cell No. 87 & SSCEP-V1-87 & $0-15.2[0-6]$ & ND & ND & 260 & ND & & Ba 0.761 \\
\hline Cell No. 90 & $\begin{array}{l}\text { SSCEP-V1-90 } \\
\text { SSCEP-V2-90 }\end{array}$ & $\begin{array}{l}0-15.2[0-6] \\
0-15.2[0-6]\end{array}$ & $\begin{array}{l}\text { ND } \\
\text { NA }\end{array}$ & $\begin{array}{l}\text { ND } \\
\text { NA }\end{array}$ & $\begin{array}{l}\text { ND } \\
\text { NA }\end{array}$ & $\begin{array}{l}\text { ND } \\
\text { ND }\end{array}$ & & $\begin{array}{r}\mathrm{Ba} 0.909 \\
\mathrm{NA}\end{array}$ \\
\hline
\end{tabular}


TABLE 6: SUMMARY OF SOUTH SCEP ZONE 2 VERIFICATION RESULTS

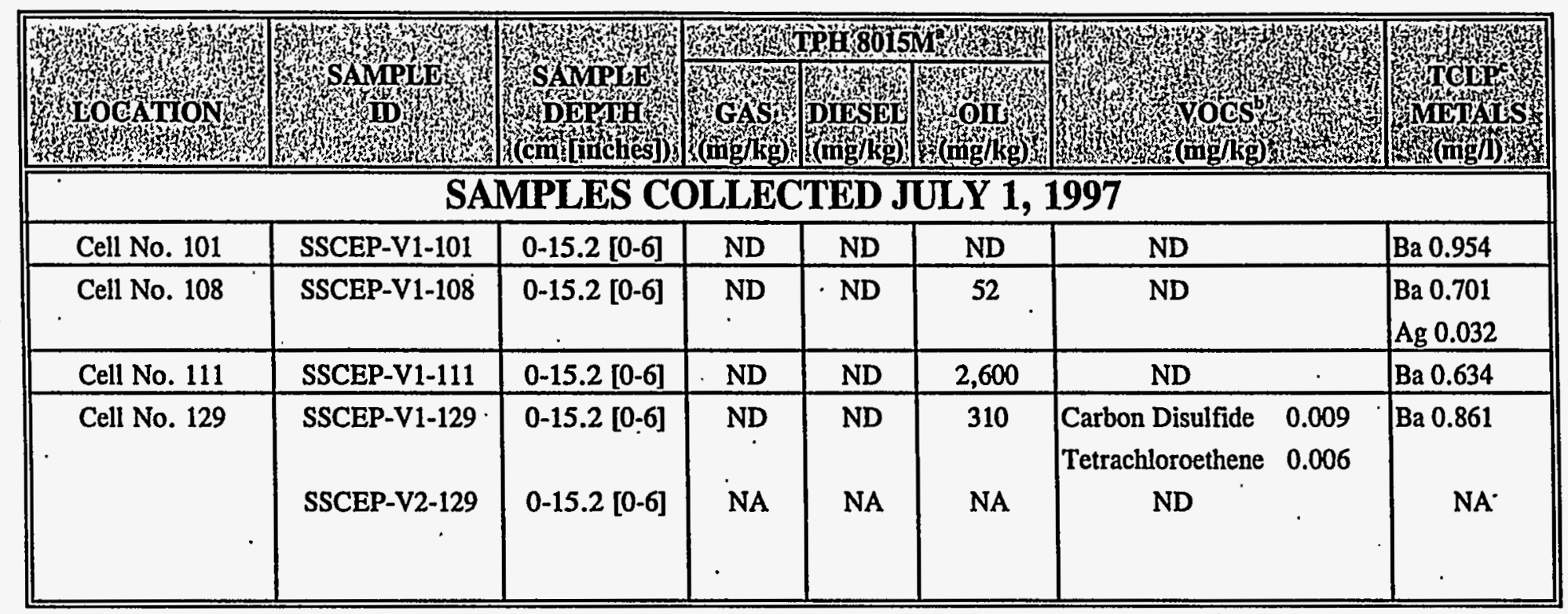

Notes:

a TPH 8015M: Total Petroleum Hydrocarbons Method 8015 Modified.

b VOCs: Volatile Organic Compounds.

' TCLP: Toxicity Characteristic Leaching Procedure.

Ag Silver, Ba Barium, Cd .Cadmium.

SCEP: Steam Cleaning Effluent Pond

ND: Not Detected

cm: centimeter

mg/kg: milligrams per kilogram

mg/l: milligrams per liter 
Lab Name: Quanterra, Mo

I b Code: ITMO Case No.:

Dutrix : (soil/water) SOIL

g-mple $\omega t / v o l: ~ 1.0 \quad(g / m l) G$

Luvel: (low/med) LOW

q Moisture: not dec.

Contract: 625.01

SAS No.:

SDG No.: V245

Lab Sample ID: 15230-005

Lab File ID:

Date Sampled: 07-01-97

Date Analyzed: 07-12-97

Dilution Factor: 1.0

CONCENTRATION UNITS:

(ug/L or $\mathrm{ug} / \mathrm{Kg}$ ) UG/KG

Q.

LEH (1)__ _ L

U: Concentration of analyte is less than the value given.

Pattern reasonably matches gasoline

pattern appears to be multi-peaks, but does not match gasoline

Concentration is primarily from a single peak

11): Low Boiling Hydrocarbon (LBH) is quantitated as if it is Gasoline. 
EPA SAMPLE NO. SSCEP-VI-001
Lab Name: QUANTERRA MO

Lab code: ITMO Case No.:

Matrix : (soil/water)

SOII

Sample wt/vol: 20.1 $(\mathrm{g} / \mathrm{ml})$ G

Level: (low/med) LOW

a Moisture: not dec. dec.

Extraction: (SepF/Cont/Sonc/Shak) SHAK GPC Cieanup: $(\mathrm{Y} / \mathrm{N})+\mathrm{N} \cdot \mathrm{pH}$

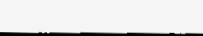

Contract: 625.01

SAS NO.:

SDG NO.: V245

Lab sample ID:

$15230-005$

Lab File ID:

Date Sampled:

$07-16-97$

Date Extracted: $07-10-97$

Date Analyzed: $07-17-97$

Dilution Factor: 10
CAS NO. Compound
CONCENTRATION UNITS:

$(\mathrm{mg} / \mathrm{L}$ or $\mathrm{mg} / \mathrm{Kg})$

\begin{tabular}{|r|}
\hline 25 \\
\hline 3200
\end{tabular}

$U$ : Concentration of analyte is less than the value given. 
b Name: QUANTERRA MO :

\& Code: ITMO

Case No.: V2300I

trix: (soil/water) soIL

ii le wt/vol:

$5.00 \cdot(\mathrm{g} / \mathrm{mL}) \mathrm{G}$

VPI: (low/med) LOW

Nuisture: not dec.

olumn: RTX-502.2 ID: 0.530 (mm)

il Extract Volume:

(uL)
Contract : $625-01$

SAS NO.:

SSCEPVIOOI

SDG No.: V245

Iab Sample ID: 15230-005

Lab File ID: $\quad$ G7482

Date Received: 07/03/97

Date Analyzed: 07/12/97

Dilution Factor: $\quad 1.0$

Soil Aliquot Volume: (uL)

CONCENTRATION UNITS:

CAS NO.

COMPOUND

Q

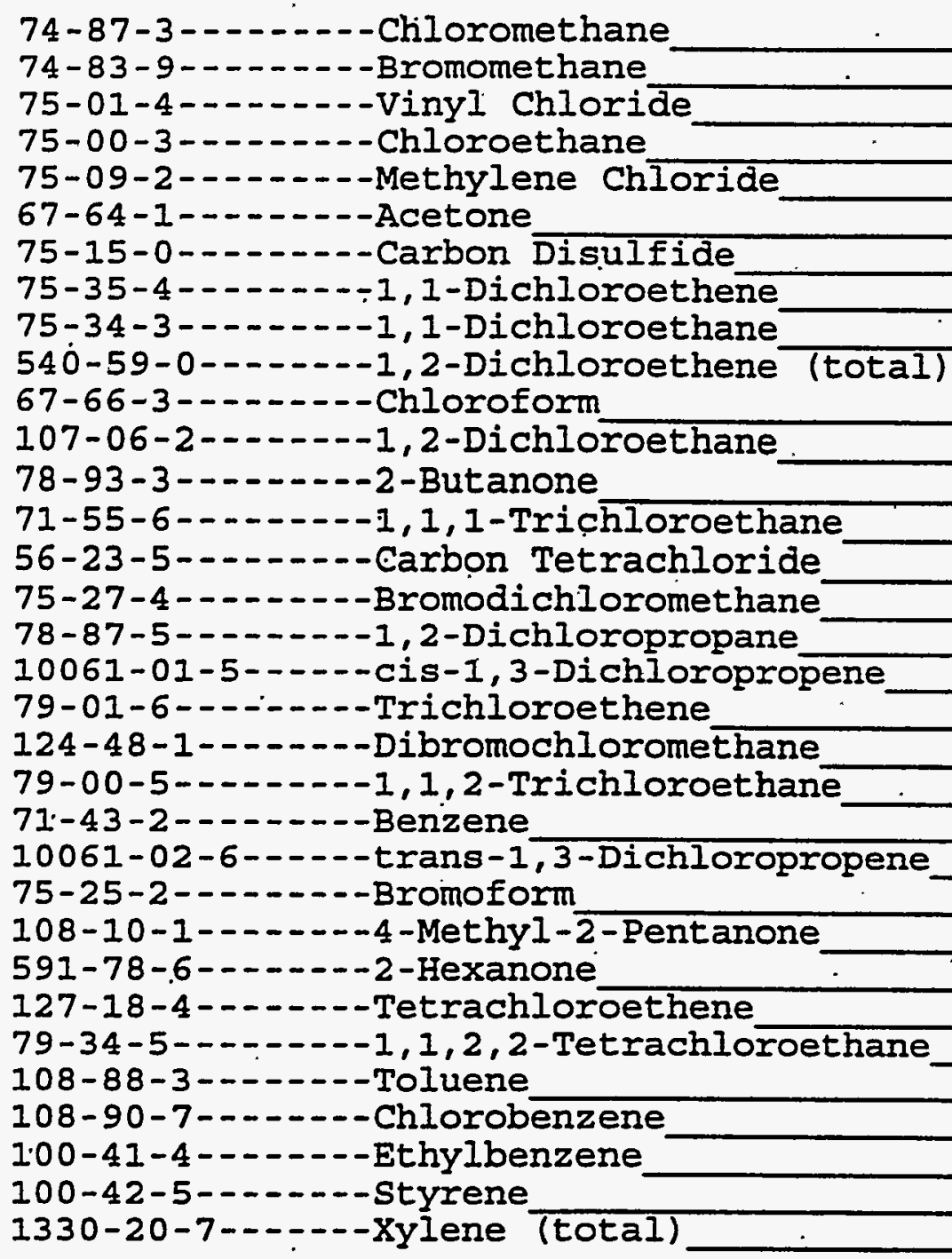


Lab Name: QUANTERRA: MO

Lab Code: ITMO

Case No.: V230001

Matrix: (soil/water) soIL

Sample wt/vol: $\quad 5.00(\mathrm{~g} / \mathrm{mL}) \mathrm{G}$

Level: (low/med) LOW

$\div$ Moisture: not dec.

GC Column: RTX-502.2 ID: 0.530 (mm)

Soil Extract Volume:

(uIs)
Contract: $625-01$

SAS NO.:

SDG NO.: V245

Lab Sample ID: 15230-005RE

Lab File ID: $\quad$ G7498

Date Received: 07/03/97

Date Analyzed: $07 / 13 / 97$

Dilution Factor: $\quad 1.0$

Soil Aliquot Volume:

(uL)

CAS NO.

COMPOUND

CONCENTRATION UNITS:

(ug/L or ug/Kg) UG/KG

Q

\begin{tabular}{|c|}
\hline 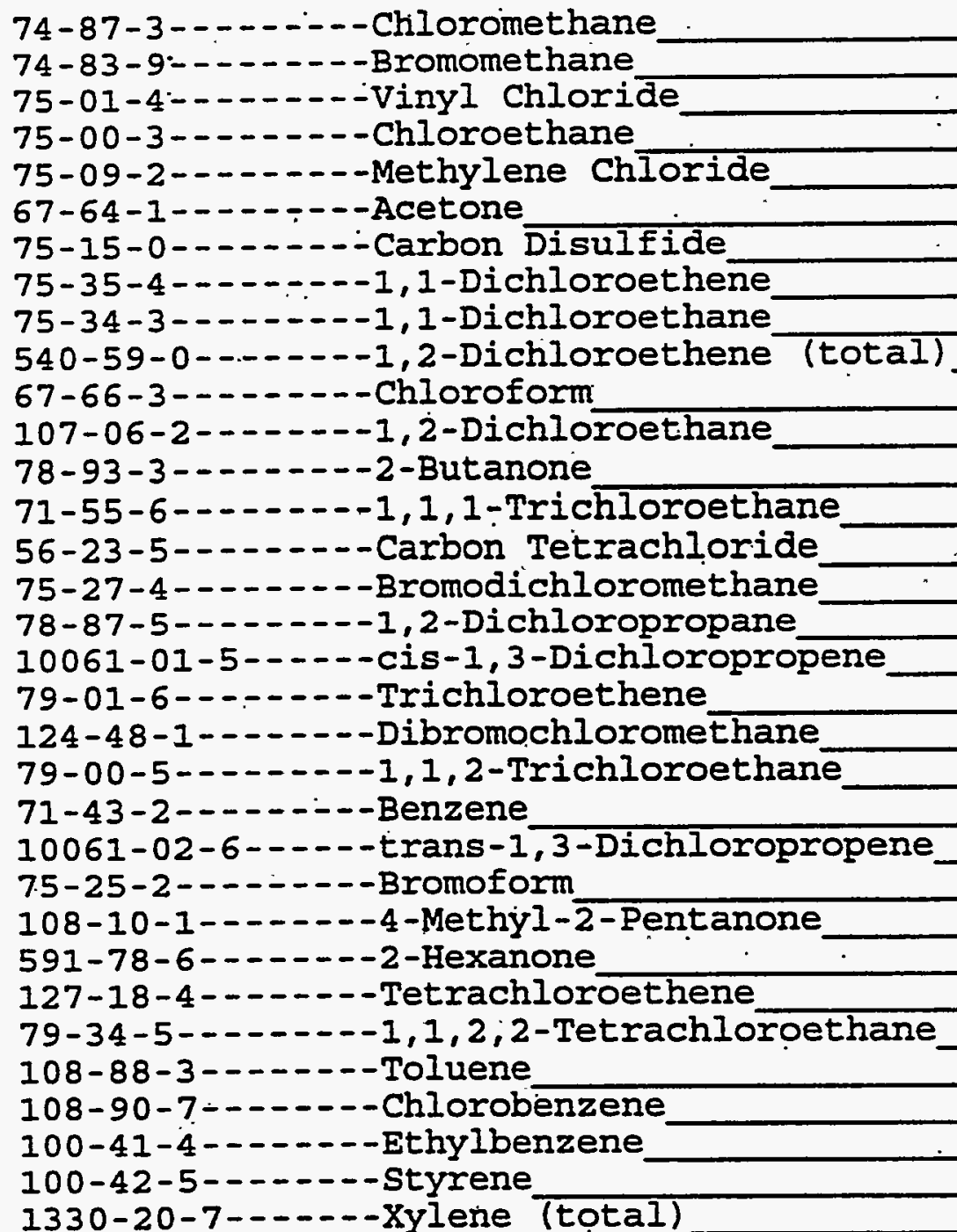 \\
\hline
\end{tabular}




\section{U.S. EPA - CLP}

INORGANIC ANALY'SES DATA SHEET

.EPA SAMPLE NO.

\section{SSCEPVIIOOI}

I me: QUANTERRA_MO

Dode: ITMO_ Case No.:SW846

i' (soil/water): WATER

L (low/med): LOW

Is :

$$
-0.0
$$

Concentration Units (ug/L or $\mathrm{mg} / \mathrm{kg}$ dry weight): UG/L_

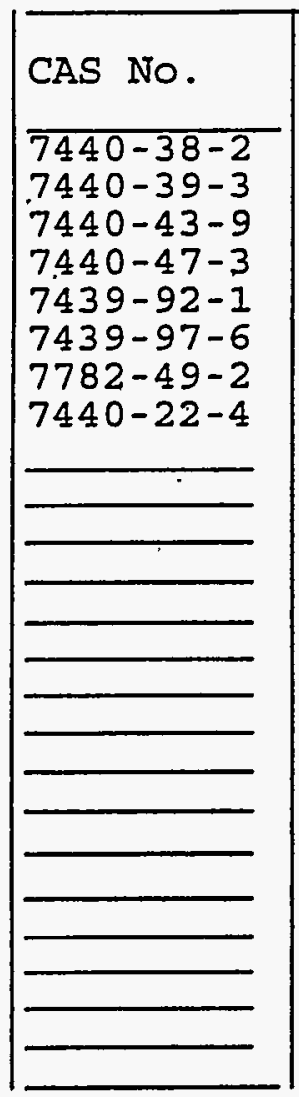

$\therefore$ 3efore:
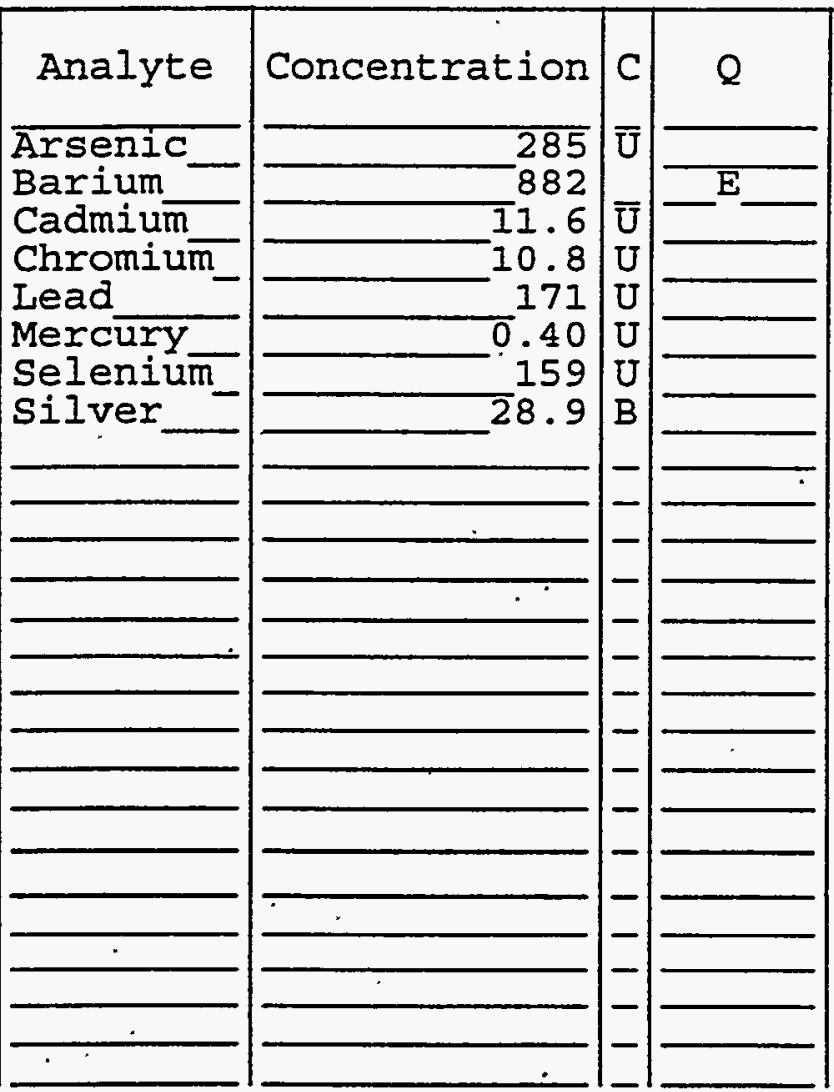

Clarity Before:

Clarity After:

\section{After:}

SDG No.: V245

Lab Sample ID: Pं15230-005

Date Received: .07/03/97

: :s:

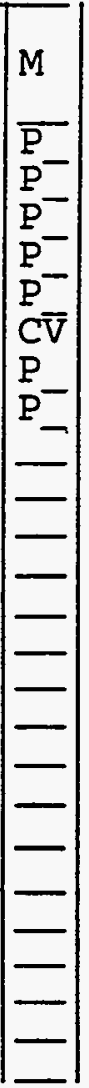

Texture:

Artifacts : 
Lab Name:

Quanterra, MO

Lab Code: ITMO Case No.:

Matrix : (soil/water) SOIL

Sample wt/vol: $1.0(\mathrm{~g} / \mathrm{ml}) \mathrm{G}$

Level: . (low/med)

LOW

웅 Moisture: not dec.
Contract: 625.01 SAS No.: SDG No.: V245

Lab Sample ID: 15230-012

Lab File ID:

Date Sampled: 07-01-97

Date Analyzed: $07-12-97$

Dilution Factor: 1.0
CAS NO.

Compound

LBH (1)

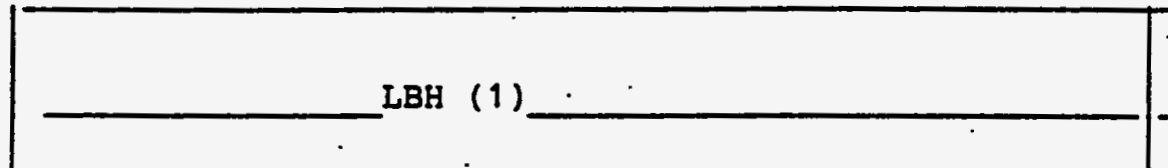

CONCENTRATION UNITS:

(ug/L or $\mathrm{ug} / \mathrm{Kg})$ UG/KG

$\mathrm{U}$ : Concentration of analyte is less than the value given.

$\mathrm{X}$ : Pattern reasonably matches gasoline

Y: Pattern appears to be multi-peaks, but does not match gasoline

z: Concentration is primarily from a single peak

(1): Low Boiling Hydrocarbon (LBH) is quantitated as if it is Gasoline. 


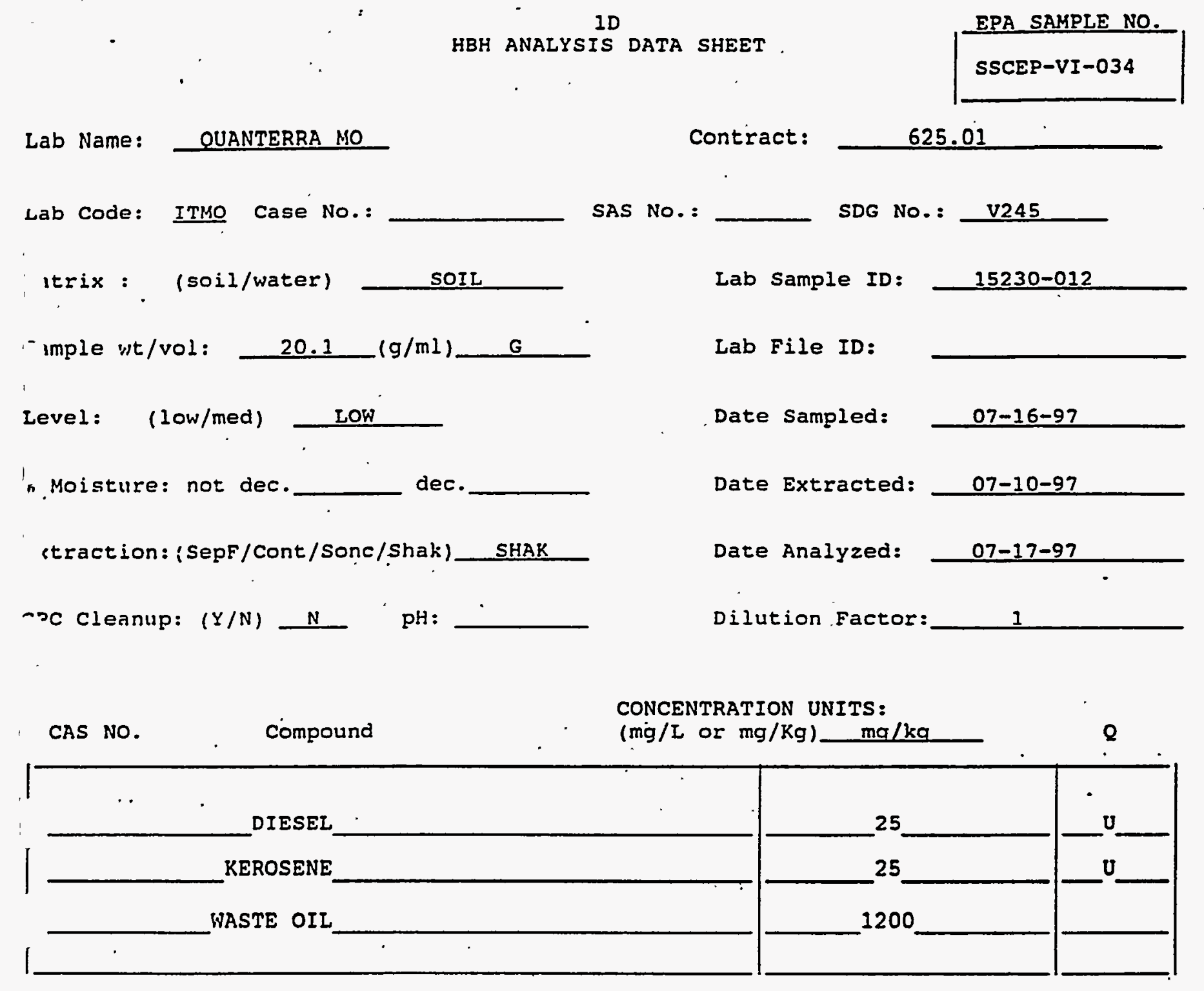

$\mathrm{U}$ : Concentration of analyte is less than the value given. 
Lab Name: QUANTERRA: MO

Lab Code: ITMO

Case No.: V23001

Matrix: (soil/water) SOIL

Sample wt/vol:

$5.00(\mathrm{~g} / \mathrm{mL}) \mathrm{G}$

Level:

(low/med) LOW.

$\%$ Moisture: not dec.

GC Column: RTX-502.2 ID: 0.530 (mm)

Soil Extract Volume:

(uL)
Contract : $625-01$

SSCEPVI034
SAS NO.:

SDG No.: V245
Lab Sample ID: 15230-012

Lab File ID: G7515

Date Received: 07/03/97

Date Analyzed: 07/13/97

Dilution Factor: $\quad 1.0$

Soil Aliquot Volume:

(UL)

CAS NO

COMPOUND

CONCENTRATION UNITS:

(ug/L or ug/Kg) UG/KG

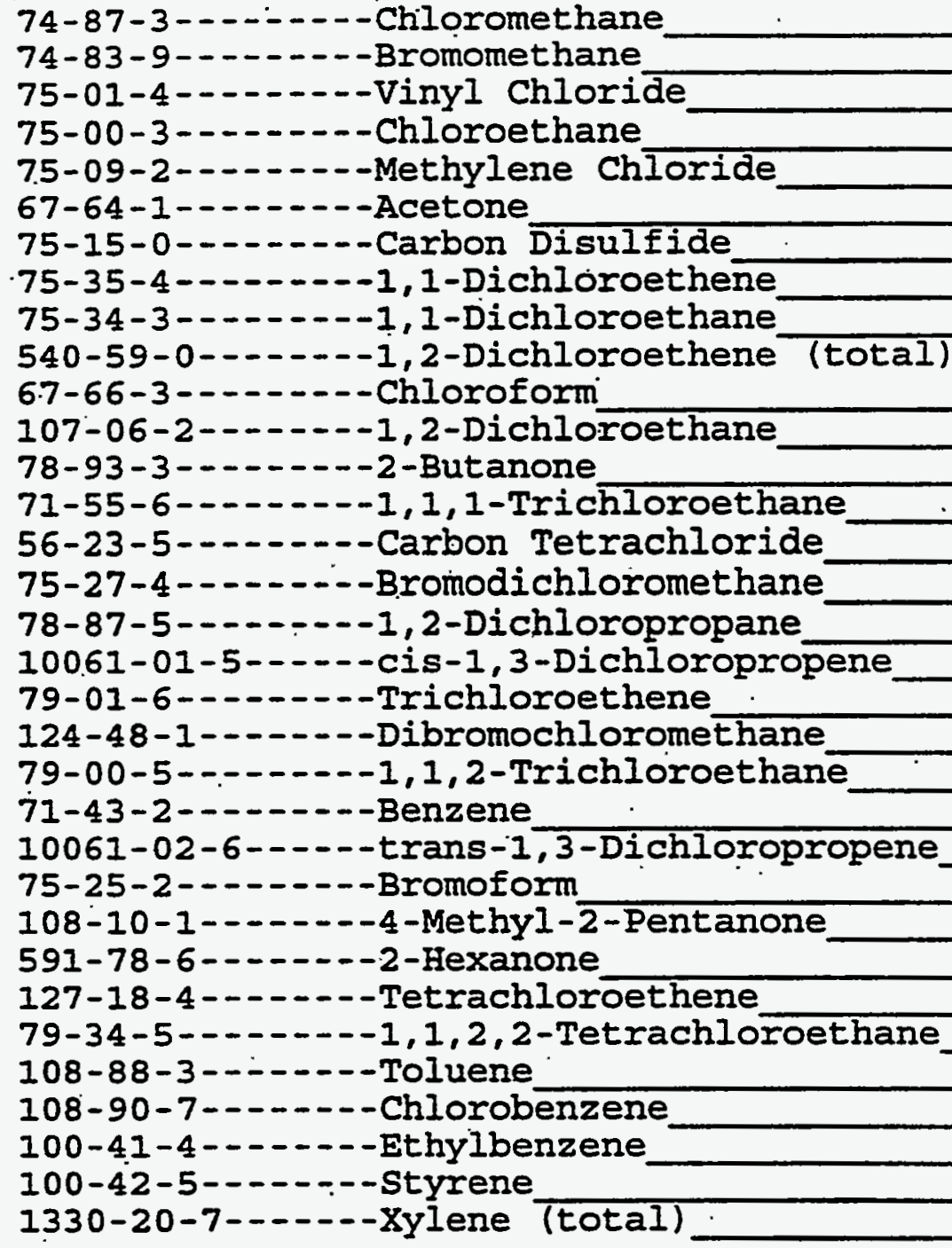

\begin{tabular}{r|l|}
10 & $U$ \\
10 & $U$ \\
10 & $U$ \\
10 & $U$ \\
5 & $U$ \\
2 & $J$ \\
5 & $U$ \\
5 & $U$ \\
5 & $U$ \\
5 & $U$ \\
5 & $U$ \\
5 & $U$ \\
20 & $U$ \\
5 & $U$ \\
5 & $U$ \\
5 & $U$ \\
5 & $U$ \\
5 & $U$ \\
5 & $U$ \\
5 & $U$ \\
5 & $U$ \\
5 & $U$ \\
5 & $U$ \\
5 & $U$ \\
20 & $U$ \\
20 & $U$ \\
5 & $U$ \\
5 & $U$ \\
5 & $U$ \\
5 & $U$ \\
5 & $U$ \\
5 & $U$ \\
5 & $U$ \\
\hline
\end{tabular}


1

INORGANIC ANALYSES DATA SHEET

c le: QUANTERRA_MO

ode : ITMO

Case No. :SW846

\& (soil/water): WATER

(low/med) : LOW

Contract: 625.01

SAS NO.:

EPA SAMPLE NO.

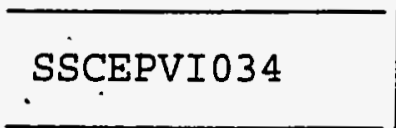

j $\mathbf{s}$ :

$$
0.0
$$

Concentration Units (ug/L or $\mathrm{mg} / \mathrm{kg}$ dry weight): UG/L

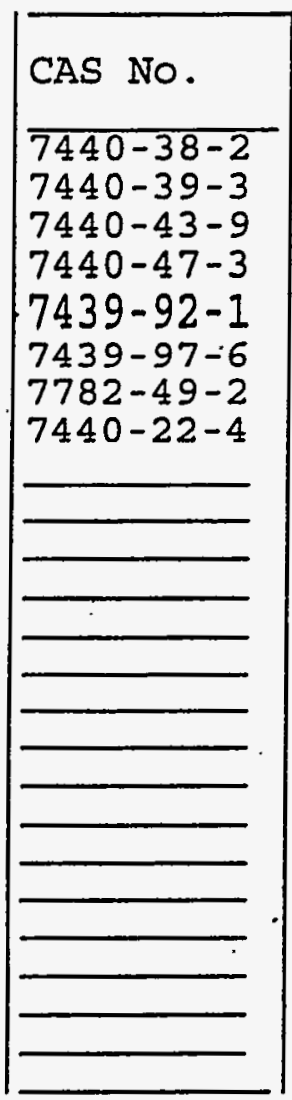

efore:

After:

r. 5 :

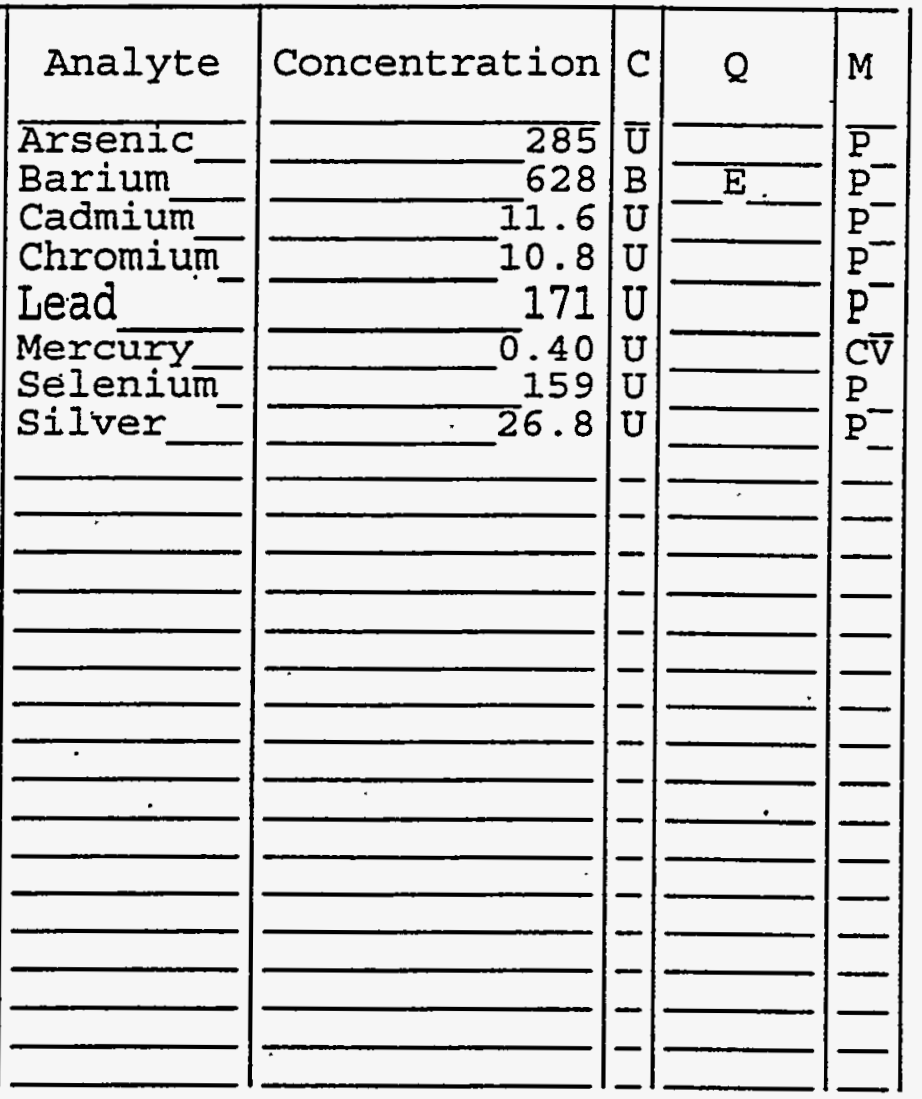

Clarity Before:

Clarițy After:
Lab Sample ID: P15230-012

Date Received: 07/03/97 
Lab Name:

Quanteria, MO

Lab Code: ITMO Case No.:

Matrix : (soil/water) SOIL

Sample wt/vol: $\frac{1.0 \quad(\mathrm{~g} / \mathrm{ml}) \_\mathrm{G}}{-}$

Level: (low/med) LOW

\& Moisture: not dec.

CAS NO.

Compound

LBH (1)
Contract: 625.01

SAS NO.: SDG No.: V245

Lab Sample ID: 15230-009

Lab File ID:

Date Sampled: 07-01-97

Date Analyzed: $\quad 07-12-97$

Dilution Factor: 1.0
CONCENTRATION UNITS:

(ug/L or $u g / \mathrm{kg}) \quad U G / K G \quad Q$

\section{U: Concentration of analyte is less than the value given. \\ $\mathrm{x}$ : Pattern reasonably matches gasoline \\ Y: Pattern appears to be multi-peaks, but does not match gasoline. \\ $\mathrm{z}$ : Concentration is primarily from a single peak}

(1): Low Boiling Hydrocarbon (LBH) is quantitated as if it is Gasoline. 
ah Name: OUANTERRA MO
ab Code: ITMO Case No.:

IIx : (soil/water)

iple wt/vol: 20.1 $(\mathrm{g} / \mathrm{ml})$ G contract: SAS NO.: SDG No.: V245 Lab Sample ID: 15230-009 Lab File ID: ,evel: (low/med) LOW Date Sampled: $07-16-97$

- Moisture: not dec. dec.

Date Extracted: 07-10-97

: :raction: (Sepf/Cont/Sonc/Shak) SHAK ; : cleanup: $(Y / N) \stackrel{\mathrm{N}}{\mathrm{N}} \mathrm{pH}:$ Dilution Factor: 10
:AS NO. Compound DIESEL KEROSENE WASTE OIL
CONCENTRATION UNITS:

(mg/L or $\mathrm{mg} / \mathrm{Kg}$ ) $\mathrm{mg} / \mathrm{kg}$

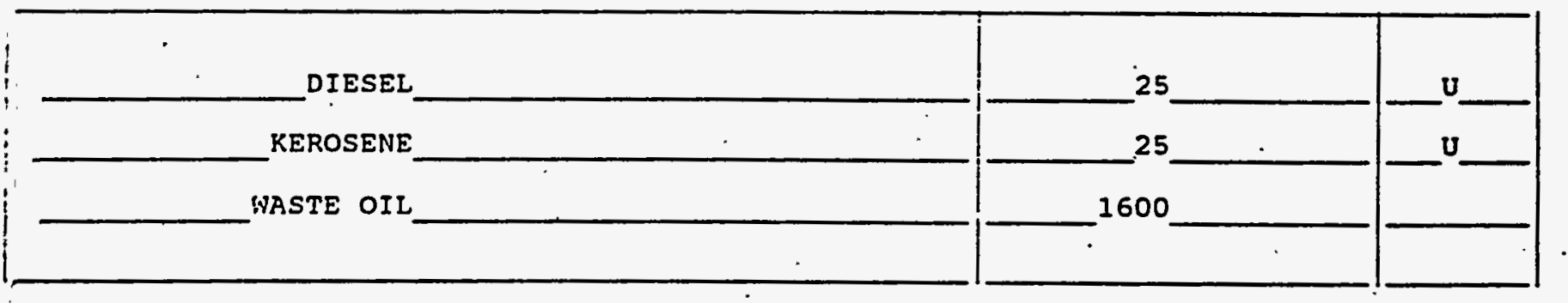

- Concentration of analyte is less than the value given. 
Lab Name: QUANTERRA: MO

Lab code: ITMO

Case No.: V23001

Matrix: (soil/water) soIL

Sample wt/vol: $\quad 5.00(\mathrm{~g} / \mathrm{mL}) \mathrm{G}$

Level : (low/med) LOW

\% Moisture: not dec.

GC Column: RTX-502.2 ID: 0.530 (mm)

Soil Extract Volume:

(UL)
Contract : 625-01

SAS - NO . :

SSCEPVI036

SDG NO.: V245

Lab Sample ID: 15230-009

Lab File ID: $\quad$ G750I.

Date Received: 07/03/97

Date Analyzed: 07/13/97

Dilution Factor: $\quad 1.0$

Soil Aliquot Volume:

(uiL)

CONCENTRATION UNITS:

CAS NO. COMPOUND.. (ug/I or ug/Kg) UG/KG

Q

74-87-3-1---Chiloromethane

74-83-9-------Bromomethane

75-01-4-...--Vinyl Chloride

75-00-3--.-----Chloroethane

75-09-2-...--.-Methylene Chloride

67-64-1-.....-...-Acetone

75-15-0-....-..-Carbón Disulfide

75-35-4------1, 1-Dichloroethene

7.5-34-3------1, 1-Dichloroethane

540-59-0-.--.-1,2-Dichloroethene (total)

67-66-3-....-.-Chloroform

107-06-2--.--1,2-Dichloroethane

78-93-3--.----2-Butanone

71-55-6-..-- i, 1, 1-Trichloroethane

56-23-5-..---.-Carbon Tetrachloride

75-27-4-1----Bromodichloromethane

78-87-5-------1, 2-Dichloropropane

10061-01-5-----cis-1,3-Dichloropropene

79-01-6-...--.-Trichloroethene

124-48-1-2.---Dibromochloromethane

79-00-5------1,1,2-Trichloroethane

$71-43-2-\ldots----B e n z e n e$

10061-02-6--.--trans-1, 3-Dichloropropene

75-25-2-.....-Bromoform

108-10-1-------4-Methy 1-2-Pentanone

591-78-6--.----2-Hexanone

127-18-4-..-- Tetrachloroethene

79-34-5--.--1, 1, 2, 2-Tetrachloroethane

1.08-88-3------Toluene

108-90-7--..--Chlorobenzene

100-41-4-.-----Ethylbenzene

100-42-5-------Styrene

1330-20-7-..--Xylene (total)

\begin{tabular}{r|l|}
10 & $\mathrm{U} \cdot$ \\
10 & $\mathrm{U}$ \\
10 & $\mathrm{U}$ \\
10 & $\mathrm{U}$ \\
5 & $\mathrm{U}$ \\
12 & $\mathrm{~J}$ \\
5 & $\mathrm{U}$ \\
5 & $\mathrm{U}$ \\
5 & $\mathrm{U}$ \\
5 & $\mathrm{U}$ \\
5 & $\mathrm{U}$ \\
5 & $\mathrm{U}$ \\
20 & $\mathrm{U}$ \\
5 & $\mathrm{U}$ \\
5 & $\mathrm{U}$ \\
5 & $\mathrm{U}$ \\
5 & $\mathrm{U}$ \\
5 & $\mathrm{U}$ \\
5 & $\mathrm{U}$ \\
5 & $\mathrm{U}$ \\
5 & $\mathrm{U}$ \\
5 & $\mathrm{U}$ \\
5 & $\mathrm{U}$ \\
5 & $\mathrm{U}$ \\
20 & $\mathrm{U}$ \\
20 & $\mathrm{U}$ \\
5 & $\mathrm{U}$ \\
5 & $\mathrm{U}$ \\
5 & $\mathrm{U}$ \\
5 & $\mathrm{U}$ \\
5 & $\mathrm{U}$ \\
5 & $\mathrm{U}$ \\
5 & $\mathrm{U}$ \\
& \\
\hline & \\
\hline
\end{tabular}


IA

VOLATIIE ORGANICS ANALYSIS DATA SHEET
EPA SAMPLE NO.

SSCEPVI036RE
J Name: QUANTERRA MO

= Code: ITMO Case No.: V23001

zrix: (soil/water) soIL
Contract: $625-01$

SDG No.: V245

Lab Sámple ID: 15230-009RE

Lab File ID: $\quad$ G7512

Date Received: 07/03/97

Date Analyzed: 07/13/97

Dilution Factor: $\quad 1.0$

Soil Aliquot Volume: (uL)

CONCENTRATION UNITS :

CAS NO.

COMPOUND

(ug/I or $\mathrm{ug} / \mathrm{Kg}$ ) UG/KG

Q

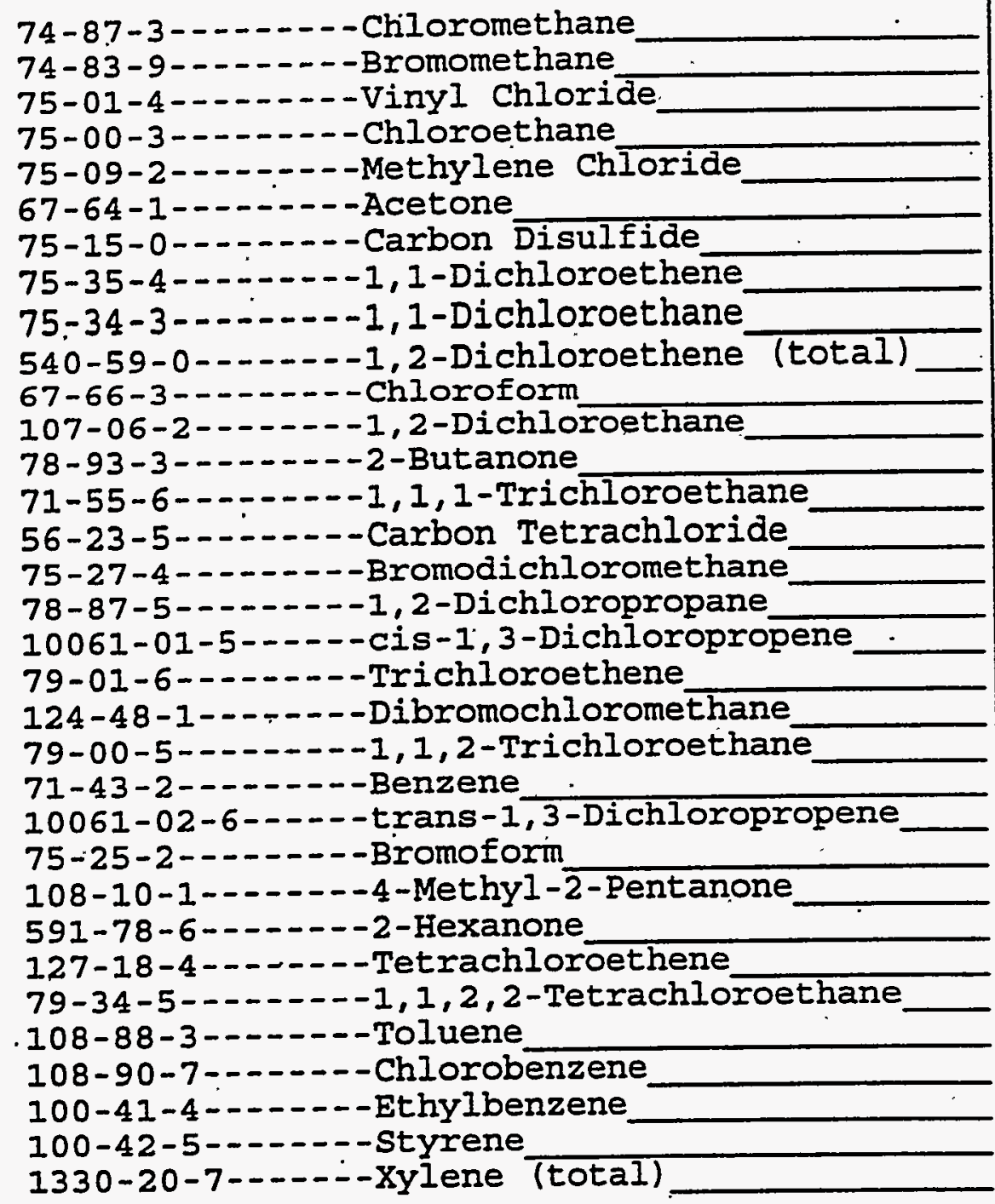

74-87-3--..--Chiloromethane

75-01-4-.....-Vinyl Chloride

75-00-3-..---Chloroethane

75-09-2--..---Methylene Chloride

67-64-1-.-----Acetone

75-15-0-.-.---Carbon Disulfide

75-35-4---n--1, 1-Dichloroethene

75-34-3-...--1, 1-Dichloroethane

540-59-0-..--1,2-Dichloroethene (total)

67-66-3-..----Chloroform

107-06-2------1,2-Dichloroethane

78-93-3-...--2-Butanone

71-55-6-...--1, 1, 1-Trichloroethane

56-23-5-...-.--Carbon Tetrachloride

78-87-5--..--1, 2-Dichloropropane

10061-01-5--.--cis-1, 3-Dichloropropene

79-01-6-...--Trichloroethene

124-48-1-..---Dibromochloromethane

79-00-5-...--1,1,2-Trichloroethane

71-43-2-..-----Benzene

10061-02-6-----trans-1,3-Dichloropropene

75-25-2-...--Bromoform

108-10-1--.---4-Methyl-2-Pentanone

591-78-6-.----2-Hexanone

127-18-4-...--Tetrachloroethene

79-34-5-..-- -1, 1, 2, 2-Tetrachloroethane

108-88-3-.----Toluene

108-90-7--.----Chlorobenzene

100-42-5--.-----Styrene

1330-20-7--.--Xylene (total)

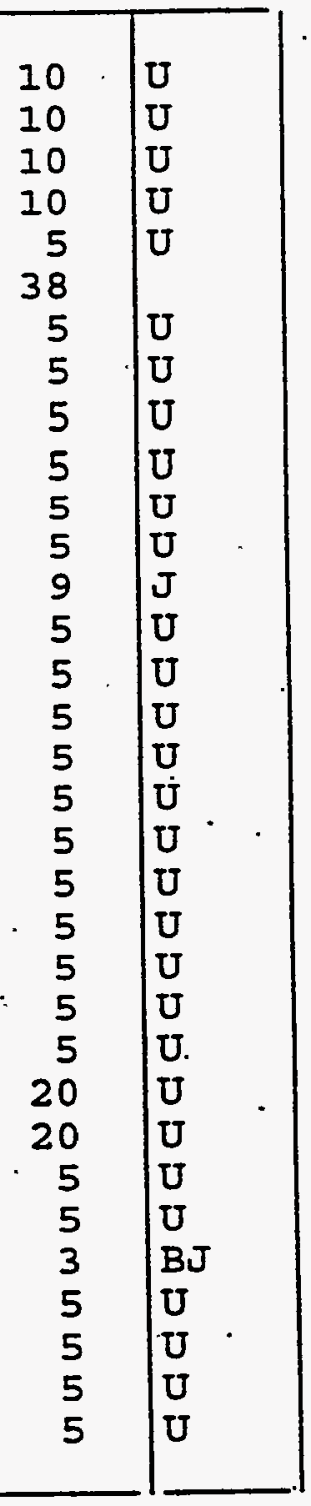

$000082 \quad 3 / 90$ 
1 . INORGANIC ANALYSES DATA SHEET

b Name : QUANTERRA_MO.

b Code: ITMO

Case No.:SW846

trix (soil/water): WATER

vel (low/med): LOW

Solids :

0.0
EPA SAMPLE NO.

\section{SSCEPVI036}

Contract: 625.01

SAS NO. :

SDG No.: V245
Lab Sample ID: Pi5230-009

Date Received: 07/03/97

Concentration Units (ug/L or $\mathrm{mg} / \mathrm{kg}$ dry weight) : UG/L_

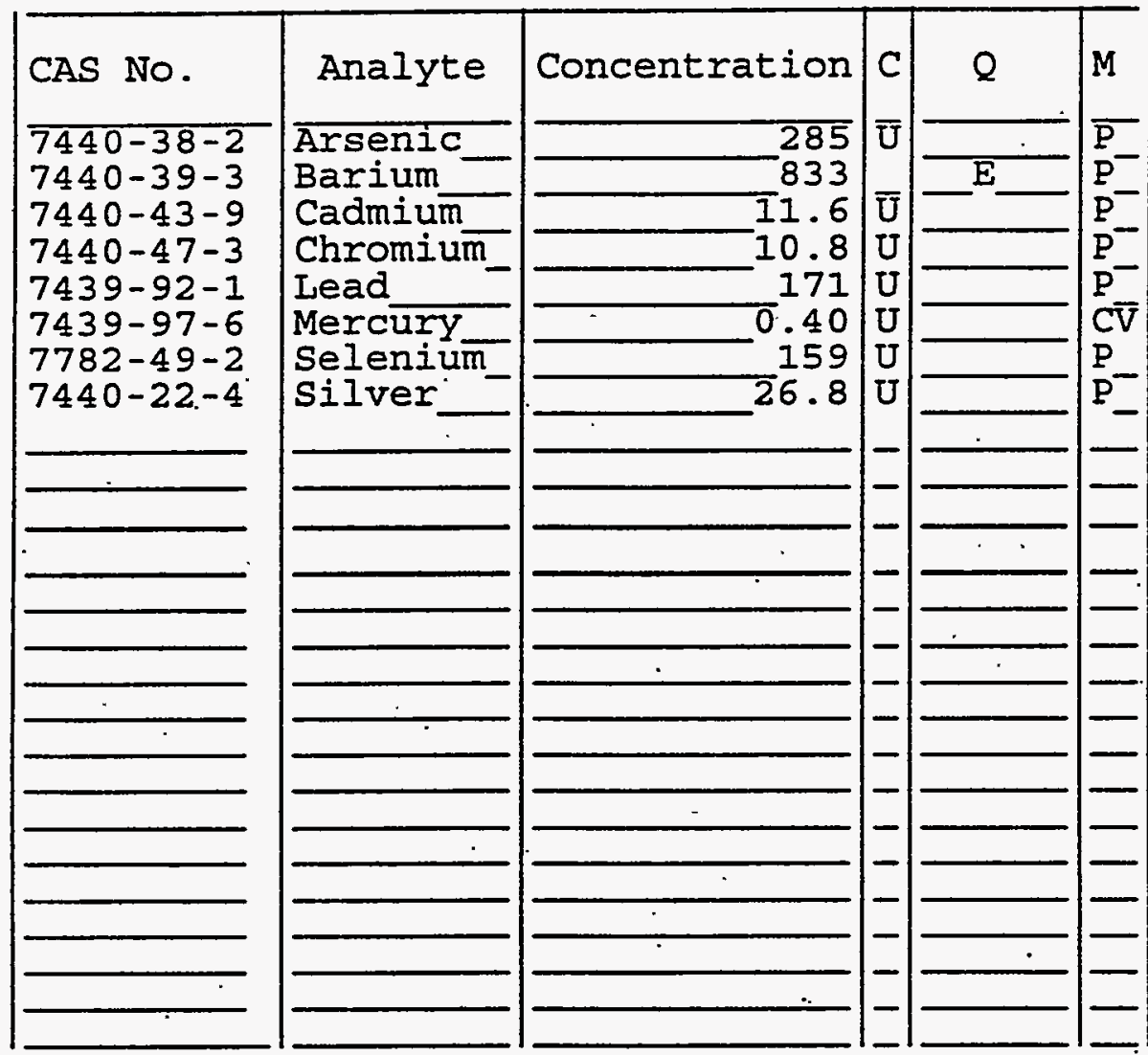

lor Before:

Clarity Before:

Texture:

lor After:

Clarity After:

Artifacts : nments : 
$1 \mathrm{~A}$

VOLATILE ORGANICS ANALYSIS DATA SHEET
EPA SAMPLE NO.

SSCEPV2036 th Name: QUANTERRA MO

an code: ITMO

Case No.: V41401

i rix: (soil/water) soIL

ample wt/vol:

$5.00(\mathrm{~g} / \mathrm{mL}) \mathrm{G}$

I) 2 : (low $/ \mathrm{med}$ ) LOW

Moisture: not dec.

Iolumn: $\operatorname{RTX}-502.2$ ID: 0.530 (mm)

ว I Extract Volume:

(uI)
Contract : $625-01$

SAS NO.:
SDG No.: V258

Lab Sample ID: 15414-001

Lab File ID: F1149

Date Received: 07/25/97

Date Analyzed: 08/06/97

Dilution Factor: $\quad 1.0$

Soil Aliquot Volume:

(uL)
CAS NO.
COMPOUND
(ug/L or $\mathrm{ug} / \mathrm{Kg}$ ) UG/KG
Q

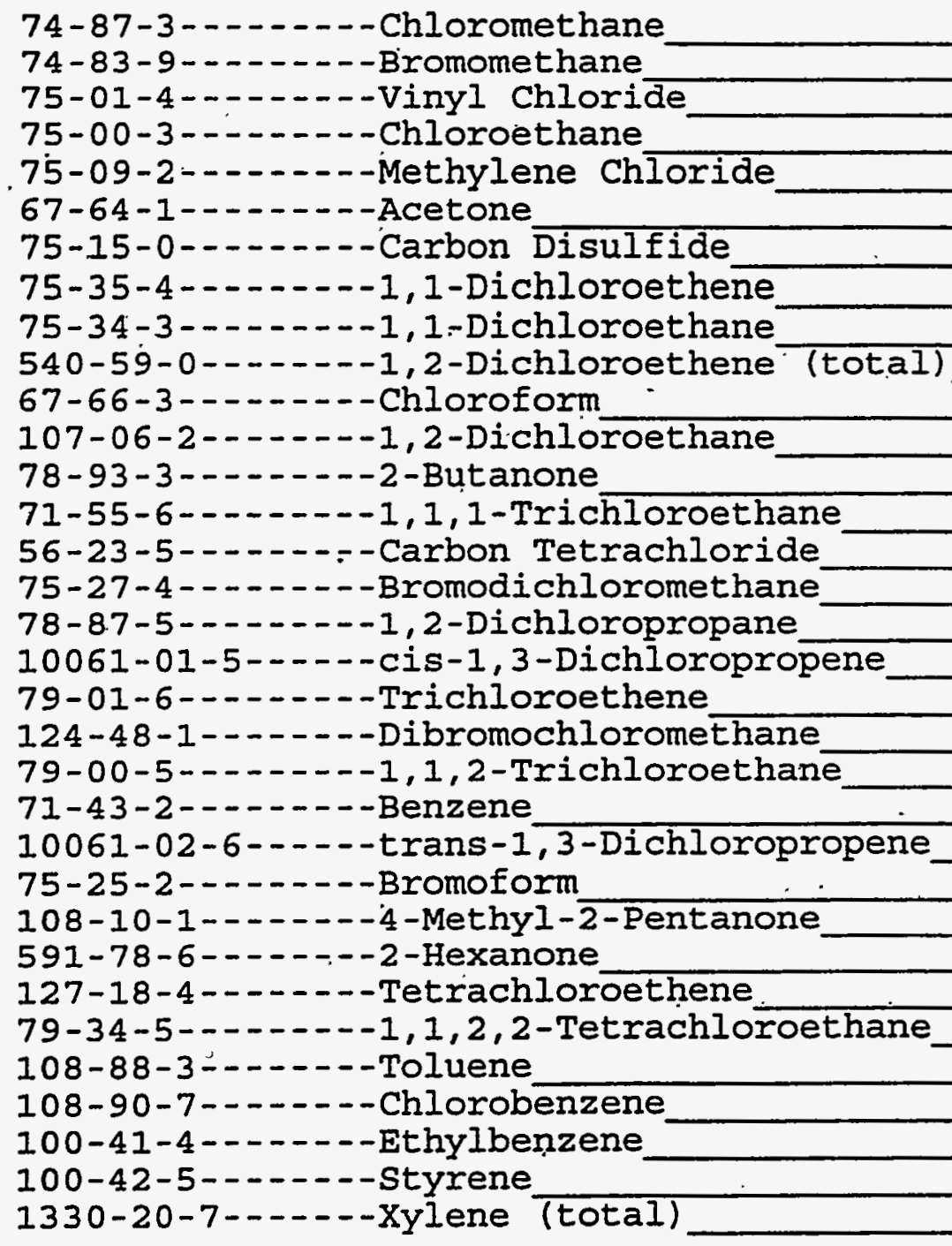


Lab Name: Quanterra, MO

Lab Code: ITMO Case No.:

Matrix : (soil/water) SoIL

Sample $w t / v o l:-1.0 \quad(g / m I) \quad G$

Level: (low/med)

LOW

\% Moisture: not dec.

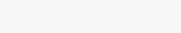

CAS No.

Compound

IBH (1)
Contract: $\quad 625.01$

SAS NO.:

SDG No.: $\mathrm{V} 245$

Lab Sample ID: 15230-007

Lab File ID:

Date Sampled: $\quad 07-01-97$

Date Analyzed: $\quad 07-12-97$

Dilution Factor: 1.0

CONCENTRATION UNITS:

(ug/L or $\mathrm{ug} / \mathrm{Kg}$ ) UG/KG

$Q$

$\mathrm{u}$ : Concentration of analyte is less than the value given.

$\mathrm{X}$ : Pattern reasonably matches gasoline

Y: Pattern appears to be multi-peaks, but does not match gasoline

Z: Concentration is primarily from a single peak

(1): Low Boiling Hydrocarbon ( $\mathrm{LBH}$ ) is quantitated as if it is Gasoline. 


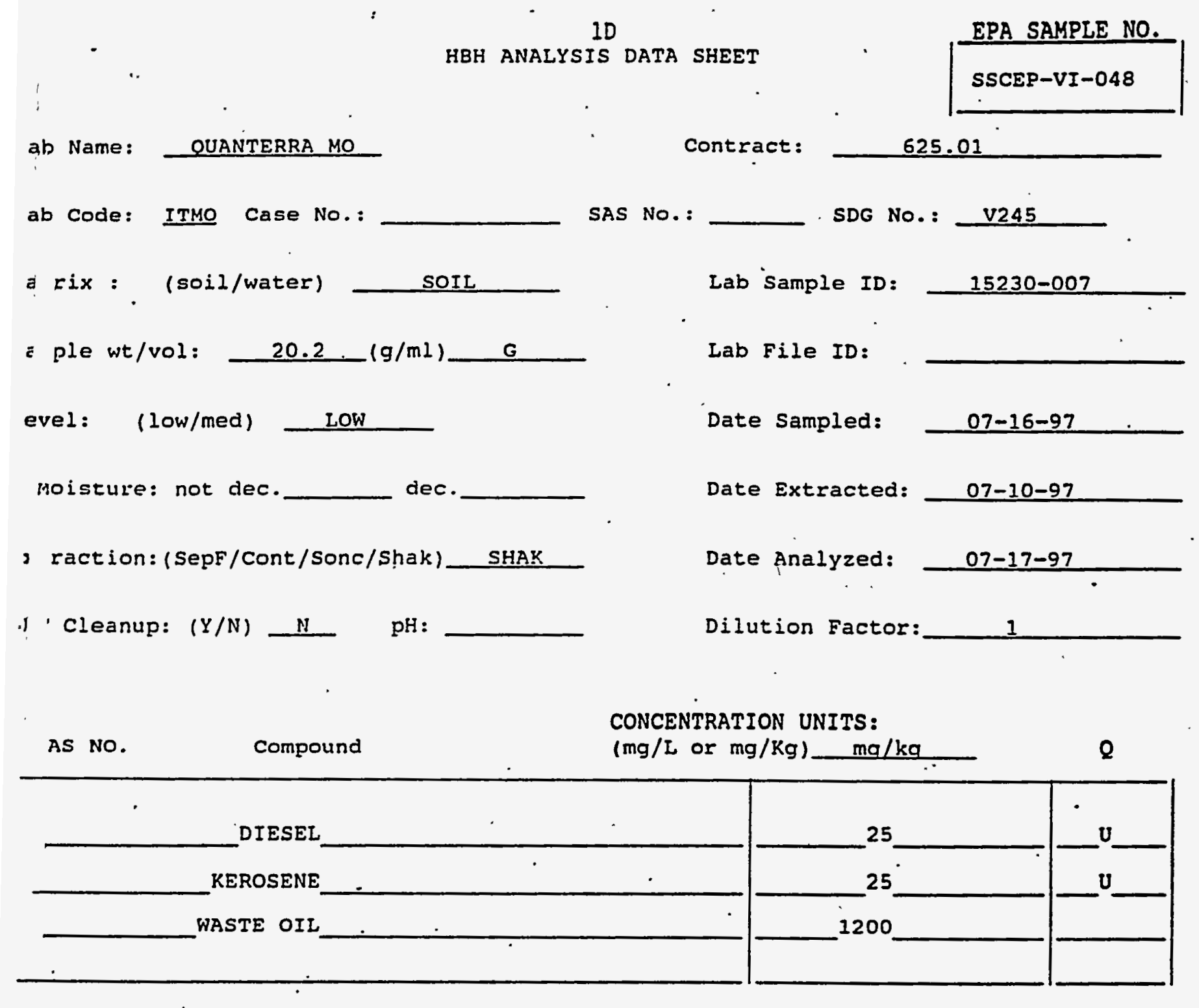

r Concentration of analyte is less than the value given.

FORM I HBH 
Lab Name: QUANTERRA'MO

Lab Code: ITMO

Case No.: V23001

Matrix: (soil/water) soIL

Sample wt/vol:

$5.00(\mathrm{~g} / \mathrm{mL}) \mathrm{G}$

Level: (low/med) LOW

\% Moisture: not dec.

GC Column: RTX-502.2 ID: 0.530 (mm)

Soil Extract Volume:

(uL)
Contract: $625-01$

SAS NO.:
SSCEPVI048

SDG No.: V245
Lab Sample ID: 15230-007

Lab File ID: $\quad$ G7499

Date Received: 07/03/97

Date Analyzed: $07 / 13 \% 97$

Dilution Factor: ' 1.0

Soil Aliquot Volume:

(uL)

CAS NO.

COMPOUND

CONCENTRATION UNITS :

(ug/I or $u g / \mathrm{Kg}$ ) UG/KG

74-87-3--..--Chloromethane

74-83-9-------Bromomethane

75-01-4-.....-.-Vinyl Chloride

75-00-3-..----Chloroethane

75-09-2-....-.-Methylene Chloride

67-64-1--.---Acetone

75-15-0-....-.-Carbon Disulfide

75-35-4-1---1, 1-Dichloroethene

75-34-3-..--1,1-Dichloroethane

540-59-0------1,2-Dichloroethene (total)

67-66-3-.-.--Chloroform

107-06-2-----1,2-Dichloroethane

78-93-3-...--2-Butanone

71-55-6-...-.-1, 1, 1-Trichloroethane

56-23-5-..----Carbon Tetrachloride

75-27-4------Bromodichloromethane

78-87-5-_----1,2-Dichloropropane

10061-01-5-----cis-1,3-Dichloropropene

79-01-6-...-- Trichloroethene

1.24-48-1-2.---Dibromochloromethane

79-00-5--.---1,1,2-Trichloroethane

71-43-2-------Benzene

10061-02-6-----trans-1,3-Dichloropropene

75-25-2--.-----Bromoform

108-10-1--.-.-4-Methyl-2-Pentanone

591-78-6-------2-Hexanone

127-18-4-...-.-Tetrachloroethene

79-34-5-...--1, 1, 2, 2-Tetrachloroethane

108-88-3-.----Toluene

108-90-7--.---Chlorobenzene

100-41-4-------Ethylbenzene

100-42-5-.------Styrene

1330-20-7-...--Xylerie (total)

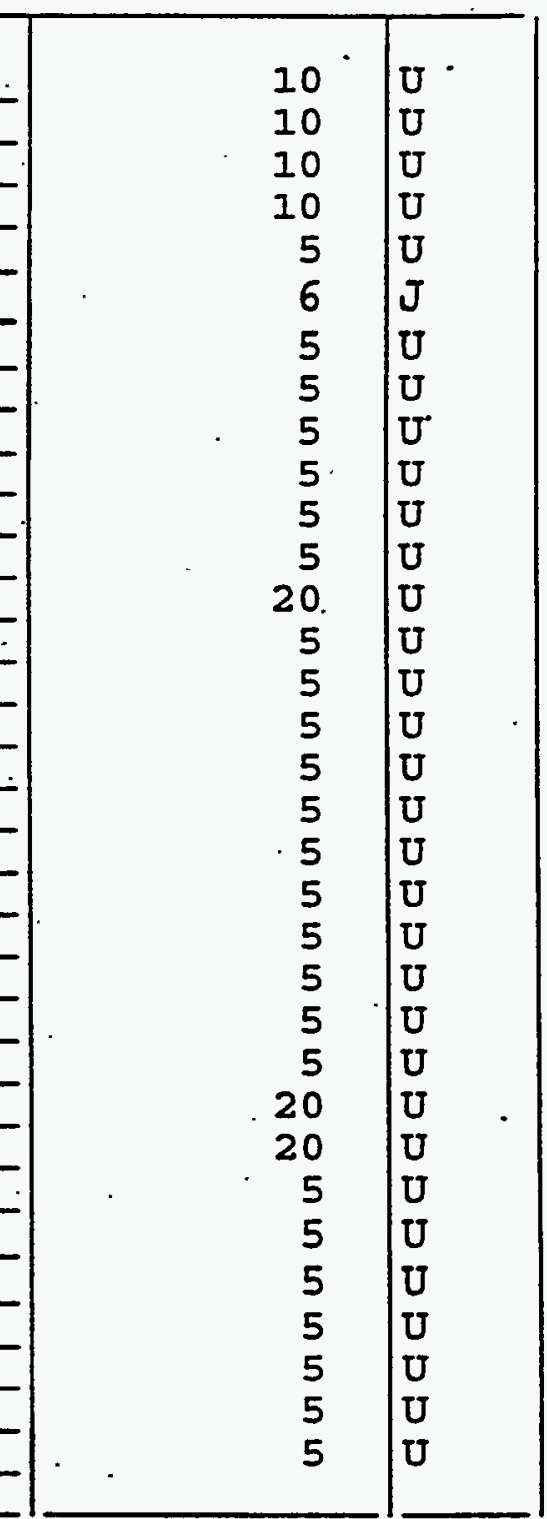

FORM I VOA 
1

INORGANIC ANALYSES .DATA SHEET $\checkmark$ me: QUANTERRA MO

Jode: ITMO__ Case No.:SW846

i (soil/water) : 'WATER

1. (low/med): LOW

$1 \mathrm{ds}$ :

$-0.0$
EPA SAMPLE NO.

\section{SSCEPVI0 48}

SDG No.: V245

Lab Sample ID: Pं15230-007

Date Received: 07/03/97

Concentration Units (ug/L or mg/kg dry weight): UG/L_

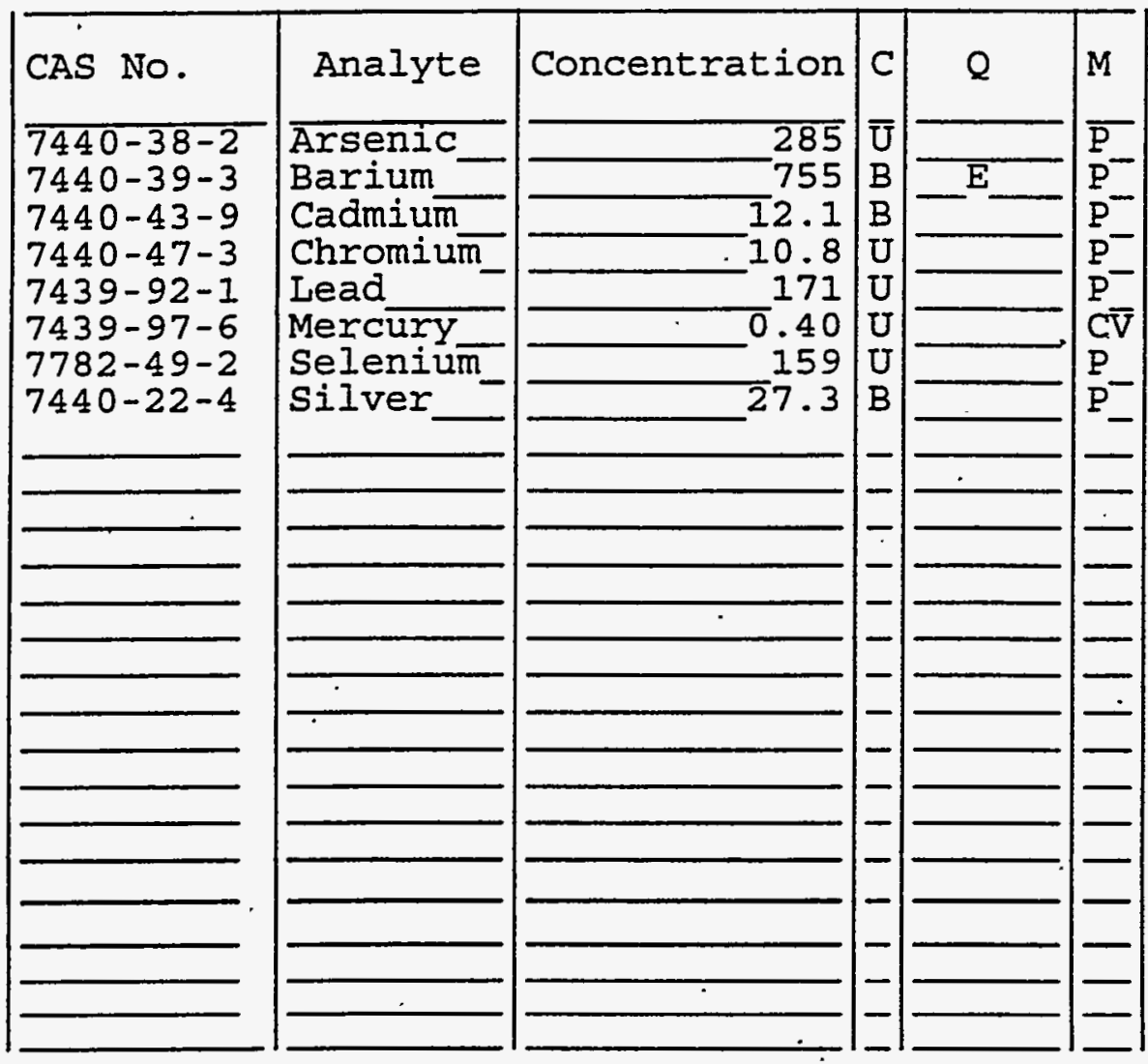

$=$ Before:

= After:

e ts :
Clarity Before:

Clarity After:
Texture:

Artifacts : 
1D

Lab Name: Quanterra,MO

Lab code: ITMO Case No.:

Matrix : (soil/water) SOIL

Sample wt/vol: $(g / m l)$

G

Level: (low/med) LOW

\% Moisture: not dec.

CAS NO.

Compound
Contract: 625.01

SAS NO.:

SDG No.: V245

Lab Sample ID: $15230-008$

Lab File ID:

Date Sampled: $\quad 07-01-97$

Date Analyzed: $\quad 07-12-97$

Dilution Factor: 1.0
CONCENTRATION UNITS:

(ug/L or $u g / K g$ ) UG/KG

LBH (1) 500 U

$\mathrm{U}$ : Concentration of analyte is less than the value given.

$x$ : Pattern reasonably matches gasoline

Y: Pattern appears to be multi-peaks, but does not match gasoline

z: Concentration is primarily from a single peak

(1): Low Boiling Hydrocarbon (LBH) is quantitated as if it is Gasoline. 


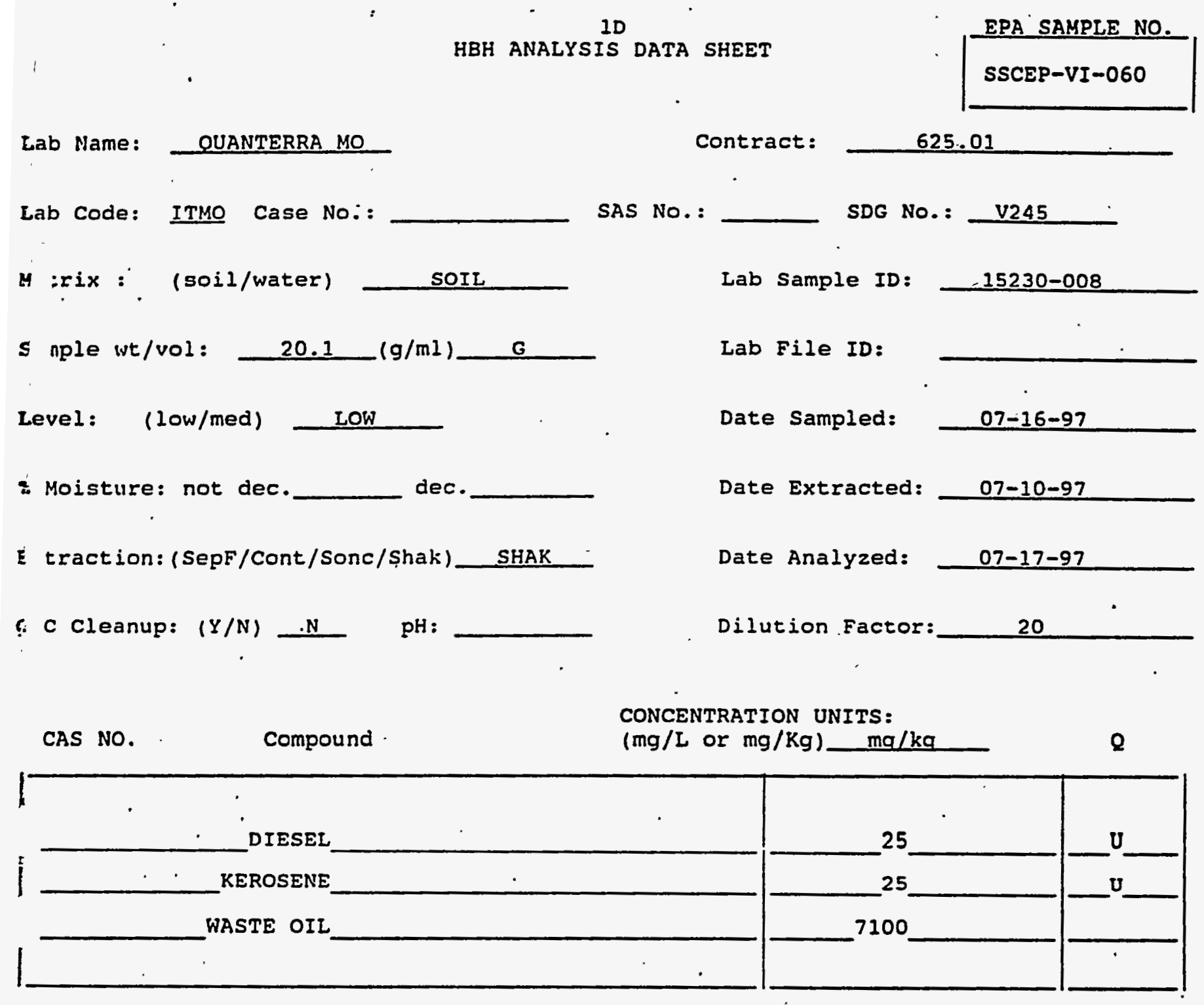

$\%$ Concentration of analyte is less than the value given.

FORM I HBH 
Lab Name: QUANTERRA: MO

Lab Code: ITMO
Contract: $625-01$

SAS NO.:

SSCEPVI060

Matrix: (soil/water) soIL

Sample wt/vol:

Level:. (low/med) LOW

$\because$ Moisture: not dec.

GC Column: RTX-502.2 ID: 0.530 (mm)

Soil Extract Volume:

CAS NO. $5.00(\mathrm{~g} / \mathrm{mL}) \mathrm{G}$

(uL)
Lab Sample ID: 15230-008

Lab File ID: $\quad$ G7500

Date Received:. 07/03/97

Date Analyzed: 07/13/97

Dilution Factor: $\quad 1.0$

Soil Aliquot Volume:

(UL)
CONCENTRATION UNITS:

(ug/L or ug/Kg) UG/KG

Q

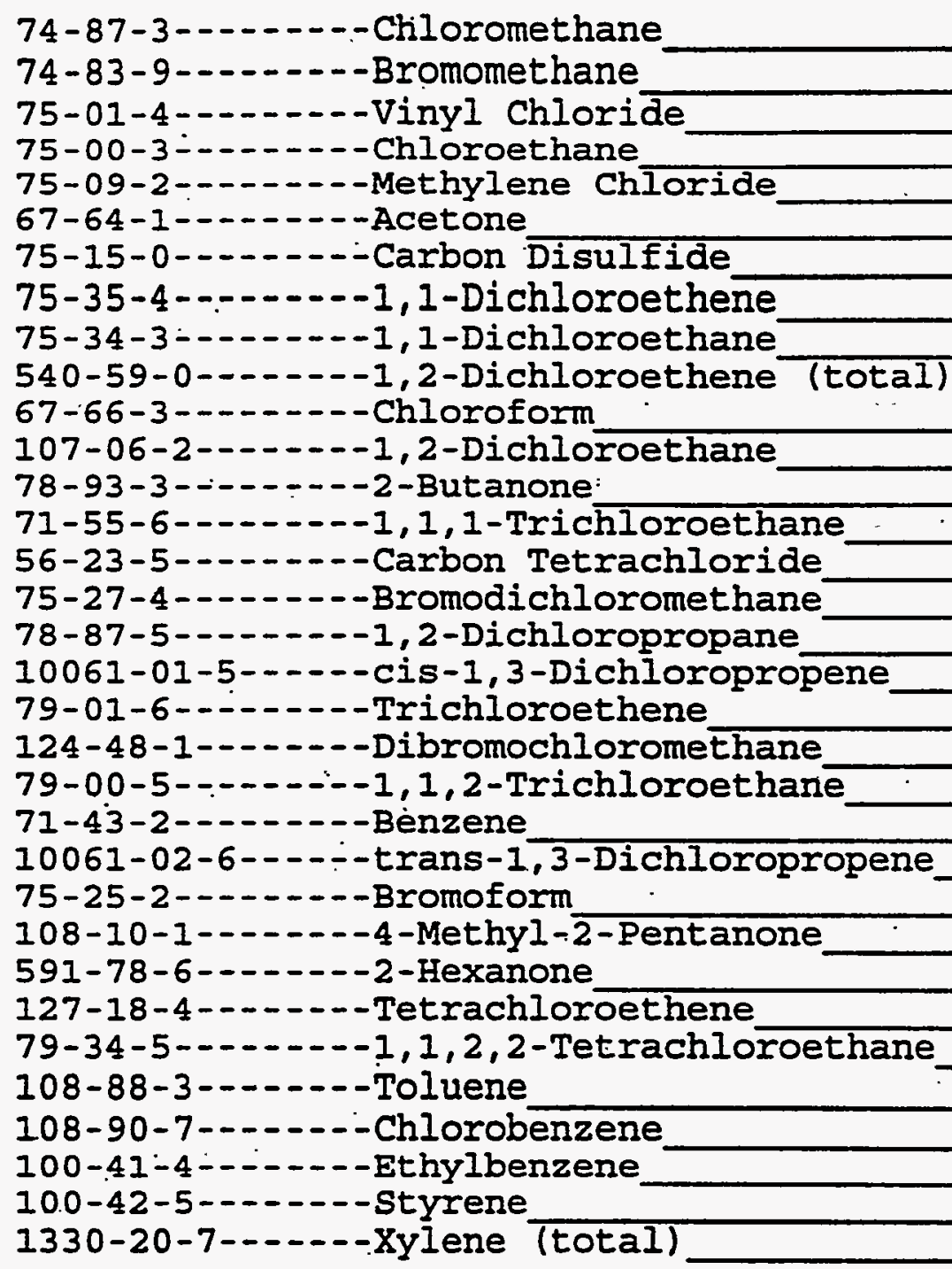


EPA SAMPLE NO.

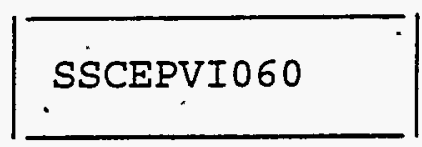

Ii ne: QUANTERRA_MO :ode: ITMO Case No.:SW846 $\therefore$ (soil/water) : WATER (low/med): .LOW Contract: 625.01 SAS NO.: SDG No.: V245 Lab Sample ID: P15230-008 Date Received: 07/03/97

is :

$$
-0.0
$$

Concentration Units (ug/L or.mg/kg dry weight): UG/L_

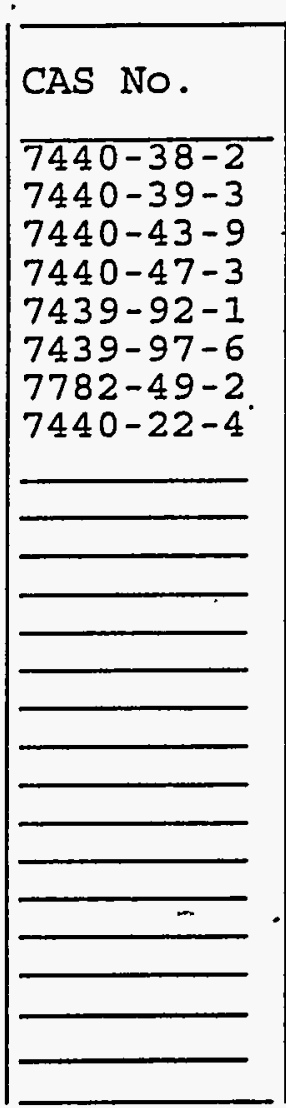

iefore:

After:

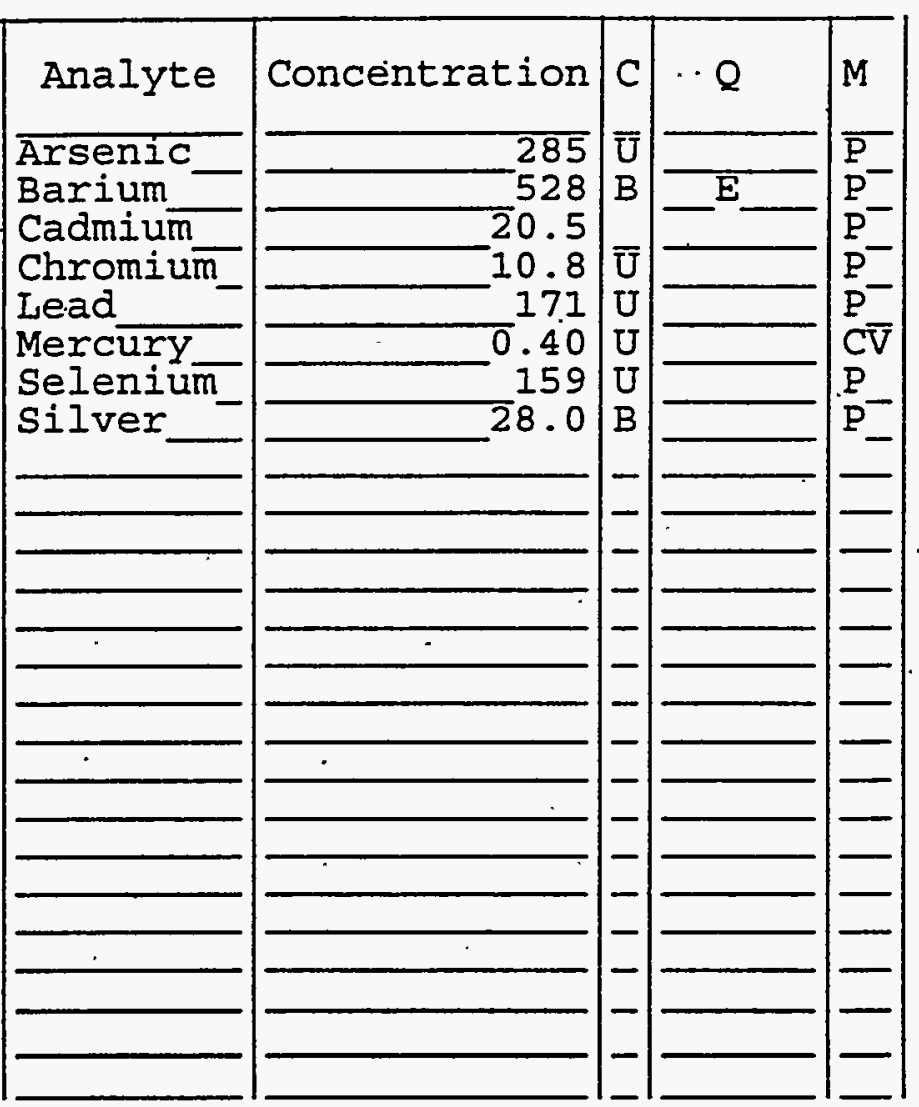

Clarity Before: Clarity After:
Texture:

Artifacts : 
1D

LBH ANALYSIS DATA SHEET
EPA SAMPLE NO.

SSCEP-VI-67
Lab Name: Quanterra, MO

Lab Code: ITMO Case No.:

Matrix : (soil/water) SOIL

Sample wt/vol:

$1.0 \quad(g / m l)$

G

Level: (low/med) LLW

\% Moisture: not dec.

CAS NO.

Compound

LBH (1)

SAS No.:

Contract: $\quad 625.01$

SDG No.: $\mathrm{V} 245$

Lab Sample ID: 15230-015

Lab File ID:

Date Sampled:

$07-01-97$

Date Analyzed: $\quad 07-12-97$

Dilution Factor: 1.0

CONCENTRATION UNITS:

(ug/L or $u g / \mathrm{Kg}$ ) UG/KG : $Q$

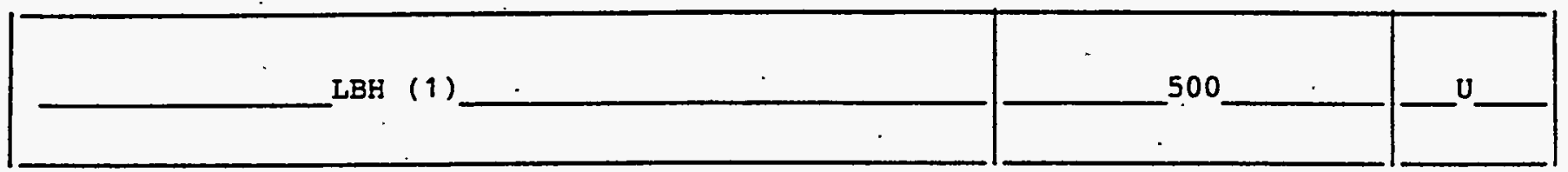

$\mathrm{U}$ : Concentration of analyte is less than the value given.

$\mathrm{x}$ : Pattern reasonably matches gasoline

Y: Pattern appears to be multi-peaks, but does not match gasoline

$\mathrm{z}$ : Concentration is primarily from a single peak

(1): Low Boiling Hydrocarbon ( $\mathrm{LBH}$ ) is quantitated as if it is Gasoline. 


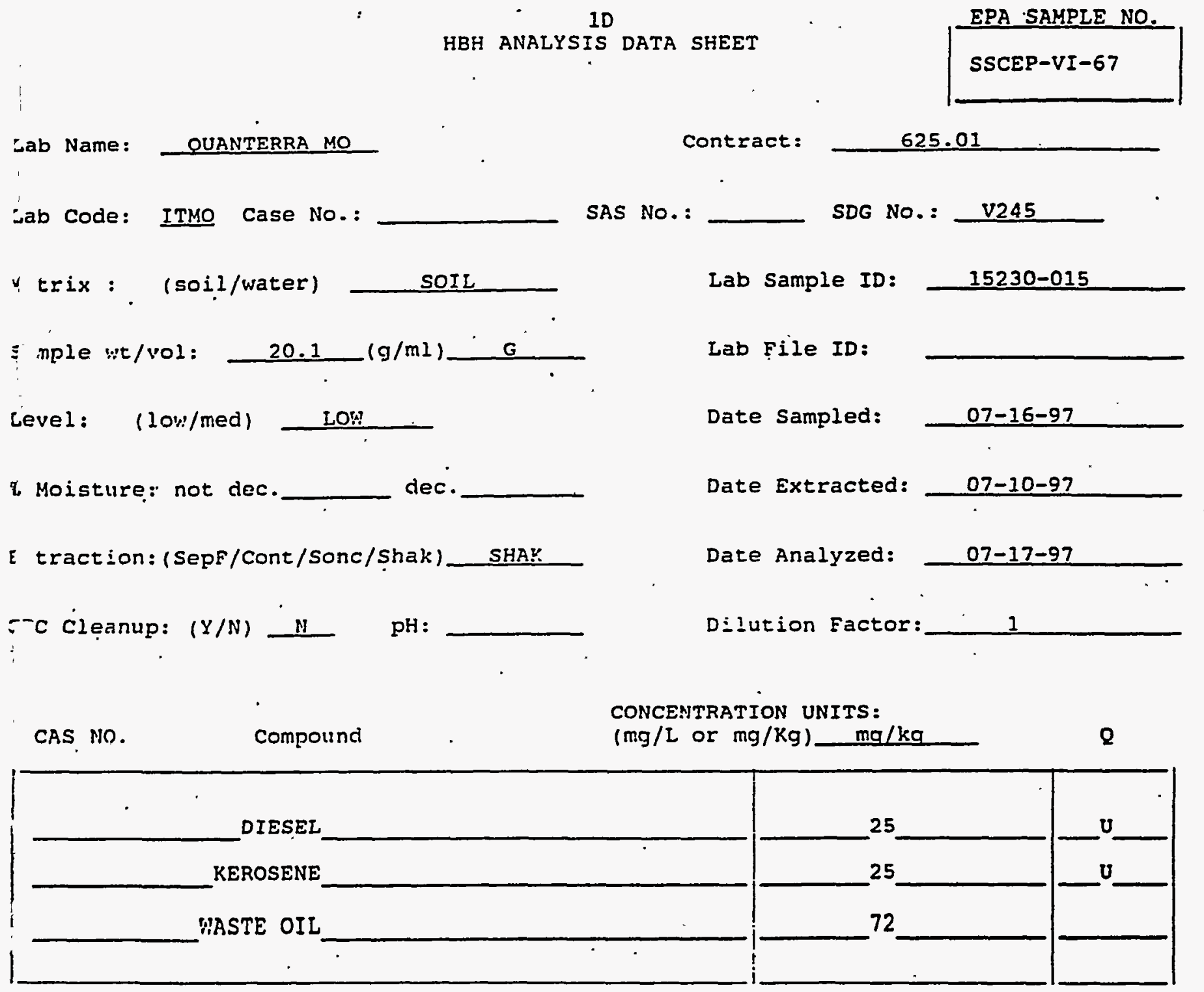

": Concentration of analyte is less than the value given.

FORM I $\mathrm{HBH}$ 
Lab Name: QUANTERRA MO

Lab Code: ITMO

Case No.: V23001

Matrix: (soil/water) SOIL

Sample wt/vol: $\quad 5.00(\mathrm{~g} / \mathrm{mL}) \mathrm{G}$

Level: (low/med) LOW

\% Moisture: not dec.

GC Column: RTX-502.2 ID: 0.530 (mm)

Soil Extract Volume:

CAS NO.
(uI)
Contract: $625-01$

SSCEPVI67
SAS No. :

SDG No.: V245
Lab Sample ID: 15230-015

Lab File ID: $\quad$ G7518

Date Received: 07/03/97

Date Analyzed: 07/13/97

Dilution Factor: $\quad 1.0$

Soil Aliquot Volume:

(uL)
CONCENTRATION UNITS:

(ug/I or ug/Kg) UG/KG

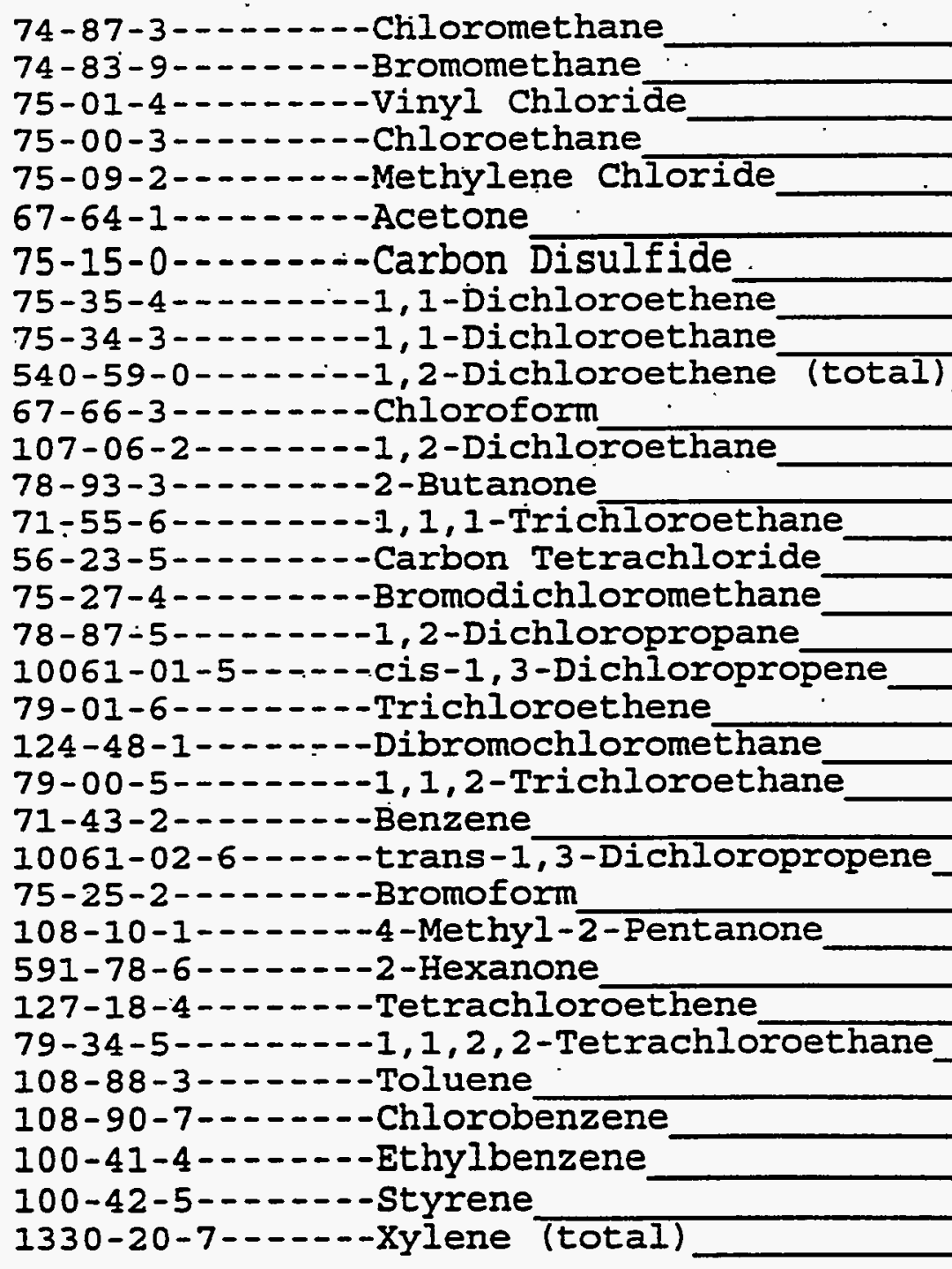




\section{5 \\ U.S. EPA - CLP \\ 1 \\ INORGANIC ANALYSES DATA SHEET}

* : QUंANTERRA_MO

sde: ITMO_ Case No.:SW846

\{ (soil/water) : WATER

(low/med): : LOW

Contract: 625.01

SAS NO.:

EPA SAMPLE NO.

SSCEPVI67
Lab Sample ID: P15230-015

Date Received: 07/03/97.
SDG NO.: V245

s :

$-0.0$

Concentration Units (ug/L or $\mathrm{mg} / \mathrm{kg}$ dry weight): UG/L_

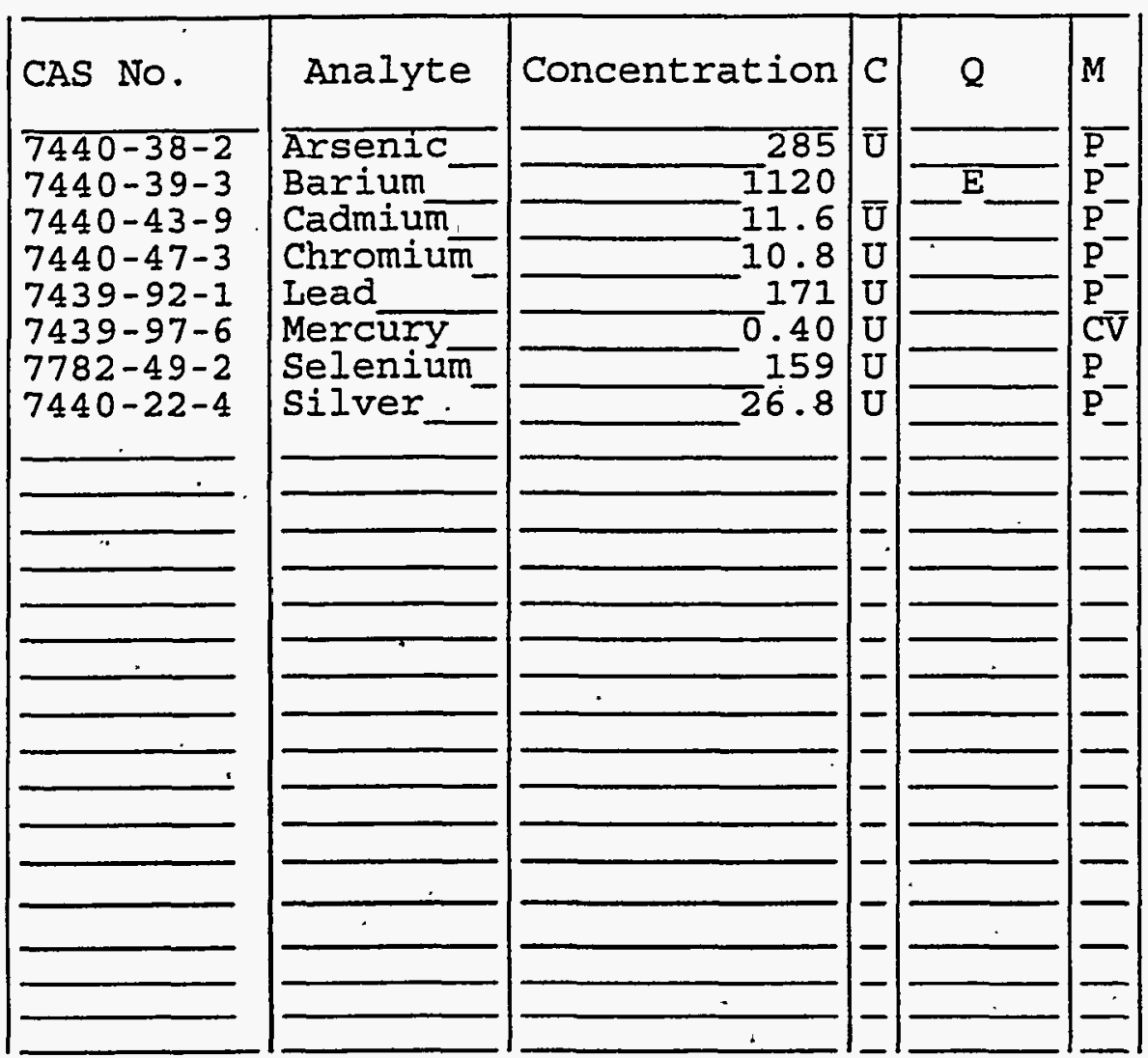

efore:

After:
Clarity Before:

Clarity After:

J

FORM I - IN

TCLP .

$000 \div 16$ 
Lab Name: Quanterra, MO

Lab Code: ITMO Case No.:

Matrix : (soil/water) SOIL

Sample wt/vol: $1.0 \ldots(\mathrm{g} / \mathrm{ml})$

G

Level: (low/med) LOW

웅 Moisture: not dec.

CAS NO.

Compound

LBH (1)
Contract: .625 .01

SAS NO.:

SDG No.: V245

Lab Sample ID: 15230-016

Lab File ID:

Date Sampled: $\quad 07-01-97$

Date Analyzed: $07-12-97$

Dilution Factor: 1.0

CONCENTRATION UNITS:

$(\mathrm{ug} / \mathrm{L}$ or $\mathrm{ug} / \mathrm{Kg})$ UG/KG Q

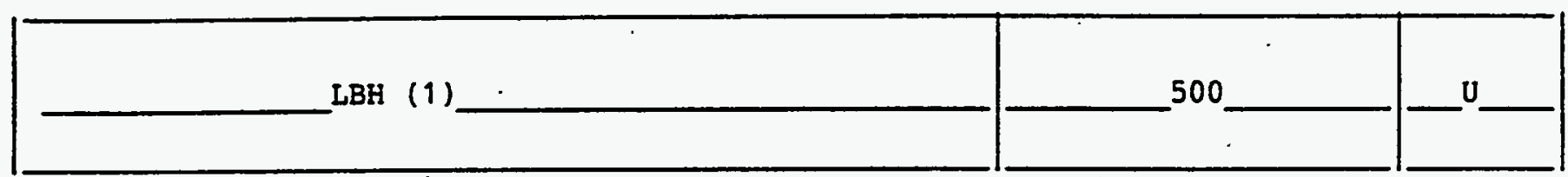

U: Concentration of analyte is less than the value given.

$\mathrm{x}$ : Pattern reasonably matches gasoline

Y: Pattern appears to be multi-peaks, but does not match gasoline

z: Concentration is primarily from a single peak

(1): Low Boiling Hydrocarbon (IBH) is quantitated as if it is Gasoline. 
ID

HBH ANALYSIS DATA SHEET
EPA SAMPLE NO. SSCEP-VI-70
Lab Name: OUANTERRA MO

Lab Code: ITMO Case No.:

atrix : (soil/water)

SOII

imple wt/vol:

$20.1 \quad(\mathrm{~g} / \mathrm{ml})$

G

Level: (low/med)

LOW

\%. Moisture: not dec. dec.

(traction: (SepF/Cont/Sonc/shak) SHAK

PC Cleanup: $(Y / N)$ N

CAS MO.

Compound

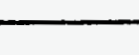

1 contract:

SAS NO.:

Lab Sample ID:

$15230-016$

Lab File ID:

Date Sampled:

$07-16-97$

Date Extracted:

$07-10-97$

Date Analyzed:

$07-17-97$

Dilution. Factor:

10
CONCENTRATION UNITS:

$(\mathrm{mg} / \mathrm{L}$ or $\mathrm{mg} / \mathrm{Kg}) \quad \mathrm{mg} / \mathrm{kg} \quad Q$

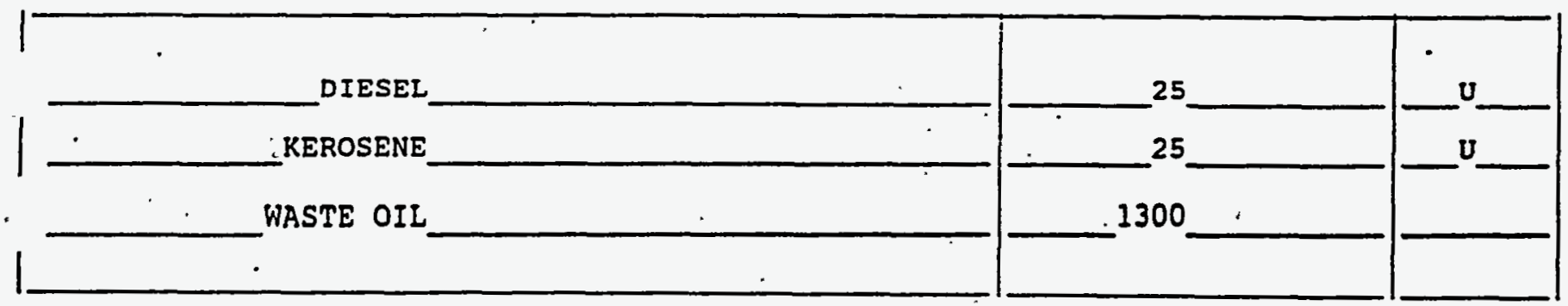

$\mathrm{U}$ : Concentration of analyte is less than the value given. 
.Lab Name: QUANTERRA MO

Lab Code: ITMO

Case No.: V23001

Matrix: (soil/water) SOIL

Sample wt/vol:

$5.00(\mathrm{~g} / \mathrm{mL}) \mathrm{G}$

Level: (low/med) LOW

$\because$ Moisture: not dec.

GC Column: RTX-502.2 ID: 0.530 (mm)

Soil Extract Volume:

(UI)
Contract: $625-01$

SSCEPVI70
CAS NO.
COMPOUND
SAS NO.:

SDG No.: V245
Lab Sample ID: 15230-016

Lab File ID: $\quad$ G7551

Date Received: 07/03/97

Date Analyzed: 07/15/97

Dilution Factor: $\quad 1.0$

Soil Aliquot Volume:

(uL)

CONCENTRATION UNITS:

(ug/L or $\mathrm{ug} / \mathrm{Kg}$ ) UG/KG 
1 INORGANIC ANALYSES DATA SHEET
EPA SAMPLE NO.

\section{SSCEPVI70}

¿ 1 : Q QUANTERRA_MO

ode: ITMO

Case No.:SW846

> (soil/water): WATER

(low/med): LOW

ids :

0.0
Contract: 625.01 SAS NO.:

SDG No.: : V245

Lab Sample ID: P15230-016

Date Received: 07/03/97

Concentration Units (ug/L or $\mathrm{mg} / \mathrm{kg}$ dry weight): UG/L_

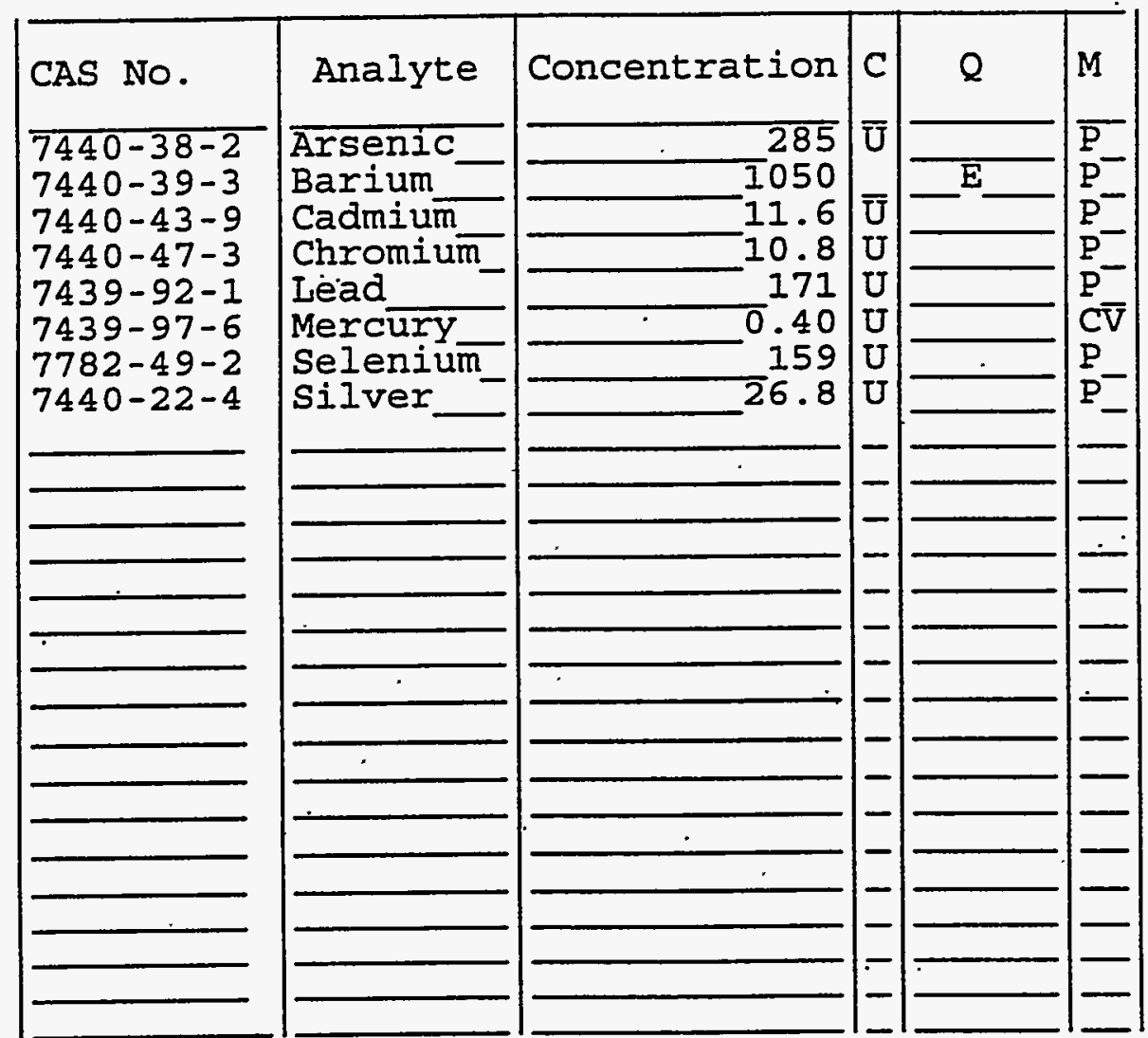

iefore:

After:
Clarity Before:

Clarity After:
Texture:

Artifacts : 
Lab Name: Quanterra, MO

Lab Code: ITMO Case No:

Matrix : (soil/water) SOIL

Sample wt/vol: $(\mathrm{g} / \mathrm{ml})$

G

Level: (low/med)

LOW

․ㅡㄴ Moisture: not dec.

Compound
Contract: 625.01

SAS NO.:

SDG No.: V245

Lab Sample ID: 15230-006

Lab File ID:

Date Sampled: $\quad 07-01-97$

Date Analyzed: 07-12-97

Dilution Factor: 1.0
CONCENTRATION UNITS:

(ug/L or $u g / \mathrm{kg}$ ) UG/KG

LBH $(1)$

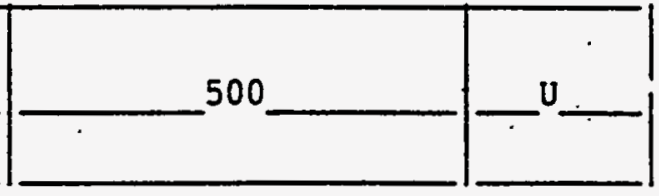

$\mathbf{U}$ : Concentration of analyte is less than the value given.

$\mathrm{X}$ : Pattern reasonably matches gasoline

Y: Pattern appears to be multi-peaks, but does not match gasoline

Z: Concentration is primarily from a single peak

(1): Low Boiling Hydrocarbon ( $\mathrm{LBH}$ ) is quantitated as if it is Gasoline. 
10

HBH ANALYSIS DATA SHEET
EPA SAMPLE NO. SSCEP-VI-076 jab Name: QUANTERRA MO Iab Code: ITMO Case No.:

SOIL

1 :rix : (soil/water) $20.0 \quad(\mathrm{~g} / \mathrm{ml})$ G

s. iple wt/vol: LO: Level: $\quad$ (low/med)

b Moisture: not dec. dec.

$E$ :raction:(SepF/Cont/Sonc/Shak) SHAK 3 : Cleanup: $(Y / \mathrm{N})+\mathrm{N} \quad \mathrm{pH}:$

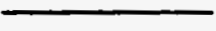

Contract:

SAS NO.:
625.01

SDG No.: V245
Lab Sample ID: $15230-006$

Lab File ID:

Date' Sampled:

$07-16-97$

Date Extracted: $\quad 07-10-97$

Date Analyzed:

Dilution Factor: . 10
ZAS NO.

Compound

DIESEI

KEROSENE

WASTE OIL

$\checkmark$ : Concentration of analyte is less than the value given.
CONCENTRATION UNITS:

$(\mathrm{mg} / \mathrm{L}$ or $\mathrm{mg} / \mathrm{Kg}$ ) $\mathrm{mg} / \mathrm{kq}$
Q

$+{ }^{25}+{ }^{25}+-$


Lab·Name: QUẠNTERRA : MO

Lab code: ITMO

Case No.: V23001

Matrix: (soil/water) soIL

Sample wt/ỵol:

$5.00(\mathrm{~g} / \mathrm{mL}) \mathrm{G}$

Lével: (low/med) LOW

\% Moisture: not dec.

GC Column: RTX-502.2 ID: 0.530 (mm)

Soil Extract Volume:

(uL)
Contract: $625-01$

SAS NO.:
SSCEPVI076

SDG NO.: V245

Lab Sample ID: 15230-006

Lab File ID: $\quad$ G7483

Date Received: 07/03/97

Date Analyzed: 07/12/97

Dilution Factor: $\quad 1.0$

Soil Aliquot Volume:

(uL)

CAS NO.

COMPOUND

CONCENTRATION UNITS:

(ug/L or.ug/Kg) UG/KG

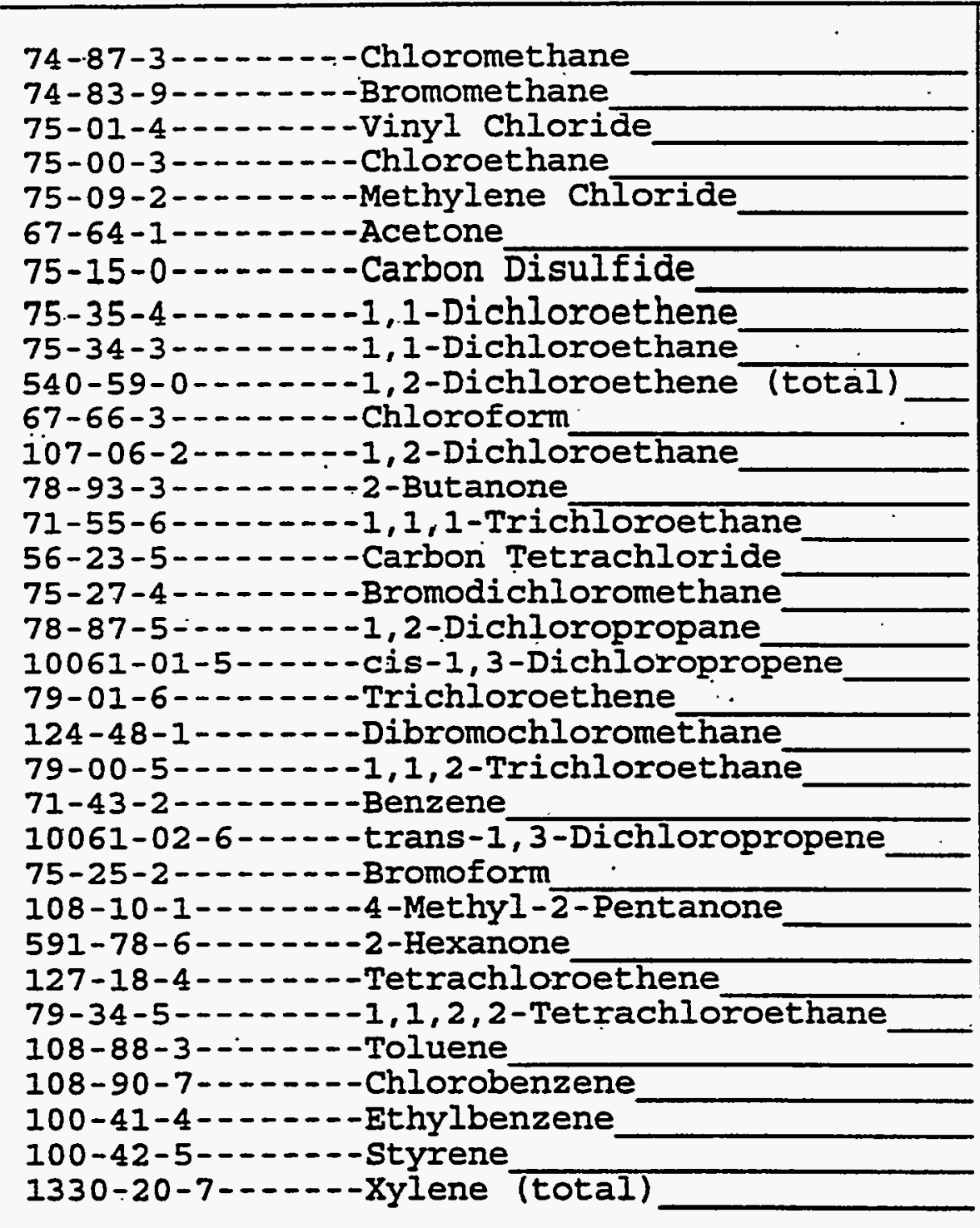


EPA SAMPLE NO.

\section{SSCEPVI076}

a $\mathrm{e}$ : QUANTERRA_MO

Jde: ITMO_ Cáse No.:SW846_

< (soil/water) : WATER

(low/med) : LOW
Contract: 625.01 SAS NO.: SDG NO.: V245 Lab Sample ID: P15230-006 Date Received: 07/03/97

i-s:

$$
-0.0
$$

Concentration Units (ug/L or $\mathrm{mg} / \mathrm{kg}$ dry weight): UG/L_

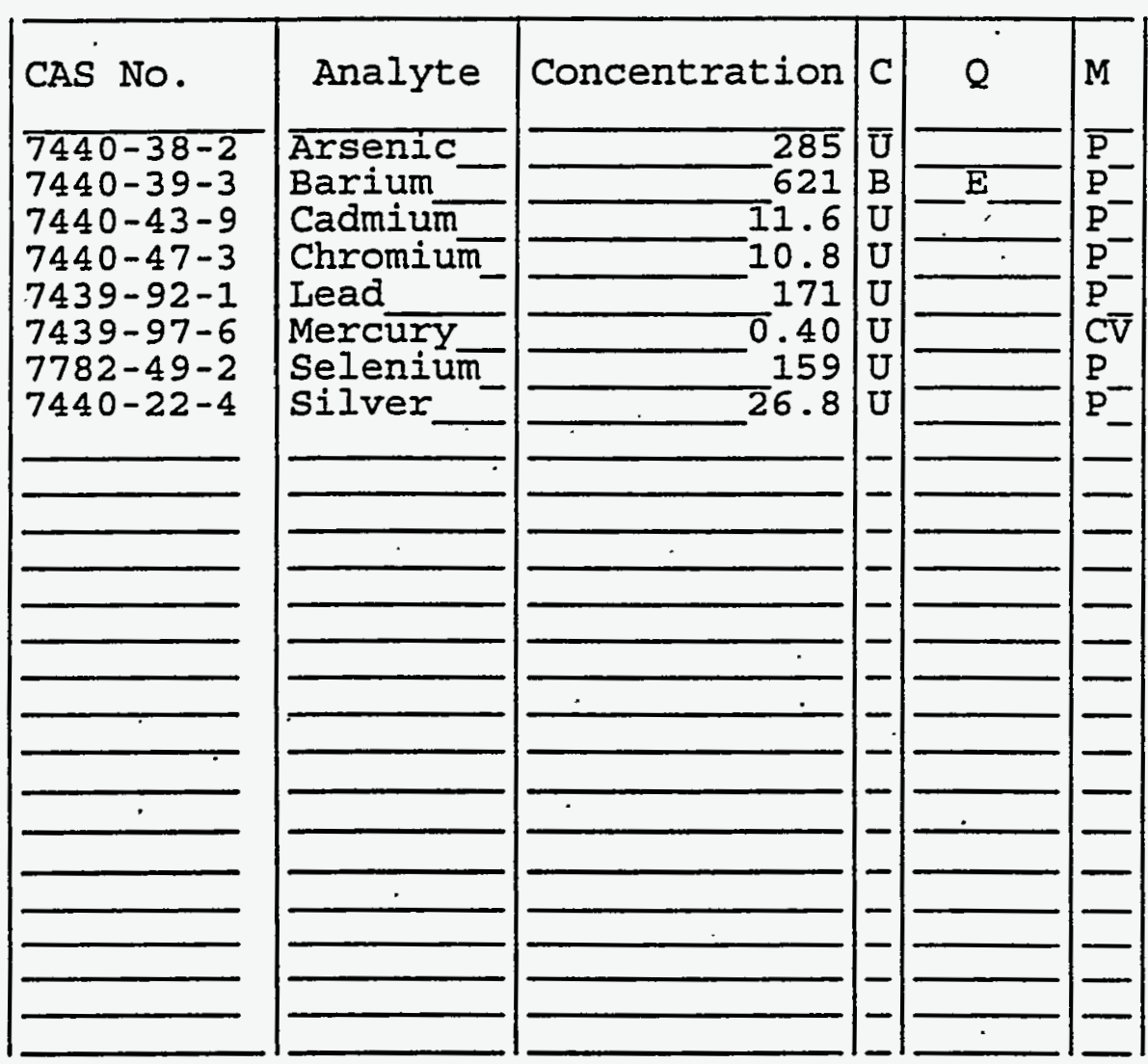

efore:

After:
Clarity Before:

Clarity After:
Texture:

Artifacts: 
Lab Name: QUANTERRA MO

Lab Code: ITMO

Case No.: V41401

Matrix: (soil/water) soIL

Sample wt/vol:

$5.00(\mathrm{~g} / \mathrm{mL}) \mathrm{G}$

Level: $\quad$ (low/med) ' LOW

$\div$ Moisture: not dec.

GC Column: RTX-502.2 ID: 0.530 (mm)

Soil Extract Volume:

(uL)
Contract : 625-01

SDG No. : V258

Lab Sample ID: 15414-002

Lab File ID: $\quad$ FI150

Date Received: $07 / 25 / 97$

Date Analyzed: 08/06/97

Dilution Factor: 1.0

Soil Aliquot Volume:

(uL CONCENTRATION UNITS :

CAS NO.

COMPOUND

(ug/L or ug/Kg) UG/KG

$Q$

74-87-3-..---Chloromethane

74-83-9--------Bromomethane

75-01-4--.-.--vinyl Chloride

75-00-3-.-.--Chloroethane

75-09-2--.----Methylene Chloride

67-64-1-...-.-Acetone

75-15-0-1-----Carbon Disulfide

75-35-4------1, 1-Dichloroethene.

75-34-3----.-1, 1-Dichloroethane

540-59-0-...-1,2-Dichloroethene (total)

67-66-3--..--Chloroform

107-06-2--.--1,2-Dichloroethane

78-93-3-...---2-Butanone

71-55-6--..--1, 1, 1-Trichloroethane

56-23-5-------Carbon Tetrachloride

75-27-4-------Bromodichloromethane

78-87-5------1,2-Dichloropropane

10061-01-5-----cis-1,3-Dichloropropene

79-01-6--.---Trichloroethene

124-48-1------Dibromochloromethane

79-00-5--..-1,1,2-Trichloroethane

$71-43-2-\ldots-\cdots-$ Benzene

10061-02-6-----trans-1,3-Dichloropropene

75-25-2---.---Bromoform

108-10-1-..---4-Methyl-2-Pentanone

591-78-6-..---2-Hexanone

127-18-4------ Tetrachloroethene

79-34-5--..--1,1,2,2-Tetrachloroethane

108-88-3-.----Toluene

108-90-7--.----Chlorobenzene

100-41-4------Ethylbenzene

100-42-5-------styrene

1330-20-7--.---xylene (total) 
Lub Name:- Quanterra, MO

I-b code: ITMO Case No.:

1.trix : (soil/water) SOIL

snmple wt/vol:

1.0 $(g / m l)$

I_vel: (low/med) LOW

; Moisture: not dec.

CAS NO

Compound

CONCENTRATION UNITS:

( $u g / \mathrm{I}$ or $\mathrm{ug} / \mathrm{Kg}$ ) UG/KG
Contract: "625.01

SAS NO.:

Lab Sample ID: 15230-00i

Lab File ID:

Date Sampled: $\quad 07-01-97$

Date Analyzed: $07-12-97$

Dilution Factor: 1.0

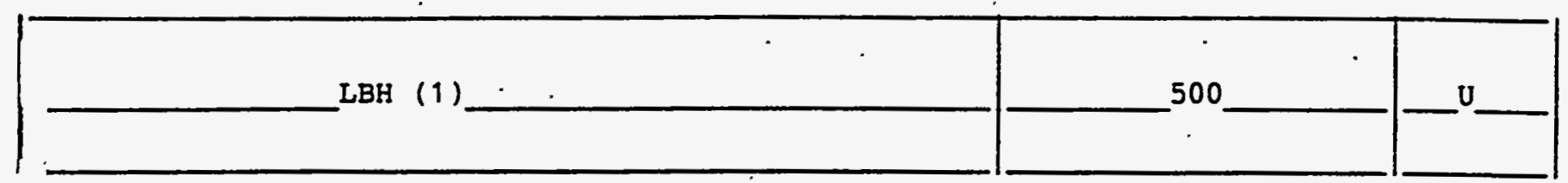

$\mathrm{U}$ : Concentration of analyte is less than the value given.

$\mathrm{x}$ : Pattern reasonably matches gasoline

: Pattern appears to be multi-peaks, but does not match gasoline

: Concentration is primarily from a single peak

(1): Low Boiling Hydrocarbon (IBH) is quantitated as if it is Gasoline. 
- . 10 .

HBH ANALYSIS DATA SHEET
EPA SAMPLE NO. SSCEP-VI-079
Lab Name: OUANTERRA MO

Lab code: ITMO Case No.:

Matrix : (soil/water)

SOIL

Sample we/vol:

$20.1 \cdot(\mathrm{g} / \mathrm{ml})$

G

Level: (low/med)

LOW

9. Moisture: not dec.

dec.

Extraction: (SepF/Cont/Sonc/Shak)

SHAK

GPC Cleanup: $(Y / \mathrm{N})$

CAS NO.

Compound

CONCENTRATION UNITS

$(\mathrm{mg} / \mathrm{L}$ OI $\mathrm{mg} / \mathrm{Kg}$ ) $\mathrm{mg} / \mathrm{kg}$

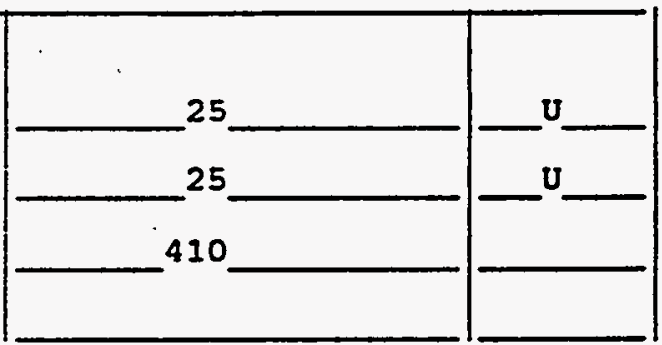

$\mathrm{U}$ : Concentration of analyte is less than the value given. 
b. Name: QUANTERRA MO :

t Code: ITMO

Case No.: V23001

trix: (soil/water) soII
n lle wt/vol:
$5.00(\mathrm{~g} / \mathrm{mL}) \mathrm{G}$

ral: (low/med) LOW

Muisture: not dec.

:olumn: RTX-502.2. ID: $0.530(\mathrm{~mm})$

il Extract Volume:

(UL)
Contract: $625-01$

SAS NO.:
SSCEPVI079

SDG NO.: V245

Lab Sample ID: 15230-001

Lab File ID: $\quad$ G7478

Date Received: 07/03/97

Date Analyzed: 07/12/97

Dilution Factor: $\quad 1.0$

Soil Aliquot Volume:

(uL)

CAS N NO.

COMPOUND

(ug/L or ug/Kg) UG/KG

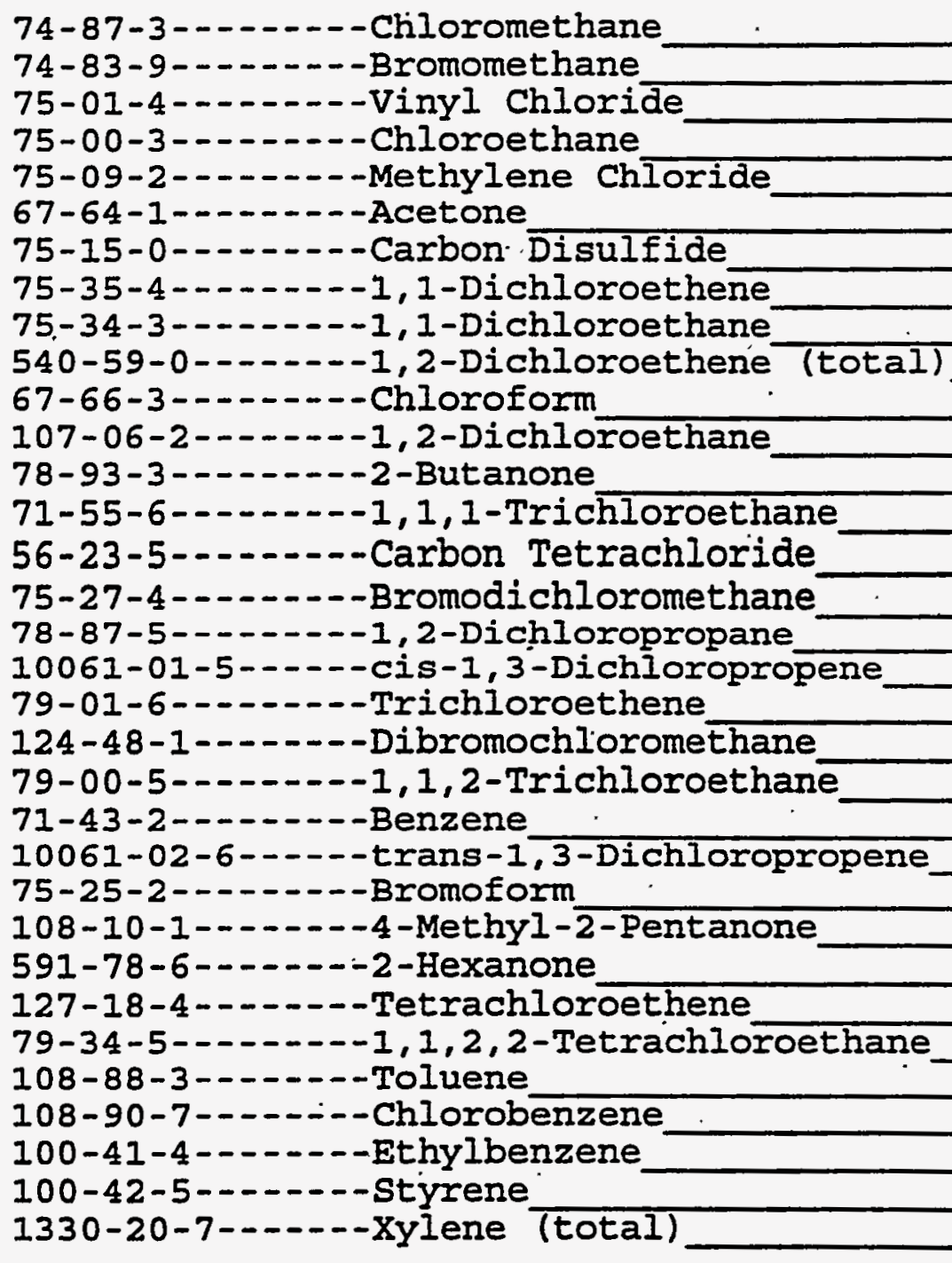

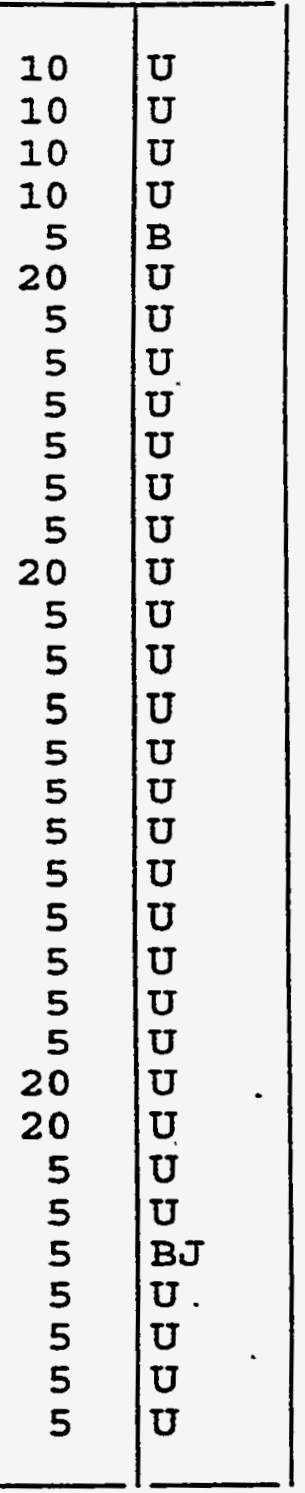


1

INORGANIC ANALYSES DATA SHEET

, Name: QUANTERRA_MO

ว Code: ITMO__ Case No. :SẈ846 :rix (soil/water): WATER

rel (low/med) : LOW

jolids :

$-0.0$
Contract: 625.01

SAS NO.:

SDG No.: V245

Lab Sample ID: P15230-001

Date Received: 07/03/97

SSCEPVI079

Concentration Units (ug/L or $\mathrm{mg} / \mathrm{kg}$ dry weight) : UG/L_

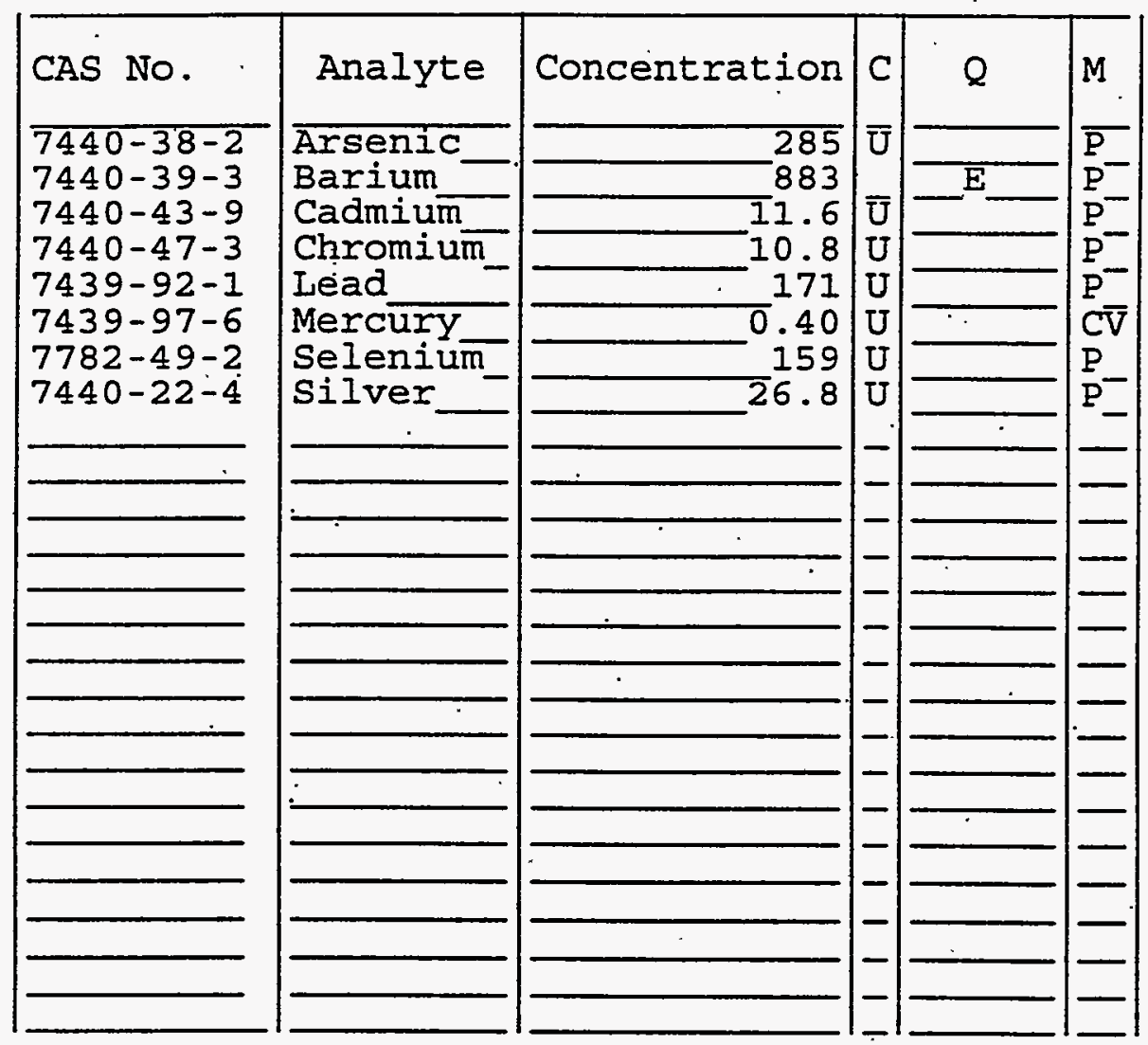

Lor Before:

Clarity Before:

Texture:

lor After:

Clarity After:

Artifacts : nments : 
uab Name: Quanterra, MO

- ab Code: ITMO Case No.: matrix : (soil/water) SOIL

rample wt/vol: $1.0(\mathrm{~g} / \mathrm{ml}) \mathrm{G}$

-evel: (low/med) LOW

Q Moisture: not dec.

CAS NO.

Compound

LBH (1)

1

U: Concentration of analyte is less than the value given.

x: Pattern reasonably matches gasoline

$l$ : Pattern appears to be multi-peaks, but does not match gasoline

1: Concentration is primarily from a single peak

(1): Low Boiling Hydrocarbon (LBH) is quantitated as if it is Gasoline.
Contract: $\quad 625.01$

SAS NO.:

SDG No.: V245

Lab Sample ID: $15230-002$

Lab File ID:

Date Sampled: $\quad 07-01-97$

Date Analyzed: 07-12-97

Dilution Factor: 1.0
CONCENTRATION UNITS:

( $u g / L$ or $u g / K g)$ UG/KG 500

Q U

$\longrightarrow$


Lab Name: OUANTERRA MO

Contract:

625.01

Lab code: ITMO Case No.:

SAS NO.:

SDG NO.: V245

Matrix : (soil/water)

SOIL

Lab Sample ID:

15230-002

Sample wt/vol:

20.0

$(\mathrm{g} / \mathrm{ml})$

Lab File ID:

Level: (low/med)

LOW

Date Sampled:

$07-16-97$

4. Moiscure: not dec. dec.

Date Extracted:

$07-10-97$

Extraction: (SepF/Cont/Sonc/Shak) ___ SHAK

Date Analyzed:

$07-16 \div 97$

GPC C:esnup: $(Y / N)-\mathrm{N} \quad \mathrm{pH}$ :

Dilution Factor:

1

CAS NO.

Compound

CONCENTRATION UNITS:

(mg/L or $\mathrm{mg} / \mathrm{Kg}$ ) $\mathrm{mg} / \mathrm{kg}$

$\mathbf{Q}$

\begin{tabular}{|c|c|c|}
\hline KEROSENE & \\
\hline WASTE OIL & & \\
\hline
\end{tabular}

$\mathrm{U}$ : Concentration of analyte is less than the value given. 
= Name: QUANTERRA MO :

: Code: ITMO

Case No.: V23001

Erix: (soil/water) SOIL

7 le wt/vol:

$5.00(\mathrm{~g} / \mathrm{mL}) \mathrm{G}$

vel: (low/med) LOW

v-isture: not dec.

lolumn: RTX-502.2 ID: 0.530 (mm)

il Extract Volume:

(uL)
Contract : 625-01

SAS NO.:
SSCEPVI790

SDG No.: V245

Lab Sample ID: 15230-00.2

Irab File ID: $\quad$ G7488

Date Received: 07/03/97.

Date Analyzed: 07/12/97

Dilution Factor: 1.0

- Soil Aliquot Volume:

(uL)

CONCENTRATION UNITS:

CAS NO. COMPOUND

(ug/I or $\mathrm{ug} / \mathrm{Kg}$ ) UG/KG

Q

74-87-3-...--Chloromethane

74-83-9-....--Bromomethane

75-01-4-..---vinyl Chloride

75-00-3-..--.-Chloroethane

75-09-2-.-.---Methylene Chloride

67-64-1-..----Acetone

75-15-0-..---Carbon Disulfide

75-35-4-...-1, 1-Dichloroethene

75-34-3-...-1, 1-Dichloroethane

540-59-0-..--1,2-Dichloróthene (total)

67-66-3-...---Chloroform

107-06-2-..-1, 2-Dichloroethane

78-93-3-2.---2-Butanone

71-55-6-...-1, 1, 1-Trichloroethane

56-23-5.-...--Carbon Tetrachloride

75-27-4-.....-Bromodichloromethane

78-87-5-...--1,2-Dichloropropane

10061-01-5--.--cis-1, 3-Dichloropropene

79-01-6-..---Trichloroethene

124-48-1-..--Dibromochloromethane

79-00-5-...--1,1,2-Trichloroethane

71-43-2-.-----Benzene

10061-02-6--.--trans-1, 3-Dichloropropene

75-25-2--.----Bromoform

108-10-1-...--4-Methyl-2-Pentanone

591-78-6-..--2-Hexanone

127-18-4-..---Tetrachloroethene

79-34-5-..--1, 1, 2, 2-Tetrachloroethane.

108-88-3-.----Toluene

108-90-7--..---Chlorobenzene

100-41-4-.-.-.-Ethylbenzene

100-42-5--.-----Styrene

1330-20-7-..-.-Xylene (total)

\begin{tabular}{r|r}
10 & $U$ \\
10 & $U$ \\
10 & $U$ \\
10 & $U$ \\
6 & $B$ \\
74 & \\
5 & $U$ \\
5 & $U$ \\
5 & $U$ \\
5 & $U$ \\
5 & $U$ \\
5 & $U$ \\
20 & $U$ \\
5 & $U$ \\
5 & $U$ \\
5 & $U$ \\
5 & $U$ \\
5 & $U$ \\
5 & $U$ \\
5 & $U$ \\
5 & $U$ \\
5 & $U$ \\
5 & $U$ \\
5 & $U$ \\
20 & $U$ \\
20 & $U$ \\
5 & $U$ \\
5 & $U$ \\
5 & $U$ \\
5 & $U$ \\
5 & $U$ \\
5 & $U$ \\
5 & $U$ \\
& \\
& \\
\hline
\end{tabular}


ab Name: QUANTERRA_MO

ab Code: ITMO

Case No. : SW846

atrix (soil/water): WATER

evel (low/med): LOW.

Solids :

$$
-0.0
$$

Contract: 625.01 SAS NO. :

SDG No.: V245

Lab Sample ID: P15230-002

Date Received: 07/03/97
SSCEPVI790

Concentration Units (ug/L or $\mathrm{mg} / \mathrm{kg}$ dry weight): UG/L_

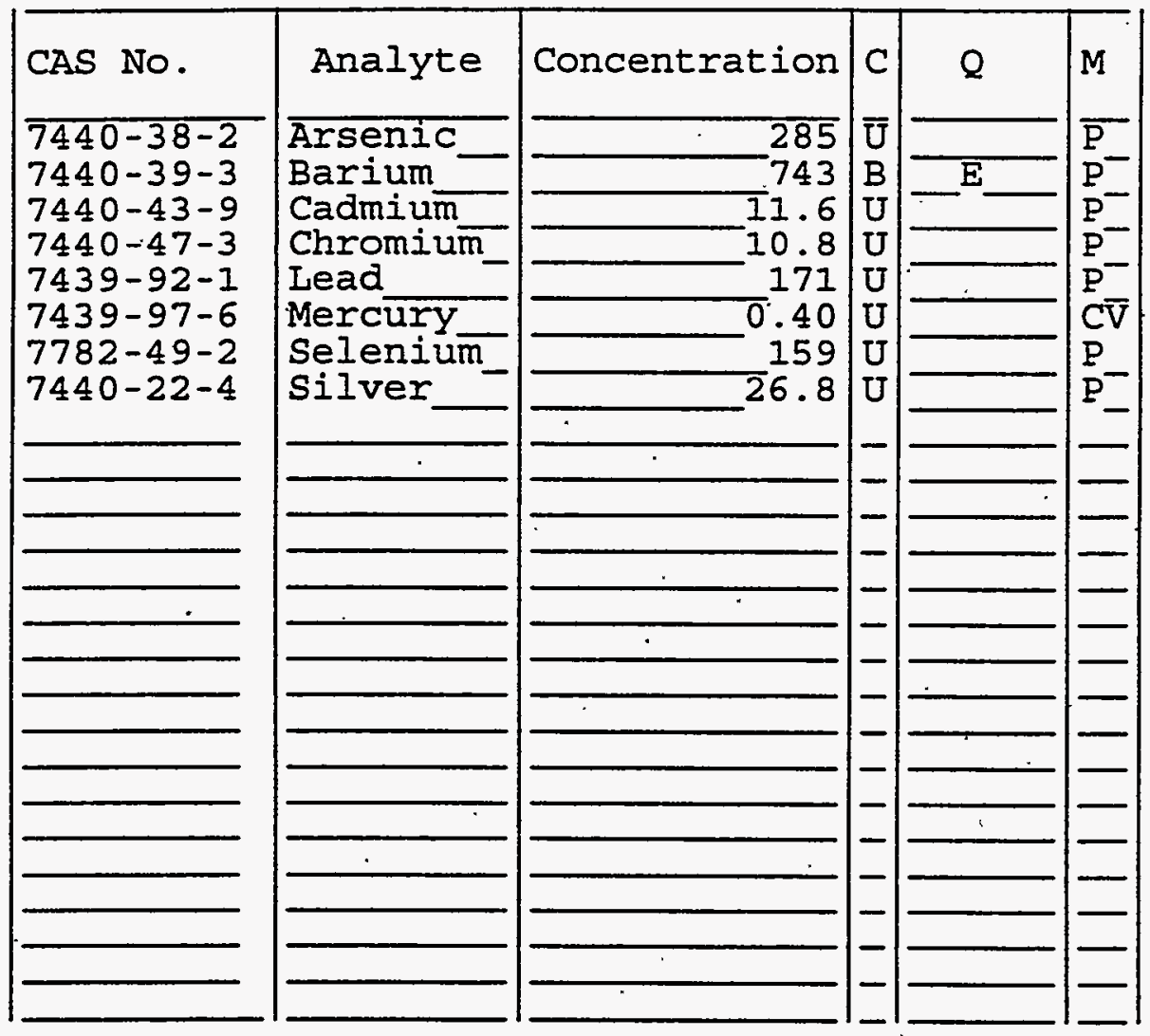

Jlor Before:

Jlor After:
Clarity Before:

Clarity After:
Texture:

Artifacts : omments : 
Lab Name: Quanterra, MO

I b Code: ITMO Case No.:

Matrix : (soil/water) SOIL

5 mple $w t / v o l:$

1.0 $(g / m l) \_G$

Level: (low/med) LOW

s Moisture: not dec.
Contract: 625.01 SAS NO.: SDG NO.: V245

Láb Sample ID: 15230-014

Lab File ID:

Date Sampled: 07-01-97

Date Analyzed: $07-12-97$

Dilution Factor: 1.0

CONCENTRATION UNITS:

CAS NO. Compound

(ug/L or $u g / K g$ ) UG/KG

$Q$

IBH $(1)$

500

U.

U: Concentration of analyte is less than the value given.

Pattern Ieasonably matches gasoline

Pattern appears to be multi-peaks, but does not match gasoline

Concentration is primarily from a single peak

(1): Low Boiling Hydrocarbon ( $\mathrm{LBH}$ ) is quantitated as if it is Gasoline. 


$$
\because \because
$$

1D

\section{HBH ANALYSIS DATA SHEET}

EPA SAMPLE NO. SSCEP-VI-87
Lab Name: OUANTERRA MO

Lab Code: ITMO Case No.: SOIL

Matrix : (soil/water)

Sample st/vol: $20.2(\mathrm{~g} / \mathrm{mi})$ G Level: (low/med) LOW

\$ Moisture: not dec. dec:

Extraction: (SepF/Cont/Sonc/shak) SHAK GPC cleanup: $(Y / N)+\mathrm{N} \quad \mathrm{pH}:$

Contract: SAS NO.: SDG No.: $\quad$ V245

Lab Sample ID: $15230-014$ Lab File ID:

Date Sampled:

$07-16-97$

Date Extracted: $07-10-97$

Date Analyzed: $07-17-97$ Dilution sactor: 1

CONCENTRATION UNITS:

CAS NO. Compound (mg/L or $\mathrm{mg} / \mathrm{Kg}$ ) $\mathrm{mg} / \mathrm{kg}$ $\mathbf{q}$

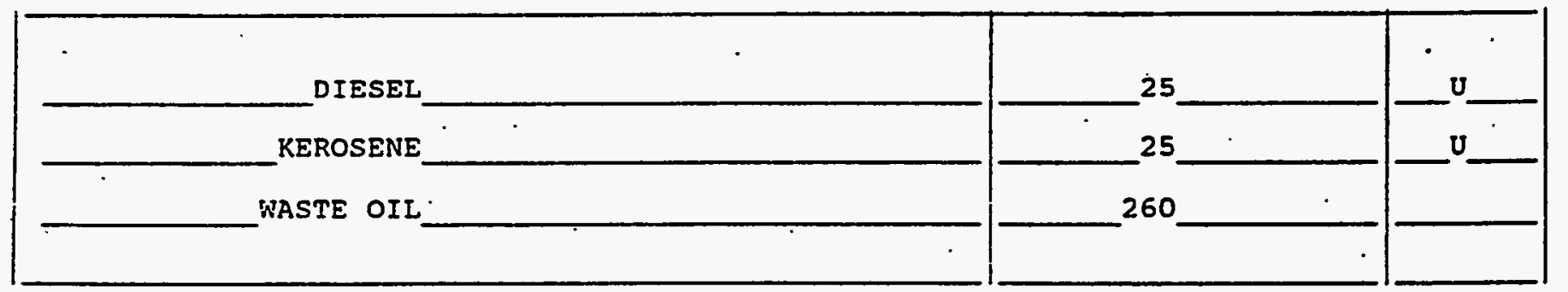

$U$ : Concentration of analyte is less than the value given. 
2Name : QUANTERRA MO :

i) Code : ITMO

six: (soil/water) soIL

n le wt/vol:

$5.00(\mathrm{~g} / \mathrm{mL}) \mathrm{G}$

pol: (low/med) LOW

ruisture: not dec.

olumn: RTX-502.2 ID: $0.530(\mathrm{~mm})$

il Extract Volume:

(UI)
Contract: $625-01$

SAS NO.:

SDG No.: V245

Lab Sample ID: 15230-014

Lab File ID: $\quad$ G7517

Date Received: 07/03/97

Date Analyzed: 07/13/97

Dilution Factor: $\quad 1.0$

Soil Aliquot Volume:

(UI)

CONCENTRATION UNITS:

CAS NO. COMPOUND (ug/L or ug/Kg) UG/KG

Q

74-87-3-..---Chiloromethane

74-83-9-...--Bromomethane

75-01-4-...--Vinyl Chloride

75-00-3-....-.-Chloroethane

75-09-2--...--Methylene ChIoride

67-64-1-...--Acetone

75-15-0-...-.-Carbon Disulfide

75-35-4---.--1,1-Dichloroethene

75-34-3-...--1,1-Dichloroethane

540-59-0-..--1,2-Dichloroethene (total)

67-66-3-....--Chloroform

107-06-2--.---1,2-Dichloroethane

78-93-3-...--2-Butanone

71-55-6-...--1, 1, 1-Trichloroethane

56-23-5-..----Carbon Tetrachloride

75-27-4-_..-- Bromodichloromethane

78-87-5-..----1,2-Dichloropropane

10061-01-5-.-.-cis-1; 3-Dichloropropene

79-01-6-..---Trichloroethene

124-48-1-...-.-Dibromochloromethane

79-00-5-2.--1,1,2-Trichloroethane

71-43-2-..--.--Benzene

10061-02-6-----trans-1,3-Dichloropropene

75-25-2--.---Bromoform

108-10-1-..-.-4-Methyl-2-Pentanone

591-78-6-2.---2-Hexanone

127-18-4-...--Tetrachloroethene

79-34-5-...--1,1,2, 2-Tetrachloroethane

108-88-3-..--.-Toluene

108-90-7--...-Chlorobenzene

100-41-4-..-.--Ethylbenzene

100-42-5--.----styrene

1330-20-7--..--Xylene (total)

\begin{tabular}{r|l}
10 & $U$ \\
10 & $U$ \\
10 & $U$ \\
10 & $U$ \\
5 & $U$ \\
20 & $U$ \\
5 & $U$ \\
5 & $U$ \\
5 & $U$ \\
5 & $U$ \\
5 & $U$ \\
5 & $U$ \\
20 & $U$ \\
5 & $U$ \\
5 & $U$ \\
5 & $U$ \\
5 & $U$ \\
5 & $U$ \\
5 & $U$ \\
5 & $U$ \\
5 & $U$ \\
5 & $U$ \\
5 & $U$ \\
5 & $U$ \\
20 & $U$ \\
20 & $U$ \\
5 & $U$ \\
5 & $U$ \\
5 & $U$ \\
5 & $U$ \\
5 & $U$ \\
5 & $U$ \\
5 & $U$ \\
\end{tabular}




\section{U.S. EPA - CLP}

\section{- INORGANIC ANALÝSES DATA SHEET}

EPA SAMPLE NO.

b Name: QUANTERRA MO

b Code: ITMO Case No.: SW846
Contract: 625.01 SAS NO.: SDG No.: V245 trix (soil/water) : WATER Lab Sample ID: P15230-014 vel (low/med): . LOW

\section{SSCEPVI 87}

Solids : $-0.0$

Concentration Units (ug/L or $\mathrm{mg} / \mathrm{kg}$ dry weight): UG/L_

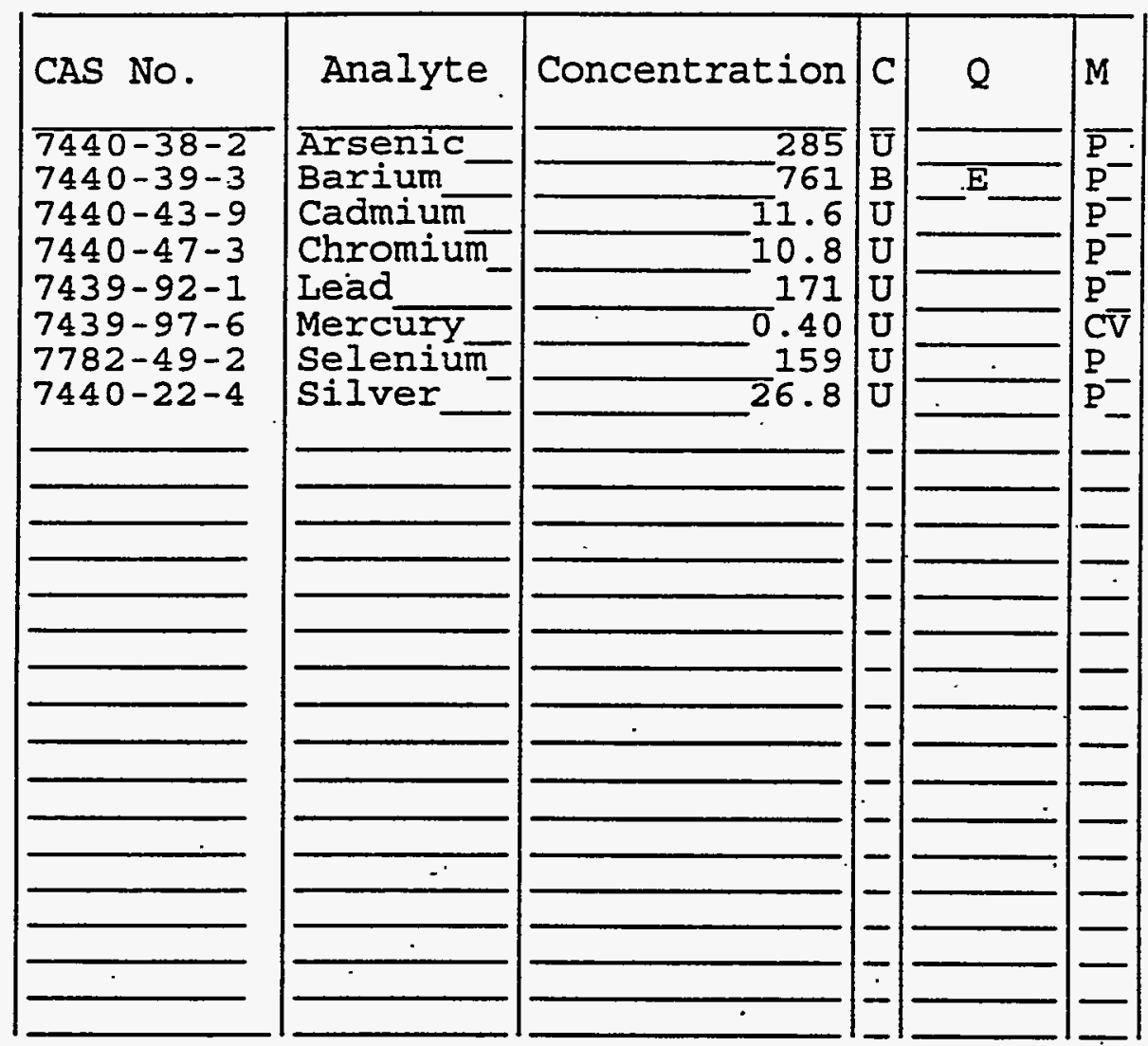

lor Before:

lor After:
Clarity Before:

Clarity After:
Date Received: 07/03/97

mments : 
EPA SAMPLE NO. SSCEP-VI-090
I b Name: Quanterra, MO

I. 'b Code: ITMO Case No.:

Ltrix : (soil/water) SOIL

sample wt/vol: $1.0 \quad(\mathrm{~g} / \mathrm{ml}) \mathrm{G}$

I vel: . (low/med) LOW

Moisture: not dec.
Contract: 625.01

SAS NO.:

SDG No. : V245

Lab Sample ID: 15230-011

Lab File ID:

Date Sampled: $07-01-97$

Date Analyzed: $07-12-97$

Dilution Factor: 1.0

\section{CONCENTRATION UNITS:}

(ug/L or $\mathrm{ug} / \mathrm{Kg}$ ) UG/KG

Q

500

$\mathrm{U}$

$\square 00 \_-1$

$\mathrm{u}$ : Concentration of analyte is less than the value given.

$\mathrm{x}$ : Pattern reasonably matches gasoline

Pattern appears to be multi-peaks, but does not match gasoline

Concentration is primarily from a single peak

(1): Low Boiling Hydrocarbon (LBH) is quantitated as if it is Gasoline. 
Lab Name: OUANTERRA MO

contract:

625.01

Lab Code: ITMO Case No.:

SAS NO.:

SDG NO.: $\mathrm{V} 245$

Matrix : (soil/water)

SOIL

Lab Sample ID:

$15230-011$

sample wt/vol:

20.0

$(\mathrm{g} / \mathrm{ml})$

Lab File ID:

Level: (low/med)

LOW

Date Sampled:

$07-16-97$

: Moisture: not dec. dec.

Date Extracted:

$07-10-97$

Extraction: (SepF/Cont/Sonc/Shak)

SHAK

Date Analyzed:

$07-17-97$

GPC cleanup: $(\mathrm{Y} / \mathrm{H}) \mathrm{N}$

Dilution Factor:

1

CONCENTRATION UNITS:

CAS NO. Compound

$(\mathrm{mg} / \mathrm{L}$ or $\mathrm{mg} / \mathrm{kg})$

$\mathbf{Q}$

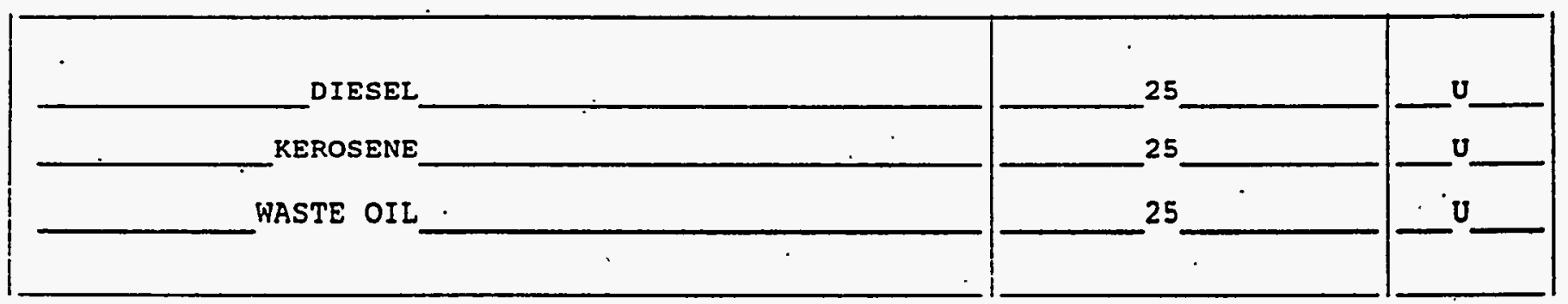

$U$ : Concentration of analyte is less than the value given.

FORIS I HBH 
b-Name: QUANTERRA MO :

t) Code: ITMO

Case No.: V23001

trix: (soil/water) soIL

mie $w t / v o l$ :

$5.00(\mathrm{~g} / \mathrm{mL}) \mathrm{G}$

vel: (low/med) LOW

Vi-isture: not dec.

olumn: RTX-502.2 ID: 0.530 (mm)

il Extract Volume:

(uL)
Contract: $625-01$

SAS NO.:
SSCEPVI090

SDG No.: V245

Lab Sample ID: 15230-011

Lab File ID: ' G7514

Date Received: 07/03/97

Date Analyzed: 07/13/97

Dilution Factor: $\quad 1.0$

Soil Aliquot Volume:

(uI)

CONCENTRATION UNITS:

CAS NO.

COMPOUND

(ug/L or ug/Kg) UG/KG

\begin{tabular}{|c|}
\hline 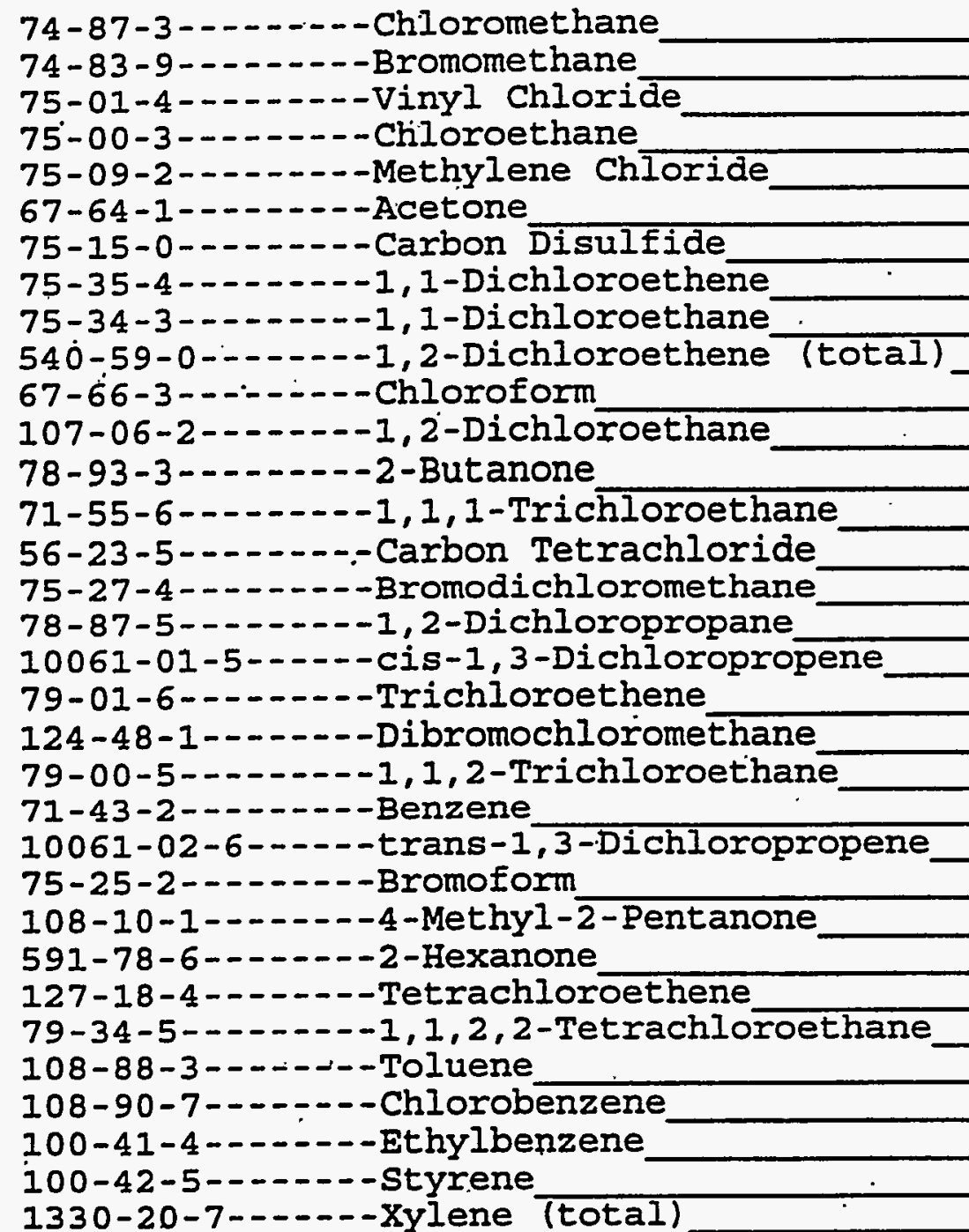 \\
\hline
\end{tabular}


EPA SAMPLE NO.

ab Name: QUANTERRA_MO

ab Code: ITMO

Case No.:SW846_

atrix (soil/water): WATER

evel (low/med): LOW
Contract: 625.01

SAS NO.:

\section{SSCEPVI090}

SDG No.: V245

Lab Sample ID: P15230-011

Date Received: 07/03/97

Solids :

$$
-0.0
$$

Concentration Units (ug/L or $\mathrm{mg} / \mathrm{kg}$ dry weight): UG/I_

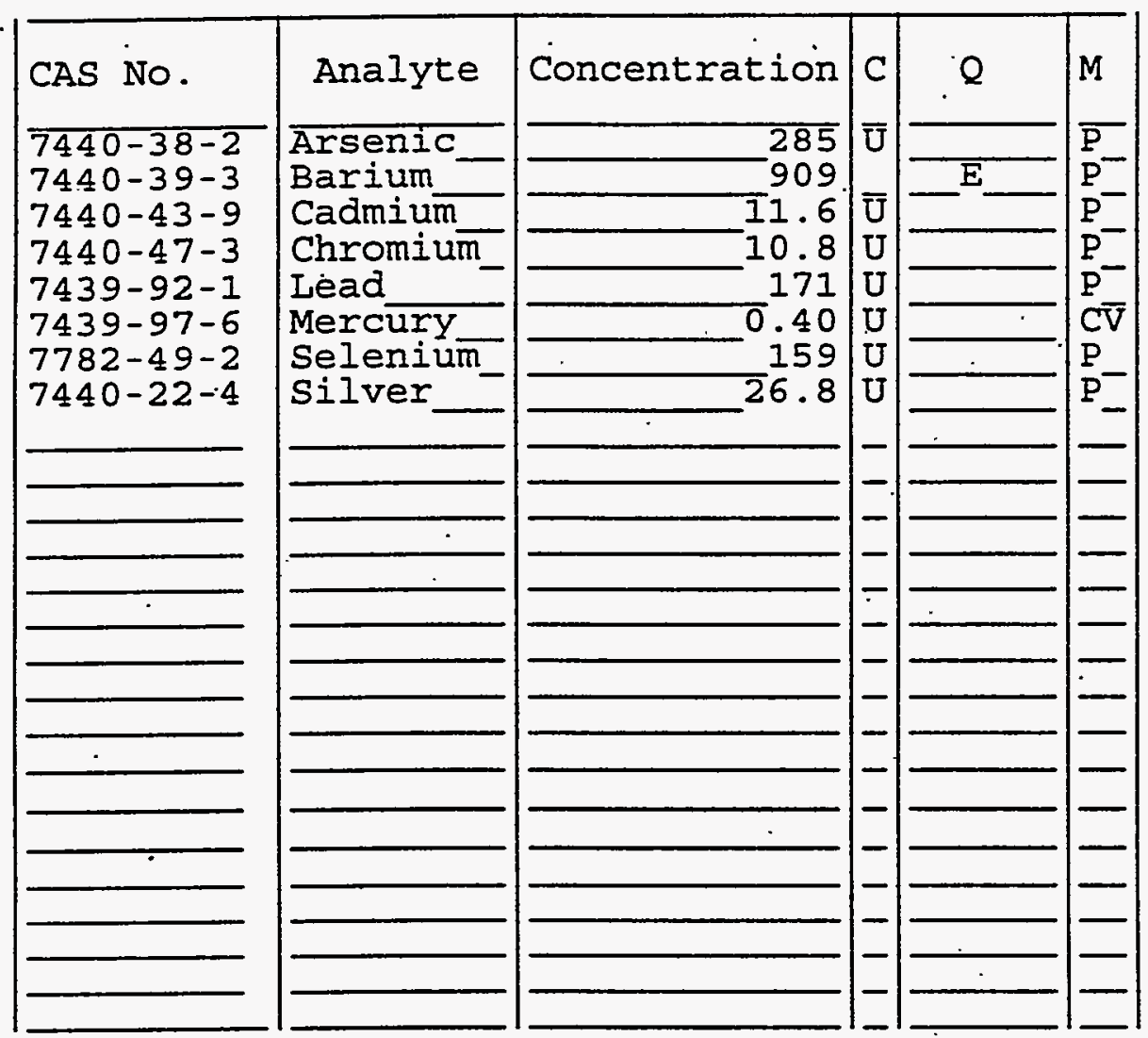

Jlor Before:

Clarity Before:

Texture:

olor After:

Clarity After:

Artifacts : Jmments : 
ah Name: QUANTERRA MO

a Code: ITMO Case No.: V41401 SAS No.:
Contract : $625-01$

SSCEPV2090
F rix: (soil/water) soIL

ample wt/vol:

E el: (low/med) LOW

Moisture: not dec.

C Column: RTX-502.2 ID: 0.530 (mm)

I I Extract Volume:

(uL)
Lab Sample ID: 15414-003

Lab File ID: F1151

Date Received: 07/25/97

Date Analyzed: 08/06/97

Dilution Factor: $\quad 1.0$

Soil Aliquot Volume:

(uL)

CAS NO.

COMPOUND

CONCENTRATION UNITS:

(ug/L or $\mathrm{ug} / \mathrm{Kg}) \mathrm{UG} / \mathrm{KG}$

\begin{tabular}{|c|}
\hline 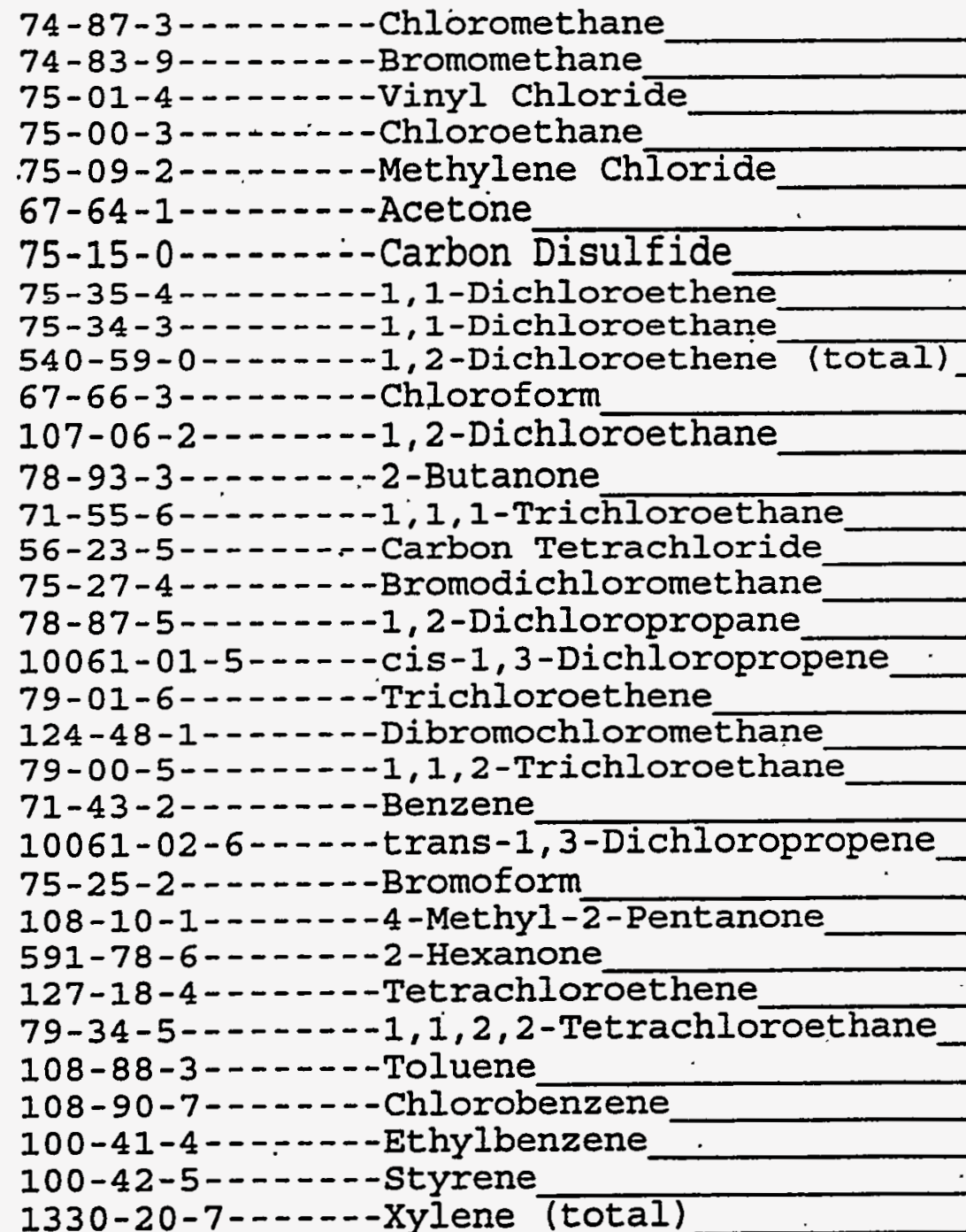 \\
\hline
\end{tabular}


Lab Name: Quanterra,MO

Lab Code: ITMO Case No.:

Matrix : (soil/water) SOIL

Sample wt/vol:

1.0 $(\mathrm{g} / \mathrm{ml}$

Level: (low/med) LOW

$\therefore$ Moisture: not dec.

CAS NO. Compound LBH (1)
Contract: 625.01

SAS NO.:

SDG No.: V245

Lab Sample ID: $15230-010$

Lab File ID:

Date Sampled:

$07-01-97$

Date Analyzed: $\quad 07-12-97$

Dilution Factor: 1.0
CONCENTRATION UNITS:

(ug/L or ug/Kg) UG/KG

Q

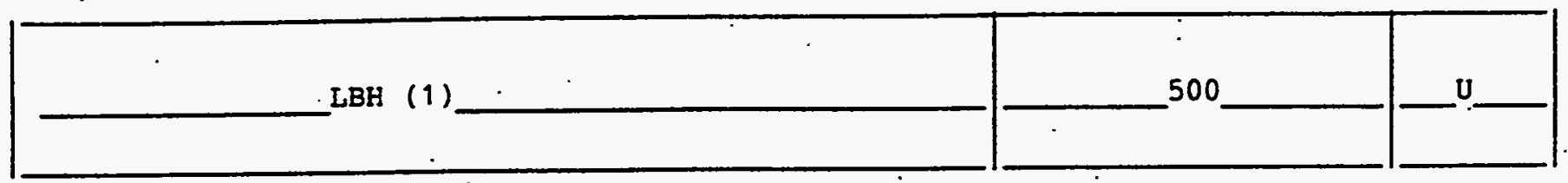

$\mathrm{U}$ : Concentration of analyte is less than the value given.

$\mathrm{X}$ : Pattern reasonably matches gasoline

Y: Pattern appears to be multi-peaks, but does not match gasoline

$\mathrm{z}$ : Concentration is primarily from a single peak

(1): Low Boiling Hydrocarbon (IBH) is quantitated as if it is Gasoline. 


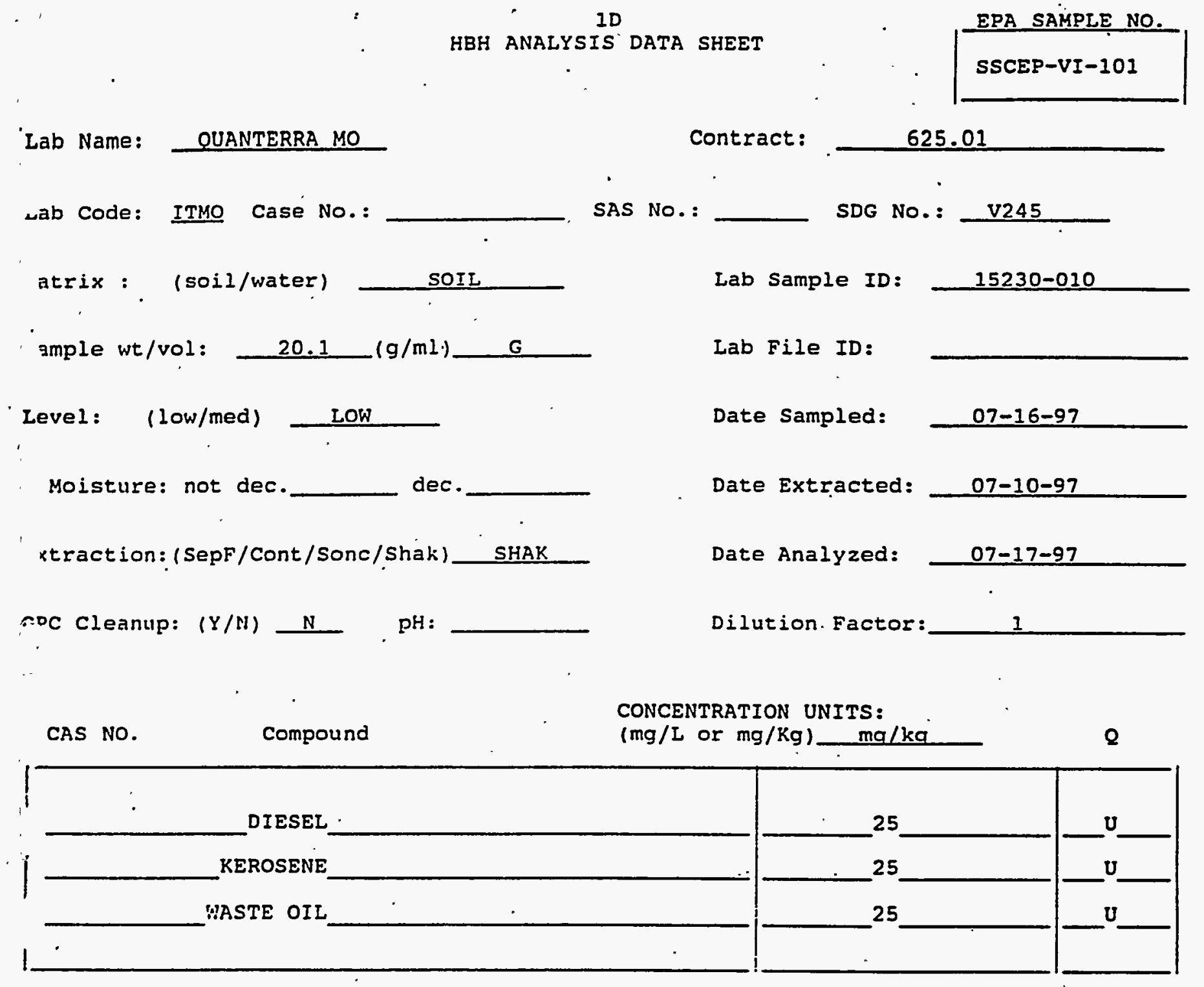

I: Concentration of analyte is less than the value given. 
Lab Name: QUANTERRA MO

Lab Code: ITMO

Case No.: V2300I

Matrix: (soil/water) SOIL

Sample wt/vol:

$5.00(\mathrm{~g} / \mathrm{mL}) \mathrm{G}^{\circ}$

Level: (low/med) LOW

: Moisture: not dec.

GC Column: RTX-502.2. ID: 0.530 (mm)

Soil Extract Volume:

(uI)
Contract: $625-01$

SAS NO.:

SDG No.: V245
SSCEPVI101

Lab Sample ID: 15230-010

Lab File ID: G7513

Date Received: 07/03/97

Date Analyzed: 07/13/97

Dilution Factor: $\quad 1.0$

Soil Aliquot Volume:

(uI)

CAS NO.

COMPOUND

CONCENTRATION UNITS:

. (ug/L or $\mathrm{ug} / \mathrm{Kg}$ ) UG/KG

Q

\begin{tabular}{|c|}
\hline 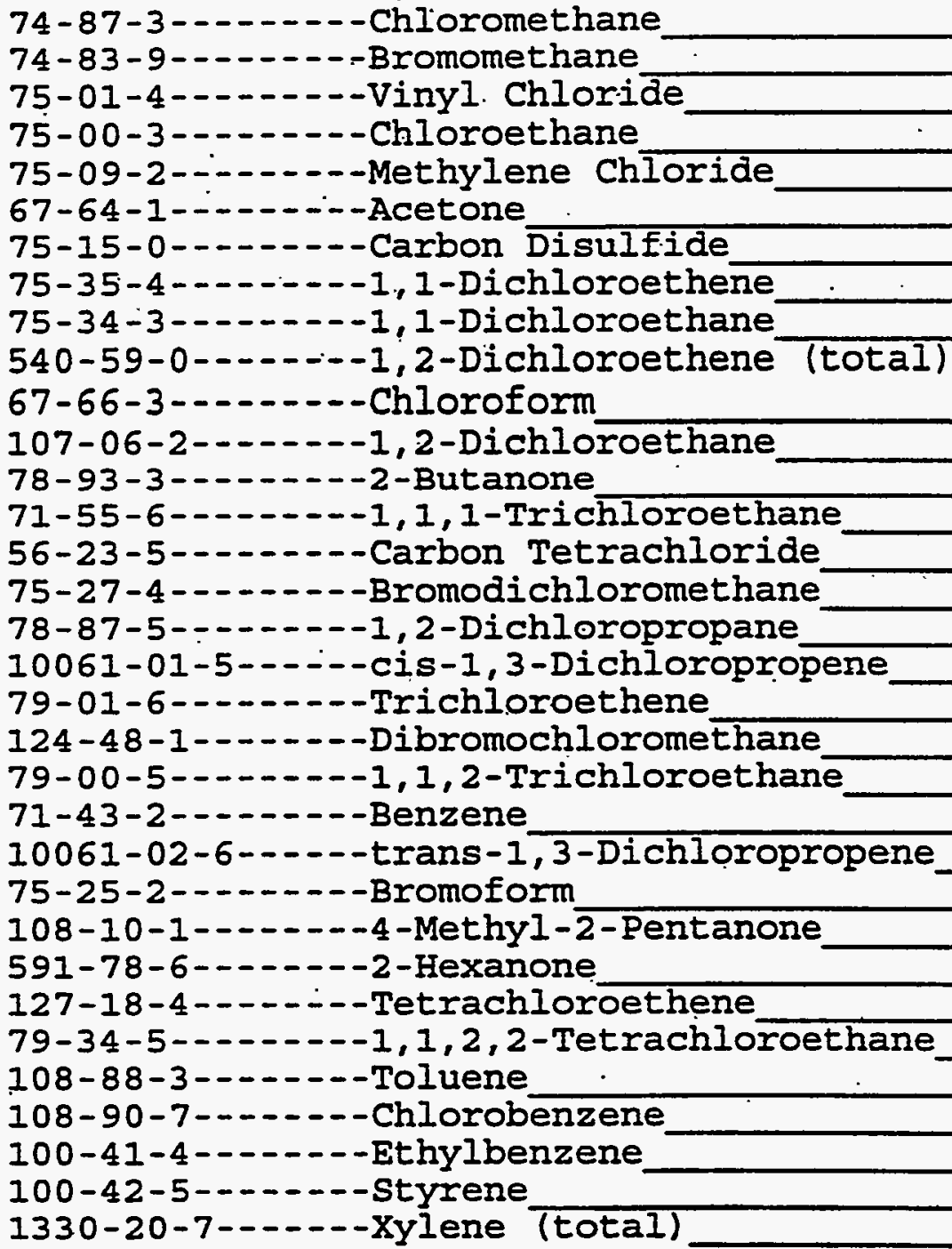 \\
\hline
\end{tabular}




\section{U.S. EPA - CLP}

1

INORGANIC ANALYSES DATA SHEET
EPA SAMPLE NO.

\section{SSCEPVII01}

'c le: QUANTERRA_MO

:ode: ITMO__ Case No.:SW846

$\dot{x}$ (soil/water): WATER

(low/med) : L LOW
Contract: 625.01

SAS NO.:
SDG No.: V245

Lab Sample ID: P15230-010

Date Received: 07/03/97

ins :

0.0

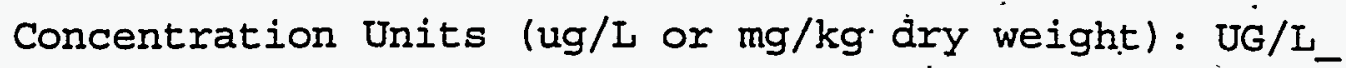

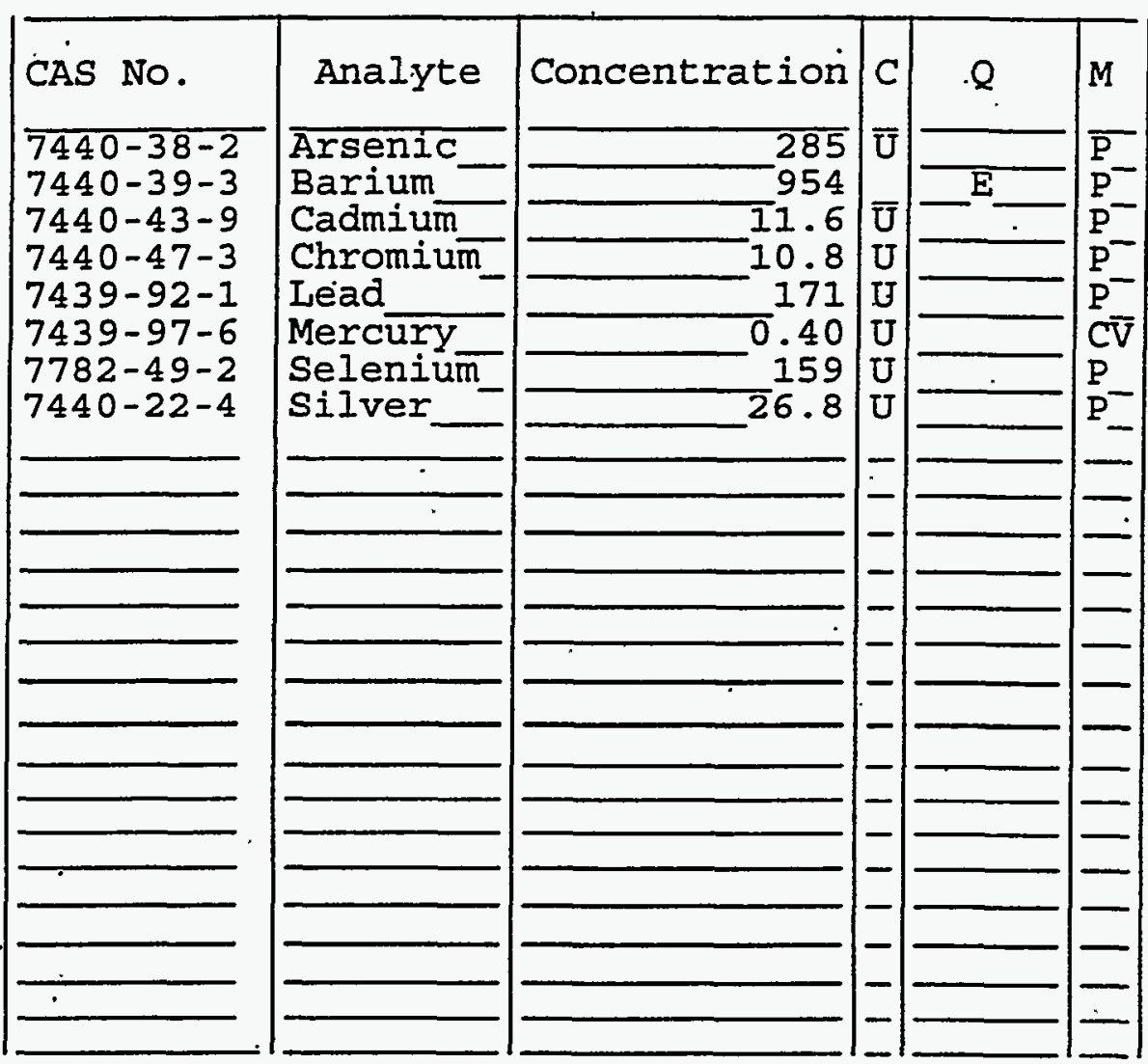

iefore:

After:
Clarity Before:

Clarity After:
Texture:

Artifacts :

\section{r_-s :}


10

\section{LBH ANALYSIS DATA SHEET}

EPA SAMPLE NO. SSCEP-VI-108
Lab Name: Duanterra, Mo

Lab Code: ITMO Case No.:

Matrix : (soil/water) SOIL

Sample wt/vol: $1.0 \quad(\mathrm{~g} / \mathrm{ml}) \mathrm{G}$

Level: $\cdot($ low/med $)$ LOW

\% Moisture: not dec.

CAS NO.

Compound
Contract: $\quad 625.01$

SAS NO.:

SDG No.: V245

Lab Sample ID: 15230-004

Lab File ID:

Date Sampled: 07-01-97

Date Analyzed: $\quad 07-12-97$

Dilution Factor: 1.0
CONCENTRATION UNITS:

(ug/I or $u g / \mathrm{Kg})$ UG/KG _. Q
LBH (1)

1)

500

$\mathbf{U}$

$\mathrm{U}:$ - Concentration of analyte is less than the value given.

$\mathrm{x}$ : Pattern reasonably matches gasoline

Y: Pattern appears to be multi-peaks, but does not match gasoline

Z: Concentration is primarily from a single peak.

(1): Low Boiling Hydrocarbon (LBH) is quantitated as if it is Gasoline. 
Lab Name: QUANTERRA: MO

Lab Code: ITMO

Case No.: V2300I

Matrix: (soil/water) SOII

Sample wt/vol: $\quad 5.00(\mathrm{~g} / \mathrm{mL})$ G

Level: (low/med) LOW

\% Moisture: not dec.

GC Column: RTX-502.2 ID: 0.530 (mm)

Soil Extract Volume:

(uI)
Contract: 625-01

SAS NO.:

SSCEPVII08

Lab Sample ID: 15230-004

Lab File ID: .G7481

Date Received: 07/03/97

Date Analyzed: $07 / 12 \% 97$

Dilution Factor: $\quad 1.0$

Soil Aliquot Volume:

(uL)

(ug/I or ug/Kg) UG/KG • Q $Q$

\begin{tabular}{|c|}
\hline 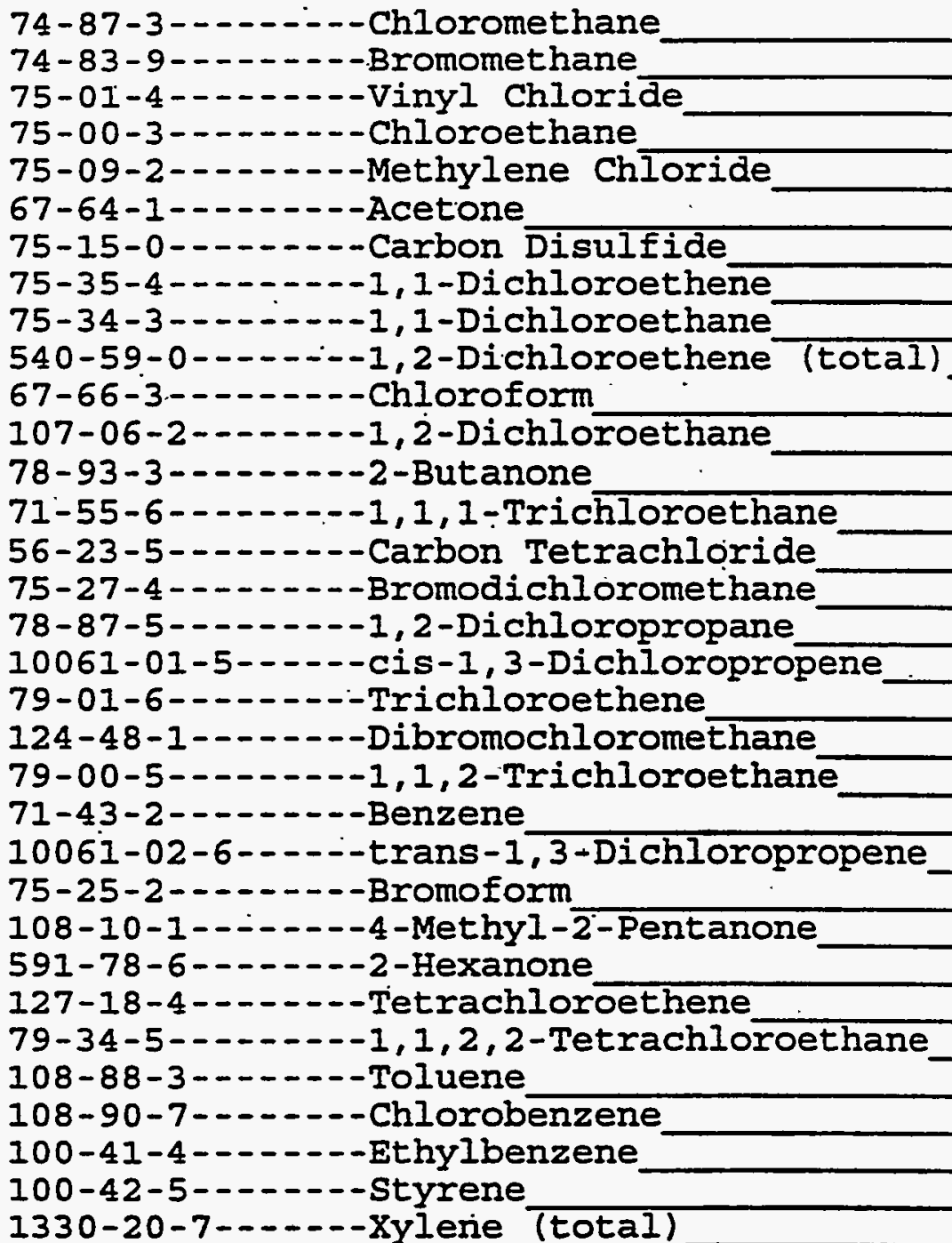 \\
\hline
\end{tabular}

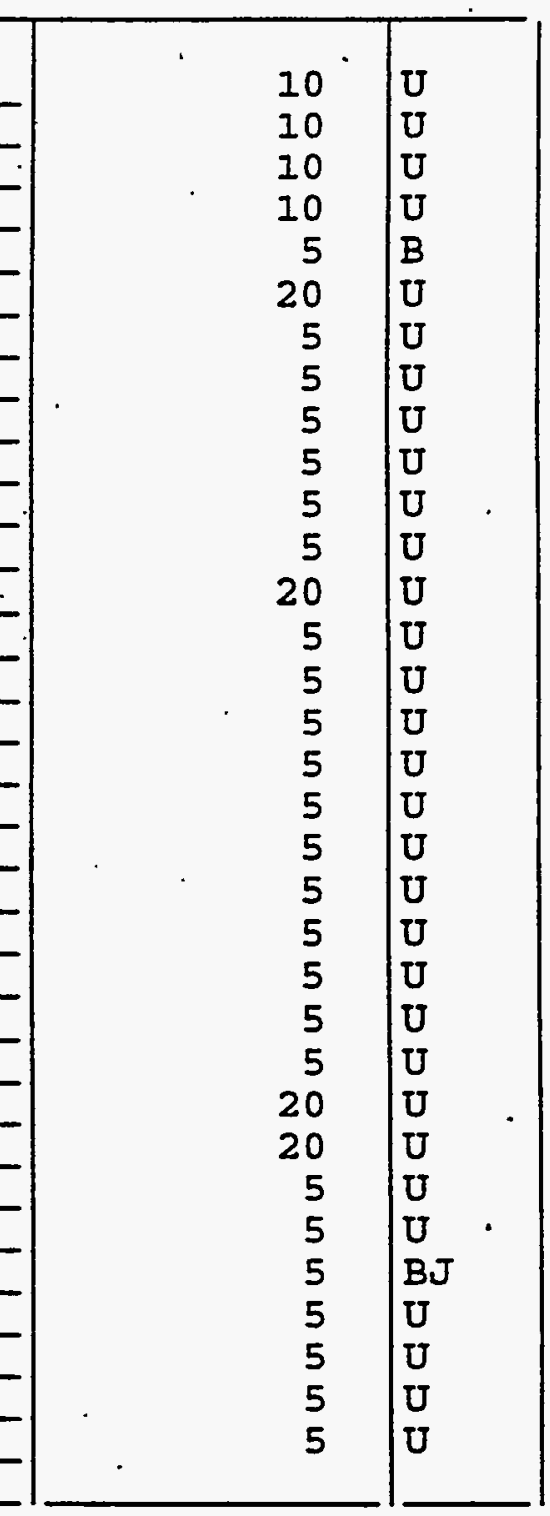


U.S. EPA - CLP

1

INORGANIC ANALYSES DATA SHEET
EPA SAMPLE NO.

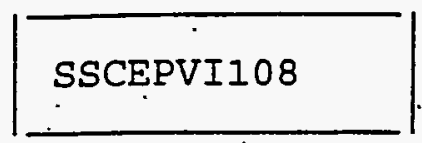

$\frac{1}{c}$ le: QUANTERRA_MO

ore: ITMO_ Case No.:SW846

× (soil/water): WATER

(low/med) : . LOW

is :

0.0
Contract: 625.01

SAS No.

SDG No.: V245

Lab Sample ID: Pi5230-004

Date Received: 07/03/97

Concentration Units (ug/L or $\mathrm{mg} / \mathrm{kg}$ dry weight): UG/L_

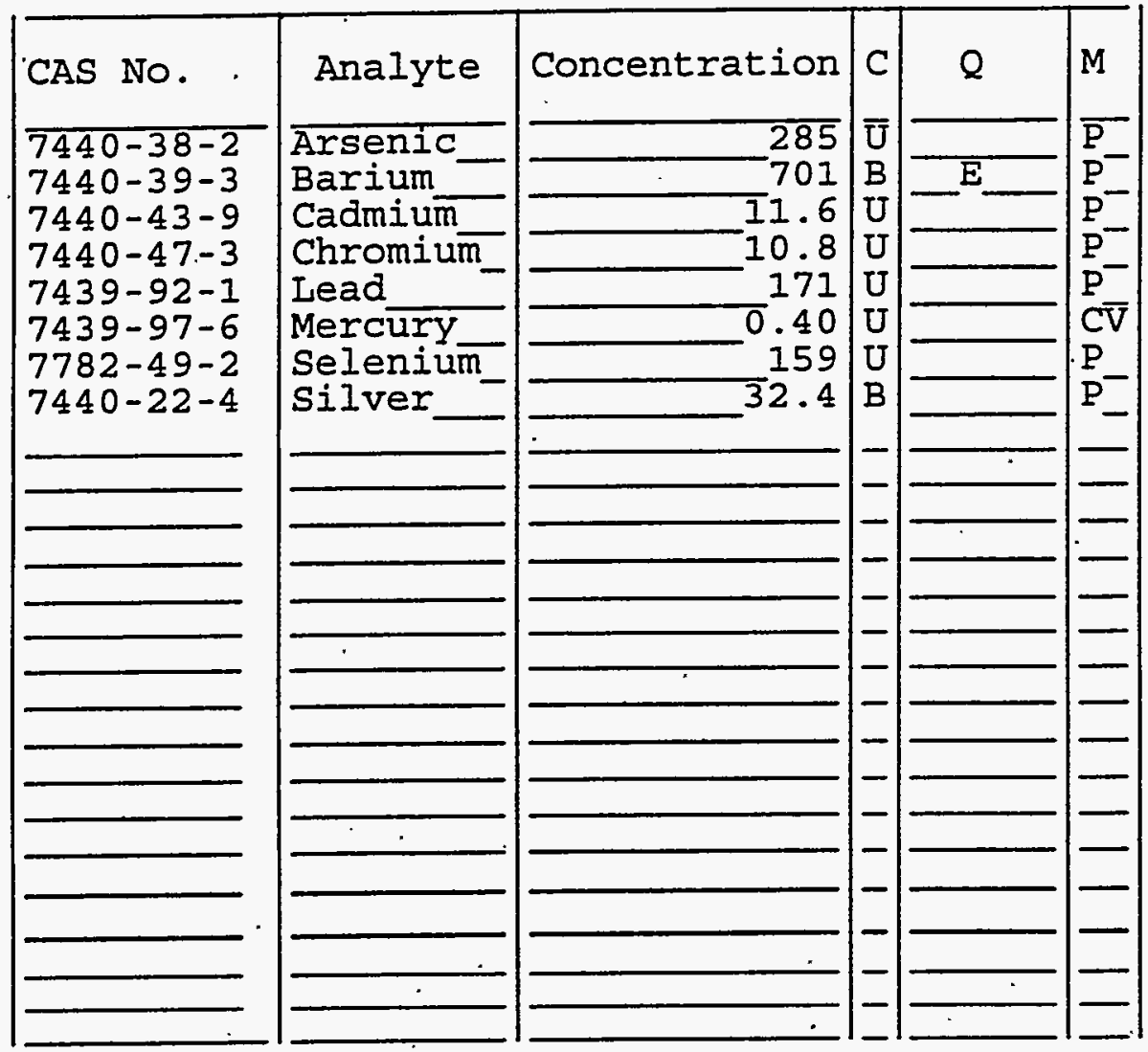

lefore:

After:
Clarity Before:

Clarity After:
Texture:

Artifacts: 
Lab Name: Quanterra, MO

Lab Code: ITMO Case No.:

Matrix : (soil/water) SOIL

Sample wt/vol:

$(\mathrm{g} / \mathrm{ml})-\mathrm{G}$

Level: . (low/med) LOW

: Moisture: not dec.

CAS NO.

Compound

LBH (1)

(1)

.

500 U'

CONCENTRATION UNITS:

$(\mathrm{ug} / \mathrm{L}$ or $\mathrm{ug} / \mathrm{Kg})$ UG/KG

$\mathbf{Q}$

SAS NO.:

SDG No.: V245

Lab Sample ID: 15230-013

Lab File ID:

Date Sampled: 07-01-97

Date Analyzed: $07-12-97$

Dilution Factor: 1.0

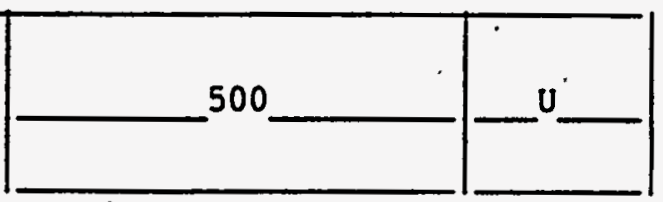

v: Concentration of analyte is less than the value given.

$\mathrm{X}$ : Pattern reasonably matches gasoline

$Y$ : Pattern appears to be multi-peaks, but does not match gasoline

z: Concentration is primarily from a single peak

(1): Low Boiling Hydrocarbon (LBH) is quantitated as if it is Gasoline. 


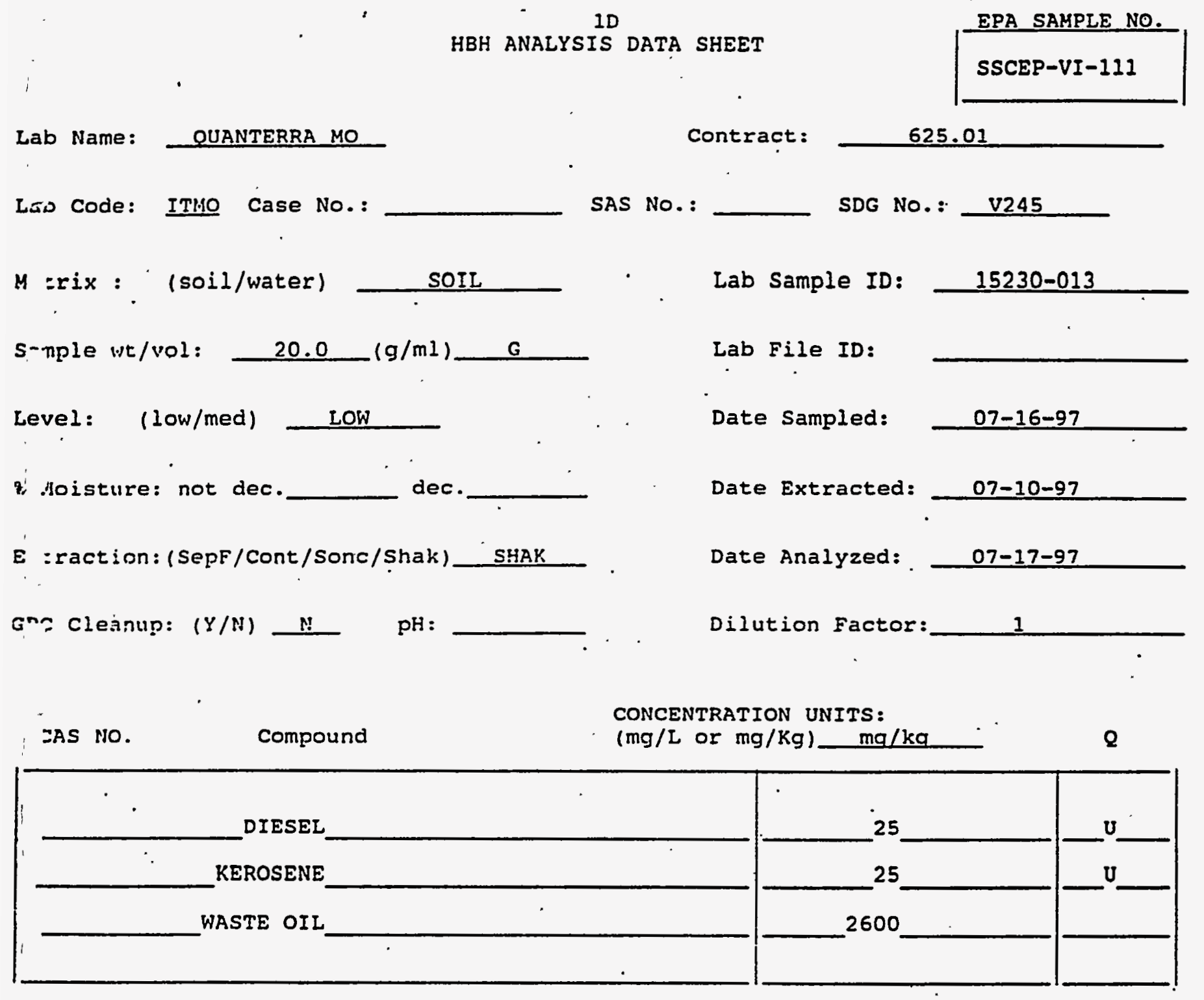

II: Concentration of analyte is less than the value given. 
Iab Name: QUANTERRA MO

Iab Code.: ITMO

Case No.: : V23001

Matrix: (soil/water) SOII

Sample wt/vol: $\quad 5.00 \cdot(\mathrm{g} / \mathrm{mL}) \mathrm{G}$

Level: (low/med) LOW

음 Moisture: not dec.

GC Column: RTX-502.2 ID: $0.530(\mathrm{~mm})$

Soil Extract Volume:

CAS NO.
Contract: $625-01$

SAS NO.:
SDG No.: V245

Lab Sample ID: 15230-013

Irab File ID: $\quad$ G7550

Date Received: 07/03/97

Date Analyzed: 07/15/97

Dilution Factor: $\quad 1.0$

Soil Aliquot Volume:

(uL)

CONCENTRATION UNITS:

(ug/L or $\mathrm{ug} / \mathrm{Kg}$ ) UG/KG

74-87-3-..---Chloromethane

74-83-9--.-----Bromomethane

75-01-4-.-.-.--Vinyl Chloride

75-00-3-..----Chloroethane

75-09-2-..----Methylene Chloride

67-64-1-...-.-.-Acetone

75-15-0-....-Carbon Disulfide

75-35-4-..-- - 1, 1-Dichloroethene

75-34-3-..---1,1-Dichloroethane

540-59-0-..---1,2-Dichloroethene (total)

67-66-3-...-.-.-Chloroform

107-06-2-----1,2-Dichloroethane

78-93-3-..---2--2utanone

71-55-6-...-. 1, 1, 1-Trichloroethane

56-23-5-..--.--Carbon Tetrachloride

75-27-4------Bromodichloromethane

78-87-5--.---1, 2-Dichloropropane

10061-01-5-.-.--cis-1,3-Dichloropropene

79-01-6-....-.-Trichloroethene

124-48-1--.---Dibromochloromethane

79-00-5-.-.--1, 1,2-Trichloroethane

71-43-2-..----Benzene

10061-02-6-...-trans-1,3-Dichloropropene

75-25-2--..---Bromoform

108-10-1-..--4-Methyl-2-Pentanone

591-78-6-...--2-Hexanone

127-18-4-..---Tetrachloroethene

79-34-5-...--1, 1, 2, 2-Tetrachloroethane

108-88-3--.----Toluene

108-90-7-------Chlorobenzene

100-41-4-------Ethylbenzene

100-42-5-------styrene

1330-20-7-.-.--Xylene (total)

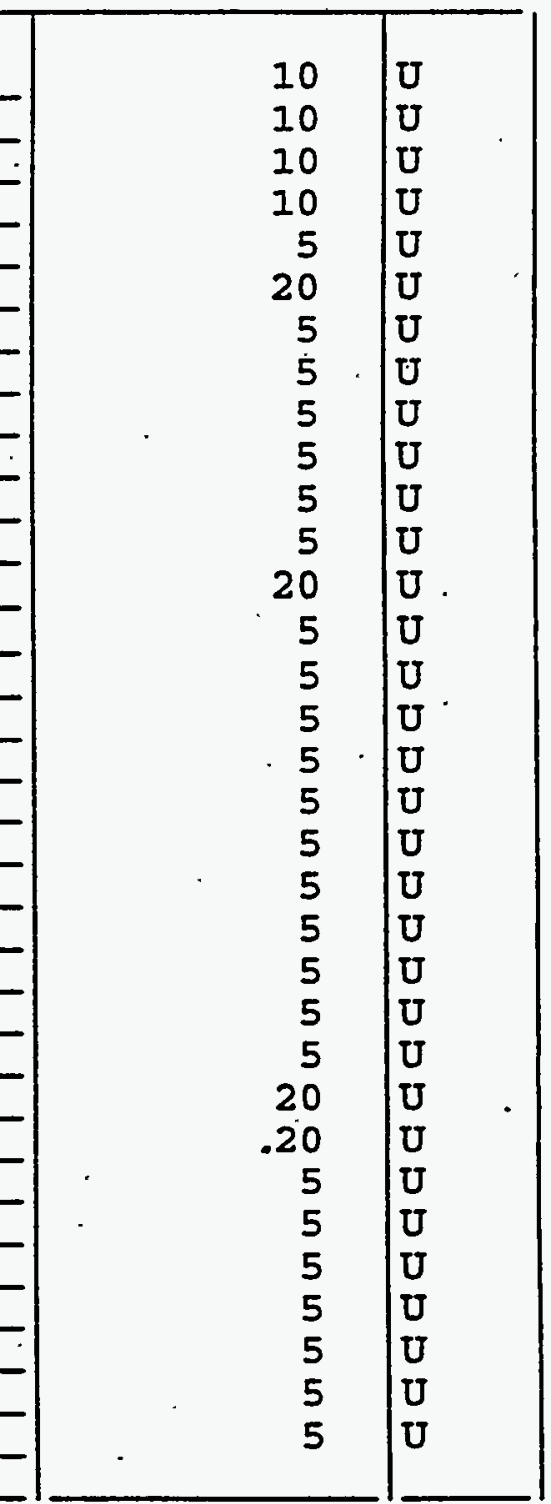




\section{INORGANIC ANALYSES DATA SHEET}

ne: QUANTERRA_MO

ode: ITMO

Contract: 625.01 SAS NO. : EPA SAMPLE NO. SSCEPVIIII (soil/water): WATER Lab Sample ID: P15230-013

(low/med): LOW Date Received: 07/03/97

$$
-0.0
$$

Concentration Units (ug/L or $\mathrm{mg} / \mathrm{kg}$ dry weight): UG/L_

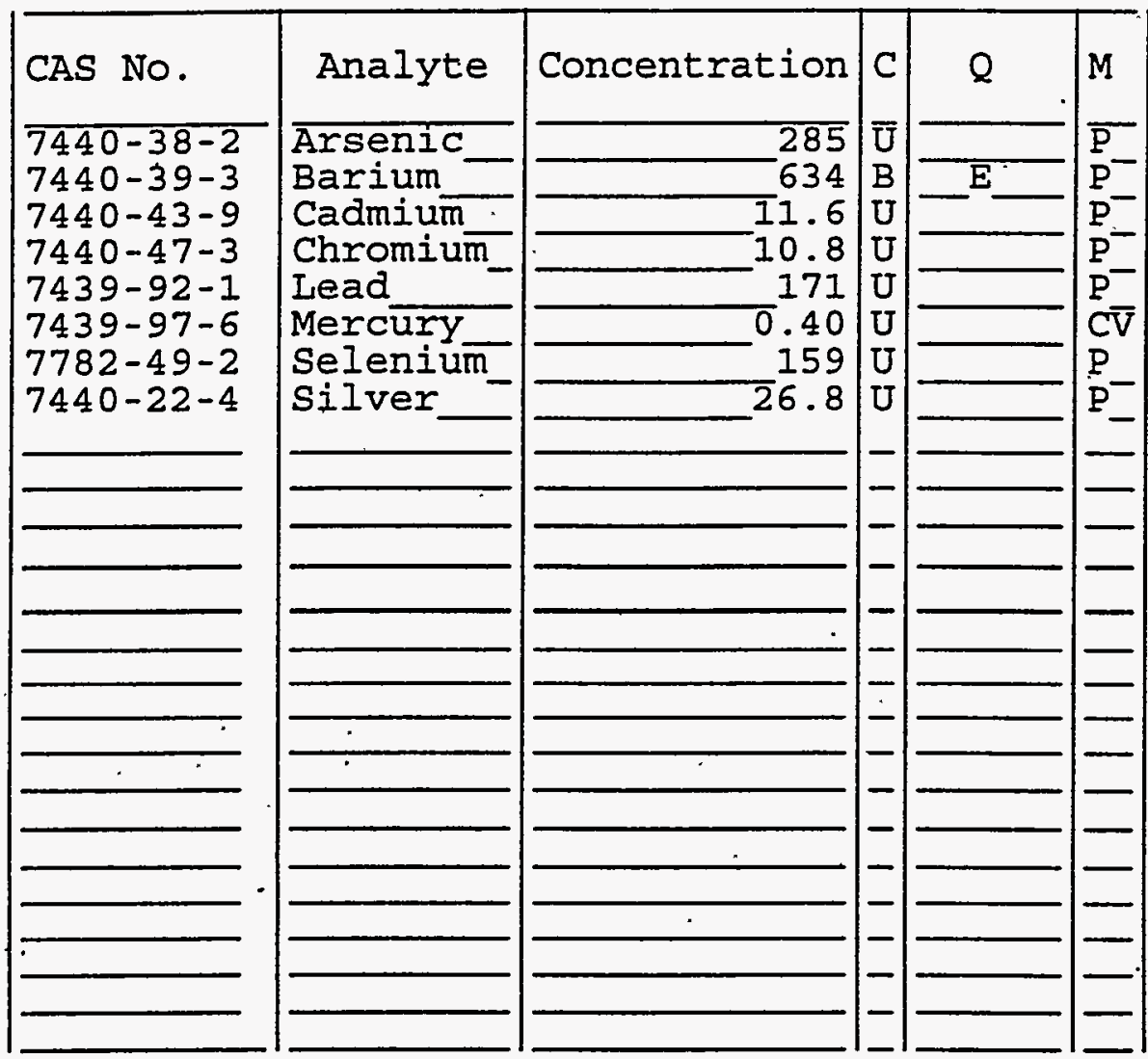

3efore:

After: :s :
Clarity Before:

Clarity After:
Texture:

Artifacts: 
1D

LBH ANALYSIS DATA SHEET
EPA. SAMPLE NO

SSCEP-VI-129
Lab Name: Quanterra,MO

Lab Code: ITMO Case No.:

Matrix : (soil/watèr) SOIL

Sample wt/vol: $1.0(\mathrm{~g} / \mathrm{ml}) \mathrm{G}$

Level: (low/med) LOW

\% Moisture: not dec

CAS NO.
Contract: 625.01

SAS NO.:

SDG No.: V245

Lab Sample ID: 15230-003

Lab File ID:

Date Sampled: 07-01-97.

Date Analyzéd: 07-12-97

Dilution Factor: 1.0

CONCENTRATION UNITS:

( $\mathrm{ug} / \mathrm{L}$ or $\mathrm{ug} / \mathrm{Kg}$ ) UG/KG

Q

LBH (1)

500

U
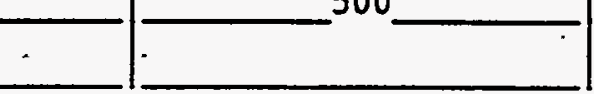

U: Concentration of analyte is less than the value given.

$\mathrm{x}$ : Pattern reasonably matches gasoline

Y: Pattern appears to be multi-peaks, but does not match gasoline

$\mathrm{z}$ : Concentration is primarily from a single peak

(1): Low Boiling Hydrocarbon. (LBH) is quantitated as if it is Gasoline. 


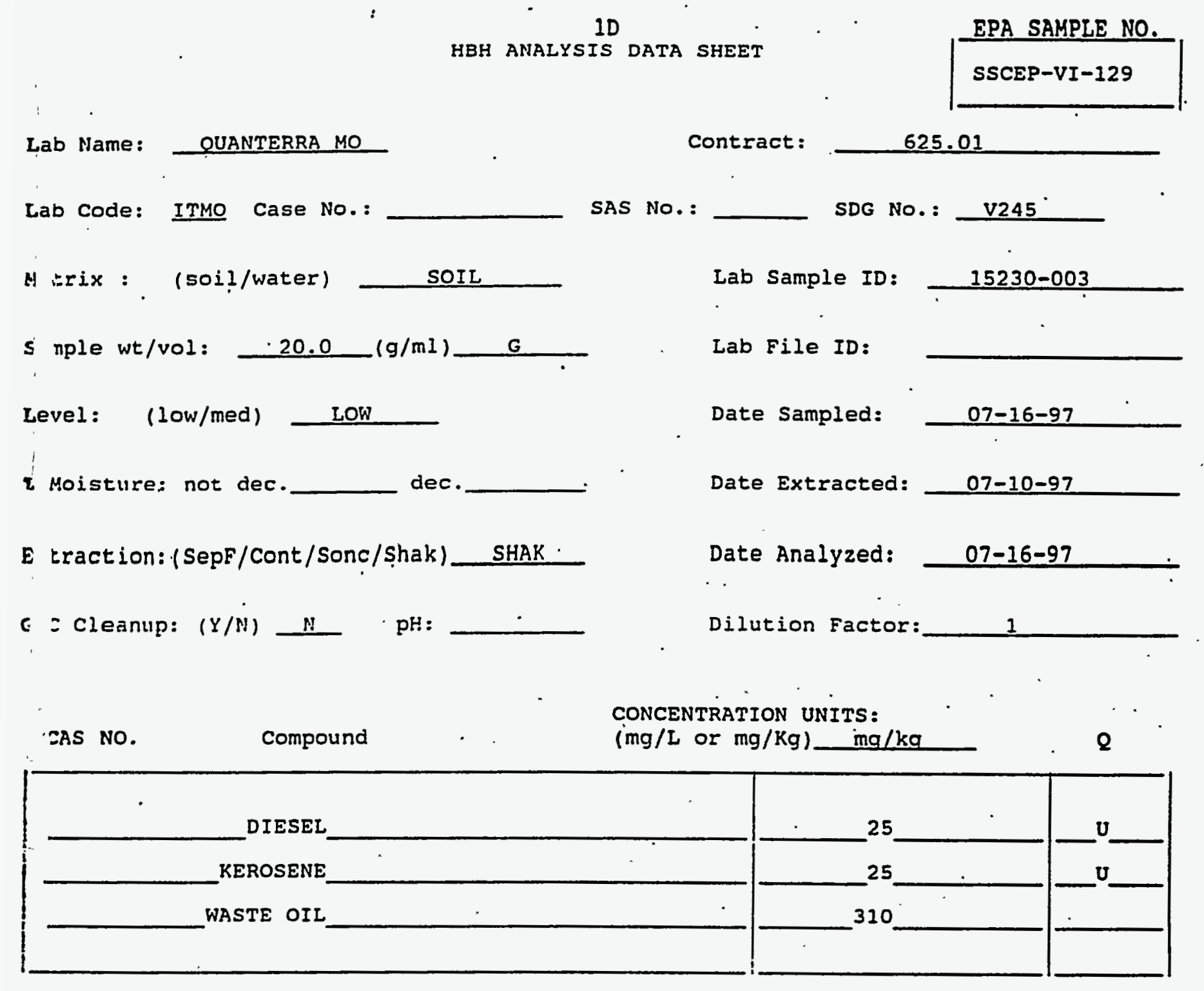

": Concentration of analyte is less than the value given.

FORM I HBH 
Lab Name: QUANTERRA:MO

Lab Code: ITMO

Case No.: V2300I

Matrix: (soil/water) soIL

Sample wt/vol:

$5.00 \cdot(\mathrm{g} / \mathrm{mL}) \mathrm{G}$

Level: (low/med) LOW

\% Moisture: not dec.

GC Column: RTX-502.2 ID: 0.530 (mm)

Soil Extract Volume:

(uL)
Contract: 625-01

SAS NO.:

SDG No.: V245
Lab Sample ID: 15230-003

Lab File ID: $\quad$ G7480.

Date Received: 07/03/97

Date Analyzed: 07/12/97

Dilution Factor: $\quad 1.0$

Soil Aliquot Volume:

(uL)

. CAS NO.

COMPOUND

CONCENTRATION UNITS:

(ug/L or ug/Kg) UG/KG

Q

74-87-3-1-.---Chloromethane

74-83-9--..----Bromomethane.

75-01-4-.-.---Vinyl Chloride

75-00-3-------Chloroethane

75-09-2-...-- Methylene Chloride

67-64-1--.-.---Acetone

75-15-0-..---Carbon. Disulfide

75-35-4-..---1, 1-Dichloroethene

75-34-3-...-. 1, 1-Dichloroethane

540-59-0--.--1,2-Dichloroethene (total)

67-66-3-.-.---Chloroform

107-06-2-----1, 2-Dichloroethane

78-93-3-..-.-2-Butanone

71-55-6--.---1, 1, 1-Trichloroethane

56-23-5-...---Carbon. Tetrachloride

75-27-4--..---Bromodichloromethane

78-87-5------1, 2-Dichloropropane

10061-01-5--..-cis-1,3-Dichloropropene

79-01-6-..---.-Trichloroethene

124-48-1--.---Dibromochloromethane

79-00-5--.---1, 1, 2-Trichloroethane

71-43-2---.-.-Benzene

10061-02-6-----trans-1,3-Dichloropropene

75-25-2--.-.--Bromoform

108-10-1--.---4-Methyl-2-Pentanone

591-78-6-..--.-2-Hexanone

127-18-4-...--Tetrachloroethene

79-34-5.-.-.--1, 1, 2, 2-Tetrachloroethane

108-88-3-...--Toluene

108-90-7-...-Chlorobenzene

100-41-4-------Ethylbenzene

100-42-5--.-----styrene

1330-20-7-..--.xylene (total) 
1

INORGANIC ANALYSES DATA SHEET

¿ $e:$ QÜANTERRA_MO

ode: ITMO_ Case No. :SW846

$x$ (soil/water) : WATER

(low/med) : LNOW

$i_{-s}$ :

$-0.0$

Concentration Units (ug/L or $\mathrm{mg} / \mathrm{kg}$ dry weight): UG/L_

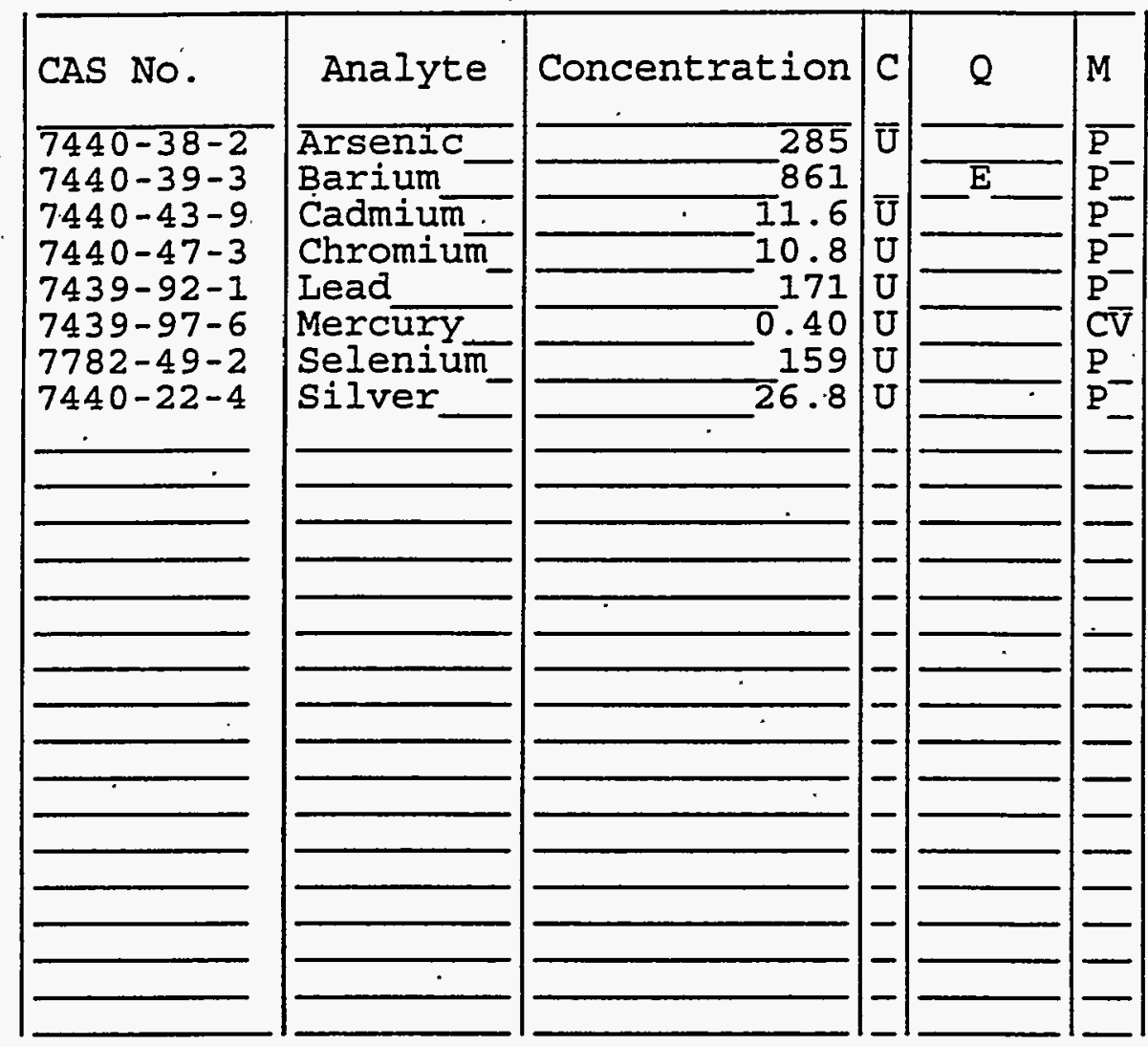

efore:

Clarity Before:

Texture:

clarity After:

\section{After:}

EPA SAMPLE NO.

\section{SSCEPVI129.}

SDG No.: V245

Lab Sample ID: P15230-003

Date Received: 07/03/97

n s :

FORM I - IN

TCLP · 
Lab Name: QUANTERRA MO

Iab Code: ITMO

Case No.: V41401

Matrix: (soil/water) soIL

Contract : 625-01

SAS No.:
SDG No.: V258
Sample wt/vol:

Level:

(low/med)

$5.00(\mathrm{~g} / \mathrm{mL}) \mathrm{G}$

LOW

$\%$ Moisture: not dec.

GC Column: RTX-502.2 ID: 0.530 (mm)

Soil Extract Volume:

CAS NO.
(uL)
Lab Sample ID: 15414-004

Lab File ID: . F1152

Date Received: 07/25/97

Date Analyzed: 08/06/97

Dilution Factor: $\quad 1.0$

Soil Aliquot volume:

(uL:
CONCENTRATION UNITS:

(ug/L or.ug/Kg) UG/KG

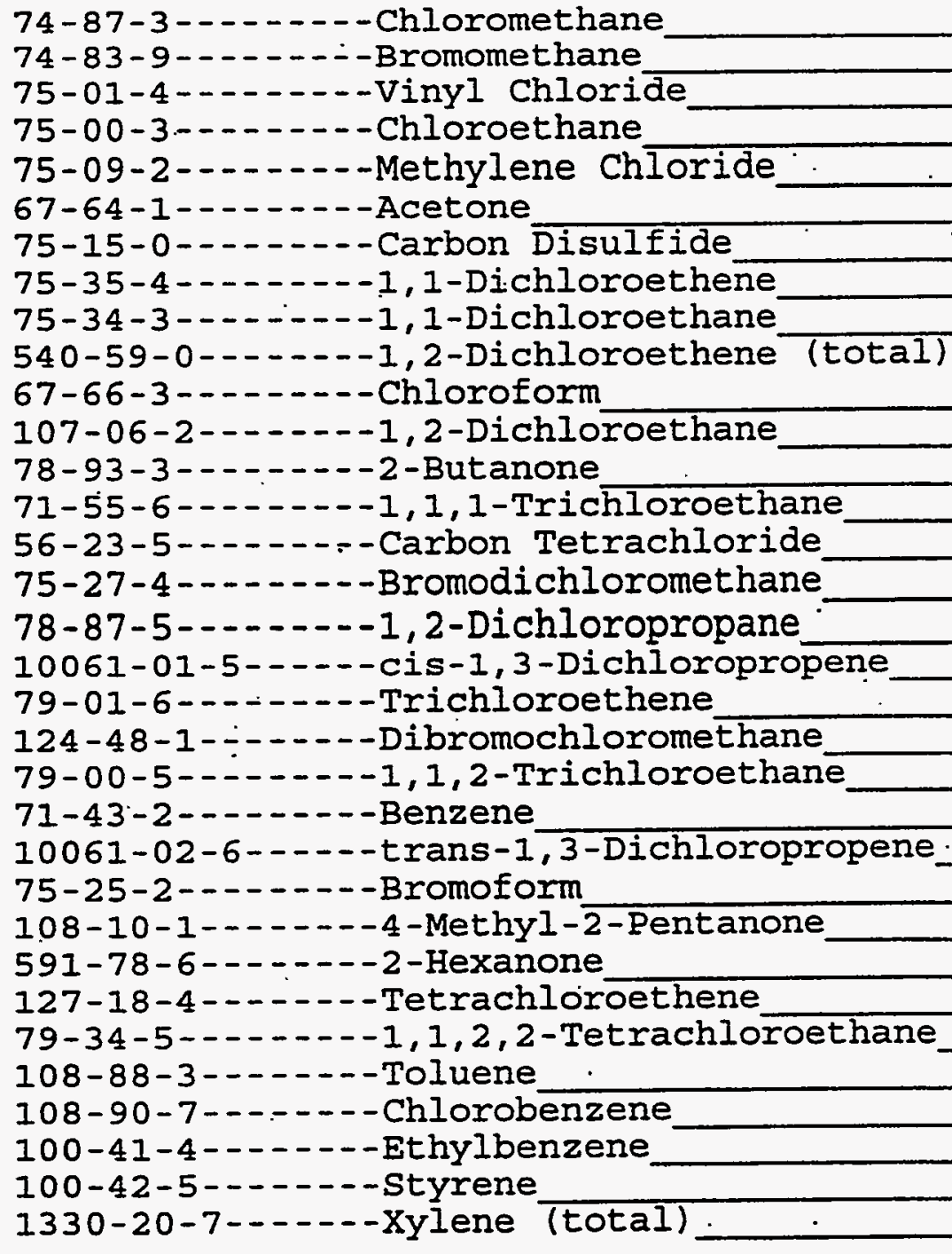




\section{VOLUME 2 APPENDIX B}

TABLE 7 ANALYTICAL DATA 


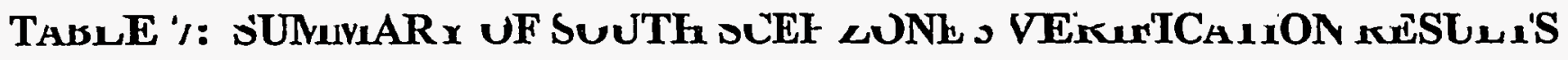

\begin{tabular}{|c|c|c|c|c|c|c|c|}
\hline 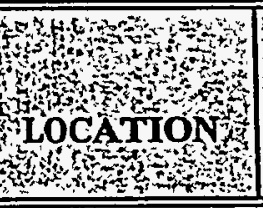 & 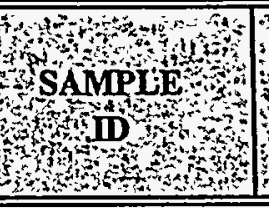 & 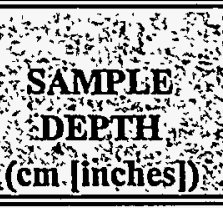 & 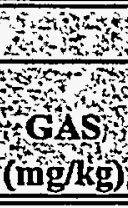 & Hifing & 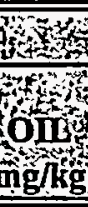 & 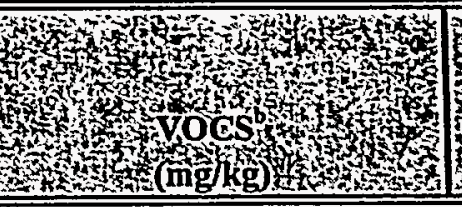 & 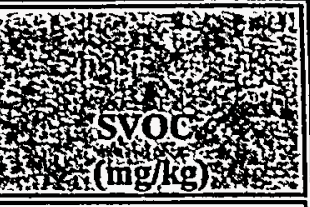 \\
\hline & & SAM & ES CC & TEI & ST 2 & & \\
\hline Cell No. 20 & SSCEP-V3-20 & $0-15.2[0-6]$ & ND & $\mathrm{ND}$ & ND & $\mathrm{NA}$ & NA \\
\hline Cell No. 24 & SSCEP-V3-24 & $0-15.2[0-6]$ & ND & ND & ND & NA & $\mathrm{NA}$ \\
\hline Cell No. 25 & SSCEP-V3-25 & $0-15.2[0-6]$ & $\cdot \mathrm{ND}$ & ND & ND & NA & NA \\
\hline & SSCEP-V4-25 & $0-15.2[0-6]$ & ND & ND & ND & NA & NA \\
\hline Cell No. 30 & SSCEP-V3-30 & $0-15.2[0-6]$ & ND & ND & ND & NA & NA \\
\hline . & SSCEP-V4-30 & $0-15.2[0-6]$ & ND & ND & ND & NA & $\mathrm{NA}$ \\
\hline Cell No. 44 & SSCEP-V3-44 & $0-15.2[0-6]$ & ND & ND & ND & NA & NA \\
\hline & SSCEP-V4-44 & $0-15.2[0-6]$ & $\mathrm{ND}$ & ND & ND & NA & NA \\
\hline Cell No. 59 & SSCEP-V3-59A & $0-15.2[0-6]$ & ND & ND & $\mathrm{ND}$ & ND & ND \\
\hline Cell No. 63 & SSCEP-V3-63A & $0-15.2[0-6]$ & $\mathrm{NA}$ & NA & NA & Methylene Chloride $\mathbf{0 . 0 0 5 8}$ & ND \\
\hline Cell No. 68 & SSCEP-V3-68 & $0-15.2[0-6]$ & ND & ND & ND & NA & NA . \\
\hline Cell No. 82 & SSCEP-V3-82 & $0-15.2[0-6]$ & ND & ND & ND & NA & .NA \\
\hline Cell No. 108 & SSCEP-V3-108 & $0-15.2[0-6]$ & ND & ND & $\mathrm{ND}$ & NA & NA \\
\hline Cell No. 115 & SSCEP-V3-115 & $0-15.2[0-6]$ & ND & ND & ND & NA & NAA \\
\hline & SSCEP-V4-115 & $0-15.2[0-6]$ & $\mathrm{ND}$ & ND & ND & NA & $\mathrm{NA}$ \\
\hline Cell No. 115 & SSCEP-V3-115A. & $0-15.2[0-6]$ & ND & ND & ND & ND & ND \\
\hline Cell No. 116 & SSCEP-V3-116 & $0-15.2[0-6]$ & ND & ND & ND & NA & NA \\
\hline & SSCEP-V4-116 & $0-15.2[0-6]$ & ND & ND & ND & NA & NA \\
\hline Cell No. 119 & SSCEP-V3-119A & $0-15.2[0-6]$ & NA & NA & $\mathrm{NA}$ & ND & ND \\
\hline Cell No. $130^{\circ}$ & SSCEP-V3-130 & $0-15.2[0-6]$ & ND & ND & 44 & NA & NA \\
\hline
\end{tabular}


TABLE 7: SUMMARY OF SOUTH SCEP ZONE 3 VERIFICATION RESULTS

\begin{tabular}{|c|c|c|c|c|c|c|c|}
\hline 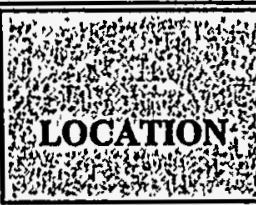 & 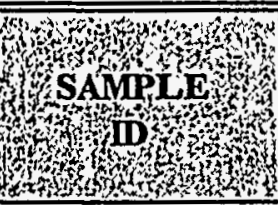 & 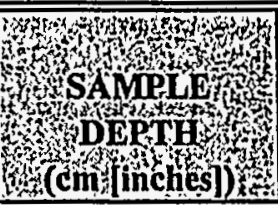 & 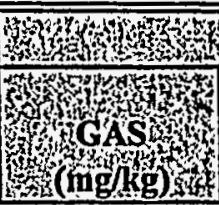 & 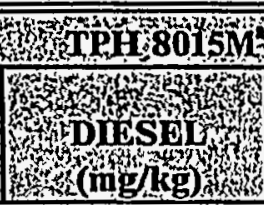 & 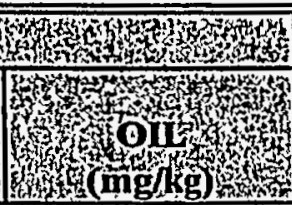 & 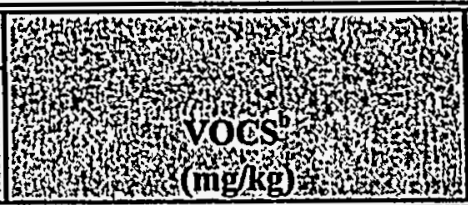 & 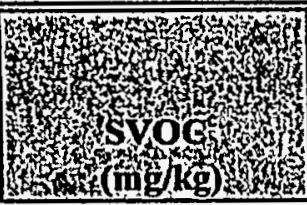 \\
\hline \multicolumn{8}{|c|}{ SAMPLES COLLECTED AUGUST 27, 1997} \\
\hline \multirow[t]{2}{*}{ Cell No. 131} & SSCEP-V3-131 & $0-15.2[0-6]$ & ND & ND & ND & NA & $\mathrm{NA}$ \\
\hline & SSCEP-V4-131 & $0-15.2[0-6]$ & ND & ND & ND & NA & NA. \\
\hline Cell No. 133 & SSCEP-V3-133 & $0-15.2[0-6]$ & ND & $\mathrm{ND}$ & ND & NA & $\mathrm{NA}$ \\
\hline Cell No. 154 & SSCEP-V3-154 & $0-15.2[0-6]$ & ND & ND & ND் & NA: & NA \\
\hline
\end{tabular}

\section{Notes: ·}

"TPH 8015M: Total Petroleum Hydrocarbons Method 8015 Modified.

${ }^{\text {b }}$ VOCs: Volatile Organic Compounds.

'SVOC:Semi-Volatile Organic Compounds SCEP: Steam Cleaning Effluent Pond

ND: Not Detected

ND: Not Analyzed

cm: centimeter

mg/kg: milligrams per kilogram 


\section{; LABORATORIES}

i \& PETROLEUM HYDROCARBONS (TPH)

IM - TPH

- Iient Sample ID: Date Collected:

Date Analyzed:

Jate Extracted:

Matrix:

Percent Moisture:',

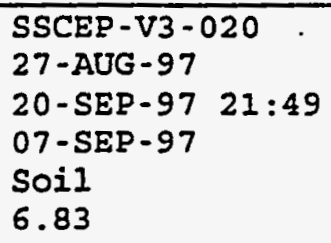

Soil

6.83

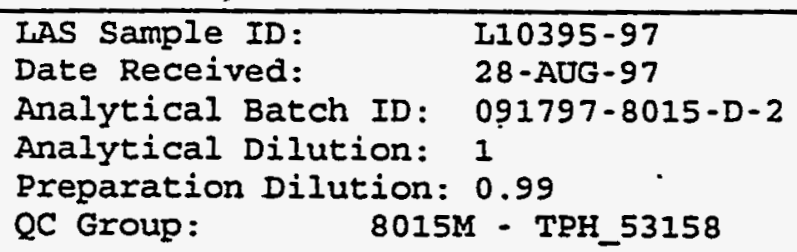
STITUENT 25-162

\begin{abstract}
sel Range Organics oline Range Organics i ! OIL
\end{abstract}
DAFA. Q 21,0 OUAJTETERTS RESUT: ito $1 \mathrm{~kg}$
32.
32 .
32 .

n-OCTACOSANE $32 \%$

\section{3}

I7682STANDARD N R20474 Page 1


TOTAL PETROLEUM HYDROCARBONS (TPH) 8015M - TPH

$\begin{array}{llll}\text { Client Sample ID: } & \text { SSCEP-V3-024 } & \text { IAS Sample ID: } & \text { I10395-72 } \\ \text { Date Collected: } & 27-A O G-97 & \text { Date Received: } & 28-A U G-97 \\ \text { Date Analyzed: } & 17-\text { SEP-97 01:27 } & \text { Analytical Batch ID: } 091597-8015-D-3 \\ \text { Date Extracted: } & 07-\text { SEP-97 } & \text { Analytical Dilution: } 1 \text { 1 } \\ \text { Matrix: } & \text { Soil } & \text { Preparation Dilution: 1:0 } \\ \text { Percent Moisture: } & 8.45 & \text { QC Group: 80I5M - TPH_53132 }\end{array}$

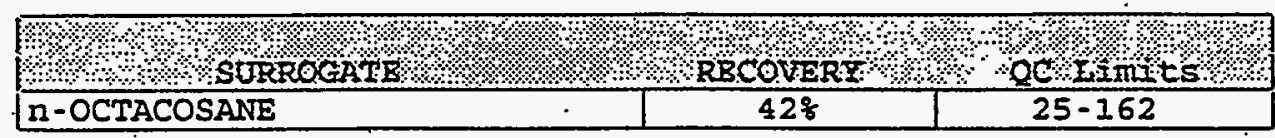

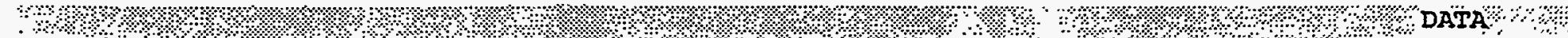

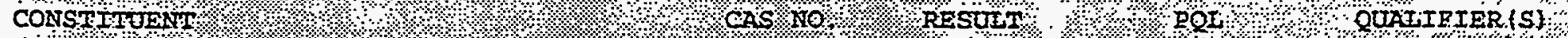
$\therefore \because \%$. mg f

Diesel Range Organics Gasoline Range Organics MOTOR OIL
TPH

$<33$.

$<33$.

$<33$.

33.

33.

33. 


\section{1) LABORATORIES}

$\therefore$ PETROLEUM HYDROCARBONS (TPH)

$5 \square-T P H$

$\begin{array}{ll}\text { Client Sample ID: } & \text { SSCEP-V3-025 } \\ \text { Date Collected: } & 27-A U G-97 \\ \text { Date Analyzed: } & 17-\text { SEP-97 02:56 } \\ \text { Date Extracted: } & 07-\text { SEP-97 } \\ \text { Matrix: } & \text { Soil } \\ \text { Percent Moisture: } & 1.44\end{array}$

IAS Sample ID:

Date Received:

Analytical Batch ID: 091597-8015-D-3

Analytical Dilution: 1

Preparation Dilution: 1.0

QC Group :
L10395-74

28-AUG-97

8015M - TPF_53132
(n)
ISTITUENT
RESUTET
का
: I Range Organics
TPH
$<30$.
Mo/kg
ioline Range Organics
$<30$.
30 .
:n, oII
$<30$.
30 .
30 .

$41 \frac{1}{6}$ $25-162$

QUATIRIER (S)

7682STANDARD $\quad$ N R20472: Páge 1




\section{LAS LABORATORIES}

TOTAL PETPROLEUM. HYDROCARBONS (TPH) 8015M - TPH

$\begin{array}{llll}\text { Client Sample ID: } & \text { SSCEP-V4-25 } & \text { IAS Sample ID: } & \text { I10479-4 } \\ \text { Date Collected: } & 09-\text { SEP-97 } & \text { Date Received: } & 10-\text { SEP-97 } \\ \text { Date Analyzed: } & 25-\text { SEP-97 } & \text { Analytical Batch ID: } 091797-8015-D-6 \\ \text { Date Extracted: } & 12-\text { SEP-97 } & \text { Analytical Dilution: } 1 \\ \text { Matrix: } & \text { Soil } & \text { Preparation Dilution: 0.99 } \\ \text { Percent Moisture: } & 7.45 & \text { QC Group: } & \end{array}$

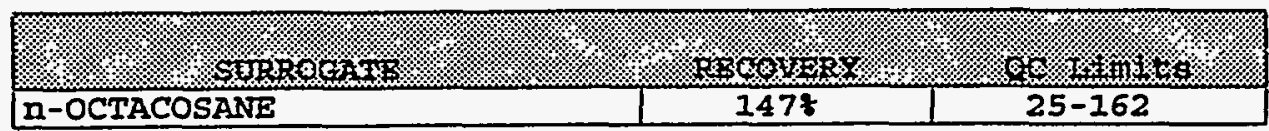

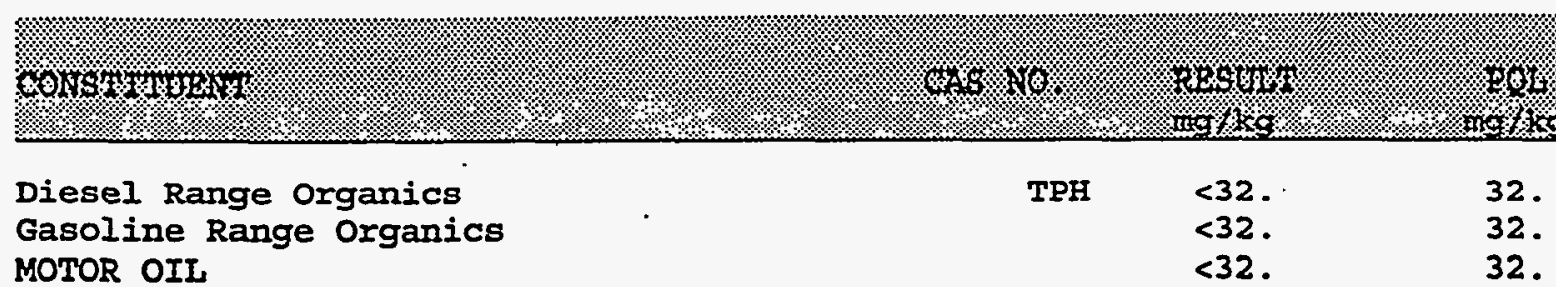

32 .

32 . 


\section{; LABORATORIES}

$i$, PETROLEUM HYDROCARBONS (TPH)

$M-$ TPH

- Iient Sample ID: SSCEP-V3-030 Jate Collected:

Date Analyzed:

Date Extracted:

Latrix:

percent Moisture:
27-AUG-97

20-SEP-97 $23: 18$

$07-$ SEP-97

Soil

1.73
LAS Sample ID:

Date Received:

Analytical Batch ID:

Analytical Dilution:

Preparation Dilution:
L10395-99

28-AUG-97

091797-8015-D-2

1

$8015 M$ - TPH_53158

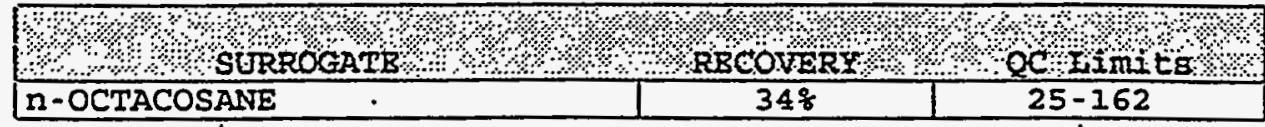

m

ITITUENC.

RESULT

PQI

QUATSTRIERIS\}

and

I 1 Range Organics Jline Range Organics

$\because$ OII
TPH

$<30$.

$<30$.

$<30$.
30 .

30 .

30 . 


\title{
LAS LABORATORIES
}

\section{TOTAL PETROLEUM HYDROCARBONS (TPH)}

\author{
8015M - TPH
}

$\begin{array}{ll}\text { Client Sample ID: } & \text { SSCEP-V4-30 } \\ \text { Date Collected: } & 09-S E P-97 \\ \text { Date Analyzed: } & 25-\text { SEP-97 } \\ \text { Date Extracted: } & 12-\text { SEP-97 } \\ \text { Matrix: } & \text { Soil } \\ \text { Percent Moisture: } & 7.92\end{array}$

LAS Sample ID:

Date Received:

Analytical Batch ID: 091797-8015-D-6

Analytical Dilution: 1

Preparation Dilution: 0.97

Percent Moisture: 7.92

QC Group:

8015M - TPH_53397
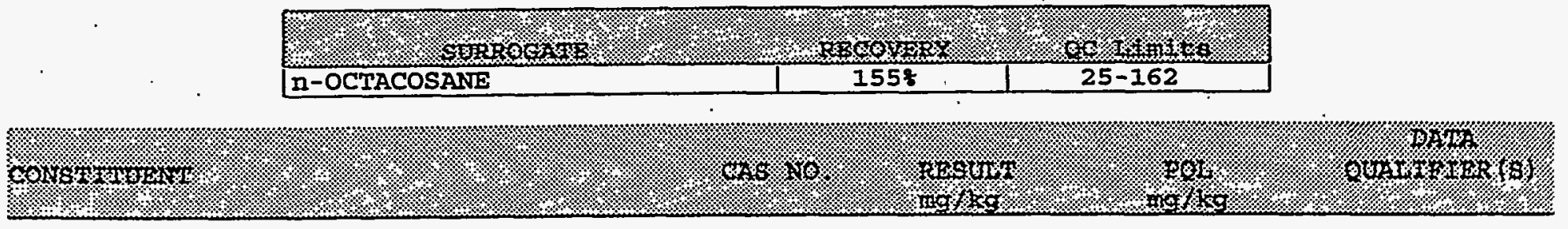

Diesel Range Organics Gasoline Range Organics MOTOR OIL
TPH $<32$.

$<32$.

$<32$.

32.

32.

32 . 


\section{‘ i LABORATORIES}

$i$, PETROLEUM HYDROCARBONS (TPH)

iM - TPH

\begin{tabular}{llll}
\hline Ilient Sample ID: & SSCEP-V3-044 & IAS Sample ID: & I10395-101 \\
Jate CoIlected: & $27-$ AUG-97 & Date Received: & $28-A U G-97$ \\
Date Analyzed: & $21-$ SEP-97 00:47 & Analytical Batch ID: $091797-8015-D-2$ \\
Jate Extracted: & $07-$ SEP-97 & Analytical Dilution: 1 &. \\
Matrix: & Soil & Preparation Dilution: 0.99 \\
Percent Moisture: & 6.13 & QC Group: & 8015M - TPH_53158
\end{tabular}

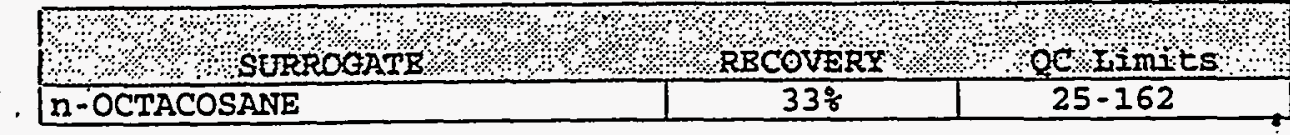

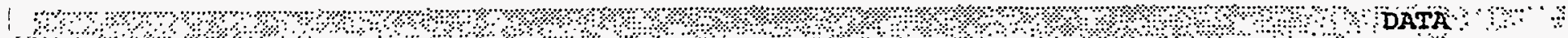
STITUENT

CASHOH, HRSUTH

ROH $०$ QUALIFIER (ST.

$\because \because 4$

m,

Sul Range Organics oline Range Organics c , OII
TPH

$<32$.

$<32$.

$<32$.
32 .

32 .

32 .

000987 


\section{LAS LABORATORIES}

TOTAL PETROLEUM HYDROCARBONS (TPH) 8015M - TPH

$\begin{array}{ll}\text { Client Sample ID: } & \text { SSCEP-V4-44 } \\ \text { Date Collected: } & 09-\text { SEP-97 } \\ \text { Date Analyzed: } & 26-\text { SEP-97 } \\ \text { Date Extracted: } & 12-\text { SEP-97 } \\ \text { Matrix: } & \text { Soil } \\ \text { Percent Moisture: } & 11.64\end{array}$

LAS Sample ID: Date Received:

I10479-6

Analytical Batch ID: 091797-8015-D-6

Analytical Dilution: 1

Preparation Dilution: 1.0

QC Group: $\quad 8015 \mathrm{M}$ - TPH_53397
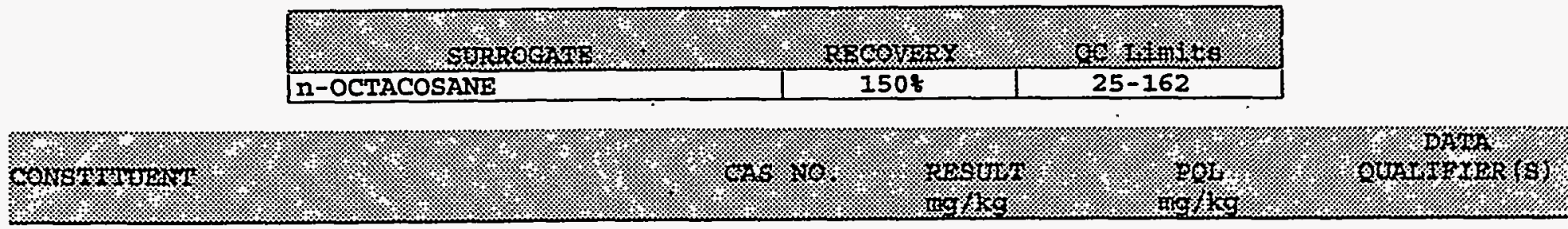

Diesel Range Organics Gasoline Range Organics MOTOR OIL
TPH

$<34$.

$<34$.

$<34$.

34.

34.

34. 


\section{LABORATORIES}

\section{AL PETROLEUM HYDROCARBONS (TPH)}

IM - TPH

\begin{tabular}{llll}
\hline -Iient Sample ID: & SSCEP-V3-59A & LAS Sample ID: & L10395-66 \\
Date Collected: & $27-$ AUG-97 & Date Received: & $28-A U G-97$ \\
Tate Analyzed: & $19-$ SEP-97 08:04 & Analytical Batch ID: 091597-8015-D-6 \\
late Extracted: & $06-$ SEP-57 & Analytical Dilution: 1 \\
Matrix: & Soil & Preparation Dilution: 1.0 \\
Percent Moisture: & 6.24 & QC Group: & B015M - TPH_53029 \\
\hline
\end{tabular}

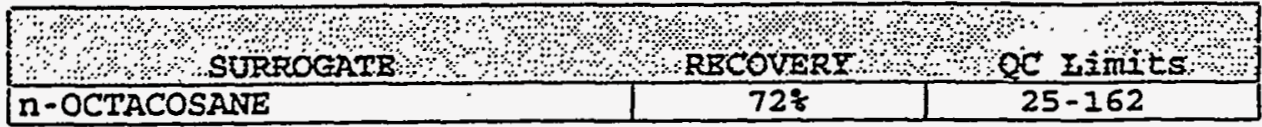

TUENT

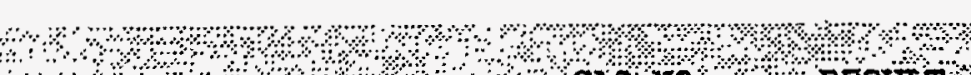

S" MIUENT

$-$

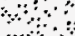

\begin{abstract}
sel Range Organics $O$ ine Range Organics ¿ OII
\end{abstract}

TPH

mg/

pol mig $/ \mathrm{kg}$
.32 .

32.

32. 


\section{LAS LABORATORIES}

\section{VOLATILE ORGANICS BY GC/MS}

8260 VOLATILES

$\begin{array}{llll}\text { Client Sample ID: } & \text { SSCEP-V3-59A } & \text { IAS Sample ID: } & \text { L10395-52 } \\ \text { Date Collected: } & 27-A U G-97 & \text { Date Received: } & 28-A U G-97 \\ \text { Date Analyzed: } & 09-\text { SEP-97 12:30 } & \text { Analytical Dilution: } & 1 \\ \text { Matrix: } & \text { Soil } & \text { Analytical Batch ID: 090997-8260-E1 } \\ \text { Percent Moisture: } & 6.24 & \text { preparation Dilution: 1.00 }\end{array}$

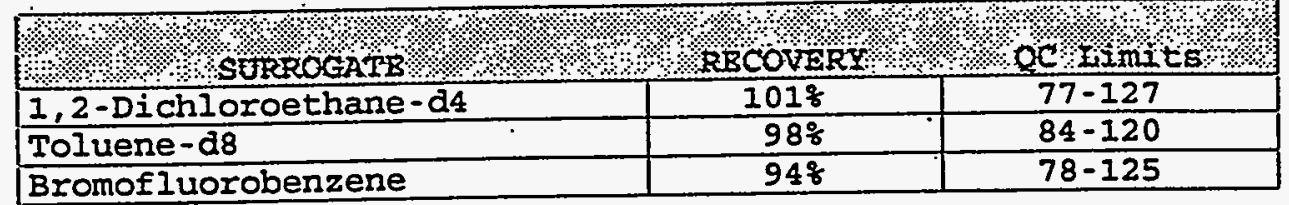

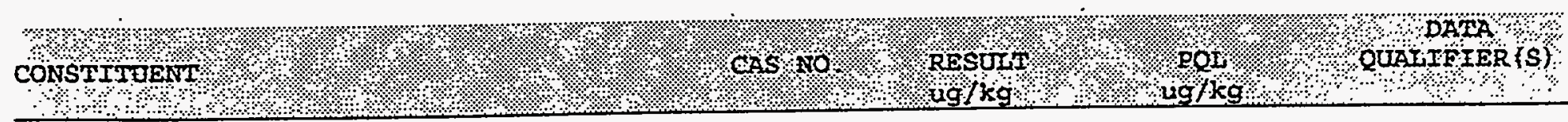

Chloromethane

vinyl Chloride

Bromomethane

Chloroethane

Trichlorofluoromethane

Acetone

1,1-Dichloroethene

Carbon Disulfide

Methylene Chloride

trans-1,2-Dichloroethene

Vinyl Acetàte

1,1-Dichloroethane

2-Butanone

cis-1,2-Dichloroethene

Chloroform

\section{2-Hexanone}

1,1,1-Trichloroethane

Carbon tetrachloride

1,2-Dichloroethane

Benzene

Trichloroethene

1,2-Dichloropropane

Bromodichloromethane

2-Chloroethylvinylether

4-Methyl-2-Pentanone

cis-1,3-Dichloropropene

Toluene

trans-1;,3-Dichloropropene

1,1,2-Trichloroethane

Tetrachloroethene

Dibromochloromethane

Chlorobenzene

Ethylbenzene

m,p-Xylene

o-Xylene

Styrene

Bromoform

$1,1,2,2$-Tetrachloroethane

1,3-Dichlorobenzene

1,4-Dichlorobenzene

1,2-Dichlorobenzene
$<5.3$

$<5.3$

75-01-4

$74-83-9$

75-00-3

$75-69-4$

$67-64-1$

$75-35-4$

$75-15-0$

75-09-2

$156-60-5$

$108-05-4$

$75-34-3$

$78-93-3$

156-59-2

$67-66-3$

591-78-6

$71-55-6$

56-23-5

107-06-2

$71-43-2$

$79-01-6$

$78-87-5$

$75-27-4$

$110-75-8$

108-10-1

10061-01-5

$108-88-3$

1006i-02-6

$79-00-5$

127-18-4

$124-48-1$

108-90-7

100-41-4

136777-61-2

95-47-6

$100-42-5$

$75-25-2$

$79-34-5$

541-73-1

106-46-7

95-50-1
$<5.3$

$<5.3$

$<5.3$

$<11$.

$<5.3$

$<5.3$

$<5.3$

$<5.3$

$<11$.

$<5.3$

$<11$.

$<5.3$

$<5.3$

$<11$.

$<5.3$

$<5.3$

$<5.3$

$<5.3$

$<5.3$

$<5.3$

$<5.3$

$<21$.

$<11$.

$<5.3$

$<5.3$

$<5.3$

$<5.3$.

$<5.3$

$<5.3$

$<5.3$

$<5.3$

$<5.3$

$<5.3$

$<5.3$

$<5.3$

$<5.3$

$<5.3$

$<5.3$

$<5.3$
5.3

5.3

5.3

5.3

5.3

11.

5.3

5.3

5.3

5.3

11.

5.3

11 .

5.3

5.3

11.

5.3

5.3

5.3

5.3

5.3

5.3

5.3

21.

11 .

5.3

5.3

5.3

5.3

5.3

5.3

5.3

5.3

5.3

5.3

5.3

5.3

5.3

5.3

5.3

5.3 


\section{1) LABORATORIES}
[-VOLATILE ORGANICS BY GC/MS
, SEMI-VOLATILES
$\dot{4}$
Client Sample ID
SSCEP.-V3-59A
27-AUG- 97
Date collected:
09-SEP-97
latrix:
QC Group:
Percent Moisture:
Soil
8270 SEMI-VOLATILES_52828
6.24
IAI Sample ID:
Date Received:
Date Extracted:
Analytical Batch ID: 090897-8270-K
Analytical Dilution: 1
Preparation Dilution: 1.00
L10395-57
28-AUG-97
01-SEP- 97

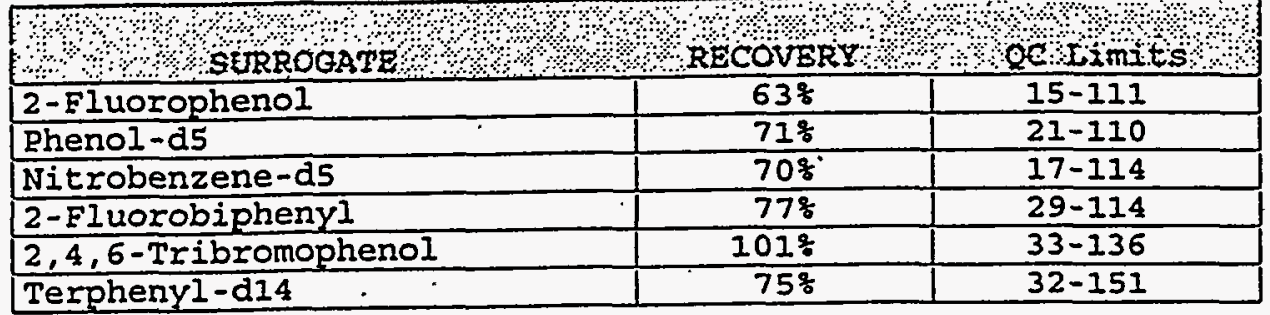

Phenol

bis (2-Chloroethyl) ether

2-Chlórophenol

1,3-Dichlorobenzene

1,4-Dichlorobenzene

Benzyl alcohol

1,2-Dichlorobenzene

2-Methylphenol

bis (2-chloroisopropyl) ether

4-Methylphenol

N-Nitroso-di-n-propylamine

Hexachloroethane

Nitrobenzene

Isophorone

2-Nitrophenol

2,4-Dimethylphenol

Benzoic acid

bis (2-Chloroethoxy) methane

2, 4-Dichlorophenol

1,2,4-Trichlorobenzene

Naphthalene

4-Chloroaniline

Hexachlorobutadiene

4-Chloro-3-methylphenol

2-Methylnaphthalene

Hexachlorocyclopentadiene

2,4, 6-Trichlorophenol

2, 4, 5-Trichlorophenol

2-Chloronaphthalene

2-Nitroaniline

Dimethylphthalate

Acenaphthylene

2,6-Dinitrotoluene

3-Nitroaniline

Acenaphthene

2,4-Dinitrophenol

4-Nitrophenol

\begin{tabular}{|c|c|c|c|}
\hline $108-95-2$ & $<710$ & & 710 \\
\hline $111-44-4$ & $<710$ & & 710 \\
\hline $95-57-8$ & $<710$ & & 710 \\
\hline $541-73-1$ & $<710$ & & 710 \\
\hline $106-46-7$ & $<710$ & & 710 \\
\hline $100-51-6$ & $<1400$ & & 1400 \\
\hline $95-50-1$ & $<710$ & & 710 \\
\hline $95-48-7$ & $<710$ & & 710 \\
\hline $108-60-1$ & $\cdot<710$ & & 710 \\
\hline $106-4 \overline{4}-5$ & $\cdot<710$ & & 710 \\
\hline $621-64-7$ & $<710$ & & 710 \\
\hline $67-72-1$ & $<710$ & & $7 i 0$ \\
\hline $98-95-3$ & $<710$ & & 710 \\
\hline $78-59-7$ & $<710$ & & 710 \\
\hline $88-75-5$ & $<710$ & & 710 \\
\hline $105-67-9$ & $<710$ & & 710 \\
\hline $65-85-0$ & $<3500$ & & 3500 \\
\hline $111-91-1$ & $<710$ & & 710 \\
\hline $120-83-2$ & $<710$ & & 710 \\
\hline $120-82-1$ & $<710$ & & 710 \\
\hline $91-20-3$ & $<710$ & . & 710 \\
\hline $106-47-8$ & $<1400$ & & 1400 \\
\hline $87-68-3$ & $<710$ & & 710 \\
\hline $59-50-7$ & $<1400$ & & 1400 \\
\hline $91-57-6$ & $<710$ & & 710 \\
\hline $77-47-4$ & $\cdot<710$ & & 710 \\
\hline $88-06-2$ & $<710$ & & 710 \\
\hline $95-95-4$ & $<710$ & & 710 \\
\hline $91-58-7$ & $<710$ & & 710 \\
\hline $88-74-4$ & $<3500$ & & 3500 \\
\hline $131-11-3$ & $<710$ & & 710 \\
\hline $208-96-8$ & $<710$ & $\therefore$ & 710 \\
\hline $606-20-2$ & $<710$ & & 710 \\
\hline $99-09-2$ & $<3500$ & & 3500 \\
\hline $83-32-9$ & $<710$ & . & 710 \\
\hline $51-28-5$ & $<3500$ & & 3500 \\
\hline $100-02-7$ & $<3500$ & & 3500 \\
\hline
\end{tabular}

\section{$\overline{1}$}




\section{LAS LABORATORIES}

SEMI-VOLATILE ORGANICS BY GC/MS

8270 SEMI-VOLATILES

- Client Sample ID:

Date Collected:

Date Analyzed:

Matrix:

QC Group:

Percent Moisture:
SSCEP-V3-59A

27-AL̈G -97

09-SEP-97

Soil

8270

6.24
IAI Sample ID:

Date Received:

Date Extracted:

Analytical Batch ID: 090897-8270-K

10395-57

28-AUG- 97

$01-$ SEP -97

Analytical Dilution: - 1

Preparation Dilution: 1.00

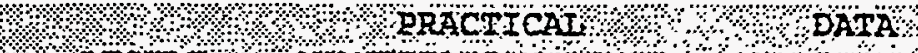
3 CONSTITUENT $+2 \%$ \%

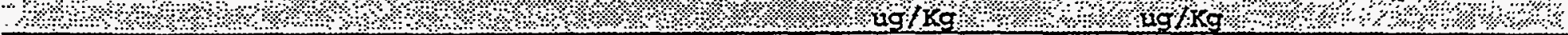

Dibenzofuran

2,4-Dinitrotoluene

Diethylphthalate

4-Chlorophenyl-phenylether

Fluorene

4-Nitroaniline

4,6-Dinitro-2-methylphenol

N-Nitrosodiphenylamine (1).

4-Bromophenyl-phenylether

Hexachlorobenzene

Pentachlorophenol

Phenanthrene

Anthracene

Carbazole

Di-n-butylphthalate

Fluoranthene.

Pyirene

Butylbenzylphthalate

3,3'-Dichlorobenzidine

Benzo (a) anthracene

Chrysene

bis (2-Ethylhexyl) phthalate

Di-n-octylphthalate

Benzo (b) fluoranthene

Benzo (k) fluoranthene

Benzo (a) pyrene

Indeno $(1,2,3-c d)$ pyrene

Dibenz $(a, h)$ anthracene

Benzo $(g, h, i)$ perylene

$\begin{array}{rll}132-64-9 & <710 & 710 \\ 121-14-2 & <710 & 710 \\ 84-66-2 & <710 & 710 \\ 7005-72-3 & <710 & 710 \\ 86-73-7 & <710 & 710 \\ 100-01-6 & <3500 & 3500 \\ 534-52-1 & <3500 & 3500 \\ 86-30-6 & <710 & 710 \\ 101-55-3 & <710 & 710 \\ 118-74-1 & <710 & 710 \\ 87-86-5 & <3500 & 3500 \\ 85-01-8 & <710 & 710 \\ 120-12-7 & <710 & 710 \\ 86-74-8 & <710 & 710 \\ 84-74-2 & <710 & 710 \\ 205-44-0 & <710 & 710 \\ 129-00-0 & <710 & 710 \\ 85-68-7 & <710 & 710 \\ 91-94-1 & <1400 & 1400 \\ 56-55-3 & <710 & 710 \\ 218-01-9 & <710 & 710 \\ 117-81-7 & <710 & 710 \\ 117-84-0 & <710 & 710 \\ 205-99-2 & <710 & 710 \\ 207-08-9 & <710 & 710 \\ 50-32-8 & <710 & 710 \\ 193-39-5 & <710 & 710 \\ 53-70-3 & <710 & 710 \\ 191-24-2 & <710 & 710\end{array}$




\section{؟ LABORATORIES}

ILE ORGANICS BY GC/MS

TTATILES

\begin{tabular}{llll}
\hline Client Sample ID: & SSCEP-V3-63A & LAS Sample ID: & L10395-48 \\
Date Collected: & $27-A U G-97$ & Date Received: & $28-A U G-97$ \\
1 the Analyzed: & $09-$ SEP-97 11:12 & Analytical Dilution: 1 & 1 \\
I trix: & Soil & Analytical Batch ID: 090997-8260-E1 \\
Percent Moisture: & 7.32 & Preparation Dilution: 1.00
\end{tabular}

Pexcent Moisture:

\begin{tabular}{|c|c|c|}
\hline 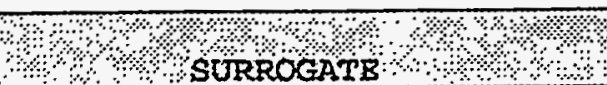 & \%ै, RCOVIRRY & ४े QCHinmits \\
\hline 1,2-Dichloroethane-d4 & 1028 & $77-127$ \\
\hline Toluene-d8 & $95 \%$ & $84-120$ \\
\hline Bromofluorobenzene & $90 \frac{9}{8}$ & $78-125$ \\
\hline
\end{tabular}

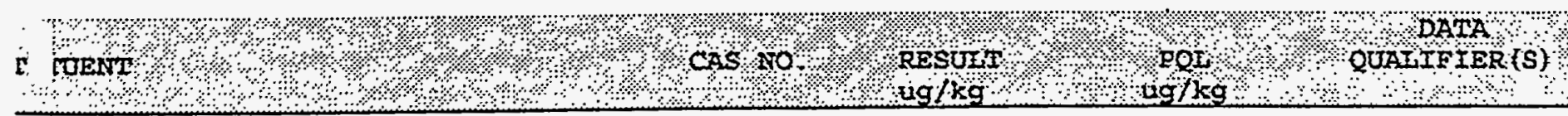

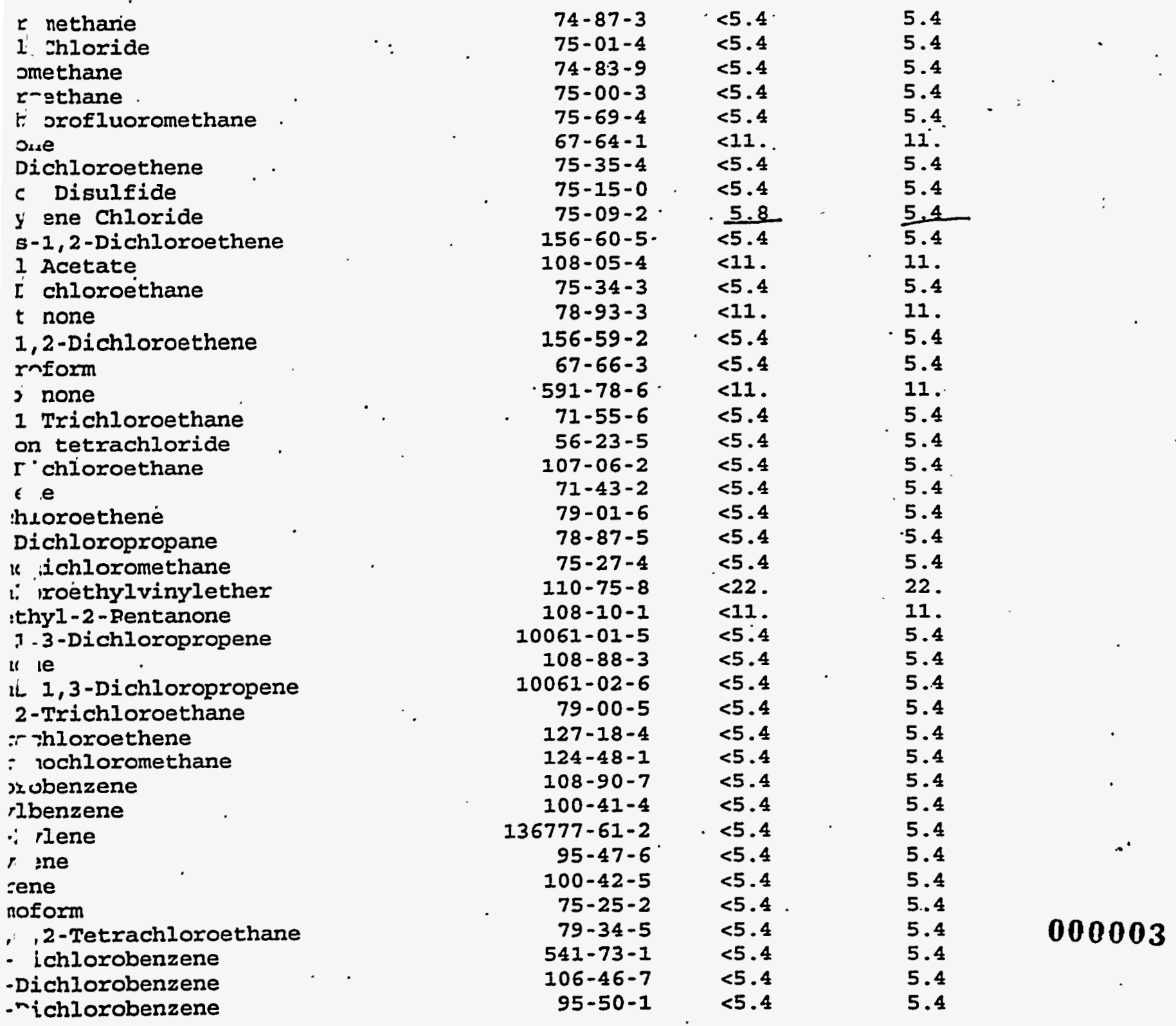




\section{LAS LABORATORIES}

SEMI-VOLATILE ORGANICS BY GC/MS 8270 SEMI-VOLATILES

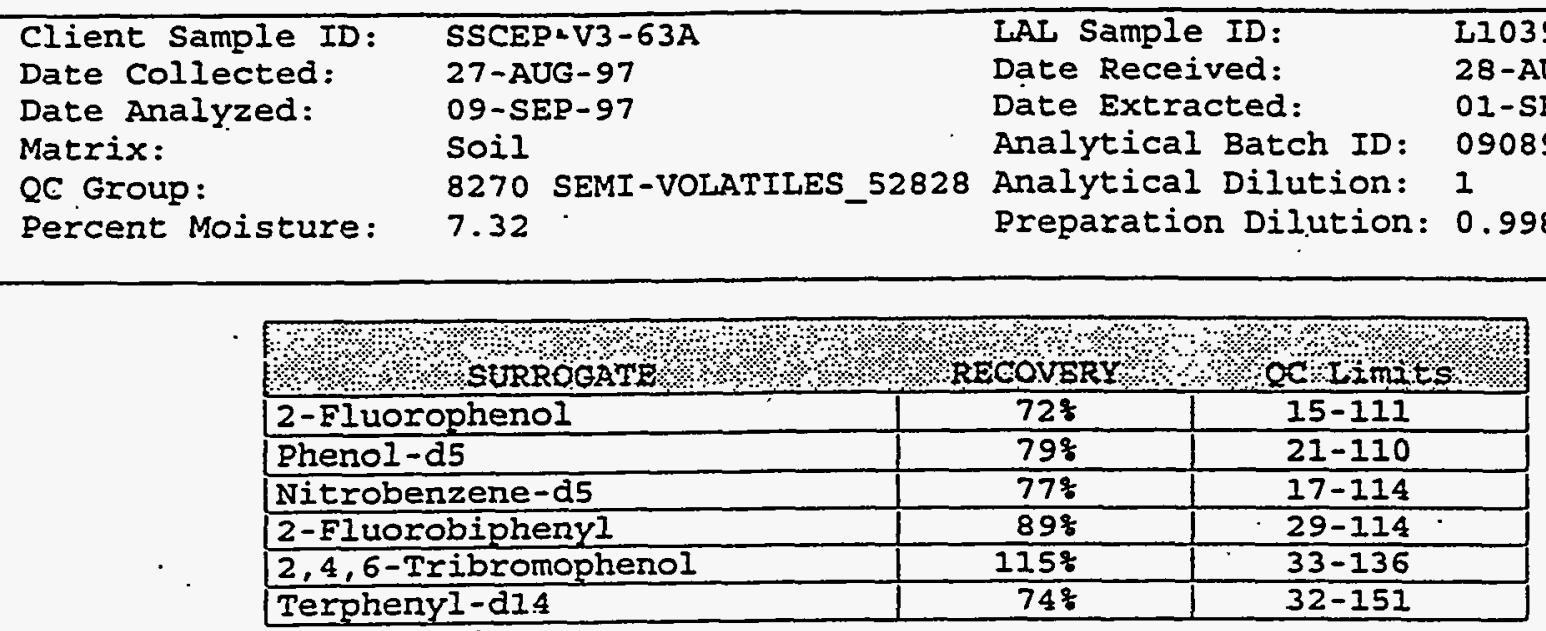

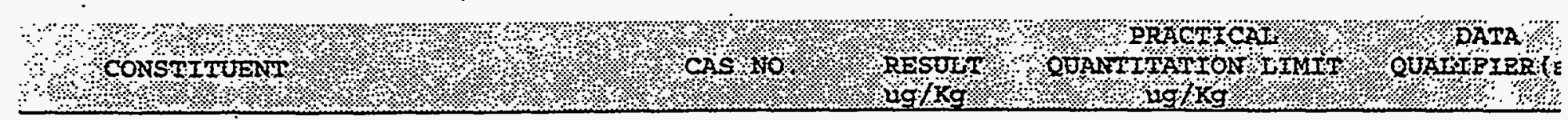

Phenol

bis (2-Chloroethyl) ether

2-Chlorophenol.

1,3-Dichlorobenzene

1,4-Dichlorobenzene

Benzyl alcohol

1,2-Dichlorobenzene

2-Methylphenol

bis (2-chloroisopropyl) ether

4-Methylphenol

N-Nitroso-di-n-propylamine

Hexachloroethane

Nitrobenzene

Isophorone

2-Nitrophenol

2,4-Dimethylphenol

Benzoic acid

bis (2-Chloroethoxy) methane

2,4-Dichlorophenol.

2,2,4-Trichlorobenzene

Naphthalene

4-Chloroaniline

Hexachlorobutadiene

4-Chloro-3-methylphenol

2-Methylnaphthalene

Hexachlorocyclopentadiene

2, 4, 6-Trichlorophenol

2,4,5-Trichlorophenol

2-Chloronaphthalene

2-Nitroaniline

Dimethylphthalate

Acenaphthylene

2,6-Dinitrotoluene

3-Nitroaniline

Acenaphthene

2,4-Dinitrophenol

4-Nitrophenol

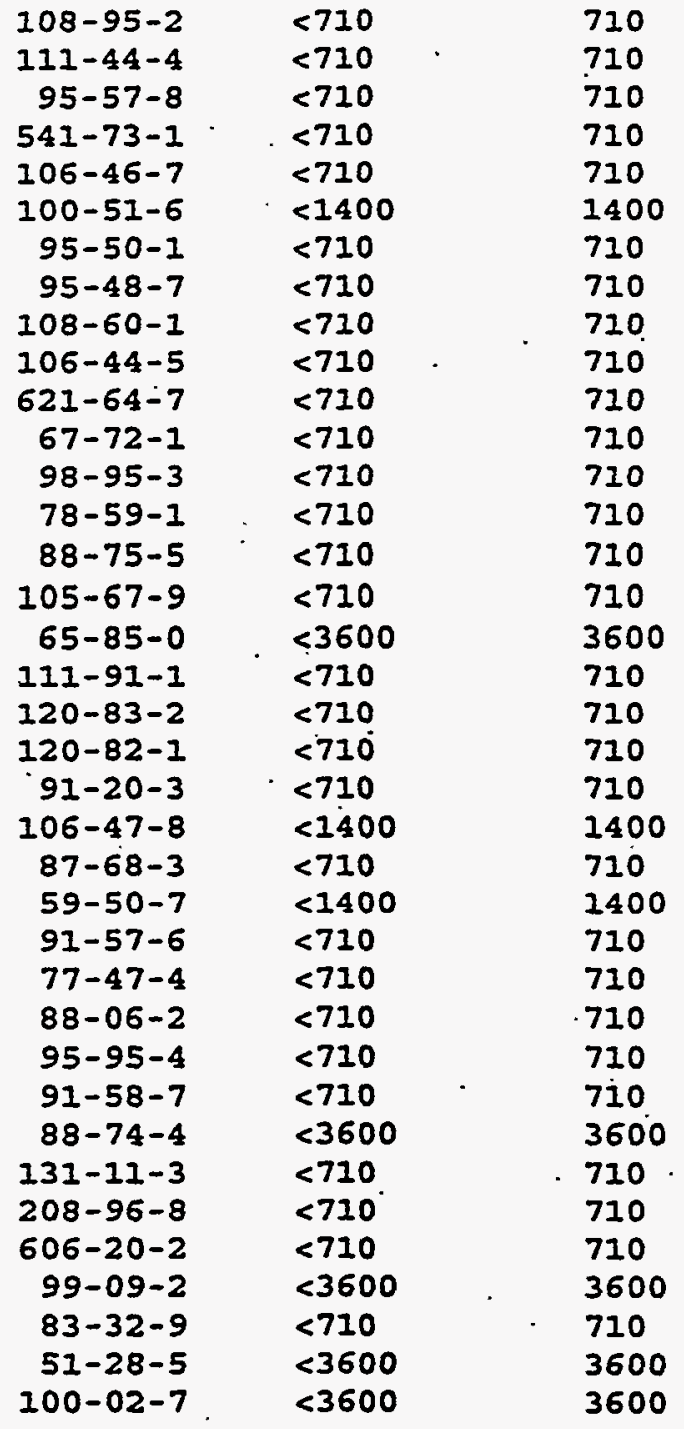

10395-55

AUG-97

090897-8270-K

0.998 


\section{I ¿ LABORATORIES}

[-VOLATILE ORGANICS BY GC/MS

, SEMI-VOLATILES

\begin{tabular}{|c|c|c|c|}
\hline $\begin{array}{l}\text { Client Sample ID: } \\
\text { Date Collected: } \\
\text { Date Analyzed: } \\
\text { Matrix: } \\
\text { QC Group: } \\
\text { Percent Moisture: }\end{array}$ & $\begin{array}{l}\text { SSCEP.V3-63A } \\
27-A U G-97 \\
\text { 09-SEP-97 } \\
\text { SOII } \\
8.270 \text { SEMI-VOLATILES_52828 } \\
7.32\end{array}$ & $\begin{array}{l}\text { LAI Sample ID: } \\
\text { Date Received: } \\
\text { Date Extracted: } \\
\text { Analytical Batch ID: } \\
\text { Analytical Dilution: } \\
\text { Preparation Dilution: }\end{array}$ & $\begin{array}{l}\text { L10395- } 55 \\
28-A U G-97 \\
01-S E P-97 \\
090897-8270-K \\
1 \\
0.998\end{array}$ \\
\hline
\end{tabular}

Dibenzofuran

2,4-Dinitrotoluene

Diethylphthalate

4-Chlorophenyl-phenylether

Fluorene

4-Nitroaniline

4,6-Dinitro-2-methylphenol

N-Nitrosodiphenylamine (1)

4-Bromophenyl-phenylether

Hexachlorobenzene

Pentachlorophenol

Phenanthrene

Anthracene

Carbazole

Di-n-butylphthalate

Fluoranthene

- Pyrene

Butylbenzylphthalate 3,3'-Dichlorobenzidine Benzo (a) anthracene

Chrysene

bis (2-Ethylhexyl) phthalate

Di-n-octylphthalate

Benzo (b) fluoranthene

Benzo (k) fluoranthene

Benzo (a) pyrene

Indeno $(1,2,3-c d)$ pyrene

Dibenz $(a, h)$ anthracene

Benzo $(g, h, i)$ perylene

$\begin{array}{rll}132-64-9 & <710 & 710 \\ 121-14-2 & <710 & 710 \\ 84-66-2 & <710 & 710 \\ 7005-72-3 & <710 & 710 \\ 86-73-7 & <710 & 710 \\ 100-01-6 & <3600 & 3600 \\ 534-52-1 & <3600 & 3600 \\ 86-30-6 & <710 & 710 \\ 101-55-3 & <710 & 710 \\ 118-74-1 & <710 & 710 \\ 87-86-5 & <3600 & 3600 \\ 85-01-8 & <710 & 710 \\ 120-12-7 & <710 & 710 \\ 86-74-8 & <710 & 710 \\ 84-74-2 & <710 & 710 \\ 206-44-0 & <710 & 710 \\ 129-00-0 & <710 & 710 \\ 85-68-7 & <710 & 710 \\ 91-94-1 & <1400 & 1400 \\ 56-55-3 & <710 & 710 \\ 218-01-9 & <710 & 710 \\ 117-81-7 & <710 & 710 \\ 117-84-0 & <710 & 710 \\ 205-99-2 & <710 & 710 \\ 207-08-9 & <710 & 710 \\ 50-32-8 & <710 & 710 \\ 193-39-5 & <710 & 710 \\ 53-70-3 & <710 & 710 \\ 191-24-2 & <710 & 710\end{array}$




\section{LAS LABORATORIES}

TOTAL PETROLEUM HYDROCARBONS (TPH)
8015M - TPH

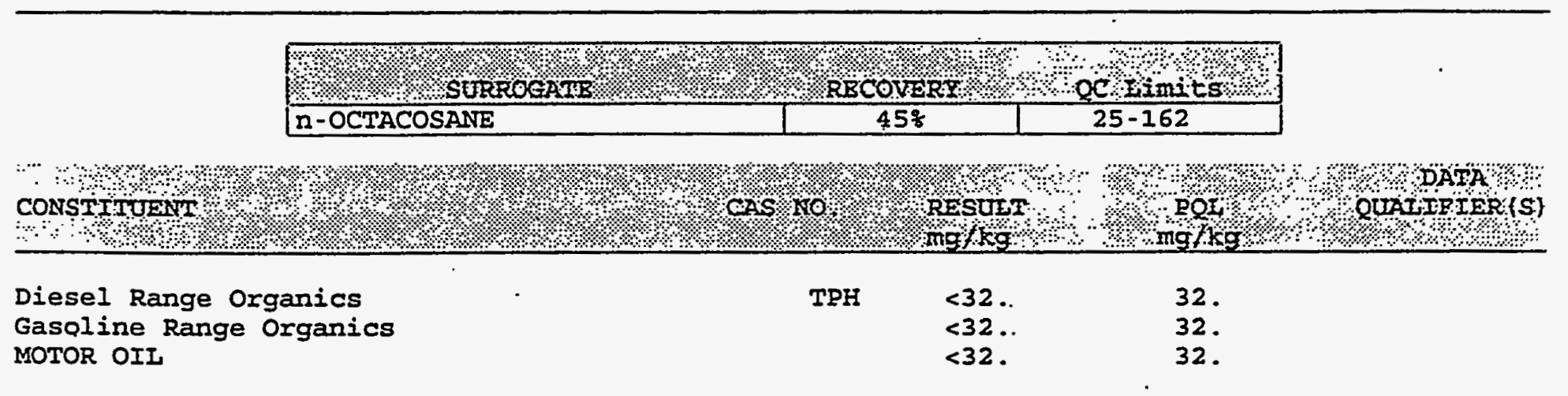

IAS Sample ID:

Analytical Batch ID: 091597-8015-D-3

Analytical Dilution: 1

Preparation Dilution: 1.0

QC Group: . 8015M - TPH_53132

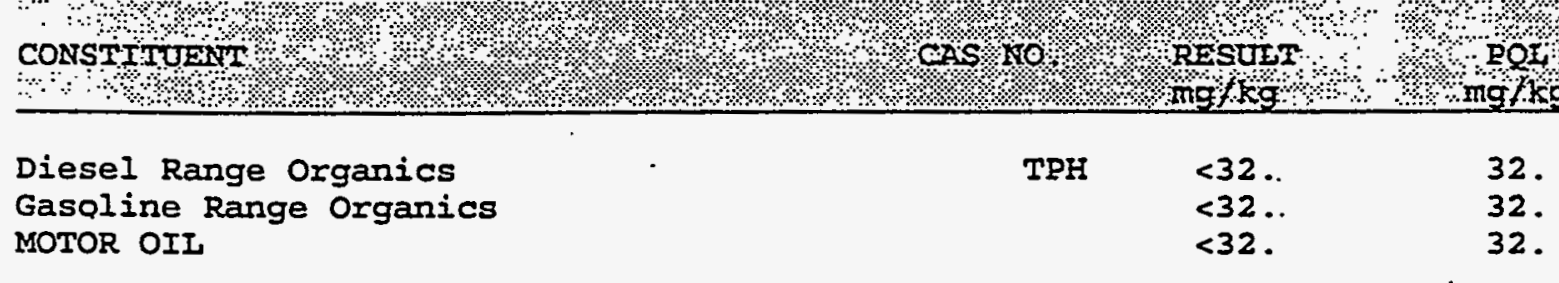




\section{LABORATORIES}

\section{PETROLEUM HYDROCARBONS (TPH)}

M - TPH

- Iient Sample ID: ate Collected: Date Analyzed:

nate Extracted: atrix: -'ercent Moisture:

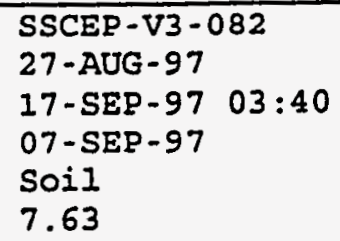
s. I Range Organics sline Range Organics J" OIL
TPEF
$<32$
$<32$.
$<32$.
32.
32.
32.

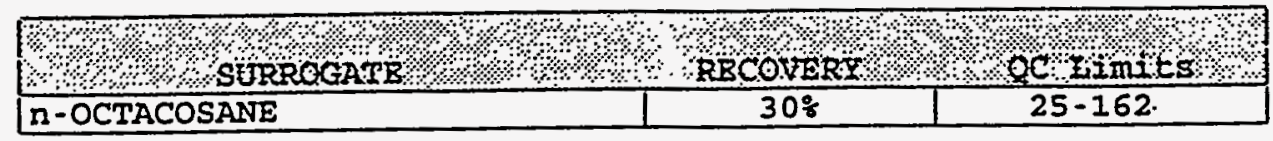




\section{LAS LABORATORIES}

TOTAL PETROLEUM HYDROCARBONS (TPH) 8015M - TPH

$\begin{array}{llll}\text { Client Sample ID: } & \text { SSCEP-V3-108 } & \text { LAS Sample ID: } & \text { L10395-76 } \\ \text { Date Collected: } & 27-\text { AUG-97 } & \text { Date Received: } & 28-A U G-97 \\ \text { Date Analyzed: } & 17-\text { SEP-97 04:25 } & \text { Analytical Batch ID: } 091597-8015-D-3 \\ \text { Date Extracted: } & 07-\text { SEP-97 } & \text { Analytical Dilution: } 1 \\ \text { Matrix: } & \text { Soil } & \text { Preparation Dilution: 0.99 } \\ \text { Percent Moisture: } & 7.45 & \text { QC Group: } & \end{array}$

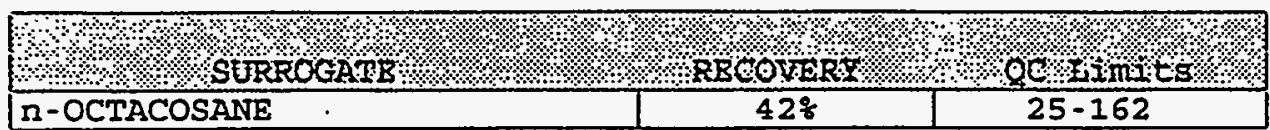

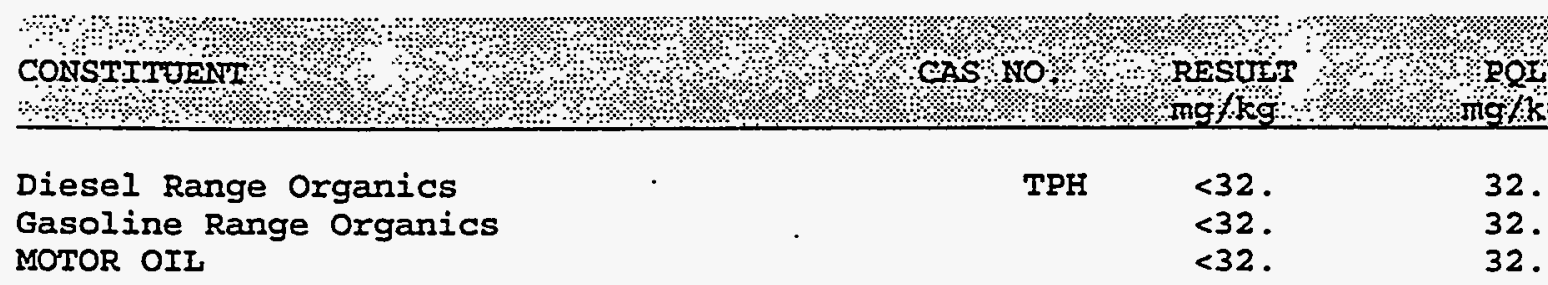

32.

32 .

32 . 


\section{: LABORATORIES}

A PETROLEUM HYDROCARBONS (TPH)

M - TPH

- Tient Sample ID: wate Collected:

Date Analyzed:

-ate Extracted:

atrix:

percent Moisture:

SSCEP-V3-115
$27-A U G-97$
$21-$ SEP-97 00:03
$07-S E P-97$
Soil
6.89

TAS Sample ID:

Date Received:

Analytical Batch ID: 091797-8015-D-2

Analytical Dilution: 1

Preparation Dilution: 0.99

QC Group:
I10395-100

28-AUG-97

8015M - TPH_53158

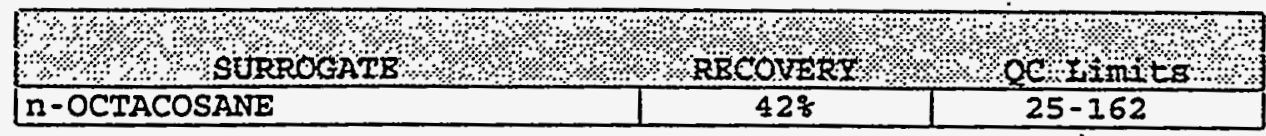

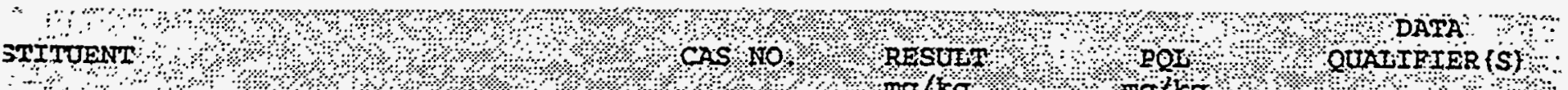

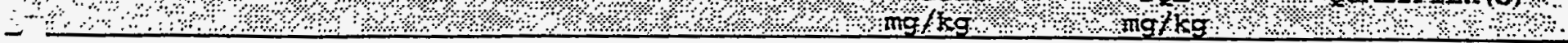

St- Range Organics sline Range Organics 3 OII
TPH: $\quad \begin{aligned} & <32 . \\ & <32 .\end{aligned}$

$<32$.

32 .

32 .

32 .

000986 


\section{$\theta$ \\ LAS LABORATORIES}

6

TOTAL PETROLEUM HYDROCARBONS (TPH) 8015M - TPH

\begin{tabular}{llll} 
& & & \\
\hline Client Sample ID: & SSCEP-V4-115 & LAS Sample ID: & II0479-7 \\
Date Collected: & 09-SEP-97 & Date Received: & 10-SEP-97 \\
Date Analyzed: & $28-$ SEP-97 & Analytical Batch ID: 092597-8015-D-3 \\
Date Extracted: & $12-$ SEP-97 & Analytical Dilution: 1 \\
Matrix: & Soil & Preparation Dilution: 0.99 \\
Percent Moisture: & 10.58 & QC Group: & 8015M - TPH_53397
\end{tabular}

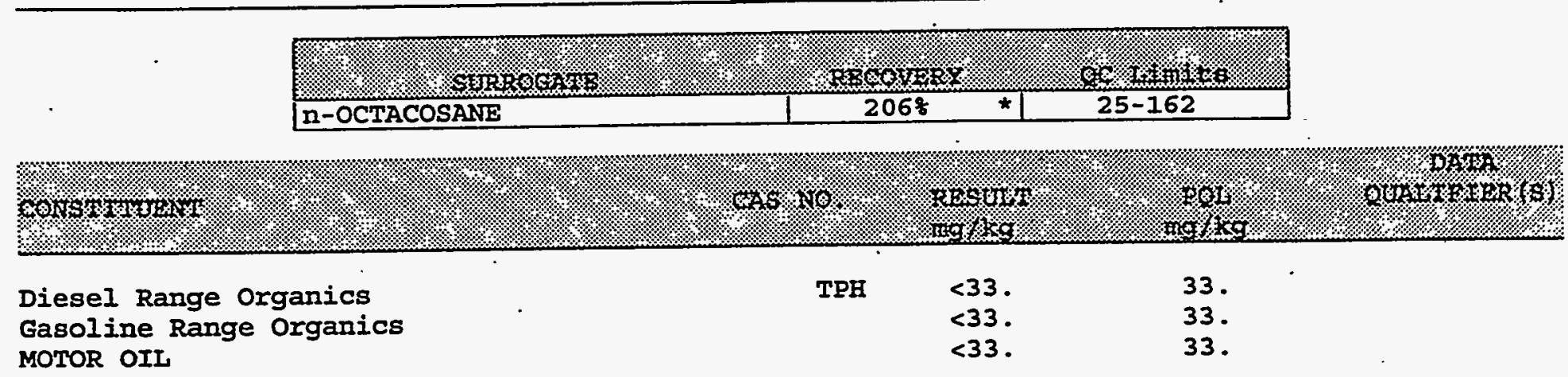




\section{AAS LABORATORIES}

OLATIIE ORGANICS BY GC/MS 260 VOLATILES

Client Sample ID: Date Collected: Date Analyzed: Matrix: Percent Moisture:
SSCEP-V3-115A

27-AUG-97

09-SEP-97 $10: 33$

Soil

5.97
LAS Sample ID:

Date Received:

Analytical Dilution: 1

Analytical Batch ID: 090997-8260-E1

Preparation Dilution: 1.00
I10395-46

28-AUG - 97

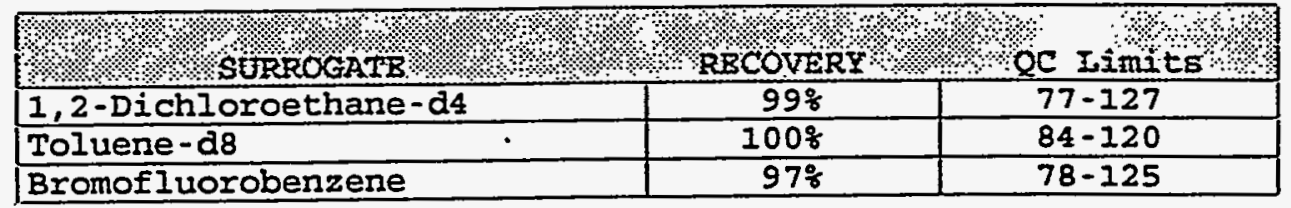

\section{2} ONSTITUENT $\because$ \%े

shloromethane

rinyl Chloride

uromomethane

thloroethane

irichlorofluoromethane

icetone

., 1-Dichloroethene

:arbon Disulfide

1ethylene chloride

:rans-1,2-Dichloroethene

Tinyl Acetate

-,1-Dichloroethane

?-Butanone

iis-1,2-Dichloroethene

thloroform

?-Hexanone

1, 1,1-Trichloroethane

Jarbon tetrachloride

L,2-Dichloroethane

3enzene

Irichloroethene

1,2-Dichloropropane

3romodichloromethane

2-Chloroethylvinylether

1 -Methyl-2-Pentanone

=is-1,3-Dichloropropene

roluene

=rans-1,3-Di chloropropene

1,1,2-Trichloroethane

retrachloroethene

Jibromochloromethane

Ihlorobenzene

Ethylbenzene

$n, p$-Xylene

o-Xylene

Styrene

Bromoform

$1,1,2,2$-Tetrachloroethane

1,3-Dichlorobenzene

1,4-Dichlorobenzene

1,2-Dichlorobenzene
CAS 20

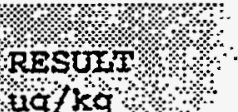

\%॥\%

pois

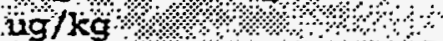

5.3

5.3

5.3

5.3

5.3

11.

5.3

5.3

5.3

5.3

11.

5.3

11.

5.3

5.3

11.

5.3

5.3

5.3

5.3

5.3

5.3

5.3

21.

11.

5.3

5.3

5.3

5.3

5.3

5.3

5.3

5.3

5.3

5.3

5.3

5.3

5.3

5.3

5.3

5.3 


\section{LABORATORIES}

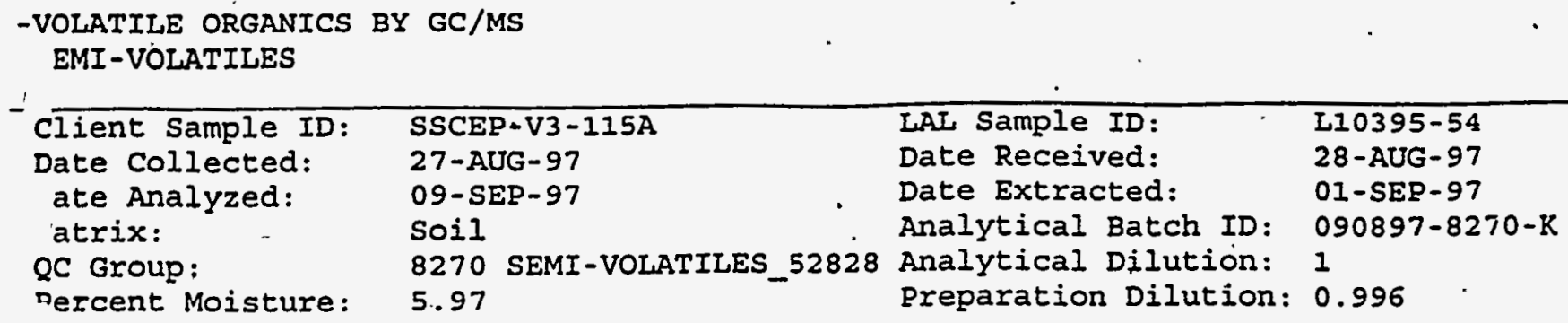

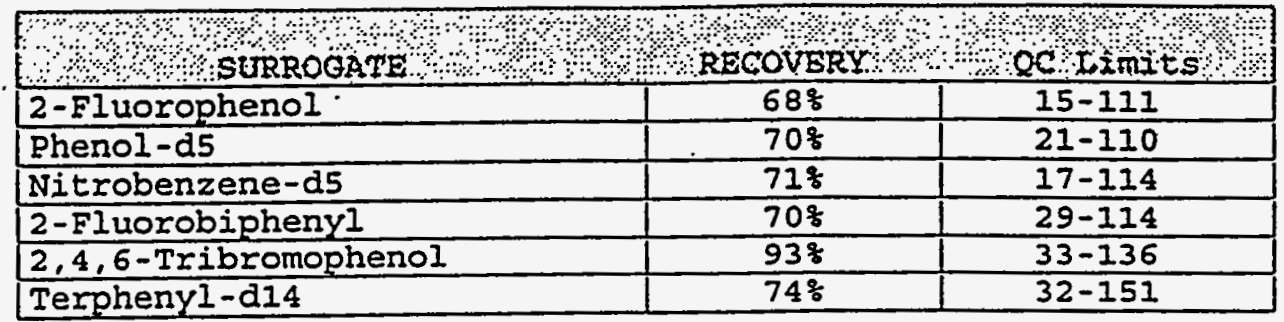

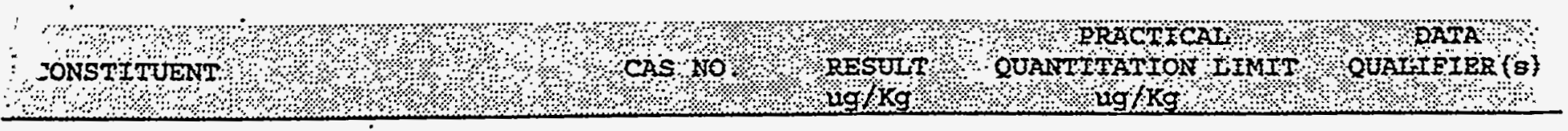

?henol

ois (2-Chloroethyl) ether

2-Chlorophenol

1,3-Dichlorobenzene

1,4-Dichlorobenzene

Benzyl alcohol

1,2-Dichlorobenzene

2-Methylphenol

bis (2-chloroisopropyl) ether

4-Methylphenol

N-Nitroso-di-n-propylamine

Hexachloroethane

Nitrobenzene

Isophorone

2-Nitrophenol

2,4-Dimethylphenol

Benzoic acid

bis (2-Chloroethoxy) methane .

2,4-Dichlorophenol

1,2,4-Trichlorobenzene

Naphthalene

4-Chloroaniline

Hexachlorobutadiene

4-Chloro-3-methylphenol

2-Methylnaphthalene

Hexachlorocyclopentadiene

2,4,6-Trichlorophenol

2,4,5-Trichlorophenol

2-Chloronaphthalene

2-Nitroaniline

Dimethylphthalate

Acenaphthylene

2,6-Dinitrotoluene

3-Nitroaniline

Acenaphthene

2,4-Dinitrophenol

4-Nitrophenol

\begin{tabular}{|c|c|c|}
\hline $108-95-2$ & $<700$ & 700 \\
\hline $111-44-4$ & $<700$ & 700 \\
\hline $95-57-8$ & $<700$ & 700 \\
\hline $541-73-1$ & $<700$ & 700 \\
\hline $106-46-7$ & $<700$ & 700 \\
\hline $100-51-6$ & $<1400$ & 1400 \\
\hline $95-50-1$ & $<700$ & 700 \\
\hline $95-48-7$ & $<700$ & 700 \\
\hline $108-60-1$ & $<700$ & 700 \\
\hline $106-44-5$ & $<700$ & 700 \\
\hline $621-64-7$ & $<700$ & 700 \\
\hline $67-72-1$ & $<700$ & 700 \\
\hline $98-95-3$ & $<700$ & 700 \\
\hline $78-59-1$ & $<700$ & 700 \\
\hline $88-75-5$ & $<700$ & 700 \\
\hline $105-67-9$ & $<700$ & 700 \\
\hline $65-85-0$ & $<3500$ & 3500 \\
\hline $111-91-1$ & $<700$ & 700 \\
\hline $120-83-2$ & $<700$ & 700 \\
\hline $120-82-1$ & $<700^{\circ}$ & 700 \\
\hline $91-20-3$ & $<700$ & 700 \\
\hline $106-47-8$ & $<1400$ & 1400 \\
\hline $87-68-3$ & $<700$ & 700 \\
\hline $59-50-7$ & $<1400$ & 1400 \\
\hline $91-57-6$ & $<700$ & 700 \\
\hline $77-47-4$ & $<700$ & 700 \\
\hline $88-06-2$ & $<700$ & 700 \\
\hline $95-95-4$ & $<700$ & 700 \\
\hline $91-58-7$ & $<700$ & 700 \\
\hline $88-74-4$ & $<3500$ & 3500 \\
\hline $131-11-3$ & $<700$ & 700 \\
\hline $208-96-8$ & $<700$ & 700 \\
\hline $606-20-2$ & $<700$ & 700 \\
\hline $99-09-2$ & $<3500$ & 3500 \\
\hline $83-32-9$ & $<700$ & 700 \\
\hline $51-28-5$ & $<3500$ & 3500 \\
\hline $.100-02-7$ & $<3500$ & 3500 \\
\hline
\end{tabular}

700

700

1400

700

700

700

700

700

700

700

700

1400

700

700

700

700

700

700

700

3500

3500

3500
000279 


\section{LAS LABORATORIES}

;EMI-VOLATILE ORGANICS BY GC/MS 1270 SEMI-VOLATILES

Client sample ID:

Date Collected:

Date Analyzed:

Matrix:

QC Group:

Percent Moisture:
SSCER-V3-115A

27-AUG- 97

09-SEP-97

Soil

8270 SEMI-VOLATILES_52828

5.97
LAI Sample ID:

Date Received:

Date Extracted:

Analytical Batch ID:

Analytical Dilution:

preparation Dilution:
L10395-54

28-AUG- 97

$01-S E P-97$

090897-8270-K

1

0.996

आ.

औ CONSTITUSNT

औे

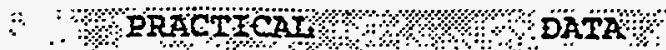

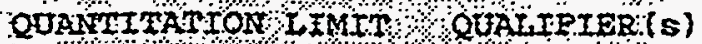
$\mathrm{m} \mathrm{ug}: \mathrm{Kg}+\%$

Dibenzofuran

2,4-Dinitrotoluene

Diethylphthalate

4-Chlorophenyl-phenylether

Fluorene

4-Nitroaniline

4, 6-Dinitro-2-methylphenol

N-Nitrosodiphenylamine (I)

4-Bromophenyl-phenylether

Hexachlorobenzene

Pentachlorophenol

Phenanthrene

Anthracene

Carbazole

Di-n-butylphthalate

Fluoranthene

Pyrene

Butylbenzylphthalate

3, 3'-Dichlorobenzidine

Benzo (a) anthracene

Chrysene

bis (2-Ethyl hexyl) phthalate

Di-n-octylphthalate

Benzo (b) fluoranthene

Benzo (k) fluoranthene

Benzo (a) pyrene

Indeno $(1,2,3$-cd) pyrene

Dibenz $(a, h)$ anthracene

Benzo $(g, h, i)$ perylene

$132-64-9$
$121-14-2$
$84-66-2$
$7005-72-3$
$86-73-7$
$100-01-6$
$534-52-1$
$86-30-6$
$101-55-3$
$118-74-1$
$87-86-5$
$85-01-8$
$120-12-7$
$86-74-8$
$84-74-2$
$206-44-0$
$129-00-0$
$85-68-7$
$91-94-1$
$56-55-3$
$218-01-9$
$117-81-7$
$117-84-0$
$205-99-2$
$207-08-9$
$50-32-8$
$193-39-5$
$53-70-3$
$191-24-2$

$<700$

$<700$

$<700$

$<700$

$<700$

$<3500$

$<3500$

$<700$

$<700$

$<700$

$<3500$

$<700$

$<700$.

$<700$

$<700$

$<700$

$<700$

$<700$

$<1400$

$<700$

$<700$

$<700$

$<700$

$<700$

$<700$

$<7.00$

$<700$

$<700$

$<700$ 


\section{ᄃ LABORATORIES}

I PETROLEUM HYDROCARBONS (TPH)

H - TPH

\begin{tabular}{llll}
\hline lient Sample ID: & SSCEP-V3:116 & IAS Sample ID: & I10395-77 \\
ate Collected: & $27-A U G-97$ & Date Received: & $28-A U G-97$ \\
Date Analyzed: & $17-$ SEP-97 05:09 & Analytical Batch ID: 091597-8015-D-3 \\
nate Extracted: & $07-$ SEP-97 & Analytical Dilution: 1 \\
atrix: & Soil & Preparation Dilution: 1:0 \\
-ercent Moisture: & 6.81 & QC Group: & 8015M - TPH_53132 \\
\hline
\end{tabular}

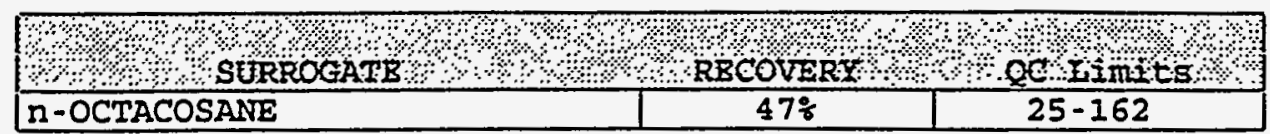

TIIUENT

?

Range Organics

Iine Range Organics

$\Gamma$ OIL

TPH

$<32$.

$<32$.

$<32$.
32 .

32 .

32 . 


\section{LAS LABORATORIES}

TOTAL PETROLEUM HYDROCARBONS (TPH)

8015M - TPH

$\begin{array}{ll}\text { Client Sample ID: } & \text { SSCEP-V4-116 } \\ \text { Date Collected: } & 09-\text { SEP-97 } \\ \text { Date Analyzed: } & 28-\text { SEP-97 } \\ \text { Date Extracted: } & 12-\text { SEP-97 } \\ \text { Matrix: } & \text { Soil } \\ \text { Percent Moisture: } & 11.06\end{array}$
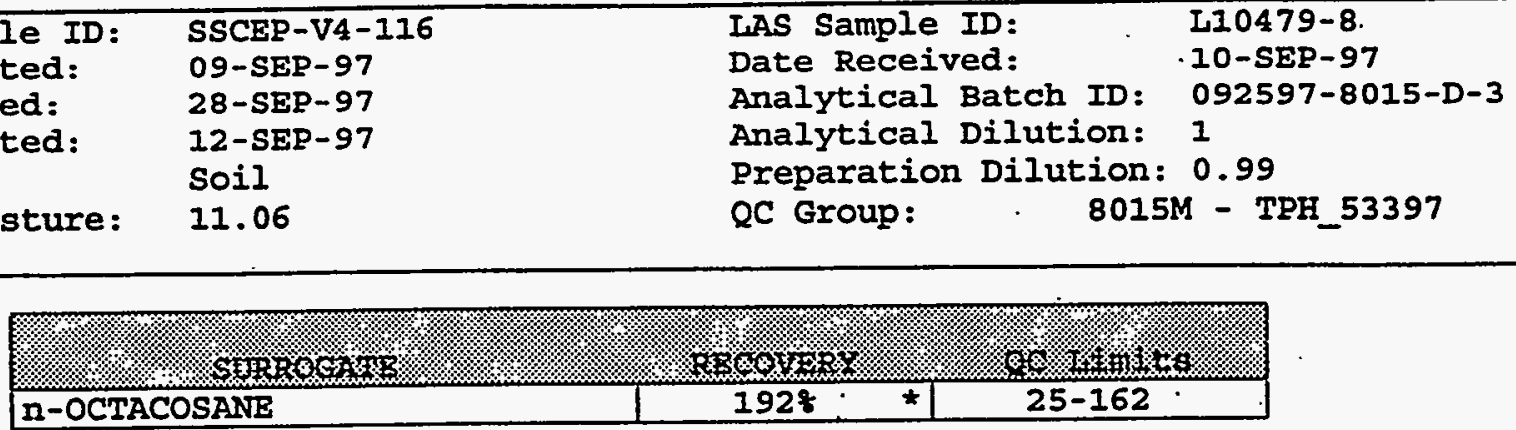

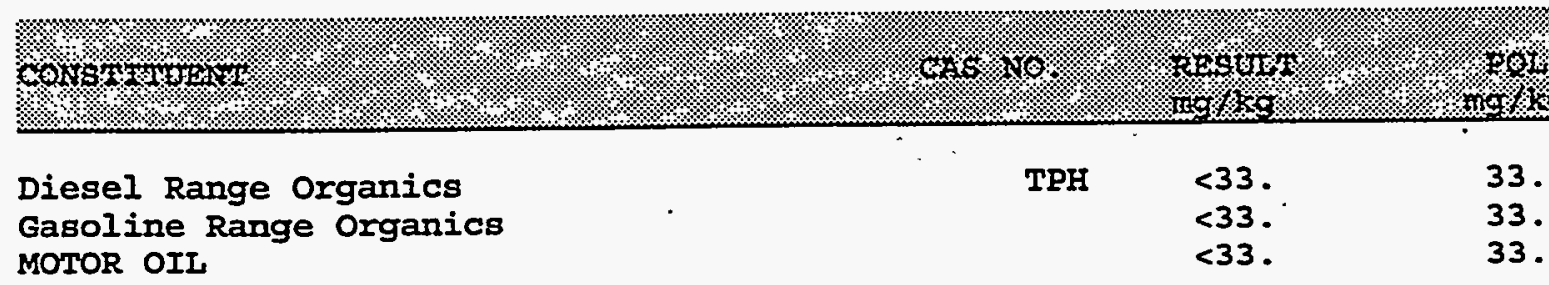




\section{S LABORATORIES}

IILE ORGANICS BY GC/MS

\section{PMLATILES}

$\begin{array}{ll}\text { CLient Sample ID: } & \text { SSCEP-V3-119A } \\ \text { Date Collected: } & 27-\text { AUG-97. } \\ \text { I te Analyzed: } & 09-\text { SEP-97 11:51 } \\ \text { I.trix: } & \text { Soil } \\ \text { Percent Moisture: } & 5.78\end{array}$

IAS Sample ID:

L10395-50

Date Received: 28-AUG-97

Analytical Dilution: 1

Analytical Batch ID: 090997-8260-E1

Percent Moisture: $\quad 5.78$

Preparation Dilution: 1.00

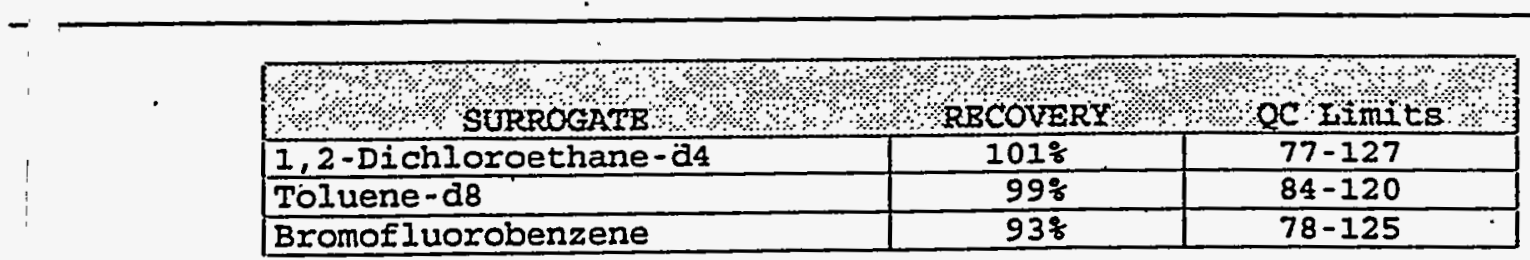

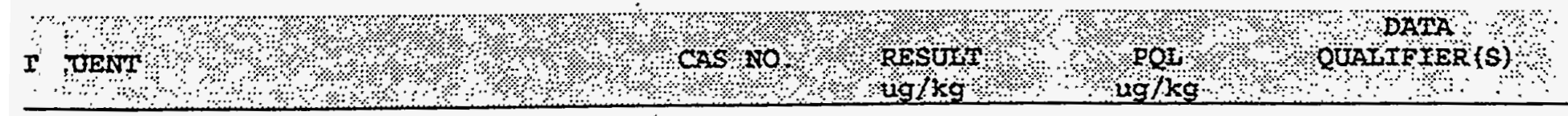

r) nethane

1 Wloride

omethane

rnathane

$h$ jrofluoromethane

o...

Dichloroethene

o Disulfide

$y$ ene Chloride

5-1,2-Dichloroethene

1 Acetate

I chloroethane

$t$ none

1,2-Dichloroethene

roform

$x$ none

1 Trichloroethane

on tetrachloride

I'chloroethane

.e $\mathrm{e}$

:tsoroethene

Dichloropropane

ic ichloromethane

1] roethylvinylether

:thyl-2-Pentanone

1.3-Dichloropropene

If $e$

18 1,3-Dichloropropene

2-Trichloroethane

arhloxoethene

$\because$ lochloromethane

xibenzene

Ilbenzene

$\because$ lene

$r$ ine

sene

noform

$\therefore$ 2-Tetrachloroethane

$\therefore$.chlorobenzene

-Dichlorabenzene

-nichlorobenzene
$<5.3$

$<5.3$

$<5.3$

$<5.3$

$<5.3$

$<11$.

$<5.3$

$<5.3$

$<5.3$

$<5.3$

$<11$.

$<5.3$

$<11$.

$<5.3$

$<5.3$

$<11$.

$<5.3$

$<5.3$

$<5.3$

$<5.3$

$<5.3$

$<5.3$

$<5.3$

$<21$.

$<11$.

$<5.3$

$<5.3$

$<5.3$

$<5.3$

$<5.3$

$<5.3$

$<5.3$

$<5.3$

$<5.3$

$<5.3$

$<5.3$

$<5.3$

$<5.3$

$<5.3$

$<5.3$

$<5.3$
5.3

5.3

5.3

5.3

5.3

11.

5.3

5.3

5.3

5.3

11 .

5.3

11.

.5 .3

5.3

11.

5.3

5.3

5.3

5.3

5.3

5.3

5.3

21.

11.

5.3

5.3

5.3

5.3

5.3

5.3

5.3

5.3

5.3

5.3

5.3

5.3

5.3

5.3

5.3

5.3 


\section{LAS LABORATORIES}

SEMI-VOLATILE ORGANICS BY GC/MS 8270 SEMI-VOLATILES

Client Sample ID: ' SSCEP.-V3-119A

Date Collected:

Date Analyzed:

Matrix:

QC Group:

Percent Moisture:

27-AUG-97

09-SEP-97

Soil

5.78
8270 SEMI-VOLATILES 52828 Analytical Dilution:
LAI Sample ID:

Date Received:

Date Extracted:

Analytical Batch ID

Preparation Dilution:
L10395-56

28-AUG-97

01-SEP-97

090897-8270-K

1

0.998

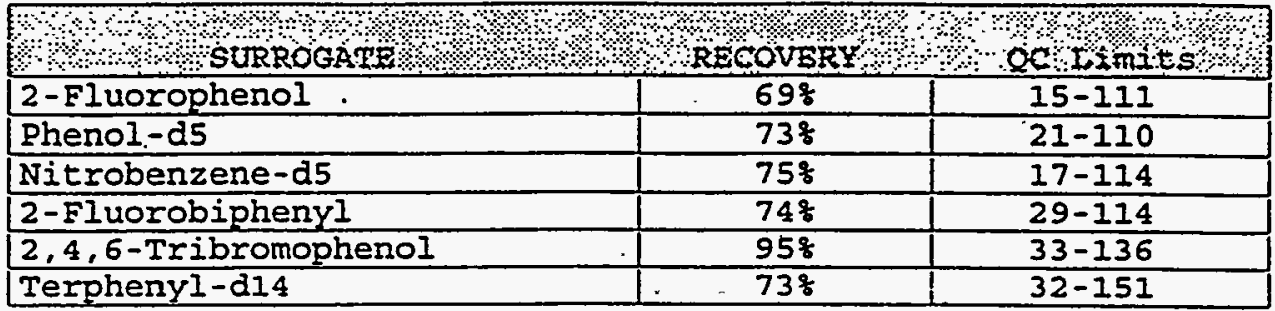

\section{CONSTITUENT}

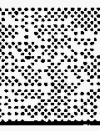

Phenol

bis (2-Chloroethyl) ether

2-Chlorophenol

1,3-Dichlorobenzene

1,4-Dichlorobenzene

Benzyl alcohol

1,2-Dichlorobenzene

2-Methylphenol

bis (2-chloroisopropyl) ether

4-Methylphenol

N-Nitroso-di-n-propylamine

Hexachloroethane

Nitrobenzene

Isophorone

2-Nitrophenol

2,4-Dimethylphenol

Benzoic acid

bis (2-Chloroethoxy) methane

2,4-Dichlorophenol

1,2,4-Trichlorobenzene

Naphthalene

4-Chloroaniline

Hexachlorobutadiene

4-Chloro-3-methylphenol

2-Methylnaphthalene

Hexachlorocyclopentadiene

2,4,6-Trichlorophenol

2,4,5-Trichlorophenol

2-Chloronaphthalene

2-Nitroaniline

Dimethylphthalate

Acenaphthylene

2,6-Dinitrotoluene

3-Nitroaniline

Acenaphthene

2,4-Dinitrophenol

4-Nitrophenol

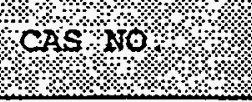

DRACTICAL

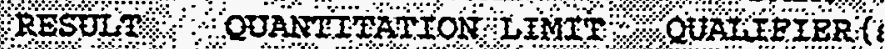
ug/ $\mathrm{xg} / \mathrm{H} /$, ug $/ \mathrm{kg}$

\begin{tabular}{|c|c|c|}
\hline $108-95-2$ & $<700$ & 700 \\
\hline $111-44-4$ & $<700$ & 700 \\
\hline $95-57-8$ & $<700$ & 700 \\
\hline $541-73-1$ & $<700$ & 700 \\
\hline $106-46-7$ & $<700$ & 700 \\
\hline $100-51-6$ & $<1400$ & 1400 \\
\hline $95-50-1$ & $<700$ & 700 \\
\hline $95-48-7$ & $<700$ & 700 \\
\hline $108-60-1$ & $<700$ & 700 \\
\hline $106-44-5$ & $<700$ & 700 \\
\hline $621-64-7$ & $<700$ & 700 \\
\hline $67-72-1$ & $<700$ & 700 \\
\hline $98-95-3$ & $<700$ & 700 \\
\hline $78-59-1$ & $<700$ & 700 \\
\hline $88-75-5$ & $<700$ & 700 \\
\hline $105-67-9$ & $<700$ & 700 \\
\hline $65-85-0$ & $<3500$ & 3500 \\
\hline $111-91-1$ & $<700$ & 700 \\
\hline $120-83-2$ & $<700$ & 700 \\
\hline $120-82-1$ & $<7000$ & 700 \\
\hline $91-20-3$ & $<700$ & 700 \\
\hline $106-47-8$ & $<1400$ & 1400 \\
\hline $87-68-3$ & $<700$ & 700 \\
\hline $59-50-7$ & $<1400$ & 1400 \\
\hline $91-57-6$ & $<700$ & 700 \\
\hline $77-47-4$ & $<700$ & 700 \\
\hline $88-06-2$ & $<700$ & 700 \\
\hline $95-95-4$ & $<700^{\circ}$ & 700 \\
\hline $91-58-7$ & $<700$ & 700 \\
\hline $88-74-4$ & $<3500$ & 3500 \\
\hline $131-11-3$ & $<700$ & 700 \\
\hline $208-96-8$ & $<700$ & 700 \\
\hline $606-20-2$ & $<700$ & 700 \\
\hline $99-09-2$ & $<3500$ & 3500 \\
\hline $83-32-9$ & $<700$ & 700 \\
\hline $51-28-5$ & $<3500$ & 3500 \\
\hline $100-02-7$. & $<3500$ & 3500 \\
\hline
\end{tabular}




\section{LABORATORIES}

-VOLATILE ORGANICS BY GC/MS

- EMI - VOLATILES

\begin{tabular}{llll}
\hline client Sample ID: & SSCEP.-V3-119A & LAL Sample ID: & L10395-56 \\
Date Collected: & $27-A U G-97$ & Date Received: & $28-A U G-97$ \\
ate Analyzed: & $09-S E P-97$ & Date Extracted: & $01-S E P-97$ \\
atrix: & Soil & Analytical Batch ID: $090897-8270-\mathrm{K}$ \\
QC Group: & 8270 SEMI-VOLATILES_52828 Analytical Dilution: 1 \\
Dercent Moisture: & 5.78 & Preparation Dilution: 0.998
\end{tabular}

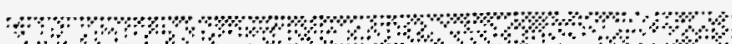

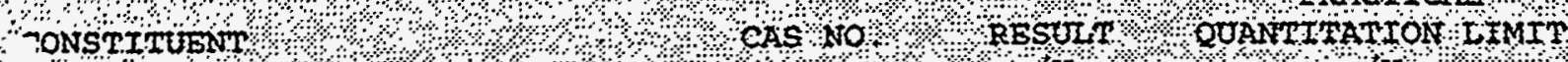
मे०

Dibenzofuran

1,4-Dinitrotoluene

liethylphthalate

4-Chlorophenyl-phenylether

Fluorene

l-Nitroaniline

1, 6-Dinitro-2-methylphenol-

N-Nitrosodiphenylamine (1)

1-Bromophenyl-phenylether

Iexachlorobenzene

zentachlorophenol

Phenanthrene

Inthracene

arbazole

Di-n-butylphthalate

Fluoranthene

Pyrene

3utylbenzylphthalate 3,31-Dichlorobenzidine

Benzo (a) anthracene

Chrysene

bis (2-Ethylhexyl) phthalate

Di-n-octylphthalate

Benzo (b) Eluoranthene

Benzo (k) fluoranthene

Benzo (a) pyrene

Indeno (1,2,3-cd) pyrene

Dibenz $(a, h)$ anthracene

Benzo $(g, h, i)$ perylene

$\begin{array}{rll}132-64-9 & <700 & 700 \\ 121-14-2 & <700 & 700 \\ 84-66-2 & <700 & 700 \\ 7005-72-3 & <700 & 700 \\ 86-73-7 & <700 & 700 \\ 100-01-6 & <3500 & 3500 \\ 534-52-1 & <3500 & 3500 \\ 86-30-6 & <700 & 700 \\ 101-55-3 & <700 & 700 \\ 118-74-1 & <700 & 700 \\ 87-86-5 & <3500 & 3500 \\ 85-01-8 & <700 & 700 \\ 120-12-7 & <700 & 700 \\ 86-74-8 & <700 & 700 \\ 84-74-2 & <700 & 700 \\ 206-44-0 & <700 & 700 \\ 129-00-0 & <700 & 700 \\ 85-68-7 & <700 & 700 \\ 91-94-1 & <1400 & 1400 \\ 56-55-3 & <700 & 700 \\ 218-01-9 & <700 & 700 \\ 117-81-7 & <700 & 700 \\ 117-84-0 & <700 & 700 \\ 205-99-2 & <700 & 700 \\ 207-08-9 & <700 & 700 \\ 50-32-8 & <700 & 700 \\ 193-39-5 & <700 & 700 \\ 53-70-3 & <700 & 700 \\ 191-24-2 & <700 & 700\end{array}$




\section{LAS LABORATORIES}

TOTAL PETROLEUM HYDROCARBONS (TPH) $8015 \mathrm{M}-\mathrm{TPH}$

$\begin{array}{ll}\text { Client Sample ID: } & \text { SSCEP-V3-130 } \\ \text { Date Collected: } & 27-A U G-97 \\ \text { Date Analyzed: } & 20-\text { SEP-97 22:33 } \\ \text { Date Extracted: } & 07-\text { SEP-97 } \\ \text { Matrix: } & \text { Soil } \\ \text { Percent Moisture: } & 8.25\end{array}$

IAS Sample ID:

Date Received:

Analytical Batch ID:

Analytical Dilution:

Analytical Dilution: 1

QC Group:
L10395-98

28-AUG-97

091797-8015-D-2

$8015 \mathrm{M}$ - TPH_53158

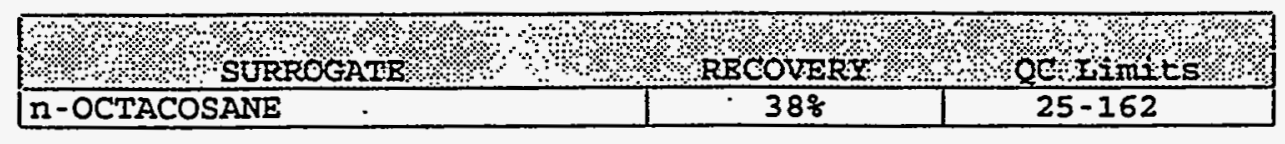

CONSTMENT

Diesel Range Organics. Gasoline Range Organics MOTOR OIL
TPH

$<32$.
$<32$.
44.

32 .

32 .

32 .

\section{4}




\section{IS LABORATORIES}

\section{$\therefore:$ PETROLEUM HYDROCARBONS (TPH)}

5 - TPH

\begin{tabular}{ll}
\hline Client Sample ID: & SSCEP-V3-131 \\
Date Collected: & $27-$ AUG-97 \\
Date Analyzed: & $17-$ SEP-97 09:35 \\
Date Extracted: & $07-$ SEP-97 \\
Matrix: & Soil \\
Percent Moisture: & 5.78
\end{tabular}
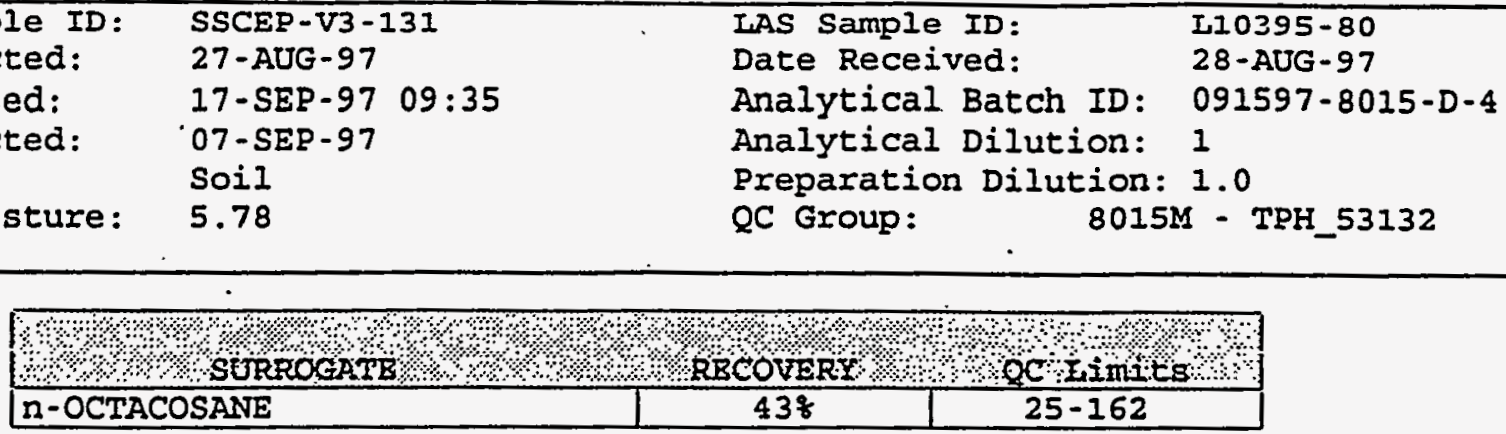

:

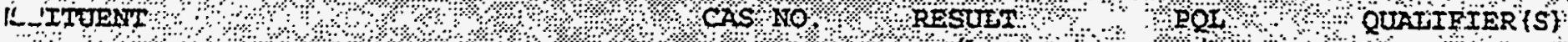

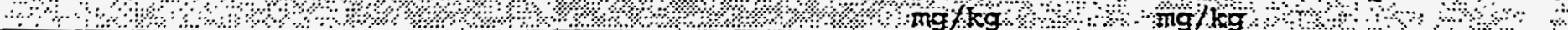

:1 - I. Range Organics iuline Range-Organics OR OII
TPH

$<32$.

$<3 \dot{2}$.

$<32^{\circ}$.

32.

32 .

32 . 


\section{LAS LABORATORIES}

TOTAL PETROLEUM HYDROCARBONS (TPH) $8015 M$ - TPH

$\begin{array}{llll}\text { Client Sample ID: } & \text { SSCEP-V4-131 } & \text { LAS Sample ID: } & \text { LI0479-9 } \\ \text { Date Collected: } & \text { 09-SEP-97 } & \text { Date Received: } & \text { 10-SEP-97 } \\ \text { Date Analyzed: } & 28-S E P-97 & \text { Analytical Batch ID: 092597-8015-D-3 } \\ \text { Date Extracted: } & 12-\text { SEP-9.7 } & \text { Analytical Dilution: 1 } \\ \text { Matrix: } & \text { SOil } & \text { Preparation Dilution: 0.97 } \\ \text { Percent Moisture: } & 10.76 & \text { QC Group: } & \text { 8015M- TPH_53397 }\end{array}$

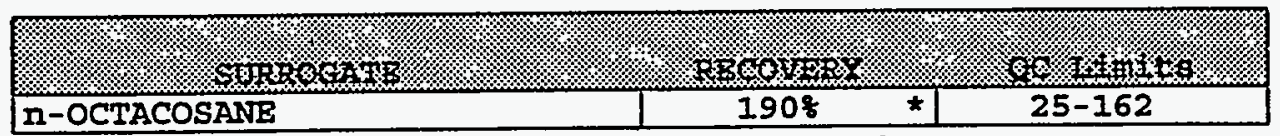

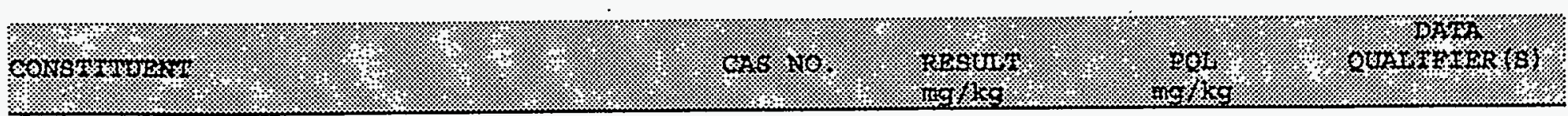

Diesel Range Organics Gasoline Range Organics MOTOR OIL

\section{TPH}

$<33$.

$<33$.

$<33$.

33.

33.

33. 
2 , PETROLEUM HYDROCARBONS (TPH)

$5: 2=\mathrm{TPH}$

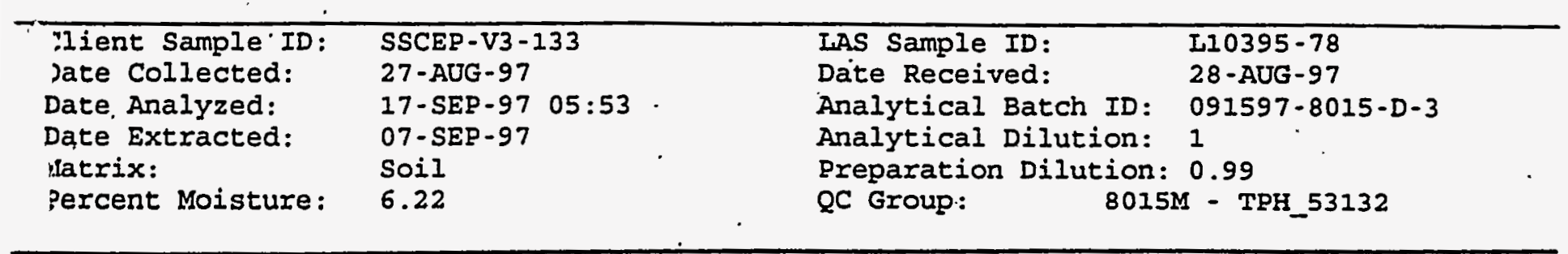

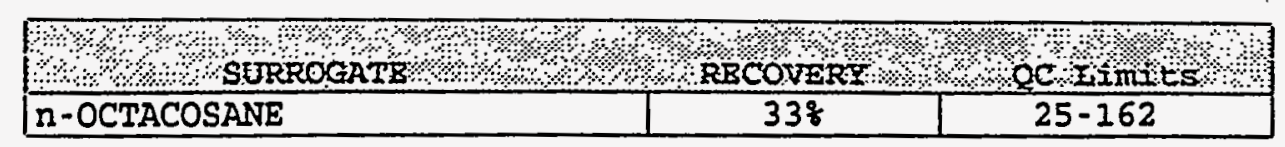

ol SxIMENT

CASHO H RESUt

PQS

QUATIFIER (S)

$\because$

s .I Range Organics oline Range Organics on OIL

$\begin{array}{cll}\text { TPH } & <32 . & 32 . \\ & <32 . & 32 . \\ & <32 . & 32 .\end{array}$

32.

32 . 


\section{LAS LABORATORIES}

TOTAL PETROLEUM HYDROCARBONS (TPH) 8015M - TPH

$\begin{array}{ll}\text { Client Sample ID: } & \text { SSCEP-V3-154 } \\ \text { Date Collected: } & 27-\text { AUG-97 } \\ \text { Date Analyzed: } & 17-\text { SEP-97 08:51 } \\ \text { Date Extracted: } & 07-\text { SEP-97 } \\ \text { Matrix: } & \text { Soil } \\ \text { Percent Moisture: } & 5.68\end{array}$

IAS Sample ID:

L10395-79

Date Received: 28-AUG-97

Analytical Batch ID: 091597-8015-D-4

Analytical Dilution: 1

Preparation Dilution: 1.0

QC Group:

$8015 M$ - TPH_53132

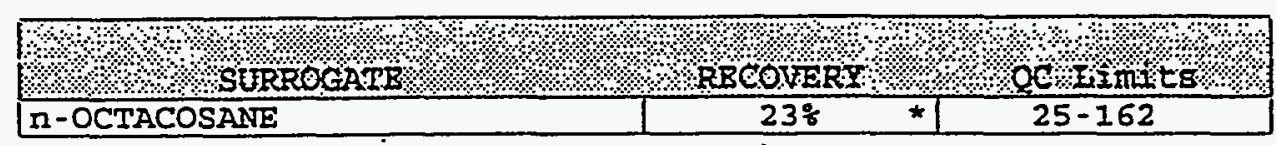

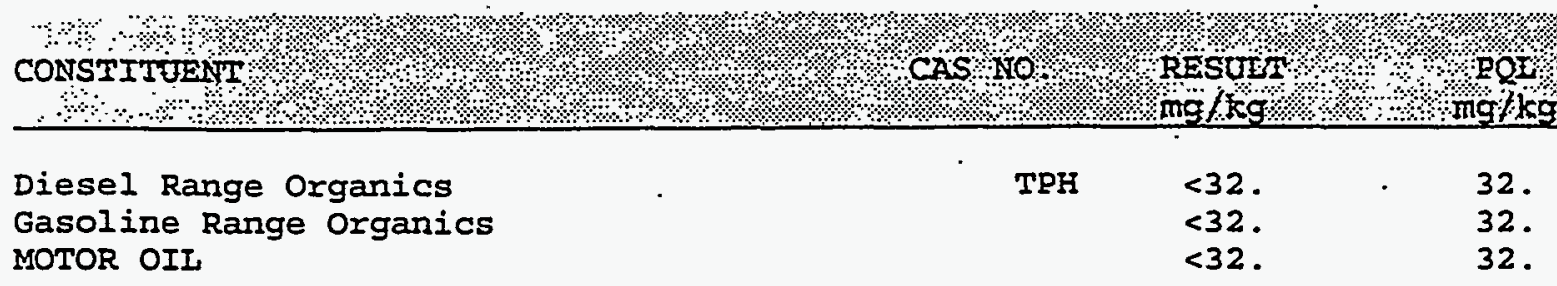




\section{VOLUME 2 APPENDIX B}

TABLE 8 ANALYTICAL DATA 
TABLE 8: NORTH SCEP SPILL H910911D CHARACTERIZATION SAMPLES

\begin{tabular}{|c|c|c|c|c|c|}
\hline 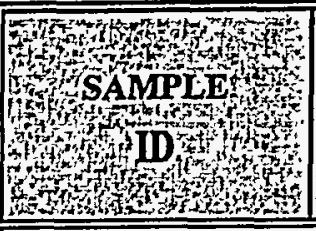 & 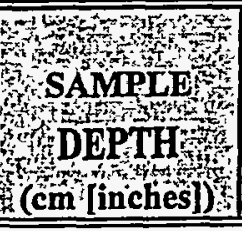 & 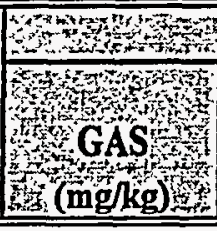 & 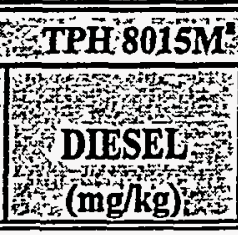 & 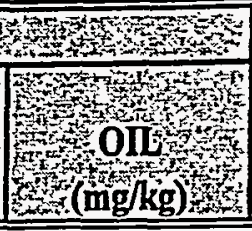 & 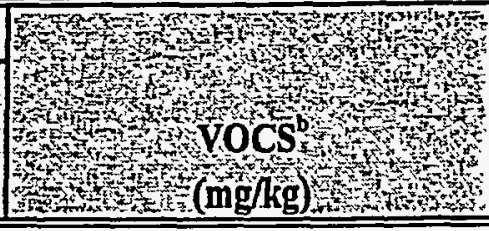 \\
\hline \multicolumn{6}{|c|}{ SAMPLES COLLECTED APRUL 30, 1997} \\
\hline NSCEP-001 & $\begin{array}{c}0-15.2 \\
{[0-6]}\end{array}$ & $\mathrm{ND}$ & $\mathrm{ND}$ & ND & ND \\
\hline NSCEP-004 & $\begin{array}{c}0-15.2 \\
{[0-6]} \\
\end{array}$ & ND & ND & ND & $\overline{N D}$ \\
\hline NSCEP-005 & $\begin{array}{r}0-15.2 \\
{[0-6]} \\
\end{array}$ & $\mathrm{ND}$ & ND & ND & $\mathrm{ND}$ \\
\hline NSCEP-029 & $\begin{array}{c}0-15.2 \\
{[0-6]}\end{array}$ & ND & $\mathrm{ND}$ & ND & ND \\
\hline NSCEP-046 & $\begin{array}{c}0-15.2 \\
{[0-6]}\end{array}$ & ND. & ND & ND & ND \\
\hline NSCEP-060 & $\begin{array}{r}0-15.2 \\
{[0-6]} \\
\end{array}$ & ND & ND & 42 & ND \\
\hline NSCEP-072 & $\begin{array}{c}0-15.2 \\
{[0-6]}\end{array}$ & ND & $\overline{\mathrm{ND}}$ & ND & $\mathrm{ND}$ \\
\hline NSCEP-075 & $\begin{array}{r}0-15.2 \\
{[0-6]} \\
\end{array}$ & ND & $\mathrm{ND}$ & $\mathrm{ND}$ & ND \\
\hline NSCEP-077 & $\begin{array}{c}0-15.2 \\
{[0-6]}\end{array}$ & ND & ND & $\overline{N D}$ & $\overline{\mathrm{ND}}$ \\
\hline NSCEP-084 & $\begin{array}{r}0-15.2 \\
{[0-6]} \\
\end{array}$ & ND & $\overline{\mathrm{ND}}$ & $\mathrm{ND}$ & ND \\
\hline NSCEP-084D & $\begin{array}{r}0-15.2 \\
{[0-6]} \\
\end{array}$ & ND & ND & ND & ND \\
\hline NSCEP-086 & $\begin{array}{c}0-15.2 \\
{[0-6]}\end{array}$ & $\mathrm{ND}$ & $\mathrm{ND}$ & ND & $\mathrm{ND}$ \\
\hline NSCEP-088 & $\begin{array}{r}0-15.2 \\
{[0-6]} \\
\end{array}$ & $\mathrm{ND}$ & $\mathrm{ND}$ & 32 & ND \\
\hline NSCEEP-098 & $\begin{array}{c}0-15.2 \\
{[0-6]} \\
\end{array}$ & ND & $\overline{N D}$ & ND & ND \\
\hline NSCEP-135 & $\begin{array}{r}0-15.2 \\
{[0-6]} \\
\end{array}$ & ND & ND & ND & ND \\
\hline NSCEP-140 & $\begin{array}{c}0-15.2 \\
{[0-6]}\end{array}$ & ND & ND & $\mathrm{ND}$ & ND \\
\hline NSCEP-154 & $\begin{array}{r}0-15.2 \\
{[0-6]} \\
\end{array}$ & ND & ND & ND & $\mathrm{ND}$ \\
\hline NSCEP-178 & $\begin{array}{c}0-15.2 \\
{[0-6]}\end{array}$ & ND & ND & $\mathrm{ND}$ & $\mathrm{ND}$ \\
\hline NSCEP-191 & $\begin{array}{r}0-15.2 \\
{[0-6]} \\
\end{array}$ & ND & $\mathrm{ND}$ & ND & ND \\
\hline
\end{tabular}


TABLE 8: NORTH SCEP SPILL H910911D CHARACTERIZATION SAMPLES

\begin{tabular}{|c|c|c|c|c|c|}
\hline Sy & 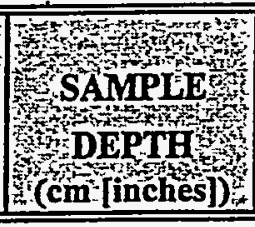 & 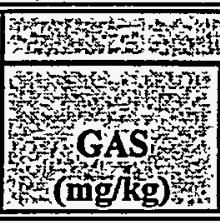 & 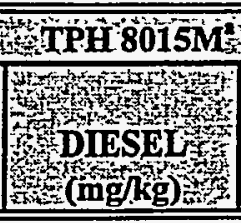 & 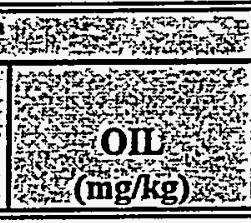 & 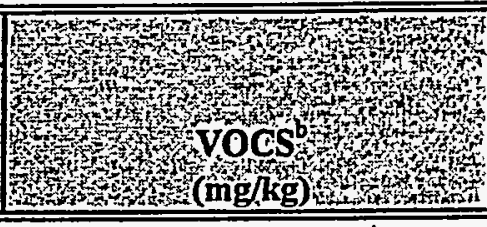 \\
\hline \multicolumn{6}{|c|}{ SAMPLES COLLECTED APRIL 30, 1997} \\
\hline NSCEP-216 & $\begin{array}{r}0-15.2 \\
{[0-6]}\end{array}$ & ND & ND & ND & ND \\
\hline NSCEP-229 & $\begin{array}{r}0-15.2 \\
{[0-6]} \\
\end{array}$ & ND & ND & ND & ND \\
\hline NSCEP-232 & $\begin{array}{r}0-15.2 \\
{[0-6]}\end{array}$ & ND & ND & ND & ND \\
\hline NSCEP-234 & $\begin{array}{r}0-15.2 \\
{[0-6]}\end{array}$ & ND & $\mathrm{ND}$ & ND & ND \\
\hline NSCEP-246 & $\begin{array}{r}0-15.2 \\
{[0-6]} \\
\end{array}$ & ND & $\mathrm{ND}$ & ND & ND \\
\hline NSCEP-246D & $\begin{array}{r}0-15.2 \\
{[0-6]}\end{array}$ & ND & ND & ND & ND \\
\hline NSCEP-249 & $\begin{array}{r}0-15.2 \\
{[0-6]}\end{array}$ & ND & ND & ND & ND \\
\hline NSCEP-262. & $\begin{array}{r}0-15.2 \\
{[0-6]} \\
\end{array}$ & ND & ND & 65 & $\mathrm{ND}$ \\
\hline NSCEP-264 & $\begin{array}{c}0-15.2 \\
{[0-6]}\end{array}$ & ND & ND & ND & ND \\
\hline NSCEP-270 & $\begin{array}{c}0-15.2 \\
{[0-6]}\end{array}$ & ND & ND & 40 & ND \\
\hline NSCEP-273 & $\begin{array}{r}0-15.2 \\
{[0-6] .}\end{array}$ & ND & $\mathrm{ND}$ & 25 & ND \\
\hline NSCEP-290 & $\begin{array}{r}0-15.2 \\
{[0-6]}\end{array}$ & $\mathrm{ND}$ & ND & ND & ND \\
\hline NSCEP-297 & $\begin{array}{r}0-15.2 \\
{[0-6]} \\
\end{array}$ & ND & ND & ND & ND. \\
\hline NSCEP-298 & $\begin{array}{r}0-15.2 \\
{[0-6]} \\
\end{array}$ & ND & ND & ND & ND \\
\hline NSCEP-304 & $\begin{array}{r}0-15.2 \\
{[0-6]} \\
\end{array}$ & ND & ND & 160 & ND \\
\hline NSCEP-305 & $\begin{array}{r}0-15.2 \\
{[0-6]}\end{array}$ & $\mathrm{ND}$ & ND & 41 & ND \\
\hline NSCEP-310 & $\begin{array}{r}0-15.2 \\
{[0-6]}\end{array}$ & ND & $\mathrm{ND}$ & ND & ND \\
\hline NSCEP-343 & $\begin{array}{r}0-15.2 \\
{[0-6]} \\
\end{array}$ & ND & $\mathrm{ND}$ & ND & ND \\
\hline NSCEP-351 & $\begin{array}{r}0-15.2 \\
{[0-6]} \\
\end{array}$ & ND & ND & ND & ND \\
\hline
\end{tabular}


TABLE 8: NORTH SCEP SPILL H910911D CHARACTERIZATION SAMPLES

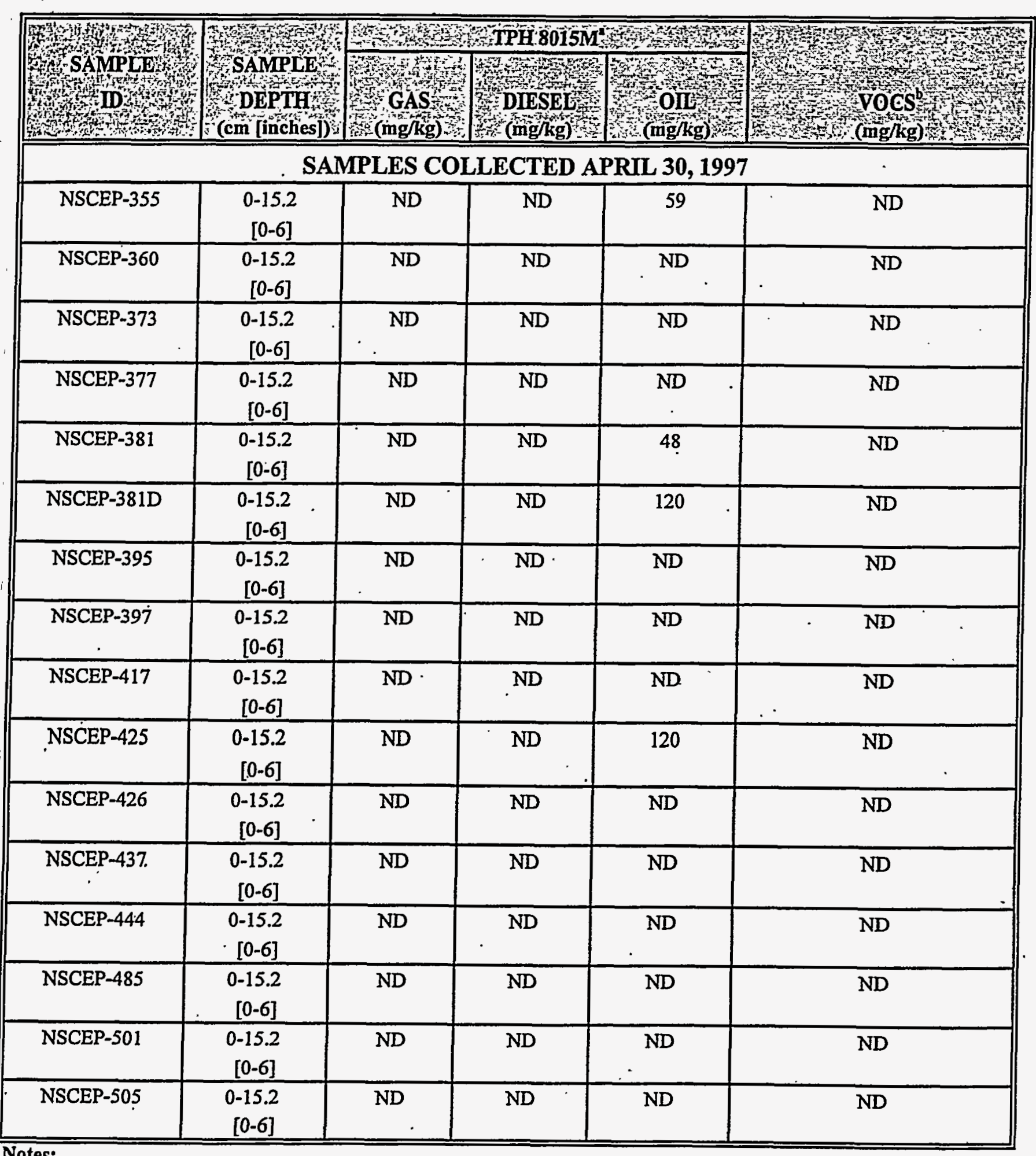

\section{Notes:}

TPH 8015M - Total Petroleum Hydrocarbons Method 8015 Modified.

${ }^{6}$ VOCs - Volatile Organic Compounds. SCEP - Steam Cleaning Effluent Pond ND - Not Detected

$\mathrm{mg} / \mathrm{kg}$ - milligrams per kilogram 
10

LBE ANALYSIS DATA SHEET
EPA SAMPIE NO NSCEP-001 ib Name:

Lab Code: ITYO Case No.:

ttrix : (8011/water) soII

sample wt/vol:

1.0 $(g / \mathrm{ml}) \quad \mathrm{G}$

: Ivel: (low/med)

LOF

* Moisture: not dec.

CAS NO.

Compound .
Contract: 625.01

SAS NO.:

SDG NO.: $\mathbf{v 2 0 4}$

Iab Sample ID: 14495-002

Lab File ID:

Date sampled: 04-29-97

Date Analyzed: 05-09-97

Dilution Factor: 1.0

CONCENTRATION UNITS:

(ug/L or ug/Rg) UG/KG

2 IBH (1) 500 $\mathbf{0}$

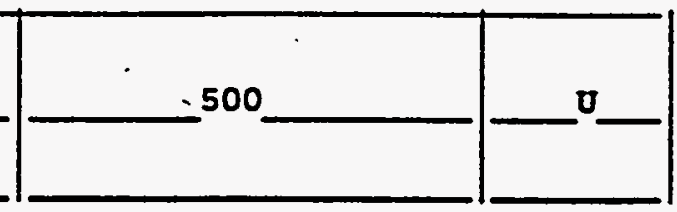

:Concentration of analyte is less than the value given. $X:$ Pattern reasonably matches gasoline ": Pattern appears to be multi-peaks, but does not match gasoline :Concentration 18 primarily from a single peak

(1) Low Boiling Hydrocarbon (LBE) is quantitated as if it ls Gasoline. 
10

HBH ANAIYSIS DATA SHEET
EPA SAMPLE NO. NSCEP-001

Lab Name: OUANTERRA MO

Contract :

62.5 .01

Lab Code: ITMO Case No.:

SAS NO.:

SDG NO.: V204

Matrix : (soil/water)

SOII

Iab Sample ID:

14495-002

Sample witvol: $20.1 \quad(g / m l)$

G

Lab File ID:

Level: - (low/med) LOW

Date Sampled:

$04-29-97$

* Moisture: not dec. dec.

Date Extracted:

05-06-97

Extraction: (SepF/Cont/Sonc/Shak) SHAK

Date Analyzed: $05-06-97$

GPC Cleanup: $(\mathrm{Y} / \mathrm{N}) \mathrm{N}$ pH:

Dilution Factor:

1

CONCENTRATION UNITS:

CAS NO.

Compound

$(\mathrm{mg} / \mathrm{L}$ or $\mathrm{mg} / \mathrm{Kg})$ $\mathrm{mg} / \mathrm{kg}$

Q

DIESEL

WASTE OIL

25

U

25

U

$U$ : Concentration of analyte is less than the value given. 
b Name: QUANTERRA MO

l Code: ITMO Case No.: V49501 SAS No.:
NSCEP-001 trix: (soil/water) SOIL

3 le wt/vol:

$5.00(\mathrm{~g} / \mathrm{mL}) \mathrm{G}$

1:1: (low/med) LOW

Moisture: not dec.

'Olumn: RTX-502.2 ID: 0.530 (mm)

iI Extract Volume:

CAS, NO.
(UI)
COMPOUND
Contract : $625-01$

SDG No.: : V204

Lab Sample ID: 14495-002

Lab File ID: $\quad$ E8463

Date Received: 05/02/97

Date Analyzed: 05/08/97

Dilution Factor: 1.0

Soil Aliquot Volume:

(uL)

CONCENTRATION UNITS:

(ug/L or $\mathrm{ug} / \mathrm{Kg}$ ) UG/KG

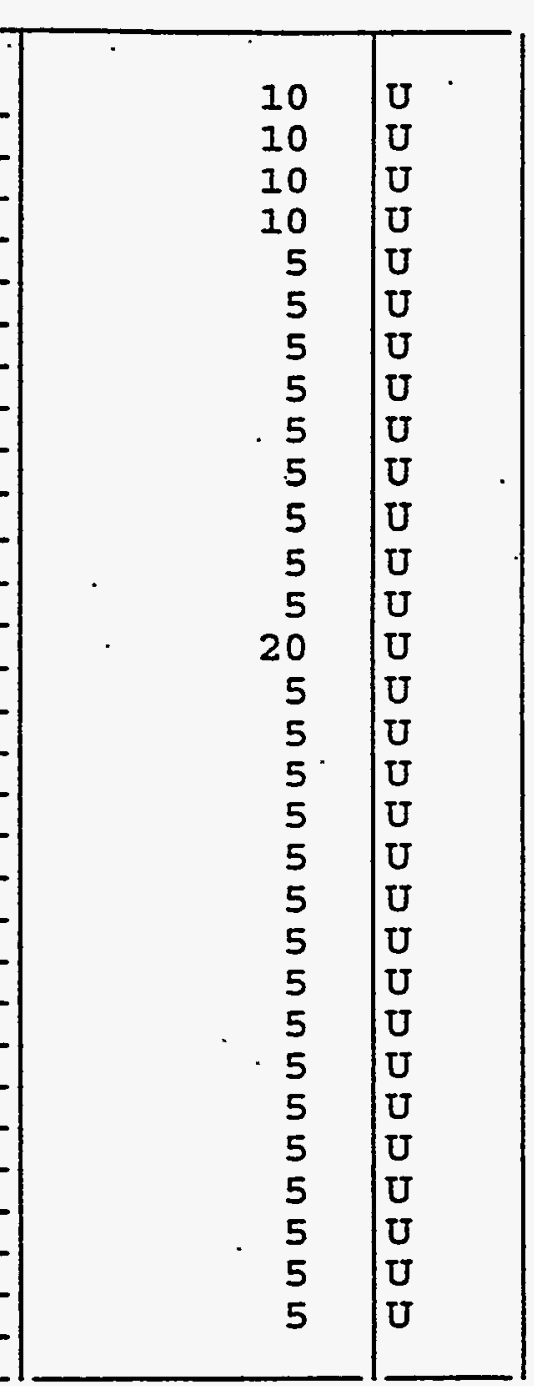

75-71-8--.--Dichlorodifluoromethane

74-83-9--.-----Bromomethane

75-01-4-...---vinyl Chloride

75-00-3-...---Chloroethane

75-09-2-...---Methylene Chloride

75-69-4-....-Trichlorofluoromethane

75-34-3----- -1, 1-Dichloroethane

156-60-5--.---trans-1,2-Dichloroethene

156-59-2---.---cis-1,2-Dichloroethene

1.07-06-2------1, 2-Dichloroethane

594-20-7--.---2,2-Dichloropropane

74-97-5-...---Bromochloromethane

71-55-6-...--1,1,1-Trichloroethane

78-93-3-..---2-Butanone

56-23-5--.----Carbon Tetrachloride

563-58-6-..--1, 1-Dichloropropene

75-27-4-------Bromodichloromethane

78-87-5-.----1,2-Dichloropropane

10061-01-5-----cis-1,3-Dichloropropene

79-01-6--...--Trichloroethene

79-00-5-.---1, 1,2-Trichloroethane

$71-43-2-\ldots-\ldots-$ - Benzene

74-95-3-...-Dibromomethane

10061-02-6----trans-1,3-Dich loropropene

108-88-3-..---Toluene

106-93-4--.---1,2-Dibromoethane

127-18-4------Tetrachloroethene

142-28-9------1, 3-Dichloropropane

124-48-1-..--Dibromochloromethane

108-90-7-.-.-.-Chlorobenzene 
Lab Name: QUANTERRA MO

Contract: $625-01$

NSCEP -001

Lab Code: ITMO Case No.: V49501. SAS No.: SDG No.: V204

Matrix: (soil/water) soII

Sample wt/vol:

$5.00(\mathrm{~g} / \mathrm{mL}) \mathrm{G}$

Level: (low/med) LOW

\% Moịsture: not dec.

GC Column: RTX-502.2 ID: 0.530 (mm) Soil Extract Volume:

(UI)
Lab Sample ID: 14495-002

Lab File ID: $\quad$ E8463

Date Received: 05/02/97

Date Analyzed: 05/08/97

Dilution Factor:

1.0

Soil Aliquot Volume:

(UL)
CAS NO.

COMPOUND
CONCENTRATION UNITS :

(ug/L or ug/Kg) UG/KG

Q
630-20-6-..--1,1,1,2-Tetrachloroethane 100-41-4-------Ethylbenzene

100-42-5-------styrene

75-25-2-..----Bromoform

108-38-3------0-Xylene

$1330-20-7-\cdots--m, p-x y l e n e s$

108-86-1--.----Bromobenzene

79-34-5--.-- 1, 1, 2, 2-Tetrachloroethane

96-18-4-------1, 2, 3-Trichloropropane

95-49-8--.--- - 2-Chlorotoluene

106-43-4------4-Chlorotoluene

541-73-1--.--1;3-Dichlorobenzene

106-46-7-----1,4-Dichlorobenzene

95-50-1--.---1,2-Dichlorobenzene

\begin{tabular}{|c|c|}
\hline $\begin{array}{r}5 \\
.5 \\
.5 \\
5 \\
5 \\
5 \\
5 \\
5 \\
5 \\
5 \\
5\end{array}$ & $\begin{array}{l}U \\
U \\
U \\
U \\
U \\
U \\
U \\
U \\
U \\
U \\
U \\
U \\
U \\
U\end{array}$ \\
\hline
\end{tabular}




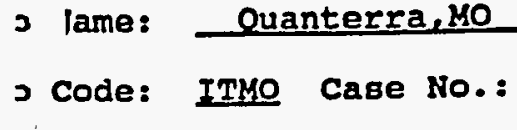

$=x:$ (øoil/water) soIL

uple wt/vol: $1.0 \quad(\mathrm{~g} / \mathrm{ml}) \mathrm{G}$

$\therefore$ (low/med) LOW

loisture: not dec.
Contract: $\quad 625.01$

SAS NO.:

SDG NO.: V204

Lab Sample ID: 14495-003

Lab File ID:

Date Sampled: $\quad 04-29-97$

Date Analyzed: 05-09-97

Dilution Factor: 1.0
CONCENTRATION UNITS:

(ug/I or ug/Rg) UG/RG

$\mathbf{2}$

IBH (1)

500

$\mathbf{0}$

:' incentration of analyte 18 less than the value given.

:Fattern reasonably matches gasoline

:p pttern appears to be multi-peaks, but does not match gasoline

: incentration is primarily from a single peak

1) Low Boiling Hydrocarbon (IBH) is quantitated as if it is Gasoline.

EORM I LBH 
Lab Name: QUANTERRA MO

Contract :

625.01

Lab Code: ITMO Case No.: SAS NO.: SDG No.: V204

Matrix : (soil/water) SOII

Iab Sample ID: $14495-003$

Sample wt/vol: $20.1 \quad(\mathrm{~g} / \mathrm{mI})$ G Lab File ID: Level: (low/med) LOW

Date Sampled:

04-29-97

\% Moisture: not dec. dec.

Date Extracted: $05-06-97$ Extraction: (SepF/Cont/Sonc/Shak) SHA'K

Date Analyzed: $05-06-97$ GPC Cleanup: $(\mathrm{Y} / \mathrm{N}) \underline{\mathrm{N}} \mathrm{pH}:$ Dilution Factor: 1 CONCENTRATION UNITS:

CAS NO. Compound (mg/L or $\mathrm{mg} / \mathrm{Kg}$ ) $\mathrm{mg} / \mathrm{kg}$

$Q$

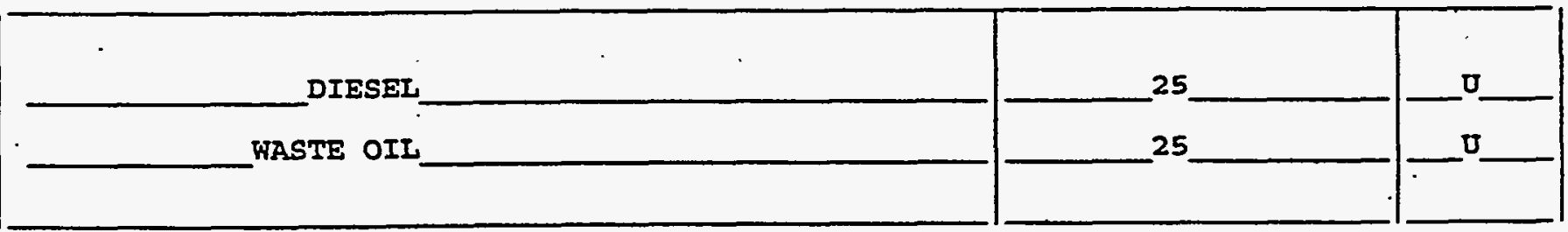

$U$ : Concentration of analyte is less than the value given. 
b Name: QUANTERRA MO

I Ċode: ITMO
Contract: $625-01$

Case No.: V49501 SAS No.:
NSCEP-004 trix: (soil/water) SOIL

n.rle wt/vol:

$5.00(\mathrm{~g} / \mathrm{mL}) \mathrm{G}$

il: (low/med) Low

Moisture: not dec.

'olumn: RTX-502.2 ID.: 0.530 (mm)

j.l Extract Volume:

(uL)
Lab Sample ID: 14495-003

Lab File ID: E8514

Date Received: 05/02/97

Date Analyzed: 05/09/97

Dilution Factor: $\quad 1.0$

Soil Aliquot Volume:

(UL)

CAS NO. COMPOUND

CONCENTRATION UNITS:

(ug/L or $u g / K g) ~ U G / K G$

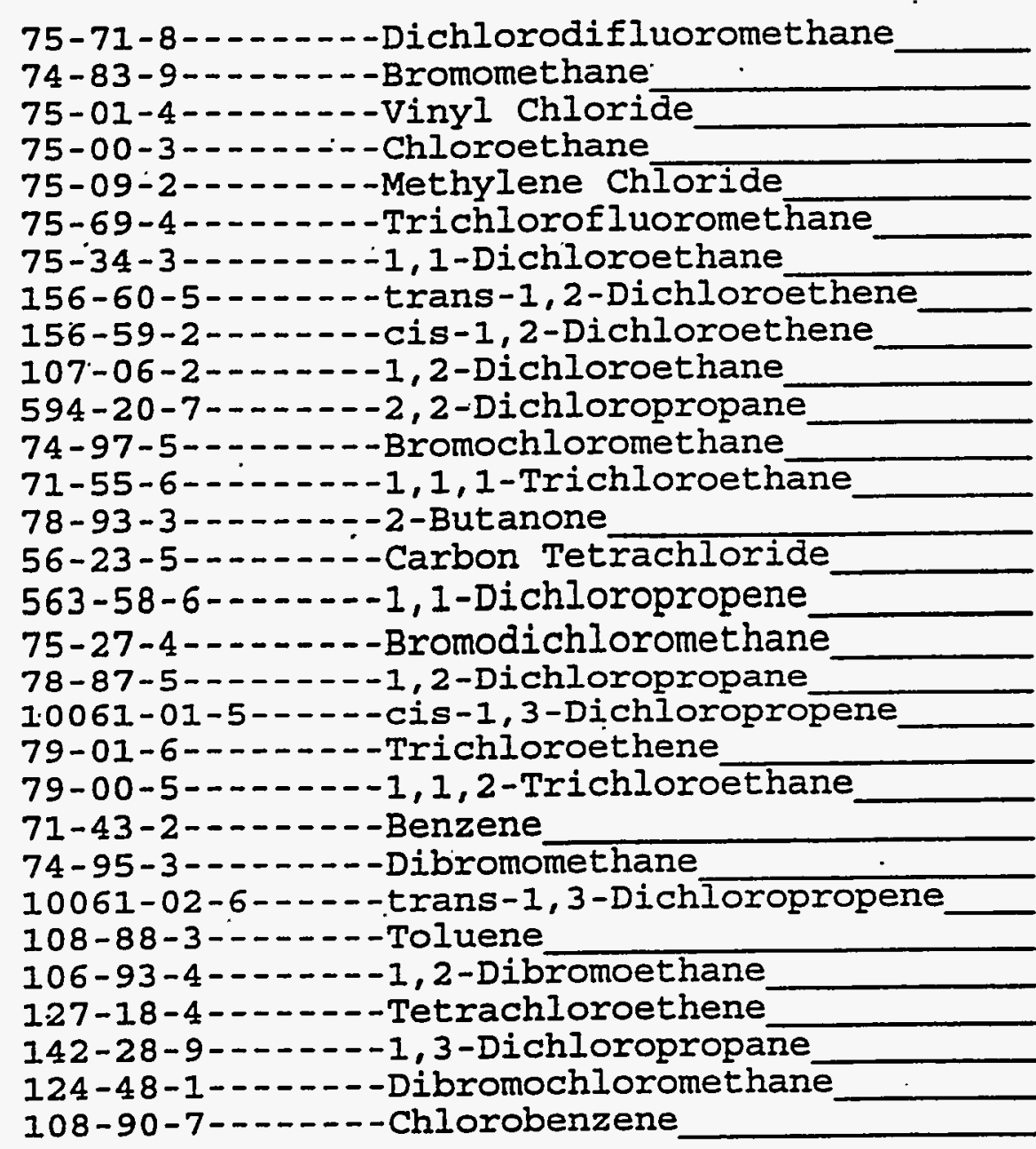

\begin{tabular}{|cc|c}
\hline 10 & $\mathrm{U}$ \\
10 & $\mathrm{U}$ \\
10 & $\mathrm{U}$ \\
10 & $\mathrm{U}$ \\
3 & $\mathrm{BJ}$ \\
5 & $\mathrm{U}$ \\
5 & $\mathrm{U}$ \\
5 & $\mathrm{U}$ \\
5 & $\mathrm{U}$ \\
5 & $\mathrm{U}$ \\
5 & $\mathrm{U}$ \\
5 & $\mathrm{U}$ \\
5 & $\mathrm{U}$ \\
& 20 & $\mathrm{U}$ \\
5 & $\mathrm{U}$ \\
5 & $\mathrm{U}$ \\
5 & $\mathrm{U}$ \\
5 & $\mathrm{U}$ \\
5 & $\mathrm{U}$ \\
5 & $\mathrm{U}$ \\
5 & $\mathrm{U}$ \\
5 & $\mathrm{U}$ \\
5 & $\mathrm{U}$ \\
5 & $\mathrm{U}$ \\
5 & $\mathrm{U}$ \\
& 5 & $\mathrm{U}$ \\
5 & $\mathrm{U}$ \\
5 & $\mathrm{U}$ \\
5 & $\mathrm{U}$ \\
& 5 & $\mathrm{U}$ \\
\hline & \\
\hline
\end{tabular}


Lab Name: QUANTERRA MO

Lab Code: ITMO

Case No.: V49501

Matrix: (soil/water) soIL

Sample wt/vol:

$5.00(\mathrm{~g} / \mathrm{mL}) \mathrm{G}$

Level: (low/med) LOW

\% Mọisture: not dec.

GC Column: RTX-502.2 ID: 0.530 (mm)

Soil Extract Volume:

(UI)
Contract: 625-01

NSCEP-004
SAS NO.:

Iab Sample ID: 14495-003

Lab File ID: $\quad$ E8514

Date Received: 05/02/97

Date Analyzed: 05/09/97

Dilution Factor:

1.0 CONCENTRATION UNITS: CAS NO. COMPOUND. (ug/L or $u g / \mathrm{Kg}$ ) UG/KG

Q

\begin{tabular}{|ll|l|} 
& 5 & $U$ \\
& 5 & $U$ \\
5 & $U$ \\
5 & $U$ \\
& 5 & $U$ \\
5 & $U$ \\
& 5 & $U$ \\
5 & $U$ \\
5 & $U$ \\
& 5 & $U$ \\
& 5 & $U$ \\
5 & $U$ \\
5 & $U$ \\
& 5 & $U$ \\
&
\end{tabular}


EPA SAMPLE NO. NSCEP-005 o Name: QUANTERRA MO

J Code: ITMO Case No.:

:ix: (soil/water)

SOIL

I' le wt/vol:

20.3

$(g / m I)$

G

vel: (low/med)

LOW

voisture: not dec.

dec.

$t$ action: (SepF/Cont/Sonc/Shak)

SHAAK

: cleanup: $(Y / N) \stackrel{N}{N} \mathrm{pH}:$

$\therefore$ NO.

Compound

CONCENTRATION UNITS

$(\mathrm{mg} / \mathrm{L}$ or $\mathrm{mg} / \mathrm{Kg}) \quad \mathrm{mg} / \mathrm{kg}$

$\left[-25+25+\frac{\mathrm{U}}{-}-\right.$

: Concentration of analyte is less than the value given.

FORM I PBBE 
Lab Name: QUANTERRA MO

Iab Code: ITMO

Case No.: V49501

Matrix: (soil/water) SOIL

Sample wt/vol: $\quad 5.00(\mathrm{~g} / \mathrm{mL})$ G

Level: (low/med) LOW

\% Moịsture:- not dec.

GC Column: RTX-502.2 ID: $0.530^{\circ}$ (mm)

Soil Extract Volume:

CAS NO.

COMPOUND
Contract: $625-01$

NSCEP-005

SDG NO.: V204

Lab Sample ID: 14495-004

Lab File ID: $\quad$ E8478

Date Received: 05/02/97

Date Analyzed: 05/08/97

Dilution Factor: $\quad 1.0$

Soil Aliquot Volume:

(UL)

CONCENTRATION UNITS:

(ug/L or $\mathrm{ug} / \mathrm{Kg}$ ) UG/KG

75-71-8-...-Dichlorodifluoromethane

74-83-9--n---Bromomethane

75-01-4-------vinyl Chloride

75-00-3-...---Chloroethane

75-09-2--.-.-- Methylene Chloride

75-69-4-------Trichlorofluoromethane

75-34-3-....-1,1-Dichloroethane:

156-60-5--.-:-trans-1,2-Dichloroethene

156-59-2------cis-1, 2-Dichloroethene

107-06-2--.---1,2-Dichloroethane

594-20-7------2, 2-Dichloropropane

74-97-5--.----Bromochloromethane

71-55-6-...-1, 1, 1-Trichloroethane

78-93-3--------2-Butanone

56-23-5-...--Carbon Tetrachloride

563-58-6------1,1-Dichloropropene

75-27-4--.----Bromodichloromethane

78-87-5-----1, 2-Dichloropropane

10061-01-5-----cis-1,3-Dichloropropene

79-01-6--.---Trichloroethene

79-00-5-..--1,1,2-Trichloroethane

$71-43-2-\ldots-\cdots-$ Benzene

74-95-3-..---Dibromomethane

10061-02-6--.--trans-1, 3-Dichloropropene

108-88-3-..--Toluene

106-93-4-------1,2-Dibromoethane

127-18-4-------Tetrachloroethene

142-28-9------1,3-Dichloropropane

124-48-1--.---Dibromochloromethane

108-90-7-------Chlorobenzene

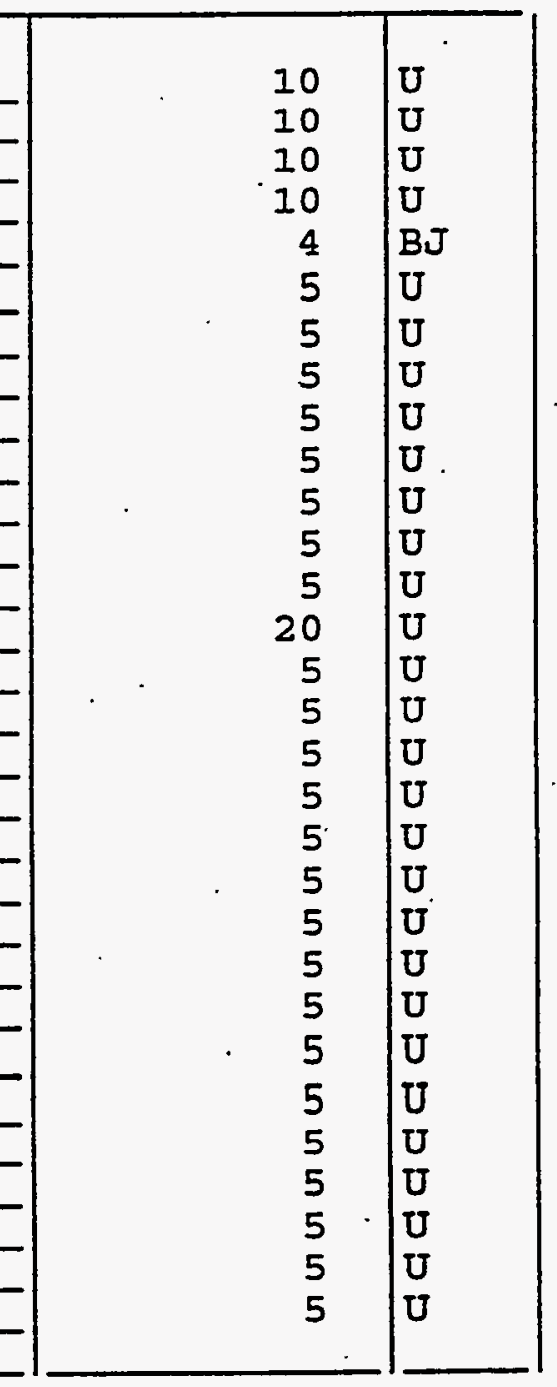


1 A

VOLATILE ORGANICS ANALYSIS DATA SHEET
EPA SAMPIE NO.

NSCEP-005
J Name: QUANTERRA MO

: Code: ITMO

Case No.: V49501

Contract: $625-01$

SDG NO..: V204

:-ix: (soil/water) soIL

niple wt/vol:

$5.00(\mathrm{~g} / \mathrm{mL}) \mathrm{G}$

1: (low/med) LOW

1oisture: not dec.

olumn: RTX-502.2 ID: $0.530^{\circ}(\mathrm{mm})$

L. Extract Volume:

(uIs)
Lab Sample ID: 14495-004

Lab File ID: E8478.

Date Received: 05/02/97

Date Analyzed: $05 / 08 / 97$

Dilution Factor: $\quad 1.0$

Soil Aliquot Volume:

(uL)

CAS NO.

COMPOUND

CONCENTRATION UNITS :

(ug/L or ug/Kg) UG/KG

630-20-6--..-1, i, 1, 2-Tetrachloroethane

100-41-4-------Ethylbenzene

100-42-5--.----Styrene

75-25-2-------Bromoform

108-38-3------o-Xylene

1330-20-7----.-m, p-Xylenes

108-86-1-2.---Bromobenzene

79-34-5- - - - 1, 1, 2,2-Tetrachloroethane

96-18-4-------1,2,3-Trichloropropane

95-49-8------2-Chlorotoluene

106-43-4------4-Chlorotoluene

541-73-1--.--1, 3-Dichlorobenzene

106-46-7-...-1,4-Dichlorobenzene

95-50-1-..---1,2-Dichlorobenzene 
1D

IBH ANAIYSIS DATA SHEET
EPA SAMPLE NO NSCEP-029

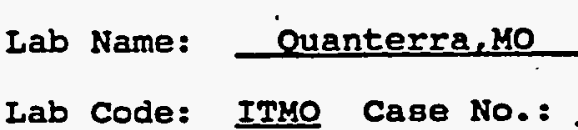

Matrix : (80il/water) soIL

Sample wt/vol: $\frac{1.0 \quad(g / m I) ـ G}{G}$

Level:" (low/med) LOW .

\& Moisture: not dec.

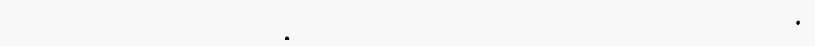

CAS NO.

compound

CONCENTRATION UNITS:

(ug/L or ug/Rg) UG/RG

SAS NO.:

Contract: 625.01

SDG NO.: $\mathbf{V} 2.04$

Lab Sample ID: $14495-005$

Lab File ID:

Date Sampled: $\quad 04-29-97$

Date Analyzed: $05-09-97$

Dilution Factor: 1.0

\begin{tabular}{|l|l|l|}
\hline IBH (1)__ & + \\
\hline
\end{tabular}

U:Concentration of analyte is less than the value given.

$X: P a t t e r n$ reasonably matches gasoline

Y; Pattern appears to be multi-peakB, but does not match gasoline

$\mathrm{z}$ : Concentration 18 primarily from a single peak

(1): Low Boiling Hydrocarbon (IBH) is quantitated as if it is Gasoline. 
ID

HBH ANALYSIS DATA SHEET
EPA SAMPLE NO NSCEP-029 b Name: OUANTERRA MO

b Code: ITMO Case No.:

t ix : (soil/water)

SOIL

i le wt/vol: $20.1(\mathrm{~g} / \mathrm{ml})$ G

vel: (low/med) LOW Muisture: not dec. dec.

I action: (SepF/Cont/Sonc/Shak) SHAR

( Cleanup: $(\mathrm{Y} / \mathrm{N}) \stackrel{\mathrm{N}}{\mathrm{pH}}$ $\longrightarrow$
SAS NO.:

Lab Sample ID:

625.01

Contract : .SDG NO.: V204

Lab File ID:

Date Sampled:

$04-29-97$

Date Extracted:

$05-06-97$

Date Analyzed:

$05-06-97$

Dilution Factor:

1

CONCENTRATION UNITS :

$(\mathrm{mg} / \mathrm{I}$ or $\mathrm{mg} / \mathrm{kg}) \mathrm{mg} / \mathrm{kg} \quad Q$

\section{DIESEL}

WASTE OIL

$\square^{25}+{ }^{25}+{ }^{0}-{ }^{0}$

$r$ : Concentration of analyte is less than the value given. 
NSCEP-029

Lab Name: QUANTERRA MO

Lab Code: ITMO
Contract: $625-01$
SAS NO. :
SDG NO.: V204
Matrix: (soil/water) SOIL

Sample wt/vol: $\quad 5.00(\mathrm{~g} / \mathrm{mL}) \mathrm{G}$

Level: (low/med) LOW

\% Moịture: not dec.

GC Column: RTX-502.2 ID: 0.530 (mm)

Soil Extract Volume:

CAS NO.

COMPOUND

Lab Sample ID: 14495-005

Lab File ID: $\quad$ E8479

Date Received: 05/02/97

Date Analyzed: $05 / 08 / 97$

Dilution Factor: 1.0

Soil Aliquot Volume:

(UL)

CONCENTRATION UNITS :

(ug/L or ug/Kg) UG/KG Q

75-71-8--..--Dichlorodifluoromethane

74-83-9-..-.--Bromomethane

75-01-4-...--Vinyl. Chloride

75-00-3--.----Chloroethane

75-09-2-.-.--Methylene Chloride

75-69-4-2.---Trichlorofluoromethane

75-34-3-..--1, 1-Dichloroethane

156-60-5----.--trans-1,2-Dichloroethene

156-59-2------cis-1,2-Dichloroethene

107-06-2------1,2-Dichloroethane

594-20-7--.----2,2-Dichloropropane

74-97-5-..----Bromochloromethane

71-55-6-...-1,1,1-Trichloroethane

78-.93-3-...-.-2-Butanone

56-23-5-..---.-Carbon Tetrachloride

563-58-6--.---1, 1-Dichloropropene

75-27-4-1.----Bromodichloromethane

78-87-5------1, 2-Dichloropropane

10061-01-5-----cis-1, 3-Dichloropropene

79-01-6-...--Trichloroethene

79-00-5-------1, 1,2-Trichloroethane

$71-43-2 \ldots-\ldots-.-$ Benzene

74-95-3------Dibromomethane

10061-02-6-----trans-1,3-Dichloropropene

108-88-3-.-----Toluene

$106-93-4-\ldots---1,2-D i b r o m o e t h a n e$

127-18-4---.---Tetrachloroethene

142-28-9-..--1, 3-Dichloropropane

124-48-1--.---Dibromochloromethane

108-90-7-...--.-Chlorobenzene

10
10
10
10
4
5
5
5
5
5
5
5
5
20
5
5
5
5
5
5
5
5
5
5
5
5
5
5
5
5

10

10

10

$10 \quad \mathrm{U}$

4 BJ

5 U

$5 \quad U$

5 U

5 U

5 U

5 U

5 U

5 U

20 U

5 U

5 U

5 U

5 U

5 U

5 U

5 U

5 U

50

5

5 U

5

5 U

5

5

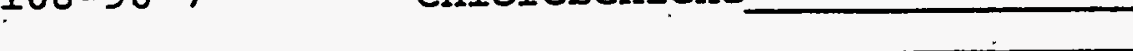


IA

VOLATILE ORGANICS ANALYSIS DATA SHEET
EPA SAMPLE NO.

NSCEP-029 b Name: 'QUANTERRA MO

I Code: ITMO

trix: (soil/water) soIL

t. Jle wt/vol:

$5.00(\mathrm{~g} / \mathrm{mL}) \mathrm{G}$

1. : (low/med) LOW

Mọisture: not dec.

Solumn: RTX-502.2 ID: 0.530 (mm)

il Extract Volume:

(uL)
Contract: $625-01$

SAS NO.:

SDG No.: V204

Lab Sample ID: 14495-005

Lab File ID: $\quad$ E8479

Date Received: 05/02/97

Date Analyzed: 05/08/97

Dilution Factor: $\quad 1.0$

Soil Aliquot Volume:

(uL)

CONCENTRATION UNITS :

CAS NO.

COMPOUND

Q

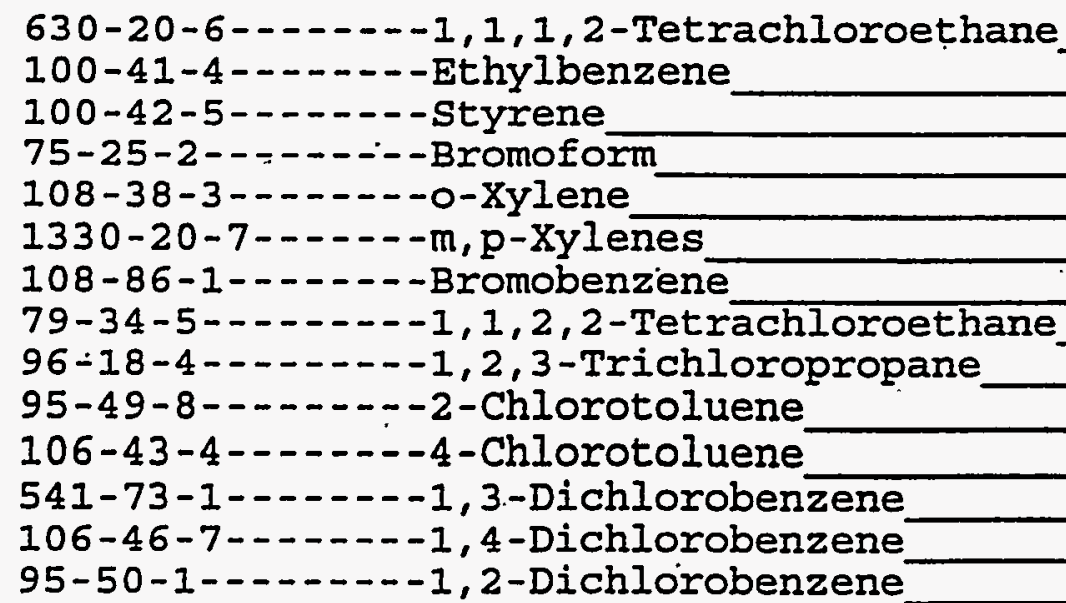


1D

IBH ANALYSIS DATA SHEET
FPA SAMPLE NO. NSCEP-046
Lab Name: $\frac{\text { Ouanterra,NO }}{\text { Lab Code: ITMO Case No.: }}$

Matrix : (soil/water) soII

Sample wt/vol: $1.0 \quad(\mathrm{~g} / \mathrm{ml}) \cdot \mathrm{G}$

Level: (low/med) LOW

\& Molsture: not dec.

CAS NO.

Compound

CONCENTRATION UNITS:

(ug/I or ug/Rg) UG/RG

500

$\mathbf{0}$

IBH (1)

SAS NO.: contract: $\quad 625.01$

SDG NO.: V204

Lab sample ID: $14495-006$

Iab FIle ID:

Date Sampled: 04-29-97

Date Analyzed: $05-09-97$

Dilution Eactor: 1.0

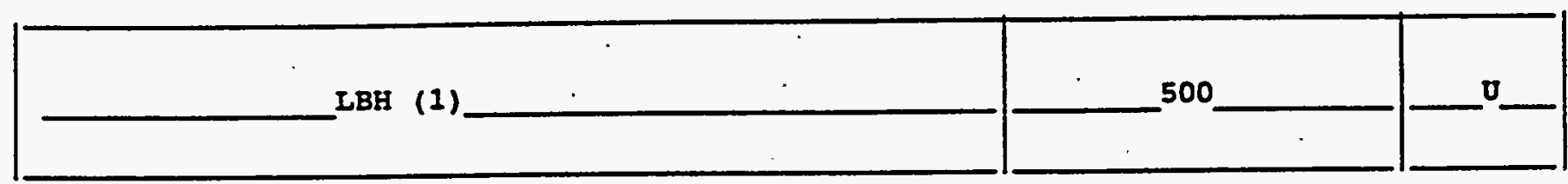

U:Concentration of analyte is less than the value given.

$X:$ Pattern reasonably matches gasoline

Y:Pattern appears to be multi-peaks, but does not match gasoline

$\mathrm{Z}$ : Concentration is primarily from a single peak

(1):Low Boiling Hydrocarbon (IBH) is quantitated as if it is Gasoline. 
ID

HBH ANALYSIS DATA SHEET
EPA SAMPLE NO.

NSCEP-046 b Name: QUANTERRA MO

b Code: ITMO Case No.:

t.ix : (soil/water)

SOIL

i' le wt/vol:

$20.1 \quad(\mathrm{~g} / \mathrm{mI})$

G

vel: (low/med)

LOW

Moisture: not dec. dec.

t action: (SepF/Cont/Sonc/Shak)

SFAR

( cleanup: $(\mathrm{Y} / \mathrm{N})+\mathrm{N} \quad \mathrm{pH}:$ N

$+$

Contract :

625.01

SAS NO.:

SDG No.: V204

Lab Sample ID:

$14495-006$

Lab File ID:

Date Sampled:

Date Extracted:

$05-06-97$

Date Analyzed:

$05-06-97$

Dilution Factor:

1

CONCENTRATION UNITS :

C S NO.

Compound

(mg/L or $\mathrm{mg} / \mathrm{kg}$ )

$\mathrm{ma} / \mathrm{kg}$

Q

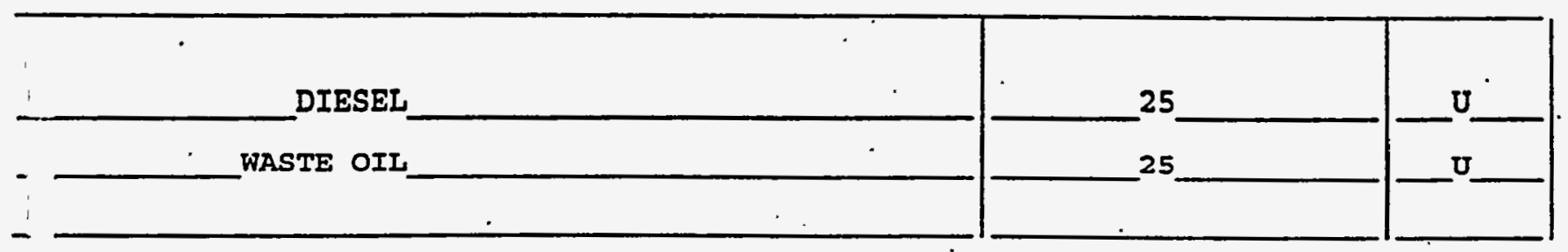

I: Concentration of analyte is less than the value given. 
$1 A$

EPA SAMPLE NO.

VOLATILE ORGANICS ANALYSIS DATA SHEET

Lab Name: QUANTERRA MO

Iab Code: · ITMO

Case No.: V49501

Matrix: (soil/water) soII

Sample wt/vol:

$5.00(\mathrm{~g} / \mathrm{mL}) \quad \mathrm{G}$

Level: $\quad$ (low/med) LOW

\% Moịture: not dec.

GC Column: RTX-502.2 ID: 0.530 (mm)

Soil Extract Volume:

CAS NO.
Contract: $625-01$

SAS NO.:
NSCEP-046

SDG .NO.: V204

Lab Sample ID: 14495-006

Lab File ID: E8480

Date Received: 05/02/97

Date Analyzed: 05/08/97

Dilution Factor: $\quad 1.0$

Soil Aliquot Volume:

(uL)

CONCENTRATION UNITS :

(ug/L or ug/Kg) UG/KG

Q

75-71-8-..--Dichlorodifluoromethane

74-83-9---.---Bromomethane

75-01-4------Vinyl Chloride

75-00-3------Chloroethane

75-09-2--------Methylene Chloride

75-69-4-------Trichlorofluoromethane

75-34-3------1, 1-Dichloroethane

156-60-5----.-trans-1,2-Dichloroethene

156-59-2--.---cis-1,2-Dichloroethene

107-06-2------1,2-Dichloroethane

594-20-7------2,2-Dichloropropane

74-97-5------Bromochloromethane

71-55-6-------1, 1, 1-Trichloroethane

78-93-3--.---2-Butanone

56-23-5--.----Carbon Tetrachloride

563-58-6------1; 1-Dichloropropene

75-27-4-------Bromodichloromethane

78-87-5--.--1,2-Dichloropropane

10061-01-5--.--cis-1,3-Dichloropropene

79-01-6-...--.-Trichloroethene

79-00-5--.---1, 1, 2-Trichloroethane

71-43-2---.---Benzene

74-95-3--.----Dibromomethane

10061-02-6---.-trans-1,3-Dichloropropene

108-88-3--.----Toluene

106-93-4------1, 2-Dibromoethane

127-18-4---.--Tetrachloroethene

142-28-9-----1, 3-Dichloropropane

124-48-1--.---Dibromochloromethane

108-90-7--.---Chlorobenzene

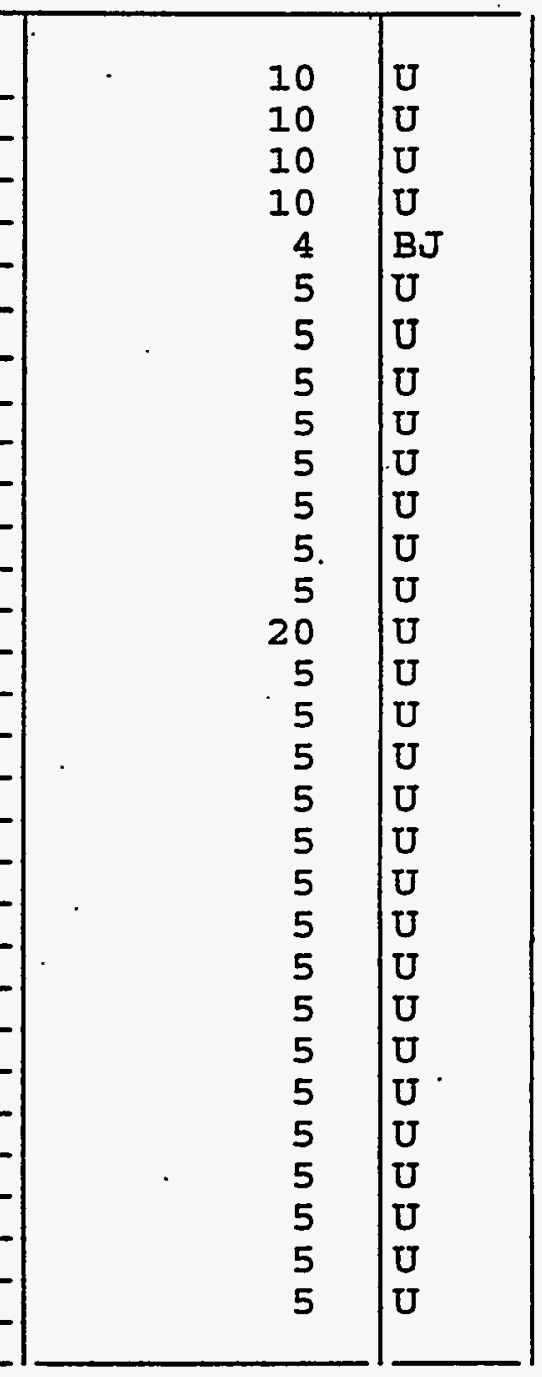


o Name: QUANTERRA MO

j Code: ITMO

zirix: (soil/water) SOIL

$\mathrm{n}_{\mathrm{r}}$ le wt/vol:

$5.00(\mathrm{~g} / \mathrm{mL}) \mathrm{G}$

1: (Iow/med) LOW

toịsture: not dec.

Dlumn: RTX-502.2 ID: 0.530 (mm)

i] Extract Volume:

(uL)
Contract: $625-01$
EPA SAMPLE NO.

NSCEP-046
Cáse No.: V49501 SAS No.:

Lab Sample ID: 14495-006

Lab File ID: $\quad$ E8480

Date Received: 05/02/97

Date Analyzed: 05/08/97

Dilution Factor: $\quad 1.0$

CAS NO.
COMPOUND $\begin{gathered}\text { CONCENTRATION } \\ \text { (ug/L or ug/ }\end{gathered}$
CONCENTRATION UNITS:

Soil Aliquot Volume:

(uI) 
10

IBH ANAIYSIS DATA SHEET
EPA SAMPLE NO. NSCEP -060
Lab Name: $\frac{\text { Ouanterra,Mo }}{\text { Lab Code: ITMO Case NO.: }}$
ITO

Matrix : (soil/water) soIL

Sample wt/vol: 1.0 . $(g / \mathrm{ml}) \quad \mathrm{G}$

Level: (low/med) LOW

\& Moisture: not dec.

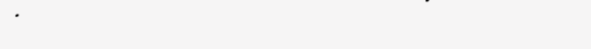

CAS NO. contract: 625.01

SAS NO.: SDG No.: V204

Lab sample ID: 14495-007

Iab File ID:

Date Sampled: 04-29-97

Date Analyzed: 05-09-97

Dilution Factor: 1.0

CONCENTRATION UNITS:

(ug/L or ug/Kg) UG/KG

$\mathbf{Q}$

\begin{tabular}{|l|l|l|}
\hline IBH (1)___ & \\
\hline
\end{tabular}

U: Concentration of analyte is less than the value given.

$\mathrm{X}$ : Pattern reasonably matches gasoline

$Y:$ Pattern appears to be multi-peaks, but does not match gasoline

$z$ : Concentration is primarily from a single peak

(1) Low Boiling Hydrocarbon (LBH) is quarititated 28 if it is Gasoline. 
1D

HBH ANALYSIS DATA SHEET
EPA SAMPLE NO.

NSCEP -060 b Name: OUANTERRA MO

b Code: ITMO Case No.:

t ix : (soil/water)

in le wt/vol:

20.1 $(g / m 1)$

G

:vel: (low/med)

LOW

SOIL

Moisture: not dec. dec.

it action: (SepF/Cont/Sonc/Shak)

SHAR

' cleanup: $(\mathrm{Y} / \mathrm{N}) \stackrel{\mathrm{N}}{\mathrm{pH}}$

c S'NO.

Compound

CONCENTRATION UNITS :

(mg/L or $\mathrm{mg} / \mathrm{Kg}$ ) $\mathrm{ma} / \mathrm{kg}$

Lab Sample ID:

14495-007

Lab File ID:

Date Sampled:

$04-29-97$

Date Extracted:

$05-06-97$

Date Analyzed:

$05-07-97$

Dilution Factor:

1 DIESEL 25 42

WASTE OIL

$\square^{25}-42 \quad 1$

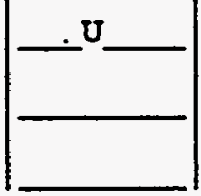

I: Concentration of analyte is less than the value given. 
VOIATILE ORGANICS ANALYSIS DATA SHEET

Lab Name: QUANTERRA MO

Lab Code : ITMO
Case No.: V49501

Matrix: (soil/water) SOIL

Sample wt/vol:

$5.00(\mathrm{~g} / \mathrm{mL}) \mathrm{G}$

Level: (low/med) LOW

\% Moisture: not dec.

GC Column: RTX-502.2 ID: $0.530(\mathrm{~mm})$

Soil Extract Volume:

(UI)
Contract: $625-01$

SAS NO.:

NSCEP- 060

SDG No.: V204

Lab Sample ID: 14495-007

Lab File ID: $\quad$ E8468

Date Received: 05/02/97

Date Analyzed: 05/08/97

Dilution Factor: $\quad 1.0$

Soil Aliquot volume:

(UI)

CONCENTRATION UNITS:

CAS NO. COMPOUND (ug/L or ug/Kg) UG/KG

\begin{tabular}{|c|}
\hline 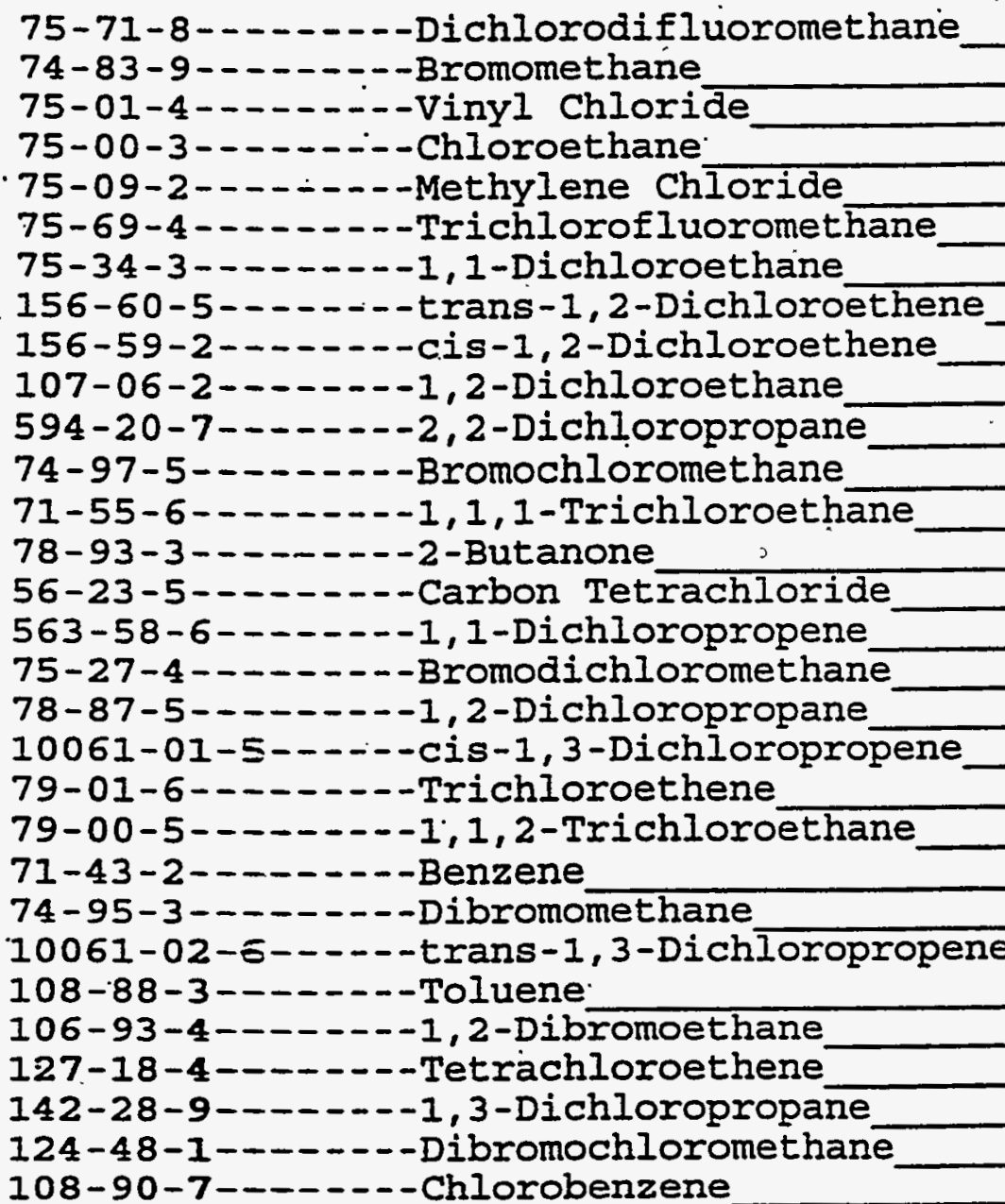 \\
\hline
\end{tabular}

\begin{tabular}{|r|r|r|}
10 & $U$ \\
10 & $U$ \\
10 & $U$ \\
10 & $U$ \\
5 & $U$ \\
5 & $U$ \\
5 & $U$ \\
5 & $U$ \\
5 & $U$ \\
5 & $U$ \\
5 & $U$ \\
5 & $U$ \\
5 & $U$ \\
20 & $U$ \\
5 & $U$ \\
5 & $U$ \\
5 & $U$ \\
5 & $U$ \\
5 & $U$ \\
5 & $U$ \\
5 & $U$ \\
5 & $U$ \\
5 & $U$ \\
5 & $U$ \\
5 & $U$ \\
5 & $U$ \\
5 & $U$ \\
5 & $U$ \\
5 & $U$ \\
5 & $U$ \\
& \\
\hline & \\
\hline \\
\hline
\end{tabular}


Name: QUANTERRA MO

:ode: ITMO

Case No.: V49501

rix: (soil/water) soIL

Fue wt/vol:

$5.00(\mathrm{~g} / \mathrm{mL}) \mathrm{G}$

: $\quad($ Iow $/ \mathrm{med})$ LOW

oisture: not dec.

( I lumn: RTX-502.2 ID: 0.530 (mm).

I Extract Volume:

(UI)
Contract: $625-01$

SAS NO.:

SDG No.: V204

NSCEP-060

Lab Sample ID: 14495-007

Lab File ID: E8468

Date Received: 05/02/97

Date Analyzed: 05/08/97

Dilution Factor: $\quad 1.0$

Soil Aliquot Volume:

(uL)

CONCENTRATION UNITS :
CAS NO.
COMPOUND
(ug/L or $\mathrm{ug} / \mathrm{Kg}$ ) UG/KG
Q

630-20-6--.---1,1,1,2-Tetrachloroethane

$100-41-4-\ldots .--$ - Ethylbenzene

100-42-5--.----Styrene

75-25-2-..-- - Bromoform

108-38-3----.--0-Xylene

1330-20-7-----m, p-Xylenes

108-86-1--------Bromobenzene

79-34-5-..---1,1,2,2-Tetrachloroethane

96-i $8-4-\cdots---1,2,3-$ Trichloropropane

95-49-8--.-.--2-Chlorotoluene

106-43-4-.-.-.-4-Chlorotoluene

541-73-1-..--1, 3-Dichlorobenzene

106-46-7-..--1;4-Dichlorobenzene

95-50-1------1,2-Dichlorobenzene 
Lab Name: Quanterra,ko .

Iab Code: ITMO Case No.:

Hatrix : (Boil/water) soIL.

Sample wt/vol: $1.0 \quad(\mathrm{~g} / \mathrm{ml}) \quad G$

Level: (low/med) LOW

\& Moisture: not dec.

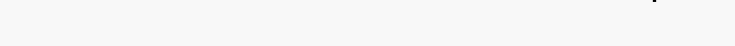

CAS NO.

Compound.
Contract: $\quad 625.01$

SAS NO.: SDG NO.: V204

Lab Sample ID: $14495-008$

Lab File ID:

Date Sampled: $\quad 04-29-97$

Date Analyzed: $05-09-97$

Dilution Factor: 1.0

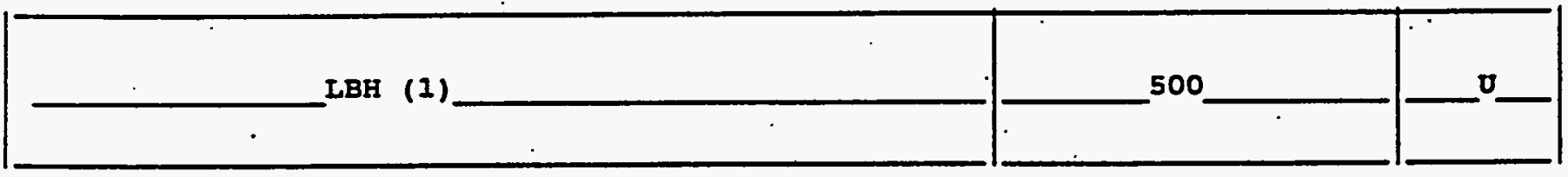

$U$ :Concentration of analyte is less than the value given. $X: P a t t e r n$ reasonably matches gasoline Y:Pattern appears to be multi-peaks, but does not match gasoline $\mathrm{z}$ :Concentration is primarily from a single peak

(1) Low Boiling Hydrocarbon (LBH) is quantitated as if. it is Gasoline. 


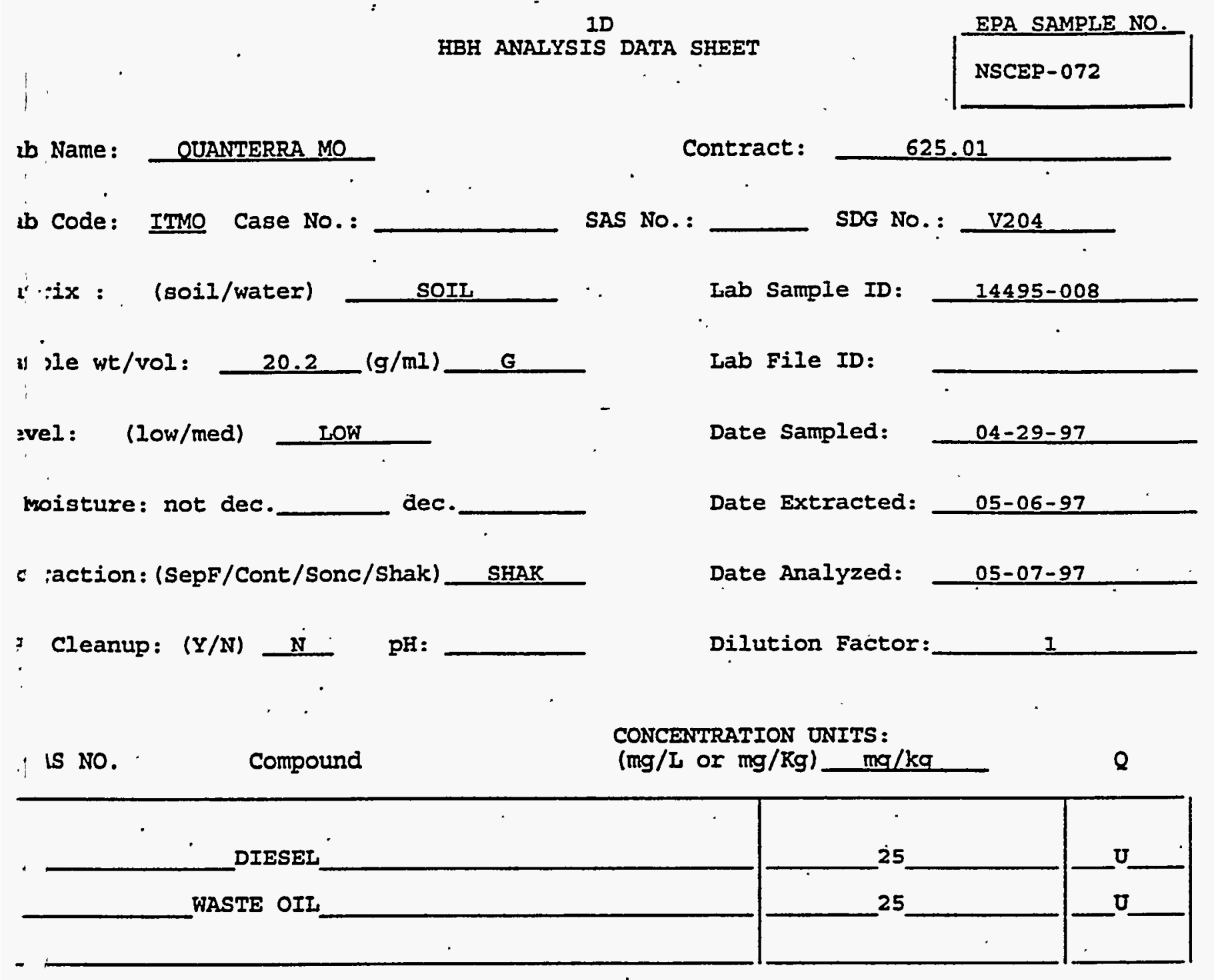

1: Concentration of analyte, is less than the value given. 
$1 \mathrm{~A}$ VOLATILE ORGANICS ANALYSIS DATA SHEET
EPA SAMPLE NO.

NSCEP-072
Lab Name: QUANTERRA MO

Lab Code: ITMO

Case No.: V49501

Matrix: (soil/water) soIL

Sample wt/vol:

$5.00(\mathrm{~g} / \mathrm{mL}) \mathrm{G}$

Level: (low/med) LOW

\% Moisture: not dec.

GC Column: RTX-502.2 ID: 0.530 (mm)

Soil Extract Volume:

(uLi)
Contract: $625-01$

SAS NO.:

SDG No.: V204

Lab Sample ID: 14495-008

Lab File ID: E8511

Date Received: 05/02/97

Date Analyzed: 05/09/97

Dilution Factor: $\quad 1.0$

Soil Aliquot Volume:

(uL)

CAS NO.

COMPOUND

CENTRATION UNITS :

(ug/L or ug/Kg) UG/KG

Q

75-71-8--.---Dichlorodifluoromethane

74-83-9--.-.--Bromomethane

75-01-4-------Vinyl Chloride

75-00-3---.---Chloroethane

75-09-2-...--Methylene Chloride

75-69-4-- - - - -Trichlorofluoromet hane

75-34-3-------1,1-Dichloroethane

156-60-5-..---trans-1,2-Dichloroethene

156-59-2------cis-1, 2-Dichloroethene

107-06-2-..--1,2-Dichloroethane

594-20-7-------2,2-Dichloropropane

74-97-5-...---Bromochloromethane

71-55-6-..--1, 1, 1-Trichloroethane

78-93-3--.---2-Butanone

56-23-5-.-----Carbon Tetrachloride

563-58-6--.--1,1-Dichloropropene

75-27-4-...--Bromodichloromethane

78-87-5-...--1, 2-Dichloropropane

10061-01-5-----cis-1,3-Dichloropropene

79-01-6------Trichloroethene

79-00-5-..--1,1,2-Trichloroethane

71-43-2---------Benzene

74-95-3--.---Dibromomethane

10061-02-6-----trans-1,3-Dichloropropene

108-88-3-..----Toluene

106-93-4------1,2-Dibromoethane

127-18-4-..---Tetrachloroethene

142-28-9--.--1,3-Dichloropropane

124-48-1------Dibromochloromethane

108-90-7--.---Chlorobenzene

\begin{tabular}{|r|r|}
10 & $\mathrm{U}$ \\
10 & $\mathrm{U}$ \\
10 & $\mathrm{U}$ \\
10 & $\mathrm{U}$ \\
4 & $\mathrm{BJ}$ \\
5 & $\mathrm{U}$ \\
5 & $\mathrm{U}$ \\
5 & $\mathrm{U}$ \\
5 & $\mathrm{U}$ \\
5 & $\mathrm{U}$ \\
5 & $\mathrm{U}$ \\
5 & $\mathrm{U}$ \\
5 & $\mathrm{U}$ \\
20 & $\mathrm{U}$ \\
5 & $\mathrm{U}$ \\
5 & $\mathrm{U}$ \\
5 & $\mathrm{U}$ \\
5 & $\mathrm{U}$ \\
5 & $\mathrm{U}$ \\
5 & $\mathrm{U}$ \\
5 & $\mathrm{U}$ \\
5 & $\mathrm{U}$ \\
5 & $\mathrm{U}$ \\
5 & $\mathrm{U}$ \\
5 & $\mathrm{U}$ \\
5 & $\mathrm{U}$ \\
5 & $\mathrm{U}$ \\
5 & $\mathrm{U}$ \\
5 & $\mathrm{U}$ \\
5 & $\mathrm{U}$ \\
& \\
\hline
\end{tabular}


$1 \mathrm{~A}$

\section{VOLATILE ORGANICS ANALYSIS DATA SHEET}

EPA SAMPLE NO.

NSCEP-072
Name: QUANTERRA MO

'ode: ITMO

Case No.: V49501

rix: (soil/water) soILi

pse wt/vol:

$5.00(\mathrm{~g} / \mathrm{mL}) \mathrm{G}$

$\epsilon:$ (low/med) LOW

oisture: not dec.

c lumn: RTX-502.2 ID: 0.530 (mm)

I Extract Volume:

(uL)
Contract: $625-01$

SAS NO.:
SDG NO.: V204

Iab Sample ID: 14495-008

Lab File ID: $\quad$ E8511

Date Received: 05/02/97

Date Ainalyzed: 05/09/97

Dilution Factor: $\quad 1.0$

Soil Aliquot Volume:

(uL) CONCENTRATION UNITS:

CAS NO.

COMPOUND

$Q$

630-20-6--.--1,1,1,2-Tetrachloroethane $100-41-4-\ldots-.-$ Ethylbenzene

100-42-5-------styrene

75-25-2-------Bromoform

108-38-3-------0-Xylene

1330-20-7--.--m, p-Xyleñes

108-86-1-...---Bromobenzene

79-34-5--..--1, 1,2,2-Tetrachloroethane

96-i8-4-...---1,2,3-Trichloropropane

95-49-8--------2-Chlorotoluere

106-43-4--.-.--4-Chlorotoluene

541-73-1-...--1, 3-Dichlorobenzene

106-46-7--.-.-1, 4-Dichlorobenzene

95-50-1-..----1,2-Dichlorobenzene

\begin{tabular}{ll|l}
5 & $\mathrm{U}$ \\
5. & $\mathrm{U}$ \\
5 & $\mathrm{U}$ \\
5 & $\mathrm{U}$ \\
5 & $\mathrm{U}$ \\
5 & $\mathrm{U}$ \\
5 & $\mathrm{U}$ \\
5 & $\mathrm{U}$ \\
5 & $\mathrm{U}$ \\
5 & $\mathrm{U}$ \\
5 & $\mathrm{U}$ \\
5 & $\mathrm{U}$ \\
5 & $\mathrm{U}$ \\
5 & $\mathrm{U}$ \\
\hline
\end{tabular}


EPA SAMPLE NO. NSCEP-075

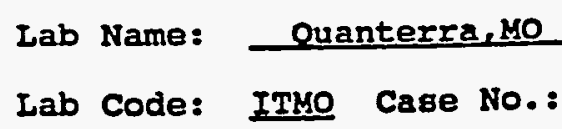

Matrix : (so11/water) SOIL

Sample wt/vol: $1,0 \quad(\mathrm{~g} / \mathrm{ml})^{\circ} G$

Levei: (low/med) Iow

soisture: not dec.

.

CAS NO.

Compound.

LBH (1)
Contract: 625.01

SÁS NO.:

SDG No.: V204

Lab Sample. ID: 14495-009

Lab File ID:

Date Sampled:

Date Analyzed: $05-09-97$

Dilution Factor: 1.0
CONCENTRATION UNITS:

(ug/L or ug/Rg) UG/RG

\begin{tabular}{|c|c|c|}
\hline LBH $(1) \_-$ & \\
\hline
\end{tabular}

v:Concentration of analyte is less than the value given.

X:Pattern reasonably matches gasoline

Y $:$ atterת appears to be multi-peaks, but does not match gasoline

$\mathrm{z}$ : Concentration is primarily from a single peak

(1):Low Boiling Eydrocarbon (IBH) is quantitated as if it is Gasoline. 
1D

HBH ANALYSIS DATA SHEET

EPA SAMPLE NO

NSCEP-075
Name: OUANTERRA MO

Code: IIMO Case No.:

: $:$ (soil/water)

SOII

$20.1(\mathrm{~g} / \mathrm{ml})$

G

SAS NO.:

Contract:

625.01

i. e wt/vol:

20.1

el: (low/med)

LOW

loisture: not dec.

dec.

lction: (SepF/Cont/Sonc/Shak)

SHAR

: Ileanup: $(\mathrm{Y} / \mathrm{N})$

pH:

SHAR

i i NO.

Compound

CONCENTRATION UNITS:

(mg/L or $\mathrm{mg} / \mathrm{kg}) \mathrm{mg} / \mathrm{kg}$

\begin{tabular}{|c|c|}
\hline 25 & 25 \\
\hline & 0 \\
\hline
\end{tabular}

Concentration of analyte is less than the value given. 
VOLATILE ORGANICS ANALYSIS DATA SHEET

Lab Name: QUANTERRA MO

Lab Code: ITMO

Case No.: V49501

Matrix: (soil/water) soIL

Sample wt/vol:

$5.00(\mathrm{~g} / \mathrm{mL}) \mathrm{G}$

Level: (low/med) LOW

\% Mọ̣sture: not dec.

GC Column: RTX-502.2 ID: 0.530 (mm)

Soil Extract Volume:

(UL)
Contract: 625-01

SAS NO.: .
NSCEP-075

SDG No.: V204

Lab Sample ID: 14495-009

Lab File ID: $\quad$ E8489

Date Received: 05/02/97

Date Analyzed: 05/08/97

Dilution Factor: $\quad 1.0$

Soil Aliquot Volume:

(uL)

CONCENTRATION UNITS:

CAS NO.

COMPOUND

(ug/I or $\mathrm{ug} / \mathrm{Kg}$ ) UG/KG

Q. 
$1 A$

VOLATILE ORGANICS ANALYSIS DATA SHEET ib Name: QUANTERRA MO

Code: ITMO

Case No.: V495.01

itrix: (soil/water) SOIL

lliple wt/vol:

$5.00(\mathrm{~g} / \mathrm{mL}) \mathrm{G}$

Moisture: not dec.

:olumn: RTX-502.2 ID: 0.530 (mm)

il Extract Volume:

(uL)
Contract: $625-01$
EPA SAMPLE NO.

NSCEP-075

\section{$\therefore$ : $:$ (low/med) LOW}

jel Extract Volume:

\begin{tabular}{|c|c|}
\hline CAS NO. & $\begin{array}{l}\text { CONCENTR } \\
\mathrm{lug} / \mathrm{L} \text { or }\end{array}$ \\
\hline $\begin{array}{l}630-20-6 \\
100-41-4 \\
100-42-5 \\
75-25-2- \\
108-38-3 \\
1330-20- \\
108-86-1 \\
79-34-5- \\
96-18-4- \\
95-49-8- \\
106-43-4 \\
541-73-1 \\
106-46-7 \\
95-50-1-\end{array}$ & $\begin{array}{l}\text { - } 1,1,1,2 \text {-Tetrachloroethane } \\
\text {-Ethylbenzene } \\
\text { - Styrene } \\
\text { - Bromoform } \\
\text {-0-Xylene } \\
\text {-m,p-Xylenes } \\
\text { - Bromobenzene } \\
\text {-1,1,2,2-Tetrachloroethane } \\
\text {-1,2,3-Trichloropropane } \\
\text {-2-Chlorotoluene } \\
\text {-4-Chlorotoluene } \\
\text {-1,3-Dichlorobenzene } \\
\text {-1,4-Dichlorobenzene } \\
\text {-1,2-Dichlorobenzene }\end{array}$ \\
\hline
\end{tabular}

Lab Sample ID: 14495-009

Lab File ID: $\quad$ E8489

Date Received: 05/02/97

Date Analyzed: . 05/08/97

Dilution Factor: $\quad 1.0$

Soil Aliquot Volume:

(UI) 


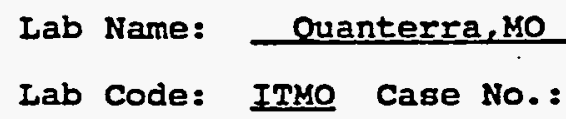

Matrix : (soil/water) soIr.

Sample wt/vol:

1.0 $(g / \mathrm{ml})$

G

Level: (low/med) Low

\& Moisture: not dec.

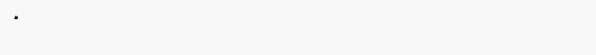

CAS NO.
Contract: 625.01

SAS NO.: SDG No.: V204

Iab sample ID: 14495-010

Iab File ID:

Date sampled: $\quad 04-29-97$

Date Analyzed: 05-09-97

Dilution Factor: 1.0
CONCENTRATION UNITS:

(ug/L or ug/Rg) UG/RG

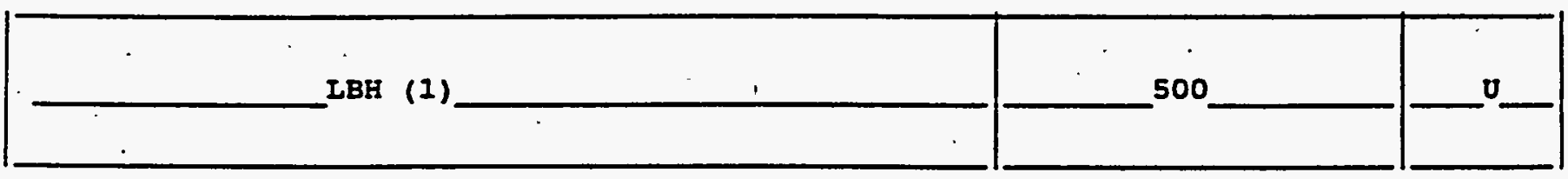

U:Concentration of analyte is less than the value given. $x$ :Pattern reasonably matches gasoline Y:Pattern appears to be multi-peaks, but does not match gasoline z:Concentration is primarily from a single peak

(1): Low Boiling Hydrocarbon (LBH) is quantitated as if it is Gasoline. 
VOLATILE ORGANICS ANALYSIS DATA SHEET

Lab Name: QUANTERRA MO

Lab Code: ITMO

Matrix: (soil/water) SOIL

Sample wt/vol: $\quad 5.00(\mathrm{~g} / \mathrm{mL}) \mathrm{G}$

Level: (low/med) LOW

\% Moịsture: not dec.

GC Column: RTX-502.2 ID: 0.530 (mm)

Soil Extract Volume:

(uL)
Contract: $625-01$

SAS NO.:

SDG No.: V204

Lab Sample ID: 14495-010

Lab File ID: $\quad$ E8490

Date Received: 05/02/97

Date Analyzed: 05/08/97

Dilution Factor: $\quad 1.0$

Soil Aliquot Volume:

(uL)

CONCENTRATION UNITS:

CAS NO. COMPOUNDD (ug/I or ug/Kg) UG/KG

75-71-8--.--Dichlorodifluoromethane

74-83-9--.---Bromomethane

75-01-4-..--.--Vinyl Chloride

75-00-3-...--Chloroethane

75-09-2--.-.---Methylene Chloride

75-69-4--.---Trichlorofluoromethane

75-34-3-...--1,1-Dichloroethane

156-60-5-..---trans-1,2-Dichloroethene

156-59-2-..---Cis-1,2-Dichloroethene

107-06-2--.--1,2-Dichloroethane

594-20-7--.--2,2-Dichloropropane

74-97-5-...---Bromochloromethane

71-55-6-...--1,1,1-Trichloroethane

$78-93-3-------2-$ Butanone

56-23-5--.----Carbon Tetrachloride

563-58-6--.---1, 1-Dichloropropene

75-27-4-...-.-Bromodichloromethane

78-87-5------1,2-Dichloropropane

10061-01-5-----cis-1,3-Dichloropropene

79-01-6.---.--Trichloroethene

79-00-5--.---1, 1, 2-Trichloroethane

71-43-2-..-...-Benzene

74-95-3-..---Dibromomethane

10061-02-6-----trans-1,3-Dichloropropene

108-88-3--.---Toluene

106-93-4-.--- 1,2-Dibromoethane

127-18-4-..----Tetrachloroethene

142-28-9-----1,3-Dichloropropane

124-48-1--.---Dibromochloromethane

108-90-7-...--Chlorobenzene

\begin{tabular}{|c|c|c|}
\hline$\cdot$ & $\begin{array}{r}10 \\
10 \\
10 \\
10 \\
5 \\
5 \\
5 \\
5 \\
5 \\
5 \\
5 \\
5 \\
5 \\
20 \\
5 \\
5 \\
5 \\
5 \\
5 \\
5 \\
5 \\
5 \\
5 \\
5 \\
5 \\
5 \\
5 \\
5 \\
5 \\
5\end{array}$ & $\begin{array}{l}U \\
U \\
U \\
U \\
U \\
U \\
U \\
U \\
U \\
U \\
U \\
U \\
U \\
U \\
U \\
U \\
U \\
U \\
U \\
U \\
U \\
U \\
U \\
U \\
U \\
U \\
U \\
U \\
U \\
U\end{array}$ \\
\hline
\end{tabular}


NSCEP-077

I Name: QUANTERRA MO

$£$ Code: ITMO

Case No.: V4950I

E ix: (soil/water) soIL

mple wt/vol:

$5.00(\mathrm{~g} / \mathrm{mL}) \mathrm{G}$

.1: $\quad$ (low/med) LOW

Moisture: not dec.

Contract: $625-01$

SAS NO.:

SDG NO.: V204
Iab Sample ID: 14495-010

Lab File ID: $\quad \mathbf{E 8 4 9 0}$

Date Received: 05/02/97

Date Analyzed: 05/08/97

Dilution Factor: $\quad 1.0$

Soil Aliquot Volume:

(uI) j- Extract Volume:

(uL)
CONCENTRATION UNITS: (ug/L or $\mathrm{ug} / \mathrm{Kg}$ ) UG/KG

Q

630-20-6------1,1,1,2-Tetrachloroethane

100-41-4--.----Ethylbenzene

100-42-5--.----styrene

75-25-2-...---Bromoform

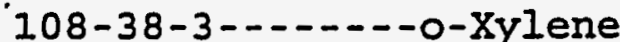

$1330-20-7 \cdots-\cdots, \mathrm{m}-\mathrm{Xy}$ lenes

108-86-1-...---Bromobenzene

79.-34-5-.....-1, 1, 2, 2-Tetrachloroethane

$96-18-4-\ldots-\ldots-1,2,3-$ Trichloropropane

95-49-8-..---2-Chlorotoluene

106-43-4-n---4-Chlorotoluene

541-73-1-...--1, 3-Dichlorobenzene

106-46-7-..--1, 4-Dichlorobenzene

95-50-1-....-1,2-Dichlorobenzene

\begin{tabular}{|ll|l|}
\hline & 5 & $U$ \\
5 & $U$ \\
5 & $U$ & $\cdots$ \\
5 & $U$ \\
5 & $U$ \\
5 & $U$ \\
5 & $U$ \\
5 & $U$ \\
5 & $U$ \\
5 & $U$ \\
5 & $U$ \\
5 & $U$ \\
5 & $U$ \\
5 & $U$ \\
\hline
\end{tabular}


Iab Name:

Iab Code:

Hatrix

Sample wt/rol:

(soil/water)

1.0

Level: (low/med) IOW

\& Moisture: not dec. $(g / m I)$

Compound

LBE (1)

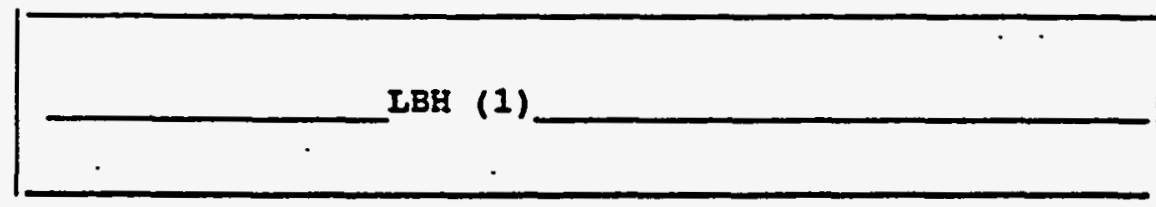

CONCENTRATION UNITS:

(ug/I or ug/Kg) UG/RG

2

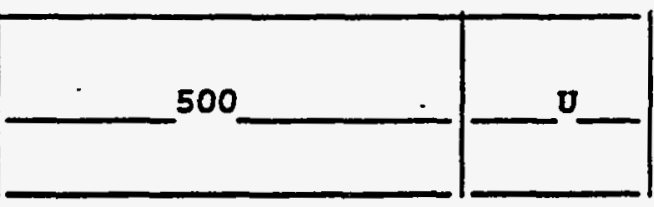

U:Concentration of analyte is less than the value given.

X:Pattern reasonably matches gasoline.

Y:Pattern appears to be multi-peaks, but does not match gasoline

$z=$ Concentration is primarily from a single peak

(1):Low Boiling Hydrocarbon (IBH) is quantitated as if it is Gasoline.

FORH I IBE 
1D

. HBH ANAIYSIS DATA SHEET
EPA SAMPLE NO

NSCEP-084 ab Name: OUANTERRA MO

ab Code: ITMO Case No.:

SAS NO.:

Contract :

625.01

- rix : (soil/water)

SOII

$(\mathrm{g} / \mathrm{mI})$

G

a ple wt/vol:

20.1

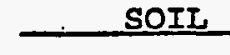

evel: (low/med)

LOW

Moisture: not dec.

dec.

j raction: (SepF/Cont/Sonc/Shak)

SHAK

1 Cleanup: $(\mathrm{Y} / \mathrm{N}) \ldots \mathrm{N} \quad \mathrm{pH}$ :

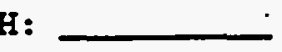

Lab Sample ID:

Lab File ID:

Date Extracted:

Date Analyzed:

Dilution Factor:
SDG NO.:

$14495-011$

Date Sampled:

$.04-29-97$

$05-06-97$

05-07-97 1

CONCENTRATION UNITS :

AS NO.

Compound

(mg/L or $\mathrm{mg} / \mathrm{kg}$ )

$\mathrm{mg} / \mathrm{kg}$

$Q$

\begin{tabular}{|c|}
25 \\
\hline 25 \\
\hline
\end{tabular}$-\mathrm{U}$

$U$ : Concentration of analyte is less than the value given. 
$1 \mathrm{~A}$

VOLATILE ORGANICS ANALYSIS DATA SHEET

Lab Name: QUANTERRA MO

Lab Code: ITMO

Case No.: V49501

Matrix: (soil/water) SOIL

Sample wt/vol:

$5.00(\mathrm{~g} / \mathrm{mL}) \mathrm{G}$

bevel: (low/med) LOW

\%oịture: not dec.

GC Column: RTX-502.2 ID: $0.530^{\circ}(\mathrm{mm})$
EPA SAMPLE NO."

NSCEP -084
Contract: $625-01$

SAS NO.:

SDG No.: V204
Soil Extract Volume:
(uL)
Soil Ext
CAS NO.
COMPOUND
Soil Aliquot Volume:
CONCENTRATION UNITS:
$(\mathrm{ug} / \mathrm{L}$ or $\mathrm{ug} / \mathrm{Kg})$ UG/KG

Lab Sample ID: 14495-011

Lab File ID: $\quad$ E8472

Date Received: 05/02/97

Date Analyzed: 05/08/97

Dilution Factor: $\quad 1.0$

(uL)

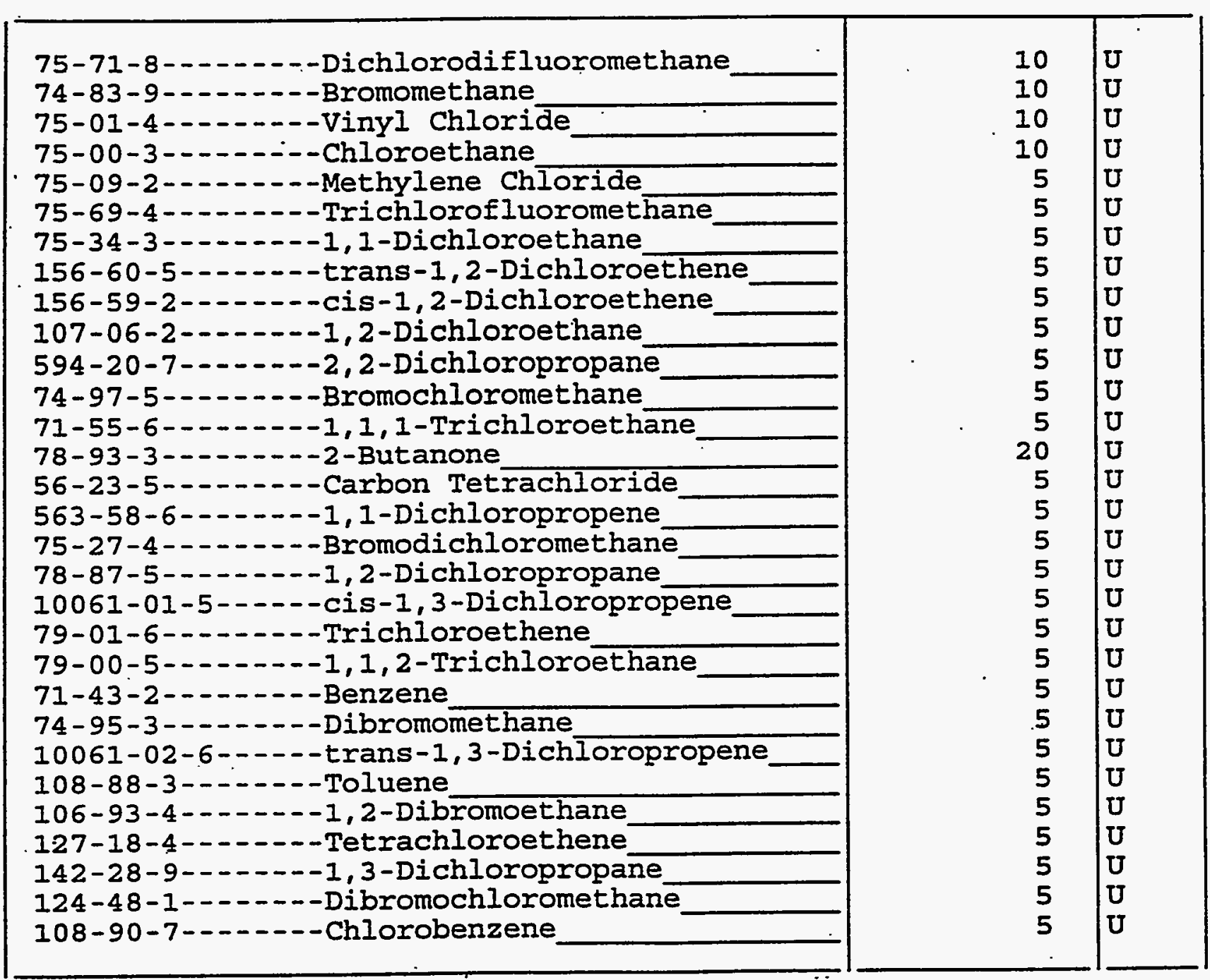


$\because$

$1 \mathrm{~A}$

VOLATILE ORGANICS ANALYSIS DATA SHEET
, Name: QUANTERRA MO

Iode: ITMO

Case No.: V49501

rix: (soil/water) soII

ple wt/vol:

$5.00(\mathrm{~g} / \mathrm{mL}) \mathrm{G}$

$\because$.: (low/med) LOW

loịsture: not dec.

Jlumn: RTX-502.2 ID: 0.530 (mm)

Extract Volume:

(UL)
Contract : $625-01$

SAS NO.:

SDG No.: V204.

Lab Sample ID: 14495-011

Lab File ID: E8472

Date Received: 05/02/97

Date Analyzed: 05/08/97

Dilution Factor: $\quad 1.0$

Soil Aliquot Volume:

(UL)

CONCENTRATION UNITS :

CAS NO.

COMPOUND (ug/L or $\mathrm{ug} / \mathrm{Kg}$ ) UG/KG

Q

630-20-6---.--1,1,1,2-Tetrachloroethane

100-41-4---..--Ethylbenzene

100-42-5--..----Styrene

75-25-2-.----Bromoform

108-38-3-.----0-Xylene

1330-20-7--.--m, p-Xylenes

108-86-1-...--Bromobenzene

79-34-5-..-..-1, 1, 2, 2-Tetrachloroethane

96-18-4-...--1,2, 3-Trichloropropane

95-49-8-....-2-Chlorotoluene

106-43-4-----4-Chlorotoluene

541-73-1-...--1,3-Dichlorobenzene

106-46-7------1,4-Dichlorobenzene

95-50-1----.--1,2-Dichlorobenzene

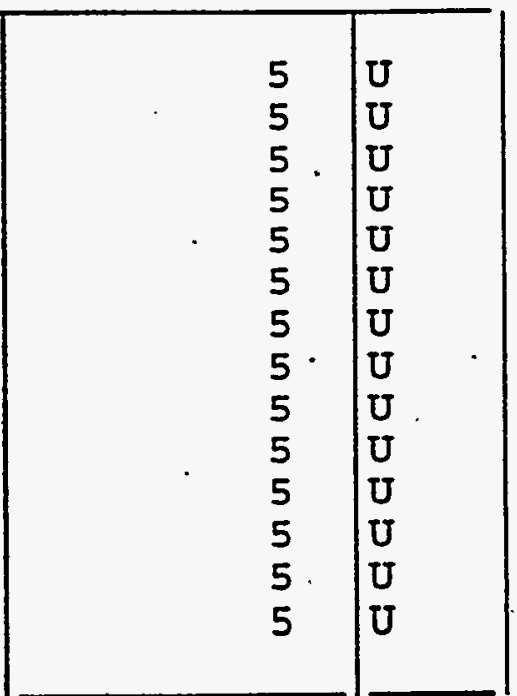




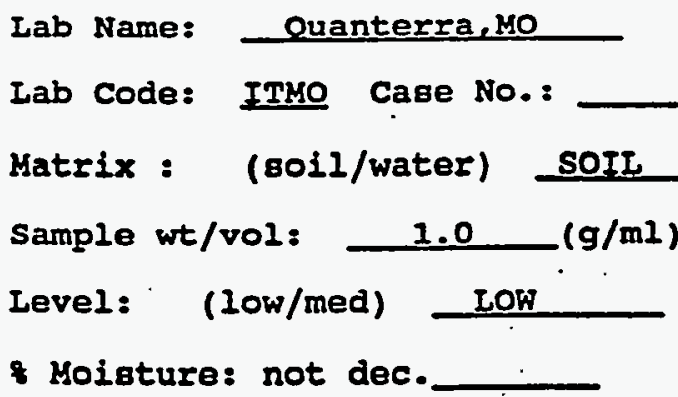

\& Moisture: not dec.

OW

CAS NO.
Contract: 625.01

SAS NO.:

SDG No.: V204

Lab Sample ID: 14495-012

Iab File ID:

Date sampled: $\quad 04-29-97$

Date Analyzed: 05-09-97

Dilution Factor: 1.0
CONCENTRATION UNITS:

(ug/L or ug/Rg) uG/RG

LBH (1) 500

$U:$ Concentration of analyte is less than the value given.

$X:$ Pattern reasonably matches gasoline

Y:Pattern appears to be multi-peaks, but does not match gasoline

2:Concentration is primarily from a single peak

(1): Low Boiling Hydrocarbon (LBB) is quantitated as if it is Gasoline. 


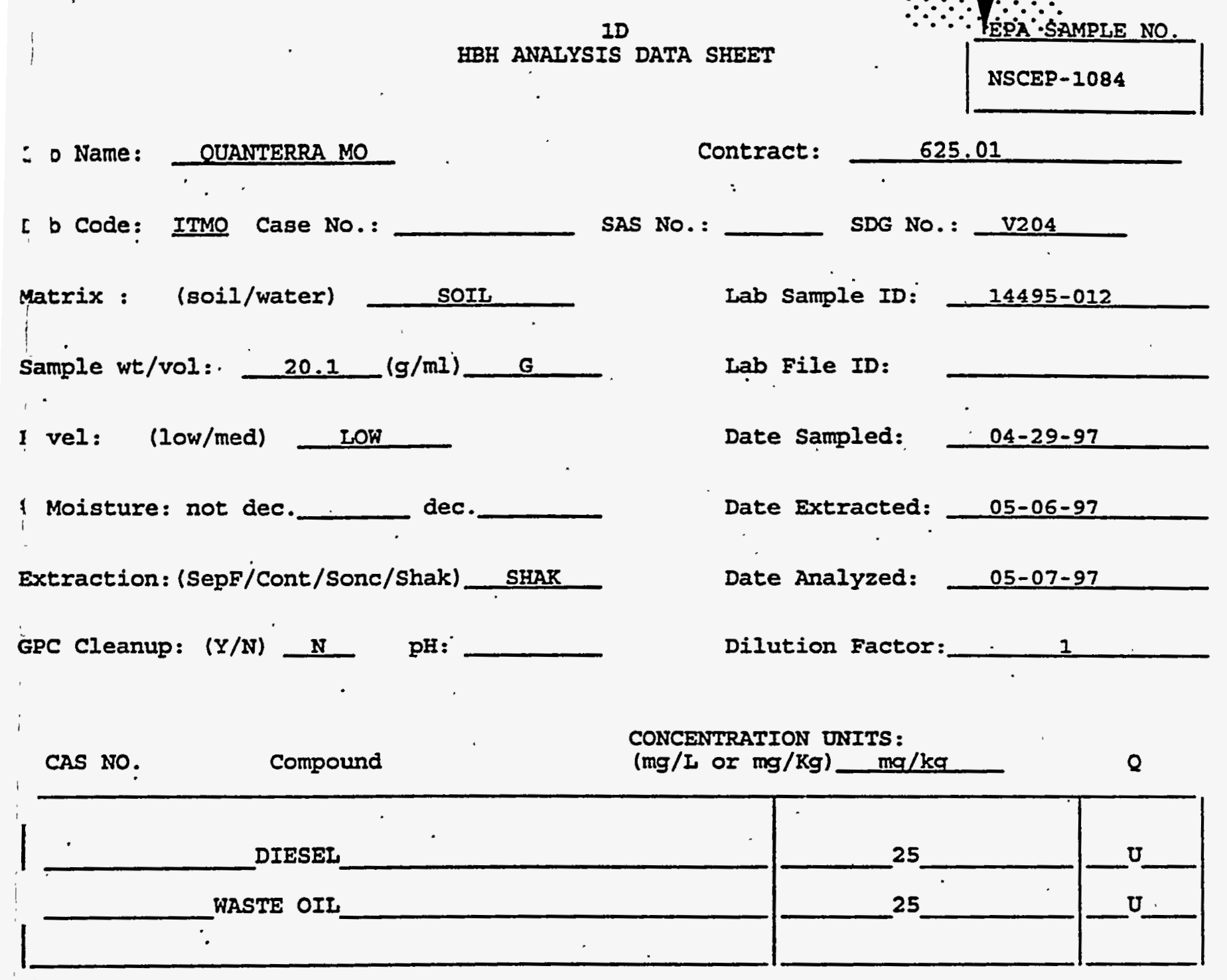

$U:$ Concentration of analyte is less than the value given. 
Lab Name: QUANTERRA MO

Lab Code: ITMO

Matrix: (soil/water) soIL

Sample wt/vol:

Level: $\quad($ low $/ \mathrm{med})$ LOW

\% Moisture: not dec.

GC Column: RTX-502.2 ID: 0.530 (mm)

Soil Extract Volume: (uis)

$5.00(\mathrm{~g} / \mathrm{mL}) \quad$ G
Contract: $625-01$

SAS NO.:
NSCEP-1084
Soll Extract Volume:

CAS NO.

Lab Sample ID: 14495-012

Lab File ID: $\quad$ E8491

Date Received: 05/02/97

Dáte Ánalyzed: 05/08/97

Dilution Factor: $\quad 1.0$

Soil Aliquot Volume:

(uL)

CONCENTRATION UNITS :

(ug/L or $\mathrm{ug} / \mathrm{Kg}$ ) UG/KG

Q 
$1 A$

VOLATILE ORGANICS ANALYSIS DATA SHEET
EPA SAMPLE NO.

NSCEP- 1084
כ Name: QUANTERRA MO

Code: ITMO Case No.: V49501 zrix: (soil/water) soII

hule wt/vol:

$5.00(\mathrm{~g} / \mathrm{mL}) \mathrm{G}$

1: (low/med) LOW

loisture: not dec.

olumn: RTX-502.2 ID: 0.530 (mm)

il Extract. Volume:

(UL)
Contract: $625-01$

SAS No.: '.

SDG No.: V204

Lab Sample ID: 14495-012

Lab File ID: $\quad \mathbf{8 4 9 1}$

Date Received: 05/02/97

Date Analyzed: 05/08/97

Dilution Factor: $\quad 1.0$

Soil Aliquot Volume:

(UL)

CONCENTRATION UNITS :

CAS NO.' COMPOUND

(ug/L or $u g / \mathrm{Kg}$ ) UG/KG

Q

630-20-6-..--1,1,1,2-Tetrachloroethane 100-41-4--------Ethylbenzene

100-42-5--.-.---Styrene

75-25-2--...--Bromoform

108-38-3------o-Xylene

$1330-20-7-\cdots-m, \mathrm{p}-\mathrm{XY}$ lenes

108-86-1--.----Bromobenzene

79-34-5-.....-1, 1, 2, 2-Tetrachloroethane

$96-18-4-\ldots-\ldots-1,2,3-T r i c h l o r o p r o p a n e$

95-49-8--.----2-Chlorotoluene

106-43-4-2.--4-Chlorotoluene

541-73-1--.--.-1, 3-Dichlorobenzene

106-46-7-_.--1., 4-Dichlorobenzene

95-50-1-..--1,2-Dichlorobenzene

\begin{tabular}{|ll|l|}
\hline & 5 & $\mathrm{U}$ \\
& 5 & $\mathrm{U}$ \\
& 5 & $\mathrm{U}$ \\
& 5 & $\mathrm{U}$ \\
& 5 & $\mathrm{U}$ \\
& 5 & $\mathrm{U}$ \\
& 5 & $\mathrm{U}$ \\
& 5 & $\mathrm{U}$ \\
& 5 & $\mathrm{U}$ \\
& 5 & $\mathrm{U}$ \\
& 5 & $\mathrm{U}$ \\
& 5 & $\mathrm{U}$ \\
& 5 & $\mathrm{U}$ \\
& 5 & $\mathrm{U}$ \\
\hline
\end{tabular}


10

IBE ANALYSIS DATA SHEET
EPA SAMPLE NO.

NSCEP-086
Lab Name: $\frac{\text { Ouanterra, MO }}{\text { Iab Code: ITYO Case No.: }}$
ITO

Matrix : (soil/water) SoIL

sample wt/vol:

1.0 $(g / m l)$

G

Level: (low/med) IOW

s Moisture: not dec.

CAS NO.

Compound.

CONCENTRATION UNITS:

(ug/I or ug/Rg) UG/RG

SÄS No.:

Contract: 625.01

SDG No.: V204

Lab Sample ID: $14495-013$

Iab File ID:

Date Sampled: 04-29-97

Date Analyzed: 05-09-97

Dilution Factor: 1.0

LBH (1) 500 0

U: Concentration of analyte is less than the value given.

$X:$ Pattern reasonably matches gasoline

Y:Pattern appears to be multi-peaks, but does not match gasoline

2:Concentration is primarily from a single peak

(1): Low Boiling Hydrocarbon (LBE) is quantitated as if it is Gasoline. 


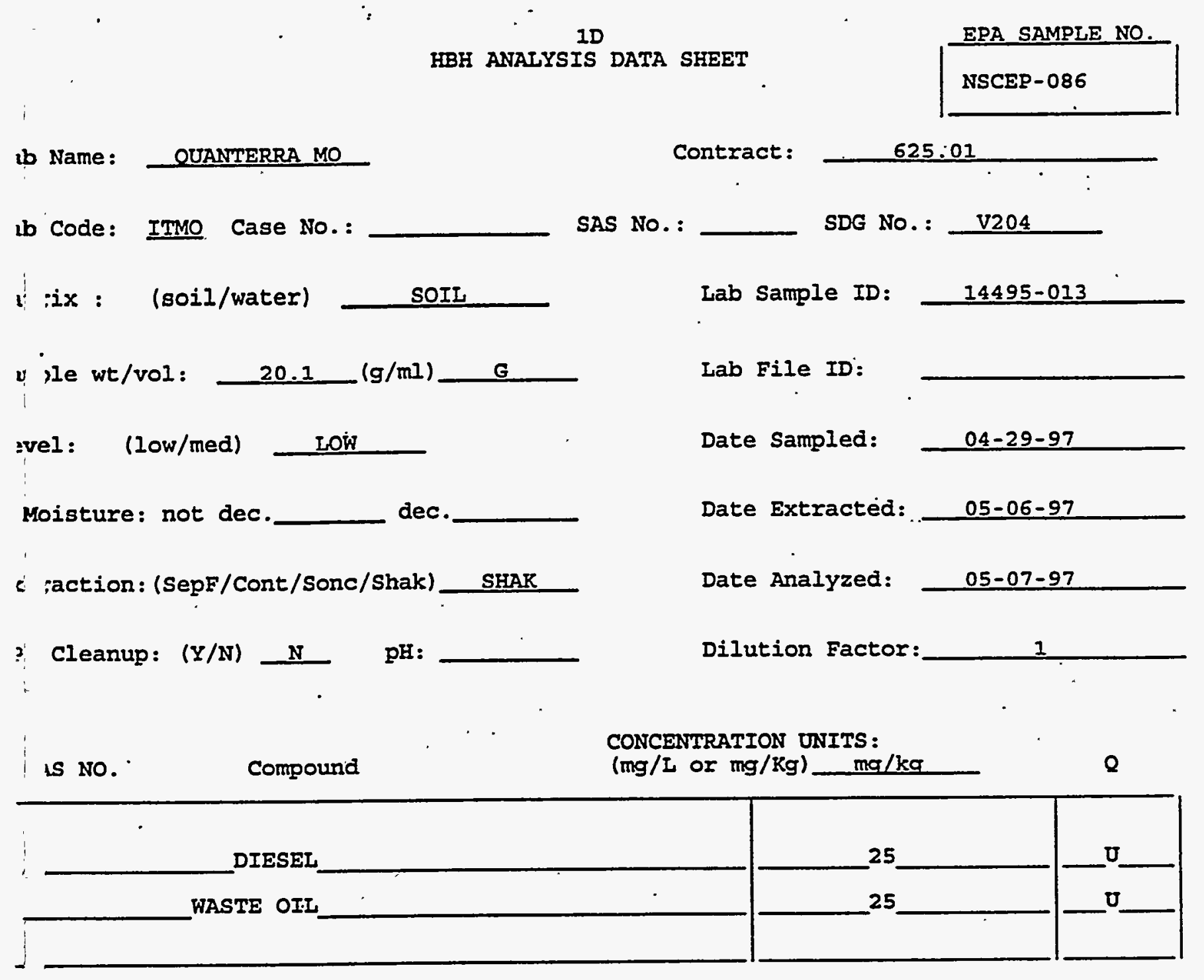

I: Concentration of analyte is less than the value given. 
Lab Name: QUANTERRA MO

Lab Code: ITMO

Case No.: V4950I

Matrix: (soil/water) SOIL

Sample wt/vol:

$5.00(\mathrm{~g} / \mathrm{mL}) \mathrm{G}$

Level: (low/med) Low

Moịsture: not dec.

GC Column: RTX-502.2 ID: 0.530 (mm)

Soil Extract Volume:

(uL)
Contract: $625-01$

SAS NO.:
SDG NO.: V204

Lab Sample ID: 14495-013

Lab File ID: $\quad$ E8512

Date Received: 05/02/97

Date Analyzed: 05/09/97

Dilution Factor: $\quad 1.0$

Soil Aliquot Volume:

(uL)

CONCENTRATION UNITS :

CAS NO. COMPOUND (ug/L or $\mathrm{ug} / \mathrm{kg}$ ) UG/KG

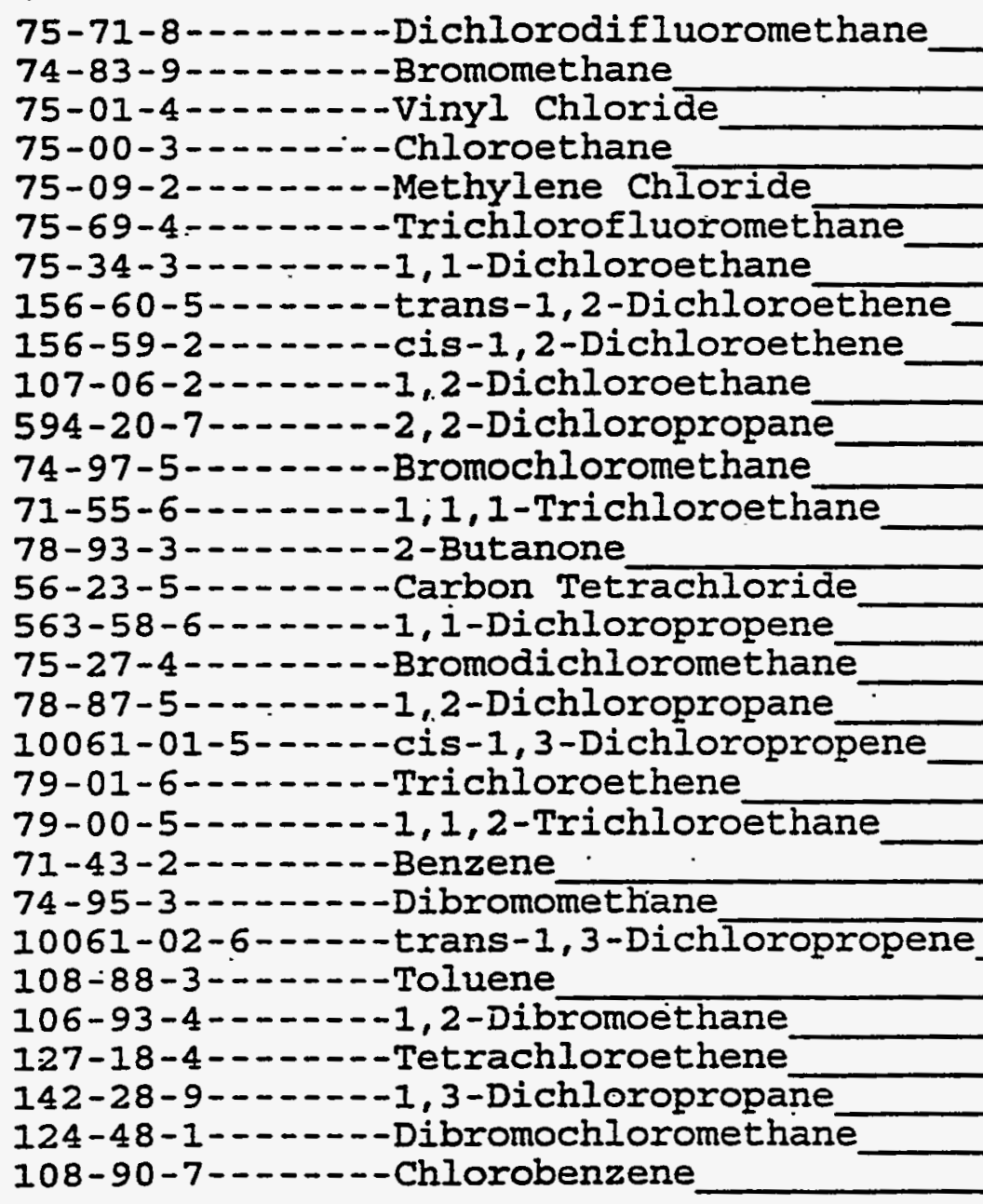

\begin{tabular}{|c|l|}
10 & $U$ \\
10 & $U$ \\
10 & $U$ \\
10 & $U$ \\
4 & $B J$ \\
5 & $U$ \\
5 & $U$ \\
5 & $U$ \\
5 & $U$ \\
5 & $U$ \\
5 & $U$ \\
5 & $U$ \\
5 & $U$ \\
20 & $U$ \\
5 & $U$ \\
5 & $U$ \\
5 & $U$ \\
5 & $U$ \\
5 & $U$ \\
5 & $U$ \\
5 & $U$ \\
5 & $U$ \\
5 & $U$ \\
5 & $U$ \\
5 & $U$ \\
5 & $U$ \\
5 & $U$ \\
5 & $U$ \\
5 & $U$ \\
5 & $U$ \\
\end{tabular}


○ Name: QUANTERRA MO

= Code: ITMO Case No.: V49501 SAS No.: trix: (soil/water) soIL nule wt/vol:

$5.00(\mathrm{~g} / \mathrm{mL}) \quad \mathrm{G}$

U 1: (low/med) LOW

Moisture: not dec.

olumn: RTX-502.2 ID: $0.530(\mathrm{~mm})$

il Extract Volume:

(uL)
Contract: $625-01$

NSCEP -086

SDG NO.: V204

Lab Sample ID: 14495-013

Lab File ID: $\quad$ E8512

Date Received: 05/02/97

Date Analyzed: 05/09/97

Dilution Factor: $\quad 1.0$

Soil Aliquot Volume:

(uL)

CONCENTRATION UNITS :

CAS NO.

COMPOUND

(ug/L or $\mathrm{ug} / \mathrm{Kg}$ ) UG/KG

Q

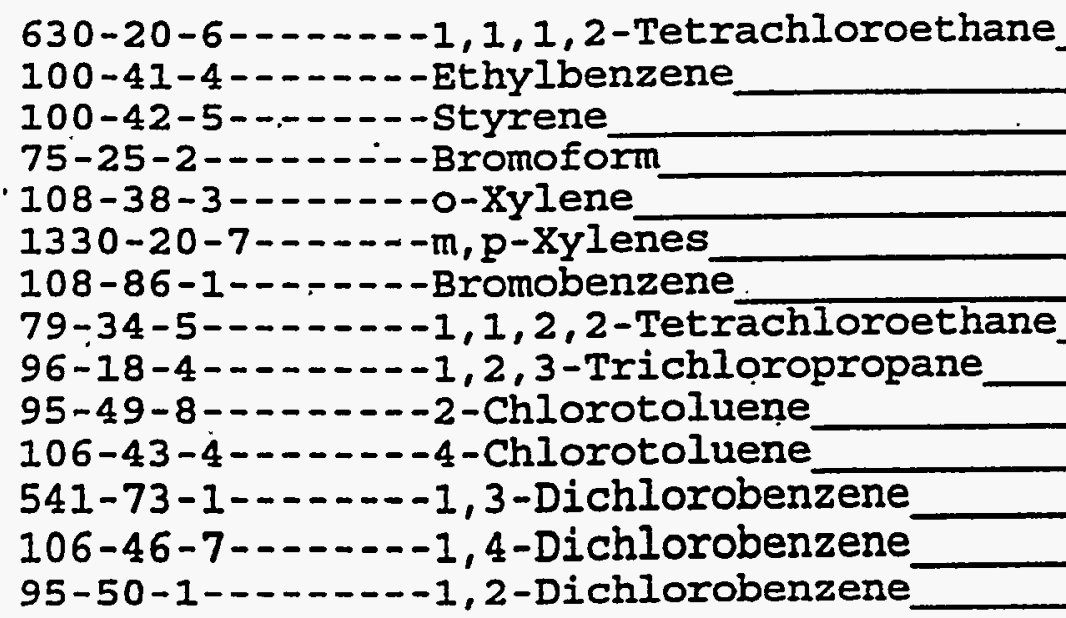


EPA SAMPLE NO.

NSCEP -088
Lab Name:

Iab code: ITxO Case No.:

Matrix : (soil/water) soII

Sample wt/vol: $1.0 \quad(\mathrm{~g} / \mathrm{ml}) \quad \mathrm{G}$

Level: (low/med) Low

\& Moisture: not dec:

CAS NO.

Compound
Contract: 625.01

SAS NO.: SDG NO.: V204

Iab Sample ID: 14495-014

Iab File ID:

Date Sampled: $\quad 04-29-97$

Date Analyzed: 05-09-97

Dilution Factor: 1.0

CONCENTRATION UNITS:

(ug/L or ug/kg) UG/KG

$\mathbf{Q}$

500

$\mathbf{0}$

IBH (1)

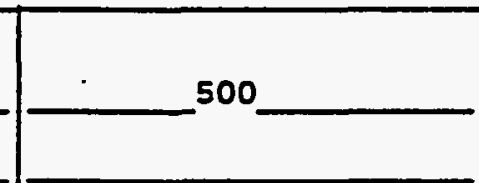

U:Concentration of analyte is less than the value given.

$x:$ Pattern reasonably matches gasoline

Y:Pattern appears to be multi-peaks, but does not match gasoline

z:Concentration is primarily from a oingle peak

(1):Low Boiling Hydrocarbon (IBH) is quantitated as if it is Gasoline.

FORM I IBH 
now Code: ITMO Case No.:

SAS NO.:

SDG NO.: V204

Lab Sample ID:

$14495-014$

Lab File ID:

Date Sampled:

$04-29-97$

Date Extracted:

$05-06-97$

Date Analyzed:

05-07-97

Dilution Factor:

1

CONCENTRATION UNITS :

. $(\mathrm{mg} / \mathrm{L}$ or $\mathrm{mg} / \mathrm{Kg}) \mathrm{mg} / \mathrm{ka}$

Q

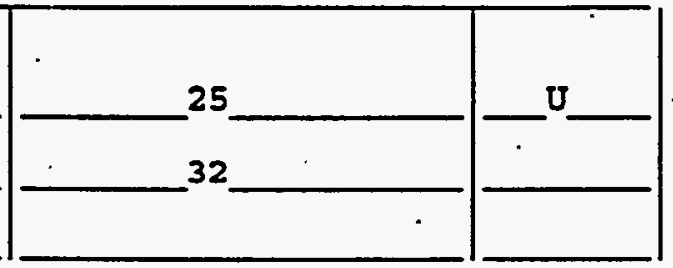

i. Concentration of analyte is less than the value given. 
VOLATILE ORGANICS ANALYSIS DATA SHEET

Lab Name: QUANTERRA MO

Lab Code: ITMO

Matrix: (soil/water) SOIL

Sample wt/vol:

$5.00(\mathrm{~g} / \mathrm{mL}) \mathrm{G}$

Irevel: : (low/med) Low

\% Moịsture: not dec.

GC Column: RTX-502.2 ID: 0.530 (mm)

(UL)
Contract: $625-01$

NSCEP-088
SAS NO.:

SDG NO.: V204
Soil Extract volume:

CAS NO.
COMPOUND
Lab Sample ID: 14495-014

Lab File ID: $\quad$ E8513

Date Received: 05/02/97

Date Analyzed: 05/09/97

Dilution Factor: $\quad 1.0$

Soil.Aliquot volume:

(uL)

\footnotetext{
75-71-8-- - - - Dichlorodifluoromethane

74-83-9-------Bromomethane

75-01-4--------Vinyl Chloride

75-00-3-...--Chloroethane

75-09-2--.----Methylene Chloride

75-69-4-..---.-Trichlorofluoromet hane

75-34-3-...--1,1-Dichloroethane

156-60-5--.---trans-1,2-Dichloroethene

156-59-2------cis-1,2-Dichloroethene

107-06-2------1,2-Dichloroethane

594-20-7--.---2, 2-Dichloropropane

74-97-5--.----Bromochloromethane

71-55-6------1, 1, 1-Trichloroethane

78-93-3----.--2-Butanone

56-23-5-------Carbon Tetrachloride

563-58-6-----1, 1-Dichloropropene

75-27-4--.-----Bromodichloromethane

78-87-5--.----1, 2-Dichloropropane

10061-01-5-----cis-1,3-Dichloropropene

79-01-6------Trichloroethene

79-00-5-...--1, 1,2-Trichloroethane

71-43-2--.-.--Benzene

74-95-3-...--Dibromomethane

10061-02-6-----trans-1,3-Dichloropropene

108-88-3-------Toluene

106-93-4--.---1,2-Dibromoethane

127-18-4------Tetrachloroethene

142-28-9-----1,3-Dichloropropane

124-48-1-----Dibromochloromethane

108-90-7---.--Chlorobenzene
}

CONCENTRATION UNITS:

$\mathrm{ug} / \mathrm{L}$ or $\mathrm{ug} / \mathrm{Kg}$ ) UG/KG 
ab Name: QUANTERRA, MO

$z$ Code : ITMO

z+rix: (soil/water) soIL

ample wt/vol:

$5.00(\mathrm{~g} / \mathrm{mL}) \mathrm{G}$

$\geqq 21:$

(low/med) LoW

Moịsture: not dec.

I Eolumn: RTX-502.2 ID: 0.530 (mim)

jil Extract Volume:

(uL)
Contract: $625-01$
NSCEP -088
SAS NO.:

SDG No.: V204

Lab Sample ID: 14495-014

Lab File ID: $\quad$ E8513

Date Received: 05/02/97

Date Analyzed: 05/09/97

Dilution Factor: 1.0

Soil Aliquot Volume:

(ụL)

CONCENTRATION · UNITS :

CAS NOO. COMPOUND (ug/L or $\mathrm{ug} / \mathrm{Kg}) \mathrm{UG} / \mathrm{KG}$. Q

\begin{tabular}{|l|l|l|}
\hline & 5 & $\mathrm{U}$ \\
& 5 & $\mathrm{U}$ \\
& 5 & $\mathrm{U}$ \\
& 5 & $\mathrm{U}$ \\
& 5 & $\mathrm{U}$ \\
& 5 & $\mathrm{U}$ \\
& 5 & $\mathrm{U}$ \\
& 5 & $\mathrm{U}$ \\
& 5 & $\mathrm{U}$ \\
& 5 & $\mathrm{U}$ \\
& 5 & $\mathrm{U}$ \\
5 & $\mathrm{U}$ \\
5 & $\mathrm{U}$ \\
& 5 & $\mathrm{U}$ \\
\hline
\end{tabular}


10

LBE ANALYSIS DATA SHEET

EPA SAYPLE NO.

NSCEP-098

Lab Name: Quanterra, Mo
Iab Code: ITMO Case No.:

Matrix : (soil/water). SoIL

Sample wt/vol:

$1.0 \quad(\mathrm{~g} / \mathrm{ml})$

Level: (low/med) LOW

\&oisture: not dec.
Contract: 625.01

SAS NO.:

SDG NO.: V205

Lab Sample ID: 14533-027

Lab File. ID:

Date sampled: $04-30-97$

Date Analyzed: 05-12-97

Dilution Factor: 1.0

CONCENTRATION ONITS:

(ug/L or ug/Rg) UG/RG

$\mathbf{Q}$

500

-

U:Concentration of analyte is 1 ess than the value given.

$X$ : Pattern reasonably matches gasoline

Y:Pattern appears to be multi-peaks, but does not match gasoline

$\mathrm{z}$ :Concentration is primarily from. a single peak

(1) : Low Boiling Hydrocarbon (LBH) is quantitated as if it is Gasoline.

FORY I LBH 
ID

HBH ANALYSIS DATA SHEET
ËPA SAMPLE NO NSCEP-098
Tb Name: QUANTERRA MO

Lab code: ITMO Case No.:

L.dtrix : (soil/water)

SOIL

imple wt/vol:

20.1

$(g / m 1)$

G

T.ovel: (low/med)

LOW

- Moisture: not dec. dec. itraction: (SepF/Cont/Sonc/Shak) SHAK

?C Cleanup: $(Y / N)-\mathrm{N}$

CAS NO. Compound
Contract:

SAS NO.:

Iab sample ID:

14533-027

Iab File ID:

Date Sampled:

$04-30-97$

Date Extracted:

$05-09-97$

Date Analyzed:

05-13-97

Dilution Factor: 1

CONCENTRATION UNITS:

(mg/L or $\mathrm{mg} / \mathrm{Kg}$ ) _ mg/ka

$\mathbf{2}$

\begin{tabular}{|r|}
\hline 25 \\
\hline
\end{tabular}

$v$ : Concentration of analyte is less than the value given. 
$1 A$

VOLATILE ORGANICS ANALYSIS DATA SHEET

Lab Name: QUANTERRA MO

Lab Code: ITMO ' Case No.: V5330I

Matrix: (soil/water) soIL

Sample wt/vol: $\quad 1.00(\mathrm{~g} / \mathrm{mL}) \mathrm{G}$

Level: (low/med) LOW

$\%$ Moisture: not dec.

GC Column: RTX-502.2 ID: 0.530 (mm)

Soil Extract Volume:

(UI)
Contract: 625-01

SAS NO.:

SDG No.: V205

NSCEP-098

Lab Sample ID: 14533-027

Lab File ID: . F0027

Date Received: 05/06/97

Date Analyzed: 05/13/97

Dilution Factor: $\quad 1.0$

Soil Aliquot Volume:

(uL) -

CONCENTRATION UNITS :

CAS NO.

COMPOUND

(ug/L or ug/Kg) UG/KG

Q

75-71-8--.--Dichlorodifluoromethane

74-83-9-...---Bromomethane

75-01-4-..-.--vinyl Chloride

75-00-3-.-----Chloroethane

75-09-2-...---Methylene Chloride

75-69-4-...-.-Trichlorofluoromethane

75-34-3-....-1;1-Dichloroethane

156-60-5--.-.--trans-1, 2-Dichloroethene

156-59-2-...--cis-1, 2-Dichloroethene

107-06-2-1---1, 2-Dichloroethane

594-20-7--.---2, 2-Dichloropropane

74-97-5-------Bromochloromethane

71-55-6----.-i, 1, 1-Trichloroethane

78-93-3--.---.-2-Butanone

56-23-5-------Carbon Tetrachloride

563-58-6-----1, 1-Dichloropropene

75-27-4--.-----Bromodichloromethane

78-87-5--.----1, 2-Dichloropropane

10061-01-5-----Cis-1,3-Dichloropropene

79-01-6-...-.-Trichloroethene

79-00-5--.---1, 1,2-Trichloroethane

71-43-2--.---Benzene

74-95-3--.----Dibromomethane

10061-02-6--.--trans-1,3-Dichloropropene

108-88-3--.---Toluene

106-93-4--.---1,2-Dibromoethane

108-10-1-..--4-Methyl-2-Pentanone

127-18-4------Tetrachloroethene

142-28-9------1,3-Dichloropropane

124-48-1--.--Dibromochloromethane

\begin{tabular}{|c|c|c|}
100 & $U$ \\
100 & $U$ \\
100 & $U$ \\
100 & $U$ \\
50 & $U$ \\
50 & $U$ \\
50 & $U$ \\
50 & $U$ \\
50 & $U$ \\
50 & $U$ \\
50 & $U$ \\
50 & $U$ \\
50 & $U$ \\
200 & $U$ \\
50 & $U$ \\
50 & $U$ \\
50 & $U$ \\
50 & $U$ \\
50 & $U$ \\
50 & $U$ \\
50 & $U$ \\
50 & $U$ \\
50 & $U$ \\
50 & $U$ \\
50 & $U$ \\
50 & $U$ \\
100 & $U$ \\
50 & $U$ \\
50 & $U$ \\
50 & $U$ \\
& \\
\hline \\
\hline
\end{tabular}


to Name :" QUUANTERRA MO"

; Code: ITMO

Case No.: V5330I

atrix: (soil/water) sOIL

ale wt/vol:

$1.00(\mathrm{~g} / \mathrm{mL}) \mathrm{G}$

эrel: (low/med) IOW

moisture: not dec.

: Column: RTX-502.2 ID: 0.530 (mm)

ill Extract Volume:

(uL)
Contract: $625-01$

SAS NO: :

NSCEP-098

SDG NO.: V205

Lab Sample ID: 14533-027

Lab File ID: F0027

Date Received: 05/06/97

Date Analyzed: 05/13/97

Dilution Factor: $\quad 1.0$

Soil Aliquot Volume:

(uL)

CONCENTRATION UNITS:

CAS NO. COMPOUND. $\quad(u g / L$ or $\mathrm{ug} / \mathrm{Kg}$ ) UG/KG

Q

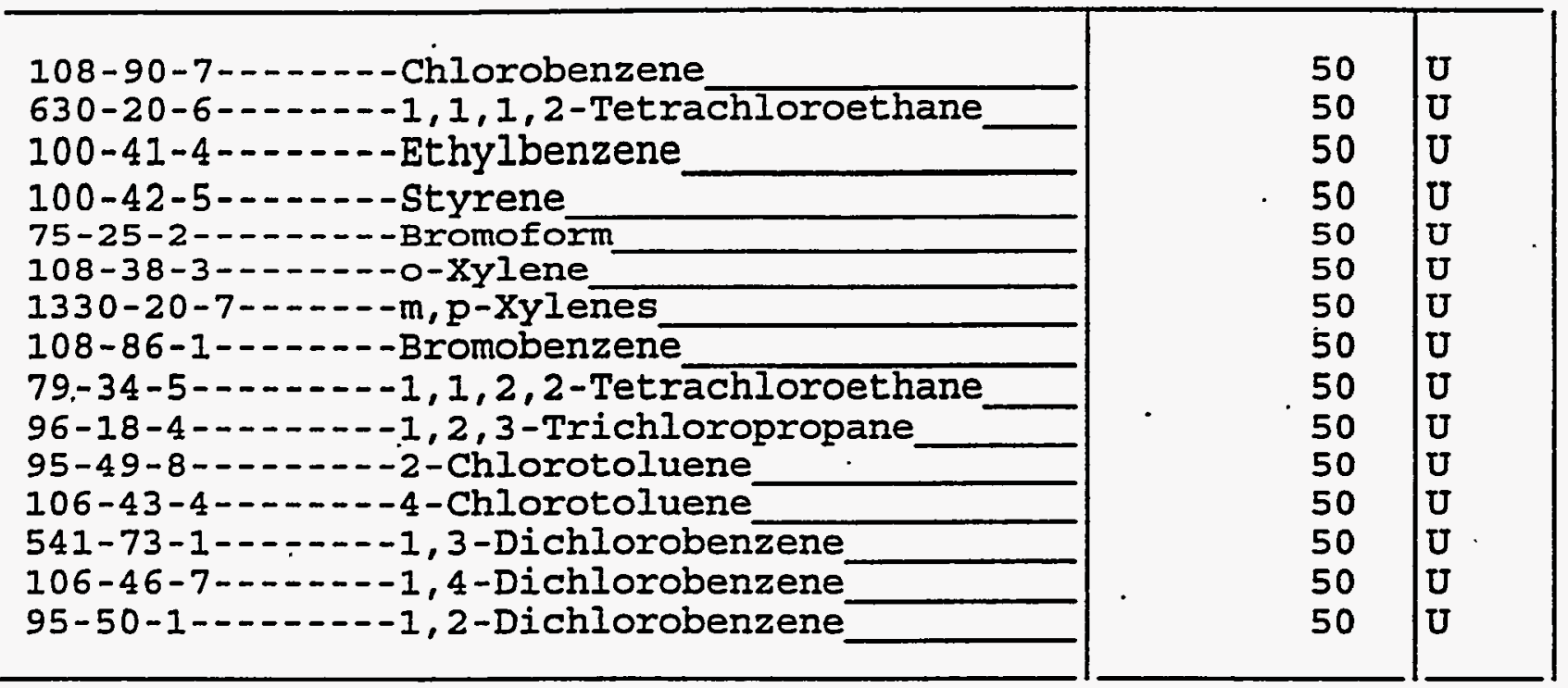


Iab Name: Quanterra, MO

Lab code: ITMO Case No.:

Matrix : (soil/water) soIL

Sample wt/vol:

1.0 $(\mathrm{g} / \mathrm{ml})$

G

Level: (low/med) IOW

soisture: not dec.

Contracts $\cdots \quad 625.01$

SAS NO.: SDG NO.: V205

Iab Sample ID: 14533-029

Iab Filo ID:

Date Sampled:

$04-30-97$

Date Analyzed: $05-12-97$

Dilution Factor: 1.0

CONCENTRATION UNITS:

(ug/I or ug./Kg) UG/KG

$\mathbf{Q}$

CAS No.

compound.

500

$\mathbf{0}$

U:Concentration of analyte is less than the value given.

$X: P a t t e r n$ reasonably matches gasoline

Y:Pattern appears to be multi-peaks, but does not match gasoline

z:Concentration is primarily from a single peak

(1) Low Boiling Hydrocarbon (IBH) is quantitated as if it 18 Gasoline. 
10

HBH ANALYSIS DATÁ SHEET
BPA SAMPLE NO.

NSCEP - 135

Lab Name: OUANTERRA MO

Contract:

625.01

Lab code: ITMO Case No.:

SAS NO.:

SDG No.: V205

Matrix : (8011/water)

SorL

Iab Sample ID:

14533-029

Sample wt/vol: $20.2(\mathrm{~g} / \mathrm{ml})$

Lab File ID:

Level: (Low/med)

IOW

Date Sampled: .

04-30-97

Moistura: not dec. dec.

Date Extracted:

05-09-97

Extraction: (SepF/Cont/Sonc/Shak) SHAR

Date Analyzed:

$05-13-97$

GPC Cleanup: $(\Psi / N)-\mathrm{N} \quad \mathrm{pH}:$

Dilution Factor:

1

CONCENTRATION OUITS:

CAS NO.

Compound

(mg/L or $\mathrm{mg} / \mathrm{Kg}$ )

$\mathrm{mg} / \mathrm{kg}$

Q

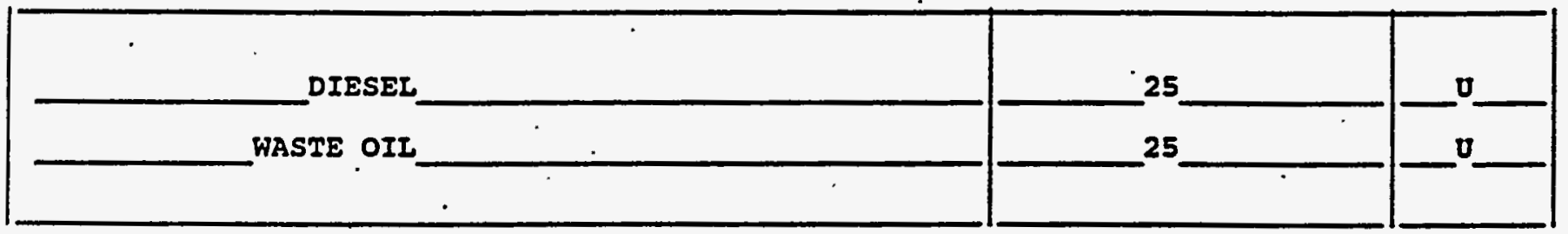

$U$ : Concentration of analyte is less than the value given. 
Lab Name:-“Q̄UANTERRÁ MO

Lab Code: ITMO

Case No.: V53301

Matrix: (soil/water) soIL

Sample wt/vol: $\quad 1.00(\mathrm{~g} / \mathrm{mL}) \mathrm{G}$

Level: (low/med) Low

\% Moisture: not dec.

GC Column: RTX-502.2 ID: 0.530 (mm)

Soil Extract Volume:

CAS NO.
(uL)
Contract : $625-01$

SAS NO.:

NSCEP- 135

SDG NO.: V205

Lab Sample ID: 14533-029

Lab File ID: E8567.

Date Received: 05/06/97

Date Analyzed: 05/13/97

Dilution Factor: , 1.0

Soil Aliquot Volume:

(uL)

CONCENTRATION UNITS :

(ug/L or ug/Kg) UG/KG

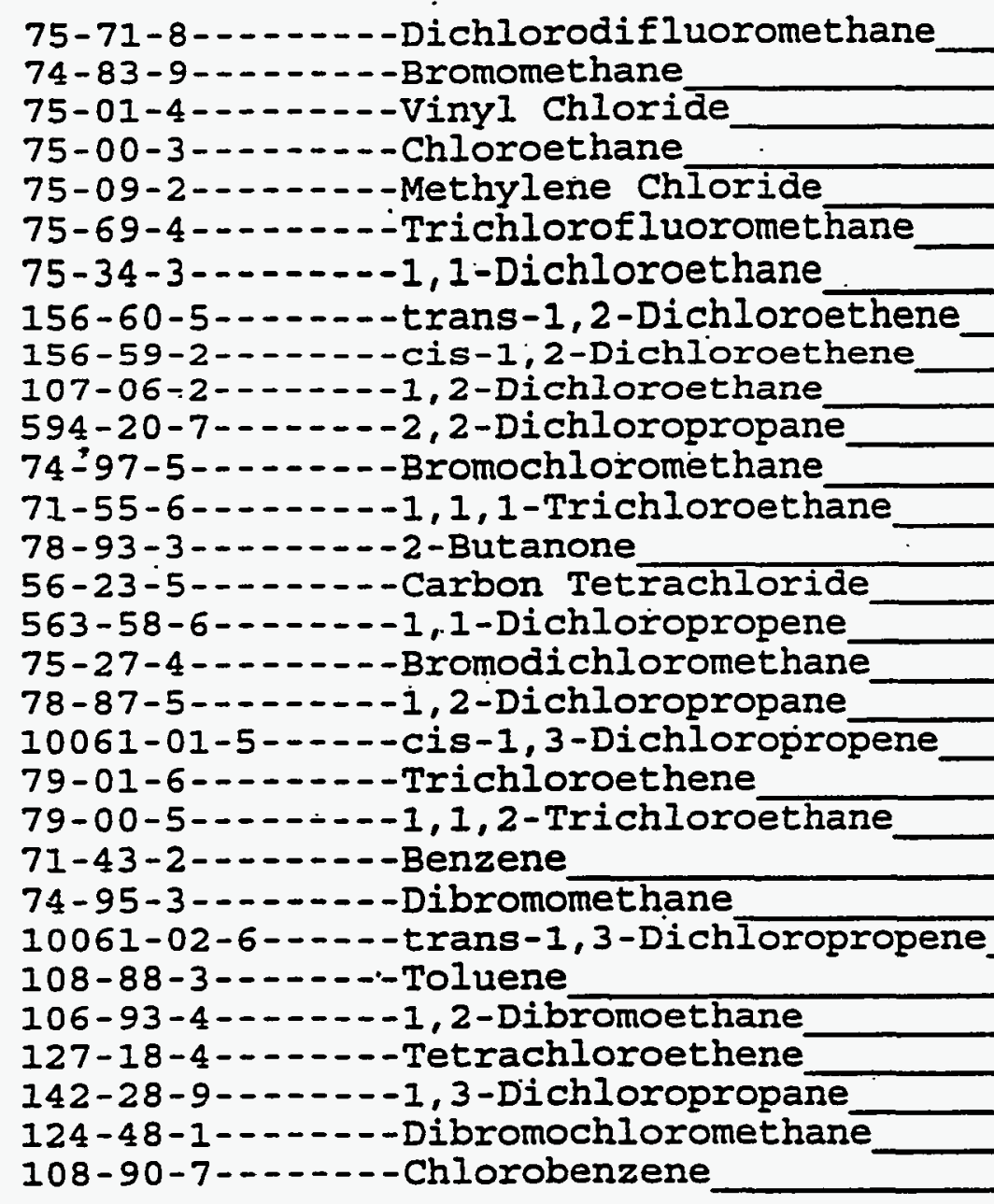


IA

VOLATILE ORGANICS ANALYSIS DATA SHEET

EPA SAMPIE NO.

b Name:“QÜANTERRA MO

Contract: 625-01

NSCEP-135

b Code: ITMO Case No.: V53301 SAS No.:

SDG NO.: V205

เtrix: (soil/water) SoIL

mple wt/vol: $\quad 1.00(\mathrm{~g} / \mathrm{mL}) \mathrm{G}$

Iab Sample ID: 14533-029

'vel: (low/med) LOW

Lab File ID: $\quad$ E8567

Moisture: not dec.

Date Received: 05/06/97

Date Analyzed: 05/13/97

Column: RTX-502.2 ID: 0.530 (mm)

Dilution Factor: $\quad 1.0$

iil Extract Volume:

(uL)

Soil Aliquot Volume:

(uL)

CONCENTRATION UNITS:

CAS NO.

COMPOUND

(ug/I or $\mathrm{ug} / \mathrm{kg}$ ) UG/KG

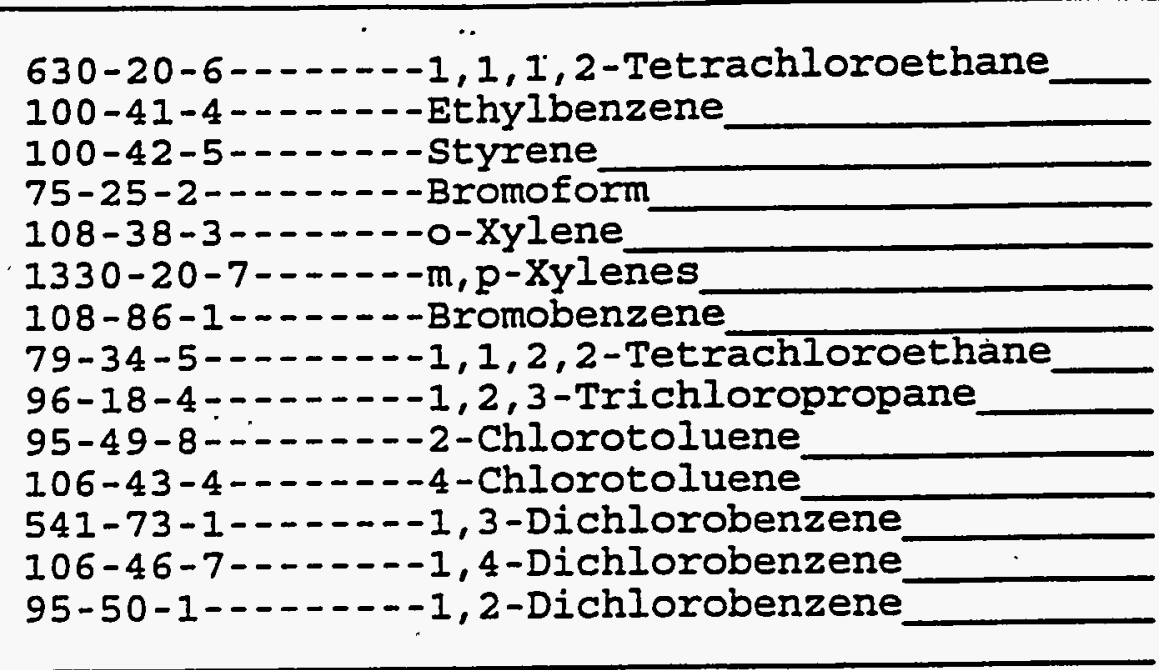

\begin{tabular}{|l|l|l|}
\hline 25 & $U$ \\
25 & $U$ \\
25 & $U$ \\
25 & $U$ \\
25 & $U$ \\
25 & $U$ \\
25 & $U$ \\
25 & $U$ \\
25 & $U$ \\
25 & $U$ \\
25 & $U$ \\
25 & $U$ \\
25 & $U$ \\
25 & $U$ \\
\hline
\end{tabular}


Lab Kame:

Lab code:

Ouanterrako

ITYO Case No.:

Matrix : (soll/water) sorr

Sample wt/vol:

1.0

$(g / m)$

G

Level: (low/med). ION

- Molsture: not dec.

CAS No.

Compound contract: 625,01

SAS NO.: SDG No.: Y205

Iab Sample ID: 14533-028

Iab Fie ID:

Date Sampleds 04-30-97

Dato Analyzed: 05-12-97

Dilution Factor: 1.0
CONCENTRATIOA UNITS:

(ug/I or ug/Kg) ug/Ke

LBH (1) 500

Uaconcentration of andyte is lose than tho value given.

X:Pattern raasonably matches gacoline

Y: Pattern appeare to be multi-peaks, but does not match gasolina

z:Concentration is primarily from a oingle peak

(1):Low Boiling Hydrocarbon (LBB) is quantitated as if it is Gasoline. 
Lab Name: OUANTERRA Mo

Contract: 625.07

Lab Code: ITMO Case No.: SAS NO.: SDG No.: V205

Matrix : (so11/water)

SOIL

Iab Sample ID: $14533-028$

Sample wt/vol: 20.1 (g/ml) G Lab File ID: Level: (low/med) LOW Date Sampled: $04-30-97$

Moisture: not dec. dec. Date Extracted: 05-09-97 Extraction: (SepF/Cont/Sonc/Shak) SHAK

Date Analyzed:

$05-13-97$

GPC Cleanup: $(Y / N) \perp N$ pH:

Dilution Factor: 1

CONCENTRATION UNITS:

CAS NO. Compound (mg/L or $\mathrm{mg} / \mathrm{kg}$ ). $\mathrm{ma} / \mathrm{ka}$ Q

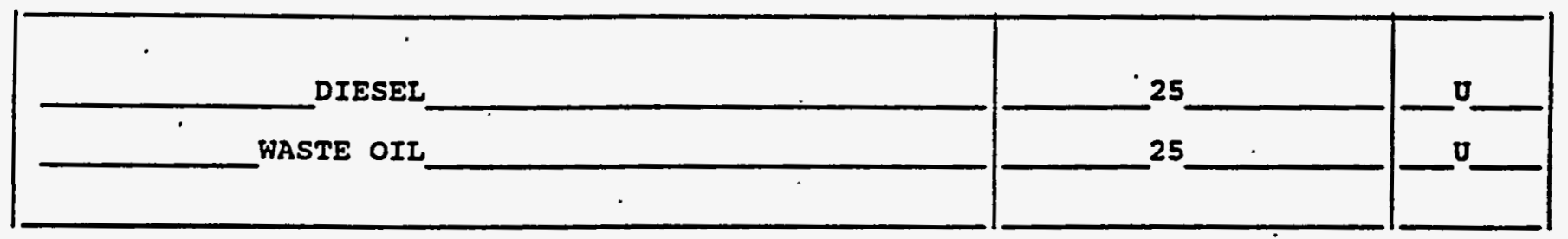

$U$ : Concentration of analyte is less than the value given. 
NSCEP-140

Lab Name: "QUUANTERRA" MO

Lab Code: ITMO

Case No.: V53301

Matrix: (soil/water) soIL

Sample wt/vol:

$1.00(\mathrm{~g} / \mathrm{mL}) \mathrm{G}$

Level: (low/med) LOW

\% Moisture: not dec.

GC Column: RTX-502.2 ID: 0.530 (mm)

Soil Extract Volume:

(uI)
Contract: $625-01$

SAS NO.:

SDG NO.: V205

Lab Sample ID: 14533-028

Lab File ID: $\quad$ E8566

Date Received: 05/06/97

Date Analyzed: 05/13/97

Dilution Factor: $\quad 1.0$

Soil Aliquot Volume:

(uL)

CAS NO.

COMPOUND

CONCENTRATION UNITS:

(ug/L or $\mathrm{ug} / \mathrm{Kg}$ ) UG/KG

Q

\begin{tabular}{|c|}
\hline 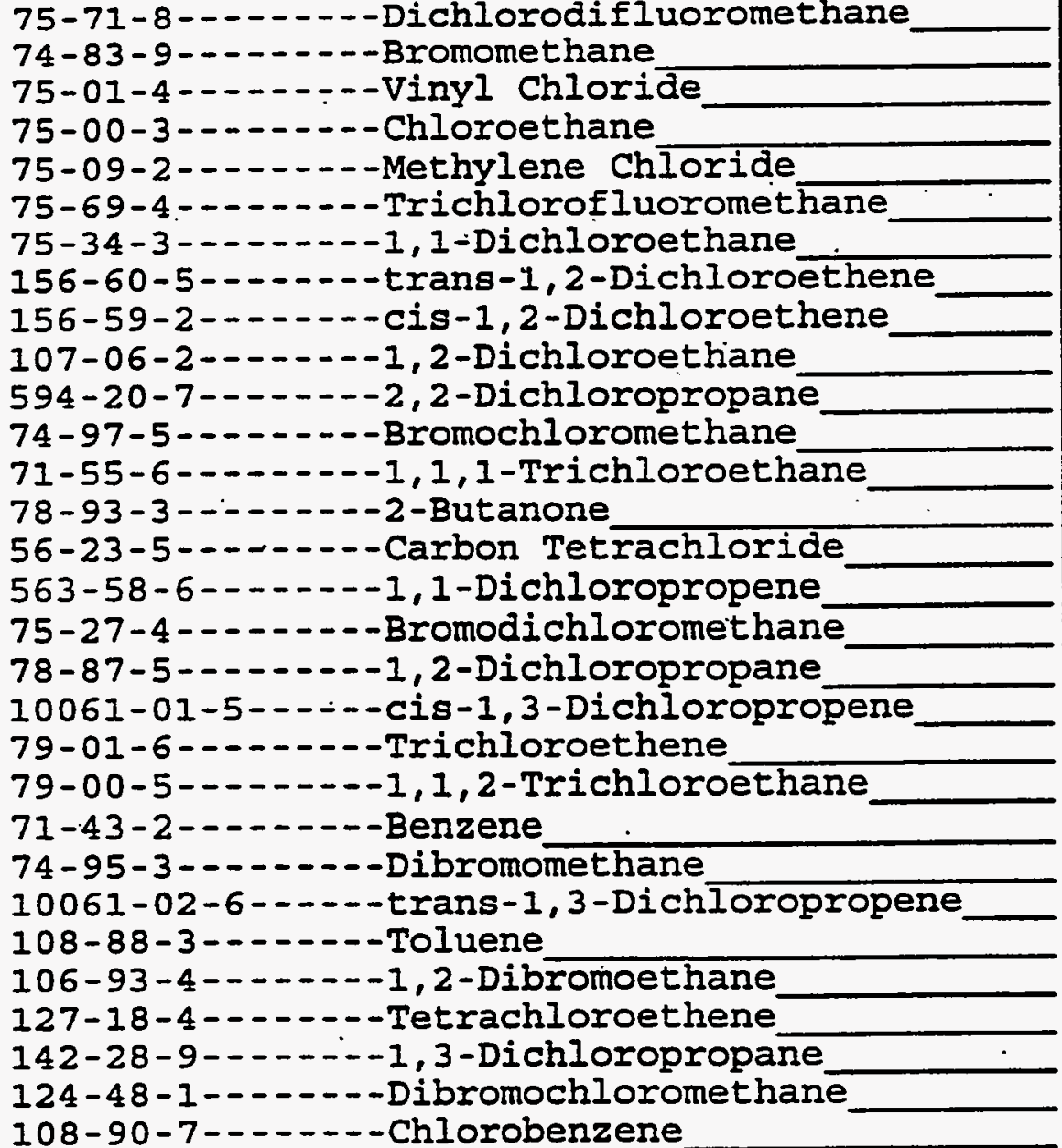 \\
\hline
\end{tabular}

-

\begin{tabular}{|l|l|}
\hline 50 & $U$ \\
50 & $U$ \\
50 & $U$ \\
50 & $U$ \\
11 & $B J$ \\
25 & $U$ \\
25 & $U$ \\
25 & $U$ \\
25 & $U$ \\
25 & $U$ \\
25 & $U$ \\
25 & $U$ \\
25 & $U$ \\
100 & $U$ \\
25 & $U$ \\
25 & $U$ \\
25 & $U$ \\
25 & $U$ \\
25 & $U$ \\
25 & $U$ \\
25 & $U$ \\
25 & $U$ \\
25 & $U$ \\
25 & $U$ \\
25 & $U$ \\
25 & $U$ \\
25 & $U$ \\
25 & $U$ \\
25 & $U$ \\
25 & $U$ \\
\end{tabular}


$1 A$

VOLATILE ORGANICS ANAIYSIS DATA SHEET
EPA SAMPLE NO.

NSCEP -140 ab Name: "QUUANTERRA MO"

Case No.: V53301

ab Code: ITMO

atrix: (soil/water) soí

ample wt/vol:

$1.00(\mathrm{~g} / \mathrm{mL}) \mathrm{G}$

evel: (low/med) LOW

Moisture: not dec.

こ Column: RTX-502.2 ID: 0.530 (mm)

jil Extract Volume:

(uL)
Contract: $625-01$

SAS NO.:

SDG No.: V205

Lab Sample ID: 14533-028

Lab File ID: $\quad$ E8566

Date Received: 05/06/97

Date Analyzed: 05/13/97

Dilution Factor: $\quad 1.0$

Soil Aliquot Volume:

(UL)

CONCENTRATION UNITS:

CAS NO.

COMPOUND

(ug/I or ug/Kg) UG/KG

Q

25

25

25

25

25

25

25

25 
10

Iab Name: Ouanteriano

Lab codes ITKO - Case No.:

Hatrix : (sol2/water) sorr.

Sample wt/vol:

1.0 $(g / m l)$

Level: (low/med) IOW

\& Molstures not dec. contracts 625.01

SDG NO.: Y205

SAS No.:

Iab Sample ID: $14533=030$

Iab File ID:

Date samplad: $04-30-97$

Date Analyzods 05-13-97

Dilution Factor: 1,0

CONCENTRATION URITS:

(ug/L or ug/Kg) ug/KG

$\mathbf{Q}$
CAS NO.

Compound

500

$\mathbf{0}$

U:Concentration of analyte is less than the value given.

X: Pattern reaconably matches gasoline

Y:Pattern appears to be multi-peaks, but does not match gasoline

z:Concentration is primarily from a single peak

(1):Low Boiling Bydrocarbon (LBB) is quantitated as if it is Gasoline.

FORY I LBE 


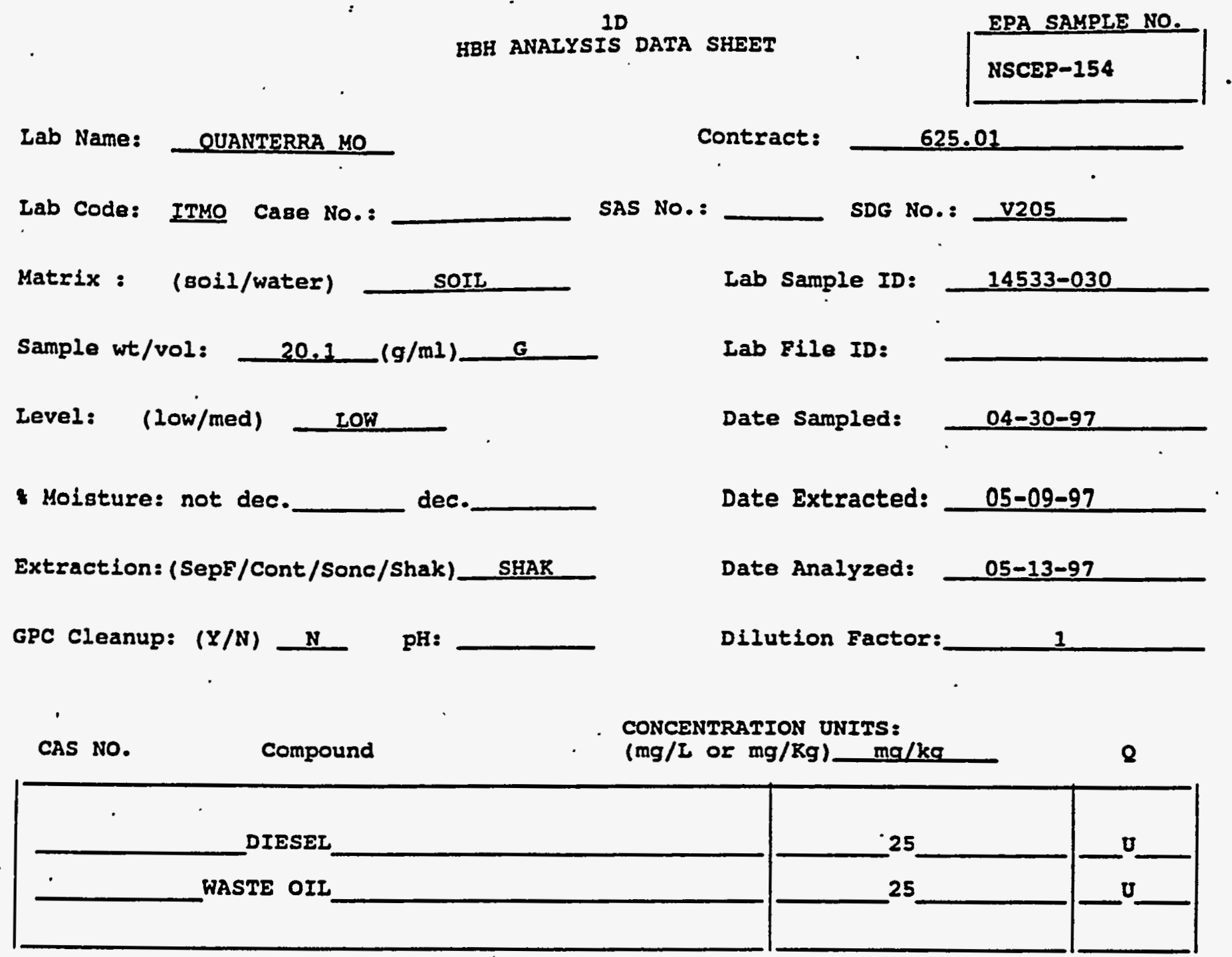

$U$ : Concentration of analyte is less.than the value given.. 
NSCEP- 154

Lab Name: “QÜANTERRÁ MO

Lab Code: ITMO

Case No.: V53301

Matrix: (soil/water) SOII

Sample wt/vol:

$1.00(\mathrm{~g} / \mathrm{mL}) \mathrm{G}$

Level: (low/med) LOW

: Moisture: not dec.

Contract: $625-01$

SAS NO.:

SDGG No.: V205

Lab Sample ID: 14533-030

Lab File ID: E8568

Date Received: 05/06/97

Date Analyzed: 05/13/97

GC Column: $\operatorname{RTX}-502.2$ ID: $0.530(\mathrm{~mm})$

Dilution Factor:

1.0

Soil Extract Volume:

(UL)
Soil Aliquot Volume:

(uL)

CAS NO. COMPOUND (ug/I or $u g / \mathrm{Kg}$ ) UG/KG

Q

\begin{tabular}{|c|}
\hline $\begin{array}{l}75-71-8-1-1- \\
74-83-9--1\end{array}$ \\
\hline
\end{tabular}


ad Name:"'QUANTERRA MO"

i ) Code: ITMO

Case No.: V53301

Contract: $625-01$
NSCEP-154 atrix: (soil/water) SOIL

; ple wt/vol:

$1.00(\mathrm{~g} / \mathrm{mL}) \mathrm{G}$

prel: (low/med) LOW

Moisture: not dec.

( Column: RTX-502.2 ID: 0.530 (mm)

oil Extract Volume:

(uL)
SAS NO..:

SDG No.: V205
Lab Sample ID: 14533-030

Lab File ID: E8568

Date Received: 05/06/97

Date Analyzed: 05/13/97

Dilution Factor: $\quad 1.0$

CAS NO. COMPOUND (ug/L or ug/Kg) UG/KG

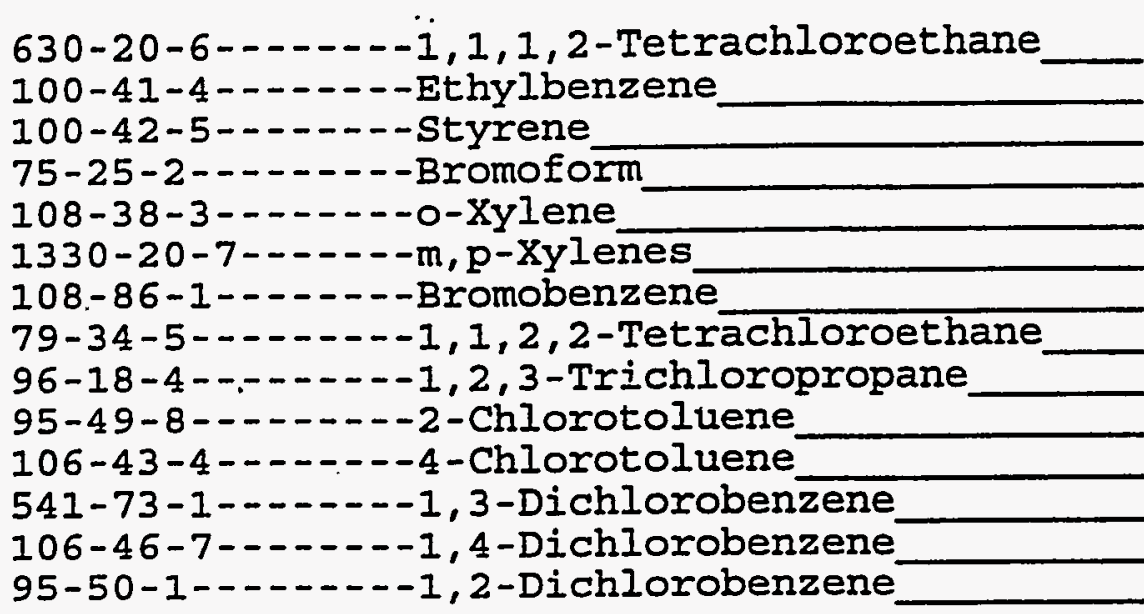


Lab Name: $\frac{\text { Ouanterra.kO }}{\text { Lab Code: ITMO Case No.: }}$
Matrix : (80il/water) soIL
Sample wt/vol: $\frac{1.0}{(\mathrm{~g} / \mathrm{ml})}$
Level: (low/med) IoW
Hoisture: not dec.

CAS NO.
Contract: 625.01

SAS NO.:

SDG NO.: V205

Iab Sample ID: 14533-031

Iab Elie ID:

Date Sampled: 04-30-97

Date Analyzed: 05-12-97

Dilution Factor: 1.0

CONCENTRATION UNITS:

$(\mathrm{ug} / \mathrm{L}$ or $\mathrm{ug} / \mathrm{Kg})$ UG/RG

\begin{tabular}{|l|l|l|}
\hline LBH (1)__ 1500 & \\
\hline
\end{tabular}

U:Concentration of analyte is 188 s than the value given. $X:$ Pattern reasonably matches gasoline

Y:Pattern appears to be multi-peaks, but does not match gasoline $z$ : Concentration is primarily from a single peak

(1):Low Boiling Hydrocarbon (LBE) is quantitated as if it is Gasoline. 


\section{0 \\ HBH ANALYSIS DATA SHEET}

EPA SAMPIE NO. NSCEP -178

NSCEP-178

Contract: $\quad 625: 01$

Lab Name: OUANTERRA MO

Contract:

Lab Code: ITMO Case No.: SAS No.:

SDG No.: V205

Matrix : (soil/water)

SOIL

Lab Sample ID:

14533-031

sample wt/vol: $20.0 \quad(\mathrm{~g} / \mathrm{ml})$

$\mathbf{G}$

Iab File ID:

Level:

(low/med)

LOW

Date Sampled:

04-30-97

\% Moisture: not dec. dec.

Date Extracted: 05-09-97

IExtraction: (SepF/Cont/Sonc/shak) SHAK

Date Analyzed: $05-13-97$

GPC cleanup $(Y / N)$ pH:

Dilution Factor: 1 CONCENTRATION UNITS: CAS NO. Compound (mg/I or $\mathrm{mg} / \mathrm{Kg}$ ) $\mathrm{mg} / \mathrm{kg}$

$\mathbf{Q}$ DIESEL WASTE OIL

\begin{tabular}{|c|c|}
\hline 25 & \\
\hline & \\
\hline & \\
\hline
\end{tabular}

$U$ : Concentration of analyte is less than the value given. 
Lab Name:" QUUANTERRA MO

Lab Code: ITMO

Case No.: V53301

Matrix: (soil/water) soIL

Sample wt/vol:

$1.00(\mathrm{~g} / \mathrm{mL}) \mathrm{G}$

Level: (low/med) LOW

$\because$ Moisture: not dec.

GC Column: RTX-502.2 ID: 0.53 .0 (mm)

Soil Extract Volume:

(uL)
Contract: $625-01$

NSCEP-178
CAS NO.
COMPOUND
SAS No.:

SDG No.: V205

Lab Sample ID: 14533-031

Lab File ID: F0035

Date Received: 05/06/97

Date Analyzed: 05/13/97

Dilution Factor: $\quad 1.0$

Soil Aliquot Volume:

(UI)

\begin{tabular}{|c|c|c|}
\hline 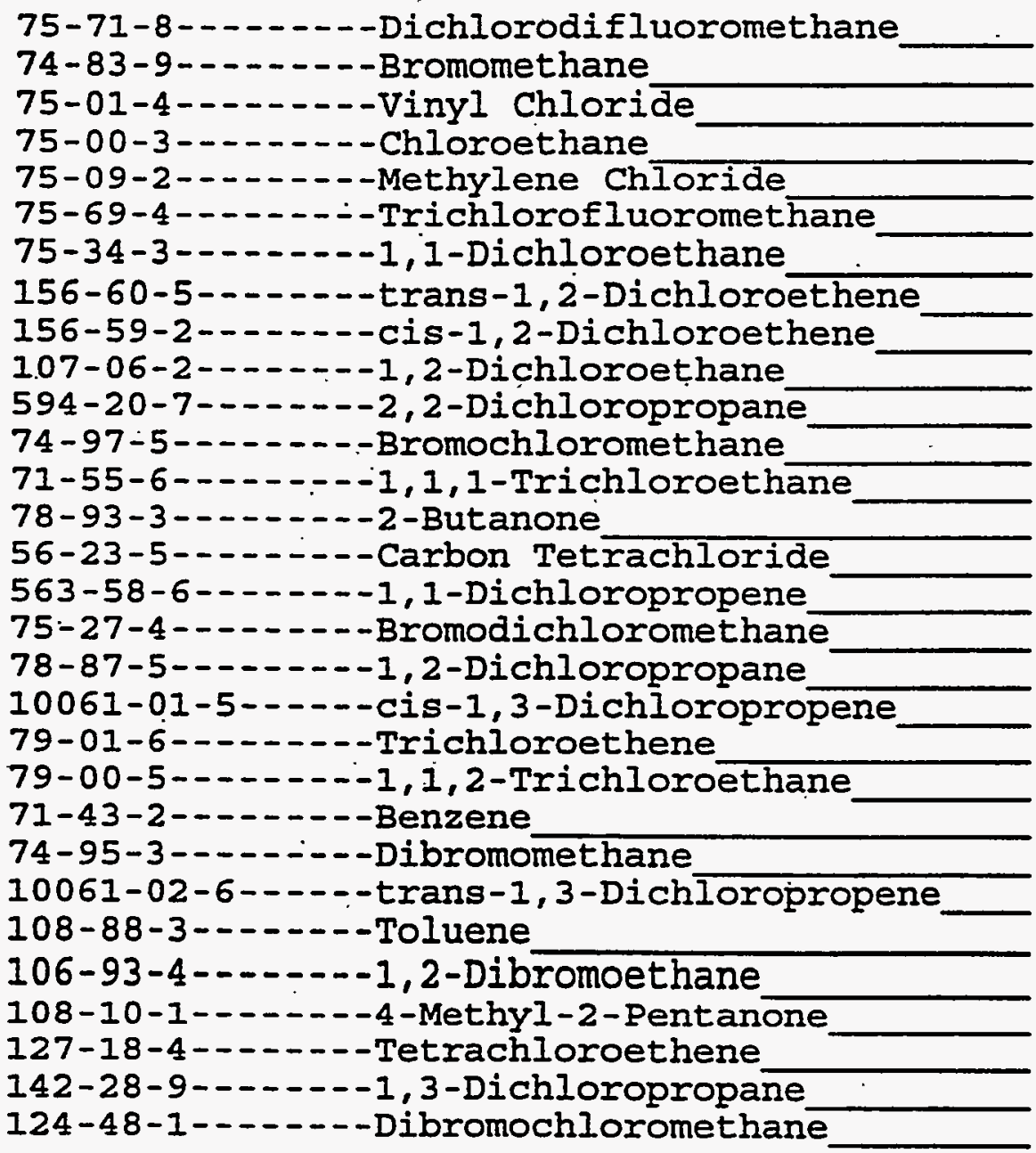 & $\begin{array}{r}100 \\
100 \\
100 \\
100 \\
50 \\
50 \\
50 \\
50 \\
50 \\
50 \\
50 \\
50 \\
50 \\
200 \\
50 \\
50 \\
50 \\
50 \\
50 \\
50 \\
50 \\
50 \\
50 \\
50 \\
50 \\
50 \\
100 \\
50 \\
50 \\
50\end{array}$ & $\begin{array}{l}U \\
U \\
U \\
U \\
U \\
U \\
U \\
U \\
U \\
U \\
U \\
U \\
U \\
U \\
U \\
U \\
U \\
U \\
U \\
U \\
U \\
U \\
U \\
U \\
U \\
U \\
U \\
U \\
U \\
U\end{array}$ \\
\hline
\end{tabular}

CONCENTRATION UNITS:

(ug/L or ug/Kg) UG/KG

Q 
Lab Name:"QÜUNTERRA MÓ

E o Code: ITMO

Case No.: V53301

Vatrix: (soil/water) soIL

3 nple wt/vol:

$1.00(\mathrm{~g} / \mathrm{mL}) \mathrm{G}$.

'jevel:. (low/med) LoW

soisture: not dec.

अ Column: RTX-502.2 ID: 0.530 (mm)

joil Extract Volume:

(uL)
Contract: $625-01$

SAS NO.:

NSCEP-178

SDG No.: V205

Lab Sample ID: 14533-031

Lab File ID: F0035

Date Received: 05/06/97

Date Analyzed: 05/13/97

Dilution Factor: $\quad 1.0$

Soil Aliquot Volume: .

(UL)

CONCENTRATION UNITS:

CAS NO:

COMPOUND

(ug/I or ug/Kg) UG/KG

$Q$

108-90-7-...--Chlorobenzene

630-20-6-...-1,1,1,2-Tetrachloroethane

100-41-4---..--Ethylbenzene

100-42-5--.----Styrene

75-25-2-...- - Bromoform

108-38-3-------o-Xylene

$1330-20-7 \ldots---m, p-x y l e n ̃ e s$

108-86-1-...-- Bromobenzene

79-34-5--..-- - 1, 1, 2, 2-Tetrachloroethane

96-18-4-n- - - - 1, 2, 3-Trichloropropane

95-49-8--- - - - 2-Chlorotoluene

106-43-4-- - - -4-Chlorotoluene

541-73-1-...--1, 3-Dichlorobenzene

106-46-7-_..-- -1, 4-Dichlorobenzene

95-50-1-- - - - - 1, 2-Dichlorobenzene
50

50

50

50

50

50

50

50

50

50

50

50

50

50

50 
1D

IBH ANALYSIS DATA SHEET

EPA SAMPLE NO. NSCEP-191

Lab Name: Ouenterra,ko
Lab Code: ITMO Case No.:
Matrix : (soll/water) soIr
Sample wt/vol: $1.0 \quad(g / \mathrm{ml}) G$

Ievel: (low/med) LOW

Molsture: not dec.

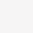

CAS NO.

compound

\author{
contract: 625.01 \\ SAS NO.: \\ SDG NO.: Y205 \\ Lab Sample ID: 14533-032 \\ Lab File ID: \\ Date sampled: $\quad 04-30-97$ \\ Date Analyzed: 05-12-97 \\ Dilution Factor: 1.0
}

CONCENTRATION UNITS:

(ug/I or ug/Rg) uG/RG

$\mathbf{Q}$

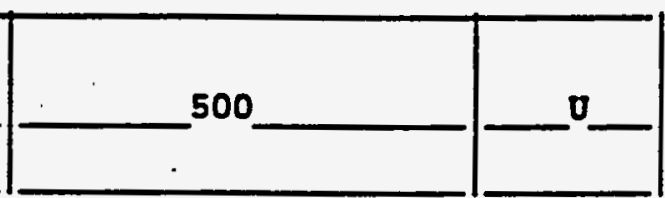

U: Concentration of analyte is less than the value given.

$X:$ Pattern reasonably matches gasoline

Y:Pattern appears to be multi-peaks, but does not match gasoline

Z:Concentration is primarily from a single peak

(1): Low Boiling Hydrocarbon (IBH) is quantitated as if it is Gasoline. 


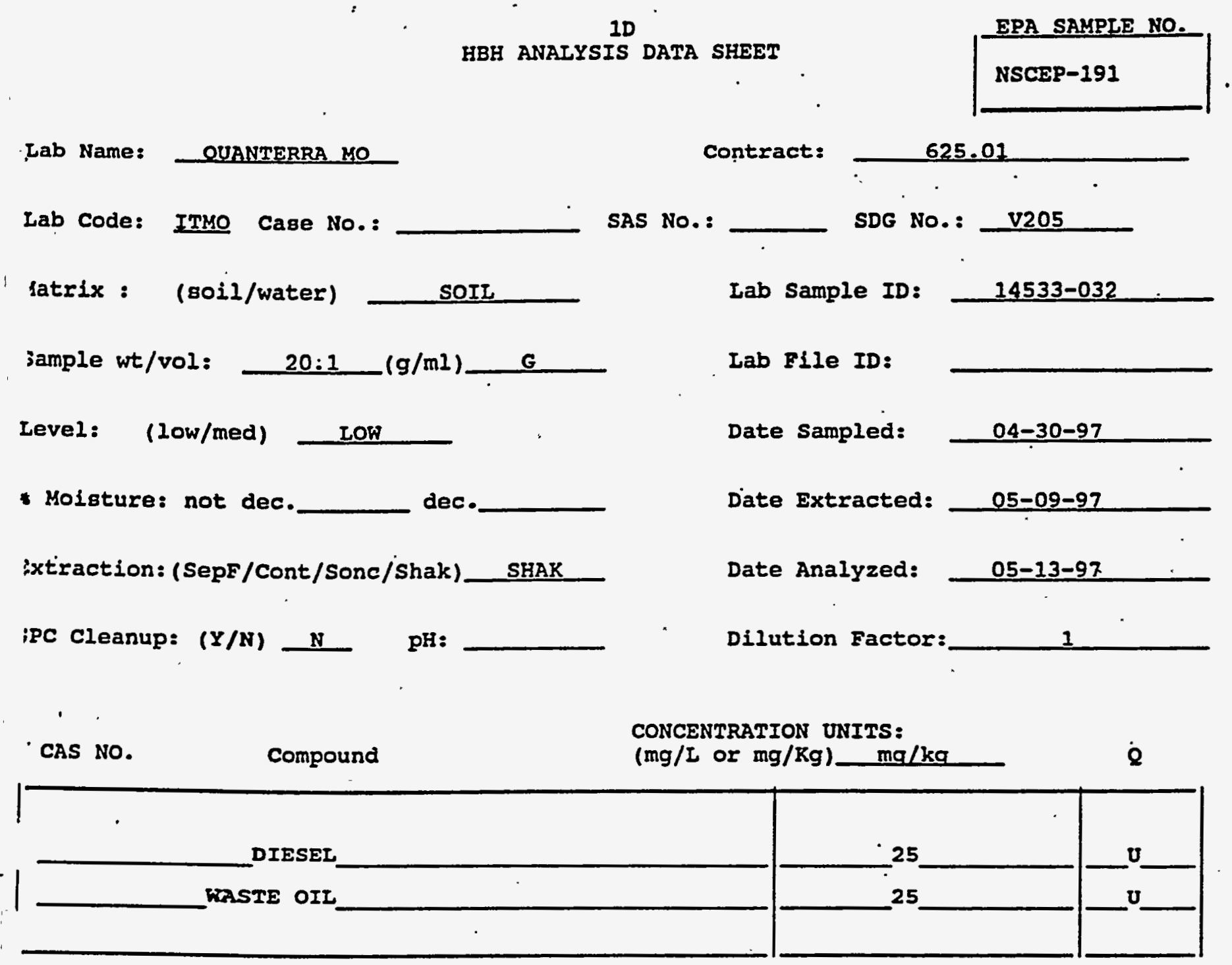

$U$ : Concentration of analyte is less than the value given. 
Lab Name:-QUUANTERRȦ MO

Lab Code: ITMO

Case No.: V5330I

Matrix: (soil/water) SOIL

Sample wt/vol:

$1.00(\mathrm{~g} / \mathrm{mL}) \mathrm{G}$

Level: (low/med) LOW

$\because$ Moisture: not dec.

GC Column: RTX-502.2 ID: 0.530 (mm)

Soil Extract Volume:

(UI)
Contract: $625-01$

SAS NO.:

SDG No.: V205

Lab Sample ID: 14533-032

Lab File ID: E8569

Date Received: 05/06/97

Date. Analyzed: 05/13/97

Dilution Factor: $\quad 1.0$

Soil Aliquot Volume: luLi

CONCENTRATION UNITS :

(ug/L or ug/Kg) UG/KG

Q

COMPOUND

-

75-71-8-2.-.-Dichlorodifluoromethane

74-83-9-...-.-Bromomethane

75-01-4-..---.-vinyl Chloride

75-00-3-..--.-Chloroethane

75-09-2-....-.-Methylene Chloride

75-69-4------- Trichlorofluoromethane

75-34-3-...--1,1-Dichloroethane

156-60-5-------trans-1,2-Dichloroethene

156-59-2-..----Cis-1,2-Dichloroethene

107-06-2------1,2-Dichloroethane

594-20-7-..--2,2-Dichloropropane

74-97-5-------Bromochloromethane

71-55-6-..---1, 1, 1-Trichloroethane

78-93-3-..---2--3utanone

56-23-5-...-.-Carbon Tetrachloride

563-58-6--.---1, 1-Dichloropropene

75-27-4-...-.-Bromodichloromethane

78-87-5-1-.--1,2-Dichloropropane

10061-01-5------cis-1,3-Dichloropropene

79-01-6--.---Trichloroethene

79-00-5-------1,1,2-Trichloroethane

71-43-2--.----Benzene

74-95-3--.---Dibromomethane

10061-02-6-----trans-1,3-Dich loropropene

108-88-3-------Toluene

106-93-4-...--1,2-Dibromoethane

127-18-4------Tetrachloroethene

142-28-9------1, 3-Dichloropropane

124-48-1--.-.-Dibromochloromethane

108-90-7-------Chlorobenzene

\begin{tabular}{|l|l|}
50 & $U$ \\
50 & $U$ \\
50 & $U$ \\
50 & $U$ \\
11 & $B J$ \\
25 & $U$ \\
25 & $U$ \\
25 & $U$ \\
25 & $U$ \\
25 & $U$ \\
25 & $U$ \\
25 & $U$ \\
25 & $U$ \\
100 & $U$ \\
25 & $U$ \\
25 & $U$ \\
25 & $U$ \\
25 & $U$ \\
25 & $U$ \\
25 & $U$ \\
25 & $U$ \\
25 & $U$ \\
25 & $U$ \\
25 & $U$ \\
25 & $U$ \\
25 & $U$ \\
25 & $U$ \\
25 & $U$ \\
25 & $U$ \\
25 & $U$ \\
& \\
& \\
25 \\
\end{tabular}


b Name: "QUUANTERRA MO

i Code: ITMO

Case No.: V53301

trix: (soil/water) soIL

r ile wt/vol:

$1.00(\mathrm{~g} / \mathrm{mL}) \mathrm{G}$

r!I: (low/med) LOW

Moisture: not dec.

:olumn: RTX-502.2 ID: 0.530 (mm)

il Extract Volume:

(UL)
Contract: $625-01$

SAS NO.:

NSCEP-191

SDG No.: V205

Lab Sample ID: 14533-032

Lab File ID: E8569

Date Received: 05/06/97

Date Analyzed: 05/13/97

Dilution Factor: $\quad 1.0$

Soil Aliquot Volume:

(uL)

CONCENTRATION UNITS :

CAS NO.

COMPOUND

(ug/L or ug/Kg) UG/KG

Q

630-20-6-..-- - 1, 1, 1, 2-Tetrachloroethane

100-41-4-..-.-.-Ethylbenzene

100-42-5-------Styrene

75-25-2--.----Bromoform

108-38-3-..---o-Xylene

$1330-20-7-\ldots--m, p-x y l e n e s$

108-86-1-.-.---Bromobenzene

79-34-5-...--1, 1, 2, 2-Tetrachloroethane

96-18-4-...--1,2, 3-Trichloropropane

95-49-8-...--2-Chlorotoluene

106-43-4-..---4-Chlorotoluene

541-73-1------1, 3-Dichlorobenzene

106-46-7------1,4-Dichlorobenzene

95-50-1--.---1,2-Dichlorobenzene
25

25

25

25

25

25

25

25

25

25

25

25

25

25 
1D IBH ANALYSIS DATA SEEET
EPA SAMPLE NO.

NSCEP -216
Lab Name:

Lab code: ITYO Case No.s

Hatrix : (go1l/water) sorr,

Sample wt/vol:

1.0 $(g / m l) \quad G$

Level: (low/med)

INOW

\& Moisture: not dec.

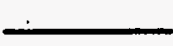

Contract: 625.01

SAS No.:

SDG No.: V205

Lab Sample ID: $14533-033$

Lab File ID:

Date Sampled: $\quad 04-30-97$

Date Analyzed: 05-12-97

Dilution Factor: 1.0
CONCENTRATION WNITS:

(ug/L or ug/Kg) UG/KG

CAS NO. 500

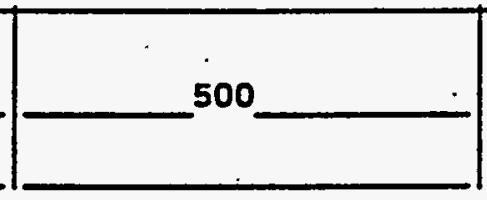

U:Concentration of analyte is less than the value given. $X: P a t t e r n$ reasonably matches gasoline Y:Pattern appears to be multi-peaks, but does not match gasoline 2: Concentration is primarily from a single peak

(1):Low Boiling Hydrocarbon (LBH) is quantitated as if it is Gasoline. 


\section{ID. \\ HBE ANALYSIS}

EPA SAMPLE NO. NSCEP -216 -ab Name: OUANTERRA MO

Lab Code: ITMO Case NO.:

latrix : (soil/water)

ample wt/vol:

$20.1 \quad(\mathrm{~g} / \mathrm{ml})$

SOIL

Ievel: (low/med)

LOW

s Moisture: not dec.

dec.

jtraction: (SepF/Cont/Sonc/Shak)__ SHAK

PC Cleanup: $(Y / N)+\mathrm{N} \quad \mathrm{pH}:$

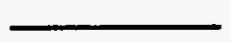

Contract:

SAS NO.:

SDG NO.: $\frac{V 205}{}$

Lab Sample ID:

$14533-033$

Iab File ID:

Date Sampled:

$04-30-97$

Date Extracted:

$05-09-97$

Date Analyzed:

$05-13-97$

Dilution Factor:

1

CONCENTRATION UNITS:

(mg/L or $\mathrm{mg} / \mathrm{Kg}$ ) $\mathrm{mg} / \mathrm{kg}$

$\mathbf{Q}$

$\square^{25}+-\frac{}{25}+{ }^{0}-$

J: Concentration of analyte is less than the value given. 
Lab Name: "-öUANTERRȦ MO

Lab Code: ITMO

Case No.: V53301

Matrix: (soil/water) SOIL

Sample wt/vol:

$1.00(\mathrm{~g} / \mathrm{mL}) \mathrm{G}$

Level: (low/med) Low

$\div$ Moisture: not dec.

GC Column: RTX-502.2 ID: 0.530 (mm)

Soil Extract Volume:

(uL)
Contract: $625-01$

SAS NO.:
NSCEP -216

SDG NO.: V205

Lab Sample ID: 14533-033

Lab File ID: . E8570

Date Received: 05/06/97

Date Analyzed: 05/13/97

Dilution Factor: $\quad 1.0$

Soil Aliquot Volume:

(uI)

CONCENTRATION UNITS:

CAS NO. COMPOUND (ug/L or ug/Kg) UG/KG

\begin{tabular}{|c|c|c|}
\hline 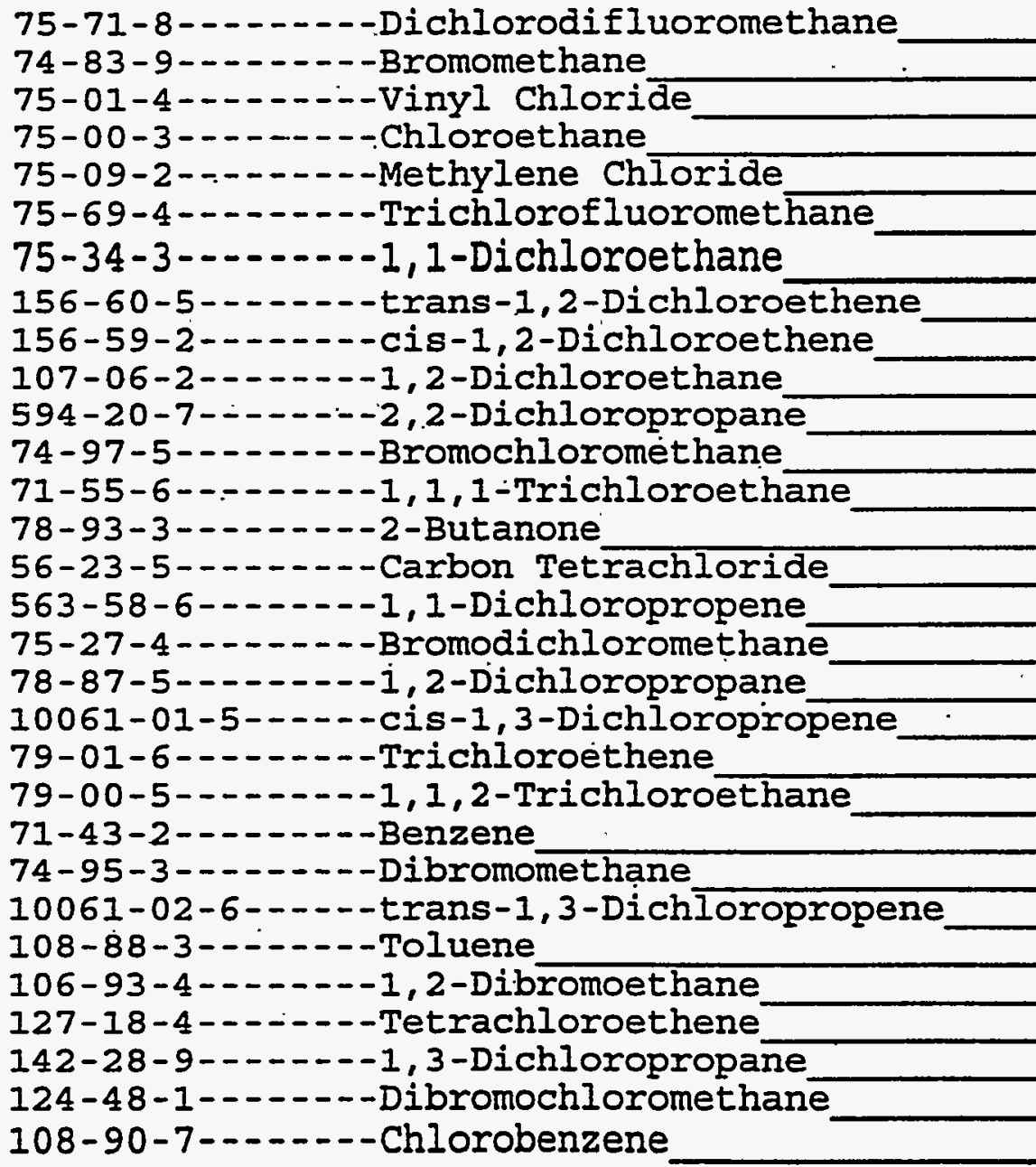 & $\begin{array}{r}50 \\
50 \\
50 \\
50 \\
12 \\
25 \\
25 \\
25 \\
25 \\
25 \\
25 \\
25 \\
25 \\
100 \\
25 \\
25 \\
25 \\
25 \\
25 \\
25 \\
25 \\
25 \\
25 \\
25 \\
25 \\
25 \\
25 \\
25 \\
25 \\
25\end{array}$ & $\begin{array}{l}U \\
U \\
U \\
U \\
B J \\
U \\
U \\
U \\
U \\
U \\
U \\
U \\
U \\
U \\
U \\
U \\
U \\
U \\
U \\
U \\
U \\
U \\
U \\
U \\
U \\
U \\
U \\
U \\
U \\
U\end{array}$ \\
\hline
\end{tabular}


$1 \mathrm{~A}$

VOLATILE ORGANICS ANALYSIS DATA SHEET b Name: “QUUANTERRA MO

t code: ITMO

Case No.: V53301

trix: (soil/water) soIL

$\pi$. le wt/vol:

$1.00(\mathrm{~g} / \mathrm{mL}) \mathrm{G}$

v 1: (low/med) LOW

Moisture: not dec.

olumn: RTX-502.2 ID: 0.530 (mm)

il Extract Volume:

(uL)
Contract:, $625-01$.

SAS NO.:

SDG NO.: V205
NSCEP- 216
EPA SAMPLE NO.

Lab Sample ID: 14533-033

Lab File ID: $\quad$ E8570

Date Received: 05/06/97

-.

Date Analyzed: 05/13/97

Dilution Factor: $\quad 1.0$

Soil Aliquot Volume:

(UL)

CAS NO.

COMPOUND

CONCENTRATION UNITS:

(ug/I or $u g / \mathrm{Kg}$ ) UG/KG

Q

25

25 .

25

25

25

25

25

25

25

25

25

25

25

25 
Iab Name: Ouanterra yo

Iab code: Imeo Case No.:

Matrlx : (so11/water) sorr

Sample wt/vol: $\quad$ i.o $(\mathrm{g} / \mathrm{ml}) \quad G$

Leve1: ( low/med) LOW

* Hoisture: not dec.

Holstures not dec.

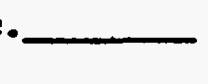

CAS NO.

Compound

CONCEINTRATION UNITS:

(ug/I or ug/Rg) UG/KG

$\mathbf{Q}$

IBH

(1)
Contract: 625.01

SDG No.: Y205

Iab Sample ID: 14533-001

Iab F110 ID:

Date sampled: 04-30-97

Date Analyzed: 05-09-97

Dilution Factor: 1.0

IBH $(1) \ldots$

\begin{tabular}{|c|}
\hline $500+0$ \\
\hline
\end{tabular}

U:Concentration of analyte is 1088 than the value given. $\mathrm{X}$ : Pattern reasonably matches gasoline $Y$ : Rattern appears to be multi-peaks, but does not match gasoline $z=$ Concentration is primarily from a single peak

(1) :Low Boliing Hydrocarbon (IBE) is quantitated as if it is GaBoline. 
1D

HBH ANALYSIS DATA SEEET
EPA SAMPLE NO.

NSCEP-229

Lab Name: QUANTERRA MO

Contract:

625.01

Lab Code: ITHO Caвe No.:

SAS NO.:

SDG NO.: V205

Matrix : (soil/water)

soIL

Lab Sample ID:

14533-001

Sample wt/vol:

$20.1(\mathrm{~g} / \mathrm{mI})$

$G$

Lab File ID:

Level: (low/med)

IOW

Date Sampled:

$04-30-97$

Date Extracted:

05-08-97

Date Analyzed:

$05-09-97$

Extraction: (SepF/Cont/Sonc/Shak) SHAK

GPC Cleanup: $(Y / N) \perp$ N

Dilution Factor:

1

CONCENTRATION UNITS:

CAS No.

Compound

(mg/L or $\mathrm{mg} / \mathrm{kg}$ )

$\mathrm{mg} / \mathrm{kg}$

Q

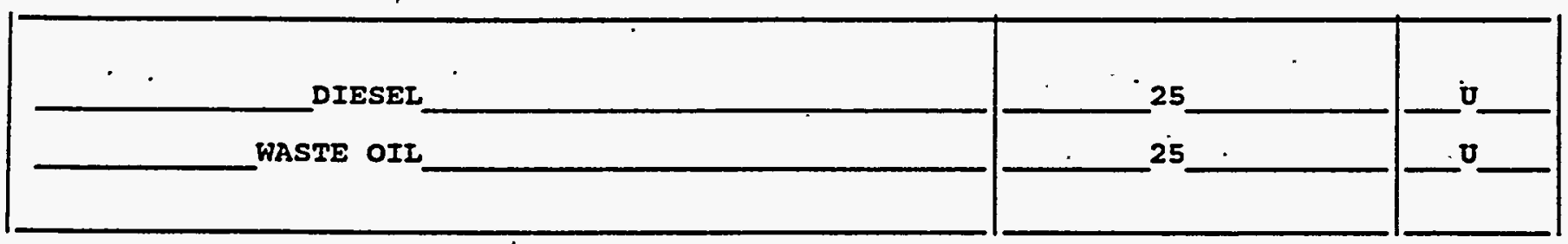

U: Concentration of analyte is less than the value given.

FORY I HBH 
Lab Name: "QUUANTERRA் MO

Irab Code: ITMO

Case No.: V53301

Matrix: (soil/water) SoIL

Sample wt/vol:

$5.00(\mathrm{~g} / \mathrm{mL}) \mathrm{G}$

Level: (low/med) LOW

\% Moisture: not dec.

GC Column: RTX-502.2 ID: 0.530 (mm)

Soil Extract Volume:

(uL)
Contract: $625-01$

NSCEP-229
SAS NO.:

SDG No.: V205
Lab Sample ID: '14533-001

Lab File ID: $\quad$ E8537

Date Received: 05/06/97

Date Analyzed: 05/10/97

Dilution Factor: $\quad 1.0$

Soil Aliquot Volume:

(uL)

CAS NO.

COMPOUND

CONCENTRATION UNITS:

(ug/L or $u g / \mathrm{kg}) \cdot \mathrm{UG} / \mathrm{KG}$

$\mathbf{Q}$

75-71-8-..--Dichlorodifluoromethane

74-83-9-....--Bromomethane

75-01-4-..----Vinyl Chloride

75-00-3-.------Chloroethane

75-09-2-...--Methylene Chloride

75-69-4--.--- Trichlorofluoromethane

75-34-3-..-- - 1, 1-Dichloroethane

156-60-5--..--trans-1,2-Dichloroethene

156-59-2--.---cis-1,2-Dichloroethene

107-06-2--.-.-1, 2-Dichloroethane

594-20-7-...-2,2-Dichloropropane

74-97-5-..----Bromochloromethane

71-55-6-- - - - 1, 1, 1-Trichloroethane

78-93-3-....-2-Butanone

56-23-5-------Carbon Tetrachloride

563-58-6-----1,1-Dichloropropene

75-27-4--.----Bromodichloromethane

78-87-5------1,2-Dichloropropane

10061-01-5-.---cis-1,3-Dichloropropene

79-01-6---.---Trichloroethene

79-00-5-..--1, 1,2-Trichloroethane

$71-43-2-\ldots----$ Benzene

74-95-3--.---Dibromomethane

10061-02-6-..--trans-1, 3-Dichloropropene

108-88-3-...-.-Toluene

106-93-4------1,2-Dibromoethane

127-18-4------Tetrachloroethene

142-28-9-----1,3-Dichloropropane

124-48-1------Dibromochloromethane

108-90-7------Chlorobenzene

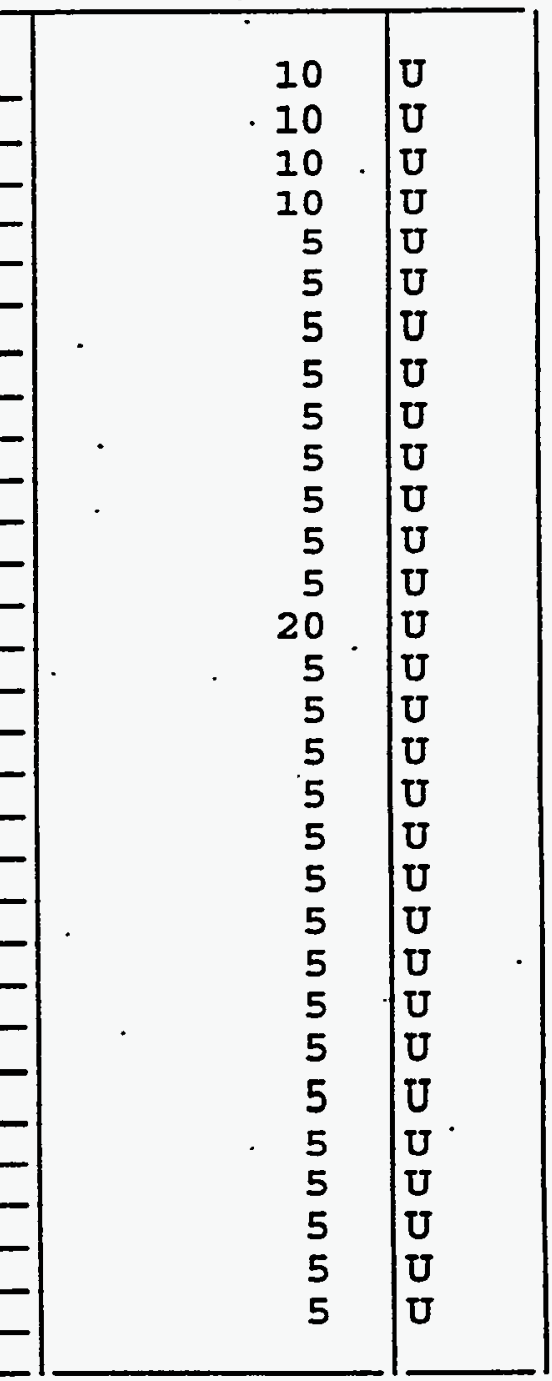


2. Name : " QUUANTERRA MO

: Code: ITMO

Case No.: V53301

srix: (soil/water) SOIL

i. le wt/vol:

$5.00^{\circ}(\mathrm{g} / \mathrm{mL}) \mathrm{G}$

s 1:. (low/med) LOW

Noisture: not dec.

olumn: RTX-502.2 ID.: 0.530 (mm)

il Extract Volume:

(UI)
Contract: $625-01$

SAS NO. :
EPA SAMPLE NO.

NSCEP-229

SDG No.: V205

Lab Sample ID: 14533-001

Lab File ID: E8537

Date Received: 05/06/97

Date Analyzed: 05/10/97

Dilution Factor: $\quad 1.0$

' Soil Aliquot Volume:

(UL)

CAS NO. COMPOUND $\quad$ (ug/L or ug/Kg) UG/KG $\quad Q$

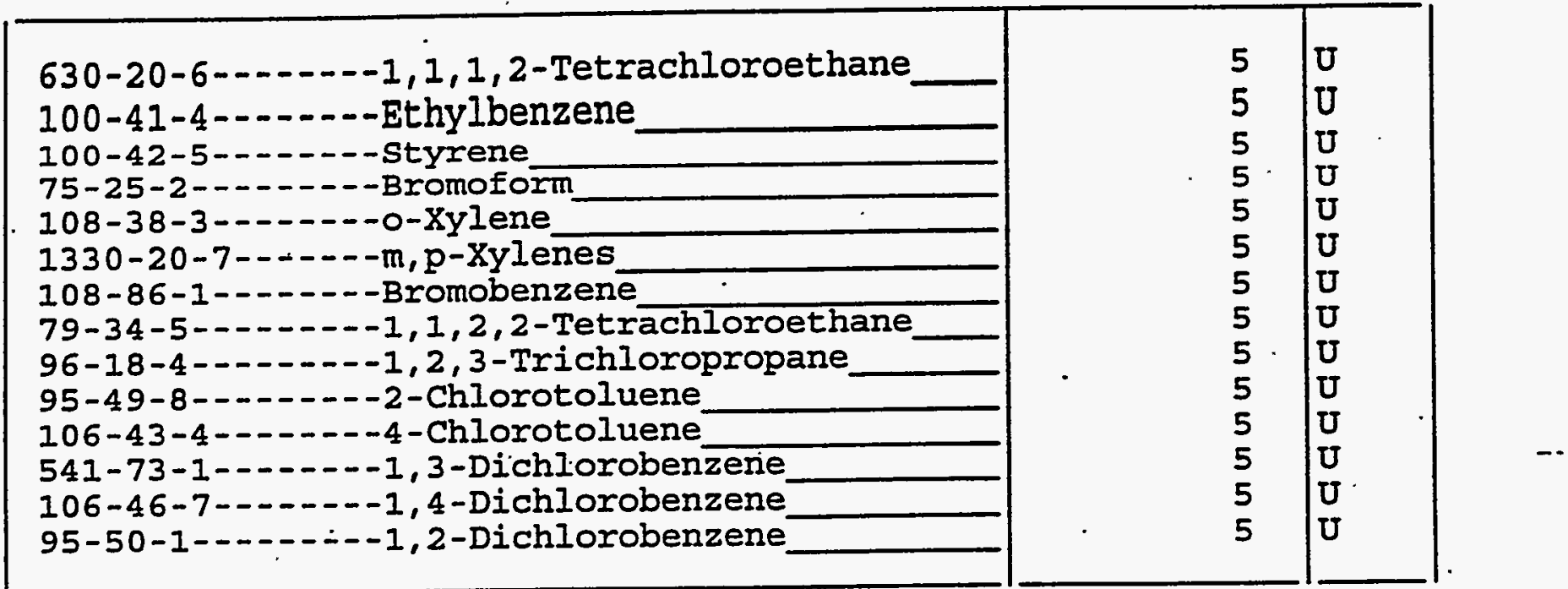


10

IBE ANAIYSIS DATA SHEET
EPA SAYPLE NO,

NSCEP-232
Lab Name:

Iab code:

Matrix

Sample wt/vol:

(soil/water) sors

1.0

$(g / m l)$

Level: (low/med) IOW

\& Moisture: not dec.

CAS NO.

compound
(1)

Contract: 625.01

SAS NO.:

SDG No.: Y205

Iab Sample ID: 14533-035

Iab F110 ID:

Date Sampled: 04-30-97

Date Analyzed: $05-13-97$

Dilution Pactorz 1.0

CONCENTRATION UNITS:

(ug/L or ug/Kg) ug/Rg

$\mathbf{Q}$

500

$\mathbf{0}$

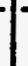

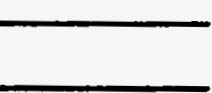

U:Concentration of analyte is 1 ess than the value given.

$\mathrm{X}$ :Pattern reasonably matches gasoline

Y: Pattern appears to be multi-peaks, but does not match gasoline

$\mathrm{Z}$ :Concentration is primarily from a dingle peak

(1):Low Bolling Hydrocarbon (LBH) is quantitated as if it is Gasoline.

FORE I LBB 
10

HBH ANALYSIS DATA SHEET
EPA SAMPLE NO.

NSCEP-232
Lab Name: ' QUANTERRA MO

Lab Code: ITMO Case No.:

latrix : (soil/water)

SOIL

ample wt/vol:

$20.1(\mathrm{~g} / \mathrm{ml})$

G

Level: (low/med)

LOW

- Moisture: not dec.

dec.

ixtraction: (SepF/Cont/Sonc/Shak)

SHAR

PC Cleanup: $(\mathrm{Y} / \mathrm{N})$ N $\mathrm{NH}$

CAS NO.

Compound

DIESEI

WASTE OIL
Contract:

SAS NO.:

SDG NO.: V205

Lab Sample ID:

$14533-035$

Iab Rile ID:

Date Sampled:

$04-30-97$

Date Extracted:

$05-09-97$

Date Analyzed:

05-13-97

Dilution Factor:

1

CONCENTRATION UNITS:

(mg/L or $\mathrm{mg} / \mathrm{Kg}$ ). $\mathrm{mg} / \mathrm{kg}$

Q

$\mid$\begin{tabular}{l|l|l|}
\hline WASTE OIL_. & DIESEL__ \\
\hline
\end{tabular}

U: Concentration of analyte is less than the value given. 
Lab Name:- QUANTERRÁ MO

Iab Code: ITMO

Case No.: V53301

Matrix: (soil/water) soII

Sample wt/vol:

$1.00(\mathrm{~g} / \mathrm{mL}) \mathrm{G}$

Level: (low/med) LOW

$\div$ Moisture: not dec.

GC Column: RTX-502.2 ID: 0.530 (mm)

Soil Extract Volume:

(uL)
Contract : $625-01$

NSCEP-232
SAS NO.:

SDG No.: V205
Lab Sample ID: 14533-035

Lab File ID: F0037

Date Received: 05/06/97

Date Analyzed:" 05/13/97

Dilution Factor: $\quad 1.0$

Soil Aliquot Volume:

(UL)

CAS NO.

COMPOUND

CONCENTRATION UNITS:

(ug/L or ug/Kg) UG/KG

75-71-8-..---Dichlorodifluoromethane

74-83-9--------Bromomethane

75-01-4-..--.--Vinyl Chloride

75-00-3--.----Chloroethane

75-09-2-..-...-Methylene Chloride

75-69-4-...-.-Trichlorofluoromethane

75-34-3-.--:--1, 1-Dichloroethane

156-60-5--.----trans-1,2-Dichloroethene

156-59-2-.----cis-1, 2-Dichloroethene

107-06-2--.---1, 2-Dichloroethane

594-20-7-------2,2-Dichloropropane

74-97-5-1.-----Bromochloromethane

71-55-6-...-1, 1, 1-Trichloroethane

78-93-3-..----2-Butanone

56-23-5--------Carbon Tetrachloride

563-58-6--.--1,1-Dichloropropene

75-27-4-..-.--Bromodichloromethane

78-87-5-..---1,2-Dichloropropane

10061-01-5-----cis-1, 3-Dichloropropene

79-01-6--.----Trichloroethene

79-00-5 -...-.-1,1,2-Trichloroethane

71-43-2-------Benzene

74-95-3--.---Dibromomethane

10061-02-6-----trans-1,3-Dichloropropene

108-88-3-..--. Toluene

106-93-4-...-1, 2-Dibromoethane

108-10-1--.--4-Methyl-2-Pentanone

127-18-4------Tetrachloroethene

142-28-9-----1, 3-Dichloropropane

124-48-1-...--Dibromochloromethane

\begin{tabular}{r|l}
100 & $U$ \\
100 & $U$ \\
100 & $U$ \\
100 & $U$ \\
50 & $U$ \\
50 & $U$ \\
50 & $U$ \\
50 & $U$ \\
50 & $U$ \\
50 & $U$ \\
50 & $U$ \\
50 & $U$ \\
50 & $U$ \\
200 & $U$ \\
50 & $U$ \\
50 & $U$ \\
50 & $U$ \\
50 & $U$ \\
50 & $U$ \\
50 & $U$ \\
50 & $U$ \\
50 & $U$ \\
50 & $U$ \\
50 & $U$ \\
50 & $U$ \\
50 & $U$ \\
100 & $U$ \\
50 & $U$ \\
50 & $U$ \\
50 & $U$ \\
& \\
50
\end{tabular}


Jab Name:-“QUUANTERRA MO

i, Code: ITMO Case No.: V53301 SAS No.:
Contract: $625-01$

. SDG NO.: V205 latrix: (soil/water) SOIL

if iple wt/vol:

$1.00(\mathrm{~g} / \mathrm{mL}) \mathrm{G}$

,r-rel: (low/med) Low

Moisture: not dec.

if Column: RTX-502.2 ID: $0.530(\mathrm{~mm})$

ioil Extract Volume:

(uI)
Lab Sample ID: 14533-035

Lab File ID: F0037

Date Received: 05/06/97

Date Analyzed: 05/13/97

Dilution Factor: $\quad 1.0$

Soil Aliquot Volume:

(UI)

CAS NO.

COMPOUND

CONCENTRATION UNITS:

(ug/L or ug/Kg) UG/KG

Q

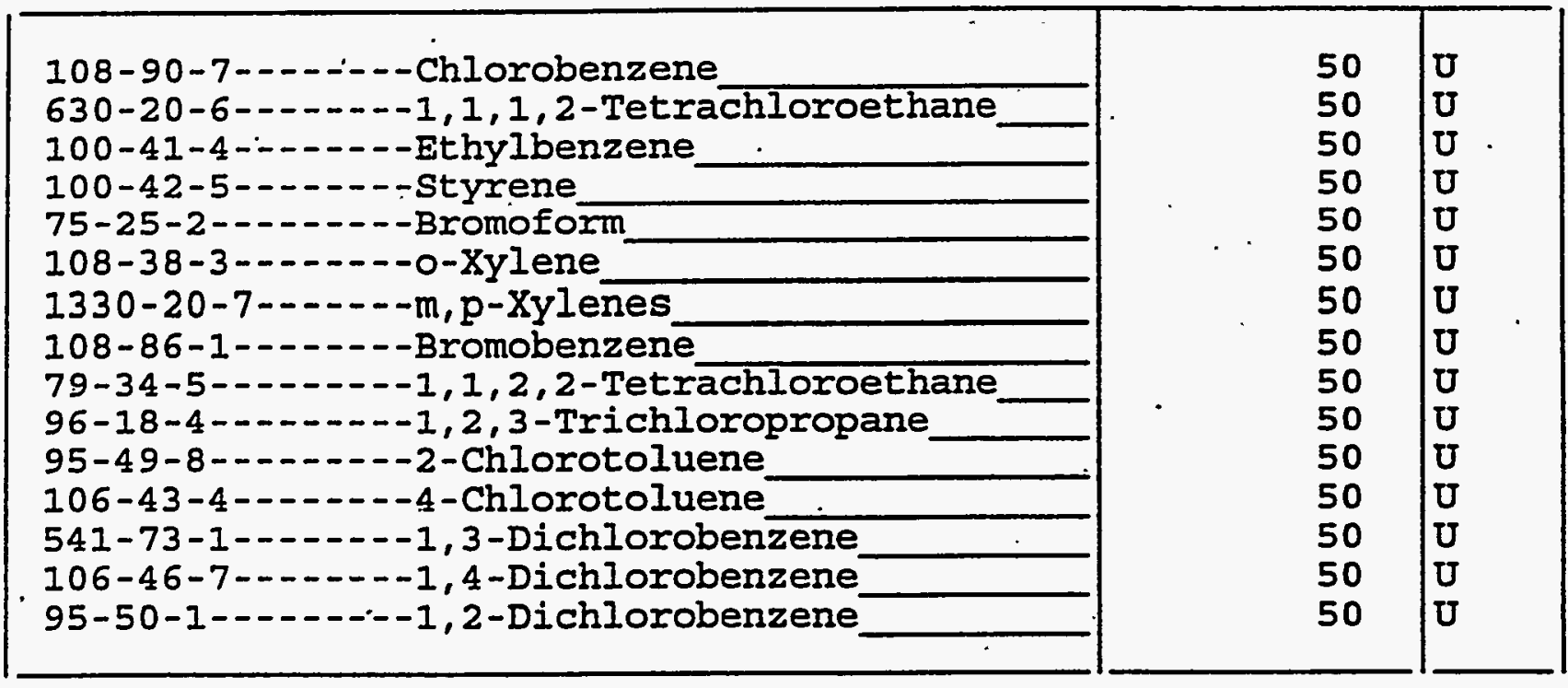


Iab Name:

Iab Code:

Ouanterefa, yo

Imyo Cage No.:

(80i1/water) sorr.

Hatrix : (soil/water)

sample wt/vol:

$$
1 . \dot{0}(\mathrm{~g} / \mathrm{ml})
$$

G

Ievel: (Low/med) IOW

4 Molsture: not dec.

$\cdot$

CAS NO.

compound
Contract: $\quad 625,01$

SAS NO.:

$$
\text { SDG NO.: Y205 }
$$

Lab Sample ID: 14533-034

Iab File ID:

Date Sampled:

04-30-97

Date Analyzeds 05-13-97

Dilution Eactors 1.0

CONCENTRATION UNITS:

(ug/I or ug/kg) UG/KG . Q

LBH (1)

500

U:Concentration of analyte 18 less than the value given.

X: Pattern reasonably matches gasoline

y. Pattern appears to be multi-peaks, but does not match gasoline

z:Concentration is primarily from a single peak

(1) :Low Boiling Bydrocarbon (LBE) is quantitated as if it is Gaṣoline. 
Lab Name: OUANTERRA MO

Contract:

625.01

Lab code: ITMO Caвe No.: SAS NO.: SDG NO.: V205

latrix : (Boil/water) SOII ample wt/vol: $20.1 \quad(\mathrm{~g} / \mathrm{ml})$ G Level: (low/med) LOW

- Moisture: not dec. dec.

Date Extracted:

$05-09-97$

xtraction: (SepF/Cont/Sonc/Shak) ___ SHAR PC cleanup: $(Y / N) \_$NH: Date Analyzed: 05-13-97 Dilution Factor: 1 CONCENTRATION UNITS: ( $\mathrm{mg} / \mathrm{L}$ or $\mathrm{mg} / \mathrm{Kg}$ ) $\mathrm{mg} / \mathrm{kg}$ 8 CAS NO. Compound

\begin{tabular}{|c|c|}
\hline 25 & -0 \\
\hline & 0 \\
- & - \\
\hline
\end{tabular}

$J$ : Concentration of analyte is less than the value given. 
Lab Name :- Q̄UANTERRAं MO

Iab Code: ITMO

Case No.: V53301

Matrix: (soil/water) soIL

Sample wt/vol:

$1.00(\mathrm{~g} / \mathrm{mL}) \mathrm{G}$

Level: (low/med) LOW

$\%$ Moisture: not dec.

GC Column: RTX-502.2 ID: 0.530 (mm)

Soil Extract Volume:

(uL)
Contract: 625-01

SAS NO.:

SDG No.: V205
Lab File ID: F0036

Date Received: 05/06/97

Date Analyzed: 05/13/97

Dilution Factor: $\quad 1.0$

Soil Aliquot Volume:

(UI)

CAS NO. COMPOUND

CONCENTRATION UNITS:

(ug/I or $\mathrm{ug} / \mathrm{Kg}$ ) UG/KG

\begin{tabular}{|c|}
\hline 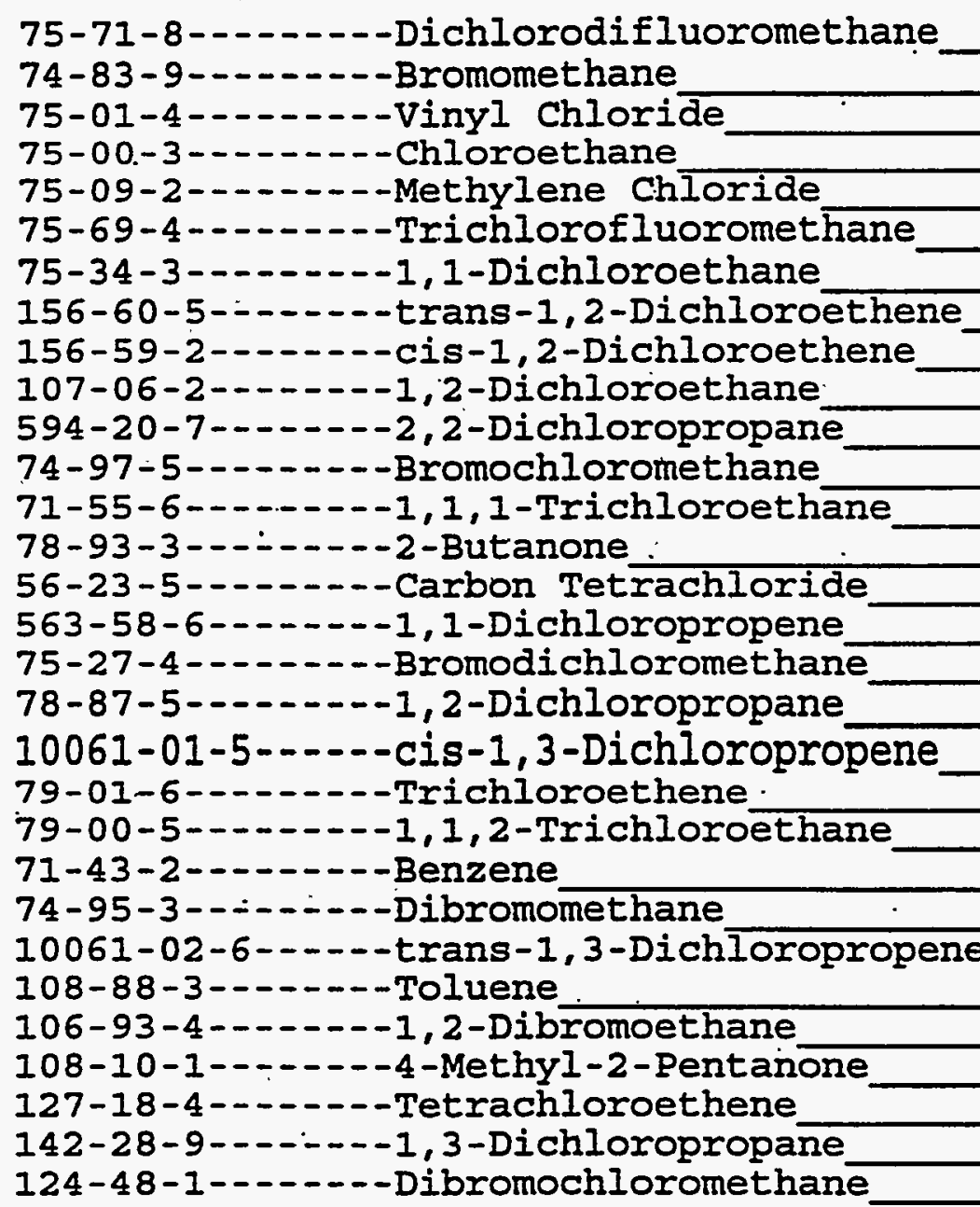 \\
\hline
\end{tabular}

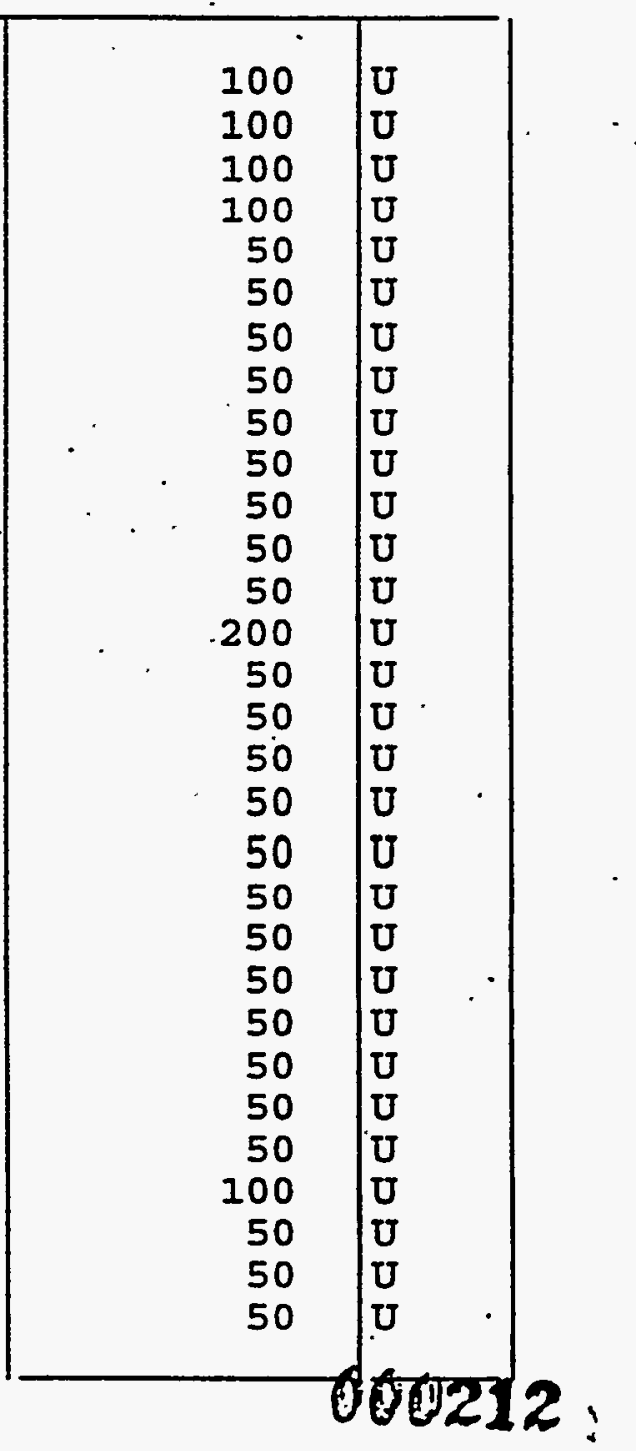


, Name : QUUANTERRA MO

) Zode: ITMO

Case No.: V53301

:rix: (soil/water) soIL

it ie wt/vol:

$1.00(\mathrm{~g} / \mathrm{mL}) \mathrm{G}$

in: (low/med) Low

loisture: not dec.

i Jlumn: RTX-502.2 ID: 0.530 (mm)

-I Extract Volume:

(uL)
Contract: $625-01$

SAS NO.:

NSCEP-234

SDG No.: V205

Lab Sample ID: 14533-034

Iab File ID: F0036

Date Received: 05/06/97

Date Analyzed: 05/13/97

Dilution Factor: $\quad 1.0$

Soil Aliquot Volume: (UL) CONCENTRATION UNITS:

CAS NO.

COMPOUND (ug/I or $\mathrm{ug} / \mathrm{Kg}$ ) UG/KG

Q

50

50

50

50

50

50

50

50

50

50

50

50

50

50

50 
Lab Name:

ouantersako

Iab Coder ITYO Case No.:

Matrix : (goil/water) soIf

Sample wt/vol: 1.0 $(g / m l$ G

Level: . (Low/med) ron

soisture: not dec. contract: 625.01

SAS No.:

SDG NO.: $\mathbf{v 2 0 5}$

Lab Sample ID: 14533-002

Iab P11e ID:

Date Sampled: 04-30-97

Date Analyzed: 05-09-97

Dilution Eactor: 1.0
CONCENTRATION UNITS:

CAS NO. Compound

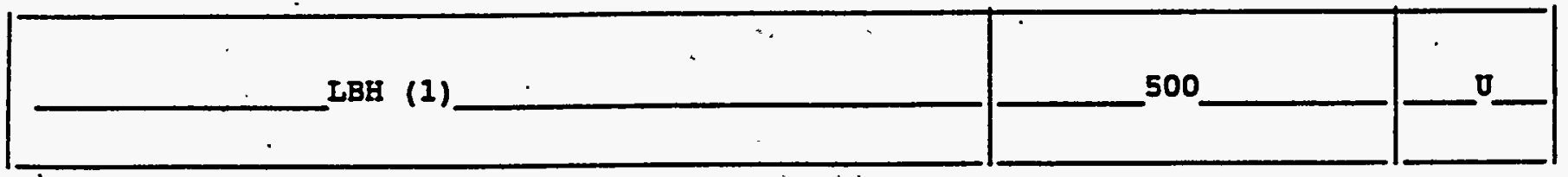

U:Concentration of analyte is 188 than the value given. $X: P a t t e r n$ reasonably matches gasoline

Y.:Pattern appears to be multi-peaks, but does not match gasoline

z:Concentration is primarily from a single peak.

(1):Low Boiling Hydrocarbon (IBE) is quantitated as if it is Gasoline.

FORY I IBE 
10

HBH ANALYSIS DATA SHEET
EPA SAMPIE NO. NSCSP 246
Lab Name: OUANTERRA MO
Iab code: ITMO Case NO.:

latrix : (soil/water)

SOIL

iample wt/vol:

$20.0 \quad(\mathrm{~g} / \mathrm{ml})$

G

Level: (Low/med)

LOW

- Moisture: not dec. dec.

ixtraction: (SepF/Cont/Sonc/Shak) ___ SHAR

iPC Cleanup: $(\mathrm{Y} / \mathrm{N}) \stackrel{\mathrm{N}}{\mathrm{pH}}$

CAS NO.

compound contract:

625.01

SAS NO.:

SDG NO.: $\mathbf{v 2 0 5}$

Lab Sample ID:

$14533-002$

Lab File ID:

Date Sampled:

$04-30-97$

Date Extracted:

$05-08-97$

Date Analyzed:

05-09-97

Dilution Factor:

1

CONCENTRATION UNITS:

(mg/L or $\mathrm{mg} / \mathrm{Kg}$ ) $\mathrm{mg} / \mathrm{kg}$

Q

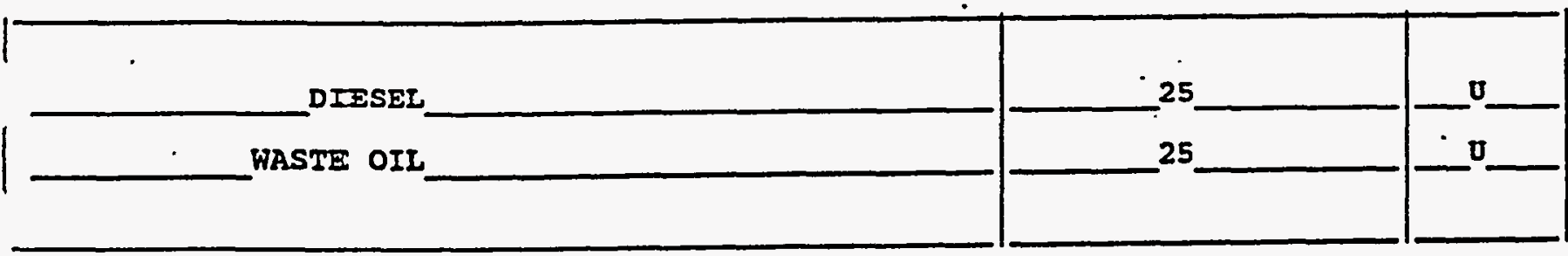

U: Concentration of analyte is less than the value given. 
Lab Name:"ⓊUANTERRẢ MO

Lab Code: ITMO

Case No.: V53301

Matrix: (soil/water) soIL

Sample wt/vol:

$5.00(\mathrm{~g} / \mathrm{mL}) \mathrm{G}$

Level: (low/med) LOW

음 Moisture: not dec.

GC Column: RTX-502.2 ID: 0.530 (mim)

Soil Extract Volume:

(uL)
Contract: $625-01$
NSCEP-246

\begin{abstract}
Soil Extract volume:
\end{abstract}
CAS NO.
COMPOUND
SAS NO.:

SDG No.: V205

Lab Sample ID: 14533-002

Lab File ID: $\quad$ E8538

Date Received: 05/06/97

Date Analyzed: 05/10/97

Dilution Factor: $\quad 1.0$

Soil Aliquot Volume:

(UI)

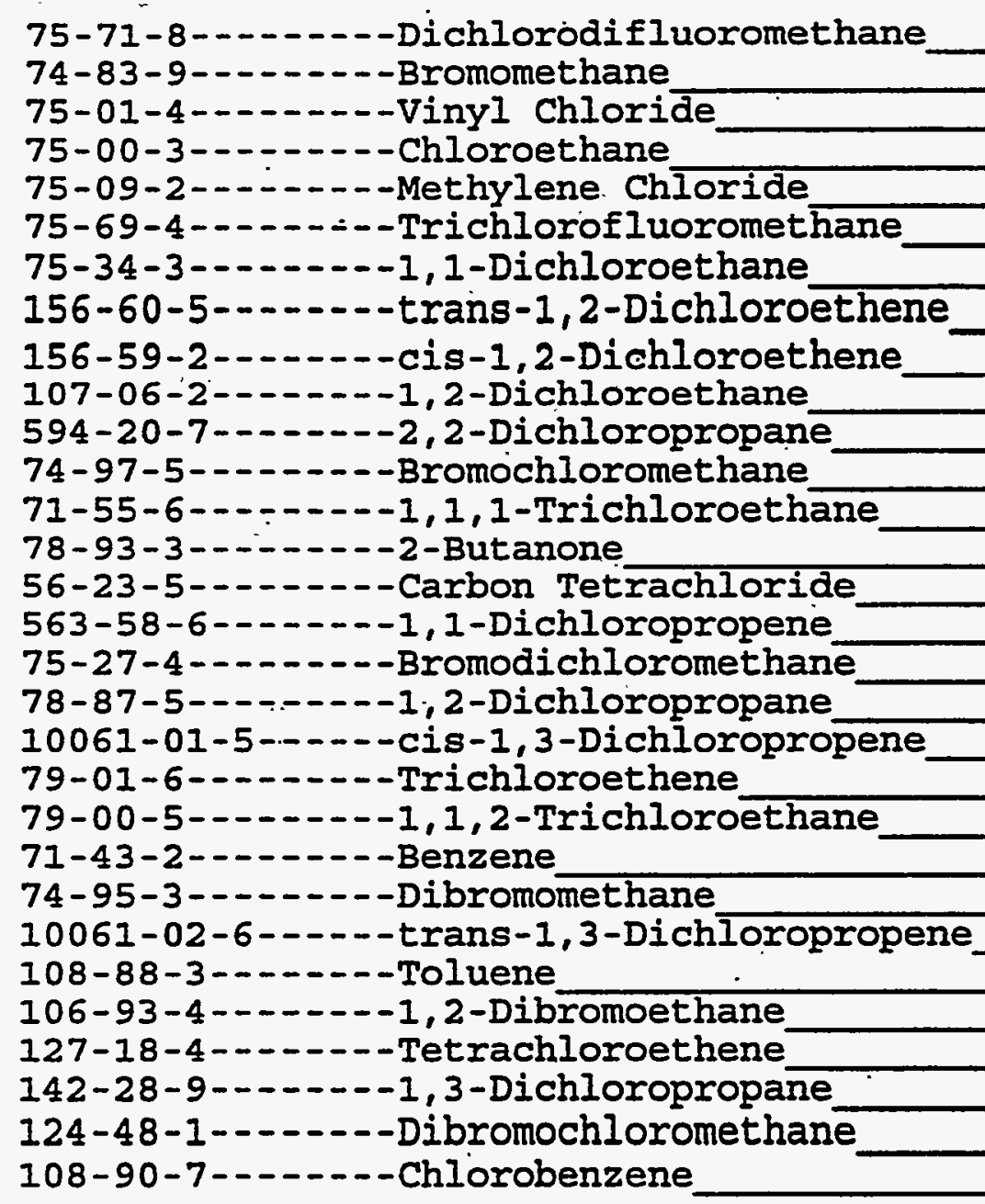


lb Name: "QÜUATERRA MO

Code: ITMO Case No.: V53301 SAS No.: ztrix: (soil/water) SOIL

zimple wt/vol: $5.00(\mathrm{~g} / \mathrm{mI}) \mathrm{G}$

el: (low $/ \mathrm{med})$ LOW

Moisture: not dec.

ב Column: RTX-502.2 ID: 0.530 (mm)

jil Extract Volume:

(uL)
Contract: $625-01$

\section{NSCEP-246}

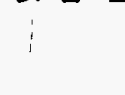

$\begin{array}{lll} & \text { CONCENTRATION UNITS: } & \text { CAS NO. } \\ \text { COMPOUND } & \text { (ug/L or ug/Kg) UG/KG }\end{array}$

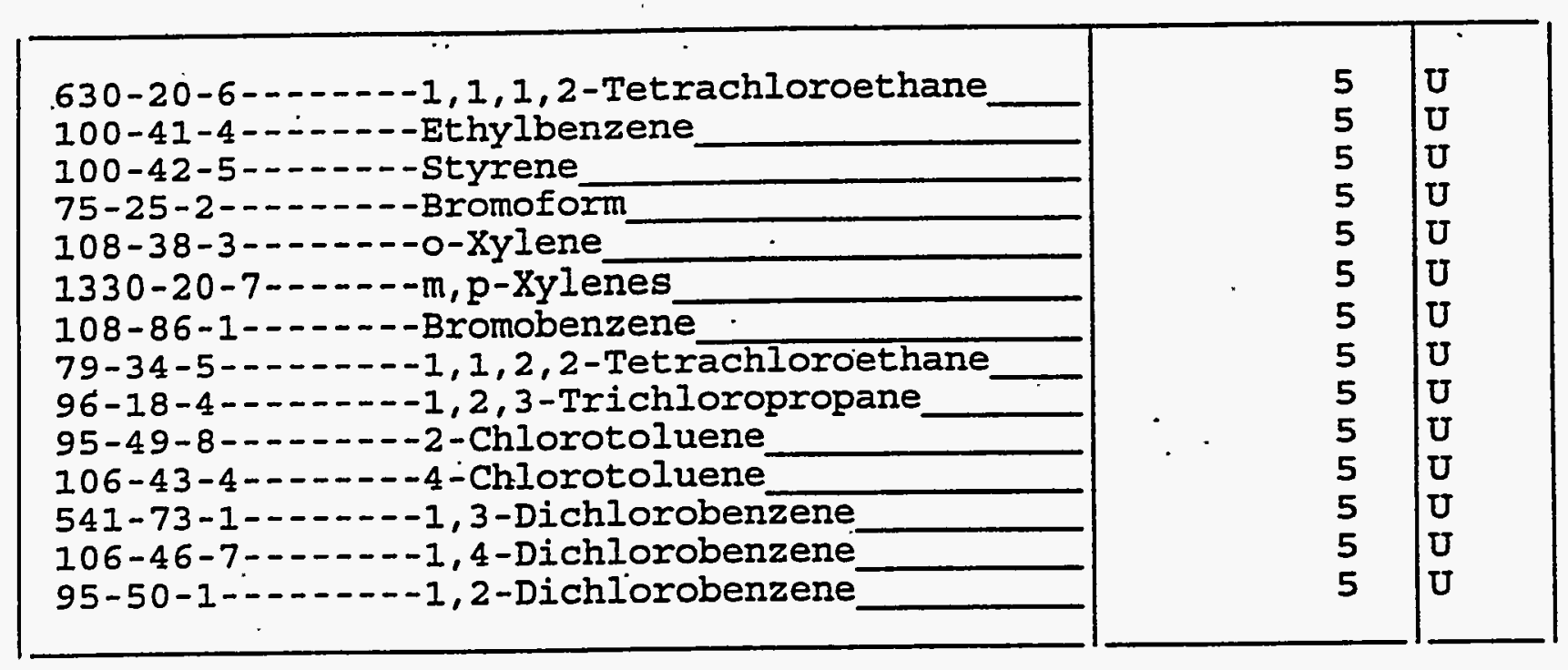

Lab Sample ID: 14533-002

Lab File ID: . E8538

Date Received: 05/06/97

Date Analyzed: 05/10/97

Dilution Factor: $\quad 1.0$

Soil Aliquot Volume:

(uI) CONCENTRATION UNITS: 
10

LBH ANALYSIS DATA SEEET
BPA SAYPI NO

NSCBP-1246

Lab Name: $\frac{\text { Ouanterra Yo }}{\text { Iab Code: Imyo Case No.: }}$
Matrix : (soil/water) soIt
Sample wt/vol: $\frac{1.0}{(\mathrm{~g} / \mathrm{ml}) \text { G }}$
Level: (low/med) Iow
\&oisture: not dec.

* Molsture: not dec.
SAS NO.:
Iab sample ID: "14533-003

Iab File ID:

Date Sampled: $\quad 04-30-97$

Date Analyzed: 05-09-97

Dilution Factor: 1.0
CONCENTRATION URITS:

(ug/I or ug/Kg) UG/KG

$\mathbf{Q}$

\begin{tabular}{|l|l|l|}
\hline IBH (1) _ _ & \\
\hline
\end{tabular}

U:Concentration of analyte is less than the value given. $X:$ Pattern reasonably matches gasoline $Y$ : Pattern appears to be multi-peaks, but does not match garoline $\mathrm{z}$ concentration 18 primarily from a-single peak
(1):Low Boiling Hydrocarbon (LBE) is quantitated as if it is Gasoline. 
ID

HBH ANALYSIS DATA SHEET
EPA SAMPLE NO.

NSCEP -1246
Tab Name: OUANTERRA MO

Iab Code: ITMO Case No.:

latrix : (soil/water)

SoII

sample wt/vol: $20.1(\mathrm{~g} / \mathrm{ml})$

G

Level: (low/med)

LOW

- Molsture: not dec. dec.

axtraction: (SepF/Cont/Sone/Shak) SHAK 3PC Cleanup: $(Y / N) \perp N$ pH:
Contract: 625.07 SAS NO.:

SDG No.: V205

Iab Sample ID:

$14533-003$

Lab File ID:

Date Sampled:

$04-30-97$

Date Extracted:

05-08-97

Date Analyzed:

05-09-97

Dilution Factor:

1

CONCENTRATION - UNITS :

CAS NO:

Compound

(mg/L or mg/kg)

$\mathrm{mg} / \mathrm{ko}$

$\dot{Q}$

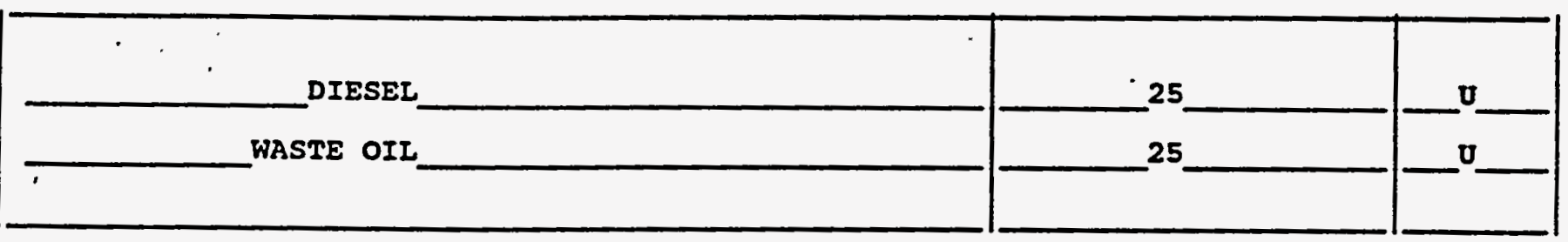

U: Concentration of analyte is less than the value given.

FORY I HBH 
Lab Name:" QUAANTERRA MO

Lab Code: ITMO

Case No.: V53301

Matrix: (soil/water) SOIL
Sample wt/vol:
$1.00(\mathrm{~g} / \mathrm{mL}) \mathrm{G}$

Level: (low/med) LOW

\% Moisture: not dec.

GC Column: RTX-502.2 ID: 0.530 (mm)

Soil Extract Volume:

(UL)
Contract: $625-01$

SAS NO.: :
NSCEP- 1246

\begin{abstract}
Soil Extract Volume:
\end{abstract}
CAS NO.
COMPOUND
Lab Sample ID: 14533-003
Lab File ID: F0002

Date Received: 05/06/97

Date Analyzed: 05/12/97

Dilution Factor: 1.0

Soil Aliquot volume:

(UL)

\begin{tabular}{|c|c|c|}
\hline 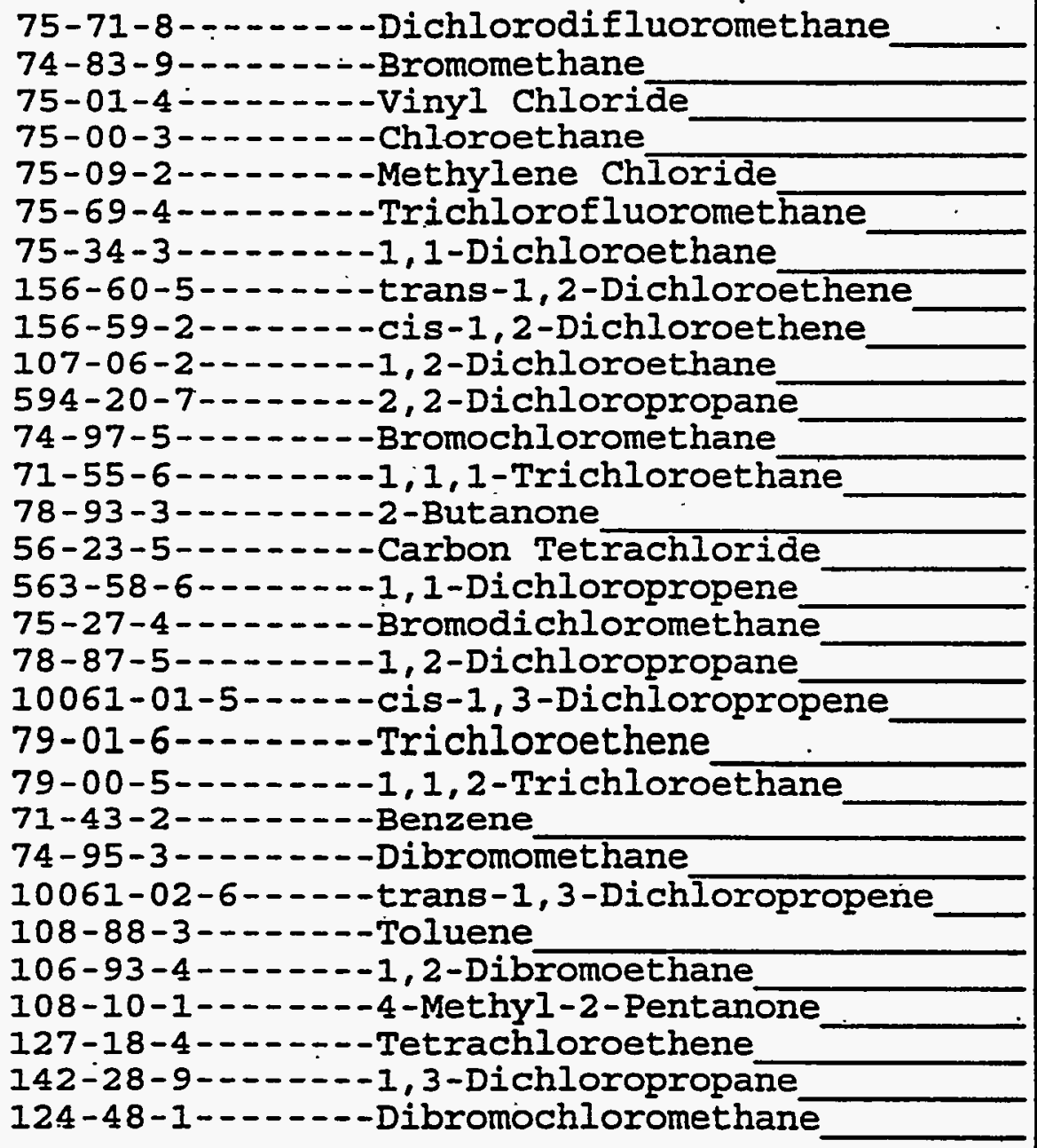 & $\begin{array}{r}100 \\
100 \\
100 \\
100 \\
50 \\
50 \\
50 \\
50 \\
50 \\
50 \\
50 \\
50 \\
50 \\
200 \\
50 \\
50 \\
50 \\
50 \\
50 \\
50 \\
50 \\
50 \\
50 \\
50 \\
8 \\
50 \\
100 \\
50 \\
50 \\
50\end{array}$ & $\begin{array}{l}U \\
U \\
U \\
U \\
U \\
U \\
U \\
U \\
U \\
U \\
U \\
U \\
U \\
U \\
U \\
U \\
U \\
U \\
U \\
U \\
U \\
U \\
U \\
U \\
J \\
U \\
U \\
U \\
U \\
U\end{array}$ \\
\hline
\end{tabular}


lb Name:“ QUUANTERRA MO

4. Code: ITMO เtrix: (soil/water) soII

( 2le wt/vol:

$1.00(\mathrm{~g} / \mathrm{mL}) \mathrm{G}$

: el: (low/med) LOW

Moisture: not dec.

$\therefore$ Iolumn: RTX-502.2 ID: 0.530 (mm)

iI Extract Volume:

(uL)
SAS NO.:

SDG NO.: V205

Lab Sample ID: 14533-003

Lab File ID: F0002

Date Received: 05/06/97

Date Analyzed: 05/12/97

Dilution Factor: $\quad 1.0$

Soil Aliquot Volume:

(UI)

CAS NO.

COMPOUND

CONCENTRATION UNITS:

(ug/I or $\mathrm{ug} / \mathrm{Kg}$ ) UG/KG

Q

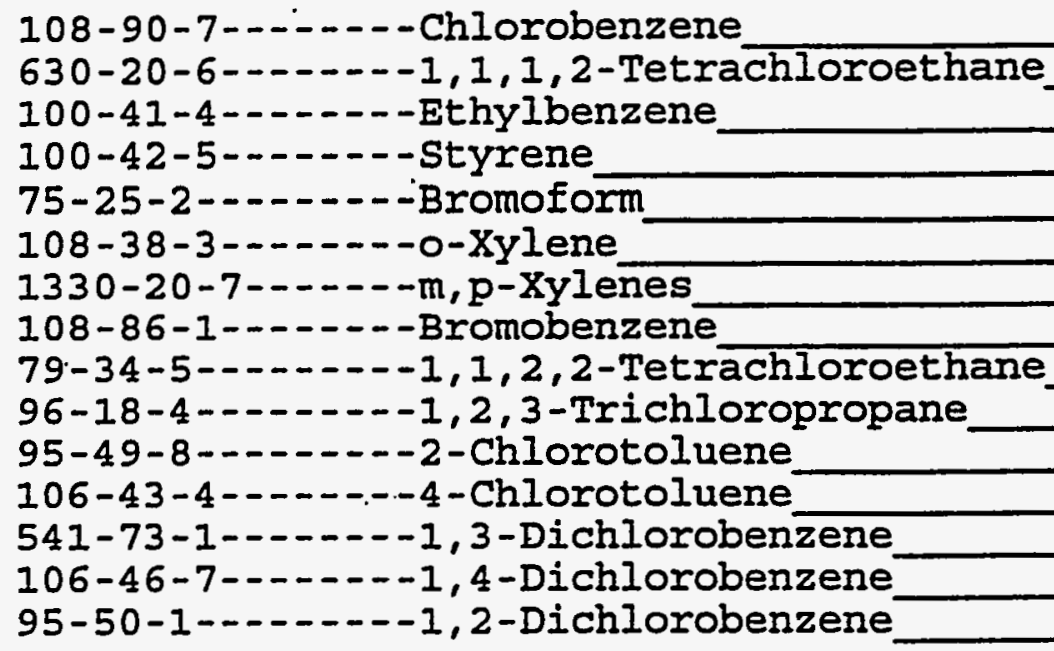


Iab Name: Ouanterra,yo

Lab Code: ITMO Case No.:

Matrix : (80il/water) soIr

Sample wt/vol:

1.0 $(g / m 1)$

$\mathbf{G}$

Level: (low/med) IOW

\& Moisture: not dec.

CAS NO. Compound
Contract: 625.01

SAS NO.: SDG NO.: V205

Irab Sample ID: 14533-004

Iab File ID:

Date sampleds

Date Analyzed: 05-10-97

Dilution Factor: 1.0
CONCENTRATION UNITS:

(ug/I or ug/Kg) UG/KG 500 $\mathbf{0}$

U:Concentration of analyte is less than the value given. $X: P a t t e r n$ reasonably matches gasoline Y:Pattern appears to be multi-peaks, but does not match gasoline z:Concentration is primarily from a aingle peak

(1):Low Boiling Hydrocarbon (LBE) is quantitated as if it is Gasoline. 


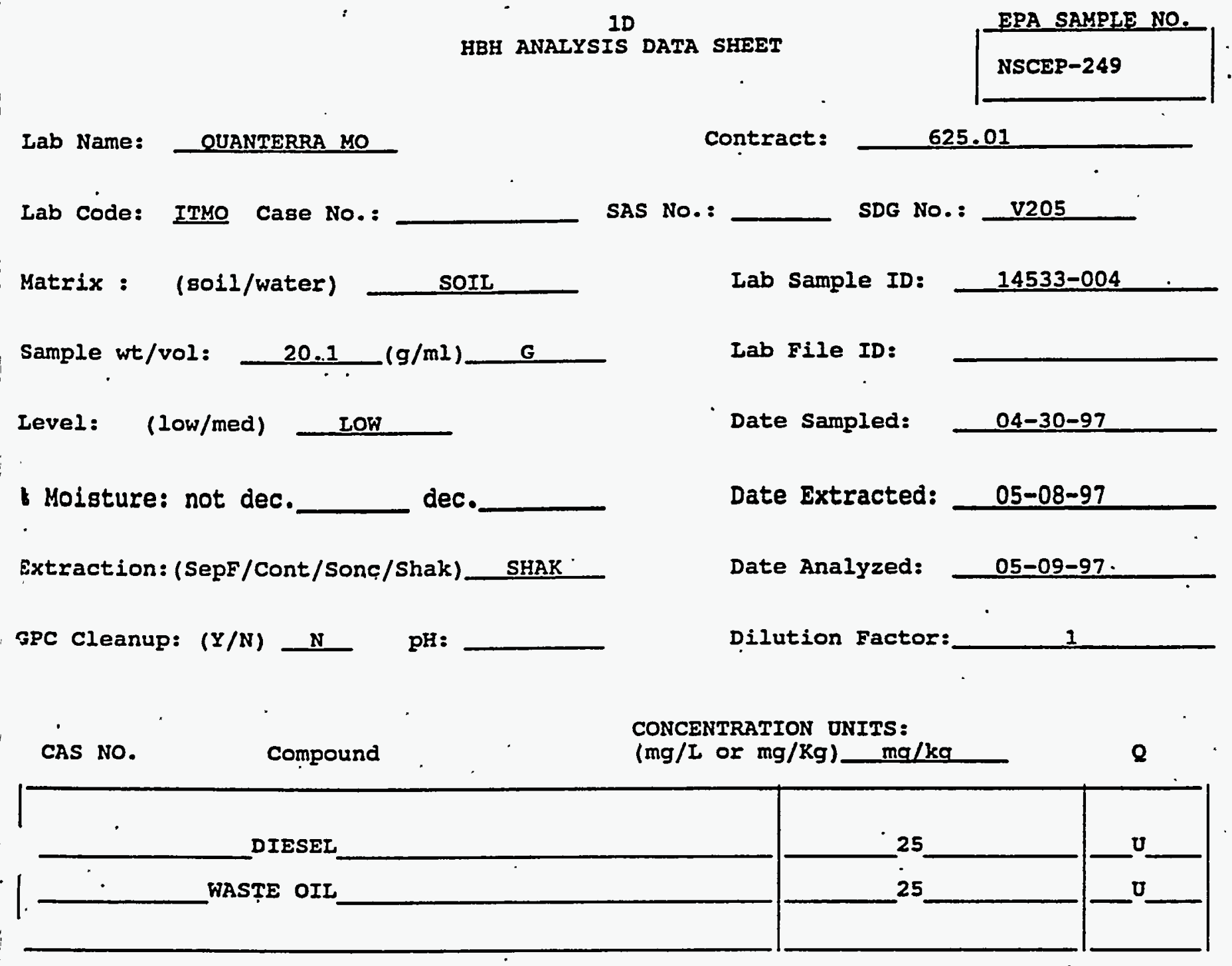

$U$ : Concentration of analyte is less than the value given. 
Lab Name:“Q̈UANTERRȦ MO

Lab Code: ITMO Case No.: V53301

Matrix: (soil/water) soII

Sample wt/vol:

$1.00 \cdot(\mathrm{g} / \mathrm{mL}) \mathrm{G}$

Level: (low/med) LOW

$\div$ Moisture: not dec.

GC Column: RTX-502.2 ID: 0.530 (mm)

Soil Extract Volume:

(uI)
Contract: 625-01

SAS NO.:

SDG NO.: V205

NSCEP -249

Lab Sample ID: 14533-004

Lab File ID: F0003

Date Received: 05/06/97

Date Analyzed: 05/12/97

Dilution Factor: $\quad 1.0$

Soil Aliquot Volume:

(uL:

CONCENTRATION UNITS :

CAS NO. COMPOUND (ug/L or ug/ $\mathrm{Kg}$ ) UG/KG

Q

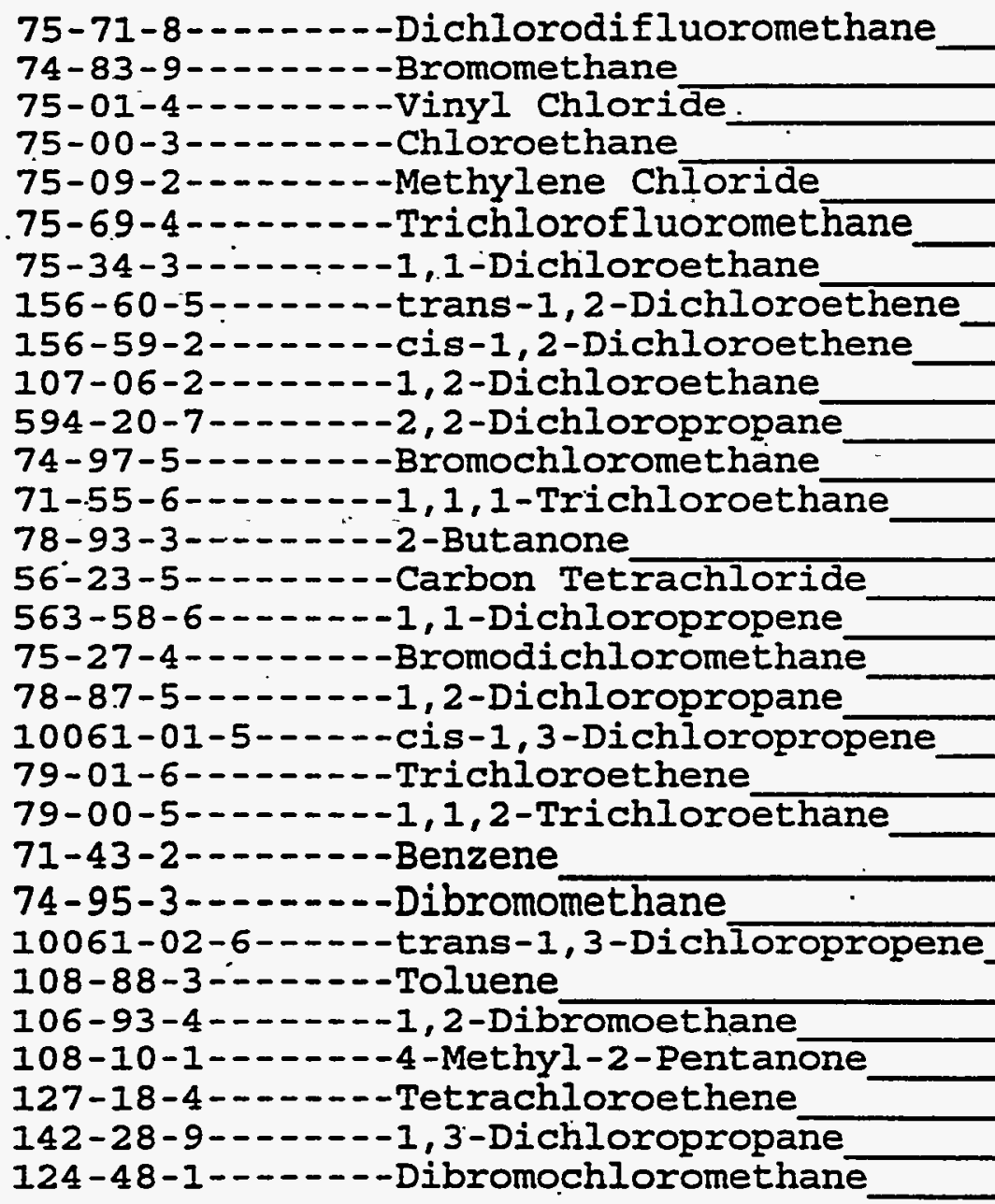




\section{VOLATILE ORGANICS ANALYSIS DATA SHEET}

ว Name:" QUUANTERRA MO

: Code: ITMO

Case No.: V53301

Erix: (soil/water) SOIL

i.jle wt/vol:

$1.00(\mathrm{~g} / \mathrm{mL}) \mathrm{G}$

1.1: (low/med) LOW

Moisture: not dec.

Solumn: RTX-502.2 ID: 0.530 (mm)

il Extract Volume:

(UI)
Contract: $625-01$

SAS NO.:

SDG NO.: V205
NSCEP -249

Lab Sample ID: 14533-004

Lab File ID: F0003

Date Received: 05/06/97

Date Analyzed: 05/12/97

Dilution Factor:

1.0

Soil Aliquot Volume:

(UIs)
CONCENTRATION UNITS:

(ug/I or $\mathrm{ug} / \mathrm{Kg}$ ) UG/KG

$\mathbf{Q}$

CAS' NO.

COMPOUND

\begin{tabular}{|ll|l|}
\hline & 50 & $U$ \\
& 50 & $U$ \\
& 50 & $U$ \\
& 50 & $U$ \\
& 50 & $U$ \\
& 50 & $U$ \\
& 50 & $U$ \\
& 50 & $U$ \\
& 50 & $U$ \\
& 50 & $U$ \\
& 50 & $U$ \\
& 50 & $U$ \\
& 50 & $U$ \\
& 50 & $U$ \\
& 50 & $U$ \\
\hline
\end{tabular}

108-90-7--.---Chlorobenzene

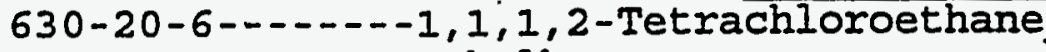

100-41-4------Ethylbenzene

100-42-5-------Styrene

75-25-2-...--Bromoform

108-38-3----.--0-Xylene

1330-20-7-...-m, p-xylenes

108-86-1--.---Bromobenzene

79-34-5-..-- 1, 1, 2, 2-Tetrachloroethane

96-18-4------1,2,3-Trichloropropane

95-49-8-...--2-Chlorotoluene

106-43-4-n---4-Chlorotoluene

541-73-1--.-.-1, 3-Dichlorobenzene

106-46-7--.--1,4-Dichlorobenzene

95-50-1---.---1,2-Dichlorobenzene 
Lab Name:

Iab code:

Matrix

Sample wt/vol: Case No.s (aoil/water) soIT

Level: (low/med) 1.0 $(g / m 1)$ G

\& Molsture: not dec.

IOA

Compound.

LBE (1)
Contract: 625.01

SAS NO.:

$$
\text { SDG NO.: Y205 }
$$

Iab Sample ID: 14533-005

Iab FIIe ID:

Date sampled:

Date Analyzed: 05-10-97

Dilution Factor: 1.0

CONCENTRATION UNITS:

$(u g / L$ or $u g / \mathrm{kg})$ UG/KG 500

U: Concentration of analyte is less than the value given.

$\mathrm{X}$ : Pattern reasonably matches gasoline

Y:Pattern appears to be multi-peaks, but does not match gasoline

z:Concentration is primarily from a single peak

(1):Low Boiling Hydrocarbon (LBH) is quantitated as if it is Gasoline. 
Lab Name: OUANTERRA MO

Contract:

625.01

Lab Code: ITMO Case No.:

SAS NO.:

SDG NO.: V205

satrix : (soil/water)

SOIL

Lab Sample ID:

$14533-005$

Sample wt/vol:

$20.1(\mathrm{~g} / \mathrm{ml})$

Lab File ID:

Level: (low/med) LOW

Date Sampled:

$04-30-97$

b Moisture: not dec. dec.

Date Extracted:

$05-08-97$

ixtraction: (SepF/Cont/Sonc/Shak) SHAK TPC Cleanup: $(\mathrm{Y} / \mathrm{N})$ N $\mathrm{NH}:$

Dilution Factor:

1

CONCENTRATION UNITS :

CAS NO.

Compound

(mg/L or $\mathrm{mg} / \mathrm{Kg}$ )

$\mathrm{ma} / \mathrm{kg}$

$\mathbf{Q}$

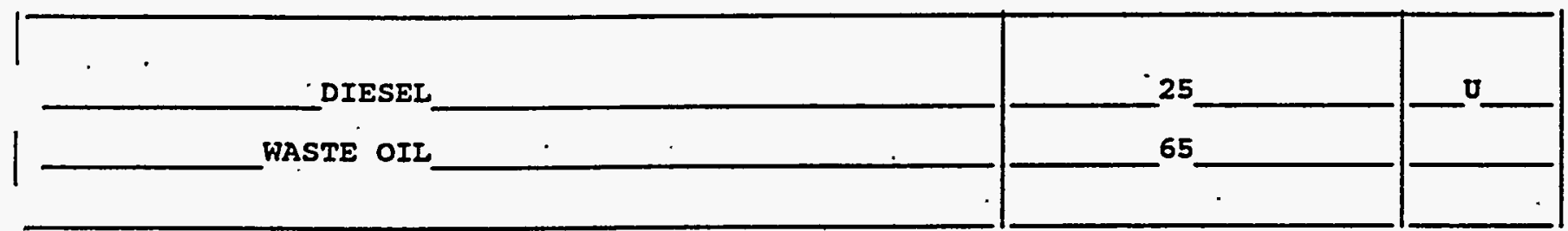

$U$ : Concentration of analyte is less than the value given. 
NSCEP-262

Lab Name: -QUUANTERRA MO

Lab Code: ITMO

Case No.: V53301

Matrix: (soil/water) soIL

Sample wt/vol:

$5.00(\mathrm{~g} / \mathrm{mL}) \mathrm{G}$

Level: (low/med) LOW

\% Moisture: not dec.

GC Column: RTX-502.2 ID: 0.530 (mm)

Soil Extract Volume:

(uL)
Contract: $625-01$

SAS NO.:

SDG No.: V205

Lab Sample ID: 14533-005

Lab File ID: $\quad$ E854i

Date Received: 05/06/97

Date Analyzed: 05/10/97

Dilution Factor: 1.0

Soil Aliquọt Volume:

(uI)

CONCENTRATION UNITS :

CAS NO.

COMPOUND

(ug/I or ug/Kg) UG/KG

Q

75-71-8-...--Dichlorodifluoromethane

74-83-9-....--Bromomethane

75-01-4-...-.-vinyl Chloride

75-00-3-..----Chloroethane

75-09-2-...-.-Methylene Chloride

75-69-4-.-----Trichlorofluoromethane

75-34-3-...--1, 1-Dichloroethane

156-60-5-------trans-1,2-Dichloroethene

156-59-2--.-.-cis-1, 2-Dichloroethene

107-06-2-----1,2-Dichloroethane

594-20-7---.--2;2-Dichloropropane

74-97-5-..----Bromochlorömethane

71-55-6-..---1, 1, 1-Trichloroethane

78-93-3-.---.--2-Butanone

56-23-5-.....-Carbon Tetrachloride

563-58-6------1, 1-Dichloropropene

75-27-4-...-.-Bromodichloromethane

78-87-5---.--1, 2-Dichloropropane

10061-01-5--.-cis-1,3-Dichloropropene

79-01-6-...--.-Trichloroethene

79-00-5-..---1, 1,2-Trichloroethane

$71-43-2-\ldots---B e n z e n e$

74-95-3-...--Dibromomethane

10061-02-6--.--trans-1,3-Dichloropropene

108-88-3-..-.-Toluene

106-93-4--.----1,2-Dibromoethane

127-18-4-...-.-Tetrachloroethene

142-28-9-----1,3-Dichloropropane

124-48-1-...--Dibromochloromethane

108-90-7-...--Chlorobenzene

\begin{tabular}{r|l|}
10 & $U$ \\
10 & $U$ \\
10 & $U$ \\
10 & $U$ \\
5 & $U$ \\
5 & $U$ \\
5 & $U$ \\
5 & $U$ \\
5 & $U$ \\
5 & $U$ \\
5 & $U$ \\
5 & $U$ \\
5 & $U$ \\
20 & $U$ \\
5 & $U$ \\
5 & $U$ \\
5 & $U$ \\
5 & $U$ \\
5 & $U$ \\
5 & $U$ \\
5 & $U$ \\
5 & $U$ \\
5 & $U$ \\
5 & $U$ \\
5 & $U$ \\
5 & $U$ \\
5 & $U$ \\
5 & $U$ \\
5 & $U$ \\
5 & $U$ \\
& \\
\hline &
\end{tabular}

000051 
O Name:"̄QUANTERRA MO"

c Code: ITMO Case No.: V53301 trix: (soil/water) SOIL

irsle wt/vol:

$5.00(\mathrm{~g} / \mathrm{mL}) \mathrm{G}$

I: (Iow/med) LOW

Moisture: not dec.

olumn: RTX-502.2 ID: 0.530 (mm)

il Extract Volume:

(uI)
Contract: $625-01$

SAS NO.:

SDG No.: V205

NSCEP-262

Lab Sample ID: 14533-005

Lab File ID: E854i

Date Received: 05/06/97

Date Analyzed: 05/10/97

Dilution Factor: $\quad 1.0$

Soil Aliquot Volume:

(UL)

CONCENTRATION UNITS:

CAS NO. COMPOUND $\quad(\mathrm{ug} / \mathrm{L}$ or $\mathrm{ug} / \mathrm{Kg}) \mathrm{UG} / \mathrm{KG}$

Q

630-20-6-..- - 1, 1, 1,2-Tetrachloroethane

100-41-4---.-.-Ethylbenzene

100-42-5-------styrene

75-25-2-....--Bromoform

108-38-3-..--- - Xylene

$1330-20-7-\ldots-m, p-x y l e n e s$

108-86-1-...-.-Bromobenzene

79-34-5--.---1,1,2,2-Tetrachloroethane

96-18-4-..--1,2,3-Trichloropropane

95-49-8--.-.--2-Chlorotoluene

106-43-4--.--4-Chlorotoluene

541-73-1-..---1, 3-Dichlorobenzene

106-46-7--.--1,4-Dichlorobenzene

95-50-1-------1,2-Dichlorobenzene

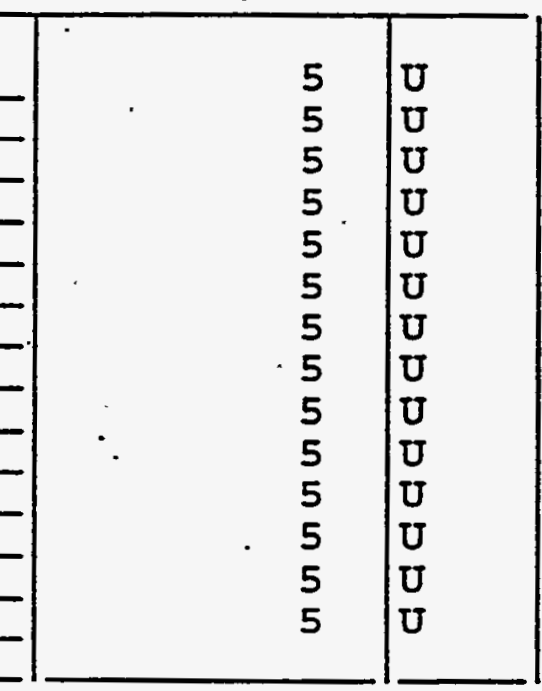


EPA SAYPLE NO

NSCEP-264
Iab Name:

Ouanterraryo

Lab code: ITyO Cage No.:

Matrix : (80il/water) soIr

Sample wt/vol:

1.0 $(\mathrm{g} / \mathrm{ml})$

Level: (low/med) LOW

* Moisture: not dec.
Contract: 625.01

SAS NO.:

SDG No.: V205

Iab Sample ID: 14533-007

Lab File ID:

Date Sampled: $\quad 04-30-97$

Date Analyzed: 05-10-97

Dilution Factors 1.0

CONCENTRATION UNITS:

(ug/L or $u g / R g)$ UG/RG

LBH (1)

500

U:Concentration of analyte is less than the value given.

X:Pattern reasonably matches gasoline

Y: Pattern appears to be multi-peaks, but does not match gasoline

$z$ :Concentration is primarily from a single peak

(1):Low Boiling Bydrocarbon (LBB) is quantitated as if it is Gasoline. 


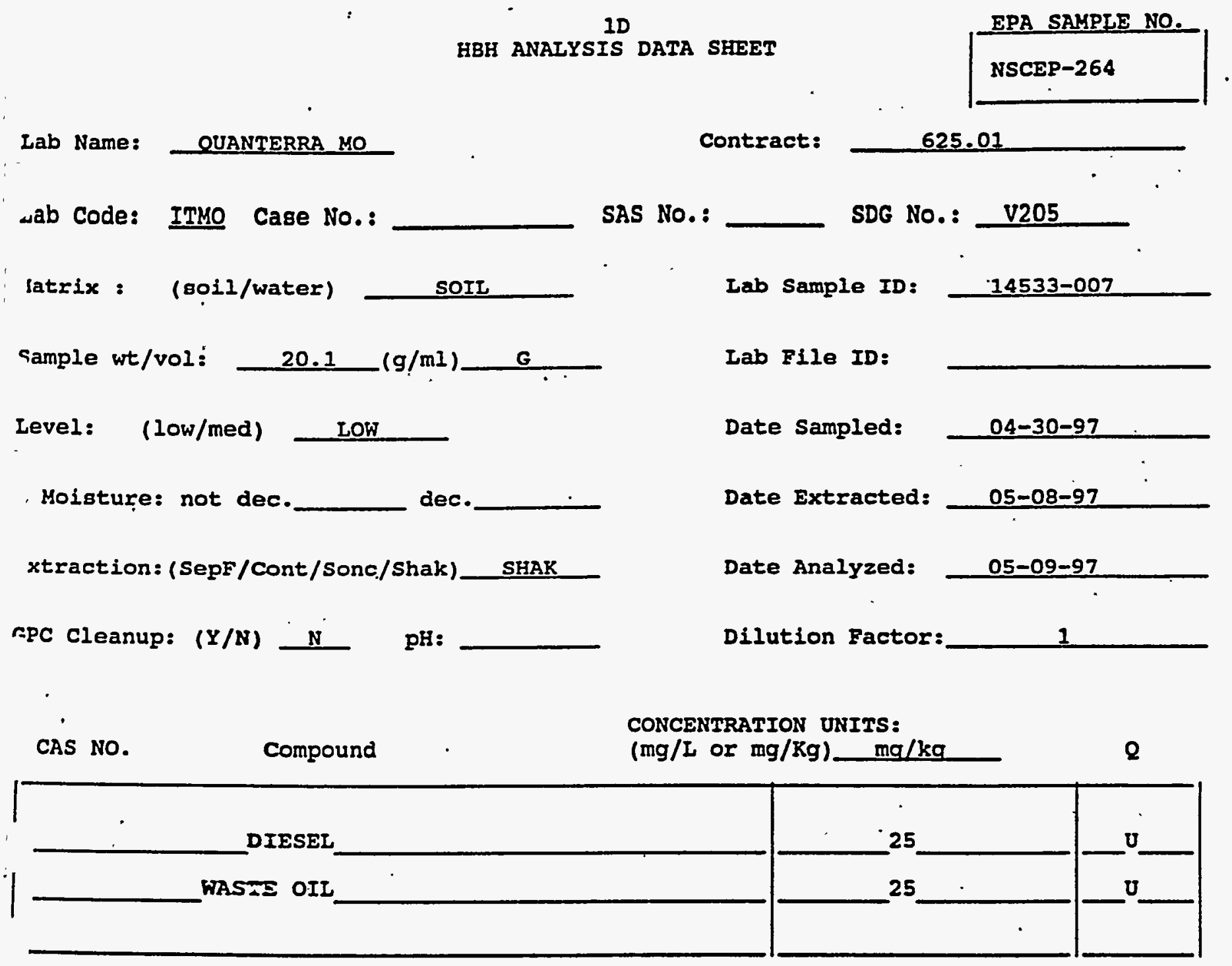

': Concentration of analyte is less than the value given. 
VOLATIIE ORGANICS ANAIYSIS DATA SHEET

Iab Name:- QUUANTERRA' MO

Lab Code: ITMO Case No.: V53301

Matrix: (soil/water) soII

- Sample wt/vol:

$1.00(\mathrm{~g} / \mathrm{mL}) \mathrm{G}$

Level: (low/med) LOW

- \% Moisture: not dec.

Contract: $625-01$

SAS NO.:

Lab Sample ID: 14533-007

Lab File ID: F0005

Date Received: 05/06/97

Date Analyzed: 05/12/97

Dilution Factor: $\quad 1.0$

(uL)

Soil Aliquot Volume:

(UI)
Soil Extract Volume:

CÁS NO.

COMPOUND

CONCENTRATION UNITS :

(ug/L or ug/Kg) UG/KG

Q

75-71-8---.--Dichlorodifluoromethane

74-83-9--.----Bromomethane

75-01-4-..-.---Vinyl Chloride

75-00-3-..---Chloroethane

75-09-2-...-.-Methylene Chloride

75-69-4-.....-Trichlorofluoromethane

75-34-3-..---1,1-Dichloroethane

156-60-5--..--trans-1,2-Dichloroethene

156-59-2-.----cis-1, 2-Dichloroethene

107-06-2-..--1,2-Dichloroethane

594-20-7--.---2,2-Dichloropropane

74-97-5-...-.-Bromochloromethane

71-55-6-..---1,1,1-Trichloroethane

78-93-3-..---2-Butanone

56-23-5-..----Carbon Tetrachloride

563-58-6--.--1,1-Dichloropropene

75-27-4-.-----Bromodichloromethane

78-87-5-----1,2-Dichloropropane

10061-01-5--.--cis-1,3-Dichloropropene

79-01-6--..---Trichloroethene

79-00-5--.---1, 1, 2-Trichloroethane

71-43-2--..---Benzene

74-95-3-...--Dibromomethane

10061-02-6--.---trans-1,3-Dich loropropene

108-88-3-...-.-Toluene

106-93-4--..--1,2-Dibromoethane

108-10-1-...--4-Methyl-2-Pentanone

127-18-4--...-Tetrachloroethene

142-28-9-----1,3-Dichloropropane

124-48-1-..--Dibromochloromethane 
ib Name:"-QUANTERRA MO"

L. Code: ITMO

itrix: (soil/water) SOIL

l Ile wt/vol:

$1.00(\mathrm{~g} / \mathrm{mL}) \mathrm{G}$

:-1: (low/med) LOW

Moisture: not dec.

: zolumn: RTX-502.2 ID: 0.530 (mm)

il Extract Volume:

(UI)
Contract: $625-01$

SAS NO.:
NSCEP-264

SDG No.: V205

Lab Sample ID: 14533-007

Lab File ID: F0005

Date Received: 05/06/97

Date Analyzed: 05/12/97

Dilution Factor: $\quad 1.0$

Soil Aliquot volume:

(uL)

CAS NO. . COMPOUND (ug/L or ug/Kg) UG/KG

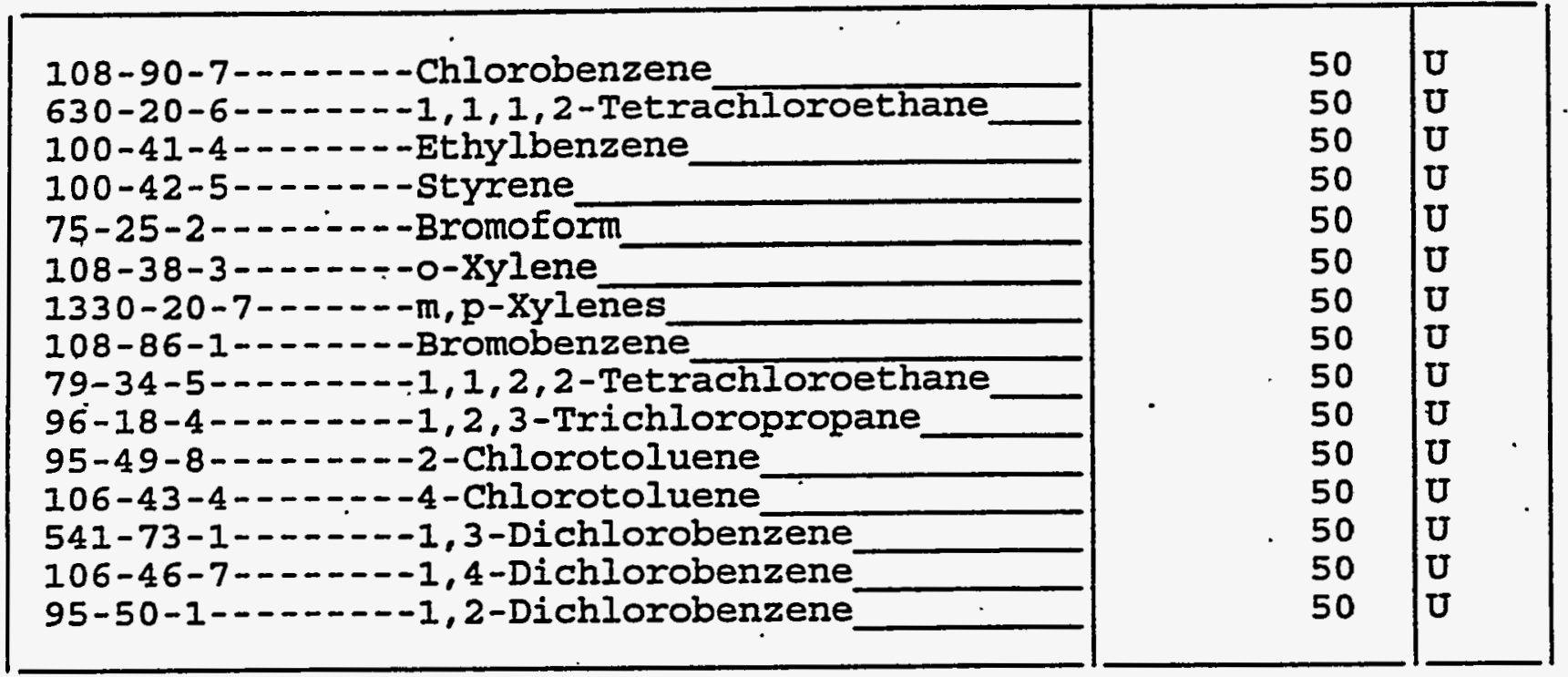


1D

IBH ANALYSIS DATA SEEET
EPA SAMPLE NO. NSCEP-270
Lab Name:

Iab code:

Matrix Ifyo Case No.:

: (Boil/water) SoII

Sample wt/vol:

1.0 $(g / m 1)$

G

Level: (low/med)

LOW

soisture: not dec.
Contract: -625.01

SAS NO.:

SDG NO.: V205

Lab Sample ID: 14533-006

Iab F110 ID:

Date Sampled:

$04-30-97$

Date Analyzed: : 05-10-97

Dilution Factor: 1,0
CONCENTRATION UNITS:

(ug/L or ug/ $\mathrm{kg}$ ) UG/KG

CAS No. Compound.

\begin{tabular}{|c|c|}
\hline \\
\hline
\end{tabular}
IBE (1)

$$
\text { 1) }
$$

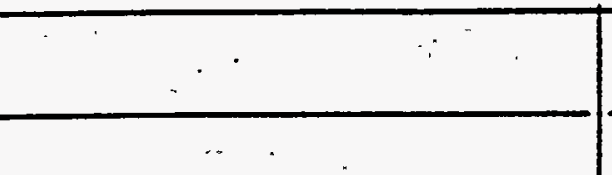

\section{0}

U: Concentration of analyte is less than the value given. $\mathrm{X}:$ Pattern reasonably matches gasoline $Y:$ Pattern appears to be multi-peaks, but does not match gasoline z:Concentration is primarily from a single peak

(1):Low Boiling Hydrocarbon (LBH) is quantitated as if it is Gasoline. 


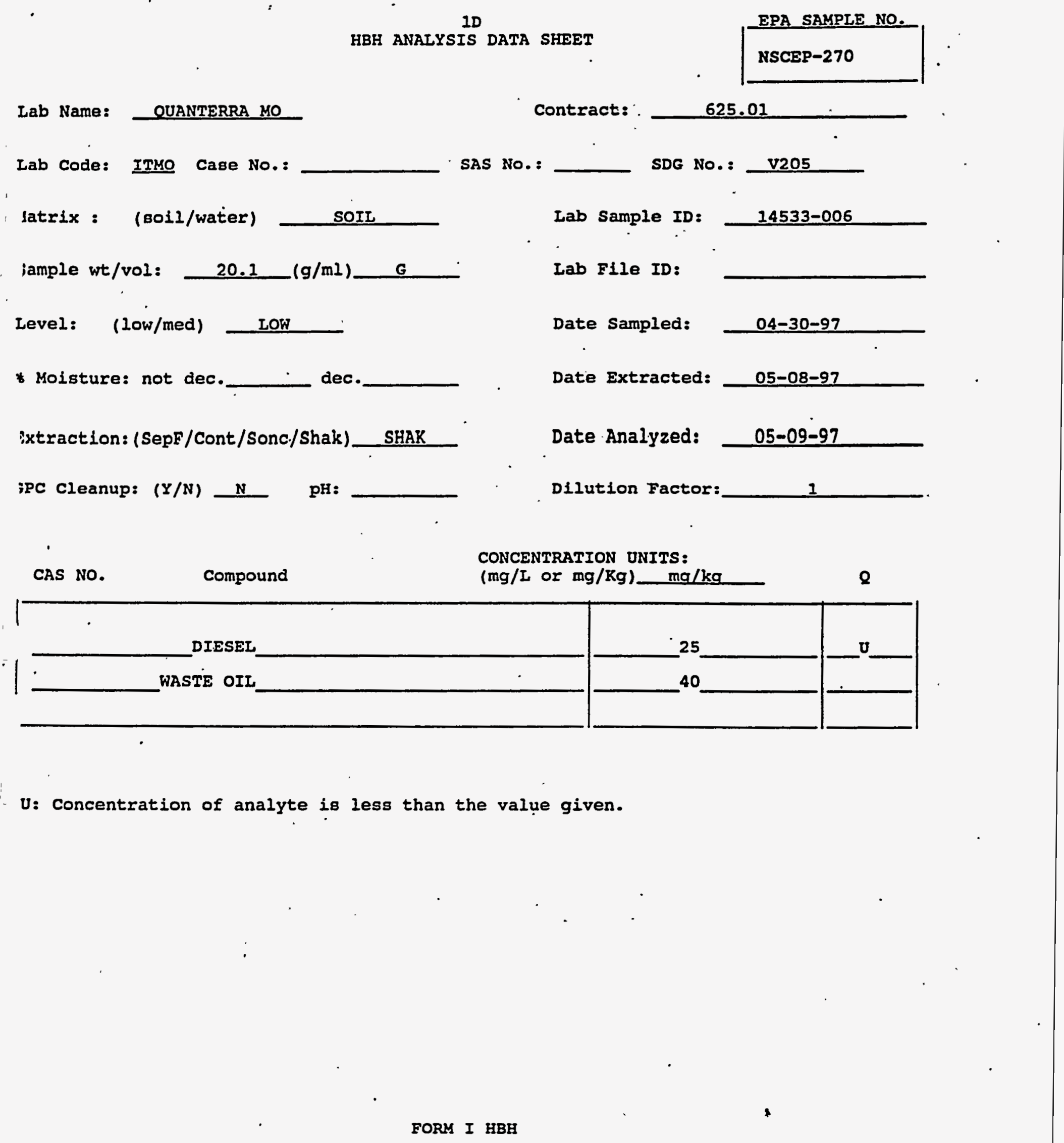


VOLATILE ORGANICS ANALYSIS DATA SHEET

Lab Name:"“QUANTERRA MO

Lab Code: ITMO

Case. NO.: V53301 SAS NO.:
NSCEP-270
Matrix: (soil/water) soIL

Sample wt/vol:

$1.00(\mathrm{~g} / \mathrm{mL}) \mathrm{G}$

Level: (Iow/med) LOW

$\%$ Moisture: not dec.

GC Columin: RTX-502.2 ID: 0.530 (mm)

Soil Extract Volume:

(uI.)
Contract: $625-01$

SDG No.: V205
Lab Sample ID: 14533-006.

Lab File ID: F0004

Date Received: 05/06/97

Date Analyzed: 05/12/97

Dilution Factor: $\quad 1.0$

Soil Aliquot Volume:

(UI)

CAS NO.

COMPOUND

CONCENTRATION UNITS:

(ug/L or ug/Kg) UG/KG

75-71-8-------Dichlorodifluoromethane

74-83-9--.----Bromomethane

75-01-4--.-----vinyl Chloride

75-00-3--.----Chloroethane

75-09-2----.---Methylene Chloride

75-69-4-...-- Trichlorofluoromethane

75-34-3--.----1, 1-Dichloroethane

156-60-5-------trans-1,2-Dichloroethene

156-59-2-...-.-cis-1, 2-Dichloroethene

107-06-2--.--1, 2-Dichloroethane

594-20-7-------2,2-Dichloropropane

74-97-5--.----Bromochloromethane

71-55-6-...-- 1, 1, 1-Trichloroethane

78-93-3--.---2-Butanone

56-23-5--.---Carbon Tetrachloride

563-58-6------1, 1-Dichloropropene

75-27-4--..--Bromodichloromethane

78-87-5------1, 2-Dichloropropane

10061-01-5-.-.-cis-1,3-Dichloropropene

79-01-6--.---.-Trichloroethene

79-00-5--.---1, 1,2-Trichloroethane

71-43-2--------Benzene

74-95-3-.-.--Dibromomethane

10061-02-6-----trans-1,3-Dich loropropene

108-88-3-...-.-Toluene

106-93-4-..-1, 2-Dibromoethane

108-10-1-------4-Methyl-2-Pentanone

127-18-4--.----Tetrachloroethene

142-28-9------1, 3-Dichloropropane

124-48-1-------Dibromochloromethane

\begin{tabular}{r|l|}
100 & $U$ \\
100 & $U$ \\
100 & $U$ \\
100 & $U$ \\
50 & $U$ \\
50 & $U$ \\
50 & $U$ \\
50 & $U$ \\
50 & $U$ \\
50 & $U$ \\
50 & $U$ \\
50 & $U$ \\
50 & $U$ \\
200 & $U$ \\
50 & $U$ \\
50 & $U$ \\
50 & $U$ \\
50 & $U$ \\
50 & $U$ \\
50 & $U$ \\
50 & $U$ \\
50 & $U$ \\
50 & $U$ \\
50 & $U$ \\
50 & $U$ \\
50 & $U$ \\
100 & $U$ \\
50 & $U$ \\
50 & $U$ \\
50 & $U$ \\
& \\
& \\
50
\end{tabular}




\section{$1 \mathrm{~A}$}

VOLATILE ORGANICS ANALYSIS DATA SHEET
EPA SAMPLE NO.

NSCEP -270 b Name :" QUANTERRA MO

1 Code: ITMO

Case No.: V53301 trix: (soil/water) soII

i sle wt/vol:

$1.00(\mathrm{~g} / \mathrm{mL}) \mathrm{G}$

1 II: (low/med) LOW

Moisture: not dec.

lolumn: RTX-502.2 ID: 0.530 (mm)

il Extract Volume:

(UI)
Contract: $625-01$

SAS NO.:

.SDG No.: V205

Lab Sample ID: 14533-006

Lab File ID: F0004

Date Received: 05/06/97

Date Analyzed: 05/12/97

Dilution Factor: $\quad 1.0$

Soil Aliquot Volume:

(uL)

CONCENTRATION UNITS:

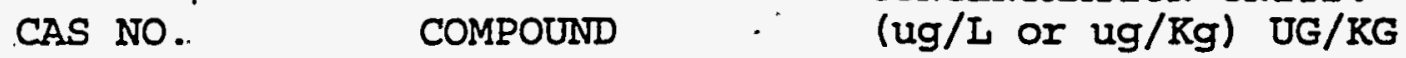

Q

\begin{tabular}{|c|}
\hline 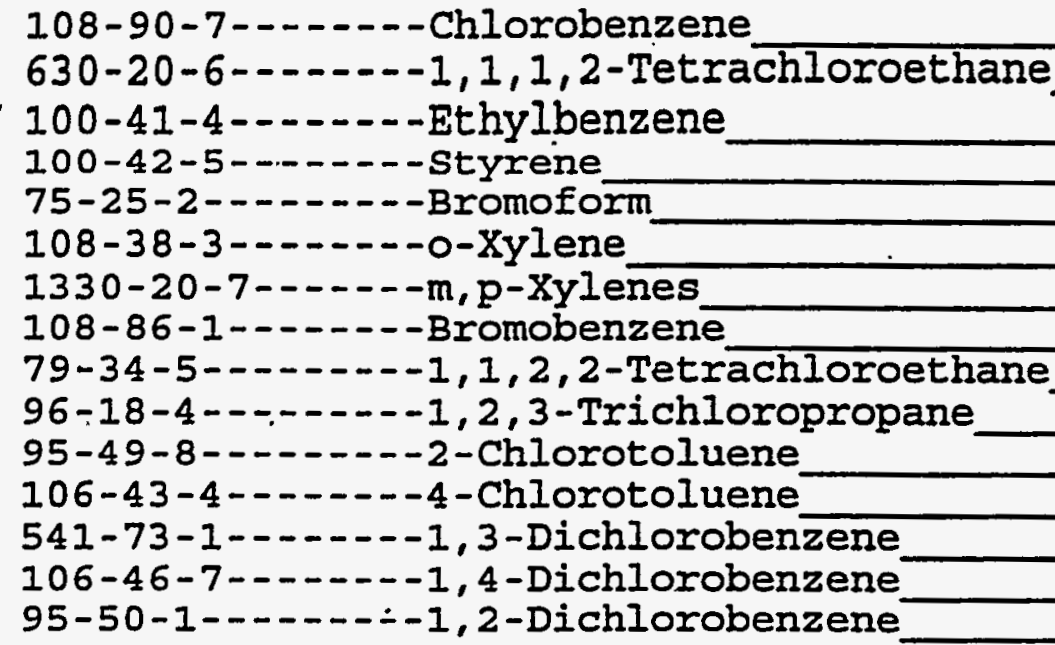 \\
\hline
\end{tabular}

\begin{tabular}{|l|l|}
\hline 50 & $U$ \\
50 & $U$ \\
50 & $U$ \\
50 & $U$ \\
50 & $U$ \\
50 & $U$ \\
50 & $U$ \\
50 & $U$ \\
50 & $U$ \\
50 & $U$ \\
50 & $U$ \\
50 & $U$ \\
50 & $U$ \\
50 & $U$ \\
50 & $U$ \\
\hline
\end{tabular}


10

LBH ANATYSIS DATA SHBBT
EPA SAMPLE NO.

NSCEP-273
Lab Name: Quanterra, kO

Iab Code: ITMO Case No.:

Matrix : (80il/water) soIr.

Sample wt/vol: $1.0(\mathrm{~g} / \mathrm{ml}) \mathrm{G}$

Level: (low/med) LOW

roisture: not dec.
Contract: 625.01

SAS NO.:

SDG NO.: V205

Lab Sample ID: $14533-010$

Lab File ID:

Date Sampled: $04-30-97$

Date Analyzed: 05-10-97

Dilution Factor: 1.0
CONCENTRATION UNITS:

( ug/L or ug/Rg) UG/KG

LBH (1)

500

U

$U$ :Concentration of analyte is less than the value given.

$X: P a t t e r n$ reasonably matches gasoline

Y:Pattern appears to be multi-peaks, but does not match gasoline

z:Concentration is primarily from a single peak

(1):Low Boiling Hydrocarbon (IBH) is quantitated as if it is Gasoline. 
ID

HBH ANALYSIS DATA SHEET
EPA SAMPLE NO.

NSCEP-273
Lab Name: QUANTERRA MO

Lab Code: ITMO Case No.:

atrix : (soil/water)

SOIL

ample wt/vol:

20.1

$(g / \mathrm{ml})$

G

Level: (low/med)

LOW

Moisture: not dec. dec.

xtraction: (SepF/Cont/Sonc/Shak)

SHAK

PC Cleanup: $(Y / N)+N$
Contract:

SAS NO.:

Lab Sample ID:

625.01

Lab File ID:

Date Sampled:

$04-30-97$

Date Extracted:

05-08-97

Date Analyzed:

05-09-97

Dilution Factor:

1

CONCENTRATION UNITS:

(mg/L or $\mathrm{mg} / \mathrm{Kg}$ ) $\mathrm{ma} / \mathrm{kg}$

$\mathbf{Q}$

\begin{tabular}{|l|l|l|}
\hline DIESEL__LSTE OIL__ & - \\
\hline
\end{tabular}

U: Concentration of analyte is less than the value given.

FORM I HBH 
Lab Name:" QUUANTERRÁ MO

Lab Code: ITMO

Case No.: V5330I

Matrix: (soil/water) SOIL

Sample wt/vol:

$$
5.00(\mathrm{~g} / \mathrm{mL}) \mathrm{G}
$$

Level: (low/med). LOW

\% Moisture: not dec.

GC Column: RTX-502.2 ID: 0.530 (mm)

Soil Extract Volume:

(uI)
Contract: $625-01$

SAS NO.:

NSCEP-273

Lab Sample ID: 14533-010

Lab File ID: $\quad$ F9979

Date Received: 05/06/97

Date Analyzed: 05/10/97

Dilution Factor: 1.0

Soil Aliquot volume: (uL CONCENTRATION UNITS:
CAS NO.
COMPOUND
(ug/L or $\mathrm{ug} / \mathrm{Kg}$ ) UG/KG

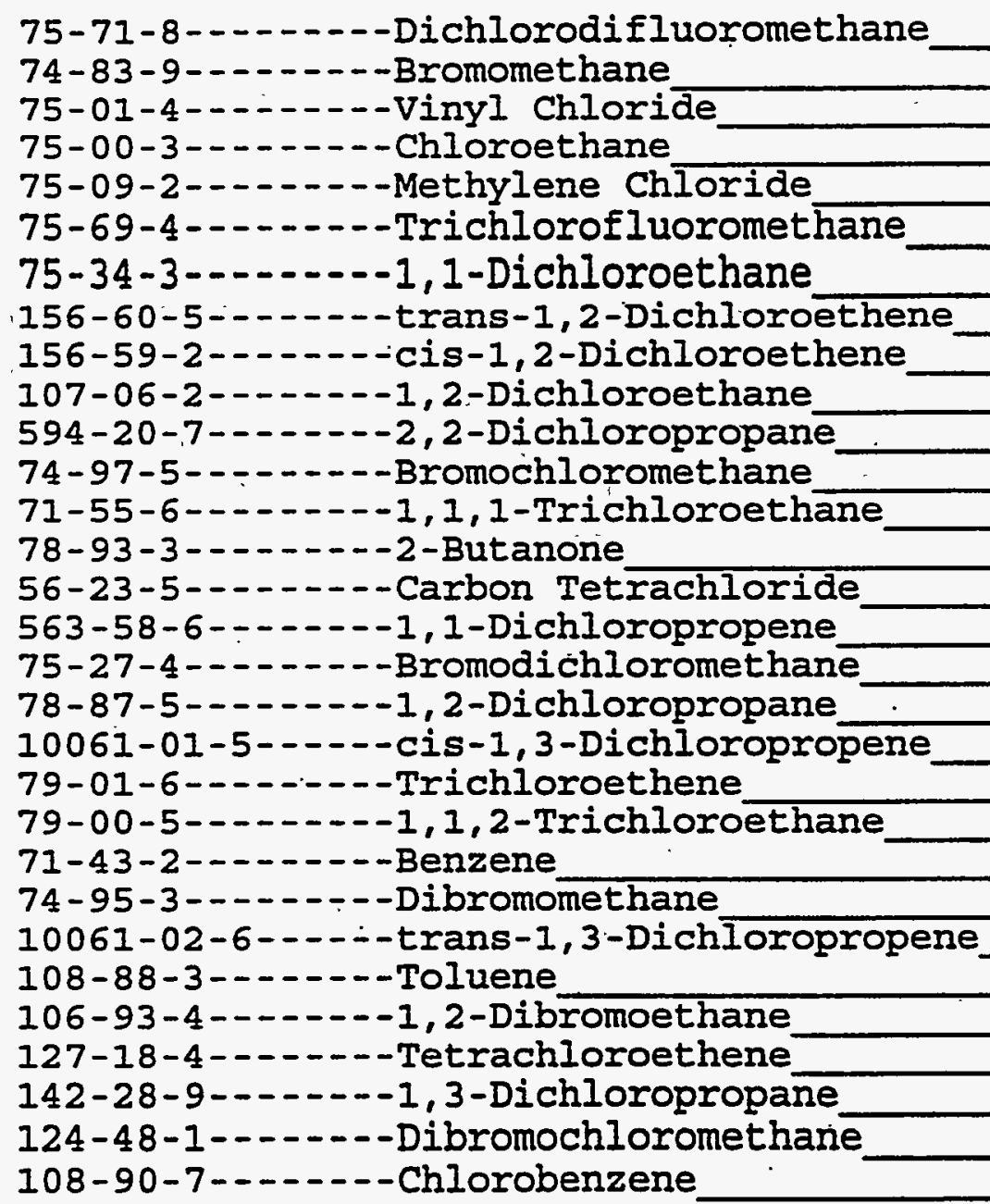

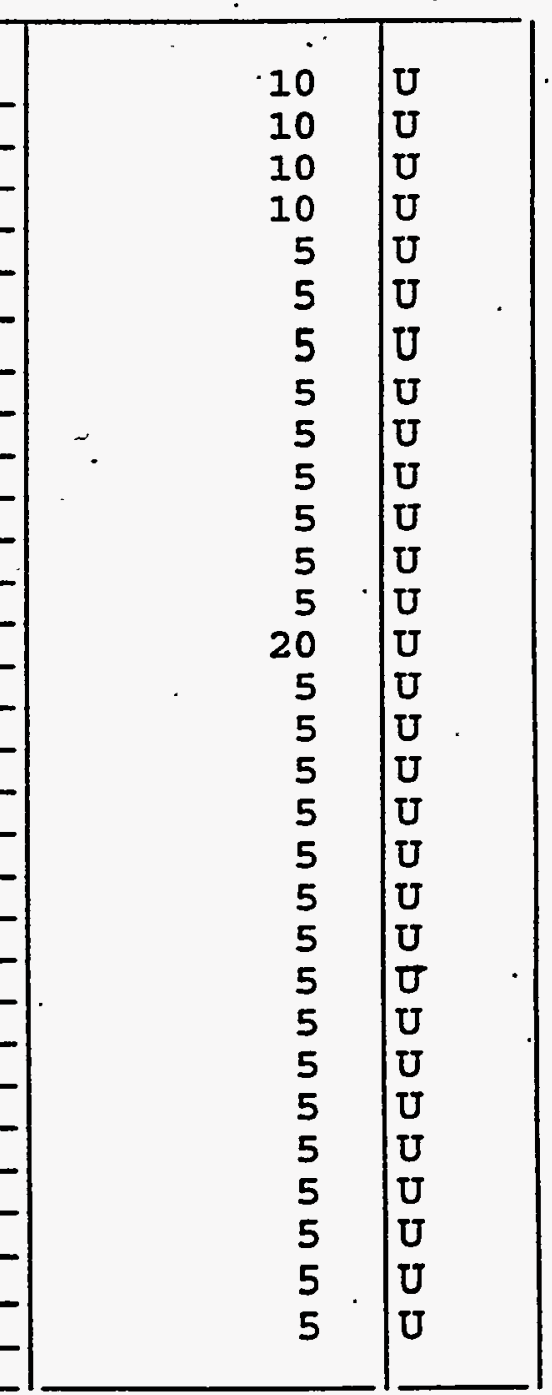


$1 \mathrm{~A}$

VOLATILE ORGANICS ANALYSIS DATA SHEET ib Name:"QUUANTERRA MO

i. Code: ITMO

ttrix: (soil/water) SOIL

H._le wt/vol:

$5.00(\mathrm{~g} / \mathrm{mL}) \mathrm{G}$

Contract: $625-01$

Case No.: V53301 SAS No.:

Lab Sample ID: 14533-010

$\because \geqq 1$ :

(low/med) Low

Moisture: not dec.

:olumn: RTX-502.2 ID: 0.530 (mm)

il Extract Volume:

(UI)
Lab File ID: F9979

Date Received: 05/06/97

Date Analyzed: 05/10/97

Dilution Factor: $\quad 1.0$

Soil Aliquot Volume:

(uL)
EPA SAMPLE NO.

NSCEP - 273
CAS NO.

CAs NO.

\begin{tabular}{|c|}
\hline 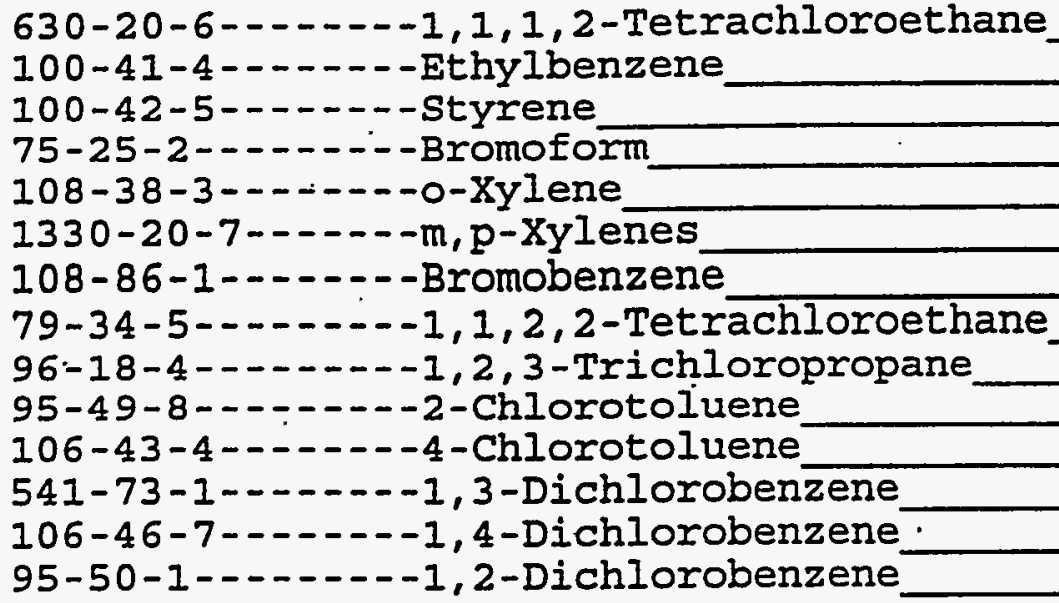 \\
\hline
\end{tabular}

630-20-6------1,1,1,2-Tetrachloroethane

100-42-5-.-.---styrene

75-25-2--.--- Bromoform

108-38-3-..---o-Xylene

108-86-1-...---Bromobenzene

79-34-5 -....-1, 1, 2, 2-Tetrachloroethane

95-49-8-...--2-Chlorotoluene

106-43-4--.--4-Chlorotoluene

541-73-1-..-1,3-Dichlorobenzene

95-50-1-..--1,2-Dichlorobenzene
CONCENTRATION UNITS:

(ug/I or ug/Kg) UG/KG 
Lab Name: ' Ouanterra, MO

Lab code: ITMO Case No.:

Matrix : . (soil/water) sorL

Sample wt/vol: $1.0 \quad(g / \mathrm{ml}) \quad G$

LeveI: (low/med) Iow.

* Moisture: not dec. Compound.
Contract: 625.01

SAS No.:

SDG NO.: V205

Iab sample ID: 14533-009

Iab File ID:

Date Sampleds 04-30-97

Date Analyzed: 05-10-97

Dilution Factor: 1.0

CONCENTRATION DNITS:

(ug/I or ug/Rg) UG/KG

$\mathbf{Q}$ 500 0 LBH (1)

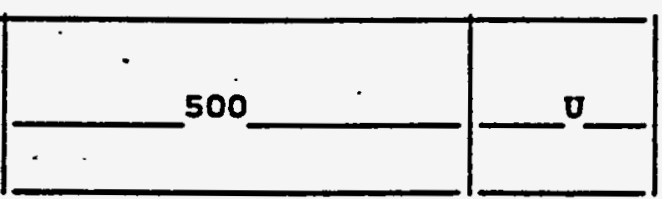

$\mathrm{U}$ :Concentration of analyte is less than the value given:

$X:$ Pattern reasonably matches gasoline

Y:Pattern appears to be multi-peaks, but does not match gasoline

2:Concentration is primarily from a single peak

(1):Low Boiling Hydrocarbon (LBH) is quantitated as if it is Gasoline. 


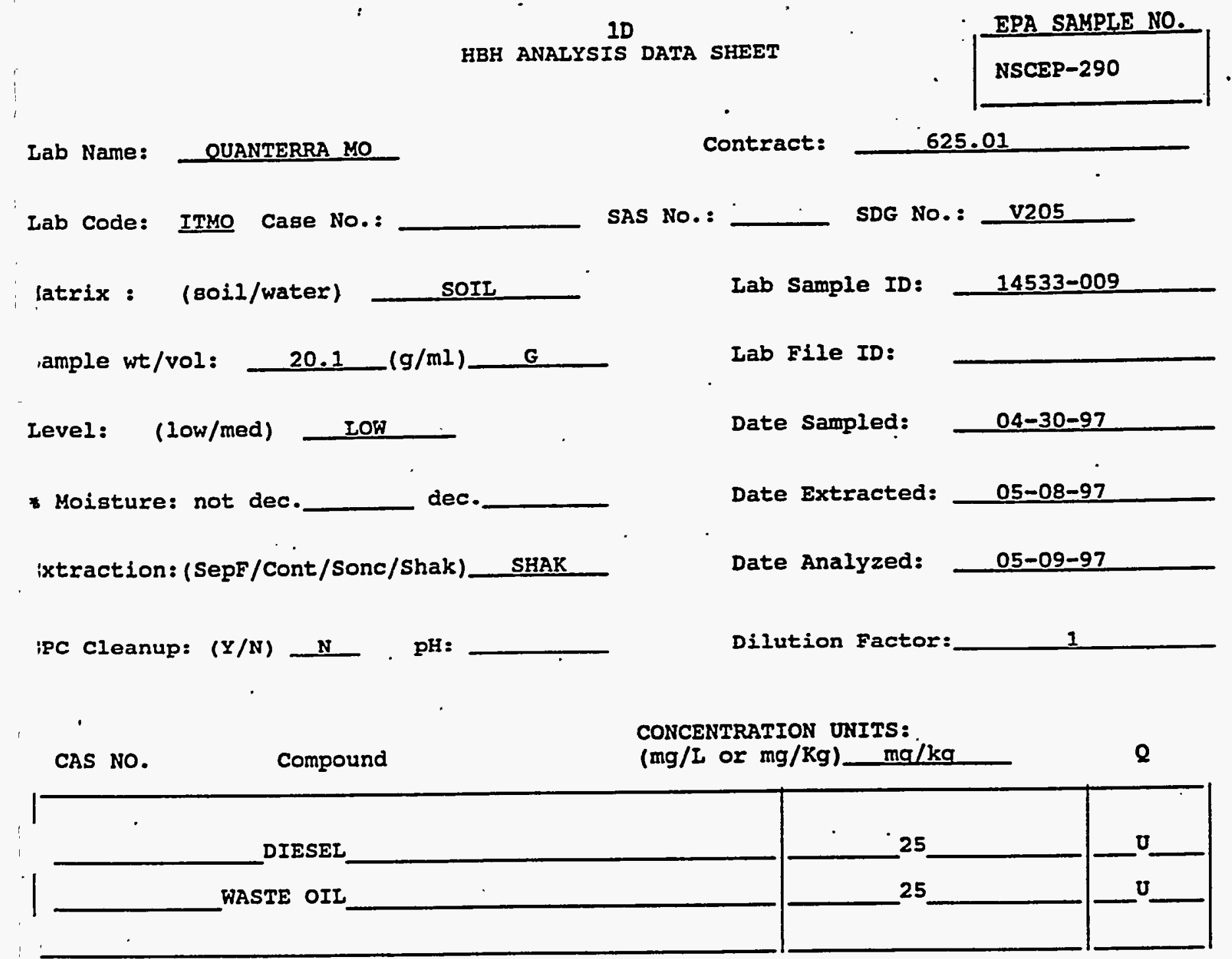

U: Concentration of analyte is less than the value given.

FORM I HBH 
NSCEP -290

Lab Name :--QUUANTERRÄ MO

Lab Codè : ITMO

Case No.: V53301

Matrix: (soil/water) soII

Sample wt/vol:

$1.00(\mathrm{~g} / \mathrm{mL}) \mathrm{G}$

Level: (low/med) LOW

$\%$ Moisture: not dec.

GC Column: RTX-502.2 ID: 0.530 (mm)

Soil Extract Volume:

(uL)
Contract: $625-01$

SAS No.:

SDG No.: V205

Lab Sample ID: 14533-009

Lab File ID: F0007

Date Received: 05/06/97

Date Analyzed: 05/12/97

Dilution Factor: $\quad 1.0$

Soil Aliquot Volume:

(uI)

CAS NO.

COMPOUND

CONCENTRATION UNITS:

(ug/L or $\mathrm{ug} / \mathrm{Kg}$ ) UG/KG

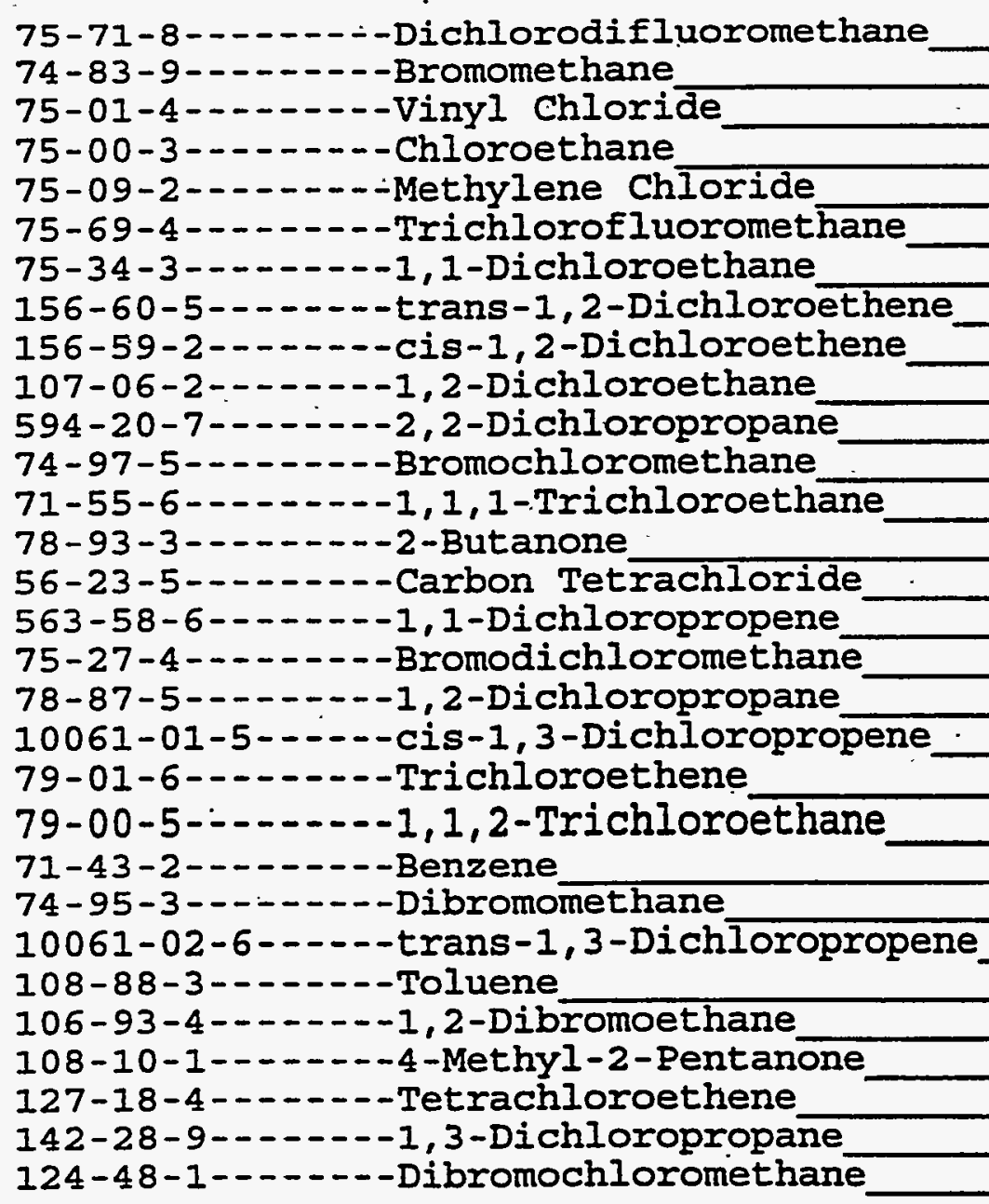

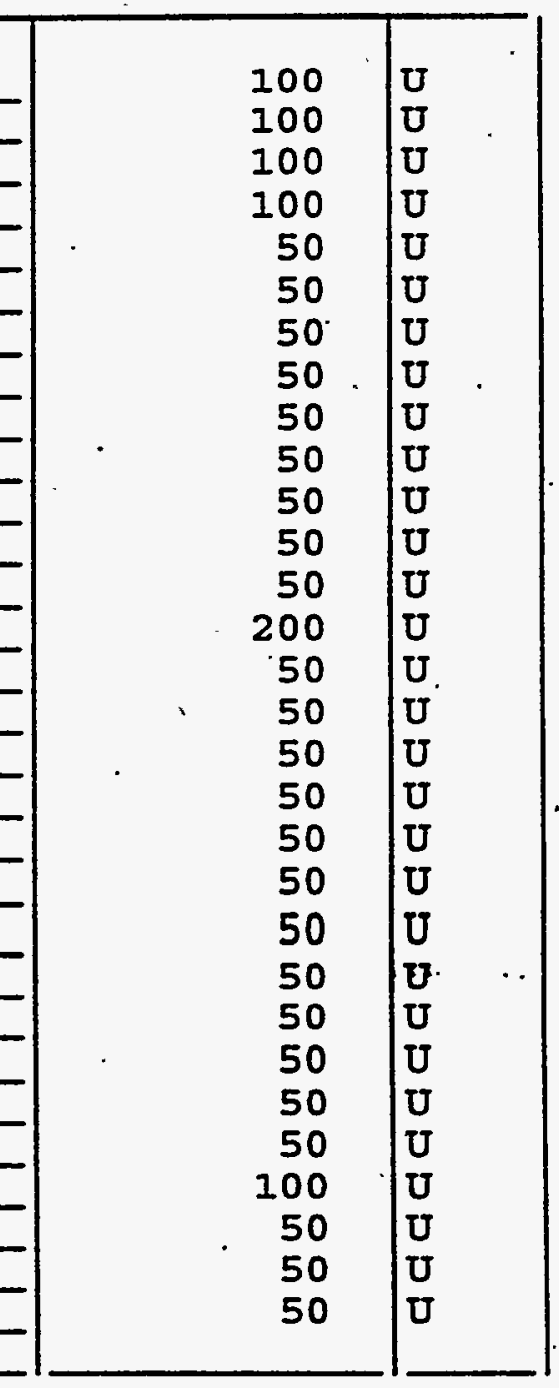


JaO Name:" QUUANTERRA MÓ

o Code: ITMO

Case No.: V53301 SAS No::

Contract: $625-01$ latrix: (soil/water) SOIL

inple wt/vol:

$1.00(\mathrm{~g} / \mathrm{mL}) \mathrm{G}$

,pvel: (low/med) LOW

Moisture: not dec.

"Column: RTX-502.2. ID: 0.530 (mm)

oil Extract Volume:

(uL)
NSCEP-290

SDG NO.: V205

Lab Sample ID: 14533-009

Lab File ID: F0007

Date Received: 05/06/97

Date Analyzed: 05/12/97

Dilution Factor: $\quad 1.0$

Soil Aliquot Volume:

(UL) CONCENTRATION UNITS :

CAS NO.

COMPOUND (ug/L or $\mathrm{ug} / \mathrm{Kg}$ ) UG/KG

Q

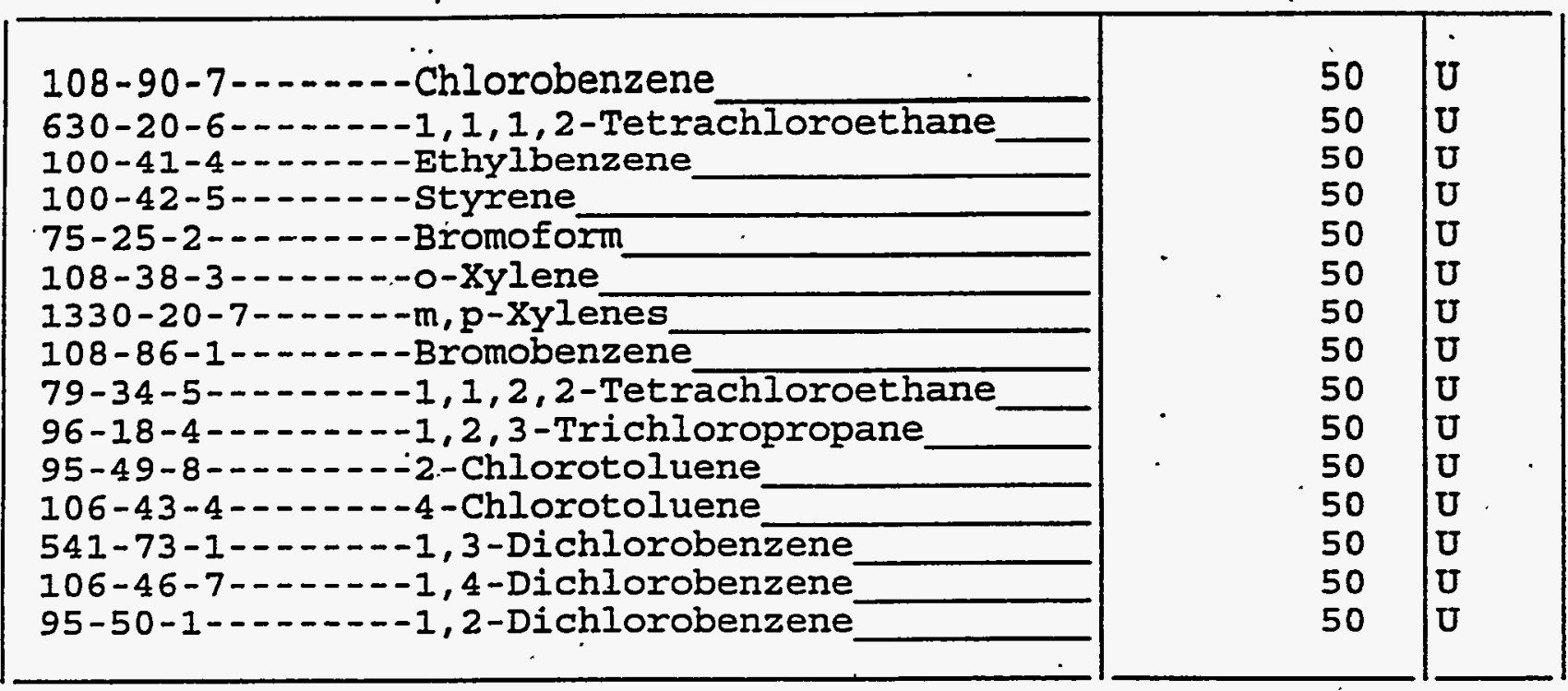


1D

LBH ANALYSIS DATA SHEBT
EPA SAMPLE NO.

NSCRP-297
Lab Name:

Lab Code:

Matr $1 x$

Sample wt/vol:

sort,

Level: (Low/med) ION

s Moisture: not dec.

CAS NO.
Contract: 625.01

SDC No.: Y205

SAS No.?

Iab Sample ID: 14533-008

Iab File ID:

Date Sampled: 04-30-97

Date Analyzed: 05-10-97

Dilution Pactor: 1.0
CONCENITATIOH UNITS:

(ug/L or ug/kg) UG/RG
Q

IBH (1)

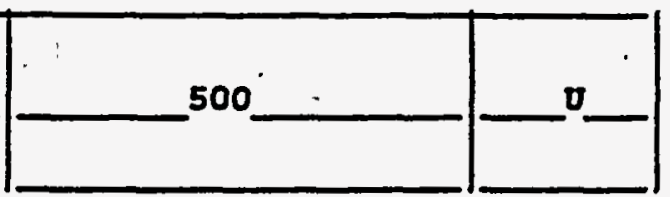

0:Concentration of analyte is less than the value given. X:Pattern reasonably matches gasoline Y:Pattern appears to be multi-peaks, but does not match gasoline Z:Concentration is primarily from a eingle peak

(1):Low Boiling Hydrocarbon (LBH) is quantitated as if it is Gasoline.

FORY I IBE 
$3 D$

HBH ANALYSIS DATA SHEET
EPA SAMPIE NO.

NSCEP-297
Lab Name: OUANTERRA MO

Lab Code: ITMO Case No::

atrix : (soil/water)

SOIL

ample wt/vol:

$20.0(\mathrm{~g} / \mathrm{ml})$

G

Level: (low/med)

LOW

* Moisture: not dec. dec.

xtraction : (SepF/Cont/Sonc/Shak) - SHAK.

PC Cleanup: $(Y / N) \perp N$ pH:

CAS NO.

Compound

CONCENTRATION UNITS:

$(\mathrm{mg} / \mathrm{L}$ or $\mathrm{mg} / \mathrm{kg}) \ldots \mathrm{ma} / \mathrm{kg}$

$\mathbf{Q}$

${ }^{25}-{ }^{25}-{ }^{0}-$

U: Concentration of analyte is less than the value given. 
VOLATILE ORGANICS ANALYSIS DATA SHEET

Lab Name: "Q QUANTERRA MO

Lab Code: ITMO

Case No.: V53301

Matrix: (soil/water) soII.

Sample wt/vol: $\quad 1.00(\mathrm{~g} / \mathrm{mL}) \mathrm{G}$

Level: (low/med) LOW

\% Moisture: not dec.

GC Column: RTX-502.2 ID: 0.530 (mm)

Soil Extract Volume:

(uL)
Contract: 625-01

SAS NO.:

NSCEP-297

SDG No.: V205

Lab Sample ID: 14533-008

Lab File ID: F0006

Date Received: 05/06/97

Date Analyzed: 05/12/97

Dilution Factor: $\quad 1.0$

Soil Aliquot Volume:

(uL.

CONCENTRATION UNITS:

CAS NO. COMPOUND (ug/L or $u g / \mathrm{Kg}$ ) UG/KG

$Q$

100

100

100

100

50

50

50

50

50

50

50

50

50

200

50

50

50

50

50

50

50

50

50

50

50

50

100

50

50

50 
zb Name:" QUUANTERRA MO.

z Code: ITMO

Case No.: V53301 ztrix: (soil/water) soIL

Auple wt/vol:

$1.00(\mathrm{~g} / \mathrm{mL}) \mathrm{G}$

e el: (Low/med) LOW

Moisture: not dec.

ב Column: RTX-502.2 ID: 0.530 (mm)

गil Extract Volume:

(uI)
Contract: $625-01$
NSCEP-297

SDG No.: V205

Iab Sample ID: 14533-008

Lab File ID: F0006

Date Received: 05/06/97

Date Analyzed: 05/12/97

Dilution Factor: $\quad 1.0$

Soil Aliquot Volume:

(UL)

CAS NO.

COMPOUND

CONCENTRATION UNITS:

(ug/L or $\mathrm{ug} / \mathrm{Kg}$ ) UG/KG

\begin{tabular}{|c|c|c|}
\hline 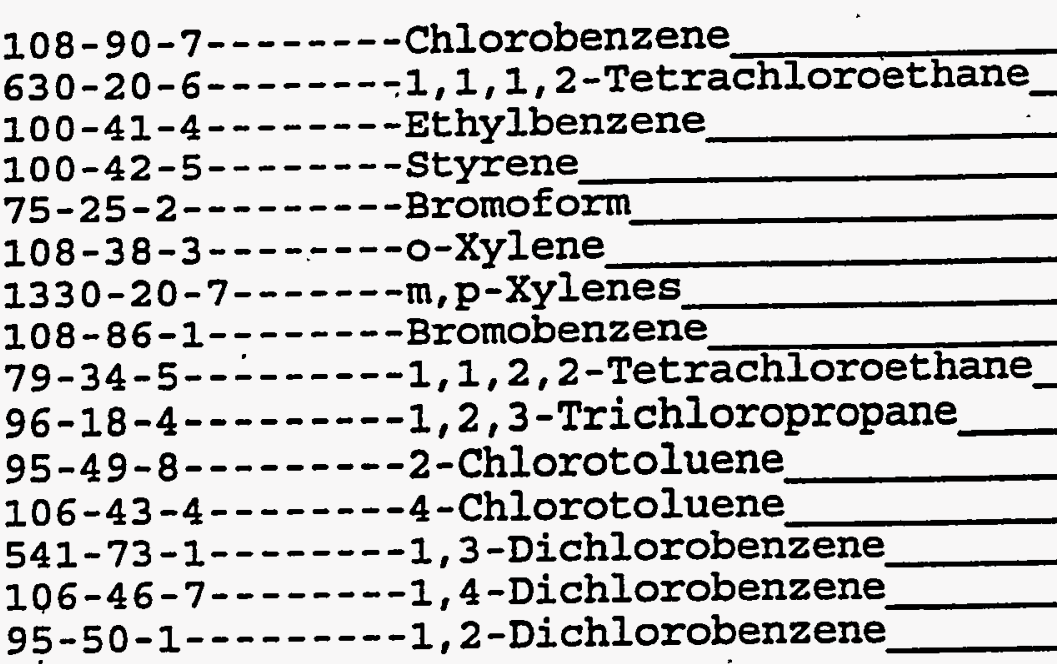 & $\begin{array}{l}50 \\
50 \\
50 \\
50 \\
50 \\
50 \\
50 \\
50 \\
50 \\
50 \\
50 \\
50 \\
50 \\
50 \\
50\end{array}$ & $\begin{array}{l}U \\
U \\
U \\
U \\
U \\
U \\
U \\
U \\
U \\
U \\
U \\
U \\
U \\
U \\
U\end{array}$ \\
\hline
\end{tabular}




Iab Name: $\frac{\text { Ouanterra,MO }}{\text { ITMO Case No.: }}$
Lab Code:
Matrix : (Boil/water) soIr
Sample wt/vol: $1.0 \quad(g / m I) \quad G$

Level: (low/med) IOW

\& Moisture: not dec.

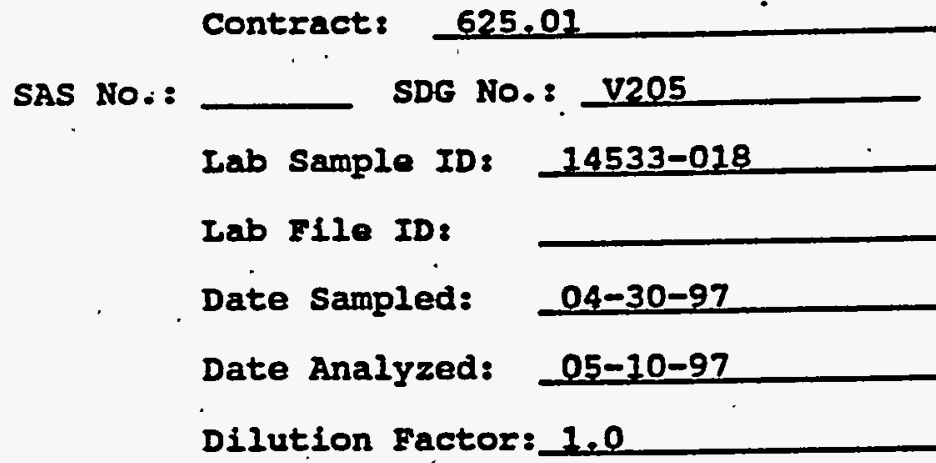

CONCENTRATION UNITS:
CAS NO.

Compōund $(\mathrm{ug} / \mathrm{L}$ or $\mathrm{ug} / \mathrm{Kg})$ UG/RG

Q.

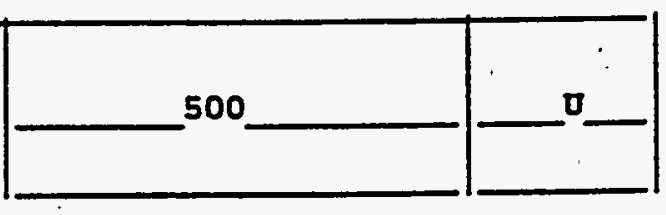

U:Concentration of analyte is less than the value given. $X:$ Pattern reasonably matches gasoline Y:-Pattern appears to be multi-peaks, but does not match gasoline $\mathrm{z}$ :Concentration is primarily from a single peak

(1) :Low Boiling Hydrocarbon (LBH) is quantitated as if it is Gasoline. 


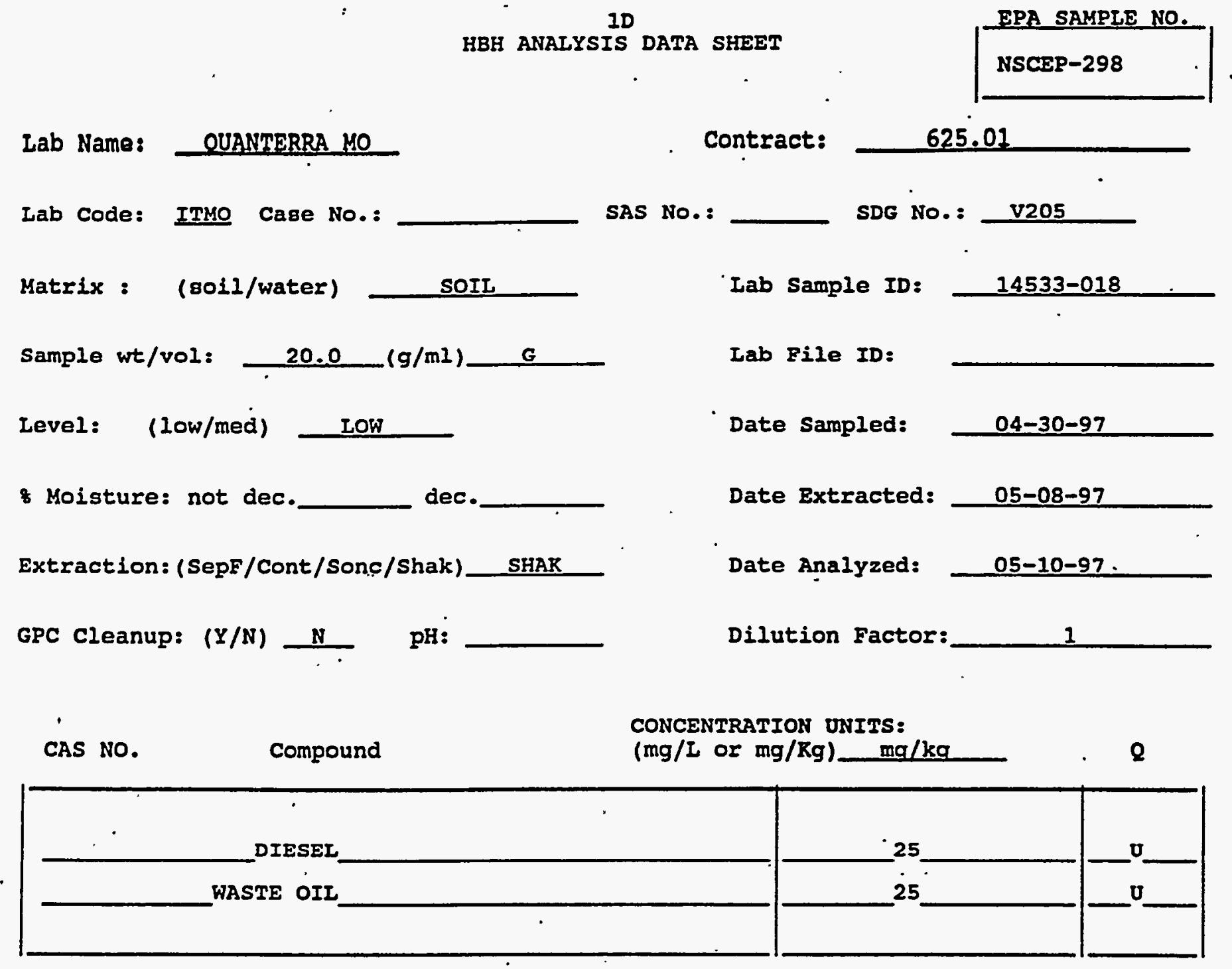

U: Concentration of analyte is less than the value given. 
Lab Name:" QUAATTERRÁ MO

Lab code: ITMO

Case No.: V53301

Matrix: (soil/water) SOIL

Sample wt/vol:

$5.00(\mathrm{~g} / \mathrm{mL}) \mathrm{G}$

Level: (Iow/med) LOW

$\because$ Moisture: not dec.

GC Column: RTX-502.2 ID: 0.530 (mm)

Soil Extract Volume:

(UL)
Contract: $625-01$

SAS NO.:

SDG No.: V205

NSCEP-298

Lab Sample ID: 14533-018

Lab File ID: F9987

Date Received: 05/06/97

Date. Analyzed: 05/10/97

Dilution Factor: $\quad 1.0$

Soil Aliquot Volume:

(UL)

CONCENTRATION UNITS:

CAS NO.

COMPOUND

(ug/L or ug/Kg) UG/KG

\begin{tabular}{|c|}
\hline 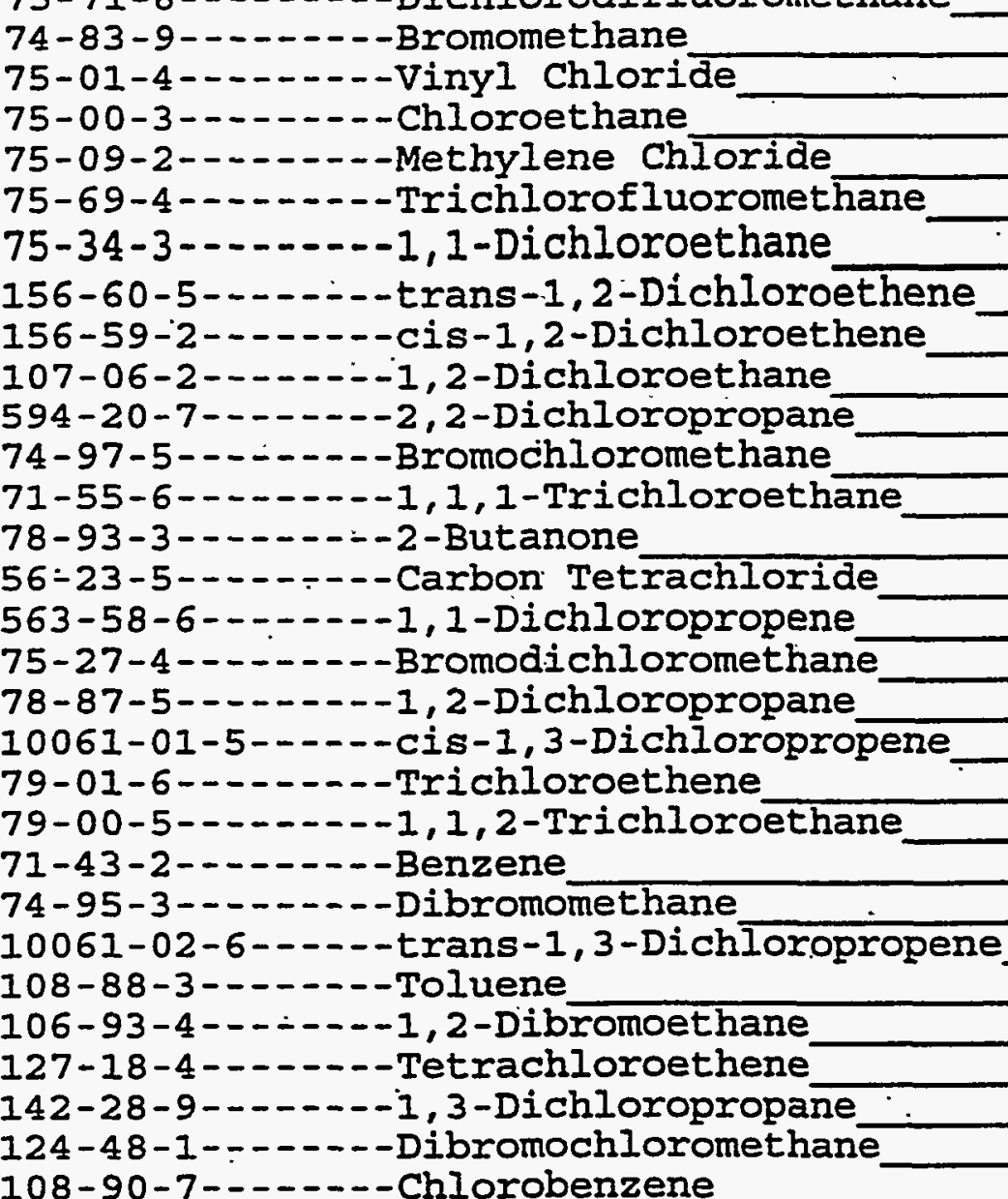 \\
\hline
\end{tabular}


$1 A$

VOLATILE ORGANICS ANALYSIS DATA SHEET
EPA SAMPLE NO.

NSCEP -298
aD Name:"Q̄UUANTERRA MO"

, Code: ITMO

Case No.: V53301 SOII

$5.00(\mathrm{~g} / \mathrm{mL}) \mathrm{G}$

nple wt/vol:

prel: (low/med) LOW

Moisture: not dec.

Column: RTX-502.2 ID: 0.530 (mm)

oil Extract Volume:

(UI)
Contract: $625-01$

SAS NO.:
SDG NO:: V205

Lab Sample ID: - 14533-018

Lab File ID: F9987

Date Received: 05/06/97

Date Analyzed: 05/10/97

Dilution Factor: $\quad 1.0$

Soil Aliquot Volume:

(uL)

CAS NO.

COMPOUND

CONCENTRATION UNITS :

( $\mathrm{ug} / \mathrm{L}$ or $\mathrm{ug} / \mathrm{Kg}$ ) UG/KG

Q

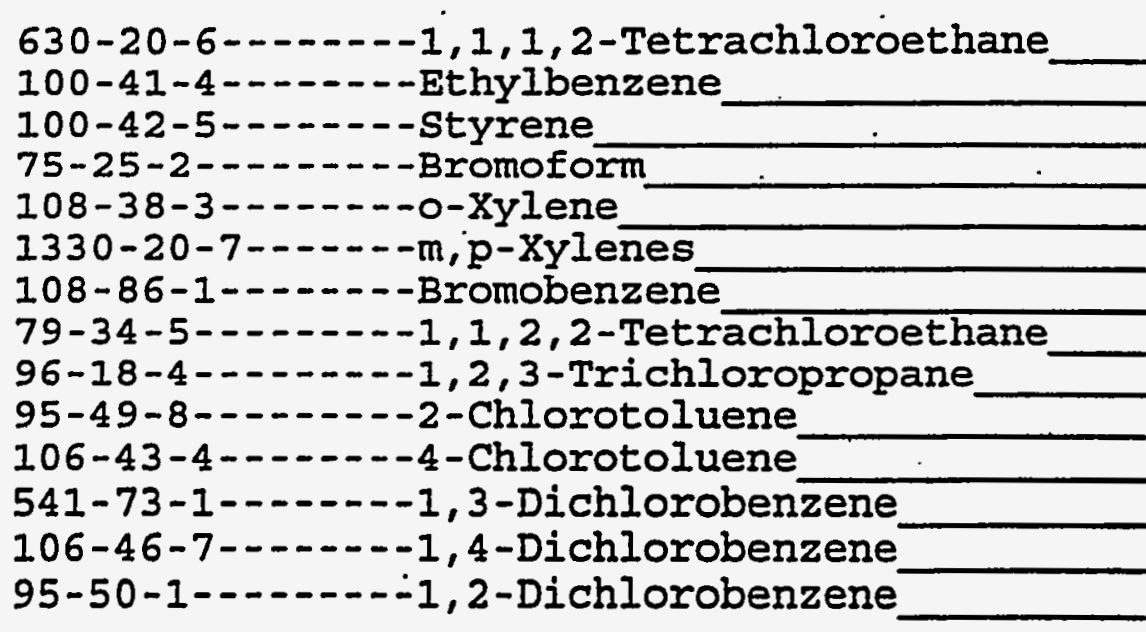


EPA SAMPLE NO. NSCEP-304
Lab Name: $\frac{\text { Quanterra,MO }}{\text { Lab Code: ITMO Case NO.: }}$
ITO

Hatrix : (goil/water) soIf

Sample wt/vol: $1.0 \quad(g / \mathrm{ml})$

$\mathbf{G}$

Level: (low/med) IOW

* Moisture: not dec.

(1)

Compound

CAS NO.

LBE $(1) \ldots$

contract

625.01

SAS NO.: SDG No.: $\mathbf{V 2 0 5}$

Iab Sample ID: -14533-012

Lab File ID:

Date Sampled:

$04-30-97$

Date Analyzed: 05-10-97

Dilution Factor: 1.0
CONCENTRATION UNITS:

(ug/I or ug/Rg) UG/KG

$\mathbf{Q}$

$\mathrm{U}$ :Concentration of analyte 18 less than the value given. $X:$ Pattern reasonably matches gasoline Y:Pattern appears to be multi-peaks, but does not match gasoline z:Concentration is primarily from a single peak 500

(1):Low Boiling Hydrocarbon (LBE) is quantitated as if it is Gasoline. 
1D

HBH ANALYSIS DATA SHEET
EPA SAMPLE NO.

NSCEP-304

Lab Name: OUANTERRA MO

Contract:

625.01

Lab code: ITMO Case No.:

SAS NO.:

SDG NO.: V205

Matrix : (soil/water)

SOII

Lab Sample ID:

$14533-012$

Sample wt/vol: $20.1(\mathrm{~g} / \mathrm{ml})$

$G$

Level: (low/med)

LOW

Lab File ID:

Date Sampled:

$04-30-97$

\&oisture: not dec. dec.

Date Extracted:

05-08-97

Extraction: (SepF/Cont/Sonc/Shak)

SHAK

Date Analyzed:

$05-09-97$

GPC Cleanup: $(Y / N)+\mathrm{N} \quad \mathrm{pH}:$

Dilution Factor:

1

CONCENTRATION UNITS:

CAS NO.

Compound

$(\mathrm{mg} / \mathrm{L}$ or $\mathrm{mg} / \mathrm{kg}$ )_ $\mathrm{mg} / \mathrm{kg}$

$\mathbf{2}$

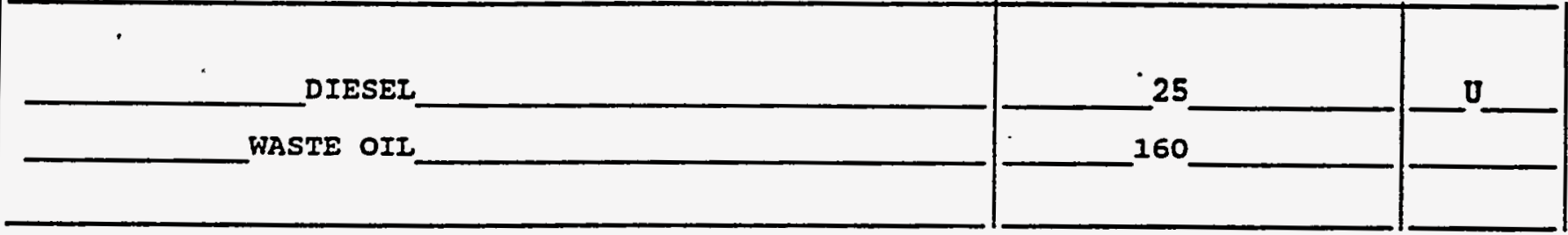

U: Concentration of analyte is.less than the value given. 
Lab Name:" Q̈UANTERRA MO

Irab Code: ITMO

Case No.: V53301

Matrix: (soil/water) soIL

Sample wt/vol: $\quad 1.00(\mathrm{~g} / \mathrm{mL}) \mathrm{G}$

Level: (low/med) Low

\% Moisture: not dec.

GC Column: RTX-502.2 ID: 0.530 (mm)

Soil Extract Volume:

(uI)
Contract: $625-01$

SAS NO: :
EPA SAMPIE NO

NSCEP -304

SDG No.: V205

Lab Sample ID: 14533-012 .

Lab File ID: F0008

Date Received: 05/06/97

Date Analyzed: 05/12/97

Dilution Factor: 1.0

Soil Aliquot Volume:

(uI)

CONCENTRATION UNITS :

CAS NO.

COMPOUND

(ug/L or ug/Kg) UG/KG

$Q$

\begin{tabular}{|c|}
\hline 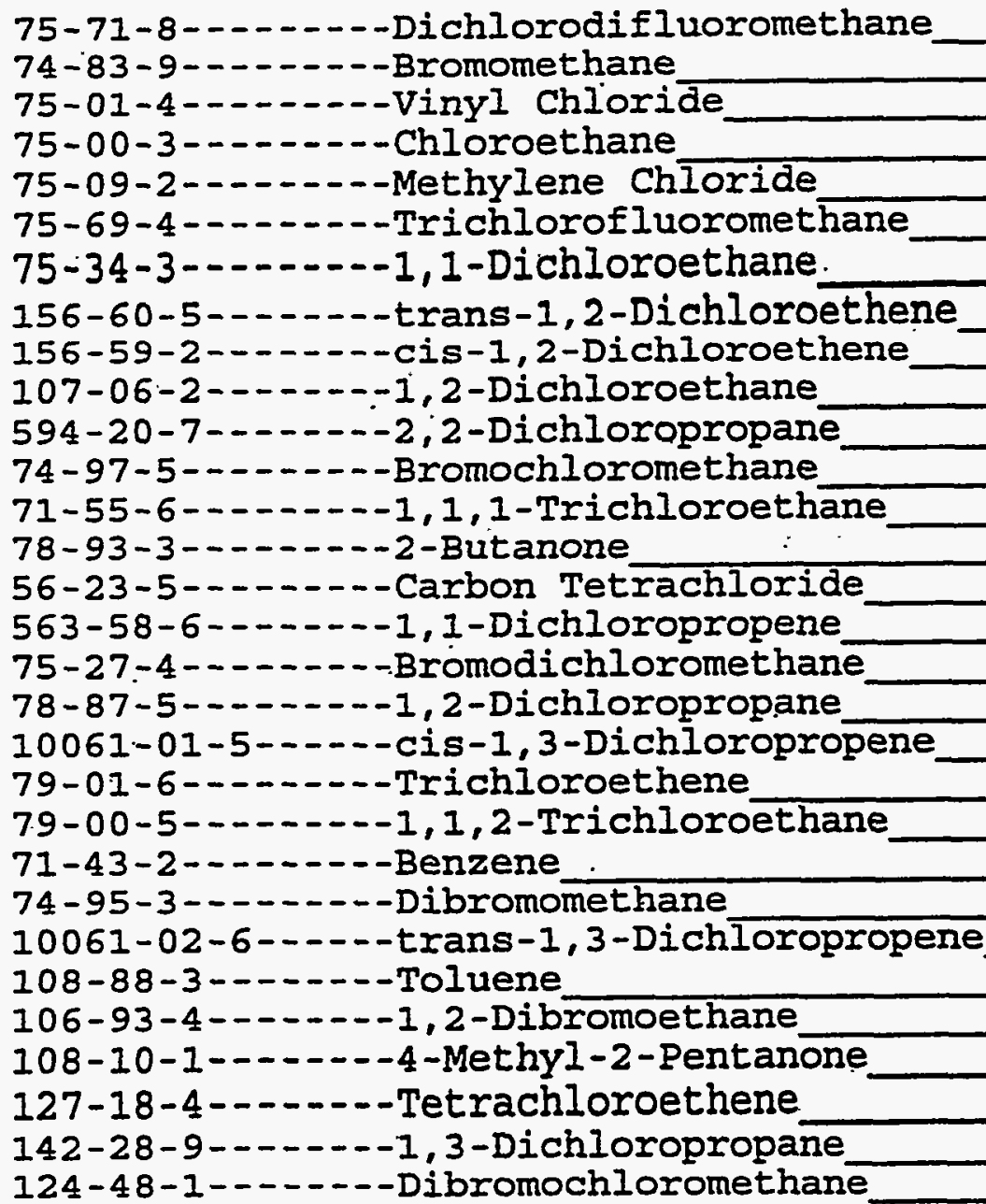 \\
\hline
\end{tabular}

100

100

100

100

50

50

50

50

50

50

50

50

50

200

50

50

$50^{\circ}$

50

50

50

50

50

50

50

50

50

100

50

50

50 
IA

VOLATILE ÓRGANICS ANALYSIS DATA SHEET
EPA SAMPLE NO.

NSCEP-304 b Name:" QUUANTERRA MO

I Code: ITMO r. lie wt/vol:

$1.00(\mathrm{~g} / \mathrm{mL}) \mathrm{G}$

i il: (low/med) LOW

Moisture: not dec.

lolumn: RTX-502.2 ID: $0.530(\mathrm{~mm})$

il Extract Volume:

(uL)
Contract : $625-01$

SAS NO.:
SDG No.: V205

Lab Sample ID: 14533-012

Iab File ID: F0008

Date Received: 05/06/97

Date Analyzed: 05/12/97

Dilution Factor: $\quad 1.0$

Soil Aliquot Volume:

(UL)

CONCENTRATION UNITS:

(ug/L or $u g / K g$ ) UG/KG

$\mathbf{Q}$

CAS NO.

COMPOUND

\begin{tabular}{|ll|l|}
\hline & 50 & $U$ \\
& 50 & $U$ \\
& 50 & $U$ \\
& 50 & $U$ \\
& 50 & $U$ \\
& 50 & $U$ \\
& 50 & $U$ \\
& 50 & $U$ \\
& 50 & $U$ \\
& 50 & $U$ \\
& 50 & $U$ \\
& 50 & $U$ \\
& 50 & $U$ \\
& 50 & $U$ \\
& 50 & $U$ \\
& \\
\hline
\end{tabular}




\begin{abstract}
Lab Name: Quanterra,ko.
Lab code: ITMO Case No.:

Hatrix : (goil/water) soII

Sample wt/vol: $1.0 \quad(9 / \mathrm{ml})$ G

Ievel: (Iow/med) IOW

* Hoisture: not dec.
\end{abstract}

CAS NO.

Compound

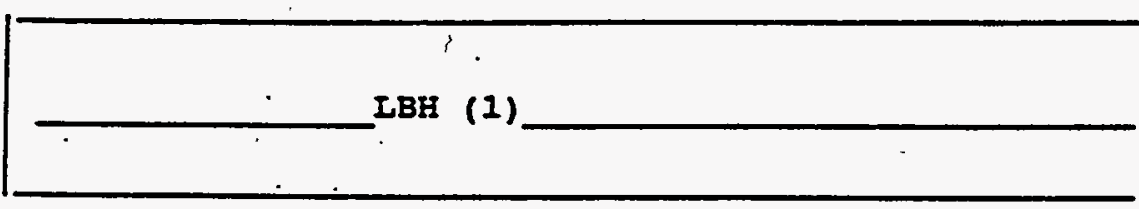

Contract: 625.01 SAS NO.:

SDE NO.: V205

Iab Sample ID: · 14533-011

Lab File ID:

Date Sampled: 04-30-97

Date Analyzed: 05-12-97

Dilution Factor: 1.0
CONCENTRATION URITS: (ug/L or ug/Kg) UG/KG

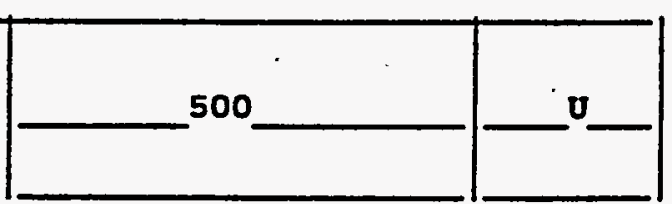

U:Concentration of analyte is less than the value given. $X:$ Pattern reasonably matches gasoline

Y:Pattern appears to be multi-peaks, but does not match gasolino

z:Concentration is primarily from a single peak

(1): Low Boiling Hydrocarbon (IBE) is quantitated as if it is Gasoline. 
EPA SAMPLE NO. NSCEP-305
Lab Name: OUANTERRA KO

Lab Code: ITMO Case NO.:

Matrix : (soil/water)

soII

Sample wt/rol: 20.1 G

Level: (low/med) LOW

\& Moisture: not dec. dec. SAS NO.:

Extraction: (SepF/Cont/Sonc/Shak) ___ SHAK GPC Cleanup: $(\mathrm{Y} / \mathrm{N}) \stackrel{\mathrm{N}}{\mathrm{NH}}$ 1 CONCENTRATION UNITS: CAS NO. Compound (mg/L or $\mathrm{mg} / \mathrm{Kg}$ ) $\mathrm{mg} / \mathrm{kg}$

$\square^{25}+{ }^{0}-$

$U$ : Concentration of analyte is less than the value given. 
Lab Name:“Q̈UANTERRA MO

Lab Code: ITMO

Matrix: (soil/water) SOIL

Sample wt/vol:

$5.00(\dot{g} / \mathrm{mL}) \quad G$

Level: (low/med) LOW

\% Moisture: not dec.

GC Column: RTX-502.2 ID: $0.530(\mathrm{~mm})$

Soil Extract Volume:

(uI)
Contract: $625-01$

SAS NO.:
NSCEP-305

SDG NO.: V205

Iab Sample ID: 14533-011

Lab File ID: F9980

Date Received: $05 / 06 / 97$

Date Analyzed: 05/10/97

Dilution Factor: $\quad 1.0$

Soil Aliquot Volume: (uL

CONCENTRATION UNITS :

CAS NO.

COMPOUND

(ug/I or $\mathrm{ug} / \mathrm{Kg}$ ) UG/KG

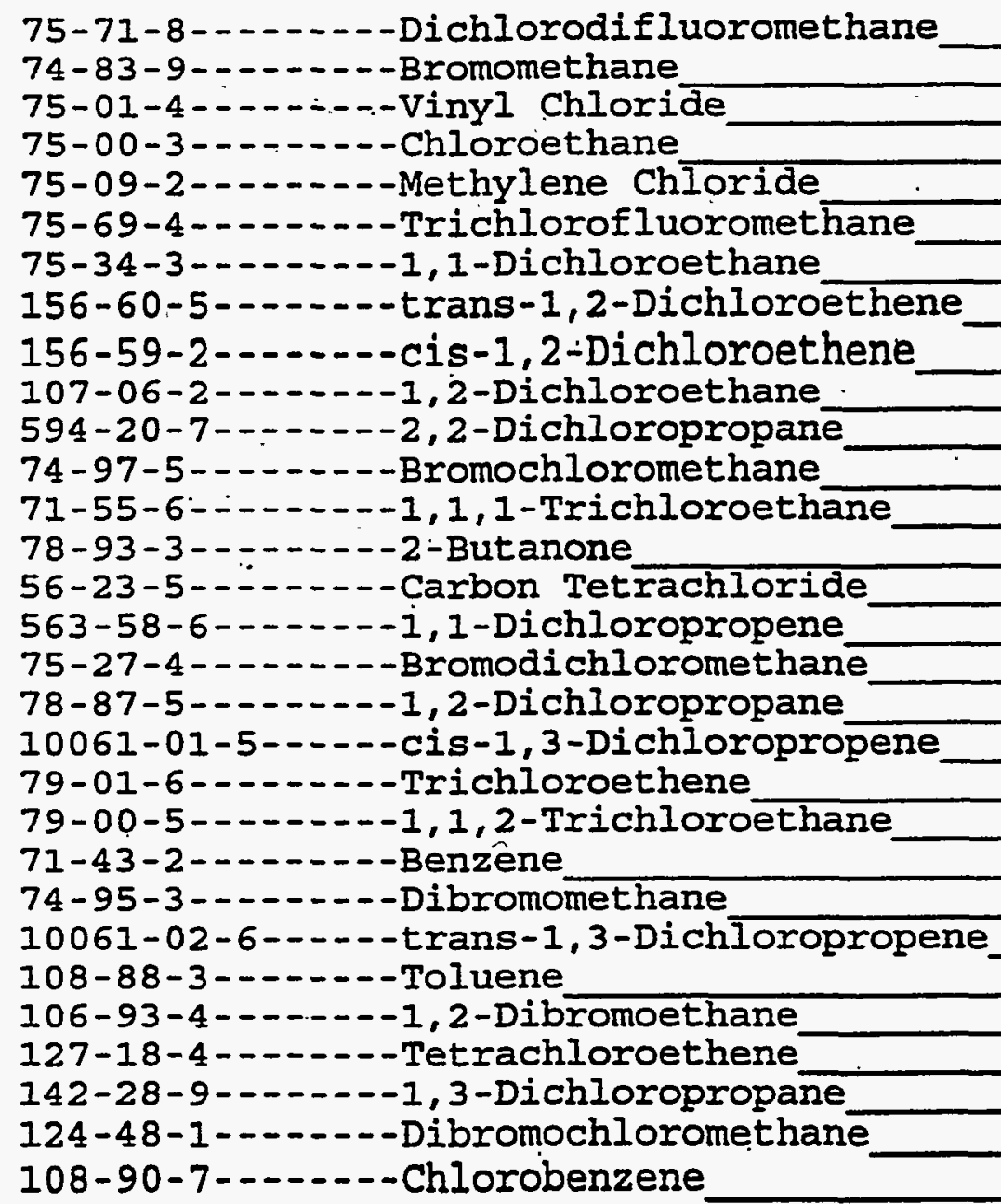


ib Name:" QUUANTERRA MO

i Code : ITMO

Case No.: V53301

atrix: (soil/water) soIL

1...ple wt/vol:

$5.00(\mathrm{~g} / \mathrm{mL}) \mathrm{G}$

$\geq$ I: (low/med) LOW

Woisture: not dec.

Column: RTX-502.2 ID: 0.530 (mm)

sil Extract Volume:

(UI)
Contract: $625-01$
EPA SAMPLE NO.

NSCEP -305

SDG NO.: V205

Lab Sample ID: 14533-011

Lab File ID: F9980

Date Received: 05/06/97

Date Analyzed: 05/10/97

Dilution Factor: $\quad 1.0$

Soil Aliquot Volume:

(uL)

CAS NO. COMPOUND CONCENTRATION UNITS: (ug/I or $\mathrm{ug} / \mathrm{Kg}$ ) UG/KG

Q

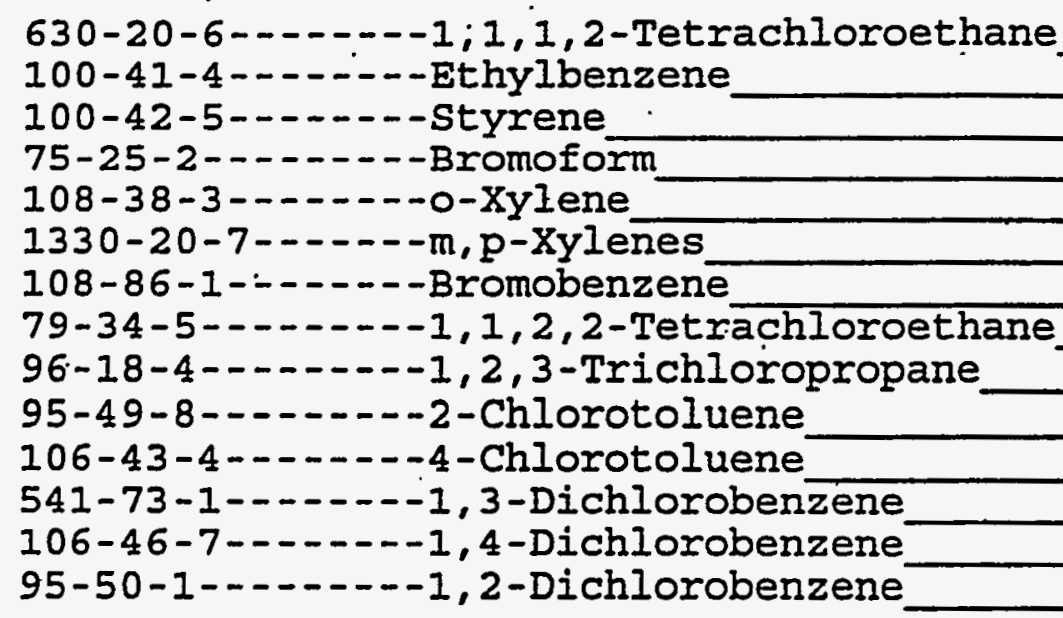


Iab Name:

Lab Code:

- Quantersa,MO

Matrix : (soil/water) soIL

Sample wt/vol:

$1.0 \quad(g / \mathrm{ml})$

G

Level: (low/med) IOW

* Moisture: not dec.

CAS NO. Compound IBH (1)

Contract: 625,01

SAS NO.:

SDG HO.: V205

Iab Sample ID: 14533-019

Lab File ID:

Date Sampled:

$04-30-97$

Date Analyzed: 05-12-97

Dilution Factor: 1.0
CONCENTRATION UNITS:

(ug/L or ug/Kg) uG/RG

$\mathbf{Q}$

\begin{tabular}{|l}
\hline $\operatorname{LBH}(1) \_\ldots$ \\
$\therefore$
\end{tabular}

U:Concentration of analyte is less than the value given.

$X:$ Pattern reasonably matches gasoline

Y: Pattern appears to be multi-peaks, but does not match gasoline

$z$ :Concentration is primarily from a single peak

(1) Low Boiling Hydrocarbon (IBH) is quantitated as if it is Gasoline. 
HBH ANALYSIS DATA SHEET
EPA SAMPLE NO. NSCEP-310
Lab Name:

OUANTERRA MO

Lab Code: ITMO Case No.:

Matrix : (Boil/water)

sample wt/vol: $20.1 \cdot(\mathrm{g} / \mathrm{ml})$

G

Level: (low/med) Low

t Moisture: not dec. dec.

3xtraction: (SepF/Cont/Sonc/Shak)

SHAK

aPC cleanup: $(Y / N)$ N $\mathrm{N}$.

H:

$+2$
SAS NO.:

Contract:

625.01

SDG No.: V205

Iab Sample ID: $14533-019$

Iab R2le ID:

Date Sampled:

$04-30-97$

Date Extracted:

Date Analyzed:

Dilution Factor:

1

CONCENTRATION ONITS:

CAS NO.

Compound

(mg/L or $\mathrm{mg} / \mathrm{Kg}$ )

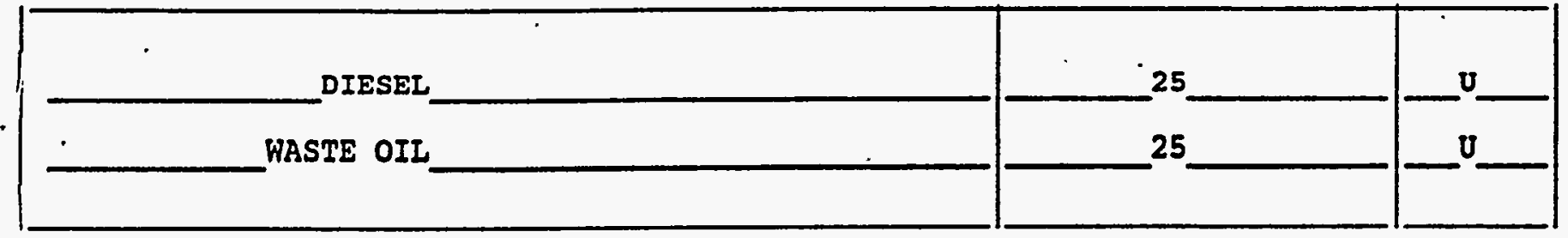

$U$ : Concentration of analyte is less than the value given. 
VOLATILE ORGANICS ANALYSIS DATA SHEET

Lab Name:" QUUANTERRA' MO

Lab Code: ITMO

Case No.: V53301

Matrix: (soil/water) SOII

Sample wt/vol: $\quad 1.00(\mathrm{~g} / \mathrm{mL})$ G

Level: (low/med) LOW

$\%$ Moisture: not dec.

GC Column: RTX-502.2 ID: 0.530 (mm)

Soil Extract Volume:

CAS NO.

COMPOUND
Contract : 625-01

SAS NO.:

NSCEP -310

SDG NO.: V205

Lab Sample ID: 14533-019

Lab File ID: Fo019

Date Received: $05 / 06 / 97$

Date Analyzed: 05/13/97.

Dilution Factor: $\quad 1.0$

Soil Aliquot Volume:

(ui)

CONCENTRATION UNITS:

(ug/L or $\mathrm{ug} / \mathrm{Kg}$ ) UG/KG

\begin{tabular}{r|l|}
100 & $U$ \\
100 & $U$ \\
100 & $U$ \\
100 & $U$ \\
50 & $U$ \\
50 & $U$ \\
50 & $U$ \\
50 & $U$ \\
50 & $U$ \\
50 & $U$ \\
50 & $U$ \\
50 & $U$ \\
50 & $U$ \\
200 & $U$ \\
50 & $U$ \\
50 & $U$ \\
50 & $U$ \\
50 & $U$ \\
50 & $U$ \\
50 & $U$ \\
50 & $U$ \\
50 & $U$ \\
50 & $U$ \\
50 & $U$ \\
50 & $U$ \\
50 & $U$ \\
100 & $U$ \\
50 & $U$ \\
50 & $U$ \\
50 & $U$ \\
& \\
& \\
50
\end{tabular}

75-7I-8--...-Dichlorodifluoromethane

74-83-9--..---Bromomethane

75-01-4-...-.--Vinyl Chloride

75-00-3-------Chloroethane

75-09-2....-.-Methylene Chloride

75-69-4-..--.-Trichlorofluoromethane

75-34-3--.-.-1,1-Dichloroethane

156-60-5------trans-1,2-Dichloroethene

156-59-2-.....-Cis-1, 2-Dichloroethene

107-06-2------1,2-Dichloroethane

594-20-7------2,2-Dichloropropane

74-97-5--.----Bromochloromethane

71-55-6-..-- 1, 1, 1-Trichloroethane

78-93-3--.----2-Butanone

56-23-5--------Carbon Tetrachloride

563-58-6----1, 1-Dichloropropene

75-27-4-------Bromodichloromethane

78-87-5------1,2-Dichloropropane

10061-01-5-----cis-1, 3-Dichloropropene

79-01-6-...--. Trichloroethene

79-00-5-..--- 1, 1,2-Trichloroethane

$71-43-2-\ldots .---$ Benzene

74-95-3-..---Dibromomethane

10061-02-6-.---trans-1,3-Dichloropropene

108-88-3-.---- Toluene

106-93-4--.--1,2-Dibromoethane

108-10-1-----4-Methyl-2-Pentanone

127-18-4-..-.-Tetrachloroethene

142-28-9-...-1, 3-Dichloropropane

124-48-1-...--Dibromochloromethane 
to Name:"QUUANTERRA MO"

Code: ITMO

Case No.: V53301

Contract: $625-01$
NSCEP-310 ttrix: (soil/water) SOII

। 'ole wt/vol:

$1.00(\mathrm{~g} / \mathrm{mL}) \mathrm{G}$

"rel: $\quad$ (low/med) LOW

woisture: not dec.

: Folumn: RTX-502.2 ID: 0.530 (mm)

il Extract Volume:

(uI)
SAS NO.:

.SDG No.: V205
Lab Sample ID: 14533-019

Lab File ID: F0019

Date Received: 05/06/97

Date Analyzed: 05/13/97

Dilution Factor: $\quad 1.0$

Soil Aliquot Volume:

(UL)

CAS NO.

COMPOUND CONCENTRATION UNITS:

\begin{tabular}{|c|c|c|}
\hline 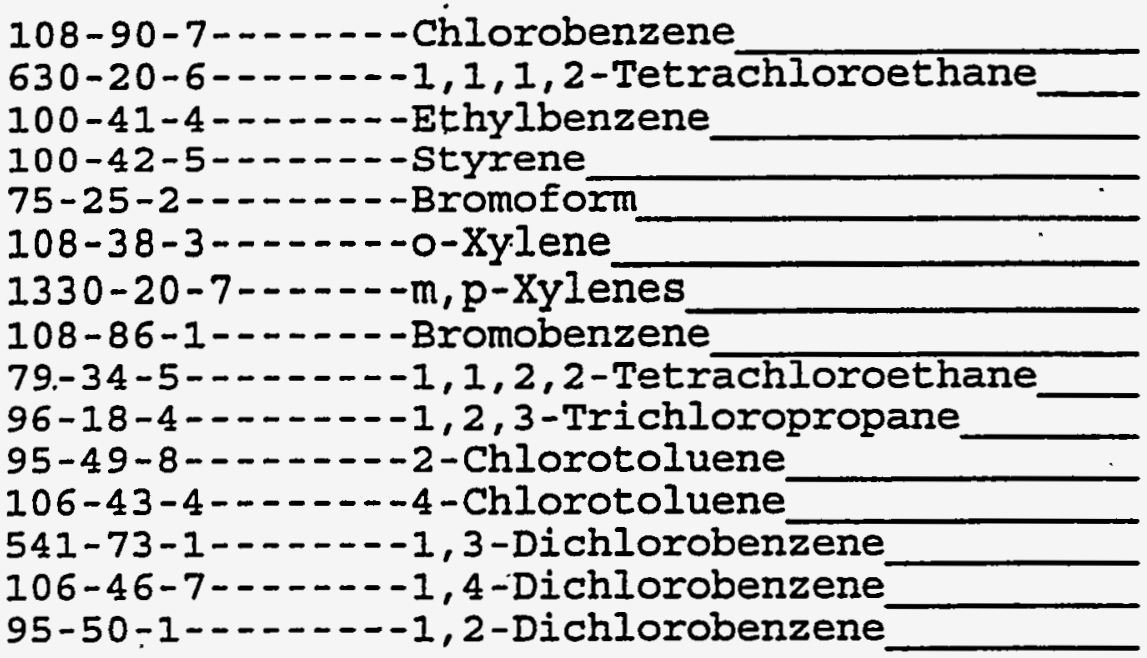 & $\begin{array}{l}50 \\
50 \\
50 \\
50 \\
50 \\
50 \\
50 \\
50 \\
50 \\
50 \\
50 \\
50 \\
50 \\
50 \\
50\end{array}$ & $\begin{array}{l}U \\
U \\
U \\
U \\
U \\
U \\
U \\
U \\
U \\
U \\
U \\
U \\
U \\
U \\
U\end{array}$ \\
\hline
\end{tabular}


EPA SAYPTE NO.

HSCEP-343
Iab Name: Ouanterra, Mo

Iab code: ITKO Caвe No.s

Matrix : (вoil/water) soII

Sample wt/vol: $1.0 \quad(\mathrm{~g} / \mathrm{ml}) \quad G$

Level: (low/med) IOW . .

8 Moisture: not dec.

CAS NO.

Compound
Contract: 625.01 SAS No.: SDG No.: Y205

Iab Sample ID: 14533-020

Iab File ID?

Date Sampled: 04-30-97

Date Analyzed: $\quad 05-12-97$

Dilution Factor: 1.0

\begin{tabular}{|l|l|l|}
\hline $\operatorname{LBH}(1)+\ldots$ \\
\hline
\end{tabular}

U:Concentration of analyte is less than the value given. X:Pattern reasonably matches gasoline Y:Pattern appears to be multi-peaks; but does not match gasoline 2:Concentration is primarily from a single peak

(1) Low Boiling Hydrocarbon (IBH) is quantitated as if it is Gasoline. 
¿ab Name: OUANTERRA MO

Lab Code: ITMO Case No.: atrix: (soil/water) SOIL ample wt/vol: $20.2(g / \mathrm{ml})$ G Level: (low/med) LOW contract: SAS NO.: Lab Sample ID: SDG NO.: V205 625.01 Lab File ID: Date Sampled: $04-30-97$

* Moisture: not dec. dec.

Date Extracted: $05-08-97$ Ktraction: (SepF/Cont/Sonc/Shak) SHAR Date Analyzed: $05-10-97$ PC Cleanup: $(\mathrm{Y} / \mathrm{N})-\mathrm{N} \quad \mathrm{pH}:$ Dilution Factor: 1 CONCENTRATION UNITS CAS NO. Compound (mg/L or $\mathrm{mg} / \mathrm{Kg}$ ) $\mathrm{ma} / \mathrm{ko}$ 2

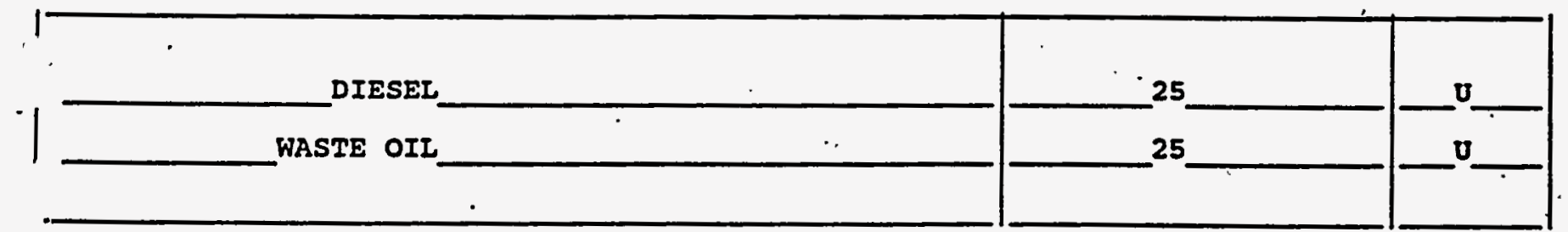

U: Concentration of analyte is 1 ess than the value given. 
Lab Name:" Q̈UANTERRA் MO

Lab Code: ITMO

Case No.: V53301.

Matrix: (soil/water) soIL

Sample wt/vol: $\quad 1.00(\mathrm{~g} / \mathrm{mL}) . \mathrm{G}$

Level: (low/med) LOW

$\%$ Moisture: not dec.

GC Column: RTX-502.2 ID: $0.530(\mathrm{~mm})$

Soil Extract Volume:

(uL)
Contract: $625-01$

SAS . NO.:
EPA SAMPLE NOL.

NSCEP -343

SDG No.: V205 .

Lab Sample ID: 14533-020

Iab File ID: F0020

Date Received: 05/06/97

Date Ánalyzed: 05/13/97.

Dilution Factor: $\quad 1.0$

Soil Aliquot Volume: (uL:

CONCENTRATION UNITS:

CAS NO. COMPOUND (ug/L or $\mathrm{ug} / \mathrm{Kg}$ ) UG/KG

Q

75-71-8--.-Dichlórodifluoromethane

74-83-9--.----Bromomethane

75-01-4--.-----Vinyl Chloride

75-00-3-..--.-Chloroethane

75-09-2-..---.-Methylene Chloride

75-69-4-...-. Trichlorofluoromethane

75-34-3-...--1,1-Dichloroethane

156-60-5--..--trans-1,2-Dichloroethene

156-59-2------cis-1, 2-Dichloroethene

107-06-2-..--1,2-Dichloroethane

594-20-7--.--2, 2-Dichloropropane

74-97-5--.----Bromochloromethane

71-55-6-...--1, 1, 1-Trichloroethane

78-93-3--.--:-2-Butanone

56-23-5--1----Carbon Tetrachloride

563-58-6--.--1, 1-Dichloropropene

75-27-4-1-----Bromodichloromethane

78-87-5--.---1, 2-Dichloropropane

10061-01-5-----cis-1,3-Dichloropropene

79-01-6-....-Trichloroethene

79-00-5-..---1, 1,2-Trichloroethane

71-43-2-...-.-.-Benzene

74-95-3-...--Dibromomethane

10061-02-6-----trans-1,3-Dich loropropene

108-88-3-...--Toluene

$106-93-4-\ldots-1,2-D i b r o m o e t h a n e$

108-10-1--.---4-Methyl-2-Pentanone

127-18-4-...-.-Tetrachloroethene

142-28-9-...--1, 3-Dichloropropane

124-48-1-----Dibromochloromethane

\begin{tabular}{|c|c|}
\hline 100 & TT \\
\hline 100 & 0 \\
\hline 100 & U \\
\hline 100 & U \\
\hline 50 & U \\
\hline 50 & $U$ \\
\hline 50 & $\mathrm{U}$ \\
\hline 50 & U \\
\hline 50 & U \\
\hline 50 & $U$ \\
\hline 50 & U \\
\hline 50 & U \\
\hline 50 & $\mathrm{U}$ \\
\hline 200 & U \\
\hline 50 & U \\
\hline 50 & U \\
\hline 50 & 0 \\
\hline 50 & 0 \\
\hline 50 & U \\
\hline 50 & $U$ \\
\hline 50 & $U$ \\
\hline 50 & U \\
\hline 50 & $U$ \\
\hline 50 & $U$ \\
\hline 50 & U \\
\hline 50 & $U$ \\
\hline 100 & $\mathrm{U}$ \\
\hline 50 & $U$ \\
\hline 50 & U \\
\hline 50 & U \\
\hline
\end{tabular}


b Name :"QUAANTERRA MO

Case No.: V53301

] Code: ITMO

trix: (soil/water) SOIL

5. Ie wt/vol:

$1.00(\mathrm{~g} / \mathrm{mL}) \mathrm{G}$

rol: (low/med) LOW

A.jisture: not dec.

DOlumn: RTX-502.2 ID: 0.530 (mm)

il Extract Volume:

(UI)
Contract: 625-01

SAS NO. :
NSCEP -343

SDG No.: V205

Lab Sample ID: 14533-020

Lab File ID: F0020

Date Received: 05/06/97

Date Analyzed: 05/13/97

Dilution Factor: $\quad 1.0$

Soil Aliquot Volume:

(uI) CONCENTRATION UNITS :

CAS NO.

COMPOUND (ug/L or $\mathrm{ug} / \mathrm{Kg}$ ) UG/KG

Q

108-90-7-...--Chlorobenzene

630-20-6-...-1,1,1,2-Tetrachloroethane

100-41-4--.-.-Ethylbenzene

100-42-5-..--.-Styrene

75-25-2-.....--Bromoform

108-38-3-...--o-Xylene

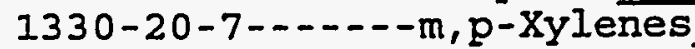

108-86-1-- - - - Bromobenzene

79-34-5-....- 1, 1, 2,2-Tetrachloroethane

96-18-4-..-- - 1, 2, 3-Trichloropropane

95-49-8-...-.--2-Chlorotoluene

106-43-4-...--4-Chlorotoluene

541-73-1-....- 1, 3-Dichlorobenzene

106-46-7-- - - - 1, 4-Dichlorobenzene

95-50-1-..---1, 2-Dichlorobenzene

\begin{tabular}{|l|l|l|}
\hline 50 & $\mathrm{U}$ \\
50 & $\mathrm{U}$ \\
50 & $\mathrm{U}$ \\
50 & $\mathrm{U}$ \\
50 & $\mathrm{U}$ \\
50 & $\mathrm{U}$ \\
50 & $\mathrm{U}$ \\
50 & $\mathrm{U}$ \\
50 & $\mathrm{U}$ \\
50 & $\mathrm{U}$ \\
50 & $\mathrm{U}$ \\
50 & $\mathrm{U}$ \\
50 & $\mathrm{U}$ \\
50 & $\mathrm{U}$ \\
50 & $\mathrm{U}$ \\
\hline
\end{tabular}


Lab Name:

Quanterra, Mo

Iab code: ITMO Case No.:

Matrix : . (so11/water) soIL

Sample wt/vol:

1.0 $(\mathrm{g} / \mathrm{ml})$

G

Level: (low/med) LOW _

\& Hoisture: not dec.

Compound

LBH (1)
Contract: 625.01

SAS NO.:

SDG NO.: V205

Iab Sample ID: 14533-013

Iab Plle ID:

Date Sampled: 04-30-97

Date Analyzed: 05-10-97

Dilution Factor: 1.0

CONCENTRATION ONITS:

(ug/L or ug/Kg) UG/RG

$\mathbf{2}$

500

to

U:Concentration of analyte is less than the value given.

$X:$ Pattern reasonably matches gasoline

Y:Pattern appears to be multi-peakB, but does not match gasoline

z:Concentration is primarlily from a single peak

(1):Low Boiling Hydrocarbon (IBH) is quantitated as if it ls Gasoline. 


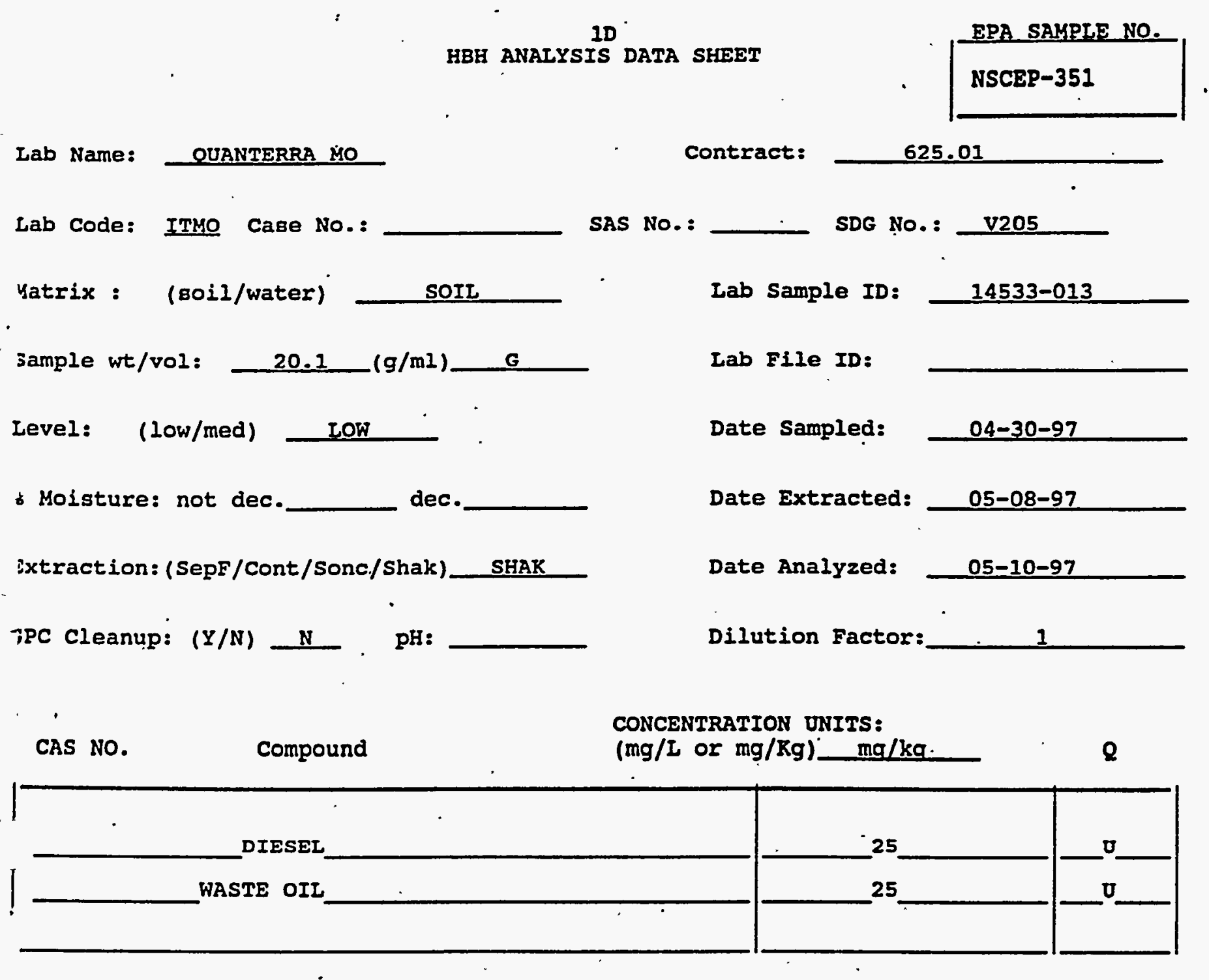

$U$ : Concentration of analyte is less than the value given. 
Lab Name:" QUUANTERRA MO

Lab Code: ITMO

Case No.: V53301

Matrix: (soil/water) SoIL

Sample. wt/vol:

$1.00(\mathrm{~g} / \mathrm{mL}) \mathrm{G}$

Level: (low/med) Low

$\%$ Moisture: not dec.

GC Column: RTX-502.2 ID: 0.530 (mm)

Soil Extract Volume:

(UI)
Contract: $625-01$

NSCEP-351
Soil Extract Volume:

CAS NO.
COMPOUND
SAS NO.:

SDG No.: V205.

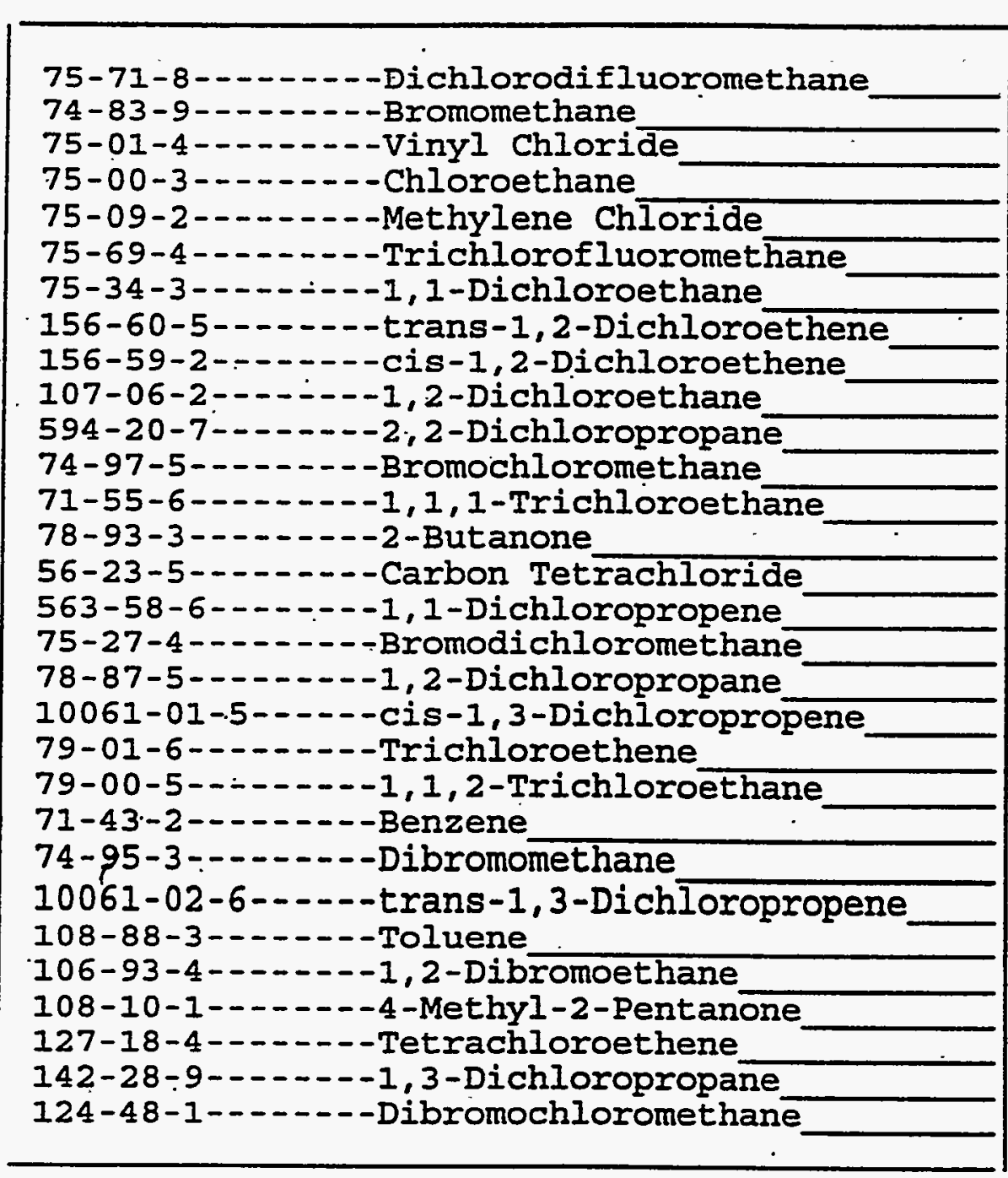

Lab Sample ID: 14533-013

Lab File ID: F0009

Date Received: 05/06/97

Date Analyzed: 05/12/97

Dilution Factor: $1.0^{\circ}$

Soil Aliquot Volume:

(UI)
CONCENTRATION UNITS:

(ug/I or ug/Kg) UG/KG 
$1 \mathrm{~A}$

VOLATILE ORGANICS ANAIYSIS DATA SHEET ab Name: "QüANTERRA MO

¿ Code: ITMO

Case No.: V53301

at.rix: (soil/water) soIL

äulple wt/vol:

$1.00(\mathrm{~g} / \mathrm{mL}) \mathrm{G}$

€ rel: (low/med) Low

Moisture: not dec.

0 Column: RTX-502.2 ID: 0.530 (mm)

o,il Extract Volume:

(uI)
Contract: 625-01

SAS NO.:

SDG NO.: V205

: Lab Sample ID: 14533-013

Iab File ID: Fo009

Date Received: 05/06/97

Date Analyzed: 05/12/97

Dilution Factor: $\quad 1.0$

Soil Aliquot Volume:

(UL)
CAS NO.

COMPOUND
CONCENTRATION UNITS:

(ug/L or ug/Kg) UG/KG

Q

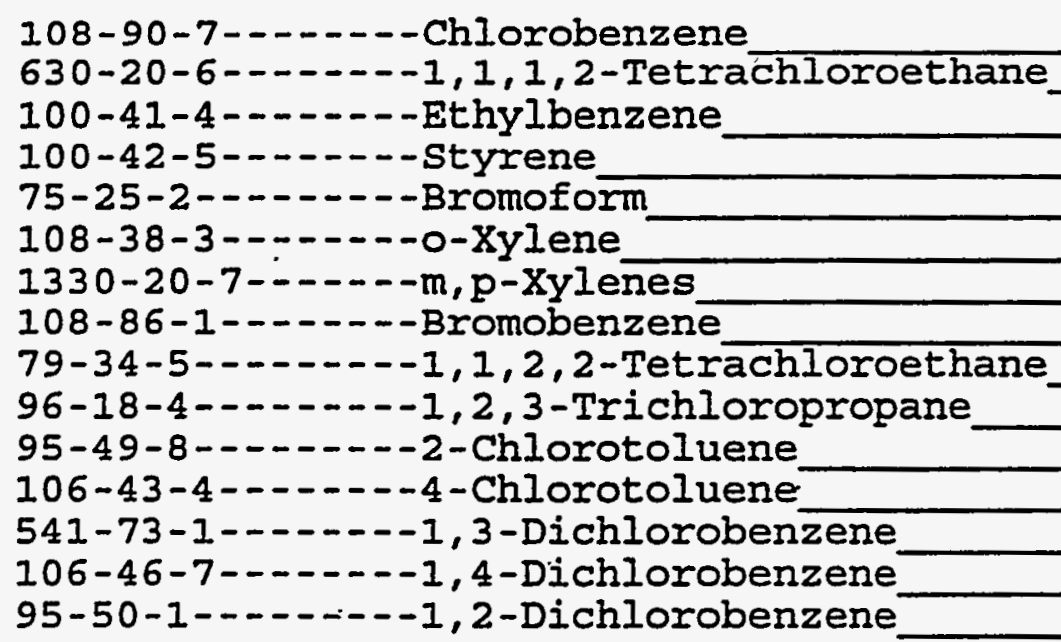


Lab Name: Quanterrarko

Lab Code: ITMO Case No.:

Matrix : (soil/water) soIL

Sample wt/vol:

1.0 $(g / m l)$

Level: (Low/med.)

ION

\&oisture: not dec.

LBH (1)

CAS NO.

Compound
Contract: 625.01

SAS No.:

SDG NO.: V205

Iab Sample ID: 14533-016

Lab File ID:

Date Sampled: $\quad 04-30-97$

Date Analyzed: 05-10-97

Dilution Factor: 1.0

CONCENTRATION URITS:

(ug/L or ug/Rg) UG/RG

$\mathbf{Q}$

500

$\mathbf{0}$

U:Concentration of analyte is less than the value given.

$X$ : Pattern reasonably matches gasoline

$Y=$ Pattern appears to be multi-peaks, but does not match gasoline

$\mathrm{z}$ :Concentration is primarily from a single peak

(1):Low Boiling Hydrocarbon (LBH) is quantitated as if it is Gasoline. 
Iab Name:

OUANTERRA MO

Contract:

625.01

Lab Code: ITMO Case No.: SAS NO.:

SDG NO.: : V205

Matrix : (soil/water)

SOII

Iab Sample ID:

$14533-016$

Sample wt/vol:

$\frac{20.1}{.}(\mathrm{g} / \mathrm{ml})$

Lab File ID:

Level: $\quad$ (low/med) IOW

Date Sampled:

$04-30-97$

\& Moisture: not dec. dec.

Date Extracted: $05-08-97$

Extraction: (SepF/Cont/Sonc/Shak) SHAK

Date Analyzed: $05-10-97$

GPC Cleanup: $(Y / N) \perp N$ pH:

Dilution Factor:

1

CONCENTRATION UNITS:

cas No.

Compound

(mg/L or $\mathrm{mg} / \mathrm{Kg}$ )

$\mathrm{mg} / \mathrm{kg}$

Q

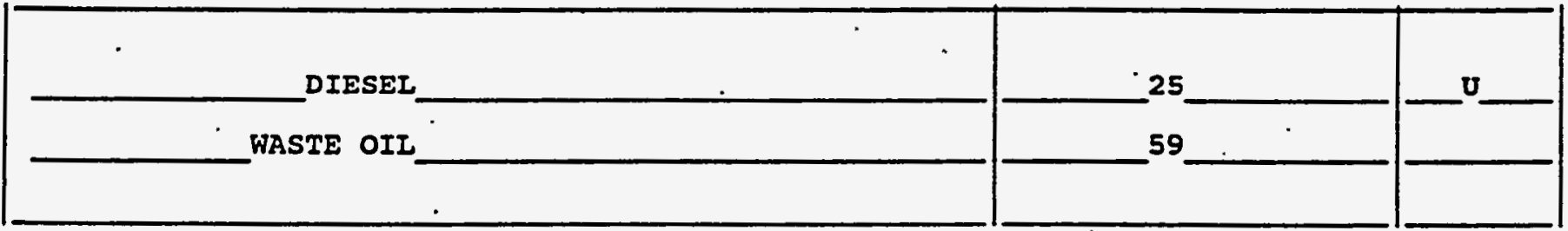

$U$ : Concentration of analyte is less than the value given. 
Lab Name:" QUUANTERRÁ MO

Lab Code: ITMO

Case No.: V53301

Matrix: (soil/water) soII

Sample wt/vol:

$1.00(\mathrm{~g} / \mathrm{mL}) \mathrm{G}$

Level: (low/med) LOW

$\%$ Moisture: not dec.

GC Cólumn: RTX-502.2 ID: 0.530 (mm)

Soil Extract Volume:

(uL)
Contract: $625-01$

SAS NO.:
NSCEP-355

SDG No.: V205

Lab Sample ID: 14533-016

Iab File ID: Fo017

Date Received: 05/06/97

Date Analyzed: 05/13/97

Dilution Factor: $\quad 1.0$

Soil Aliquot Volume: (uI,

CONCENTRATION UNITS:

CAS NO. COMPOUND

(ug/L or ug/Kg) UG/KG

Q

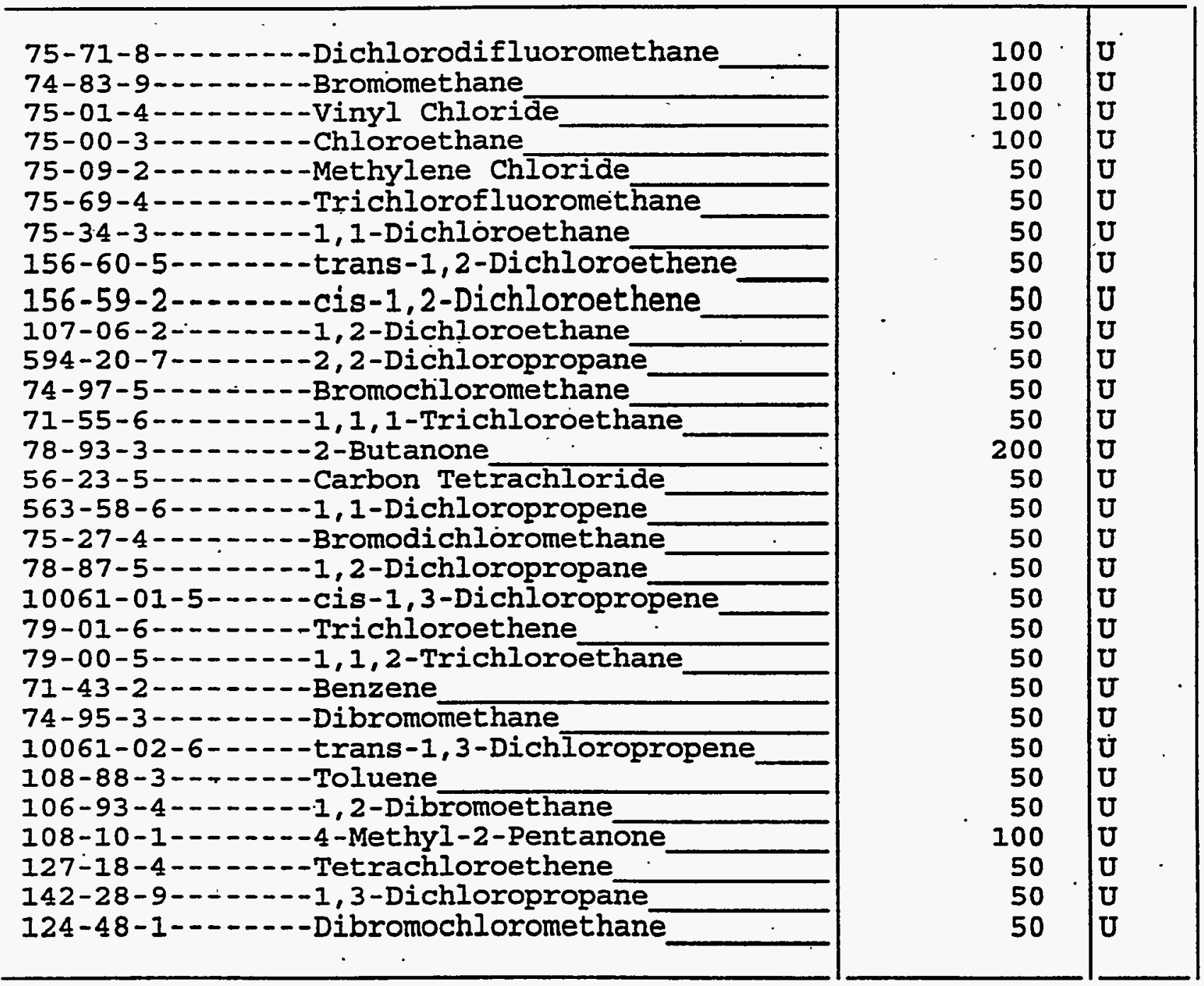


b Name:" QUUANTERRA MO :

Case No.: V53301

trix: (soil/water) soIL

Contract: $625-01$

- Code: ITMO

NSCEP -355

. Ple wt/vol:

$1.00(\mathrm{~g} / \mathrm{mL}) \mathrm{G}$

:-1: (low/med) LOW

moisture: not dec.

Column: RTX-502.2 ID: 0.530 (mm)

il Extract Volume:

(uL)
SAS NO.:

SDG No.: V205
Lab Sample ID: 14533-016

Lab File ID: Fo017

Date Received: 05/06/97

Date Analyzed: 05/13/97

Dilution Factor:

1.0 .

Soil Aliquot Volume:

(UI)

CAS NO.

COMPOUND

CONCENTRATION UNITS:

(ug/L or ug/Kg) UG/KG

Q

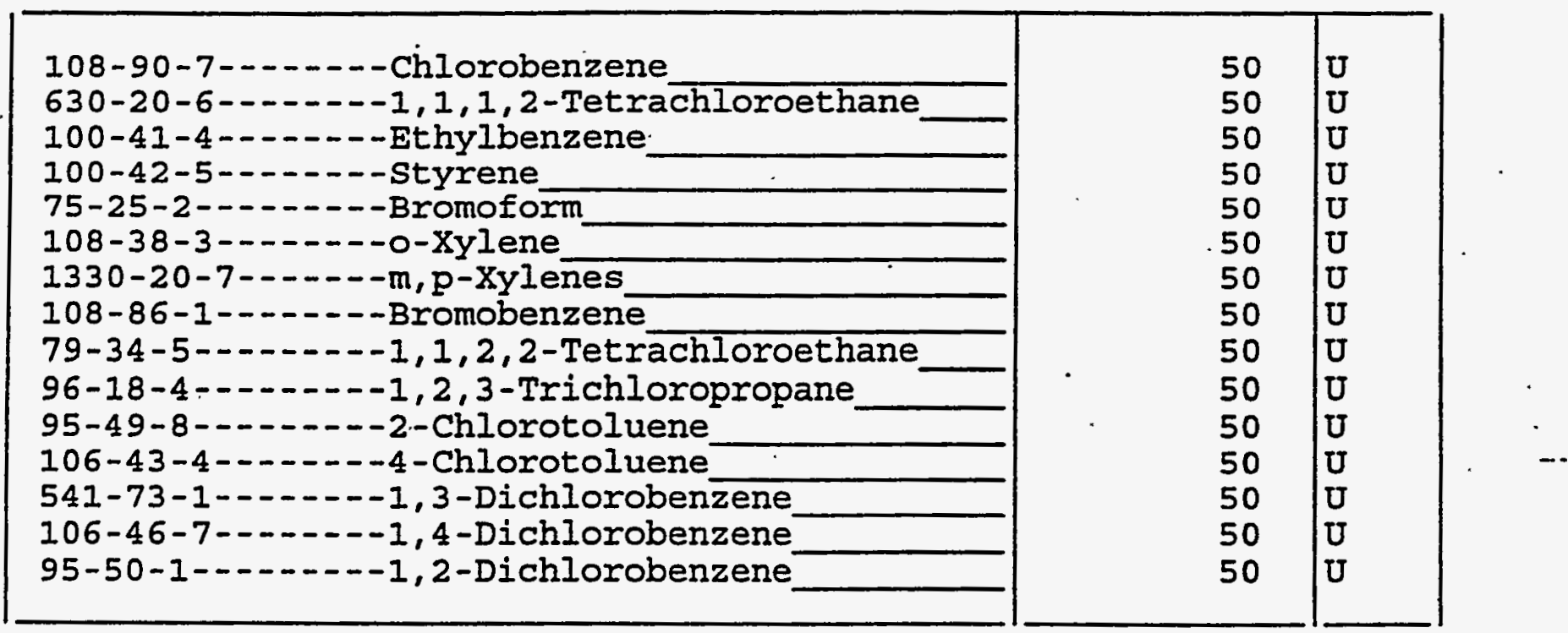


Iab Name:

Iab code: Ifyo Case No.:

Matrix : (Boil/water) sort

Sample wt/vol:

$1.0 \quad(g / \mathrm{ml})$

G

Level: (low/med) IOW

\& Moisture: not dec.

Contract: 625.01

SAS NO.:

SDG NO.: V205

Iab Sample ID: 14533-014

Iab File ID:

Date Samplad:

$04-30-97$

Date Analyzed: 05-10-97

Dilution Pactor: 1.0

CONCENTRATION UNITS:

(ug/L or ug/Kg)_ UG/RG_.

$\mathbf{Q}$ 500

IBE (1)

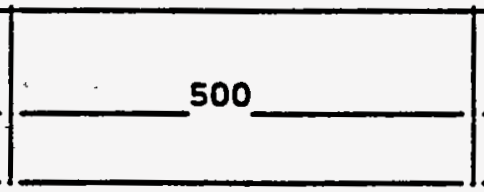

U:Concentration of analyte is less than the value.given.

$X:$ Pattern reasonably matches gasoline

$Y$ :Pattern appears to be multi-peaks, but does not match gasoline

$z$ :Concentration is primarily from a single peak

(1) : Low Boiling Hydrocarbon (LBH) is quantitated as if it is Gasoline. 
1D

HBH ANALYSIS DATA SHEET
EPA SAMPIE NO. NSCEP -360 ¿ab Name: OUANTERRA YO

Iab Code: ITMO Case NO.:

datrix : (8011/water)

SOIL

Sample wt/vol:

-20.1 $(\mathrm{g} / \mathrm{ml})$

G

Ieve 1: (Low/med)

LOW

\% Moisture: not dec. dec.

Extraction: (SepF/Cont/Sonc/Shak) SHAK

GPC Cleanup: $(\mathrm{Y} / \mathrm{N}) \underline{\mathrm{N}} \mathrm{pH}:$ $\mathrm{PH}:$ CAS NO. Compound DIESEL WASTE OIL

$U$ : Concentration of analyte is less than the value given.
Conțract: 625.01

SAS No.:

SDG No.: V205

Iab Sample ID: _14533-014

Lab File ID:

Date Sampled:

$04-30-97$

Date Extracted:

$05-08-97$

Date Analyzed:

$05-10-97$.

Dilution Factor:

1

CONCENTRATION UNITS:

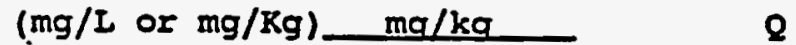

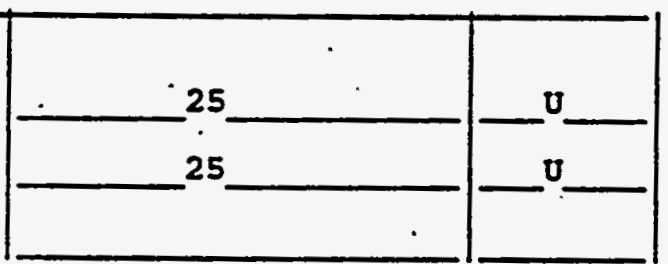


Lab Name:“QUUANTERRA MO

Lab Code: ITMO

Case No.: V53301

Matrix: (soil/water) soII

Sample wt/vol:

$5.00(\mathrm{~g} / \mathrm{mL}) \cdot \mathrm{G}$

Level: (low/med) LOW

\% Moisture: not dec.

GC Column: RTX-502.2 ID: 0.530 (mm)

Soil Extract Volume:

(uL)
Contract: $625-01$

SAS NO.:

SDG NO.: V205
Lab Sample ID: 14533-014

Lab File ID: $\quad$ F9983

Date Received: 05/06/97

Date Analyzed: 05/10/97

Dilution Factor: $\quad 1.0$

Soil Aliquot Volume:

(uL
CAS NO.
COMPOUND
CONCENTRATION UNITS:

(ug/I or ug/Kg) UG/KG
75-71-8--.--Dichlorodifluoromethane

74-83-9--.---Bromomethane

75-01-4-.-.---vinyl Chloride

75-00-3-...---Chloroethane

75-09-2--.-.--Methylene Chloride

75-69-4-..--.-Trichlorofluoromethane

75-34-3-...-1, 1-Dichloroethane

156-60-5-------trans-1, 2-Dichloroethene

156-59-2------cis-1,2-Dichloroethene

107-06-2-----1, 2-Dichloroethane

594-20-7------2, 2-Dichloropropane

74-97-5------Bromochloromethane

71-55-6-...-1, 1,1-Trichloroethane

78-93-3--.-----2-Butanone

56-23-5-------Carbon Tetrachloride

563-58-6-..--1, 1-Dichloropropene

75-27-4-...--Bromodichloromethane

78-87-5--...-1, 2-Dichloropropane

10061-01-5------cis-1,3-Dichloropropene

79-01-6--.----Trichloroethene.

79-00-5-...--1,1,2-Trichloroethane

71-43-2-------Benzene

74-95-3-.---Dibromomethane

10061-02-6-----trans-1, 3-Dichloropropene

108-88-3-------Toluene

106-93-4-.-.--1, 2-Dibromoethane

127-18-4-------Tetrachloroethene

142-28-9------1, 3-Dichloropropane

124-48-1-..---Dibromochloromethane

108-90-7-------Chlorobenzene

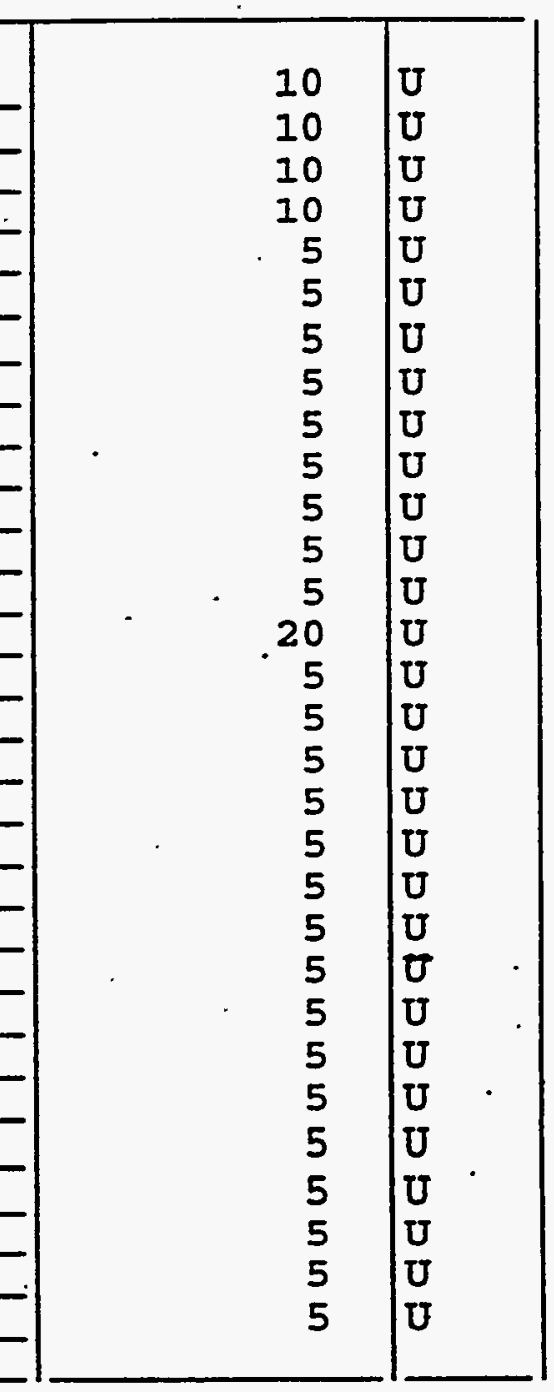


b Name;" QUANTERRA MO

Code : ITMO

Case No.: V53301

trix: (soil/water) SOIL

huple wt/vol:

$5.00(\mathrm{~g} / \mathrm{mL}) \mathrm{G}$

el: (low/med) LOW

Moisture: not dec.

Contract: $625-01$

NSCEP-360 .
: Column: RTX-502.2 ID: $0.530(\mathrm{~mm})$

il Extract Volume:

(UL)
SAS NO.:

SDG No.: V205

Lab Sample ID: 14533-014

Lab File ID: F9983

Date Received: 05/06/97

Date Analyzed: 05/10/97

Dilution Factor:

1.0

Soil Aliquot Volume:

(UI)
CAS NO.

COMPOUND
CONCENTRATION UNITS:

(ug/L or ug/Kg) UG/KG

Q

\begin{tabular}{|c|}
\hline 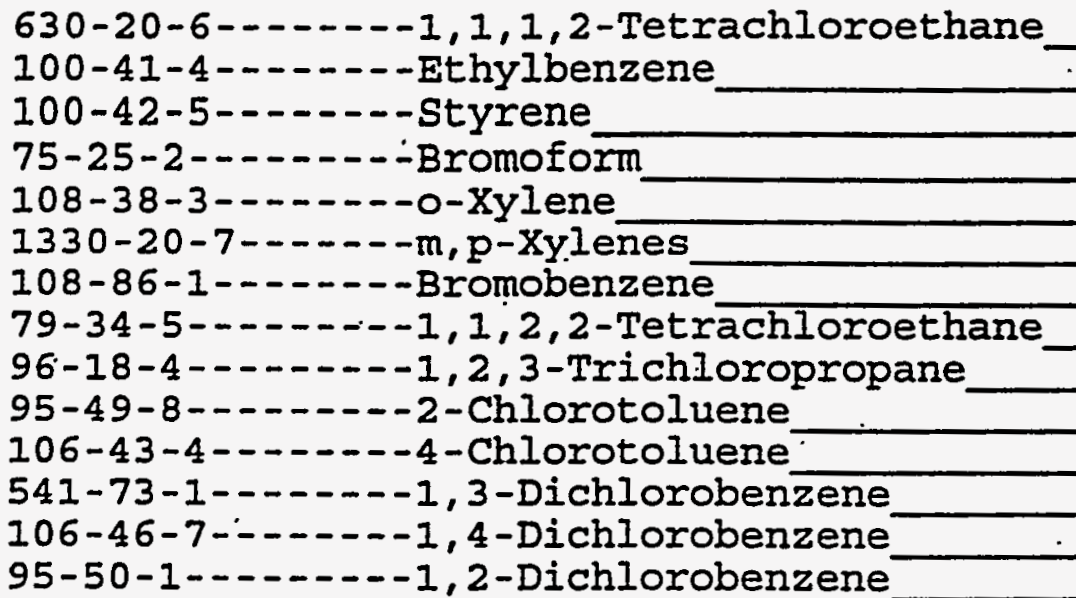 \\
\hline
\end{tabular}

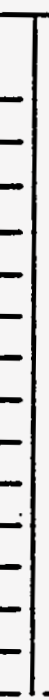


10

IBH ANAIYSIS DATA SHBET
EPA SAMPTE NO.

NSCEP -373
Iab Name:

Quanterra, MO.

Lab Code: ITxO Caвe No.:

Matrix : (soil/water) SoIr

Sample wt/rol:

$1.0 \quad(\mathrm{~g} / \mathrm{ml})$

G

Level: (low/med) LOW

* Moisture: not dec.

Contract: 625.01

SAS NO.: SDG NO.: V205.

Iab Sample ID: 14533-021

Lab File ID:

Date sampled: 04-30-97

Date Analyzed: $05-12-97$

Dilution Factor: 1.0

CONCENTRATION UNITS:

(ug/I or ug/Rg) UG/RG

$\mathbf{Q}$

CAS NO.

Compound

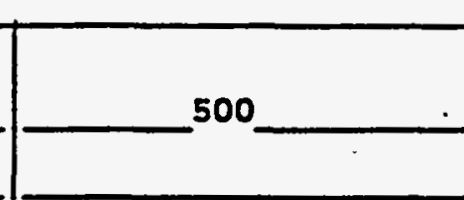

0

U:Concentration of analyte is less than the value given.

$X:$ Pattern reasonably matches gasoline

Y:Pattern appears to be mult 1 -peakB, but does not match gasoline

z:Concentration is primarily from a single peak

(1): Low Boiling kydrocarbon (LBH) is quantitated as if it is Gasoline. 
Lab Name: OUANTERRA YO

contract:

625,01

Lab Code: ITMO Case No.:

SAS NO.:

SDG NO.: V205

Matrix : (soil/water)

SOIL

Lab Sample ID:

14533-021

Sample wt/vol:

$20.1-(\mathrm{g} / \mathrm{ml})$

Iab File ID:

Level: (low/med)

LOW

Date Sampled:

$04-30-97$.

* Moisture: not dec. dec.

Date Extracted:

$05-09-97$

Extraction: (SepF/Cont/Sone/Shak) SHAK

Date Analyzed:

$05-13-97$

GPC Cleanup: $(Y / N)+N \quad \mathrm{NH}$

Dilution Factor:

1

CONCENTRATION UNITS:

CAS NO.

Compound

$(\mathrm{mg} / \mathrm{L}$ or $\mathrm{mg} / \mathrm{Kg}) \mathrm{mg} / \mathrm{kg}$

$\mathbf{Q}$

\begin{tabular}{|c|c|c|}
\hline $\begin{array}{r}2.25 \\
\end{array}$ & 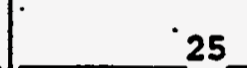 & $\mathbf{u}$ \\
\hline _ WASTE OIL_ & 25 & _ \\
\hline
\end{tabular}

$U$ : Concentration of analyte is less than the value given. 
Lab Name:" QUUANTERRA" MO

Lab Code: ITMO

Case No.: V53301

Matrix: (soil/water) soII

Sample wt/vol:

$1.00(\mathrm{~g} / \mathrm{mL}) \mathrm{G}$

Level: (low/med) LOW

$\%$ Moisture: not dec.

GC column: RTX-502.2 ID: 0.530 (mm)

Soil Extract Volume:

(uI)
Contract: 625-01

SAS NO.:

NSCEP -373

SDG NO.: V205

Lab Sample ID: 14533-021

Lab File ID: F002i

Date Received: 05/06/97

Date Analyzed: 05/13/97

Dilution Factor: 1.0

Soil Aliquot Volume:

(uI)

CONCENTRATION UNITS:

CAS NO.

COMPOUND

(ug/I or $u g / \mathrm{Kg}$ ) UG/KG

Q

75-71-8--.--Dichlorodifluoromethane

74-83-9.--..---Bromomethane

75-01-4-...---.-Vinyl Chloride

75-00-3-....-.-Chloroethane

75-09-2-....--Methylene chloride

75-69-4-1.---Trichlorofluoromethane

75-34-3-.-.--1, I-Dichloroethane

156-60-5--.---trans-1, 2-Dichloroethene

156-59-2--.---cis-1,2-Dichloroethene

107-06-2------1, 2-Dichloroethane

594-20-7----2,2-Dichloropropane

74-97-5-...-- - Bromochloromethane

71-55-6--.---1, 1, 1-Trichloroethane

78-93-3-..-.--2-Butanone

56-23-5--.----Carbon Tetrachloride

563-58-6-...-1, 1-Dichloropropene

75-27-4-...-.--Bromodichloromethane

78-87-5-----1, 2-Dichloropropane

10061-01-5-----cis-1,3-Dichloropropene

79-01-6-...-.-Trichloroethene

79-00-5-------1,1,2-Trichloroethane

71-43-2---.----Benzene

74-95-3--.---Dibromomethane

10061-02-6-----trans-1,3-Dich loropropene

108-88-3-..--.-Toluene

106-93-4-...-1,2-Dibromoethane

108-10-1-...-2-Methyl-2-Pentanone

127-18-4-..-- Tetrachloroethene

142-28-9------1,3-Dichloropropane

124-48-1-..--Dibromochloromethane

\begin{tabular}{r|l|}
100 & $U$ \\
100 & $U$ \\
100 & $U$ \\
100 & $U$ \\
50 & $U$ \\
50 & $U$ \\
50 & $U$ \\
50 & $U$ \\
50 & $U$ \\
50 & $U$ \\
50 & $U$ \\
50 & $U$ \\
50 & $U$ \\
200 & $U$ \\
50 & $U$ \\
50 & $U$ \\
50 & $U$ \\
50 & $U$ \\
50 & $U$ \\
50 & $U$ \\
50 & $U$ \\
50 & $U$ \\
50 & $U$ \\
50 & $U$ \\
50 & $U$ \\
50 & $U$ \\
100 & $U$ \\
50 & $U$ \\
50 & $U$ \\
50 & $U$ \\
& \\
\hline 5
\end{tabular}


ib Name:"QUUANTERRA MO"

I Code:. ITMO

Case No.: V5330I itrix: (soil/water) SOIL

1.ple wt/vol:

$1.00(\mathrm{~g} / \mathrm{mL}) \mathrm{G}$

$\exists e l$

(low/med) LOW

moisture: not dec.

: Column: RTX-502.2 ID: $0.530 \cdot(\mathrm{mm})$

iil Extract volume:

(uL)
Contract: $625-01$

SAS NO.:

SDG No.: V205

Lab Sample ID: 14533-021

Lab File ID: F002i

Date Received: 05/06/97

Date Analyzed: 05/13/97

Dilution Factor: $\quad 1.0$

Soil Aliquot Volume:

(UL)

CAS NO.

COMPOUND

CONCENTRATION UNITS:

(ug/L or ug/Kg) UG/KG

Q

\begin{tabular}{|c|c|c|}
\hline 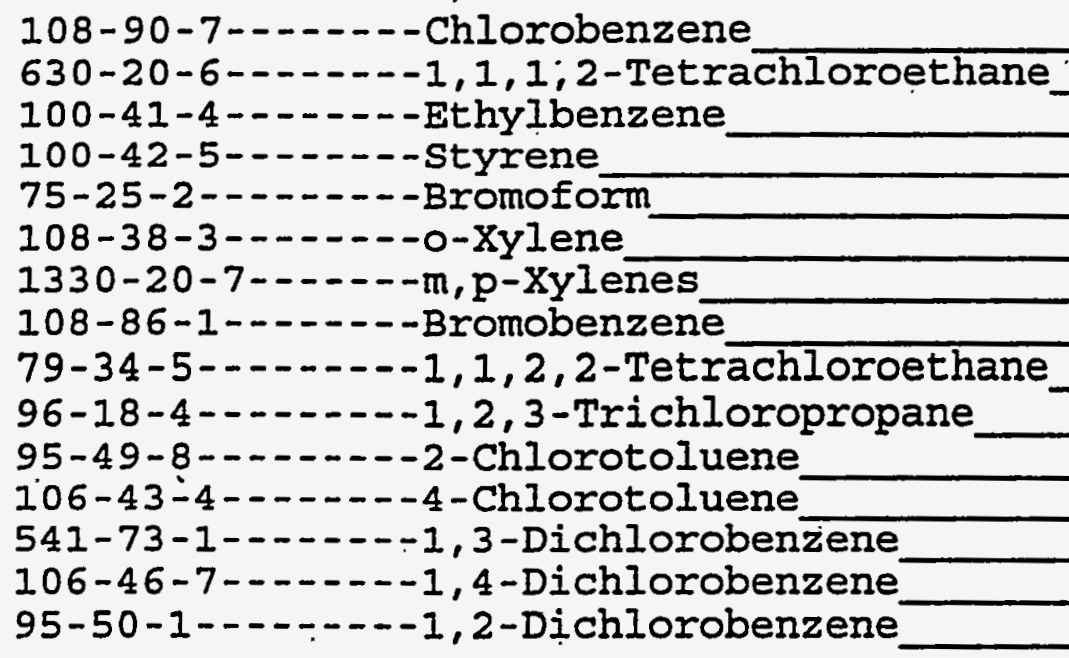 & $\begin{array}{l}50 \\
50 \\
50 \\
50 \\
50 \\
50 \\
50 \\
50 \\
50 \\
50 \\
50 \\
50 \\
50 \\
50 \\
50\end{array}$ & $\begin{array}{l}U \\
U \\
U \\
U \\
U \\
U \\
U \\
U \\
U \\
U \\
U \\
U \\
U \\
U \\
U\end{array}$ \\
\hline
\end{tabular}


Lab Name:

Ouanterra, MO

Lab Code: IтMo case No.:

Matrix : (8oil/water) soIf

Sample wt/vol:

1.0 $(\mathrm{g} / \mathrm{ml})$

Level: (low/med) IOW

\& Moisture: not dec.

SAS NO.:

contract: $\quad 625.01$

SDG NO.: V205

Iab Sample ID: 14533-015

Iab File ID:

Date Sampled: 04-30-97

Date Analyzed: 05-10-97

Dilution Factor: 1.0
CONCENTRATION UNITS:

CAS NO.

Compound
(ug/L or ug/kg) UG/KG

\section{$\mathbf{Q}$}

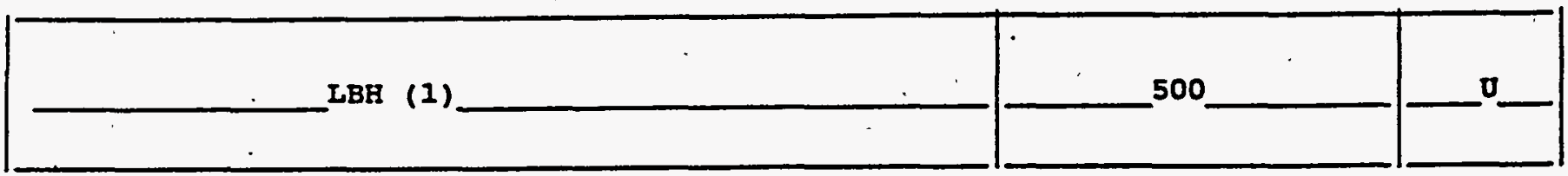

U:Concentration of analyte is less than the value given.

$\mathrm{X}$ : Pattern reasonably matches gasoline

Y: Pattern appears to be multi-peaks, but does nót match gasoline

2:Concentration is primarily from a single peak

(1): Low Boiling Hydrocarbon (IBE) is quantitated as if it is Gasoline. 


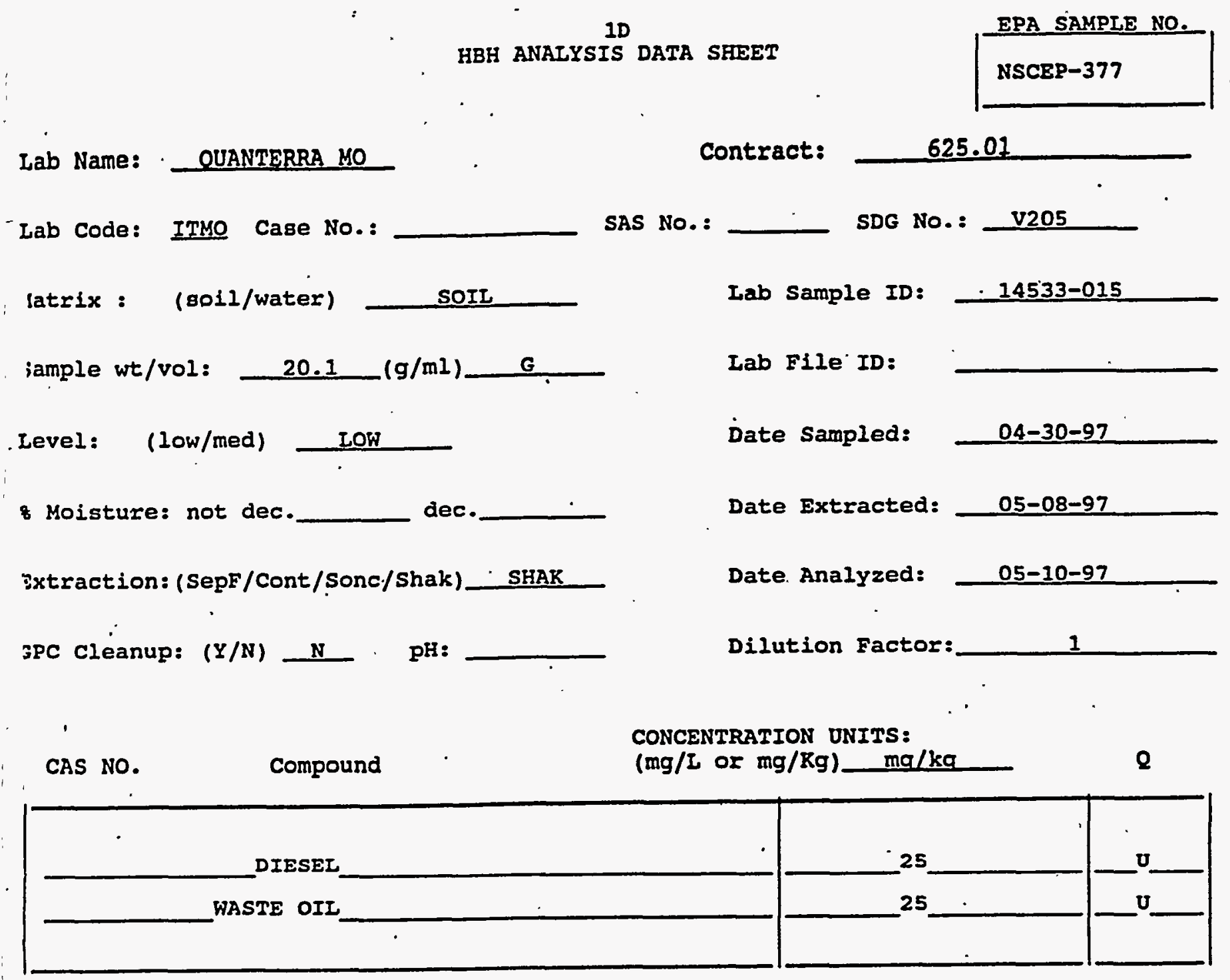

$\mathrm{U}$ : Concentration of analyte is less than the value given. 
Lab Name:"Q̈UANTERRA MO

Lab Code: ITMO

Case No.: V53301

Matrix: (soil/water) soIL

Sample wt/vol:

$1.00(\mathrm{~g} / \mathrm{mL}) \mathrm{G}$

Level: (low/med) LOW

$\because$ Moisture: not dec.

GC Column: RTX-502.2 ID: 0.530 (mm)

Soil Extract Volume:
Contract: $625-01$

SAS NO.:

SDG NO.: V205

Lab Sample ID: 14533-015

Lab File ID: F0012

Date Received: 05/06/97

Date Analyzed: 05/12/97

Dilution Factor: $\quad 1.0$

Soil Aliquot Volume:

CONCENTRATION UNITS :

CAS NO.

COMPOUND

(ug/L or $\mathrm{ug} / \mathrm{Kg}$ ) UG/KG

Q

100

100

100

100

50

50

50

50

50

50

50

50

50

200

50

50

50

50

50

50

50

50

50

50

50

50

100

50

50

50 
b Name:" Q̈UANTERRA MO

I Code: ITMO

Case No.: V53301

ttrix: (soil/water) SOIL

r. $l$ le wt/vol:

$1.00(\mathrm{~g} / \mathrm{mL}) \mathrm{G}$

:1: (low/med) LOW

Moisture: not dec.

$\therefore$ :olumn: RTX-502.2 ID: 0.530 (mm)

il Extract Volume:

(UL)
Contract: $625-01$

SAS NO.:

Iab Sample ID: 14533-015

Lab File ID: F0012

Date Received: 05/06/97

Date Analyzed: 05/12/97

Dilution Factor: $\quad 1.0$

Soil Aliquot Volume:

(uL)

CONCENTRATION UNITS :

CAS NO. COMPOUND (ug/L or ug/Kg) UG/KG $Q$

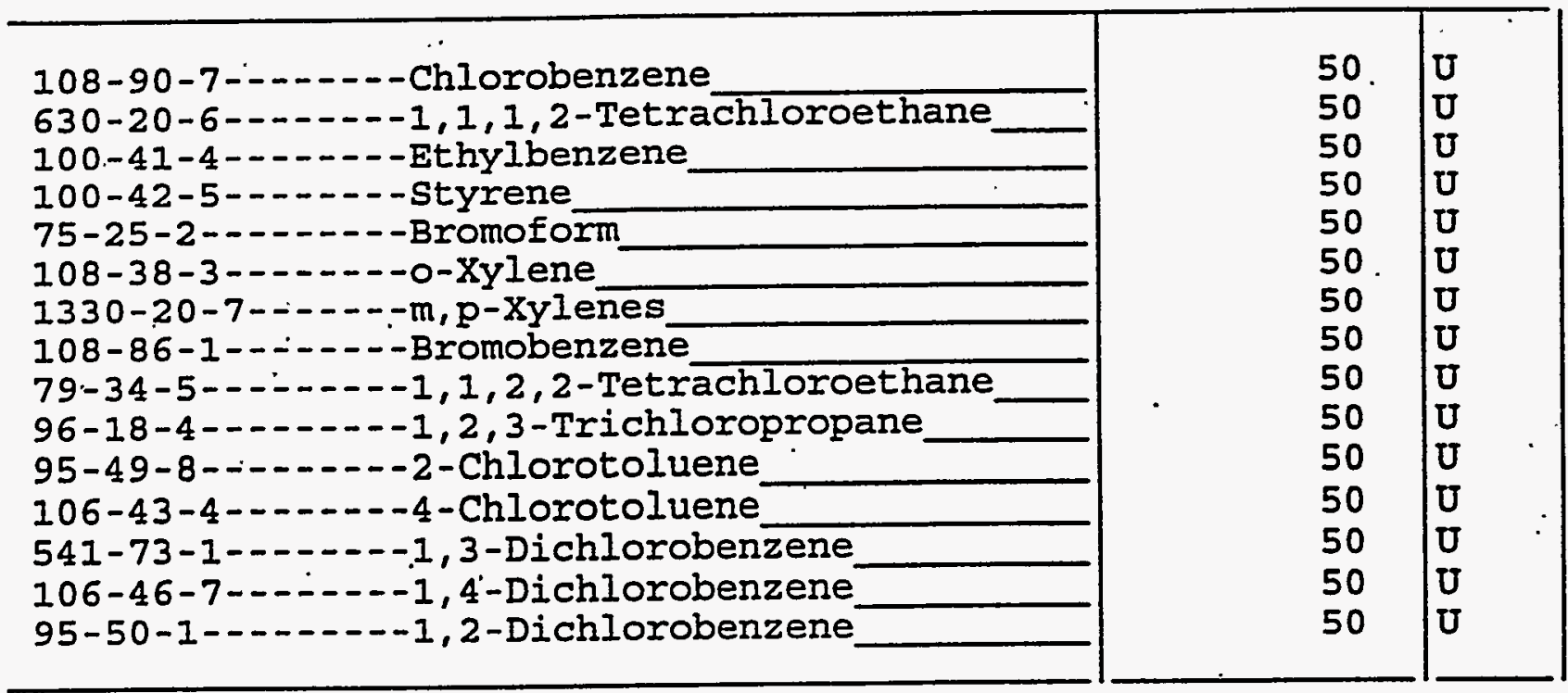




\begin{abstract}
Lab Name: Quantersamo
Lab Code: ITHO Case No.:

Matrix : ' (soil/wäter) soIL

Sample wt/vol: 1.0 $(\mathrm{g} / \mathrm{mI})$

G
\end{abstract}

Contract: 625.01

SAS NO.:

SDG NO.: V205

Iab Sample ID: 14533-017

Lab File ID:

Date Sampled:

04-30-97

Date Analyzed: 05-10-97

Dilution Factor: 1,0

CONCENTRATION ONITS:

CAS NO.

Compound

(ug/L or ug/Kg) UG/RG

Q

IBH

(1)

500

0

U:Concentration of analyte is less than the value given.

$X$ : Pattern reasonably matches gasoline

Y:Pattern appears to be multi-peaks, but does not match gasoline

z:Concentration is primarily from a single peak

(1) :Low Boiling Hydrocarbon (LBB) is quantitated as if it is Gasoline.

FORY I IBE 


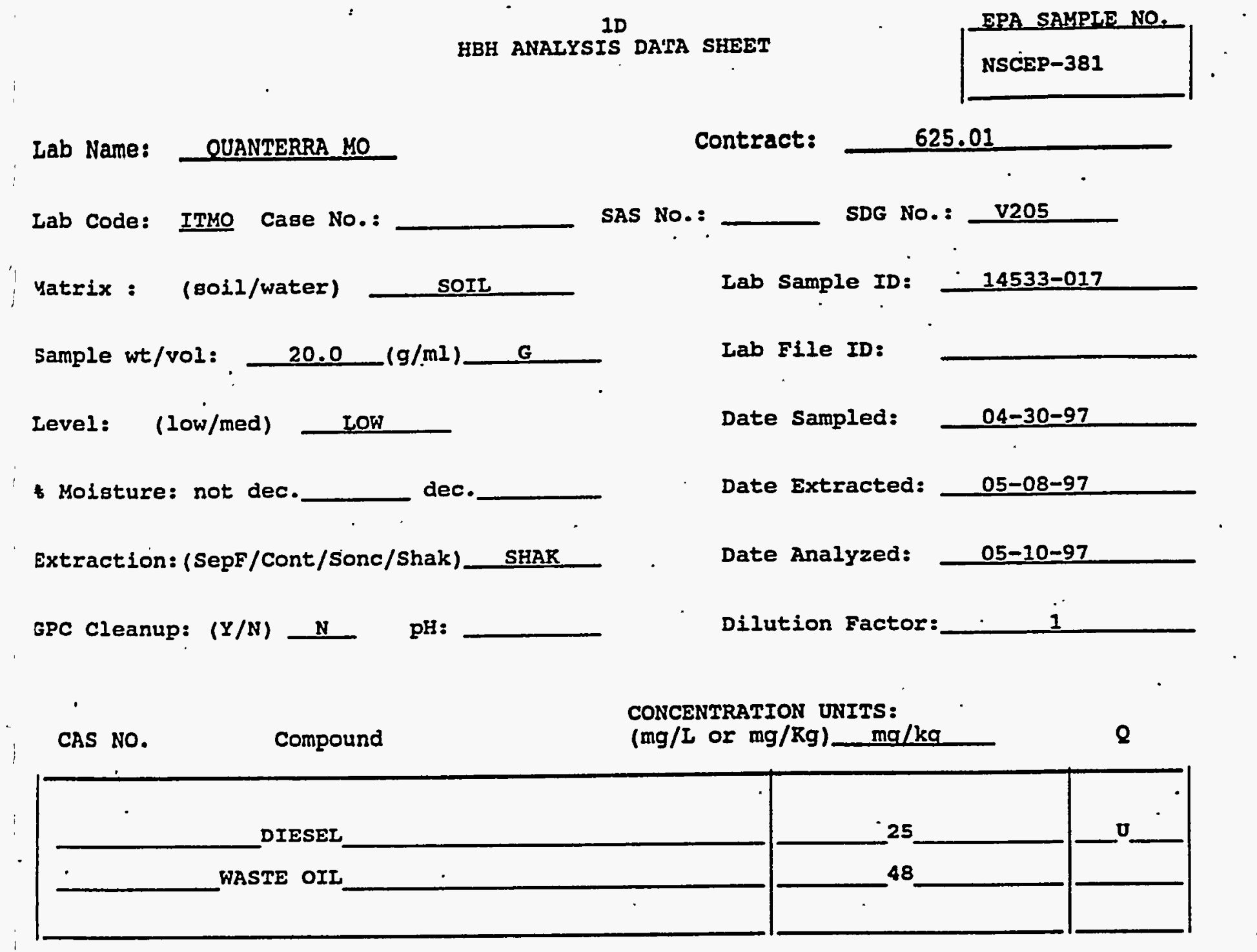

$\mathrm{U}$ : Concentration of analyte is less than the value given. 
Lab Name :- QUUANTERRÁ MO

Lab Code: ITMO

Case No.: V53301

Matrix: (soil/water) SOII

Sample wt/vol:

$1.00(\mathrm{~g} / \mathrm{mL}) \mathrm{G}$

Level: (low/med). LOW

: Moisture: not dec.

GC Column: RTX-502.2 ID: 0.530 (mm)

Soil Extract Volume:

(uL)
Contract : $625-01$

NSCEP-381 SAS NO.:

SDG No.: V205

Lab Sample ID: 14533-017

Lab File ID: F0018

Date Received: 05/0.6/97

Date Analyzed: 05/13/97

Dilution Factor: $\quad 1.0$

Soil Aliquot Volume:

(uI

CONCENTRATION UNITS :

CAS NO. $\quad$ COMPOUND

(ug/L or ug/Kg) UG/KG

Q

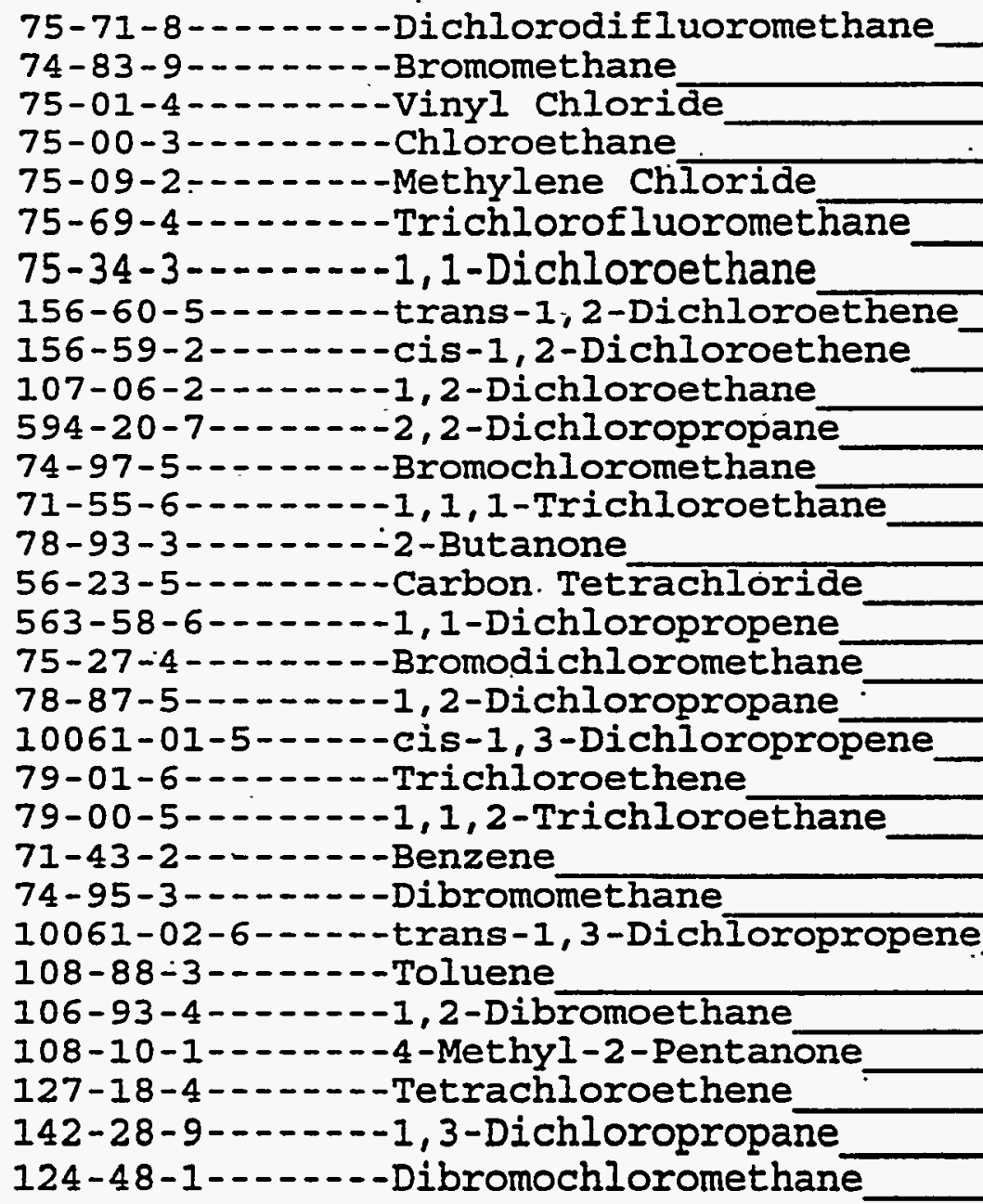

\begin{tabular}{r|l|}
100 & $\mathrm{U}$ \\
100 & $\mathrm{U}$ \\
100 & $\mathrm{U}$ \\
100 & $\mathrm{U}$ \\
50 & $\mathrm{U}$ \\
50 & $\mathrm{U}$ \\
50 & $\mathrm{U}$ \\
50 & $\mathrm{U}$ \\
50 & $\mathrm{U}$ \\
50 & $\mathrm{U}$ \\
50 & $\mathrm{U}$ \\
50 & $\mathrm{U}$ \\
50 & $\mathrm{U}$ \\
200 & $\mathrm{U}$ \\
50 & $\mathrm{U}$ \\
50 & $\mathrm{U}$ \\
50 & $\mathrm{U}$ \\
50 & $\mathrm{U}$ \\
50 & $\mathrm{U}$ \\
50 & $\mathrm{U}$ \\
50 & $\mathrm{U}$ \\
50 & $\mathrm{U}$ \\
50 & $\mathrm{U}$ \\
50 & $\mathrm{U}$ \\
50 & $\mathrm{U}$ \\
50 & $\mathrm{U}$ \\
100 & $\mathrm{U}$ \\
50 & $\mathrm{U}$ \\
50 & $\mathrm{U}$ \\
50 & $\mathrm{U}$ \\
\hline
\end{tabular}


ib Name:" Q̈UANTERRA MO :

1. Code: ITMO

Case No.: V53301 SAS No.:
NSCEP-381 itrix: (soil/water) SOIL

u ?le wt/vol:

$1.00(\mathrm{~g} / \mathrm{m} \tau) \mathrm{G}$

?-al: (low/med) LOW

moisture: not dec.

: Zolumn: RTX-502.2. ID: $0.530(\mathrm{~mm})$

sil Extract Volume:

(uL)
Contract : 625-01

SDG No.: V205
Lab Sample ID: 14533-017

Lab File ID: F0018

Date Received: 05/06/97

Date Analyzed: 05/13/97

Dilution Factor: $\quad 1.0$

Soil Aliquot Volume:

(uL)

CONCENTRATION UNITS:

CAS NO.

COMPOUND

(ug/I or ug/Kg) UG/KG

Q

\begin{tabular}{|c|}
\hline 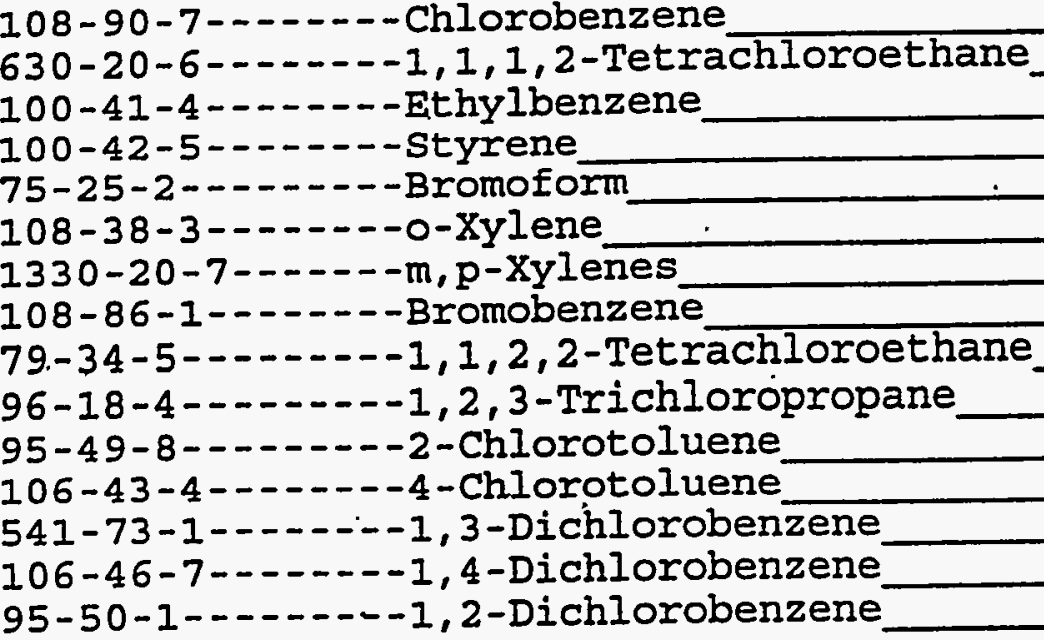 \\
\hline
\end{tabular}


ID

IBH ANALYSIS DATA SHEET
IPA SAYPLE NO.

ASCBP-1381
Iab Names Ouanterra, Mo

Iab Code: ITxO Cage NO. $3^{\prime}$

Matrix : (soil/water) sorr

Sample wt/vol: $1.0 \quad(g / m 1)$ G

Level: (low/med) LOW

solsture: not dec.

CAS NO.

Compound.

CONCENTRATION UNITS:

(ug/I or ug/Rg) ug/Rg

Q
Contracts $\quad 625.01$

SDE HO.: Y205

Iab sample IDs $14533-036$

Iab File ID:

Date Sampleds 04-30-97

Date Analyzed: 05-13-97

Dilution Factor: 1,0

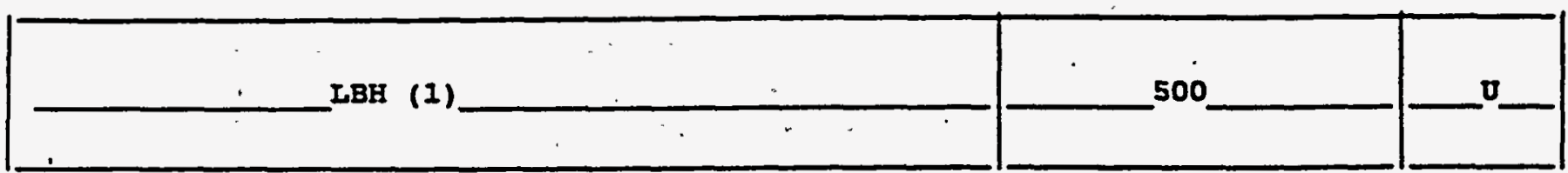

$U$ : Concentration of analyte 18 lese than the value given. $X:$ Pattern reasonably matches gasoline

Y:Pattern appears to be multi-peaks, but does not match gasoline

z:Concentration is primarily from a single peak

(1) Low Boiling Hydrocarbon (IBE) is quantitated as if it is Gasoline. 
Lab Name: ' QUANTERRA MO

contract:

625.01

Lab code: ITMO Case No.:

latrix : (soil/water)

SOIL

sample wti/vol:

20.1

$(g / m l)$

G

Level: (Low/med)

LOW

is Moisture: not dec. dec.

Extraction: (SepF/Cont/Sonc/Shak)

SHAK

sPC Cleanup: $(Y / N)-\mathrm{N} \quad \mathrm{pH}:$

$\longrightarrow$
SAS No.:

Iab Sample ID:

$14533-036$

Iab File ID:

Date Sampled:

Date Extracted:

05-09-97

Date Analyzed:

$05-13-97$

Dilution Factor:

1

CONCENTRATION UNITS:

CAS NO.

Compound

(mg/L or $\mathrm{mg} / \mathrm{Kg}$ )

$\mathrm{mg} / \mathrm{kg}$

$Q$

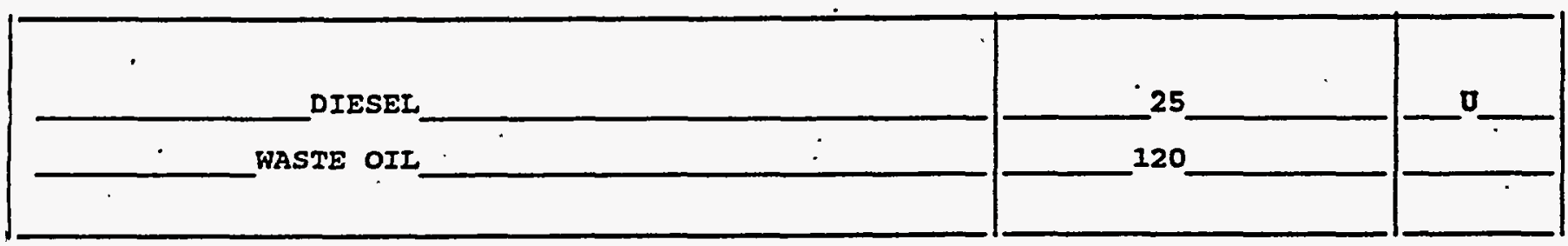

$U$ : Concentration of analyte is less than the value given. 
Lab Name:"-QUUANTERRA MO

Lab Code: ITMO

Case No.: V53301

Matrix: (soil/water) soIL

Sample wt/vol: $\quad 1.00(\mathrm{~g} / \mathrm{mL}) \mathrm{G}$

Level: (low/med) LOW

$\because$ Moisture: not dec.

GC Column: RTX-502.2 ID: 0.530 (mm)

Soil Extract Volume:

(UL)
Contract: $625-01$

SAS NO.:

SDG NO.: V205

NSCEP-1381

Lab Sample ID: 14533-036

Lab File ID: F0038

Date Received: 05/06/97

Date Analyzed: 05/13/97

Dilution Factor: 1.0

Soil Aliquot Volume: (uI

CONCENTRATION UNITS :

CAS NO. COMPOUND (ug/I or ug/Kg) UG/KG Q

100

100

100

100

50

50

50

50

50

50

50

50

50

200

50

50

50

50

50

50

50

50

50

50

50

50

100

50

50

50 
zb Name:-"QUUANTERRA MO"

; Code: : ITMO
Contract: $625-01$

Case No.: V.53301 SAS No.:
NSCEP-1381 ztrix: (soil/water) soIL

a ple wt/vol: $\quad 1.00(\mathrm{~g} / \mathrm{mL}) \mathrm{G}$

?-rel: (low/med) LOW

Moisture: not dec.

: Column: RTX-502.2 ID: $0.530(\mathrm{~mm})$

sil Extract Volume:

(UI)
Lab Sample ID: 14533-036

Lab File ID: F0038

Date Received: 05/06/97

Date Analyzed: 05/13/97

Dilution Factor: $\quad 1.0$

Soil Aliquot Volume:

(uL)
CAS NO.
COMPOUND
(ug/L or $\mathrm{ug} / \mathrm{Kg}$ ) UG/KG
$Q$

CONCENTRATION UNITS:

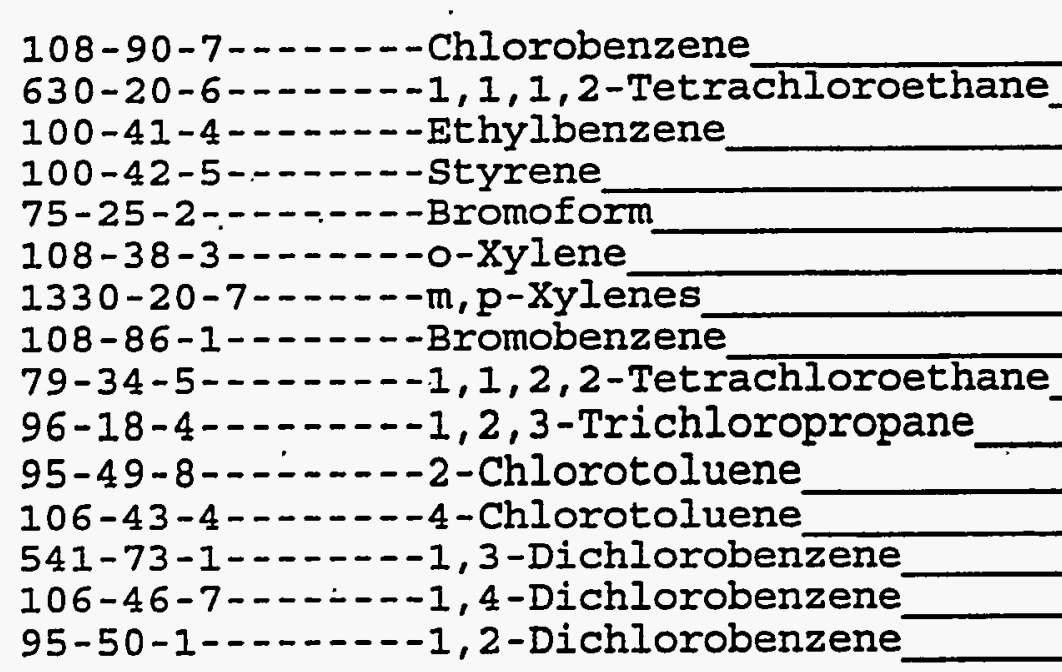


Lab Name:

Lab Code:

Quanterra $\mathrm{kO}$

ITMO Case No.:

Matrix : (8011/water) soIf

Sample wt/vol:

1.0 $(g / m l)$

Level: (low/med)

IOW

Hoisture: not dec.

CAS NO. Compound
(1)
Contract: 625,01 SAS NO.: SDG No.: V205

Lab Sample ID: 14533-02?

Iab F11e ID:

Date sampled:

04-30-97

Date Analyzad: 05-12-97

Dilution Factor: 1.0

. IBH (1) _ I

$\left[\begin{array}{r|}500 \\ \hline\end{array}\right.$

U:Concentration of analyte 18 less than the value given.

$X:$ Pattern reasonably matches gasoline

$Y$ : Pattern appears to be multi-peaks, but does not match gasoline

z:Concentration is primarily from a single peak

(1) Low Boiling Hydrocarbon (IBH)-is quantitated as if it is Gasoline. 


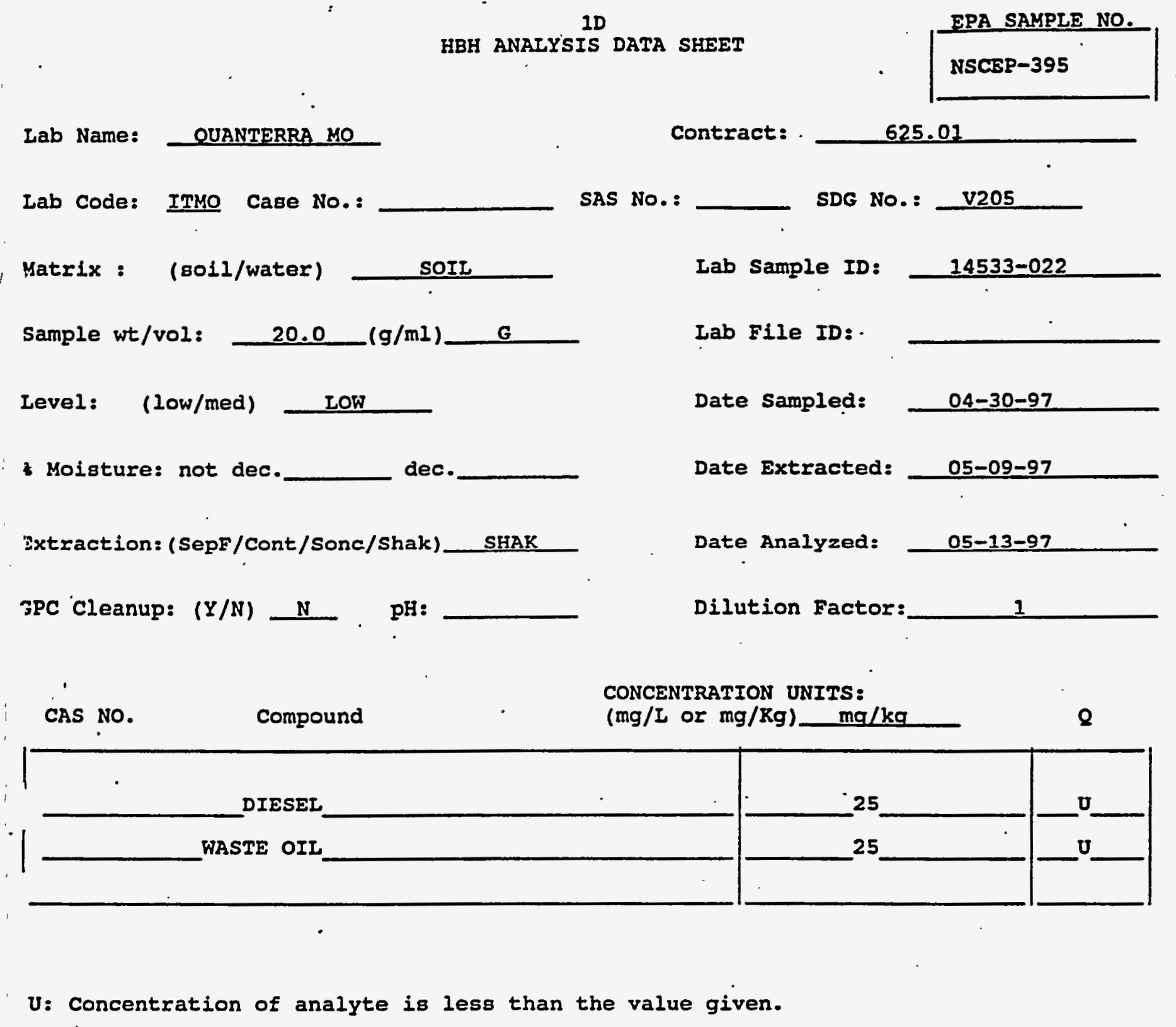

FORY I HBH 
Lab Name:"“OUUANTERRẢ MO

Lab Code: ITMO

Case No.: V53301

Matrix: (soil/water) soII

Sample wt/vol:

$1.00(\mathrm{~g} / \mathrm{mL}) \mathrm{G}$

Level: (low/med) LOW

$\%$ Moisture: not dec.

GC Column: RTX-502.2 ID: 0.530 (mm)

Soil Extract Volume:

CAS NO.
Contract : $625-01$

NSCEP-395

SDG No.: V205

Lab Sample ID: 14533-022

Lab File ID: F0033

Date Received: 05/06/97

Date Analyzed: 05/13/97

Dilution Factor: $\quad 1: 0$

Soil Aliquot Volume:

(uL)

CONCENTRATION UNITS:

(ug/L or $\mathrm{ug} / \mathrm{Kg}$ ) UG/KG

Q

75-71-8--..-Dichlorodifluoromethane

74-83-9--.-.--Bromomethane

75-01-4--.----Vinyl Chloride

75-00-3-..-.--Chloroethane

75-09-2-...--.-Methylene ChIoride

75-69-4-...-.-Trichlorofluoromethane

75-34-3-.----1, 1-Dichloroethane

156-60-5-------trans-1,2-Dichloroethene

156-59-2------cis-1,2-Dichloroethene

107-06-2-..--1, 2-Dichloroethane

594-20-7------2, 2-Dichloropropane

74-97-5-1-.---Bromochloromethane

71-55-6-...-.-1,1,1-Trichloroethane

$78-93-3-\ldots-\ldots-2-$ Butanone

56-23-5-..--.--Carbon Tetrachloride

563-58-6--.--1, 1-Dichloropropene

75-27-4-...---Bromodichloromethane

78-87-5-...-.-1,2-Dichloropropane

10061-01-5-----cis-1, 3-Dichloropropene

79-01-6--.-.--Trichloroethene

79-00-5-...-1, 1, 2-Trichloroethane

$71-43-2--------$ Benzene

74-95-3-..--.-Dibromomethane

10061-02-6-----trans-1,3-Dichloropropene

108-88-3--.----Toluene

106-93-4-..---1,2-Dibromoethane

108-10-1------4-Methyl-2-Pentanone

127-18-4------Tetrachloroethene

142-28-9-..--1,3-Dichloropropane

124-48-1---.--Dibromochloromethane

\begin{tabular}{r|l|}
100 & $U$ \\
100 & $U$ \\
100 & $U$ \\
100 & $U$ \\
50 & $U$ \\
50 & $U$ \\
50 & $U$ \\
50 & $U$ \\
50 & $U$ \\
50 & $U$ \\
50 & $U$ \\
50 & $U$ \\
50 & $U$ \\
200 & $U$ \\
50 & $U$ \\
50 & $U$ \\
50 & $U$ \\
50 & $U$ \\
50 & $U$ \\
50 & $U$ \\
50 & $U$ \\
50 & $U$ \\
50 & $U$ \\
50 & $U$ \\
50 & $U$ \\
50 & $U$ \\
100 & $U$ \\
50 & $U$ \\
50 & $U$ \\
50 & $U$ \\
& \\
50
\end{tabular}


j Name:"“ QUUANTERRA MO

= Code $:$ ITMO

Case No.: V53301

Irix: (soil/water) SOIL

r_le $w t / v o l$ :

$1.00(\mathrm{~g} / \mathrm{mL}) \mathrm{G}$

$\mathrm{v}-1: \quad$ (low/med) LOW

Voisture: not dec.

Olumn: RTX-502.2 ID: 0.530 (mm)

il Extract Volume:

(uI)
Contract: $625-01$
NSCEP-395
SAS NO.: :

Lab Sample ID: 14533-022

Lab File ID: F0033

Date Received: 05/06/97

Date Analyzed: 05/13/97

Dilution Factor: $\quad 1.0$

Soil Aliquot Volume: (UL)

CAS NO.

COMPOUND

CONCENTRATION UNITS:

(ug/L or $\mathrm{ug} / \mathrm{Kg}$ ) UG/KG

Q

\begin{tabular}{|c|}
\hline 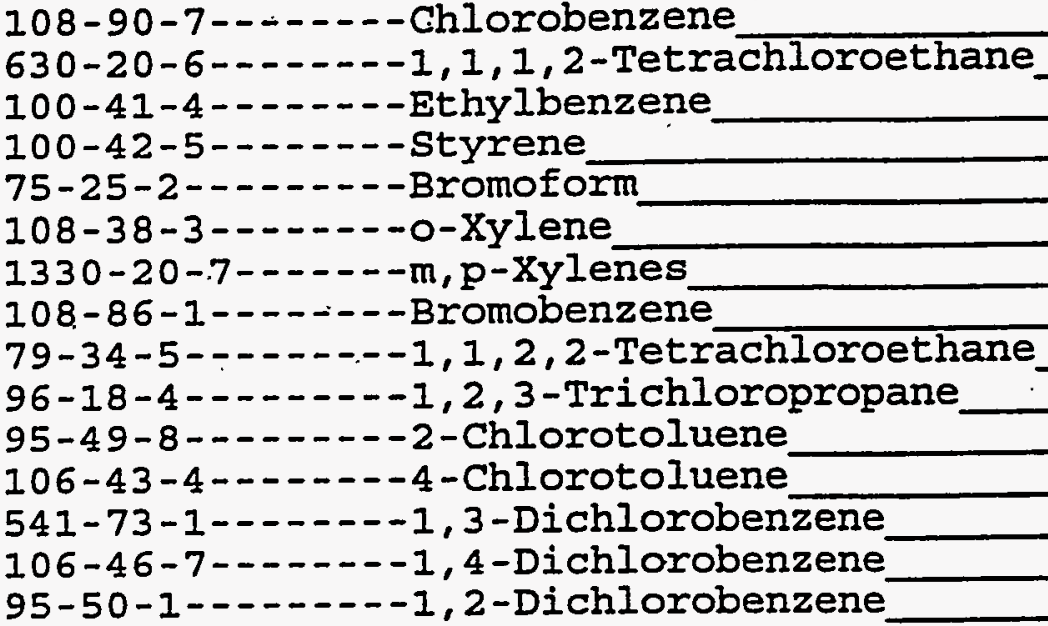 \\
\hline
\end{tabular}

\begin{tabular}{|ll|l|}
\hline & 50 & $\mathrm{U}$ \\
& 50 & $\mathrm{U}$ \\
& 50 & $\mathrm{U}$ \\
& 50 & $\mathrm{U}$ \\
& 50 & $\mathrm{U}$ \\
& 50 & $\mathrm{U}$ \\
& 50 & $\mathrm{U}$ \\
& 50 & $\mathrm{U}$ \\
& 50 & $\mathrm{U}$ \\
& 50 & $\mathrm{U}$ \\
& 50 & $\mathrm{U}$ \\
& 50 & $\mathrm{U}$ \\
& 50 & $\mathrm{U}$ \\
& 50 & $\mathrm{U}$ \\
50 & $\mathrm{U}$ \\
\hline
\end{tabular}


Lab Name:

Lab code: ITYO Case No.:

Hatrix : (ooil/water) soIL

Sample wt/vol: $1.0 \quad(\mathrm{~g} / \mathrm{m} 2)$

G

Level: (low/med) IOW

* Moisture: not dec.

Compound
Contract: $\quad 625.01$ SAS NO.: SDG No.: V205

Lab Sample ID: 14533-023

Lab File ID:

Date Sampiod:

$04-30-97$

Date Analyzed: $05-12-97$

Dilution Factor: 1.0

CONCENTRATION UNITS:

(ug/L or ug/Rg) UG/RG

$\mathbf{Q}$ $\mathbf{0}$ 500

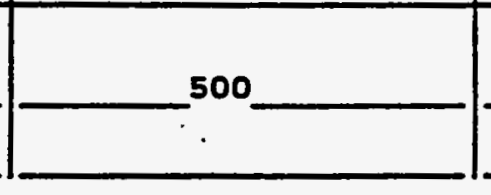

$U:$ Concentration of analyte is less than the value given. $X:$ Pattern reasonably matches gasoline

Y:Pattern appears to be multi-peaks, but does not match gasoline z:Concentration is primarily from a single peak

(1) :Low Boiling Hydrocarbon (IBB) is quantitated as if it is Gasolino. 
Lab Name: OUANTERRA MO

Iab Code: ITMO Case No.:

Matrix : (soil/water)

SOIL

Sample wt/vol: $20.2(\mathrm{~g} / \mathrm{ml})$

$\mathbf{G}$

Level: (low/med) IOW

\& Moisture: not dec. dec. Extraction: (SepF/Cont/Sonc/Shak) SHAR GPC Cleanup: $(\Psi / N)-N \quad \mathrm{NH}:$ H: Contract: SAS NO.: SDG No.: V205

Lab Sample ID: $14533-023$

Iab File ID:

Date Sampled: $04-30-97$

Date Extracted:

05-09-97

Date Analyzed:

05-13-97

Dilution Factor: 1

CONCENTRATION UNITS:

CAS NO. Compound (mg/L or $\mathrm{mg} / \mathrm{kg}$ ) $\mathrm{ma} / \mathrm{kg}$

$\mathbf{Q}$

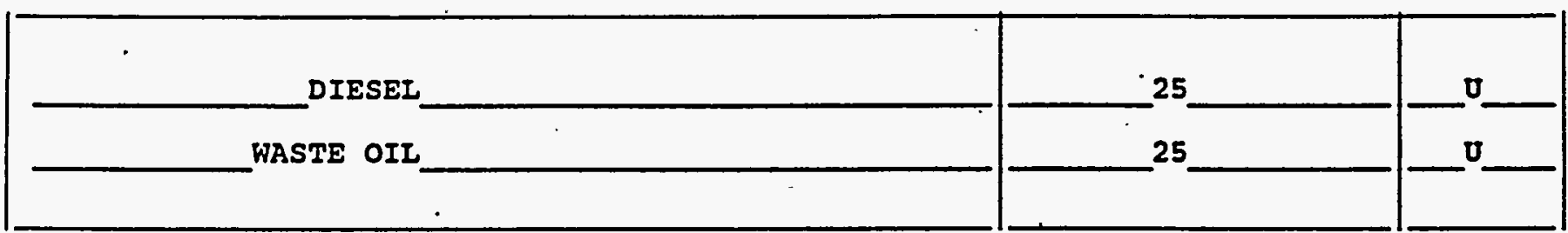

U: Concentration of analyte is less than the value given. 
Lab Name:- QUUANTERRÁ MO

Lab Code: ITMO

Case No.: V53301

Matrix: (soil/water) soII

Sample wt/vol: $\quad 1.00(\mathrm{~g} / \mathrm{mL}) \mathrm{G}$

Level: (low/med) LOW

\% Moisture: not dec.

GC Column: RTX-502.2 ID: 0.530 (mm)

Soil Extract Volume:

(uL)
Contract: $625-01$

SAS NO.:

NSCEP- 397

SDG No.: V205

Lab Sample ID: 14533-023

Lab File ID: Fo023

Date Received: 05/06/97

Date Analyzed: 05/13/97

Dilution Factor: $\quad 1.0$

Soil Aliquot Volume:

(uL

CONCENTRATION UNITS :

CAS NO.

COMPOUND

(ug/L or $\mathrm{ug} / \mathrm{Kg}$ ) UG/KG

Q

75-71-8-...-Dichlorodifluoromethane

74-83-9-...---Bromomethane

75-01-4-...--.-Vinyl Chloride

75-00-3--.----Chloroethane

75-09-2-..----Methylene Chloride

75-69-4-..----.-Trichlorofluoromethane

75-34-3-...-.-1,1-Dichloroethane

156-60-5--.-.-trans-1,2-Dichloroethene

156-59-2--.-.-cis-1, 2-Dichloroethene

107-06-2--.--1,2-Dichloroethane

594-20-7------2,2-Dichloropropane

74-97-5-...---Bromochloromethane

71-55-6--..--1, 1, 1-Trichloroethane

78-93-3-...--2-Butanone

56-23-5-------Carbon Tetrachloride

563-58-6-------1, 1-Dichloropropene

75-27-4-..----Bromodichloromethane.

78-87-5------1,2-Dichloropropane

10061-01-5----cis-1, 3-Dichloropropene

79-01-6--..--.-Trichloroethene

79-00-5-...-. 1, 1,2-Trichloroethane

71-43-2-...---Benzene

74-95-3-..--.-Dibromomethane

10061-02-6-----trans-1,3-Dichloropropene

108-88-3--.-----Toluene

106-93-4-...--1,2-Dibromoethane

108-10-1--.---4-Methyl-2-Pentanone

127-18-4--.-.--Tetrachloroethene

142-28-9-...--1, 3-Dichloropropane

124-48-1-..-.-Dibromochloromethane
100

100

100

100

50

50

50

50

50

50

50

50

50

200

50

50

50

50

50

50

50

50

50

50

50

50

100

50

50

50 
Io Name:" QUUANTERRA MO".

Case No.: V53301

a Code: ITMO

atrix: (soil/water) SOII

a ple wt/vol: $\quad 1.00(\mathrm{~g} / \mathrm{mL}) \mathrm{G}$

errel: (low/med) LoW

"oisture: not dec.

¿ Column: RTX-502.2 ID: 0.530 (mm)

oil Extract Volume:

(UL)
Contract: $625-01$

SAS NO.:
NSCEP -397

SDG No.: V205

Lab Sample ID: 14533-023

Lab File ID: F0023

Date Received: 05/06/97

Date Analyzed: 05/13/97

Dilution Factor: $\quad 1.0$

Soil Aliquot. Volume: (UL)

CONCENTRATION UNITS:

CAS NO.

COMPOUND

(ug/L or ug/Kg) UG/KG

Q

\begin{tabular}{|c|c|c|}
\hline 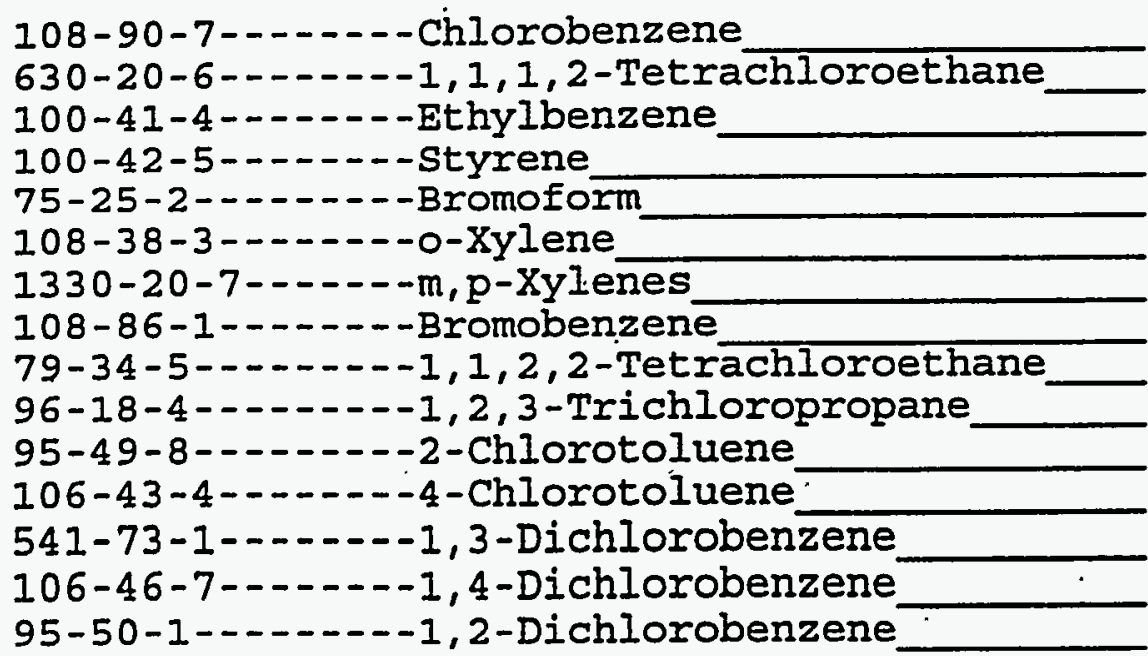 & $\begin{array}{l}50 \\
50 \\
50 \\
50 \\
50 \\
50 \\
50 \\
50 \\
50 \\
50 \\
50 \\
50 \\
50 \\
50 \\
50\end{array}$ & $\begin{array}{l}U \\
U \\
U \\
U \\
U \\
U \\
U \\
U \\
U \\
U \\
U \\
U \\
U \\
U \\
U\end{array}$ \\
\hline
\end{tabular}


Lab Name:

Quanterra, Mo

Iab code: ITYO Case No.:

Hatrix : (Boil/water) sorr

Sample wt/vol:

1.0 $(g / m l)$

$\mathbf{G}$

Level: (low/med)

* Hoisture: not dec.

IOW

CAS NO.
Contract: $\quad 625,01$

SAS NO.:

SDG No.: $\mathbf{Y 2 0 5}$

Iab Sample ID: 14533-024

Lab Flle ID:

Date Sampled: 04-30-97.

Date. Analyzed: 05-12-97

Dilution Factors_1.0

CONCENTRATION UNITS:

(ug/I or ug/Kg) UG/KG

8 $\mathbf{0}$

IBE (I)

500

$+$

U:Concentration of analyte is lesg than the value given.

$x=$ Pattern reasonably matches gasoline.

Y : Pattern appears to be multi-peaks, but does not match gasoline

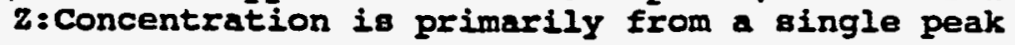

(1):Low Boiling Hydrocarbon (IBE) is quantitated as if it is Gasoline.

FORM I IBE 


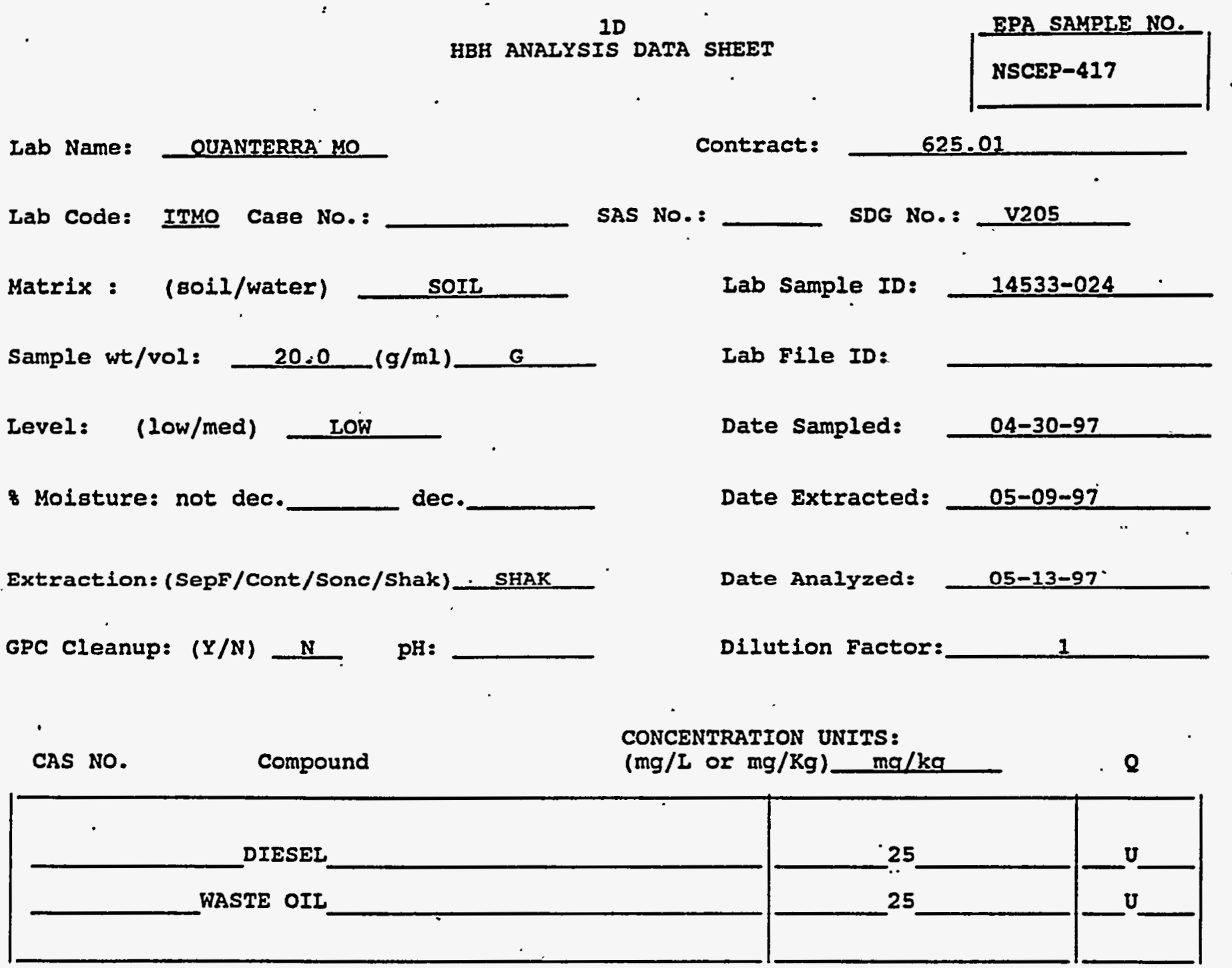

$U$ : Concentration of analyte is less thar the value given. 
VOLATILE ORGANICS ANALYSIS DATA SHEET

Lab Name:- QUUANTERRÁ MO

Lab Code: ITMO

Case No.: V53301

Matrix: (soil/water) soIL

Sample wt/vol:

$1.00(\mathrm{~g} / \mathrm{mL}) \mathrm{G}$

Level: (low/med) LOW

$\%$ Moisture: not dec.

GC Column: RTX-502.2 ID: 0.530 (mm)

Soil Extráct Volume:

(UIs)
Contract $: 625-01$

NSCEP -417
SAS NO.:

SDG No.: V205

Lab Sample ID: 14533-024

Lab File ID: FO024

Date Received: 05/06/97

Date Analyzed: 05/13/97

Dilution Factor: $\quad 1.0$

Soil Aliquot Volume:

(uL)

CONCENTRATION UNITS:

CAS NO.

COMPOUND

Q

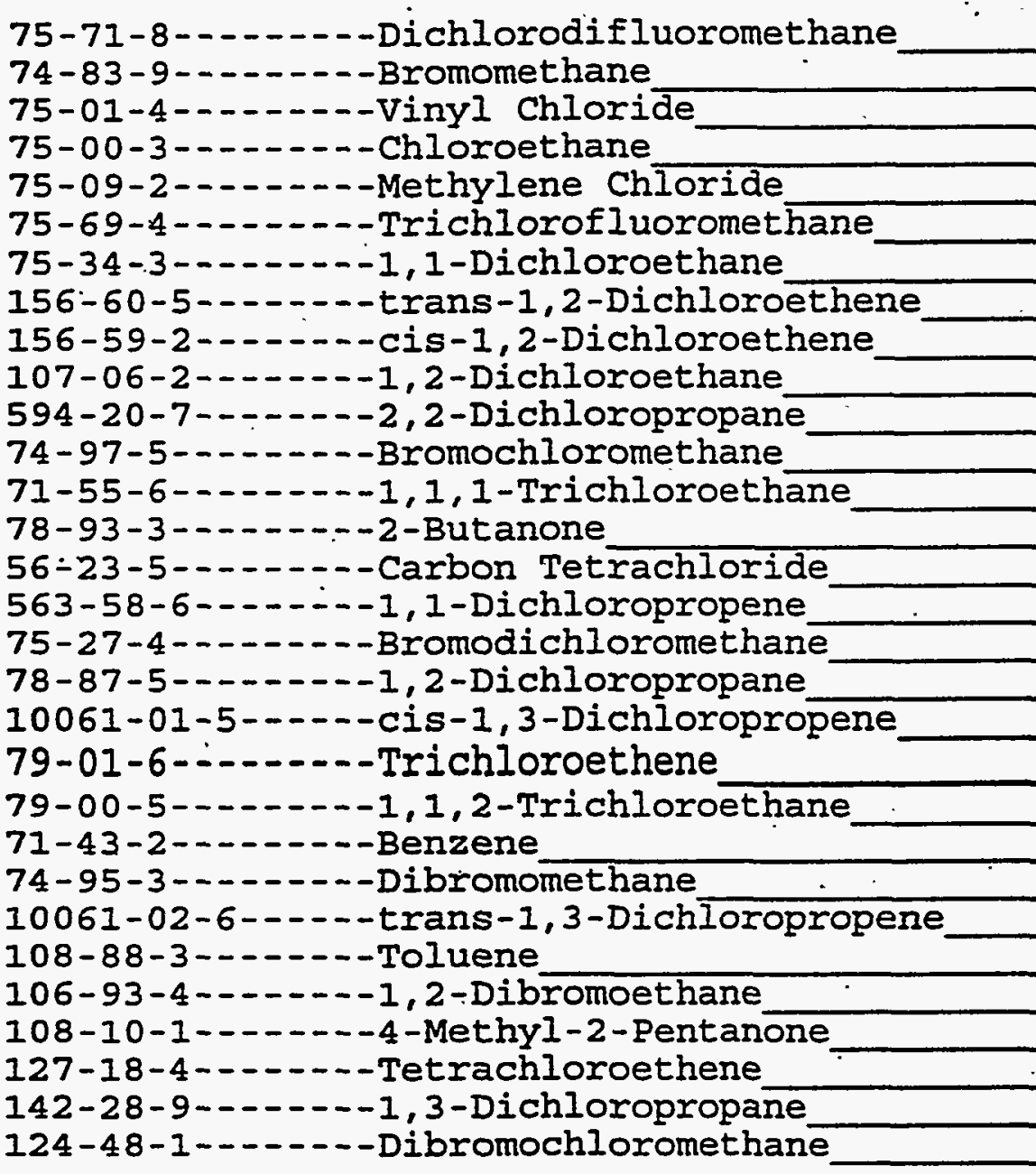


ab Name:- Q̈UANTERRA MO"

₹ Code : ITMO

Case No.: V53301

ztrix: (soil/water) SOII

э ple wt/vol:

$1.00(\mathrm{~g} / \mathrm{mL}) \mathrm{G}$

z-el:

(low/med) Low

Moisture: not dec.

- Column: RTX-502.2. ID: 0.530 (mm)

sil Extract Volume:

(uL)
Contract: 625-01
NSCEP-417
SAS NO.:

SDG No.: V205

Lab Sample ID: 14533-024

Lab File ID: F0024

Date Received: 05/06/97

Date Analyzed: 05/13/97.

Dilution Factor: $\quad . \quad 1.0$

Soil Aliquot Volume:

CAS NO. COMPOUND $\cdot(\mathrm{ug} / \mathrm{L}$ or $\mathrm{ug} / \mathrm{Kg}$ ) UG/KG

Q

\begin{tabular}{|c|c|c|}
\hline 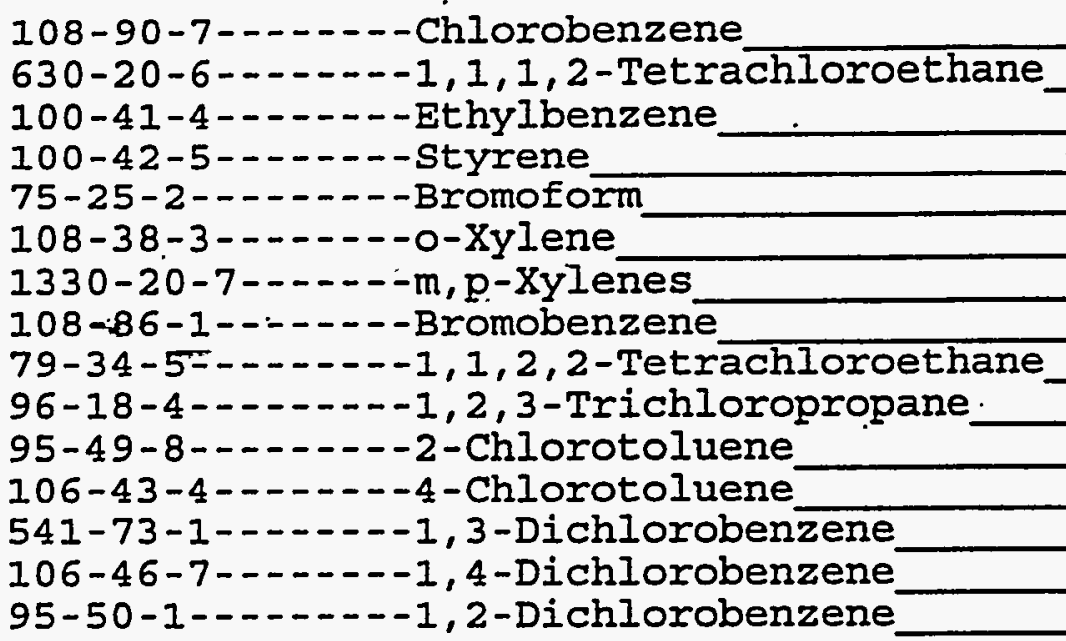 & $\begin{array}{l}50 \\
50 \\
50 \\
50 \\
50 \\
50 \\
50 \\
50 \\
50 \\
50 \\
50 \\
50 \\
50 \\
50 \\
50\end{array}$ & $\begin{array}{l}U \\
U \\
U \\
U \\
U \\
U \\
U \\
U \\
U \\
U \\
U \\
U \\
U \\
U \\
U\end{array}$ \\
\hline
\end{tabular}


1D

LBE ANALYSIS DATA SHEET
EPA SAMPLE NO.

NSCBP -425
Lab Name: Ouanterra, MO

Lab code: ITYO Case No.:

Matrix : (soil/water) soIf

Sample wt/vol:

1.0 $(g / m l)$

G

Level: (Low/med)

IOA

soleture: not dec.

CAS No. contract: 625,01

SAS NO.:

SDG NO.: V205

Iab sample ID: $14533-040$

Iab File IDs

Date Sampled: $\underline{04-30-97}$

Date Analyzed: 05-13-97

Dilution Factor: 1.0
CONCENTRATION UNITS:

(ug/L or ug/Kg) UG/RG
$\mathbf{Q}$ v

LBH (1)

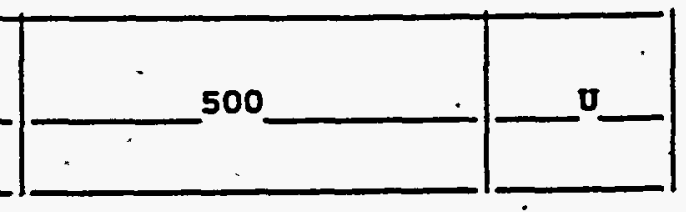

U:Concentration of analyte is less than the value given.

X: Pattern reasonably matches gasoline

$Y:$ Pattern appears to be mult 1 -peaks, but does not match gasoline

$\mathrm{z}$ : Concentration is primarily from a single peak

(1):Low Boiling Hydrocarbon (IBH) is quantitated as if it is Gasoline. 


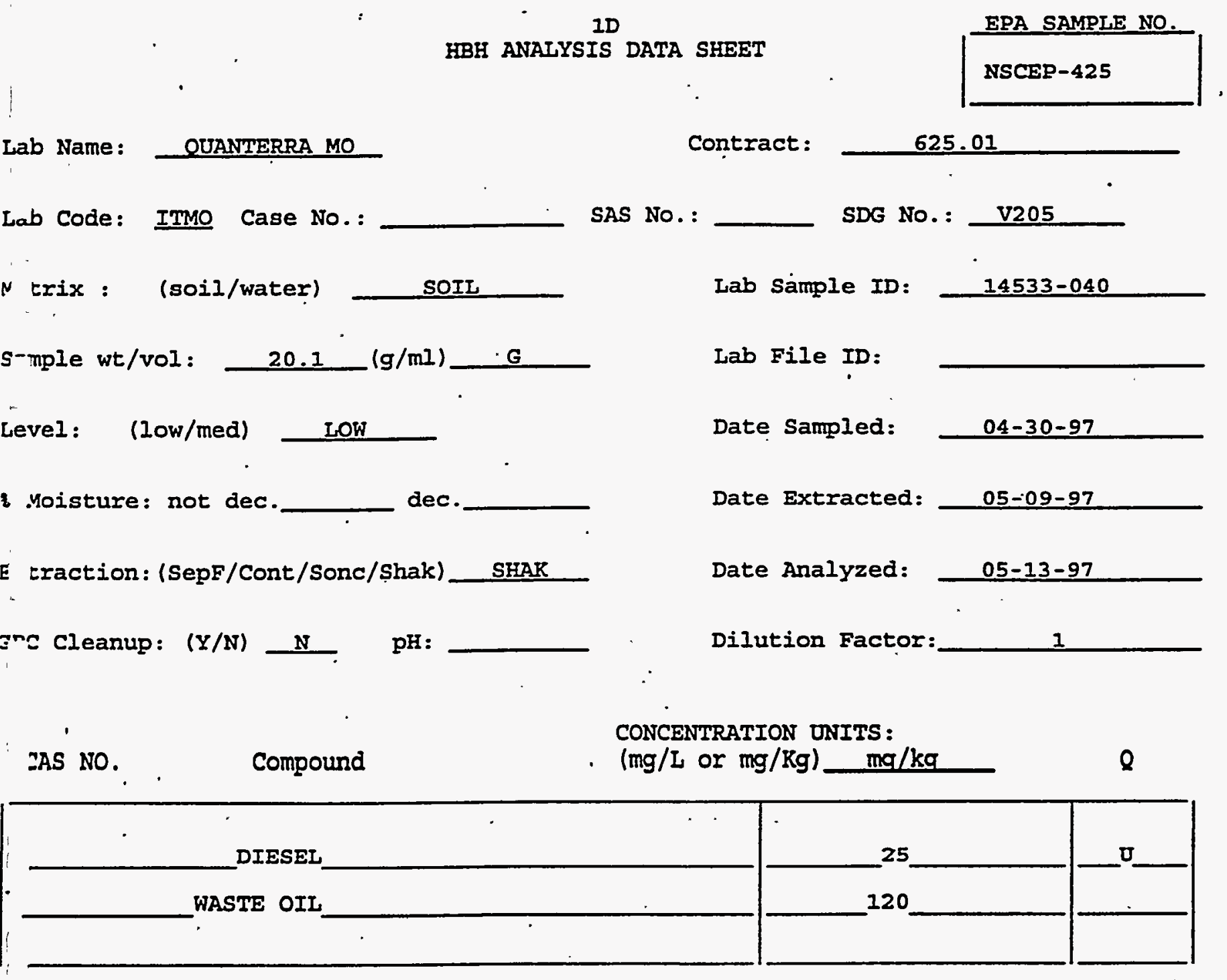

: Concentration of analyte is less than the value given. 
Lab Name:" Q̈UANTERRA MO

Lab Code: ITMO

Case No.: V53301

Matrix: (soil/water) SOIL

Sample wt/vol:

$1.00(\mathrm{~g} / \mathrm{mL}) \mathrm{G}$

Level: (low/med) LOW

\% Moisture: not dec.

GC Column: RTX-502.2 ID: 0.530 (mm)

Soil Extract Volume:

(uI)
Contract: $625-01$

NSCEP -425
SAS NO.:

SDG NO.: V205
Lab Sample ID: 14533-040

Lab File ID: F0042

Date Received: 05/06/97

Date Analyzed: 05/13/97

Dilution Factor: $\quad 1.0$

Soil Aliquot Volume:

(uI

CAS NO.

COMPOUND

CONCENTRATION UNITS :

(ug/L or ug/Kg) UG/KG

Q

75-71-8--..-Dichlorodifluoromethane

74-83-9-..-.--Bromomethane

75-01-4-..--.--Vinyl Chloride

75-00-3-..--.-Chloroethane

75-09-2--.-.-Methylene Chloride

75-69-4-..---Trichlorof luoromethane

75-34-3--.---1, 1-Dichloroethane

156-60-5------trans-1, 2-Dichloroethene

156-59-2------cis-1, 2-Dichloroethene

107-06-2------1, 2-Dichloroethane

594-20-7------2,2-Dichloropropane

74-97-5--------Bromochloromethane

71-55-6-..---1, 1, 1-Trichloroethane

$78-93-3-1-----2-$ Butanone

56-23-5--..---Carbon Tetrachloride

563-58-6-.-.-1,1-Dichloropropene

75-27-4-..-.--Bromodichloromethane

78-87-5---.--1, 2-Dichloropropane

10061-01-5----cis-1,3-Dichloropropene

79-01-6--.----Trichloroethene

79-00-5-...-1, 1,2-Trichloroethane

71-43-2---- - - Benzene

74-95-3-...--Dibromomethane

10061-02-6--.--trans-1,3-Dichloropropene

108-88-3-...---Toluene

106-93-4-...-1, 2-Dibromoethane

108-10-1--.---4-Methyl-2-Pentanone

127-18-4-------Tetrachloroethene

142-28-9--.---1, 3-Dichloropropane

124-48-1--.----Dibromochloromethane

\begin{tabular}{r|l|}
100 & $U$ \\
100 & $U$ \\
100 & $U$ \\
100 & $U$ \\
50 & $U$ \\
50 & $U$ \\
50 & $U$ \\
50 & $U$ \\
50 & $U$ \\
50 & $U$ \\
50 & $U$ \\
50 & $U$ \\
50 & $U$ \\
200 & $U$ \\
50 & $U$ \\
50 & $U$ \\
50 & $U$ \\
50 & $U$ \\
50 & $U$ \\
50 & $U$ \\
50 & $U$ \\
50 & $U$ \\
50 & $U$ \\
50 & $U$ \\
50 & $U$ \\
50 & $U$ \\
100 & $U$ \\
50 & $U$ \\
50 & $U$ \\
50 & $U$ \\
& \\
\hline 0
\end{tabular}

000237 
ab Name: "Q̈UANTERRA MO

a Code: ITMO . Case No.: V53301

atrix: (soil/water) soIL

$\exists$ ple wt/vol:

$1.00(\mathrm{~g} / \mathrm{mL}) \mathrm{G}$

ఇ-el: (Iow/med) LOW

Moisture: not dec.

$\approx$ Column: RTX-502.2 ID:

$0.530(\mathrm{~mm})$

sil Extract Volume:

(uI)
Contract: $625-01$

SAS NO.:
NSCEP -425

SDG NO.: V205

Iab Sample ID: 14533-040

Lab File ID: F004i

Date Received: · 05/06/97

Date Analyzed: 05/13/97

Dilution Factor: $\quad 1.0$

Soil Aliquot Volume:

(uI)

CONCENTRATION UNITS :

CAS NO.

COMPOUND

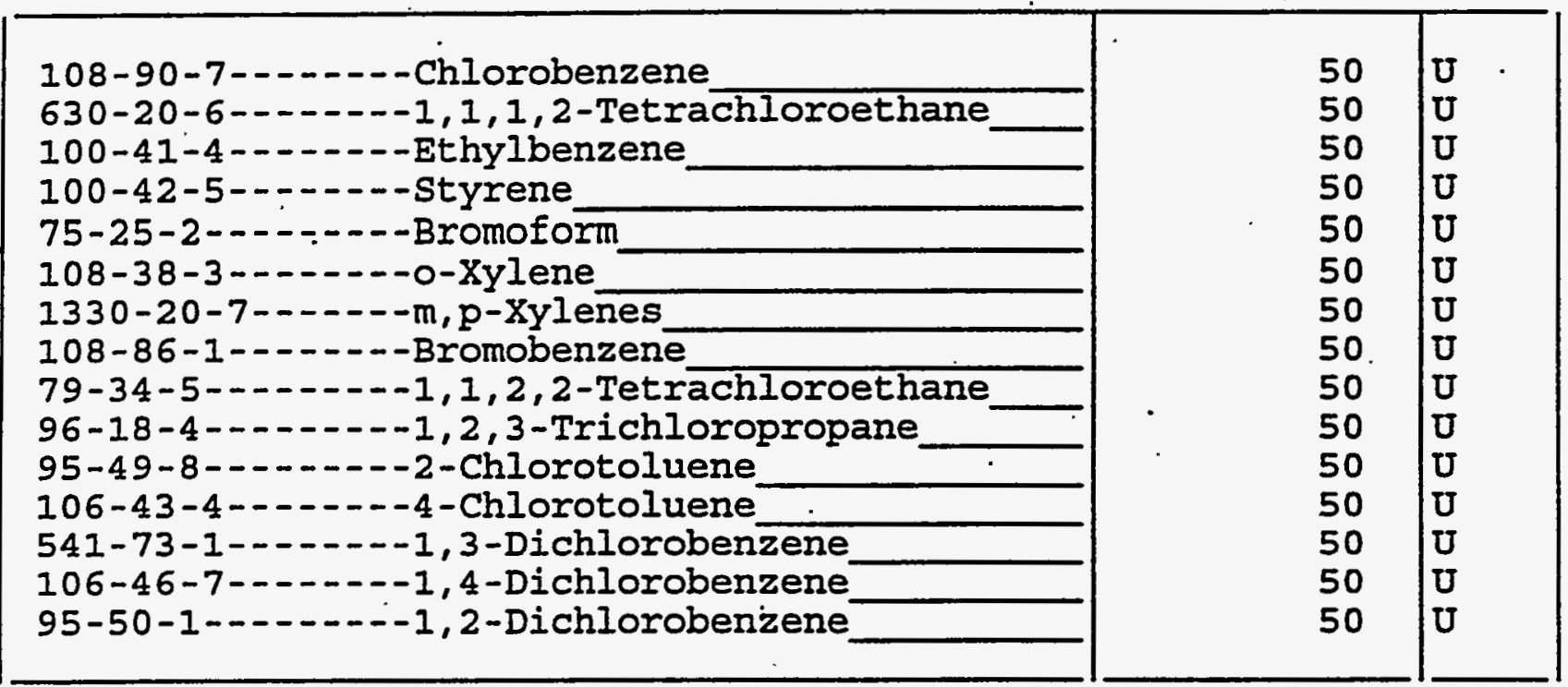


Lab Names

Ouanterra kO

Iab code: ITKO Cage No.:

Hatrix : (goil/water) soII

Sample wt/vol:

1,0 $(9 / \mathrm{ml}$ G

Ievel: (low/med) IOF

soisture: not dec.
Contract: 625.01

SAS NO.: SDG NO.: V205

Lab Sample ID: 14533-041

Lab PII0 ID:

Dato Sampled: 04-30-97

Date Analyzed: 05-13-97

Dilution Factor: 1.0
CONCENTRATION UNITS: (ug/L or ug/Rg) UG/KG

U:Concentration of analyte is less than the value given. $X:$ Pattern reasonably matches gasoline

Y:Pattern appears to be mult1-peaks, but does not match gasoline $\mathrm{z}$ : Concentration is primarily from a. Bingle peak

(1) :Low Boiling Bydrocarbon (IBH) is quantitated as if. it is Gasoline. 
BPA SAMPLE NO, NSCEP -426

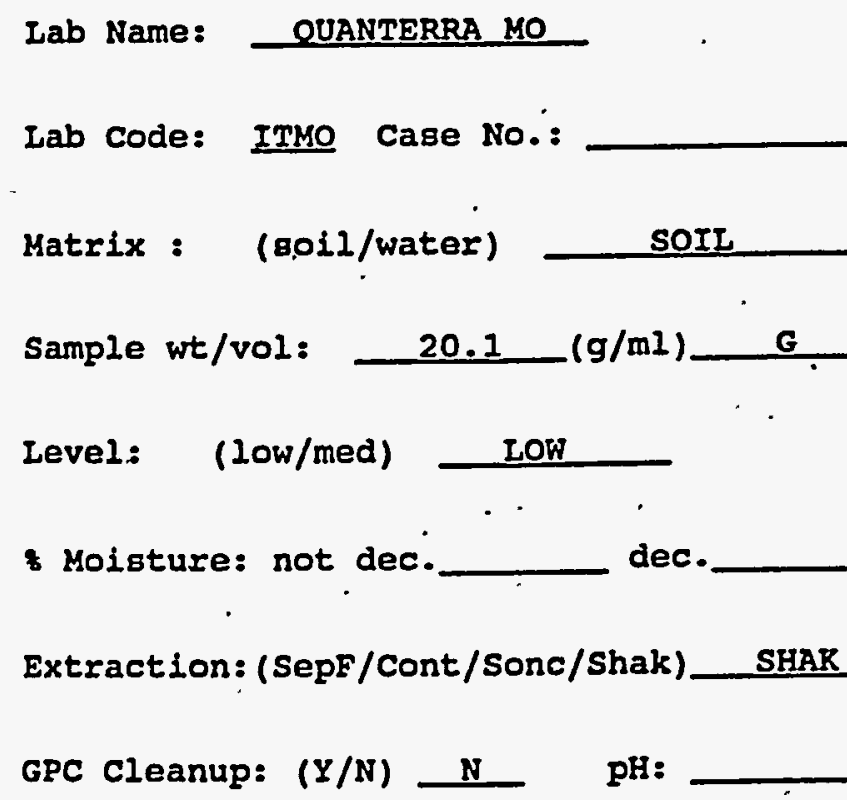

$\mathbf{2}$

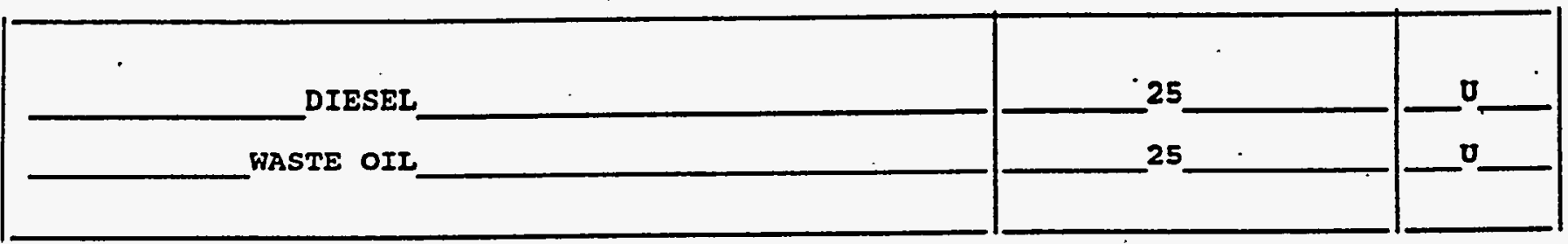

$U$ : Concentration of analyte is less than the value given. 
Lab Name :- ÖUANTERRÄ MO

Lab Code: ITMO

Case No.: V5330.1

Matrix: (soil/water) SOIL

Sample wt/vol: $\quad 1.00(\dot{g} / \mathrm{mL}) \mathrm{G}$

Level: (low/med) LOW

\% Moisture: not dec.

GC Column: RTX-502.2 ID: $0.530(\mathrm{~mm})$

Soil Extract Volume:

(UL)
Contract: $625-01$

SAS NO.:

SDG NO.: V205
Lab Sample ID: 14533-041

Lab-File ID: F0043

Date Received: 05/06/97

Date Analyzed: $05 / 13 / 97$

Dilution Factor: $\quad 1.0$

Soil Aliquot Volume:

(uI

CAS NO. COMPOUND (ug/L or ug/Kg) UG/KG

CONCENTRATION UNITS:
(ug/L or ug/ $/ \mathrm{kg}$ ) UG/KG

\begin{tabular}{|c|c|c|}
\hline 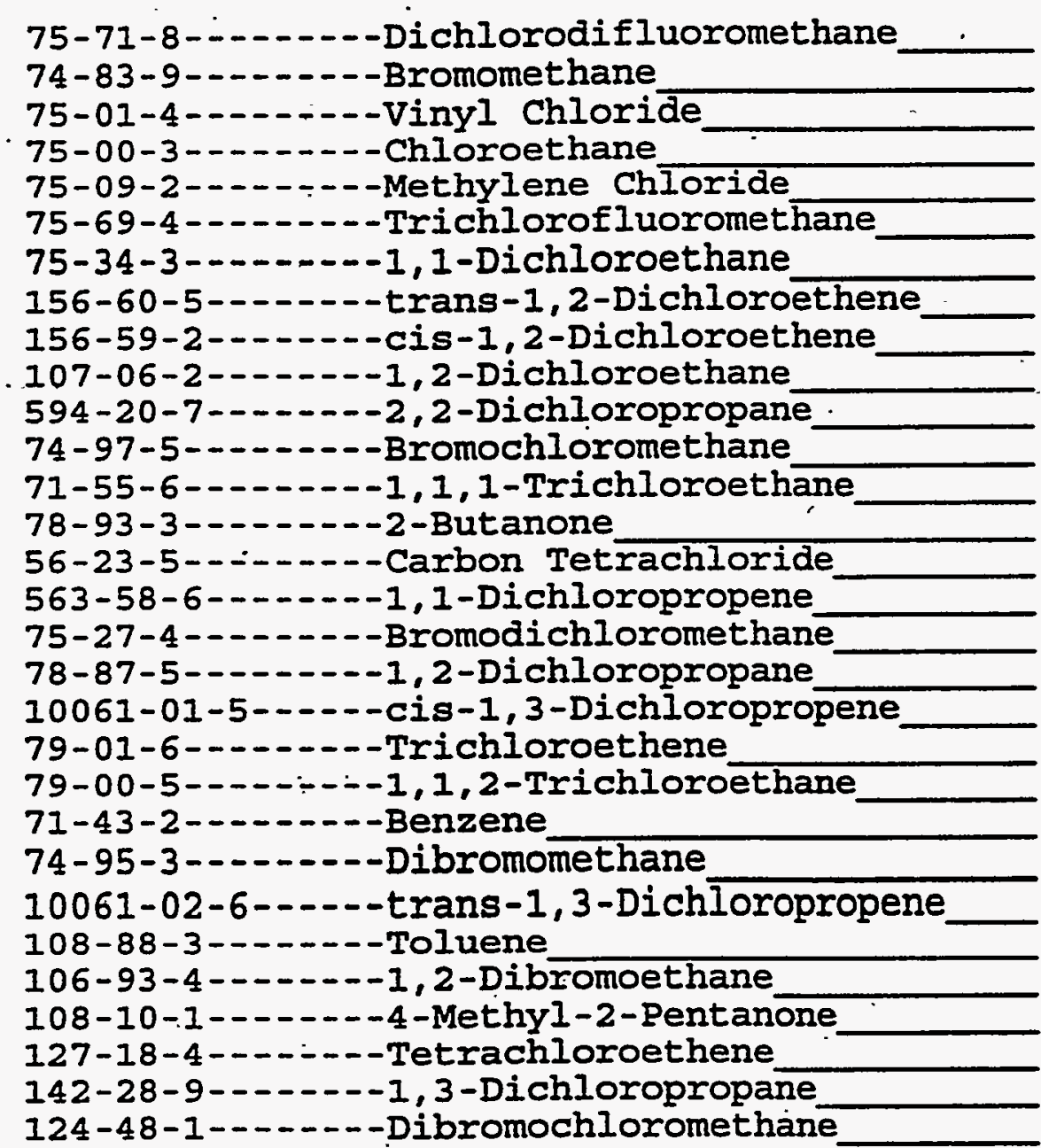 & $\begin{array}{r}100 \\
100 \\
100 \\
100 \\
50 \\
50 \\
50 \\
50 \\
50 \\
50 \\
50 \\
50 \\
50 \\
200 \\
50 \\
50 \\
50 \\
50 \\
50 \\
50 \\
50 \\
50 \\
50 \\
50 \\
50 \\
50 \\
100 \\
50 \\
50 \\
50\end{array}$ & \begin{tabular}{|l}
$U$ \\
$U$ \\
$U$ \\
$U$ \\
$U$ \\
$U$ \\
$U$ \\
$U$ \\
$U$ \\
$U$ \\
$U$ \\
$U$ \\
$U$ \\
$U$ \\
$U$ \\
$U$ \\
$U$ \\
$U$ \\
$U$ \\
$U$ \\
$U$ \\
$U$ \\
$U$ \\
$U$ \\
$U$ \\
$U$ \\
$U$ \\
$U$ \\
$U$ \\
$U$
\end{tabular} \\
\hline
\end{tabular}


aD Name:" QUUANTERRA MO"

₹ Code: ITMO

Case No.: V53301

atrix: (soil/water) soIL

a ple wt/vol: $\quad 1.00(\mathrm{~g} / \mathrm{mL}) \mathrm{G}$

əarel: (low/med) LOW.

moisture: not dec.

¿ Column: RTX-502.2 ID: $0.530 .(\mathrm{mm})$

jil Extract Volume:

(uL)
Contract: $625-01$

SAS NO.:

SDG No.: V205

Lab Sample ID: 14533-047

Lab File ID: F0043

Date Received: 05/06/.97

Date Analyzed: 05/13/97

Dilution Factor: $\quad 1.0$

Soil Aliquot volume: . (uL)

CAS NO. COMPOUND (ug/L or ug/Kg) UG/KG

Q

$108-90-7-\ldots-$ - Chlorobenzene

$630-20-6 \ldots \ldots-1,1,1,2$-Tetrachloroethane

100-41-4-.----Ethylbenzene

100-42-5-.-.---Styrene

75-25-2-...---Bromoform

108-38-3--.---o-Xylene

$1330-20-7-\cdots---m, p-x y l e n e s$

108-86-1-...--Bromobenzene

79-34-5-...-1, 1,2,2-Tetrachloroethane

96-18-4-------1,2,3-Trichloropropane

95-49-8--.---2-Chlorotoluene

106-43-4--.---4-Chlorotoluene

541-73-1------1,3-Dichlorobenzene

106-46-7--.--1, 4-Dichlorobenzene

95-50-1-2.-.-.-1,2-Dichlorobenzene 
Iab Name:

Ouanterracko

Iab Code: ITYO Case NO.:

Matrix : (soil/water) soIL

Sample wt/vol:

1.0 $(g / m l)$ G

Leve1: (Low/med) . LOW

soisture: not dec.

CAS NO.

Compound
Contract: 625.01

SDG NO.: V205

SAS NO.:

Iab Sample ID: 14533-025

Lab File ID:

Date Sampled: $\quad 04-30-97$

Date Analyzed: $\quad 05-12-97$

Dilution Factor: 1.0

CONCENTRATION UNITS:

(ug/L or ug/kg) UG/RG

$\mathbf{Q}$

LBE (1)

500 $\mathbf{0}$

$U:$ Concentration of analyte is less than the value given.

$X$ : Pattern reasonably matches gasoline.

Y:Pattern appears to be multi-peaks, but does not match gasoline

z:Concentration is primarily from a single peak

(1): Low Boiling Hydrocarbon (IBH) is quantitated as if it is Gasoline. 


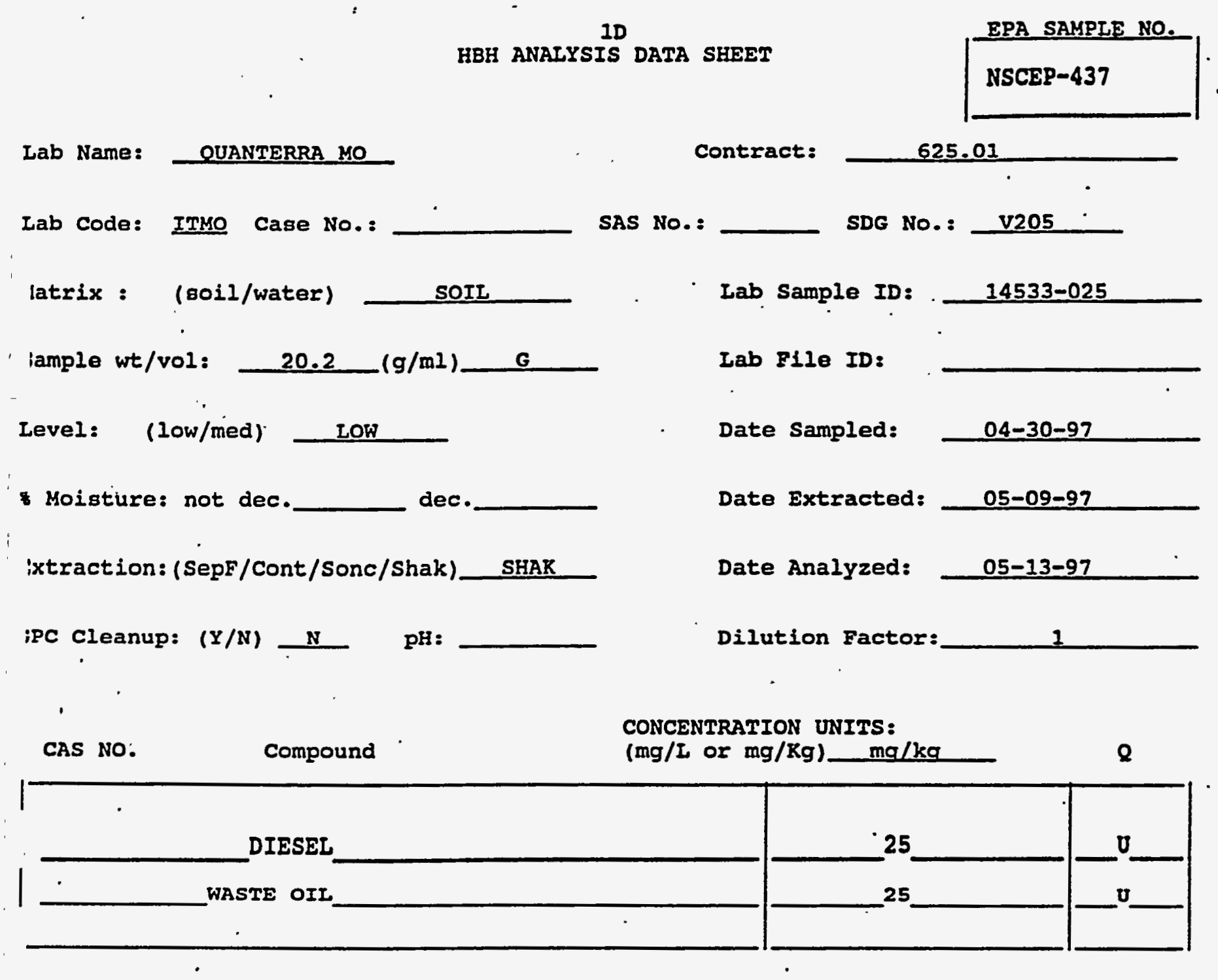

$U$ : Concentration of analyte is less than the value given. 
Lab Name:- QUUANTERRÁ MO

Lab Code: ITMO

Case No.: V53301

Matrix: (soil/water) SOIL

Sample wt/vol:

$1.00(\mathrm{~g} / \mathrm{mL}) \mathrm{G}$

Level: (low/med) LOW

\% Moisture: not dec.

GC Column: RTX-502.2 ID: 0.530 (mm) .

Soil Extract Volume:

CAS NO.

COMPOUND

(ius)
Contract: $625-01$

SAS NO.:

NSCEP -437

SDG NO.: V205

Lab Sample ID: 14533-025

Lab File ID: F0034

Date Received: 05/06/97

Date Analyzed: 05/13/97

Dilution Factor: $\quad 1.0$

Soil Aliquot Volume:

(uL)

CONCENTRATION UNITS:

(ug/L or $\mathrm{ug} / \mathrm{Kg}$ ) UG/KG

\begin{tabular}{|c|}
\hline 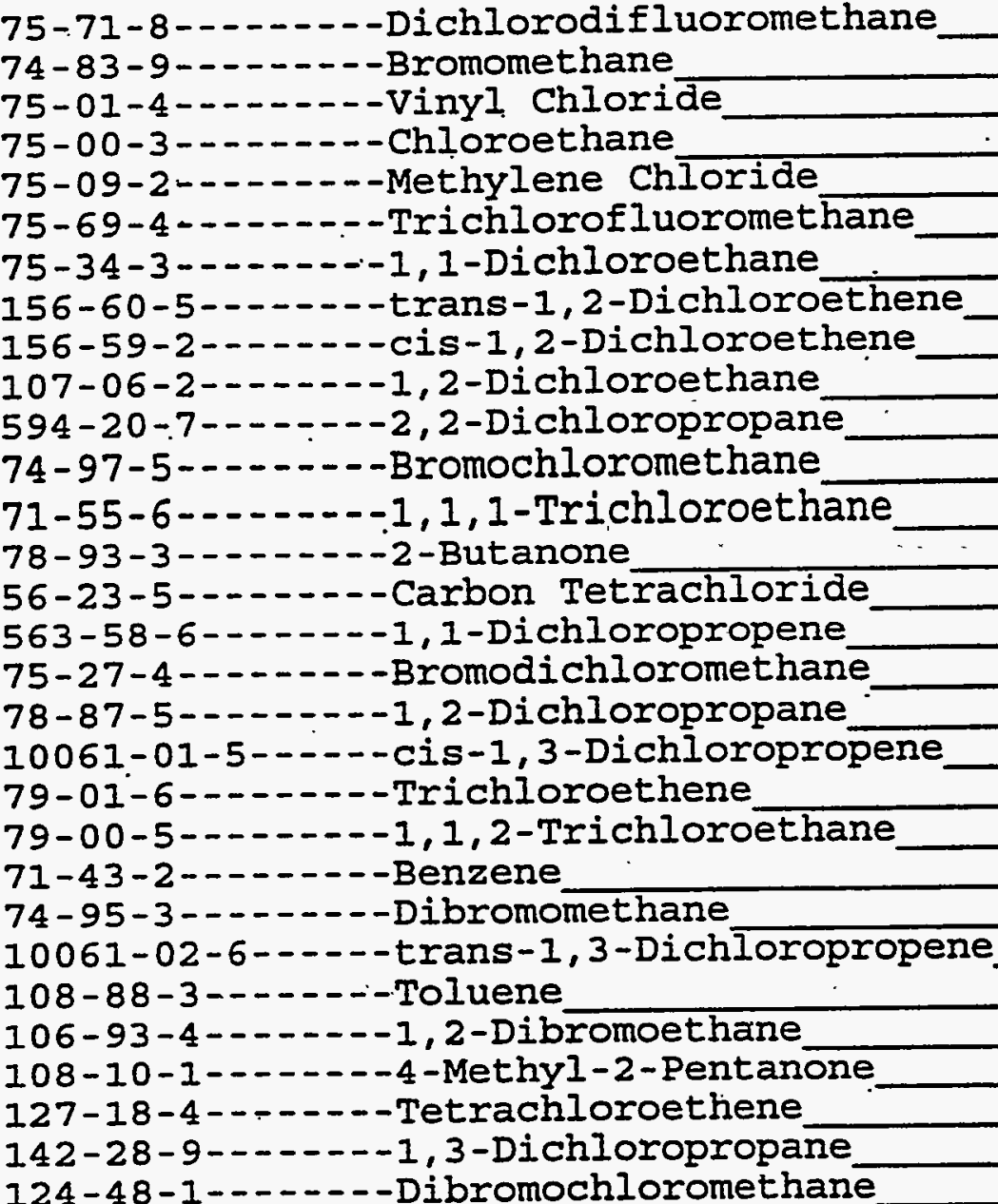 \\
\hline
\end{tabular}

100

100

100

100

50

50

50

50

50

50

50

50

50

200

50

50

5.0

50

50

50

50

50

50

50

50

50

100

50

50

50 
saw Name:- QUUANTERRA MO

¿ , Code: ITMO

Case No.: V53301

latrix: (soil/water) soIL

j, nple wt/vol:

$1.00(\mathrm{~g} / \mathrm{mL}) \mathrm{G}$

Contract: $625-01$

SAS NO.:

NSCEP -437

SDG No.: V205

Iab Sample ID: 14533-025

Lab File ID: F0034

Date Received: 05/06/97

Date Analyzed: 05/13/97

.oisture: not dec.

Dilution Factor: $\quad 1.0$

I1 Column: RTX-502.2 ID: 0.530 (mm)

(uL)

Soil Aliquot Volume:

(UI)

joil Extract Volume:

(uI)

CONCENTRATION UNITS:

CAS NO.

COMPOUND

(ug/L or $\mathrm{ug} / \mathrm{Kg}$ ) UG/KG

Q

50

50

50

50

50

50

50

50

$50^{\circ}$

50

50

50

50

50

50 
Lab Name: Quanterra,MO

Lab Code: ITMO Case No.:

Matrix : (soil/water) soII

Sample wt/vol:

1.0

$(\mathrm{g} / \mathrm{ml})$

G

Level: (low/med)

LOW

soisture: not dec.

CAS NO.

Compound

IBH (1)
Contract: 625.01

SAS No.:

SDG NO.: V205

Lab Sample ID: 14533-039

Iab File IDs

Date Sampled: $04-30-97$

Date Analyzed: 05-13-97

Dilution Factor: 1.0
CONCENTRATION UNITS:

(ug/L or ug/Kg) UG/RG

\begin{tabular}{|l|l|l|l|}
\hline IBH (3) & 500 \\
\hline
\end{tabular}

$U$ : Concentration of analyte is less than the value given.

$X:$ Pattern reasonably matches gasoline

Y: Pattern appears to be multi-peaks, but does not match gasoline

2:Concentration is primarily from a single peak

(1): Low Boiling Hydrocarbon (LBH) is quantitated as if it is Gasoline.

FORE I IBE 
1D

HBH ANALYSIS DATA SHEEI
EPA SAMPLE NO. NSCEP-444
Ib Name: QUANTERRA MO

Lab Code: ITMO Cage No.:

1trix : (Bo11/water)

SOIL

umple wt/vol:

$20.1 \quad(\mathrm{~g} / \mathrm{ml})$

G

Level: (low/med)

LOW

\& Moisture: not dec. dec.

straction: (SepF/Cont/Sonc/Shak)

SHAR

?C Cleanup: $(Y / N)$

pH: contract:

SAS NO.:

Lab Sample ID:

$14533-039$

Lab File ID:

Date Sampled:

$04-30-97$

Date Extracted:

05-09-97

Date Analyzed:

$05-13-97$

Dilution Factor: 1

CONCENTRATION UNITS:

CAS NO.'

Compound

$(\mathrm{mg} / \mathrm{L}$ or $\mathrm{mg} / \mathrm{Kg}) \ldots \mathrm{mg} / \mathrm{kg}$

2

DIESEI

WASTE OIL

•

$\left[\begin{array}{l}25 \\ \hline 25-\ldots+\end{array}\right.$

$\mathrm{U:}$ Concentration of analyte is less than the value given. 
Lab Name:-"Q̈UANTERRA MO

Lab Code: ITMO

Case No.: V53301

Matrix: (soil/water) soIL

Sample wt/vol:

$1.00(\mathrm{~g} / \mathrm{mL}) \mathrm{G}$

Level : . (low/med) LOW

$\div$ Moisture: not dec.

GC Column: RTX-502.2 ID: .0 .530 (mm)

Soil Extract Volume:

CAS NO.
Contract: $625-01$

SAS NO.:

SDG No.: V205

Lab Sample ID: 14533-039

Lab File ID: F004i

Date Received: 05/06/97

Date. Analyzed: $05 / 13 / 97$

Dilution Factor: $\quad 1.0$

Soil Aliquot Volume:

(uL
CONCENTRATION UNITS:

(ug/I or ug/Kg) UG/KG
75-71-8---.--Dichlorodifluoromethane

74-83-9-....--Bromomethane

75-01-4-..-.-.-Vinyl-Chloride

75-00-3-.-.-.--Chloroethane

75-09-2-..-.--Methylene Chloride

75-69-4------- Trichlorofluoromethane

75-34-3-..--1, 1-Dichloroethane

156-60-5-...--trans-1,2-Dichloroethene

156-59-2------Cis-1, 2-Dichloroethene

107-06-2-1.-.-1,2-Dichloroethane

594-20-7-..---2, 2-Dichloropropane

74-97-5------Bromochlorometharie

71-55-6-..---1, 1, 1-Trichloroethane

78-93-3-------2-Butanone

56-23-5-------Carbon Tetrachloride

563-58-6------1,1-Dichloropropene

75-27-4--.---Bromodichloromethane

78-87-5-------1, 2-Dichloropropane

10061-01-5-----cis-1,3-Dichioropropene

79-01-6-...--Trichloroethene

79-00-5--.---1, 1, 2-Trichloroethane

71-43-2-.-----Benzene

74-95-3-..--Dibromomethane

10061-02-6--.--trans-1,3-Dich loropropene

108-88-3--.----Toluene

106-93-4-----1,2-Dibromoethane

108-10-1--.---4-Methyl-2-Pentanone

127-18-4-...--Tetrachloroethene

142-28-9-.---1, 3-Dichloropropane

124-48-1-..--Dibromochloromethane
100

100

100

100

50

50

50

50

50

50

50

50

50

200

50

50

50

50

50

50

50

50

50

50

50

50

100

50

50

50

Q 
ao Name:"QUUANTERRA MO"

a Code: ITMO Case No.: V53301 SAS No.:
NSCEP -444 atrix: (soil/water) SOIL

a ple wt/vol:

$1.00(\mathrm{~g} / \mathrm{mL}) \mathrm{G}$

errel: (low/med) LOW

roisture: not dec.

こ Column: RTX-502.2 ID: $0.530(\mathrm{~mm})$

jil Extract Volume:

(uL)
Contract: $625-01$
SDG NO.: V205

$-444$

\begin{abstract}
Jil Extract Volume:
\end{abstract}
CAS NO.

COMPOUND

Lab Sample ID:

$14533-039$

Lab File ID: F0041

Date Received: 05/06/97

Date Analyzed: 05/13/97

Dilution Factor: 1.0

Soil Aliquot Volume:

(uI)

CONCENTRATION UNITS: (ug/L or $\mathrm{ug} / \mathrm{Kg}$ ) UG/KG

Q

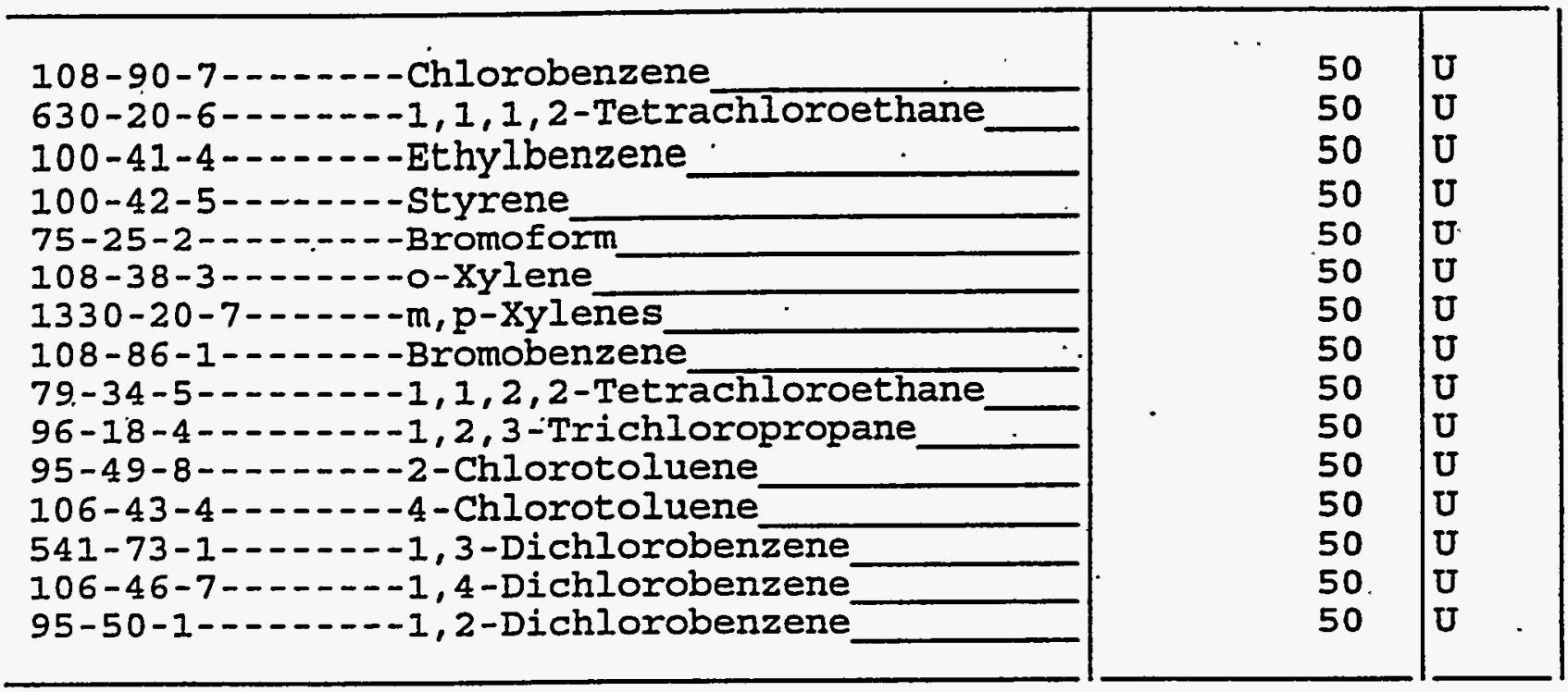


EPA SAMPIE NO. NSCBP -485
Lab Names
Lab codes Imyo Case No.:

Matrix \&. (goll/water) soIr

Sample wt/vol:

$1.0 \quad(\mathrm{~g} / \mathrm{ml})$ G

Ievel: (Low/med) IOW

\& Hoisture: not dec. ION ".

CAS NO.
Contract: 625.01

SDG HO.: V205

SAS NO.:

Iab Sample ID: 14533-026

Iab File ID:

Date sampled: $\quad 04-30-97$

Date Analyzed: 0, 05-12-97

Dilution Factor: 1.0
CONCENTRATION UNITS:

(ug/L or ug/ $\mathrm{kg}$ ) UG/RG

LBH (1)

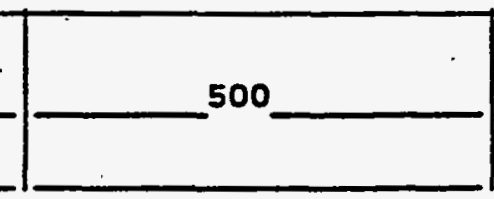

U:Concentration of analyte is legs than the value given. $X: P a t t e r n$ reasonably matches gasoline Y:Pattern appears to be multi-peaks, but does not match gasoline Z:Concentration is primarily from a single peak

(1) Low Boiling Bydrocarbon (IBE) is quantitated as if it is Gasoline. 
1D

HBE ANALYSIS DATA SHEET
EPA SAMPLE NO.

NSCEP -485
T,ab Name: OUANTERRA MO
Lab Code: ITMO Case No.:

iatrix : (soil/water)

soIt

iample wt/vol: $20.2 \quad(\mathrm{~g} / \mathrm{ml})$

G

Level: (low/med)

LOW

soisture: not dec. dec.

'xtraction: (SepF/Cont/Sonc/Shak)_ SHAK iPC Cleanup: $(\Psi / N)$ N CAS NO. Compound Contract: SAS NO.: SDG NO.:

Lab Sample ID: $14533-026$

Lab File ID:

Date sampled:

$04-30-97$

Date Extracted:

$05-09-97$

Date Analyzed:

$05-13-97$

Dilution Factor: 1

CONCENTRATION UNITS:

(mg/L or $\mathrm{mg} / \mathrm{Kg}$ ) $\mathrm{mg} / \mathrm{kg}$

$\mathbf{a}$

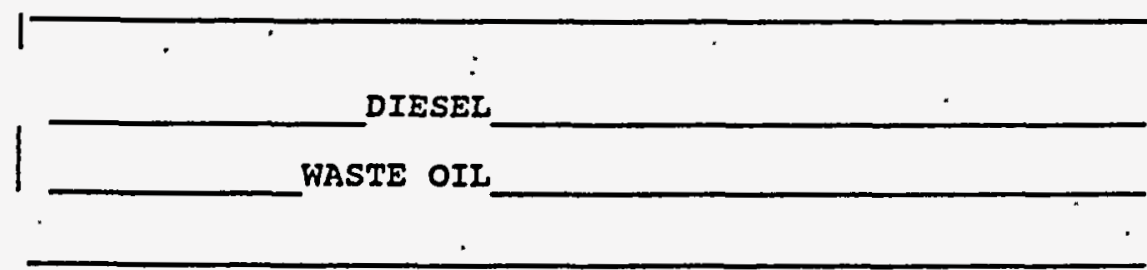

$\square^{25}+{ }_{-}^{25}+{ }_{-}$

$U$ : Concentration of analyte is less than the value given. 
Läb Name:"QUUANTERRȦ MO

Lab Code: ITMO . Case No.: V53301

Matrix: (soil/water) soIL

Sample wt/vol: $\quad 1.00(\mathrm{~g} / \mathrm{mL}) \mathrm{G}$

Level: (low/med) Low

$\%$ Moisture: not dec.

GC Column:-RTX-502.2 ID: $0.530(\mathrm{~mm})$

Soil Extract Volume:

(uL)
Contract: $625-01$.

NSCEP -485
SAS NO.:

SDG NO.: V205
Lab Sample ID: 14533-026

Lab File ID: F0026

Date Received: 05/06/97

Date Analyzed: 05/13/97

Dịlution Factor: $\quad 1.0$

Soil Aliquot Volume: (uI CONCENTRATION UNITS :-

CAS NO. COMPOUND (ug/L or ug/Kg) UG/KG

Q

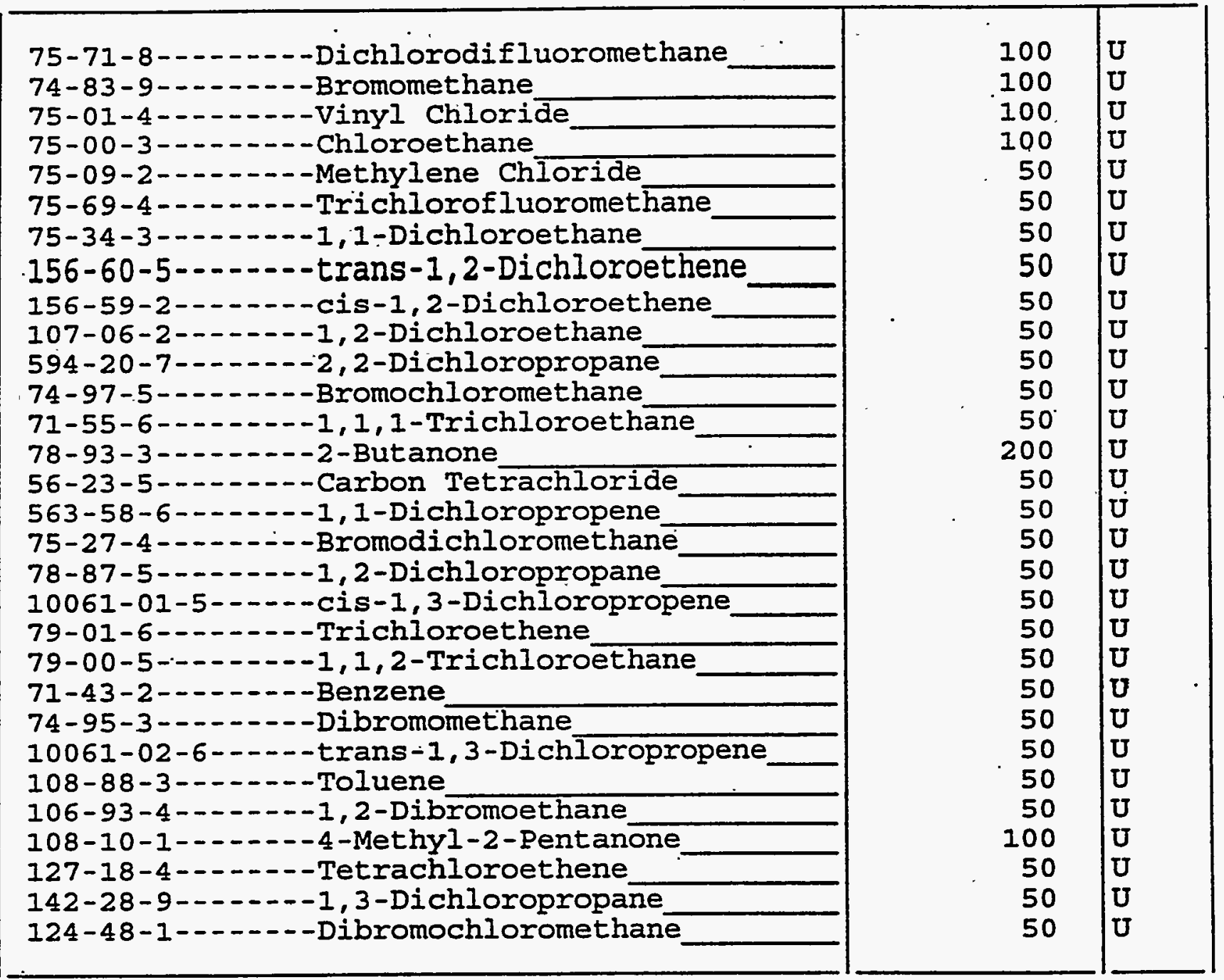


b Name:" QUUANTERRA MO

t Code: ITMO

trix: (soil/water) SOIL

ח_le wt/vol:

$1.00(\mathrm{~g} / \mathrm{mL}) \mathrm{G}$

$v-1: \quad$ (low/med) LOW

Moisture: not dec.

'olumn: RTX-502.2 ID: $0.530(\mathrm{~mm})$

il Extract Volume:

(UL)
Contract: $625-01$

SȦS No.:
SDG No.: V205

Lab Sample ID: 14533-026

Lab File ID: F0026.

Date Received: 05/06/97

Date Analyzed: 05/13/97

Dilution Factor: 1.0

Soil Aliquot Volume:

(UL)

CAS NO. COMPOUND (ug/I or ug/Kg) UG/KG

Q

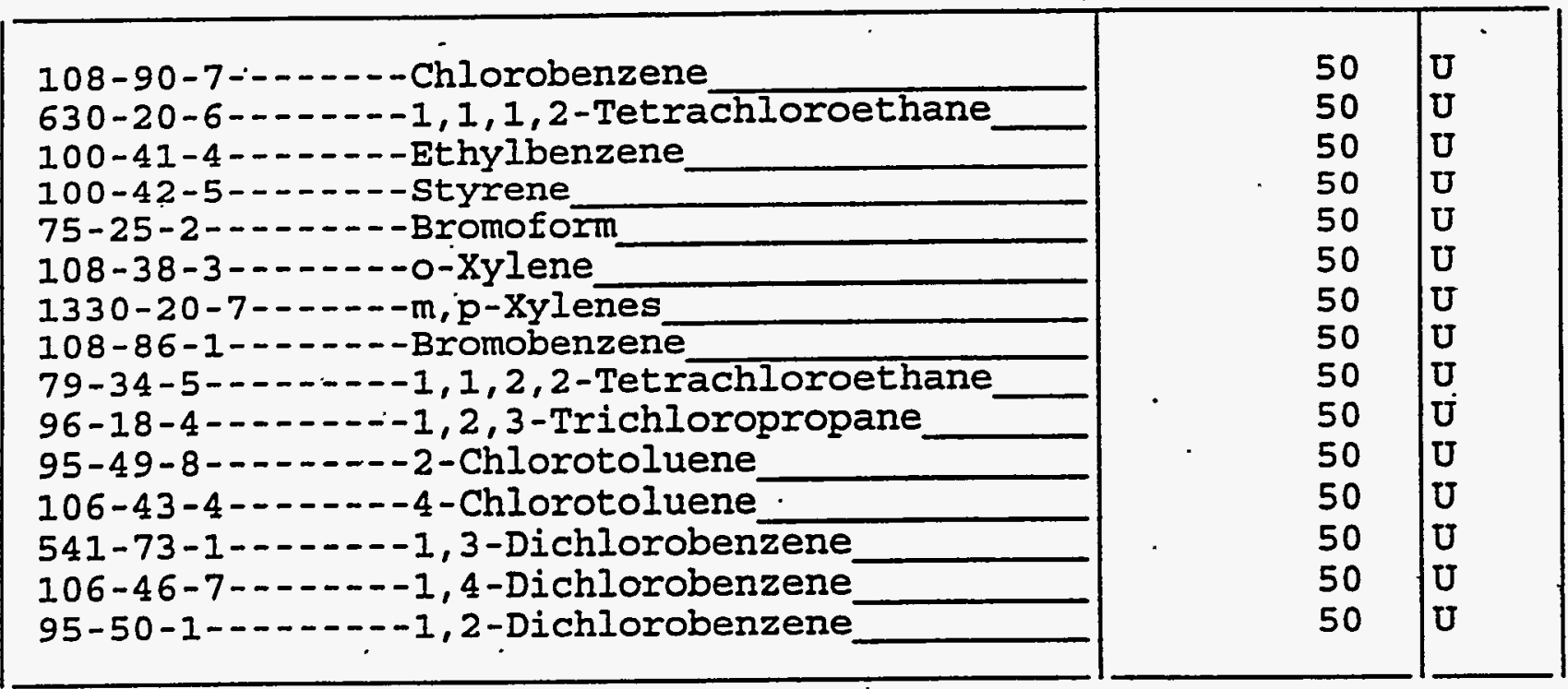


10

LBH ANALYSIS DATA SHEET
EPA SAYPLE NO. NSCEP-501

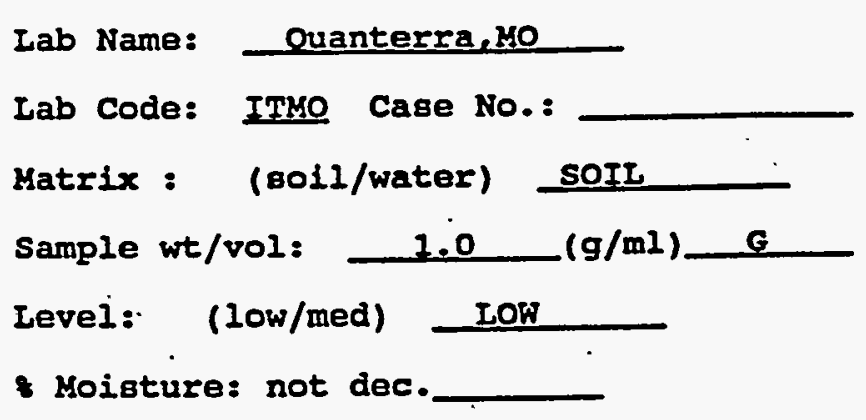

Lab code: ITMO Case No.: SAS NO.:

contract: 625.01

Iab Sample ID: 14533-037

Iab File ID:

Dàte sampled:

Date Analyzed: 05-13-97

Dilution Factors 1.0
CAS NO.

Compound
CONCENTRATION UNITS:

( $u g / L$ or $u g / R g$ ) UG/KG

$\mathbf{8}$

IBH (1)

500

v

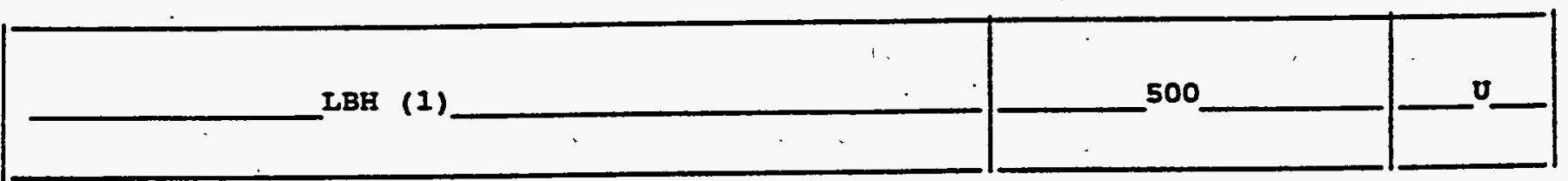

U:Concentration of analyte is less than the value given.

$x:$ Pattern reasonably matches gasoline

$Y$ : Pattern appears to be multi-peaks, but does not match gasoline

z:Concentration is primarily from a single peak

(1) Low Boiling Hydrocarbon (IBH) is quantitated as if It is Gasoline. 
EPA SAMPLE NO. NSCEP -501
Lab Name: OUANTERRA YO

Lab Code: ITMO Case NO.:

4 trix: (soil/water)

SOIL

s nple wt/vol: 20. $(g / m 1)$ G Level: (low/med) LOW

Hoisture: not dec. dec. SAS NO: :

contract: 625.01

Iab Sample ID:

$14533-037$

Iab File ID:

Daté Sampled:

$04-30-97$

Date Extracted:

$05-09-97$

Date Analyzed:

05-13-97

Dilution Factor: 1

CONCENTRATION UNITS:

CAS NO. Compound (mg/L or $\mathrm{mg} / \mathrm{kg}$ ) $\mathrm{mq} / \mathrm{kq}$

\begin{tabular}{|c|c|}
\hline 25 & 0 \\
\hline & \\
\hline & 0 \\
\hline
\end{tabular}

$u$ : Concentration of analyte is less than the value given. 
Lab Name:" QUUANTERRÁ MO

Lab Code: ITMO

Case No.: V53301

Matrix: (soil/water) SOIL .

Sample wt/vol:

$1.00(\mathrm{~g} / \mathrm{mL}) \mathrm{G}$

Level: (low/med) LOW

$\because$ Moisture: not dec.

GC Column: RTX-502.2 ID: $0.530^{\circ}(\mathrm{mm})$

Soil Extract Volume:

(ut)
Contract: $625-01$

SAS NO.:

SDG No.: V205

Lab Sample ID: 14533-037

Lab File ID: F0039

Date Received: 05/06/97

Date Analyzed: 05/13/97

Dilution Factor: $\quad 1.0$

Soil Aliquot Volume:

(uL:

CAS NO.

COMPOUND

CONCENTRATION UNITS:

(ug/I or $u g / \mathrm{Kg})$ UG/KG

75-71-8--...--Dichlorodifluoromethane

74-83-9-2.-.--Bromomethane

75-01-4-------vinyl Chloride

75-00-3-.-.---Chloroethane

75-09-2:-.---Methylene Chloride

75-69-4-..-2--Trichlorofluoromethane

75-34-3-------1,1-Dichloroethane

156-60-5--.---trans-1,2-Dichloroethene

156-59-2------cis-1,2-Dichloroethene

107-06-2------1,2-Dichloroethane

594-20-7--1---2,2-Dichloropropane

74-97-5-------Bromochloromethane

71-55-6------1, 1,1-Trichloroethane

78-93-3--.---2-Butanone

56-23-5-..---.-Carbon Tetrachloride

563-58-6-----1, 1-Dichloropropene

75-27-4-1--.---Bromodichloromethane

78-87-5-------1,2-Dichloropropane

10061-01-5-----cis-1,3-Dichloropropene

79-01-6--.-----Trichloroethene

79-00-5--.---1,1,2-Trichloroethane

71-43-2-...-.--Benzene

74-95-3-..---Dibromomethane

10061-02-6--.-trans-1, 3-Dichloropropene

108-88-3--.----Toluene

106-93-4--.---1,2-Dibromoethane

108-10-1--.---4-Methyl-2-Pentanone

127-18-4-------Tetrachloroethene

142-28-9-------1, 3-Dichloropropane

124-48-1--.---Dibromochloromethane

100

100

100

100

50

50

50

50

50

50

50

50

50

200

50

50

50

50

50

50

50

50

50

50

50

50

100

50

50

50

$Q$ 
b Name:"- QUANTERRA MO

b Code: ITMO

Case No.: V53301

trix: (soil/water) soII

$\pi_{\text {rele }}$ wt/vol:

$1.00(\mathrm{~g} / \mathrm{mL}) \mathrm{G}$

v 1: (low/med) LOW

Moisture: not dec.

olumn: RTX-502.2 ID: 0.530 (mm)

il Extract Volume:

(UL)
Contract: $625-01$

SAS NO.:
NSCEP -501

SDG No.: V205

Lab Sample ID: 14533-037

Lab File ID: F0039

Date Received: 05/06/97

Date Analyzed: 05/13/97

Dilution Factor: $\quad 1.0$

Soil Aliquot Volume:

(UI)

CAS NO.

COMPOUND

(ug/L or $\mathrm{ug} / \mathrm{Kg}$ ) UG/KG

\begin{tabular}{|c|}
\hline 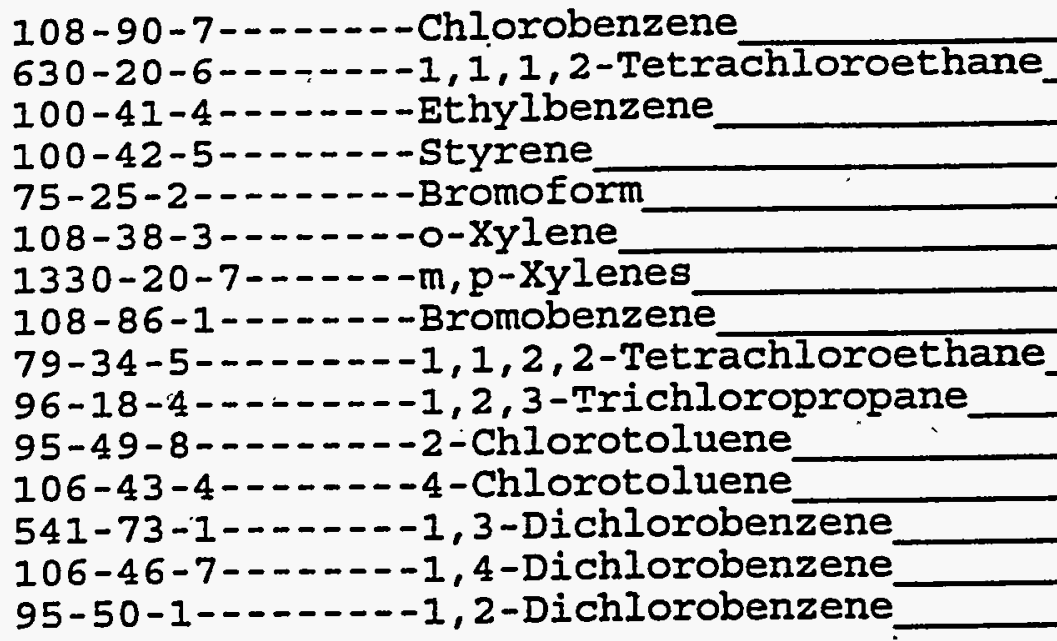 \\
\hline
\end{tabular}

\begin{tabular}{|c|c|c|}
\hline$\cdot$ & $\begin{array}{l}50 \\
50 \\
50 \\
50 \\
50 \\
50 \\
50 \\
50 \\
50 \\
50 \\
50 \\
50 \\
50 \\
50 \\
50\end{array}$ & $\begin{array}{l}U \\
U \\
U \\
U \\
U \\
U \\
U \\
U \\
U \\
U \\
U \\
U \\
U \\
U \\
U \\
U\end{array}$ \\
\hline
\end{tabular}


1D

IBH ANAIYSIS DATA SHEET
PPA SAMPLE NO. :NSCHP-505
Lab Name: Quanterra, no

Iab code: ITMO Case No.:

Matrix: (Boil/water) sorI

Sample wt/vol:

1.0 $(g / m 1)$

G

Level: (low/med) IOH

\& Moisture: not dec.

(

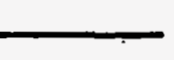

CAS NO.

$$
\text { Compound }
$$

IBH (1)
Contract: 625.01

SAS NO.:

SDG HO.: V205

Iab Sample ID: 14533-038

Iab File ID:

Date sampled: $\quad 04-30-97$

Date Analyzed: 05-13-97

Dilution Factor: 1,0

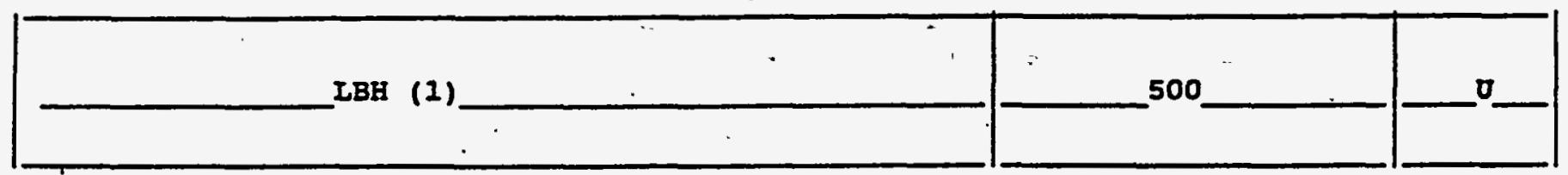

$U$ : Concentration of analyte 28 less than the value given.

X:Pattern reasonably matches gasoline

Y:Pattern appears to be multi-peaks, but does not match gasoline

z:Concentration is primarily from a single peak

(1):Low Boiling Hydrocarbon (IBE) is quantitated as if it ig Gasoline. 
NSCEP -505

Lab Name: 'QUANTERRA' MO

Iab Code: ITMO

Case No.: V53301

Matrix: (soil/water) soIL

Sample wt/vol:

$1.00(\mathrm{~g} / \mathrm{mL}) \mathrm{G}$

Level : (low/med) LOW

\% Moistūre: not dec.

GC Column: RTX-502.2 ID: 0.530 (mm)

Soil Extract Volume:

(uL).
Contract: $625-01$

SAS NO.:

SDG NO.: V205

Lab Sample ID: 14533-038

Lab File ID: G6225

Date Received: 05/06/97

Date Analyzed: 05/14/97

Dilution Factor: $\quad 1.0$

Soil Aliquot Volume:

(uL)

CONCENTRATION UNITS:

CAS NO. COMPOUND

(ug/L or ug/Kg) UG/KG

Q

75-71-8--..--Dichlorodifluoromethane

74-83-9--.-.---Bromomethane

75-01-4-....-.-Vinyl Chloride

75-00-3-...-.--Chloroethane

75-09-2-....--.-Methylene Chloride

75-69-4-....-.-Trichlorofluoromethane

75-34-3--.--1,1-Dichloroethane

156-60-5----.--trans-1,2-Dichloroethene

156-59-2--.---Cis-1, 2-Dichloroethene

107-06-2--.--1, 2-Dichloroethane

594-20-7-...--2,2-Dichloropropane

74-97-5-1.-----Bromochloromethane

71-55-6--.---1,1,1-Trichloroethane

78-93-3--.----2-Butanone

56-23-5--.----Carbon Tetrachloride

563-58-6--.--1, 1-Dichloropropene

75-27-4-...---Bromodichloromethane

78-87-5------1, 2-Dichloropropane

10061-01-5---.--cis-1,3-Dichloropropene

79-01-6-...--Trichloroethene

79-00-5----.--1, 1,2-Trichloroethane

$71-43-2--.----$ Benzene

74-95-3--..--Dibromomethane

10061-02-6-----trans-1,3-Dichloropropene

108-88-3-...-.-Toluene

106-93-4--.---1,2-Dibromoethane

127-18-4--.-.--Tetrachloroethene

142-28-9--.--1,3-Dichloropropane

124-48-1-2.---Dibromochloromethane

108-90-7-...--Chlorobenzene

\begin{tabular}{|l|l|}
50 & $U$ \\
50 & $U$ \\
50 & $U$ \\
50 & $U$ \\
25 & $U$ \\
25 & $U$ \\
25 & $U$ \\
25 & $U$ \\
25 & $U$ \\
25 & $U$ \\
25 & $U$ \\
25 & $U$ \\
25 \\
100 \\
25 & $U$ \\
25 & $U$ \\
25 & $U$ \\
25 & $U$ \\
25 & $U$ \\
25 & $U$ \\
25 & $U$ \\
25 & $U$ \\
25 & $U$ \\
25 & $U$ \\
25 \\
25 & $U$ \\
25 & $U$ \\
25 & $U$ \\
25 & $U$ \\
25 & $U$ \\
& \\
\hline
\end{tabular}


つ Name:- QUUANTERRA MO

= Code: ITMO . Case No.: V5330I =rix: (soil/water) SOIL

r lie wt/vol:

$1.00(\mathrm{~g} / \mathrm{mL}) \mathrm{G}$

1: (low/med) LOW

voisture: not dec.

Olumn: RTX-502.2 ID: 0.530 (mm)

il Extract Volume:

(UL)

Contract : $625-01$

SAS NO.:
NSCEP-505

SDG NO.: V205

Lab Sample ID: 14533-038

Lab File ID: G6225

Date Received: 05/06/97

Date Analyzed: 05/14/97

Dilution Factor: $\quad 1.0$

Soil Aliquot Volume:

(UI)

CONCENTRATION UNITS :

CAS 'NO.

COMPOUND

(ug/L or $\mathrm{ug} / \mathrm{Kg}$ ) UG/KG

Q

630-20-6--.--1,1,1,2-Tetrachloroethane

100-41-4-.---.-Ethylbenzene

100-42-5-------Styrene

75-25-2-...--Bromoform

108-38-3--.---o-Xylene

$1330-20-7-\ldots-\ldots-\mathrm{m}, \mathrm{p}-\mathrm{xy}$ lenes

108-86-1-1----Bromobenzene

79-34-5-...--1, 1,2,2-Tetrachloroethane

96-18-4-..--.-1, 2,3-Trichloropropane

95-49-8-...--2-Chlorotoluene

106-43-4-..--4-Chlorotoluene

541-73-1-..---1, 3-Dichlorobenzene

106-46-7------1,4-Dichlorobenzene

95-50-1-...-1,2-Dichlorobenzene 


\section{VOLUME 2 APPENDIX B}

TABLE 9 ANALYTICAL DATA 
TABLE 9: NSCEP SPILL-H910911D VERIFICATION RESULTS

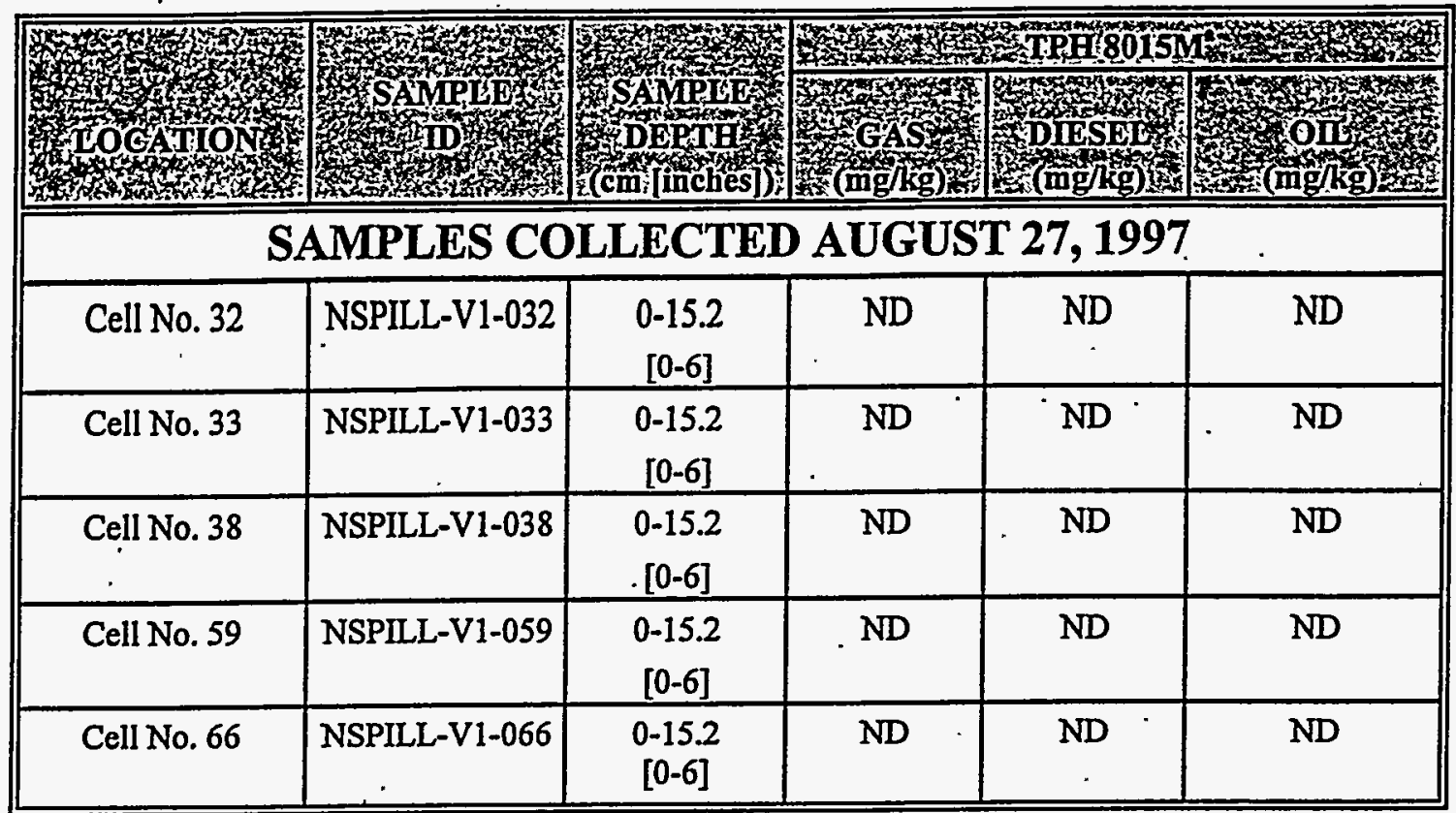

Notes:

^TPH 8015M: Total Petroleum Hydrocarbons Method 8015 Modified.

SCEP: Steam Cleaning Effluent Pond

ND: Not Detected

cm: centimeter

mg/kg: milligrams per kilogram 


\section{; LABORATORIES}

\section{$i$, PETROLEUM HYDROCARBONS (TPH)}

M - TPH

\begin{tabular}{ll}
\hline :lient Sample ID: & .NSPILL-V1-032 \\
late Collected: & $27-$ AUG -97 \\
Date Analyzed: & $19-$ SEP-97 11:48 \\
Jate Extracted: & $06-$ SEP-97 \\
latrix: & Soil \\
fercent Moisture: & 11.64
\end{tabular}

$\begin{array}{ll}\text { IAS Sample ID: } & \text { I10395-68 } \\ \text { Date Received: } & 28-A U G-97 \\ \text { Analytical Batch ID: } & 09179.7-8015-D-1 \\ \text { Analytical Dilution: } & 1 \\ \text { Preparation Dilution: } & 1.0 \\ \text { QC Group: } & \text { 8015M - TPH_53029 }\end{array}$

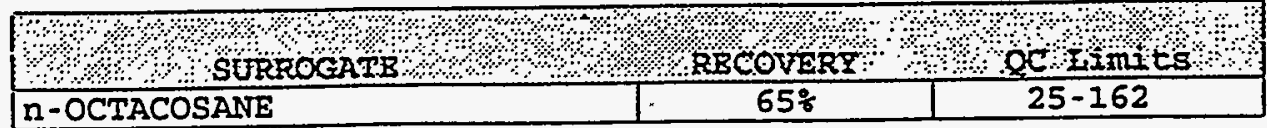

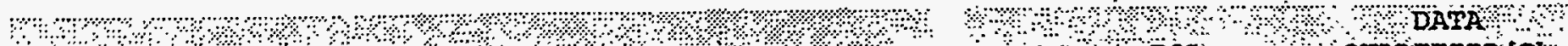
m l

PSS $10 \%$ RESUET QUATIPIER: $\{S$

:- Range Organics

Jine Range Organics

J OIL
TPH

$<34$.

$<34$.

$<34$.
34.

34 .

34 . 


\section{LAS LABORATORIES}

TOTAL PETROLEUM HYDROCARBONS (TPH) $8015 \mathrm{M}$ - TPH

Client Sample ID:

Date Collected:

Date Analyzed:"

Date Extracted:

Matrix:

Percent Moisture:

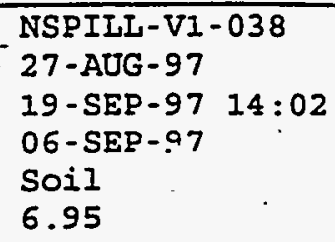

NSPILL-VI-038

27-AUG-97

19-SEP-97 14:02

06-SEP-.97

Soil

6.95

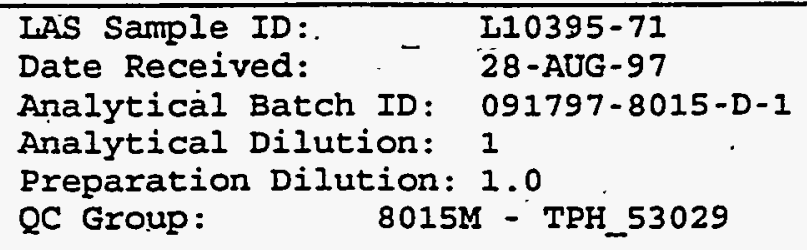

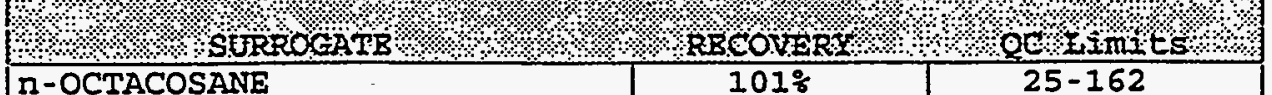

Diesel Range Organics Gasoline Range Organics MOTOR OIL

$\begin{array}{lll}\text { TPH } & <32 . & 32 . \\ & <32 . & 32 . \\ . & <32 . & 32 .\end{array}$


, PETROLEUM HYDROCARBONS (TPH)

1. - TPH

\begin{tabular}{llll}
\hline Tlient Sample ID: & NSPIIL-VI-033 & IAS Sample ID: & I10395-67 \\
late Collected: & $27-$ AUG-97 & Date Received: & $28-A U G-97$ \\
Date Analyzed: & $19-$ SEP-97 11:03 & Analytical Batch ID: $091797-8015-D-1$ \\
Date Extracted: & $06-$ SEP-97 & Analytical Dilution: 1 \\
latrix: & Soil & Preparation Dilution: 1.0 \\
'ercent Moisture: & 8.5 & QC Group: & $8015 M-$ TPH_53029 \\
\hline
\end{tabular}

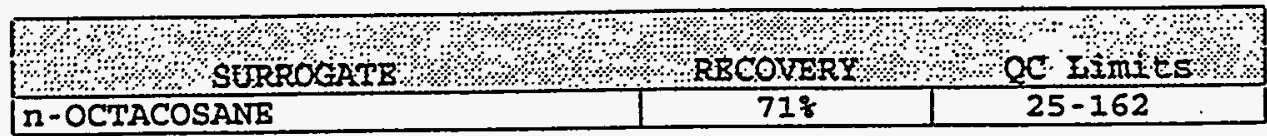
m lun ll

1. Range Organics II ine Range Organics IR OII
TPH

$\begin{array}{ll}<33 . & 33 . \\ <33 . & 33 . \\ <33 . & 33 .\end{array}$




\section{LAS LABORATORIES}

TOTAL PETROLEUM HYDROCARBONS (TPH)

$8015 \mathrm{M}-\mathrm{TPH}$

Client Sample ID: NSPILL-V1-059

IAS Sample ID:

L10395-69

Date Collected:

Date Received:

28-AUG-97

Date Analyzed:

27-AUG-97

Date Extracted:

19-SEP-97 $12: 33$

Analytical Batch ID:

$091797-8015-D-1$

Matrix:

06-SEP-97

Analytical Dilution: 1

Percent Moisture:

Soil

Preparation Dilution: 0.99

6.5

QC Group:

8015M - TPH_53029

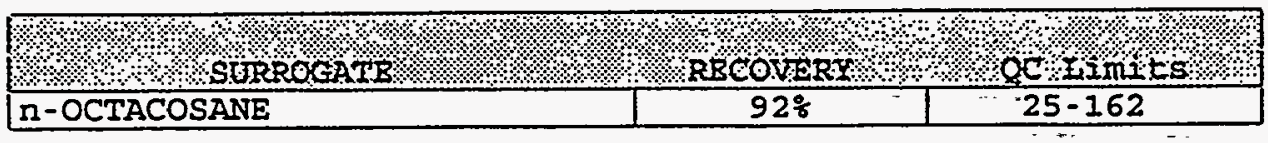

Diesel Range Órganics Gasoline Range Organics MOTOR OIL
TPH

$<32$.

$<32$.

$<32$.
32 .

32 .

32 .

\begin{tabular}{llll}
\hline LJ7682STANDARD & N & R20473 & Page 1
\end{tabular}




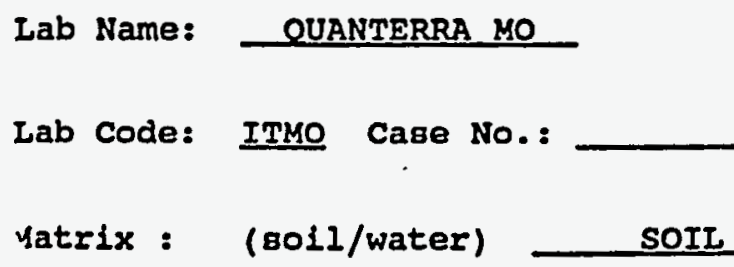
dec. Extraction: (SepF/Cont/Sonc/Shak) SHAK IPC Cleanup: $(\mathrm{Y} / \mathrm{N})+\mathrm{N} \quad \mathrm{pH}:$

Contract: SAS NO.: SDG No.: V205

Lab Sample ID: $14533-029$ Lab File ID: Date Sampled: . $04-30-97$

Date Analyzed: $\quad 05-13-97$

Dilution Factor: 1 CONCENTRATION UNITS: (mg/L or $\mathrm{mg} / \mathrm{Kg}$ ) $\mathrm{mg} / \mathrm{kg}$ 8

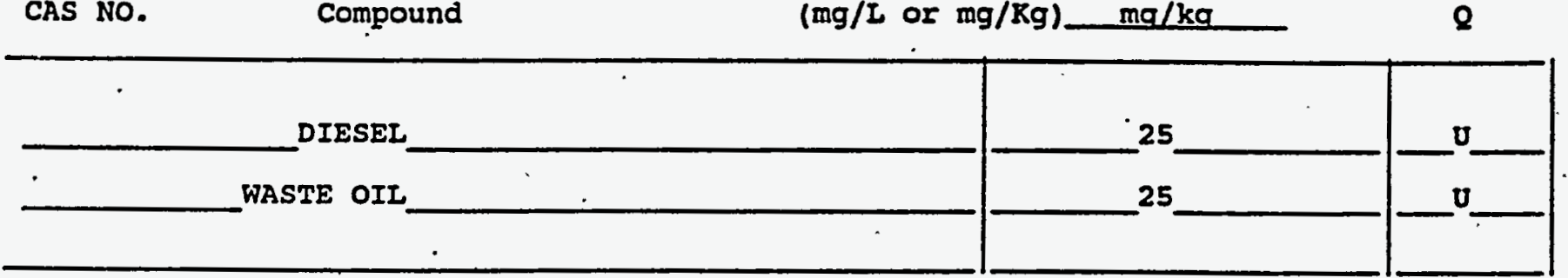

U: Concentration of analyte is less than the value given. 
Lab Name: “Q̈UANTERRȦ MO

Lab Code: ITMO

Case No.: V53301

Matrix: (soil/water) SOIL

Sample wt/vol:

$1.00(\mathrm{~g} / \mathrm{mL}) \mathrm{G}$

Level: (low/med) LOW

$\%$ Moisture: not dec.

GC Column: RTX-502.2 ID: 0.530 (mm)

Soil Extract Volume:

CAS NO.
Contract : 625-01

SAS NO.:

NSCEP -135

SDG NO.: V205

Lab Sample ID: 14533-029

Lab File ID: E8567.

Date Received: 05/06/97

Date Analyzed: 05/13/97

Dilution Factor: $\quad 1.0$

Soil Aliquot Volume:

(UL)

CONCENTRATION UNITS :

(ug/L or ug/Kg) UG/KG

Q

75-71-8-..--DDichlorodifluoromethane

74-83-9-1.---Bromomethane

75-01-4-....--vinyl Chloride

75-00-3-...--Chloroethane

75-09-2-...--- Methylene Chloride

75-69-4-....- Trichlorofluoromethane

75-34-3-..----1, 1-Dichloroethane

156-60-5------trans-1,2-Dichloroethene

156-59-2----.-cis-1;2-Dichloroethene

107-06-2-.---1, 2-Dichloroethane

594-20-7-n---2,2-Dichloropropane

74-97-5-.-----Bromochloromethane

71-55-6------1, 1, 1-Trichloroethane

78-93-3--.---.-2-Butanone

56-23-5--.-.--Carbon Tetrachloride

563-58-6-..--1,1-Dichloropropene

75-27-4-...----Bromodichloromethane

78-87-5-.-.--1, 2-Dichloropropane

10061-01-5-----cis-1,3-Dichloropropene

79-01-6-...--Trichloroethene

79-00-5-...-.-1, 1, 2-Trichloroethane

$71-43-2-----.--B e n z e n e$

74-95-3-..---Dibromomethane

10061-02-6------trans-1, 3-Dichloropropene

108-88-3-..---Toluene

106-93-4--.--1,2-Dibromoethane

127-18-4-.--- Tetrachloroethene

142-28-9-.----1,3-Dichloropropane

124-48-1------Dibromochloromethane

108-90-7-.----Chlorobenzene

\section{0}

50

50

50

10

25

25

25

25

25

25

25

25

100

25

25

25

25

25

25

25

25

25

25

25

25

25

25

25

25 
b Name:" QUUANTERRA MO "

j Code : ITMO

Case No.: V53301

trix: (soil/water) SOIL

i sle wt/vol:

$1.00(\mathrm{~g} / \mathrm{mL}) \mathrm{G}$

ril: (Iow/med) LOW

Moisture: not dec.

: :olúmn: RTX-502.2 ID: 0.530 (mm)

iI Extract Volume:

(UL)
Contract: $625-01$

SAS NO.:

NSCEP-135

SDG No.: V205

Lab Sample ID: 14533-029

Lab File ID: $\quad$ E8567

Date Received: 05/06/97

Date Analyzed: 05/13/97

Dilution Factor: $\quad 1.0$

Soil Aliquot Volume:

(uL)
CAS NO.
COMPOUND
( $(\mathrm{ug} / \mathrm{L}$ or $\mathrm{ug} / \mathrm{Kg}$ ) UG/KG
Q

CONCENTRATION UNITS:

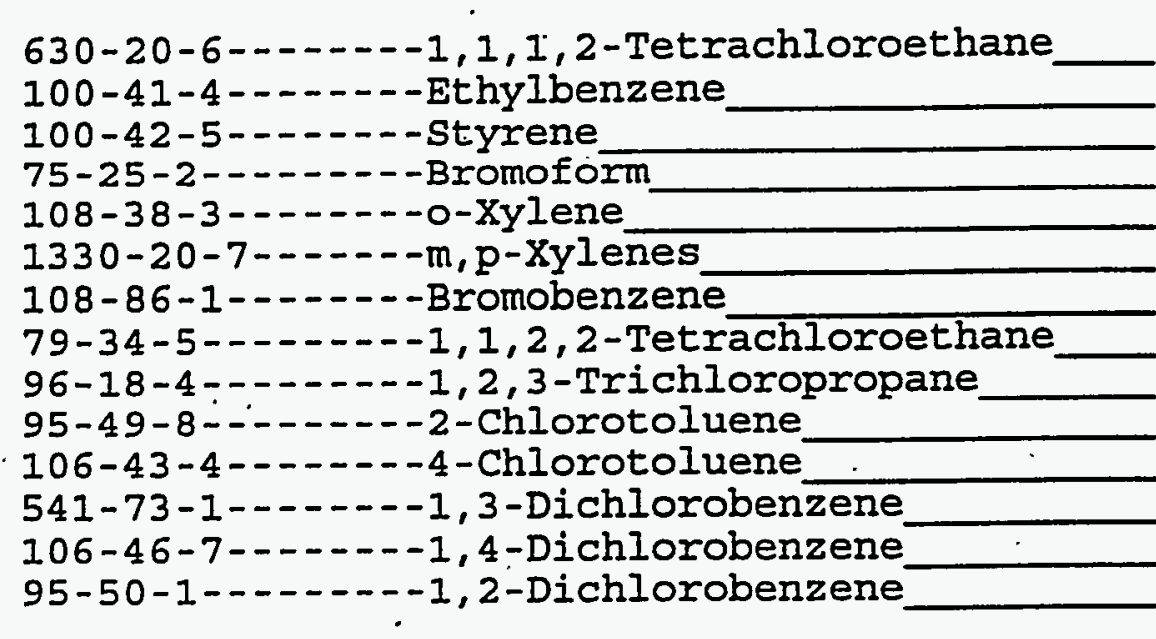


10

IBH ANALYSIS DATA SHEET
EPA SAYPTE NO. NSCSP -140
Lab Name: Quanterfa, Mo

Lab code: ITYO Case No.:

Matrix : (soil/water) soIL

Sample wt/vol: $1.0 \quad(\mathrm{~g} / \mathrm{ml})$ G

Level: (low/med). IOW

roisture: not dec.

Compound

IBH

(1)

$\cdot$

contract: $\quad 625,01$

SAS NO.:

SDG No.: V205

Lab Sample ID: 14533-028

Lab File ID:

Date Sampled: 04-30-97

Date Analyzed: 05-12-97

Dilution Factor: 1.0
CONCENTRATION UNITS:

(ug/L or ug/Kg) UG/RG

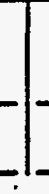

U: Concentration of analyte is less than the value given.

$X:$ Pattern reasonably matches gasoline

Y: Pattern appears to be multi-peaks, but does not match gasoline

z: Concentration is primarily from a single peak

(1): Low Boiling Hydrocarbon (LBA) is quantitated as if it is Gasoline. 
1D

HBH ANAIYSIS DATA SHEET
EPA SAMPLE NO. NSCEP-140
Lab Name: QUANTERRA MO

Lab Code: ITMO Case No.:

atrix : (soil/water)

SOIL

ample wt/vol:

$20.1 \quad(\mathrm{~g} / \mathrm{ml})$

G

Level: (low/med)

LOW

* Moisture: not dec. dec.

xtraction: (SepF/Cont/Sonc/Shak) SHAK

PC Cleanup: $(Y / N)-N \quad \mathrm{~N}$ -

CAS NO.

Compound
Contract:

SAS NO.:

SDG No.: U205

Lab Sample ID:

14533-028

Lab File ID:

Date Sampled:

$04-30-97$

Date Extracted:

$05-09-97$

Date Analyzed:

05-13-97

Dilution Factor:

1

CONCENTRATION UNITS:

(mg/L or $\mathrm{mg} / \mathrm{kg}$ ) $\mathrm{mg} / \mathrm{kg}$

2

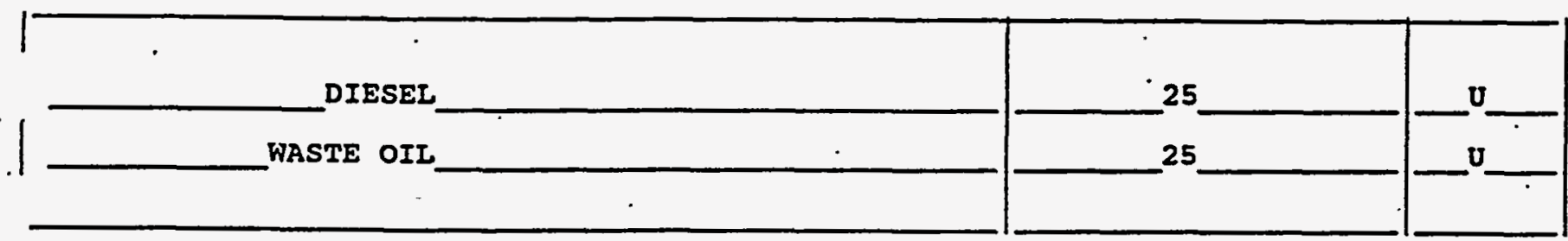

U: Concentration of analyte is less than the value given. 
$1 \mathrm{~A}$

VOLATILE ORGANICS ANALYSIS DATA SHEET
Lab Name: "QUUANTERRA' MO

Lab Code: ITMO

Case No.: V53301

Matrix: (soil/water) soIL

Sample wt/vol:

$1.00(\mathrm{~g} / \mathrm{mL}) \mathrm{G}$

Level: (low/med) LOW

$\%$ Moisture: not dec.

GC Column: RTX-502.2 ID: $0.530(\mathrm{~mm})$

Soil Extract Volume:
Contract: $625-01$

SAS NO. :

SDG NO.: V205

Lab Sample ID: 14533-028

Lab File ID: $\quad$ E8566

Date Received: $05 / 06 / 97$

Date Analyzed: $05 / 13 / 97$

Dilution Factor: $\quad 1.0$

(uL) Soil Aliquot Volume:

(uL)

CONCENTRATION UNITS:

CAS NO. COMPOUND (ug/I or $\mathrm{ug} / \mathrm{Kg}$ ) UG/KG

Q

\begin{tabular}{|c|}
\hline 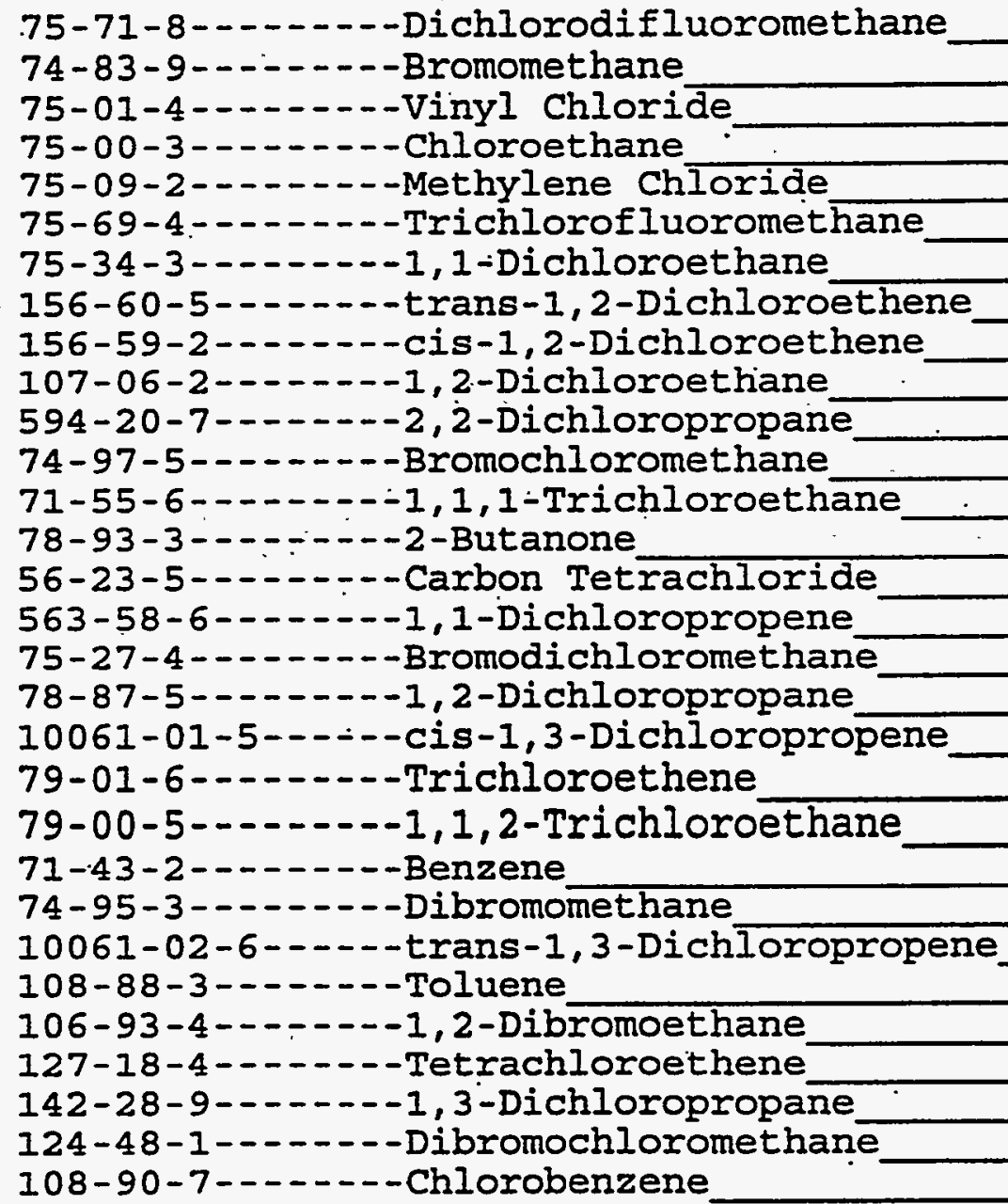 \\
\hline
\end{tabular}

\begin{tabular}{l|l|}
50 & $U$ \\
50 & $U$ \\
50 & $U$ \\
50 & $U$ \\
11 & $B J$ \\
25 & $U$ \\
25 & $U$ \\
25 & $U$ \\
25 & $U$ \\
25 & $U$ \\
25 & $U$ \\
25 & $U$ \\
25 & $U$ \\
100 & $U$ \\
25 & $U$ \\
25 & $U$ \\
25 & $U$ \\
25 & $U$ \\
25 & $U$ \\
25 & $U$ \\
25 & $U$ \\
25 & $U$ \\
25 & $U$ \\
25 & $U$ \\
25 & $U$ \\
25 & $U$ \\
25 & $U$ \\
25 & $U$ \\
25 & $U$ \\
25 & $U$ \\
& \\
\hline 5
\end{tabular}


au Name: "QÚANTERRA MO"

$\triangleq$, Code: ITMO
Case No.: V53301

Contract: $625-01$

SAS NO.:

NSCEP -140

SDG No.: V205

atrix: (soil/water) soIL

$\equiv$ ple wt/vol:

$1.00(\mathrm{~g} / \mathrm{mL}) \mathrm{G}$

evel: (low $/ \mathrm{med})$ LOW

'soisture: not dec.

こ Column: $\operatorname{RTX}-502.2$ ID: 0.530 (mm)

sil Extract Volume:

CAS NO.
(uL)
Lab Sample ID: 14533-028

Iab File ID: $\quad$ E8566

Date Received: 05/06/97

Date Analyzed: 05/13/97

Dilution Factor: $\quad 1.0$

Soil Aliquot Volume:

(uL)
CONCENTRATION UNITS :

(ug/L or $\mathrm{ug} / \mathrm{Kg}$ ) UG/KG.
630-20-6-...--1,1,1,2-Tetrachloroethane 100-41-4---.--Ethylbenzene

100-42-5-------Styrene

75-25-2-...--Bromoform

108-38-3----.--0-Xylene

$1330-20-7-\cdots-\cdots, p-x y l e n e s$

108-86-1-.----Bromobenzene

79-34-5-- - - - 1, 1, 2, 2-Tetrachloroethane

96.-18-4-..--1,2, 3-Trichloropropane

95-49-8-...--2-Chlorotoluene

106-43-4------4-Chlorotoluene

541-73-1-..--1, 3-Dichlorobenzene

106-46-7------1,4-Dichlorobenzene

95-50-1-_.--1,2-Dichlorobenzene
25.

25

25

25

25

25

25

25

25

25

25

25

25

25

Q

\begin{tabular}{l|l|l}
\multicolumn{2}{c}{ Q } \\
\hline 5. & $U$ \\
5 & $U$ \\
5 & $U$ \\
5 & $U$ \\
5 & $U$ \\
5 & $U$ \\
5 & $U$ \\
5 & $U$ \\
5 & $U$ \\
5 & $U$ \\
5 & $U$ \\
5 & $U$ \\
5 & $U$ \\
5 & $U$ \\
\end{tabular}


Lab Name: Quanterra, wo
Lab Code: ITYO Case No.:

Matrix : (soil/water) SOIL

Sample wt/vol:

1.0 $(\mathrm{g} / \mathrm{ml})$

Level: (low/med) IOW

1 Moigture: not dec.

CAS NO.

Compound

LBH (1)
Contract: $\quad 625.01$

SAS NO.: SDG KO.: V205

Iab Sample ID: 14533-030

Lab File ID:

Date Sampled:

$04-30-97$

Date Analyzeds 05-13-97

Dilution Factor: 1,0

CONCENTRATION ORITS:

(ug/L or ug/Rg) UG/RG

$\mathbf{2}$

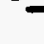

U: Concentration of analyte is 1888 than the value given. $\mathrm{X}$ : Pattern reasonably matches gasoline Y:Pattern appears to be multi-peaks, but does not match gasoline $\mathrm{z}$ : Concentration is primarily from a single peak

(1):Low Boiling Hydrocarbon (LBH) is quantitated as if it is Gasoline. 


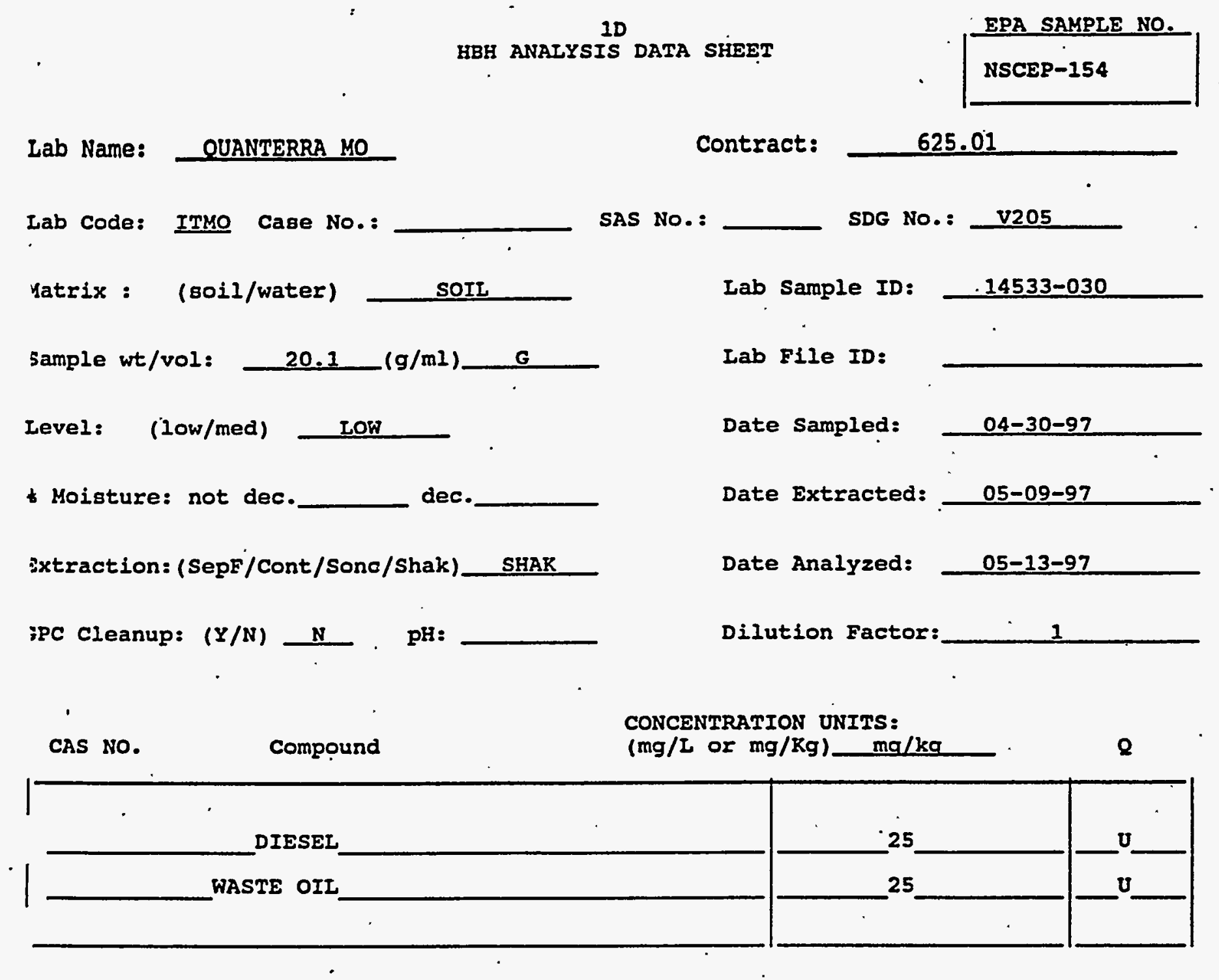

U: Concentration of analyte is less than the value given. 
Lab Name:" "QUAANTERRA” MO

Lab Code: ITMO

. Case No.: V53301

Matrix: (soil/water) SOIL

Sample wt/vol:

$1.00(\mathrm{~g} / \mathrm{mL}) \mathrm{G}$

Level: (low/med) Low

\% Moisture: not dec.

GC Column: RTX-502.2 ID: 0.530 (mm)

Soil Extract Volume:

(uL)
Contract: $625-01$

SÁS NO.:

NSCEP-154

SDG No.: V205

Lab Sample ID: 14533-030

Lab File ID: $\quad$ E8568

Date Received: 05/06/97

Date Analyzed: 05/13/97

Dilution Factor: $\quad 1.0$

- Soil Aliquot Volume:

(UL)

CONCENTRATION UNITS:

CAS NO. COMPOUND

(ug/L or ug/Kg) UG/KG

Q

75-71-8-.---Dichlorodifluoromethane

74-83-9--.-----Bromomethane

75-01-4-..----Vinyl Chloride

75-00-3-.-.---Chloroethane

75-09-2--.----Methylene Chloride

75-69-4-...-- Trichlorofluoromethane

75-34-3-..--1, 1-Dichloroethane

156-60-5-------trans-1,2-Dichloroethene.

156-59-2-...-..cis-1, 2-Dichloroethene

107-06-2--.---1,2-Dichloroethane

594-20-7--.---2,2-Dichloropropane

74-97-5--------Bromochloromethane

71-55-6-....-1, 1, 1-Trichloroethane

78-93-3-..--.-2-Butanone

56-23-5-...---Carbon Tetrachloride

563-58-6------1, 1-Dichloropropene

75-27-4-1------Bromodichloromethane

78-87-5--.---1, 2-Dichloropropane

10061-01-5-----cis-1,3-Dichloropropene

79-01-6--.-.--Trichloroethene

79-00-5--.---1, 1,2-Trichloroethane

71-43-2--.-----Benzene

74-95-3-..--Dibromomethane

10061-02-6-----trans-1,3-Dichloropropene

108-88-3-.-.-.-Toluene

106-93-4------1,2-Dibromoethane

127-18-4--.---Tetrachloroethene

142-28-9-..---1, 3-Dichloropropane

124-48-1--.---Dibromochloromethane

108-90-7--.---Chlorobenzene

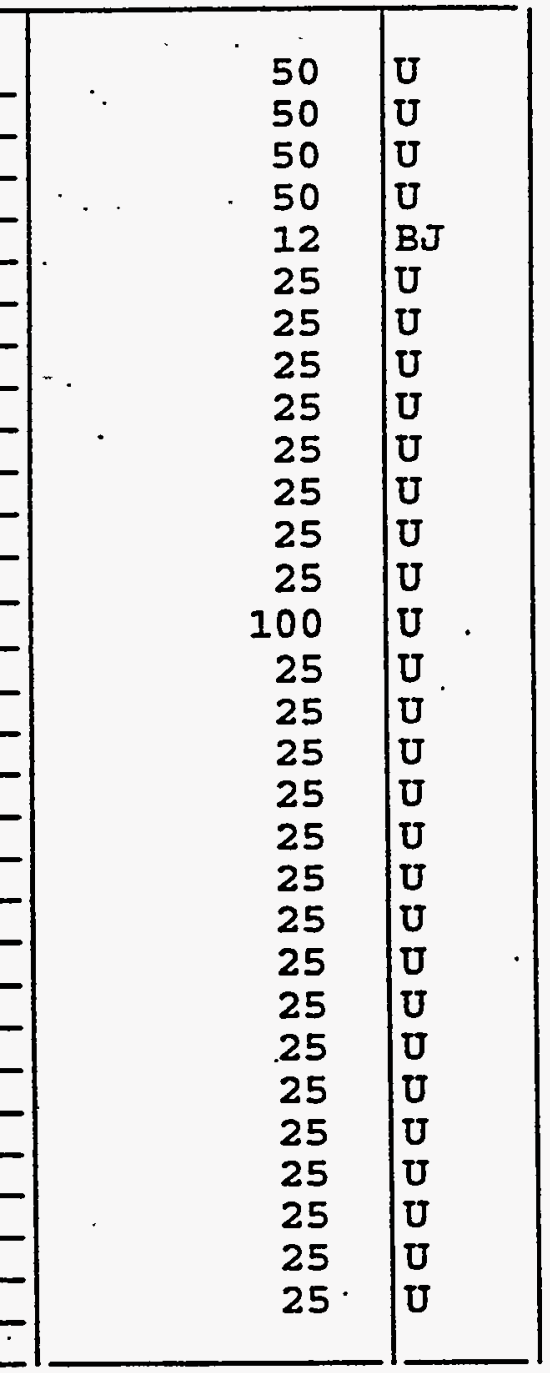




\section{VOLUME 2 APPENDIX B}

NORTH \& SOUTH SCEP NUCLEAR DENSITY DATA REPORT 


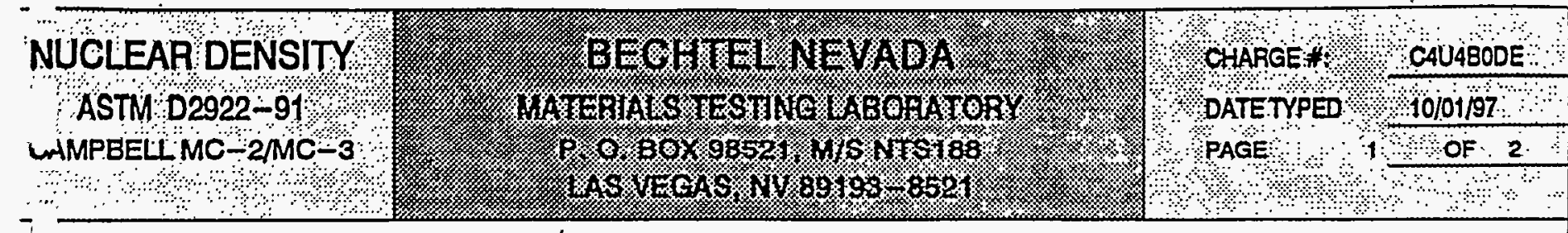

\begin{tabular}{l} 
Eyulested by \\
$n$ jet \\
\hline A/6 SCEPS
\end{tabular}
isted by D. HERRINGTON Date Tested 10/01/97 Checked by V. Whumdeal if mation transmitted to D. TOBIASON By D. HERRINGTON How COPY Date 10/01/97

\begin{tabular}{|c|c|c|c|c|c|c|}
\hline ABORATORYNO & 1 & 2 & 3 & 4 & 5 & N/A \\
\hline E ГLOCATION & 1 & 2 & 3 & 4 & 5 & \\
\hline EPTH OF PROBE & $10^{n}$ & $10^{n}$ & $10^{n}$ & $10^{n}$ & $10^{n}$ & \\
\hline E TH OF TESTS (BELOW SUBGRADE) & Subgrade & Subgrade & Subgrade & Subgrade & Subgrade & \\
\hline RY DENSITY-PCF & 91.8 & 94.1 & 96.0 & 96.6 & 95.3 & \\
\hline II STURE \% & 7.7 & 7.7 & 8.3 & 7.4 & 6.8 & \\
\hline Un DENSITY PCF & 104.0 & 104.0 & 104.0 & 104.0 & 104.0 & \\
\hline FTIMUM MOISTURE \% & 21.1 & 21.1 & 21.1 & 21.1 & 21.1 & \\
\hline E CENT COMPACTION & 88.3 & 90.5 & 92.3 & 92.9 & $91: 6$ & \\
\hline EQUIRED COMPACTION \% & 85.0 & 85.0 & 85.0 & 85.0 & 85.0 & \\
\hline JUT of SPECIFICATION & WITHIN & WITHIN & WITHIN & WITHIN & WITHIN & \\
\hline \multirow[t]{2}{*}{ AUGENO } & ANDARDIZATION & \multirow{2}{*}{\multicolumn{2}{|c|}{$10 / 01 / 97$}} & & VALUE OF $M$ & 647 \\
\hline & & & & STANDA & DIZATION D & 3070 \\
\hline
\end{tabular}

E ARKS: NATTVE PROCTOR FROM 04/03/89 USED TEST AREAS DESIGNATED BY D. TOBIASON VATIVE MATERIAL CLOSE TO THE PONDS WAS USED FOR FILIING TOP $12^{n}$
CC: J.SMITH BSTESTEL D. TOBIASON BECHTEL MTL BECHTEL FILES 


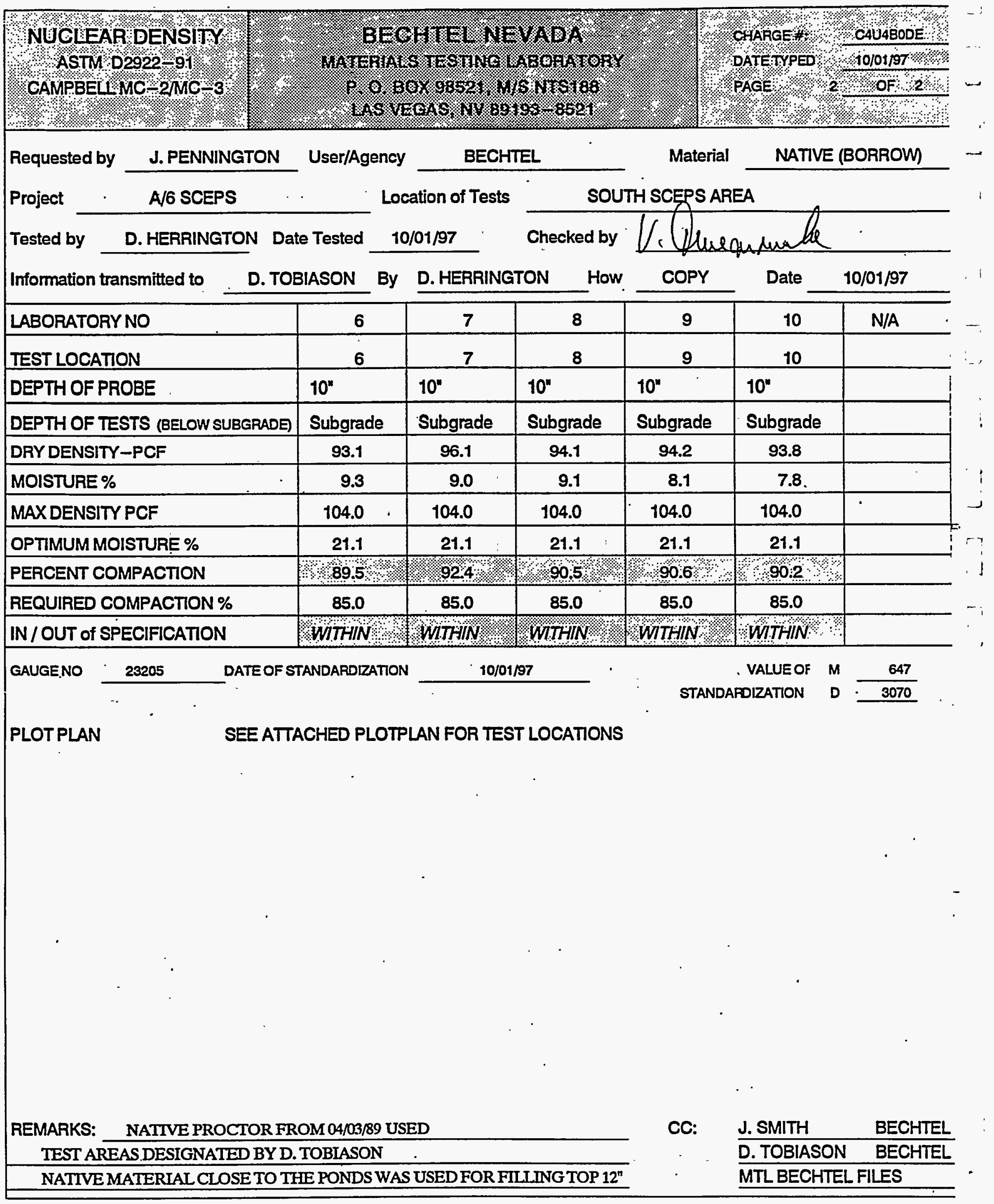


